\title{
Tethered Counterion-Directed Catalysis: Merging the Chiral Ion-pairing and Bifunctional Ligand Strategies in Enantioselective Gold(I) Catalysis
}

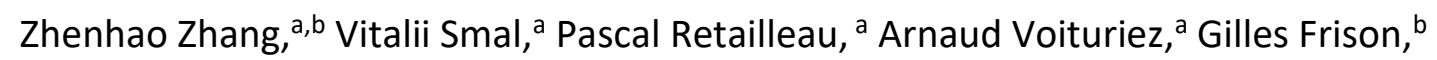 \\ Angela Marinetti, ${ }^{*, a}$ Xavier Guinchard*,a \\ a Université Paris-Saclay, CNRS, Institut de Chimie des Substances Naturelles, UPR 2301, 91198, Gif-sur- \\ Yvette, France. \\ ${ }^{\mathrm{b}}$ LCM, CNRS, Ecole Polytechnique, Institut Polytechnique de Paris, 91128 Palaiseau, France.
}

\section{Corresponding Authors}

angela.marinetti@cnrs.fr xavier.guinchard@cnrs.fr

\section{Table of Contents}

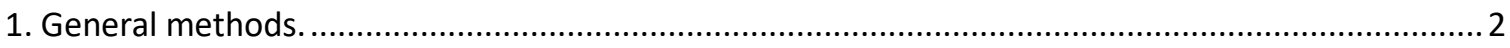

2. Synthesis and Characterization of the gold(I) complexes (S)-5, (S)-6, (S)-7 and (S)-11a..................... 2

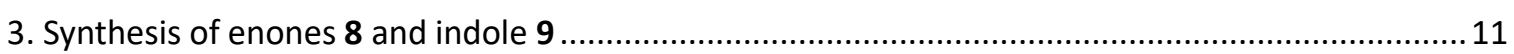

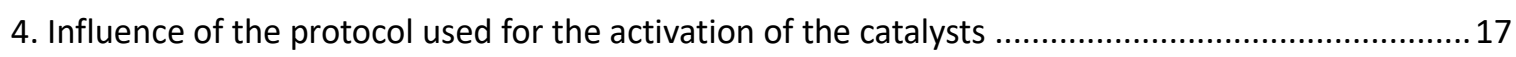

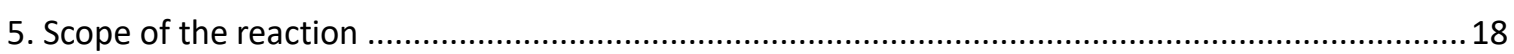

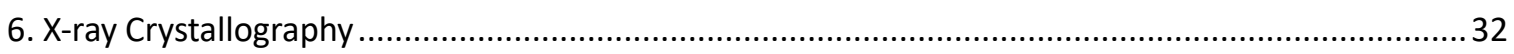

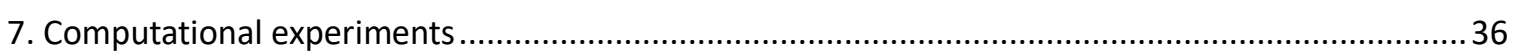

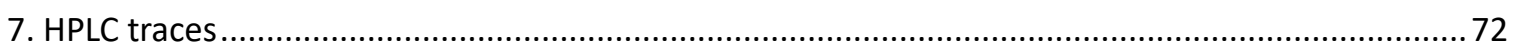

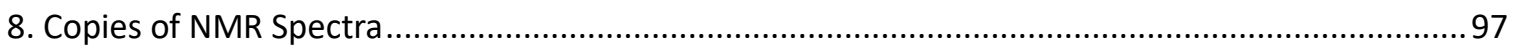




\section{General methods.}

Unless otherwise noted, all of the reactions were performed under $\mathrm{N}_{2}$ atomosphere, the solvents were distilled under $\mathrm{N}_{2}$, and the separations were carried out under flash-chromatographic conditions on silica gel (Redi Sep prepacked column, 230-400 mesh) with use of a CombiFlash Companion. Reagent-grade chemicals were obtained from diverse commercial suppliers (Sigma-Aldrich, Acros Organics, $\mathrm{TCl}$ and Alfa-Aesar) and were used as received. $n$-BuLi was used as commercially available solutions and titrated before use. ${ }^{1} \mathrm{H}$ NMR (300 and $\left.500 \mathrm{MHz}\right),{ }^{31} \mathrm{P}(120 \mathrm{MHz}$ and $200 \mathrm{MHz})$ and ${ }^{13} \mathrm{C}(75$ and $125 \mathrm{MHz}$ ) NMR spectra were recorded on Bruker Advance spectrometers. The chemical shifts ( $\delta$ ) are reported in part per million (ppm) and coupling values $(J)$ are given in hertz $(\mathrm{Hz})$. Multiplicities are abbreviated as follows: $s$ (singlet), $d$ (doublet), $t$ (triplet), q (quadruplet), bs (broad singlet) dd (doublet of doublet), dt (doublet of triplet), $m$ (multiplet). Infrared spectra (IR) were recorded on a Perkin-Elmer FT-IR system using diamond window Dura SamplIR II and the data are reported in reciprocal centimeters (cm-1). Optical rotations were measured on a Anton Paar MCP 300 polarimeter at 589 $\mathrm{nm}$. $[\alpha]_{\mathrm{D}}$ is expressed in deg. $\mathrm{cm}^{3} \cdot \mathrm{g}^{-1} \cdot \mathrm{dm}^{-1}$ and $\mathrm{c}$ is expressed in $\mathrm{g} / 100 \mathrm{~cm}^{3}$. Melting points were recorded in open capillary tubes on a Büchi B-540 apparatus and are uncorrected. High resolution mass spectra (HRMS) were recorded using a Micromass LCT Premier XE instrument (Waters) and were determined by electrospray ionization (ESI) with a TOF analyzer.

\section{Synthesis and Characterization of the gold(I) complexes $(S)-5,(S)-6,(S)-7$}

\section{and (S)-11a}

\section{Synthesis of (S)-2,2'-bis(methoxymethoxy)-1,1'-binaphthalene}<smiles>COCOc1ccc2ccccc2c1-c1c(OCOC)ccc2ccccc12</smiles>

(S)-BINOL (14.3 g, $50.0 \mathrm{mmol}, 1.0$ equiv.) was dissolved in $80 \mathrm{~mL}$ of anhydrous THF and added dropwise to a suspension of $\mathrm{NaH}$ ( $60 \% \mathrm{wt}$ in mineral oil, $6.0 \mathrm{~g}, 150 \mathrm{mmol}, 3$ equiv.) in $80 \mathrm{ml}$ of anhydrous THF at $0{ }^{\circ} \mathrm{C}$. The reaction mixture was stirred for $1 \mathrm{~h}$ at room temperature and then $\mathrm{MOM}-\mathrm{Cl}(11.4 \mathrm{~mL}, 150 \mathrm{mmol}, 3$ equiv.) was added dropwise at $0{ }^{\circ} \mathrm{C}$. The reaction mixture was stirred for another 3 hours at room temperature. When the reaction was completed, it was quenched with saturated aqueous $\mathrm{NaHCO}_{3}$ and extracted twice with $\mathrm{CH}_{2} \mathrm{Cl}_{2}$. Combined organic layers were then dried over $\mathrm{MgSO}_{4}$ and evaporated. The crude product was used directly for further synthesis without purification (18.5 g, $49.4 \mathrm{mmol}, 99 \%$ ). 
IR (neat) $v_{\max }=3057,2956,2901,2826,1621,1592,1506,1237,1147,1031,1011,920,809,749 \mathrm{~cm}^{-}$ ${ }^{1} .{ }^{1} \mathrm{H}$ NMR $\left(\mathrm{CDCl}_{3}, 300 \mathrm{MHz}\right) \delta(\mathrm{ppm})=7.92(\mathrm{~d}, J=9.0 \mathrm{~Hz}, 2 \mathrm{H}), 7.84(\mathrm{~d}, J=8.3 \mathrm{~Hz}, 2 \mathrm{H}), 7.55(\mathrm{~d}, J=9.0 \mathrm{~Hz}$, $2 \mathrm{H}), 7.33-7.28(\mathrm{~m}, 2 \mathrm{H}), 7.22-7.14(\mathrm{~m}, 4 \mathrm{H}), 5.05(\mathrm{~d}, J=6.8 \mathrm{~Hz}, 2 \mathrm{H}), 4.94(\mathrm{~d}, J=6.8 \mathrm{~Hz}, 2 \mathrm{H}), 3.11(\mathrm{~s}, 6 \mathrm{H}) .{ }^{13} \mathrm{C}$ NMR $\left(\mathrm{CDCl}_{3}, 75 \mathrm{MHz}\right) \delta(\mathrm{ppm})=152.8,134.2,130.0,129.5,128.0,126.5,125.7,124.2,121.4,117.4,95.3$, 56.0 .

Synthesis of (S)-2- (2,2'-bis(methoxymethoxy)- [1,1'-binaphthalen]-3-yl)- 4,4,5,5-tetramethyl- 1,3,2dioxaborolane $[(S)-1]$<smiles>COCOc1cc2ccccc2c(OC)c1-c1c(OCOC)ccc2ccccc12</smiles>

To a solution of (S)-2,2'-bis(methoxymethoxy)-1,1'-binaphthalene (7.84 g, $21 \mathrm{mmol}, 1.0$ equiv.) in 140 $\mathrm{mL}$ of anhydrous THF, $n$-BuLi (19.6 mL, $29.4 \mathrm{mmol}, 1.4$ equiv.) was added dropwise at $-78{ }^{\circ} \mathrm{C}$. The reaction mixture was stirred at $-78^{\circ} \mathrm{C}$ for 4 hours, then isopropoxypinacolborate $(5.6 \mathrm{~mL}, 27.3 \mathrm{mmol}, 1.3$ equiv.) was added to the flask and the resulting solution was allowed to warm to room temperature. It was stirred overnight at room temperature and then quenched with water and extracted with DCM. Combined organic layers were dried over $\mathrm{MgSO}_{4}$ and evaporated. The crude product was purified by flash column chromatography on silica gel (pure heptane to heptane:DCM = 2:1) yielding the product as a white solid (7.03 g, $14.1 \mathrm{mmol}, 67 \%){ }^{1}$

IR (neat) $v_{\max }=3411,3054,2956,2825,1909,1622,1592,1436,1241,1146,1014,988,923,696 \mathrm{~cm}^{-}$ ${ }^{1} .{ }^{1} \mathrm{H} \mathrm{NMR}\left(\mathrm{CDCl}_{3}, 300 \mathrm{MHz}\right) \delta=8.49(\mathrm{~s}, 1 \mathrm{H}), 7.92(\mathrm{~d}, J=8.9 \mathrm{~Hz}, 2 \mathrm{H}), 7.82(\mathrm{~d}, J=8.1 \mathrm{~Hz}, 1 \mathrm{H}), 7.55(\mathrm{~d}, J=9.0$ $\mathrm{Hz}, 1 \mathrm{H}), 7.39-7.29(\mathrm{~m}, 2 \mathrm{H}), 7.27-7.17(\mathrm{~m}, 4 \mathrm{H}), 5.15(\mathrm{~d}, J=6.8 \mathrm{~Hz}, 1 \mathrm{H}), 4.93(\mathrm{~d}, J=7.0 \mathrm{~Hz}, 1 \mathrm{H}), 4.89(\mathrm{~d}, J=5.8$ $\mathrm{Hz}, 1 \mathrm{H}), 4.84(\mathrm{~d}, J=5.8 \mathrm{~Hz}, 1 \mathrm{H}), 3.11(\mathrm{~s}, 3 \mathrm{H}), 2.40(\mathrm{~s}, 3 \mathrm{H}), 1.39(\mathrm{~s}, 12 \mathrm{H}) .{ }^{13} \mathrm{C} \mathrm{NMR}\left(\mathrm{CDCl}_{3}, 75 \mathrm{MHz}\right) \delta(\mathrm{ppm})=$ $156.9,153.1,139.4,135.9,134.5,130.5,129.8,129.5,128.7,127.7,127.5,126.5,126.3,126.1,125.5,124.8$, 124.1, 121.8, 116.9, 100.2, 100.1, 95.2, 84.0, 56.0, 55.9, 25.0. HRMS (ESI): $\mathrm{m} / \mathrm{z}$ : calcd for $\mathrm{C}_{30} \mathrm{H}_{33} \mathrm{BNaO}_{6}^{+}$ $523.2262[\mathrm{M}+\mathrm{Na}]^{+}$, found, 523.2313. $[\alpha]_{\mathrm{D}}=-68\left(\right.$ c 1.16, $\left.\mathrm{CHCl}_{3}\right)$.

\section{Synthesis of (2-bromophenyl)diphenylphosphine oxide ${ }^{2}$}<smiles>O=P(c1ccccc1)(c1ccccc1)c1ccccc1Br</smiles>

Diphenylphosphine oxide (4.81 g, $23.8 \mathrm{mmol}, 1.0$ equiv.) was dissolved in PhMe (60 mL), and then $\mathrm{Pd}_{2}(\mathrm{dba})_{3}(0.38 \mathrm{~g}, 0.4 \mathrm{mmol}, 1.75 \mathrm{~mol} \%), 1,3$-bis(diphenylphosphino)propane (0.34 g, $0.8 \mathrm{mmol}, 3.5 \mathrm{~mol} \%$ ),

\footnotetext{
1 L.-X. Gao; Ma, L.; Jin, R.-Z.; Lü, G.-H.; Bian, Z.; Ding, M.-X. Synthesis 2007, 2461-2470.

2 H.-L. Wang; Hu, R.-B.; Zhang, H.; Zhou, A.-X.; Yang, S.-D. Org. Lett. 2013, 15, 5302-5305.
} 
then bromoiodobenzene ( $3.36 \mathrm{~mL}, 26.2 \mathrm{mmol}, 1.1$ equiv.), and ' $\operatorname{Pr}{ }_{2} \mathrm{NEt}(4.9 \mathrm{~mL}, 29.7 \mathrm{mmol}, 1.25$ equiv.) were added and the mixture stirred at $90^{\circ} \mathrm{C}$ for 4 days. After cooling to room temperature, water was added and the solution was extracted with DCM. Organic layers were dried over $\mathrm{MgSO}_{4}$, filtered and evaporated under vacuum. The crude product was purified by flash column chromatography (Hept: EA = 4:1 to EA), giving product as a pale yellow solid $(6.0 \mathrm{~g}, 16.8 \mathrm{mmol}, 64 \%)$.

${ }^{1}{ }_{\mathrm{H}}$ NMR $\left(\mathrm{CDCl}_{3}, 500 \mathrm{MHz}\right) \delta=7.75-7.65(\mathrm{~m}, 5 \mathrm{H}), 7.58-7.53(\mathrm{~m}, 2 \mathrm{H}), 7.60-7.45(\mathrm{~m}, 4 \mathrm{H}), 7.41-7.30(\mathrm{~m}$,

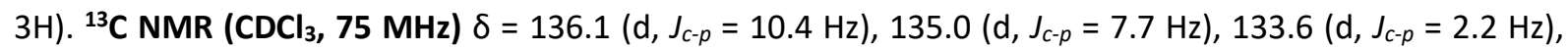
$133.2\left(\mathrm{~d}, J_{c-p}=104.3 \mathrm{~Hz}\right), 132.3\left(\mathrm{~d}, J_{c-p}=9.9 \mathrm{~Hz}\right), 132.1\left(\mathrm{~d}, J_{c-p}=2.7 \mathrm{~Hz}\right), 131.9\left(\mathrm{~d}, J_{c-p}=107.6 \mathrm{~Hz}\right), 128.7\left(\mathrm{~d}, J_{c-}\right.$ $p=12.6 \mathrm{~Hz}), 127.1\left(\mathrm{~d}, \mathrm{~J}_{c-p}=11.5 \mathrm{~Hz}\right), 127.1\left(\mathrm{~d}, J_{c-p}=4.9 \mathrm{~Hz}\right) .{ }^{31} \mathrm{P}$ NMR $\left(\mathrm{CDCl}_{3}, 200 \mathrm{MHz}\right) \delta=30.6$.

\section{Synthesis of (S)-(2-(2,2'-dihydroxy-[1,1'-binaphthalen]-3-yl)phenyl) diphenylphosphine oxide [(S)-2] ${ }^{3}$}<smiles>O=Pc1ccccc1-c1cc2ccccc2c(-c2c(O)ccc3ccccc23)c1O</smiles>

(2-bromophenyl)diphenylphosphine oxide $\left(4.44 \mathrm{~g}, 12.5 \mathrm{mmol}, 1.5\right.$ equiv.) and $\mathrm{Pd}\left(\mathrm{PPh}_{3}\right)_{4}(0.48 \mathrm{~g}, 0.42$ $\mathrm{mmol}, 5 \mathrm{~mol} \%$ ) were added to a flask, followed by anhydrous, degassed THF (100 mL) and $\mathrm{H}_{2} \mathrm{O}(50 \mathrm{~mL})$. The mixture was stirred at room temperature for $10 \mathrm{~min}$. To this solution were added (S)-2-(2,2'bis(methoxymethoxy)-[1,1'-binaphthalen]-3-yl)-4,4,5,5-tetramethyl-1,3,2-dioxaborolane (4.78 g, $8.3 \mathrm{~mol}$, 1.0 equiv.) [(S)-1] and $\mathrm{K}_{2} \mathrm{CO}_{3}\left(3.45 \mathrm{~g}, 24.9 \mathrm{mmol}, 3\right.$ equiv.), and the solution was stirred at $100{ }^{\circ} \mathrm{C}$ for $24 \mathrm{~h}$. After cooling to room temperature, water was added and the reaction mixture was extracted twice with methyl tert-butyl ether. The combined organic layers were dried over $\mathrm{MgSO}_{4}$, filtered and concentrated under vacuum.

The crude product was dissolved in a mixture of THF/MeOH 1:1 $(80 \mathrm{~mL})$, then $p-\mathrm{Ts} \mathrm{OH} \cdot \mathrm{H}_{2} \mathrm{O}$ was added to this solution and reaction mixture was stirred at $60^{\circ} \mathrm{C}$ for 3 hours. After that, it was cooled down to room temperature and solvents were evaporated under reduced pressure. Water was added and the reaction mixture was extracted twice with DCM. The combined organic layers were dried over $\mathrm{MgSO}_{4}$, filtered and concentrated under vacuum. The crude product was purified by flash column chromatography (eluent: $100 \%$ heptane to heptane/ethyl acetate, 30/70) giving the product as a white solid ( $3.43 \mathrm{~g}, 6.1 \mathrm{mmol}, 74 \%$ ).

IR (neat) $\mathbf{v}_{\max }=3058,1619,1467,1438,1142,750 \mathrm{~cm}^{-1} .{ }^{1} \mathbf{H} \mathbf{~ N M R}\left(\mathrm{CDCl}_{3}, 500 \mathrm{MHz}\right) \delta=8.11(\mathrm{br} \mathrm{s}, 1 \mathrm{H})$, $7.83(\mathrm{~d}, J=8.8 \mathrm{~Hz}, 1 \mathrm{H}), 7.77(\mathrm{~d}, J=8.2 \mathrm{~Hz}, 1 \mathrm{H}), 7.67\left(\mathrm{dd}, J_{1}=11.6 \mathrm{~Hz}, J_{2}=7.6 \mathrm{~Hz}, 2 \mathrm{H}\right), 7.61(\mathrm{t}, J=7.6 \mathrm{~Hz}, 1 \mathrm{H})$, 7.58-7.54 (m, $1 \mathrm{H}), 7.46(\mathrm{t}, J=6.9 \mathrm{~Hz}, 1 \mathrm{H}), 7.42-7.30(\mathrm{~m}, 4 \mathrm{H}), 7.29-7.06(\mathrm{~m}, 12 \mathrm{H}), 6.97(\mathrm{~s}, 1 \mathrm{H}), 6.93(\mathrm{~d}, J=7.9$

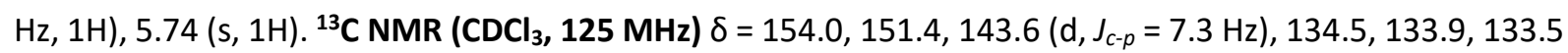
$\left(d, J_{c-p}=13.7 \mathrm{~Hz}\right), 133.0,132.6\left(\mathrm{~d}, J_{c-p}=1.8 \mathrm{~Hz}\right), 132.5\left(\mathrm{~d}, J_{c-p}=9.2 \mathrm{~Hz}\right), 132.4\left(\mathrm{~d}, J_{c-p}=158.5 \mathrm{~Hz}\right), 132.2\left(\mathrm{~d}, J_{c-}\right.$ $p=2.7 \mathrm{~Hz}), 132.0\left(\mathrm{~d}, J_{c-p}=10.1 \mathrm{~Hz}\right), 131.9,131.9,131.4,131.3,131.3,131.2\left(\mathrm{~d}, J_{c-p}=106.3 \mathrm{~Hz}\right), 130.5,130.1$, $130.1,129.0\left(\mathrm{~d}, J_{c-p}=50.4 \mathrm{~Hz}\right), 128.7\left(\mathrm{~d}, J_{c-p}=11.9 \mathrm{~Hz}\right), 128.4\left(\mathrm{~d}, J_{c-p}=12.8 \mathrm{~Hz}\right), 128.3\left(\mathrm{~d}, J_{c-p}=6.4 \mathrm{~Hz}\right), 127.4$

\footnotetext{
3 H. Sasai; Matsui, K.; Takizawa, S. Synlett 2006, 5, 761-765.
} 
$\left(d, J_{c-p}=12.8 \mathrm{~Hz}\right), 126.7,126.8,125.4,123.7,123.3,120.2,115.4,113.1 .{ }^{31} \mathrm{P}$ NMR (CDCl, $\left.200 \mathrm{MHz}\right) \delta=30.5$. HRMS (ESI): $\mathrm{m} / z$ : calcd for $\mathrm{C}_{38} \mathrm{H}_{28} \mathrm{O}_{3} \mathrm{P}^{+} 563.1771[\mathrm{M}+\mathrm{H}]^{+}$, found, 563.1770. [ $\left.\alpha\right]_{\mathrm{D}}=+71\left(c 0.76, \mathrm{CHCl}_{3}\right)$, lit +86 (c 0.6, $\mathrm{CHCl}_{3}$ ).

2.Synthesis of (S)-3-(2-(diphenylphosphaneyl)phenyl)-[1,1'-binaphthalene]-2,2'-diol [(S)-3 $]^{3}$<smiles>Oc1ccc2ccccc2c1-c1c(O)c(-c2ccccc2P)cc2ccccc12</smiles>

(S)-(2-(2,2'-dihydroxy-[1,1'-binaphthalen]-3-yl)phenyl)diphenylphosphine oxide [(S)-2] (3.43 g, 6.1 mmol, 1.0 equiv.) was dissolved in toluene $(100 \mathrm{~mL})$ at $0{ }^{\circ} \mathrm{C}$, and then $\mathrm{Et}_{3} \mathrm{~N}(8.5 \mathrm{~mL}, 61 \mathrm{mmol}, 10$ equiv.) and $\mathrm{Cl}_{3} \mathrm{SiH}\left(12.5 \mathrm{~mL}, 122.1 \mathrm{mmol}, 20\right.$ equiv.) were added to the solution dropwise at $0^{\circ} \mathrm{C}$. The reaction was then stirred at $90^{\circ} \mathrm{C}$ for $24 \mathrm{~h}$. After cooling to room temperature, the reaction solution was quenched with $2 \mathrm{M}$ $\mathrm{NaOH}$ and then THF was evaporated under reduced pressure. $2 \mathrm{M} \mathrm{NaOH}$ was added to dissolve the precipitated solid and then extracted twice with $\mathrm{DCM}$, dried over $\mathrm{MgSO}_{4}$ and evaporated. The crude product was purified by flash column chromatography (eluent: heptane to DCM) giving the product as a white solid ( $2.61 \mathrm{~g}, 4.7 \mathrm{mmol}, 78 \%$ ).

IR (neat) $\mathbf{v}_{\max }=3510,3053,1619,1596,1434,1138,734 \mathrm{~cm}^{-1} .{ }^{1} \mathbf{H}$ NMR $\left(\mathrm{CDCl}_{3}, 500 \mathrm{MHz}\right) \delta=7.94(\mathrm{~d}, J$ $=8.8 \mathrm{~Hz}, 1 \mathrm{H}), 7.87(\mathrm{~d}, J=7.9 \mathrm{~Hz}, 1 \mathrm{H}), 7.64-7.57(\mathrm{~m}, 1 \mathrm{H}), 7.55-7.42(\mathrm{~m}, 3 \mathrm{H}), 7.40-7.15(\mathrm{~m}, 17 \mathrm{H}), 7.11-7.03(\mathrm{~m}$, 2H), 5.71 (bs, 1H), 5.15 (bs, 1H). ${ }^{13} \mathrm{C} \mathrm{NMR}\left(\mathrm{CDCl}_{3}, 125 \mathrm{MHz}\right) \delta=153.2,150.1,143.2\left(\mathrm{~d}, J_{c-p}=30.2 \mathrm{~Hz}\right), 138.2$ $\left(d, J_{c-p}=30.2 \mathrm{~Hz}\right.$ ), $136.9\left(\mathrm{~d}, J_{c-p}=8.2 \mathrm{~Hz}\right), 136.7\left(\mathrm{~d}, J_{c-p}=9.2 \mathrm{~Hz}\right.$ ), 134.1, 134.0, $133.8\left(\mathrm{~d}, J_{c-p}=19.2 \mathrm{~Hz}\right), 132.3$, $131.2,130.5,130.3\left(\mathrm{~d}, J_{c-p}=3.7 \mathrm{~Hz}\right.$ ), 129.6, 129.5, 128.8, 128.6, 128.5, 128.5, 128.5, 127.6, 127.5, 127.2, $125.1,124.9,124.2,123.9,118.1\left(\mathrm{~d}, \mathrm{~J}_{c-p}=82.5 \mathrm{~Hz}\right), 112.1,112.0 .{ }^{31} \mathrm{P}$ NMR $\left(\mathrm{CDCl}_{3}, 120 \mathrm{MHz}\right) \delta=-10.6,-12.3$. HRMS (ESI): $\mathrm{m} / z$ : calcd for $\mathrm{C}_{38} \mathrm{H}_{28} \mathrm{O}_{2} \mathrm{P}^{+} 547.1821[\mathrm{M}+\mathrm{H}]^{+}$, found, 547.1801. [ $\left.\alpha\right]_{\mathrm{D}}=-80$ (c 0.94, $\mathrm{CHCl}_{3}$ ), lit -73 (c $\left.0.6, \mathrm{CHCl}_{3}\right)$.

Synthesis of (S)-(2-(2,2'-dihydroxy-[1,1'-binaphthalen]-3-yl)phenyl)diphenylphosphine gold(I) chloride $[(S)-4]$<smiles>Oc1ccc2ccccc2c1-c1c(O)c(-c2ccccc2P(=P)(c2ccccc2)C(Cl)(Cl)Cl)cc2ccccc12</smiles>

(S)-3-(2-(diphenylphosphaneyl)phenyl)-[1,1'-binaphthalene]-2,2'-diol [(S)-3] (2.63 g, $4.8 \mathrm{mmol}, 1.0$ equiv.) and $\mathrm{Me}_{2} \mathrm{~S} \bullet \mathrm{AuCl}(1.41 \mathrm{~g}, 4.8 \mathrm{mmol}, 1.0$ equiv.) were dissolved in dry DCM (130 mL), and then the reaction solution was stirred at room temperature for $1 \mathrm{~h}$. The solvent was evaporated and the crude 
product was purified by flash column chromatography (Heptane-DCM), yielding the product as a white solid (3.4 g, $4.4 \mathrm{mmol}, 89 \%)$.

IR (neat) $\mathbf{v}_{\max }=3515,3405,3057,2926,1619,1597,1437,1131,748 \mathrm{~cm}^{-1} .{ }^{1}{ }^{H}$ NMR (CDCl, $500 \mathrm{MHz}$ ) $\delta=7.87(\mathrm{~d}, J=8.8 \mathrm{~Hz}, 1 \mathrm{H}), 7.79(\mathrm{~d}, J=7.9 \mathrm{~Hz}, 1 \mathrm{H}), 7.63-7.61(\mathrm{~m}, 1 \mathrm{H}), 7.58-7.49(\mathrm{~m}, 4 \mathrm{H}), 7.50-7.47(\mathrm{~m}, 1 \mathrm{H})$, 7.46-7.40 (m, 4H), 7.39-7.34 (m, 3H), 7.34-7.23 (m, 5H), 7.23-7.16 (m, 4H), $7.00(\mathrm{~d}, J=8.2 \mathrm{~Hz}, 1 \mathrm{H}), 6.53(\mathrm{br}$ s, $1 \mathrm{H}), 4.90$ (br s, $\left.1 \mathrm{H}) .{ }^{13} \mathrm{C} \mathrm{NMR} \mathrm{CDCl}_{3}, 125 \mathrm{MHz}\right) \delta=154.3,150.2,144.0\left(\mathrm{~d}, \mathrm{~J}_{c-p}=15.6 \mathrm{~Hz}\right), 134.8\left(\mathrm{~d}, J_{c-p}=5.5\right.$ $\mathrm{Hz}), 134.5\left(\mathrm{~d}, J_{c-p}=13.7 \mathrm{~Hz}\right), 134.3\left(\mathrm{~d}, J_{c-p}=13.7 \mathrm{~Hz}\right), 134.2,133.7\left(\mathrm{~d}, J_{c-p}=8.2 \mathrm{~Hz}\right), 133.5,133.1,132.1\left(\mathrm{~d}, J_{c-}\right.$ $p=1.8 \mathrm{~Hz}), 131.7\left(\mathrm{~d}, J_{c-p}=1.8 \mathrm{~Hz}\right), 131.6,131.5\left(\mathrm{~d}, J_{c-p}=1.8 \mathrm{~Hz}\right), 130.9\left(\mathrm{~d}, J_{c-p}=62.3 \mathrm{~Hz}\right), 129.9,129.6\left(\mathrm{~d}, J_{c-p}\right.$ $=12.8 \mathrm{~Hz}$ ), 129.4, $129.2\left(\mathrm{~d}, J_{c-p}=11.9 \mathrm{~Hz}\right), 129.0,128.7,128.6,128.6, \quad 128.2,128.1,127.8,127.4,124.9$, 124.8, 124.1, 123.8, 119.4, 113.6, 110.4. ${ }^{31}$ P NMR ( $\left.\mathrm{CDCl}_{3}, 200 \mathrm{MHz}\right) \delta=24.2$. HRMS (ESI): $\mathrm{m} / \mathrm{z}$ : calculated for $\mathrm{C}_{40} \mathrm{H}_{30} \mathrm{AuNO}_{2} \mathrm{P}^{+}$: $784.1680[\mathrm{M}+\mathrm{MeCN}-\mathrm{Cl}]^{+}$, found, 784.1688; $\mathrm{m} / \mathrm{z}$ : calcd for $\mathrm{C}_{38} \mathrm{H}_{26} \mathrm{AuClO}_{2} \mathrm{P}^{-} 777.1030$ [M$\mathrm{H}]^{-}$, found, 777.1016. $[\alpha]_{\mathrm{D}}=-149\left(\mathrm{c} 1.07, \mathrm{CHCl}_{3}\right)$.

Synthesis of (S)-(2-(2,2'-diyl hydrogen phosphate-[1,1'-binaphthalen]-3-yl)phenyl) diphenylphosphine gold $(I)$ chloride $[(S)-5]$<smiles>O=P(O)(Oc1ccc2ccccc2c1-c1c(OP(=O)(O)c2ccccc2)c(-c2ccccc2P[Z17](Cl)(c2ccccc2)c2ccccc2)cc2ccccc12)c1ccccc1</smiles>

(S)-(2-(2,2'-dihydroxy-[1,1'-binaphthalen]-3-yl)phenyl)diphenylphosphine gold(I) chloride [(S)-4] (3.4 g, $4.3 \mathrm{mmol}, 1.0$ equiv.) was added to $10 \mathrm{~mL}$ pyridine, then $\mathrm{POCl}_{3}(0.48 \mathrm{~mL}, 5.11 \mathrm{mmol}, 1.2$ equiv.) was added to the solution dropwise under $0{ }^{\circ} \mathrm{C}$. The resulting solution was stirred at room temperature for $24 \mathrm{~h}$. After completion, it was quenched with water and the solvent were removed under vacuum. $2 \mathrm{M} \mathrm{HCl}$ and THF were added, and the reaction mixture was stirred overnight at $50{ }^{\circ} \mathrm{C}$. After cooling to room temperature, the reaction solution was extracted with $\mathrm{DCM}$ and $\mathrm{H}_{2} \mathrm{O}$. Crude product was purified by flash column chromatography (eluent: heptane to DCM) giving the product as a brown solid ( $3.15 \mathrm{~g}, 3.7 \mathrm{mmol}, 88 \%$ ).

m.p.: $256.2-261.5^{\circ} \mathrm{C}$. IR (neat) $v_{\max }=3515,3405,3056,2925,1619,1436,1214,1131,748 \mathrm{~cm}^{-1}$. HRMS (ESI): $\mathrm{m} / \mathrm{z}$ calcd for $\mathrm{C}_{40} \mathrm{H}_{29} \mathrm{AuNO}_{4} \mathrm{P}_{2}{ }^{+} 846.1232\left[\mathrm{M}+\mathrm{MeCN}-\mathrm{Cl}^{-}\right]^{+}$, found, 846.1245; $\mathrm{m} / \mathrm{z}$ calcd for

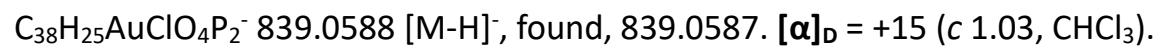

\section{-NMR data in DMSO-d6:}

${ }^{1}{ }_{\mathrm{H}}$ NMR (DMSO-d6, 343K, $500 \mathrm{MHz}$ ) $\delta=8.13-8.07(\mathrm{~m}, 2 \mathrm{H}$ ), $8.06(\mathrm{~d}, J=6.6 \mathrm{~Hz}, 1 \mathrm{H}$ ), $8.03(\mathrm{~d}, J=8.2 \mathrm{~Hz}$, $1 \mathrm{H}), 7.86(\mathrm{~d}, J=8.2 \mathrm{~Hz}, 1 \mathrm{H}), 7.72(\mathrm{t}, J=7.6 \mathrm{~Hz}, 2 \mathrm{H}), 7.62(\mathrm{~d}, J=6.3 \mathrm{~Hz}, 1 \mathrm{H}), 7.60-7.48(\mathrm{~m}, 7 \mathrm{H}), 7.43(\mathrm{~d}, J=8.8$ $\mathrm{Hz}, 1 \mathrm{H}), 7.40(\mathrm{~d}, J=9.1 \mathrm{~Hz}, 1 \mathrm{H}), 7.37-7.28(\mathrm{~m}, 4 \mathrm{H}), 7.18-7.10(\mathrm{~m}, 2 \mathrm{H}), 7.00(\mathrm{br} \mathrm{s}, 2 \mathrm{H}), 6.24(\mathrm{br} \mathrm{s}, 1 \mathrm{H})$.

${ }^{13}$ C NMR (DMSO-d6, 343K, $125 \mathrm{MHz}$ ) $\delta=146.9\left(\mathrm{~d}, J_{c-p}=9.2 \mathrm{~Hz}\right.$ ), $144.9\left(\mathrm{~d}, J_{c-p}=10.1 \mathrm{~Hz}\right.$ ), $141.5\left(\mathrm{~d}, J_{c-p}=\right.$ $13.7 \mathrm{~Hz}), 133.8\left(\mathrm{~d}, J_{c-p}=7.3 \mathrm{~Hz}\right), 133.6\left(\mathrm{~d}, J_{c-p}=13.7 \mathrm{~Hz}\right), 133.3\left(\mathrm{~d}, J_{c-p}=13.7 \mathrm{~Hz}\right), 132.9\left(\mathrm{~d}, J_{c-p}=6.4 \mathrm{~Hz}\right), 131.8$, 131.5, $131.3\left(\mathrm{~d}, J_{c-p}=5.5 \mathrm{~Hz}\right), 131.2,131.0\left(\mathrm{~d}, J_{c-p}=116.4 \mathrm{~Hz}\right), 130.7,130.3,129.8,129.5,129.4,129.1\left(\mathrm{~d}, J_{c-}\right.$ $p=11.0 \mathrm{~Hz}$ ), 128.4, $128.4\left(\mathrm{~d}, J_{c-p}=12.8 \mathrm{~Hz}\right), 128.0\left(\mathrm{~d}, J_{c-p}=9.2 \mathrm{~Hz}\right), 127.6,127.6,127.4\left(\mathrm{~d}, J_{c-p}=12.8 \mathrm{~Hz}\right)$, 
$126.9\left(\mathrm{~d}, J_{c-p}=15.6 \mathrm{~Hz}\right), 126.4,125.9,125.6,125.2,124.9,121.7,121.0,120.7,120.3$.

31P NMR (DMSO-d6, $343 \mathrm{~K}, 200 \mathrm{MHz}$ ) $\delta=$ 26.4, 1.2 (major isomer) and 25.8, 2.5 (minor isomer).

${ }^{31}$ P NMR (DMSO-d6, $383 \mathrm{~K}, 200 \mathrm{MHz}$ ) $\delta=26.8,1.1$.

-NMR data in deuterated pyridine:

$(\mathrm{PAuCl}$

(S)-5.Py-d5

${ }^{1} \mathrm{H}$ NMR (Pyridine-d5, $500 \mathrm{MHz}$ ) $\delta=11.86$ (bs, 1H), $9.26(\mathrm{t}, J=6.3 \mathrm{~Hz}, 1 \mathrm{H}$ ), $7.97(\mathrm{~s}, 1 \mathrm{H}), 7.88(\mathrm{~d}, J=8.0$ $\mathrm{Hz}, 1 \mathrm{H}), 7.84(\mathrm{t}, J=7.7 \mathrm{~Hz}, 1 \mathrm{H}), 7.82-7.75(\mathrm{~m}, 3 \mathrm{H}), 7.72(\mathrm{~d}, J=8.7 \mathrm{~Hz}, 1 \mathrm{H}), 7.63\left(\mathrm{dd}, J_{1}=13.2 \mathrm{~Hz}, J_{2}=7.7 \mathrm{~Hz}\right.$, $2 \mathrm{H}), 7.53(\mathrm{t}, J=6.9 \mathrm{~Hz}, 1 \mathrm{H}), 7.49-7.33(\mathrm{~m}, 9 \mathrm{H}), 7.20(\mathrm{t}, J=7.6 \mathrm{~Hz}, 1 \mathrm{H}), 7.15\left(\mathrm{dd}, J_{1}=12.1 \mathrm{~Hz}, J_{2}=7.9 \mathrm{~Hz}, 1 \mathrm{H}\right)$, $6.99(\mathrm{t}, J=6.8 \mathrm{~Hz}, 2 \mathrm{H}), 6.92(\mathrm{t}, J=6.9 \mathrm{~Hz}, 1 \mathrm{H})$.

${ }^{13}$ C NMR (Pyridine-d5, $\left.125 \mathrm{MHz}\right) \delta=148.6\left(\mathrm{~d}, J_{c-p}=10.1 \mathrm{~Hz}\right), 144.1\left(\mathrm{~d}, J_{c-p}=13.7 \mathrm{~Hz}\right), 136.9\left(\mathrm{~d}, J_{c-p}=7.3\right.$ $\mathrm{Hz}), 135.6\left(\mathrm{~d}, J_{c-p}=14.7 \mathrm{~Hz}\right), 134.7,134.7\left(\mathrm{~d}, J_{c-p}=13.7 \mathrm{~Hz}\right), 134.1,133.9,133.7\left(\mathrm{~d}, J_{c-p}=4.6 \mathrm{~Hz}\right), 133.5,132.8$, 132.4, 132.1, 131.7, 131.6 (d, $J_{c-p}=62.3 \mathrm{~Hz}$ ), 131.3, 130.7, 130.3 (d, $\left.J_{c-p}=11.9 \mathrm{~Hz}\right), 130.3,129.9,129.8,129.7$ (d, $J_{c-p}=11.0 \mathrm{~Hz}$ ), 129.4, $129.0\left(\mathrm{~d}, J_{c-p}=10.1 \mathrm{~Hz}\right), 128.8,128.7,128.3,127.8,127.6,127.3,126.2,126.0,123.1$.

31P NMR (Pyridine-d5, $200 \mathrm{MHz}) \delta=29.2,6.7$.

\section{-NMR data in $\mathrm{CDCl}_{3}$ :}

${ }^{1} \mathrm{H} \mathrm{NMR}\left(\mathrm{CDCl}_{3}, 500 \mathrm{MHz}\right) \delta=8.30(\mathrm{~s}, 1 \mathrm{H}), 8.04(\mathrm{~d}, J=8.2 \mathrm{~Hz}, 1 \mathrm{H}), 7.90(\mathrm{~d}, J=8.2 \mathrm{~Hz}, 2 \mathrm{H}), 7.77-7.72(\mathrm{~m}$, $1 \mathrm{H}), 7.58-7.48(\mathrm{~m}, 5 \mathrm{H}), 7.46-7.39(\mathrm{~m}, 3 \mathrm{H}), 7.35-7.19(\mathrm{~m}, 8 \mathrm{H}), 6.97\left(\mathrm{dd}, J_{1}=11.9 \mathrm{~Hz}, J_{2}=7.9 \mathrm{~Hz}, 1 \mathrm{H}\right), 6.73-$ $6.66(\mathrm{~m}, 2 \mathrm{H}), 6.66-6.60(\mathrm{~m}, 1 \mathrm{H}), 6.53(\mathrm{bs}, 1 \mathrm{H})$.

${ }^{31} \mathrm{P}$ NMR $\left(\mathrm{CDCl}_{3}, 200 \mathrm{MHz}\right) \delta=27.7,2.9$ (major isomer), 26.2, 4.6 (minor isomer).

Isolation of the intermediate phosphoroyl chloride SI-1

In some reaction batch for the synthesis of $\mathbf{5}$, one of the intermediate phosphoroyl chloride diastereoisomer was showed to be poorly reactive towards hydrolysis and could be isolated and characterized.

Data for (S)-(2-(2,2'-diylchlorogenphosphate-[1,1'-binaphthalen]-3-yl)phenyl) diphenylphosphine gold(I) chloride [SI-1]: m.p.: 193.4-194. $2^{\circ} \mathrm{C}$. IR (neat) $v_{\max }=3056$,<smiles>O=P(Cl)(Cl)Oc1ccc2ccccc2c1-c1c(-c2ccccc2P(=O)([AlH2])Cl)cc2ccccc2c1OP(=O)(Cl)Cl</smiles>
2961, 2925, 2854, 1591, 1437, 1413, 1310, 1242, 1208, 1101, 1082, 970, 954, 904, 821, 876, 750, 693. ${ }^{1} \mathrm{H}$ $\operatorname{NMR}\left(\mathrm{CDCl}_{3}, 500 \mathrm{MHz}\right) \delta=8.37(\mathrm{~s}, 1 \mathrm{H}), 8.09(\mathrm{~d}, J=8.2 \mathrm{~Hz}, 1 \mathrm{H}), 8.03(\mathrm{~d}, J=8.8 \mathrm{~Hz}, 1 \mathrm{H}), 7.97(\mathrm{~d}, J=8.2 \mathrm{~Hz}$, $1 \mathrm{H}), 7.85(\mathrm{t}, J=6.3 \mathrm{~Hz}, 1 \mathrm{H}), 7.71(\mathrm{t}, J=7.6 \mathrm{~Hz}, 1 \mathrm{H}), 7.62-7.55(\mathrm{~m}, 2 \mathrm{H}), 7.52-7.45(\mathrm{~m}, 5 \mathrm{H}), 7.45-7.40(\mathrm{~m}, 3 \mathrm{H})$, 7.40-7.31 (m, 4H), $7.25(\mathrm{~d}, J=9.1 \mathrm{~Hz}, 1 \mathrm{H}), 7.07\left(\mathrm{dd}, J_{1}=12.0 \mathrm{~Hz}, J_{2}=8.2 \mathrm{~Hz}, 1 \mathrm{H}\right), 6.74-6.69(\mathrm{~m}, 3 \mathrm{H}) .{ }^{13} \mathrm{C} \mathrm{NMR}$ $\left(\mathrm{CDCl}_{3}, 125 \mathrm{MHz}\right) \delta=146.1\left(\mathrm{~d}, J_{c-p}=11.9 \mathrm{~Hz}\right), 143.9\left(\mathrm{~d}, J_{c-p}=11.9 \mathrm{~Hz}\right), 141.1\left(\mathrm{~d}, J_{c-p}=12.8 \mathrm{~Hz}\right), 134.9\left(\mathrm{~d}, J_{c-p}=\right.$ $14.7 \mathrm{~Hz}), 134.6\left(\mathrm{~d}, J_{c-p}=14.7 \mathrm{~Hz}\right), 134.1\left(\mathrm{~d}, J_{c-p}=7.3 \mathrm{~Hz}\right), 133.8,133.2\left(\mathrm{~d}, J_{c-p}=6.4 \mathrm{~Hz}\right), 132.6,132.2,131.9$, 131.9, 131.9, 131.7, 131.7, 131.5, $130.9\left(\mathrm{~d}, J_{c-p}=1.8 \mathrm{~Hz}\right), 129.8,129.6,129.4\left(\mathrm{~d}, J_{c-p}=11.9 \mathrm{~Hz}\right), 129.0,128.9$ $\left(d, J_{c-p}=8.2 \mathrm{~Hz}\right), 128.9,128.8,128.6,128.4,128.0,127.5,127.0\left(\mathrm{~d}, J_{c-p}=68.7 \mathrm{~Hz}\right), 126.8,122.1,121.8,121.8$, $119.6\left(d, J_{c-p}=2.7 \mathrm{~Hz}\right.$ ). ${ }^{31}$ P NMR $\left(\mathrm{CDCl}_{3}, 200 \mathrm{MHz}\right) \delta=26.8$, 9.2. HRMS (ESI): $\mathrm{m} / \mathrm{z}$ calcd for $\mathrm{C}_{40} \mathrm{H}_{28} \mathrm{AuClNO}_{3} \mathrm{P}_{2}{ }^{+}$ $864.0893\left[\mathrm{M}+\mathrm{MeCN}-\mathrm{Cl}^{-}\right]^{+}$, found, 864.0898 . 
This compound SI-1 could be submitted to hydrolysis according to the following procedure.
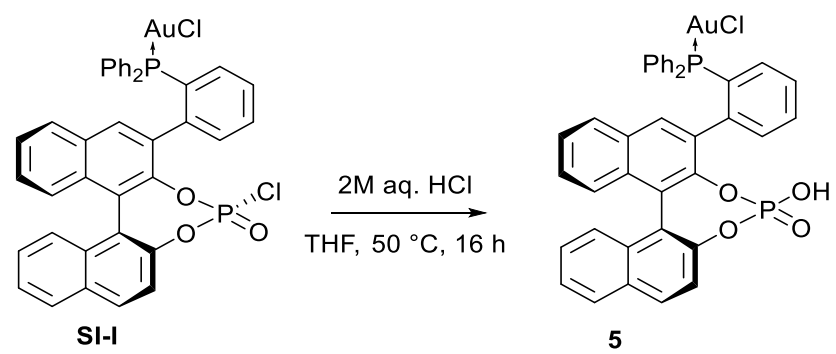

To a solution of SI-1 $(436 \mathrm{mg}, 0.51 \mathrm{mmol})$ in THF $(30 \mathrm{~mL})$ was added $2 \mathrm{M} \mathrm{HCl}(30 \mathrm{~mL})$. The resulting mixture was then stirred overnight at $50^{\circ} \mathrm{C}$. After cooling to room temperature, the THF was removed under vacuum and the resulting solution was extracted with DCM. Organic layers were dried over $\mathrm{MgSO}_{4}$, filtered and evaporated under vacuum. The crude product was purified by flash column chromatography, giving product (S)-5 as a brown solid ( $350.0 \mathrm{mg}, 0.42 \mathrm{mmol}, 82 \%$ ).

\section{Synthesis of $(S)-6$}<smiles></smiles>

To a suspension of $\mathrm{Ag}_{2} \mathrm{CO}_{3}(82.8 \mathrm{mg}, 0.3 \mathrm{mmol})$ in toluene $(2 \mathrm{~mL})$ and $\mathrm{H}_{2} \mathrm{O}(2 \mathrm{~mL}),(S)-5(84.1 \mathrm{mg}, 0.1$ $\mathrm{mmol}$ ) was added. The mixture was stirred overnight at room temperature. Then the suspension was filtered on celite. The filter was rinsed with DCM and evaporated under vacuum. The product was obtained as an amorphous gray solid (56.1 mg, $0.07 \mathrm{mmol}, 69 \%$ ). This $\mathrm{Au}(\mathrm{l})$ complex is also very cleanly generated by performing the reaction in pure $\mathrm{CDCl}_{3}$ within $1 \mathrm{~h}$.

IR (neat) $v_{\max }=3656,3410,3054,2923,2852,1621,1592,1509,1480,1436,1417,1330,1263,1227$, 1101, 1080, 1052, 997, 967, 950, 869, 850, 816, 749, 712, 694.

HRMS (ESI): $\mathrm{m} / \mathrm{z}$ calcd for $\mathrm{C}_{40} \mathrm{H}_{29} \mathrm{AuNO}_{4} \mathrm{P}_{2}{ }^{+} 846.1232[\mathrm{M}+\mathrm{H}+\mathrm{MeCN}]^{+}$, found, 846.1232;

HRMS (ESI): $m / z$ calcd for $\mathrm{C}_{39} \mathrm{H}_{26} \mathrm{AuO}_{6} \mathrm{P}_{2}-849.0876$ [M+HCOO]', found, 849.0867.

$[\alpha]_{\mathrm{D}}=+39\left(c 1.12, \mathrm{CHCl}_{3}\right)$.

\section{-NMR data in Pyridine-d5}

${ }^{1}{ }_{\mathrm{H}}$ NMR (Pyridine, $\left.500 \mathrm{MHz}\right) \delta=8.12-7.98(\mathrm{~m}, 3 \mathrm{H}), 7.92(\mathrm{~d}, J=8.9 \mathrm{~Hz}, 1 \mathrm{H}), 7.89(\mathrm{~d}, J=8.2 \mathrm{~Hz}, 1 \mathrm{H}), 7.82-$ $7.74(\mathrm{~m}, 3 \mathrm{H}), 7.70\left(\mathrm{td}, J_{1}=7.6 \mathrm{~Hz}, J_{2}=1.8 \mathrm{~Hz}, 2 \mathrm{H}\right), 7.57(\mathrm{~d}, J=9.2 \mathrm{~Hz}, 1 \mathrm{H}), 7.50-7.44(\mathrm{~m}, 3 \mathrm{H}), 7.44-7.32(\mathrm{~m}$, 7H), 7.30-7.27 (m, 1H), 7.20-7.08 (m, 4H).

${ }^{13}$ C NMR (Pyridine, $\left.125 \mathrm{MHz}\right) \delta=152.0\left(\mathrm{~d}, J_{c-p}=9.2 \mathrm{~Hz}\right), 149.4\left(\mathrm{~d}, J_{c-p}=8.2 \mathrm{~Hz}\right), 144.8\left(\mathrm{~d}, J_{c-p}=14.7 \mathrm{~Hz}\right)$, 136.4, $135.8\left(\mathrm{~d}, J_{c-p}=13.7 \mathrm{~Hz}\right), 135.6,135.4\left(\mathrm{~d}, J_{c-p}=5.5 \mathrm{~Hz}\right), 134.7\left(\mathrm{~d}, J_{c-p}=12.8 \mathrm{~Hz}\right), 134.2\left(\mathrm{~d}, J_{c-p}=8.2 \mathrm{~Hz}\right)$, $133.8,133.2,133.1,133.1\left(\mathrm{~d}, J_{c-p}=128.3 \mathrm{~Hz}\right), 132.1,131.8\left(\mathrm{~d}, J_{c-p}=45.8 \mathrm{~Hz}\right), 130.9,130.5\left(\mathrm{~d}, J_{c-p}=11.9 \mathrm{~Hz}\right)$, $130.4\left(\mathrm{~d}, J_{c-p}=79.7 \mathrm{~Hz}\right), 129.9,129.9,129.6,129.3,129.0,128.7\left(\mathrm{~d}, J_{c-p}=9.2 \mathrm{~Hz}\right), 127.9,127.4,127.1,126.7$, 
$125.9,125.0,124.9\left(\mathrm{~d}, J_{c-p}=127.4 \mathrm{~Hz}\right), 123.8,123.5,122.8$.

${ }^{31}$ P NMR (Pyridine, $200 \mathrm{MHz}$ ) $\delta=22.9,8.6$.

-NMR data in $\mathrm{CDCl}_{3}$

${ }^{1} \mathrm{H}$ NMR $\left(\mathrm{CDCl}_{3}, 500 \mathrm{MHz}\right) \delta=7.80(\mathrm{~d}, J=8.2 \mathrm{~Hz}, 1 \mathrm{H}), 7.76(\mathrm{~d}, J=8.9 \mathrm{~Hz}, 1 \mathrm{H}), 7.65(\mathrm{brs}, 2 \mathrm{H}), 7.52-7.23$ (m, 16H), 7.20-7.10 (m, 2H), 7.00-6.85 (m, 3H).

${ }^{31} \mathrm{P}$ NMR $\left(\mathrm{CDCl}_{3}, 200 \mathrm{MHz}\right) \delta=21.8,8.3$.

- $\quad$ Synthesis of (S)-(2-(2,2'-diyl hydrogen phosphate-[1,1'-binaphthalen]-3-yl)phenyl) diphenylphosphine gold(I) bis(trifluoromethanesulfonyl)imide (S)-7a<smiles>[14CH2]Pc1ccccc1-c1cc2ccccc2c2c1OP(=O)(O)Oc1ccc3ccccc3c1-2</smiles>

To a suspension of $\operatorname{AgNTf}_{2}(16.8 \mathrm{mg}, 0.030 \mathrm{mmol})$ in toluene $(1 \mathrm{~mL}),(\boldsymbol{S})-6$ (16.8 mg, $\left.0.020 \mathrm{mmol}\right)$ was added. The mixture was stirred overnight at room temperature. Then the suspension was filtered on celite. The filter was rinsed with DCM and evaporated under vacuum. The product was obtained as an amorphous gray solid (17.8 $\mathrm{mg}, 0.016 \mathrm{mmol}, 82 \%)$.

IR (neat) $v_{\max }=3061,1592,1438,1401,1353,1209,1198,1131,1082,1060,1028,958,900,830,751$. $[\alpha]_{D}=+170$ (c 1.16, $\mathrm{CHCl}_{3}$ ). HRMS (ESI): $\mathrm{m} / z$ calcd for $\mathrm{C}_{40} \mathrm{H}_{29} \mathrm{AuNO}_{4} \mathrm{P}_{2}{ }^{+} 846.1232\left[\mathrm{M}-\mathrm{NTf}{ }_{2}+\mathrm{H}+\mathrm{MeCN}\right]^{+}$, found, 846.1224 .

\section{-NMR data in $\mathrm{CDCl}_{3}$}

${ }^{1} \mathrm{H}$ NMR $\left(\mathrm{CDCl}_{3}, 500 \mathrm{MHz}\right) \delta=8.32(\mathrm{~s}, 1 \mathrm{H}), 8.17(\mathrm{~d}, J=8.2 \mathrm{~Hz}, 1 \mathrm{H}), 7.95(\mathrm{~d}, J=8.2 \mathrm{~Hz}, 1 \mathrm{H}), 7.92(\mathrm{~d}, J=$ $8.8 \mathrm{~Hz}, 1 \mathrm{H}), 7.65-7.59(\mathrm{~m}, 2 \mathrm{H}), 7.59-7.52(\mathrm{~m}, 3 \mathrm{H}), 7.52-7.37(\mathrm{~m}, 6 \mathrm{H}), 7.37-7.28(\mathrm{~m}, 3 \mathrm{H}), 7.27-7.18(\mathrm{~m}, 3 \mathrm{H})$, $6.96\left(\mathrm{dd}, J_{1}=12.3 \mathrm{~Hz}, J_{2}=7.9 \mathrm{~Hz}, 1 \mathrm{H}\right), 6.60-6.52(\mathrm{~m}, 1 \mathrm{H}), 6.50-6.38(\mathrm{~m}, 2 \mathrm{H}), 5.69(\mathrm{bs}, 1 \mathrm{H})$.

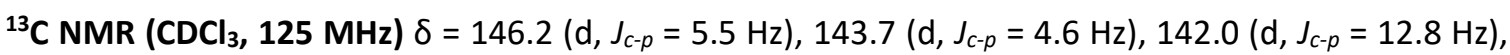
$135.3\left(\mathrm{~d}, J_{c-p}=14.7 \mathrm{~Hz}\right), 134.4\left(\mathrm{~d}, J_{c-p}=13.7 \mathrm{~Hz}\right), 133.3\left(\mathrm{~d}, J_{c-p}=7.3 \mathrm{~Hz}\right), 133.1\left(\mathrm{~d}, J_{c-p}=8.2 \mathrm{~Hz}\right), 132.6\left(\mathrm{~d}, J_{c-p}\right.$ $=108.1 \mathrm{~Hz}$ ), $132.5\left(\mathrm{~d}, J_{c-p}=1.8 \mathrm{~Hz}\right), 132.5,131.6,131.5\left(\mathrm{~d}, J_{c-p}=46.7 \mathrm{~Hz}\right), 131.5\left(\mathrm{~d}, J_{c-p}=1.8 \mathrm{~Hz}\right), 131.4,130.7$ $\left(d, J_{c-p}=6.4 \mathrm{~Hz}\right), 129.8\left(\mathrm{~d}, J_{c-p}=12.8 \mathrm{~Hz}\right), 129.3,129.0,128.9,128.9,128.6,128.0,127.8\left(\mathrm{~d}, J_{c-p}=20.2 \mathrm{~Hz}\right)$, $127.8,127.4,127.3\left(\mathrm{~d}, J_{c-p}=20.2 \mathrm{~Hz}\right), 127.0,126.7,126.4,124.4\left(\mathrm{~d}, J_{c-p}=67.8 \mathrm{~Hz}\right), 123.4,121.6\left(\mathrm{~d}, J_{c-p}=103.6\right.$ $\mathrm{Hz}), 120.8,119.5,118.3$.

${ }^{31} \mathrm{P}$ NMR $\left(\mathrm{CDCl}_{3}, 200 \mathrm{MHz}\right) \delta=22.9,2.9$.

-NMR data in deuterated pyridine

${ }^{1} \mathrm{H}$ NMR (Pyridine-d5, 500 MHz) $\delta=11.45(\mathrm{br} \mathrm{s}, 1 \mathrm{H}), 8.05-7.97(\mathrm{~m}, 3 \mathrm{H}), 7.93-7.80(\mathrm{~m}, 3 \mathrm{H}), 7.75\left(\mathrm{td}, J_{1}\right.$ 
$\left.=7.6 \mathrm{~Hz}, J_{2}=1.8 \mathrm{~Hz}, 1 \mathrm{H}\right), 7.69-7.28(\mathrm{~m}, 15 \mathrm{H}), 7.25(\mathrm{~d}, J=7.5 \mathrm{~Hz}, 1 \mathrm{H}), 7.19(\mathrm{~d}, J=7.6 \mathrm{~Hz}, 1 \mathrm{H}), 7.11(\mathrm{~d}, J=8.5$ $\mathrm{Hz}, 1 \mathrm{H})$.

${ }^{31}$ P NMR (Pyridine-d5, 200 MHz) $\delta=22.8,7.6$ (major isomer), 27.9, 7.1 (minor isomer).

\section{Synthesis of SI-2}<smiles></smiles>

(S)-(2-(2,2'-diylchlorogenphosphate-[1,1'-binaphthalen]-3-yl)phenyl) diphenylphosphine gold(I) chloride [SI-1] (122 mg, $0.14 \mathrm{mmol}, 1.0$ equiv.) was added to methanol $(5 \mathrm{~mL})$, the result solution was then stirred overnight at $35{ }^{\circ} \mathrm{C}$. The methanol was removed and the crude product was purified by flash column chromatography, giving product as a white solid $(69.0 \mathrm{mg}, 0.08 \mathrm{mmol}, 57 \%)$.

IR (neat) $\mathbf{v}_{\max }=3056,2958,2923,2856,1620,1592,1437,1300,1218,1187,1101,1051,993,974$, $955,892,829,750,694 .{ }^{1} \mathbf{H ~ N M R}\left(\mathrm{CDCl}_{3}, 500 \mathrm{MHz}\right) \delta=8.27(\mathrm{~s}, 1 \mathrm{H}), 8.01-7.95(\mathrm{~m}, 3 \mathrm{H}), 7.93(\mathrm{~d}, J=8.2 \mathrm{~Hz}$, $1 \mathrm{H}), 7.71(\mathrm{t}, J=7.6 \mathrm{~Hz}, 1 \mathrm{H}), 7.56-7.47(\mathrm{~m}, 5 \mathrm{H}), 7.47-7.39(\mathrm{~m}, 4 \mathrm{H}), 7.39-7.31(\mathrm{~m}, 4 \mathrm{H}), 7.30-7.24(\mathrm{~m}, 1 \mathrm{H}), 7.20$ $(\mathrm{d}, J=8.5 \mathrm{~Hz}, 1 \mathrm{H}), 7.05\left(\mathrm{dd}, J_{1}=11.6 \mathrm{~Hz}, J_{2}=7.9 \mathrm{~Hz}, 1 \mathrm{H}\right), 6.82-6.72(\mathrm{~m}, 3 \mathrm{H}), 3.66(\mathrm{~d}, J=11.7 \mathrm{~Hz}, 3 \mathrm{H}) .{ }^{13} \mathrm{C} \mathrm{NMR}$ $\left(\mathrm{CDCl}_{3}, 125 \mathrm{MHz}\right) \delta=147.0\left(\mathrm{~d}, J_{c-p}=11.0 \mathrm{~Hz}\right), 143.6\left(\mathrm{~d}, J_{c-p}=8.2 \mathrm{~Hz}\right), 141.6\left(\mathrm{~d}, J_{c-p}=12.8 \mathrm{~Hz}\right), 134.8\left(\mathrm{~d}, J_{c-p}=\right.$ $8.2 \mathrm{~Hz}), 134.6\left(\mathrm{~d}, J_{c-p}=9.2 \mathrm{~Hz}\right), 134.4\left(\mathrm{~d}, J_{c-p}=8.2 \mathrm{~Hz}\right), 133.4,133.2\left(\mathrm{~d}, J_{c-p}=7.3 \mathrm{~Hz}\right), 132.5\left(\mathrm{~d}, J_{c-p}=43.1 \mathrm{~Hz}\right)$, $131.9\left(\mathrm{~d}, J_{c-p}=1.8 \mathrm{~Hz}\right), 131.7\left(\mathrm{~d}, J_{c-p}=1.8 \mathrm{~Hz}\right), 131.6\left(\mathrm{~d}, J_{c-p}=2.7 \mathrm{~Hz}\right), 131.3,131.1,131.1,131.0,130.5\left(\mathrm{~d}, J_{c-}\right.$ $p=143.0 \mathrm{~Hz}$ ), 129.5, 129.4, 129.3, 128.8, 128.7, 128.6, 128.6, 128.2, 127.8 (d, $J_{c-p}=135.6 \mathrm{~Hz}$ ), 127.7, 127.6, 127.2, 126.8, $126.4\left(\mathrm{~d}, J_{c-p}=64.2 \mathrm{~Hz}\right), 122.2,121.3,119.7\left(\mathrm{~d}, J_{c-p}=2.7 \mathrm{~Hz}\right), 55.8\left(\mathrm{~d}, J_{c-p}=5.5 \mathrm{~Hz}\right) .{ }^{31} \mathrm{P}$ NMR $\left(\mathrm{CDCl}_{3}, 200 \mathrm{MHz}\right) \delta=27.2$, 1.2. $\mathrm{HRMS}$ (ESI): $\mathrm{m} / \mathrm{z}$ calcd for $\mathrm{C}_{41} \mathrm{H}_{31} \mathrm{AuNO}_{4} \mathrm{P}_{2}{ }^{+} 860.1388$ [M+MeCN-Cl$]^{+}$, found,

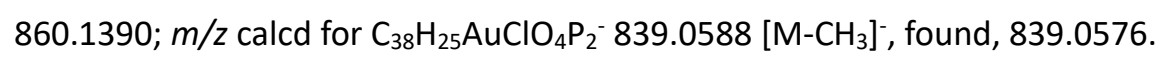

\section{Synthesis of $(S)-11$ a}<smiles></smiles>

SI-2 (16.7mg, $0.02 \mathrm{mmol}, 1.0$ equiv.) and $\mathrm{AgNTf}_{2}(7.5 \mathrm{mg}, 0.02 \mathrm{mmol}, 1.0$ equiv.) were added to $1 \mathrm{~mL}$ $\mathrm{CDCl}_{3}$, the resulting solution was then stirred at room temperature for $2 \mathrm{~h}$. The reaction solution was filtered and evaporated, giving product 11 as a white solid (18.8 $\mathrm{mg}, 0.02 \mathrm{mmol}, 79 \%$ ).

IR (neat) $v_{\max }=3059,2961,2927,2856,1537,1438,1400,1354,1300,1261,1210,1193,1131,1102$, 
1055, 975, 957, 895, 830, 751, 693. ${ }^{1} \mathrm{H}$ NMR (CDCl $\left.3,500 \mathrm{MHz}\right) \delta=8.35(\mathrm{~s}, 1 \mathrm{H}), 8.15(\mathrm{~d}, J=8.2 \mathrm{~Hz}, 1 \mathrm{H}), 7.96$ $(\mathrm{d}, J=8.8 \mathrm{~Hz}, 1 \mathrm{H}), 7.95(\mathrm{~d}, J=7.6 \mathrm{~Hz}, 1 \mathrm{H}), 7.85(\mathrm{t}, J=6.3 \mathrm{~Hz}, 1 \mathrm{H}), 7.74(\mathrm{t}, J=7.6 \mathrm{~Hz}, 1 \mathrm{H}), 7.59(\mathrm{t}, J=7.6 \mathrm{~Hz}$, $1 \mathrm{H}), 7.55(\mathrm{t}, J=7.6 \mathrm{~Hz}, 1 \mathrm{H}), 7.55(\mathrm{~s}, 1 \mathrm{H}), 7.51-7.42(\mathrm{~m}, 5 \mathrm{H}), 7.38(\mathrm{t}, J=7.6 \mathrm{~Hz}, 1 \mathrm{H}), 7.36(\mathrm{t}, J=7.6 \mathrm{~Hz}, 1 \mathrm{H})$, $7.29(\mathrm{~d}, J=8.8 \mathrm{~Hz}, 2 \mathrm{H}), 7.27-7.21(\mathrm{~m}, 3 \mathrm{H}), 6.98\left(\mathrm{dd}, J_{1}=12.6 \mathrm{~Hz}, J_{2}=7.9 \mathrm{~Hz}, 1 \mathrm{H}\right), 6.58(\mathrm{t}, J=6.9 \mathrm{~Hz}, 1 \mathrm{H}), 6.48$ $(\mathrm{t}, J=6.6 \mathrm{~Hz}, 2 \mathrm{H}), 3.71(\mathrm{~d}, J=11.7 \mathrm{~Hz}, 3 \mathrm{H}) .{ }^{13} \mathrm{C} \mathrm{NMR}\left(\mathrm{CDCl}_{3}, 125 \mathrm{MHz}\right) \delta=146.9\left(\mathrm{~d}, J_{c-p}=11.0 \mathrm{~Hz}\right), 143.2(\mathrm{~d}$, $\left.J_{c-p}=8.2 \mathrm{~Hz}\right), 141.9\left(\mathrm{~d}, J_{c-p}=12.8 \mathrm{~Hz}\right), 135.2\left(\mathrm{~d}, J_{c-p}=15.6 \mathrm{~Hz}\right), 134.4\left(\mathrm{~d}, J_{c-p}=13.7 \mathrm{~Hz}\right), 134.2\left(\mathrm{~d}, J_{c-p}=7.3 \mathrm{~Hz}\right)$, $132.9\left(\mathrm{~d}, J_{c-p}=9.2 \mathrm{~Hz}\right), 132.8,132.6\left(\mathrm{~d}, J_{c-p}=106.3 \mathrm{~Hz}\right), 132.4\left(\mathrm{~d}, J_{c-p}=2.7 \mathrm{~Hz}\right), 131.9\left(\mathrm{~d}, J_{c-p}=2.7 \mathrm{~Hz}\right), 131.8$ $\left(d, J_{c-p}=1.8 \mathrm{~Hz}\right), 131.6,131.3,131.3,130.6\left(\mathrm{q}, J_{c-p}=2.7 \mathrm{~Hz}\right), 129.7\left(\mathrm{~d}, J_{c-p}=11.9 \mathrm{~Hz}\right), 129.1,128.9\left(\mathrm{~d}, J_{c-p}=\right.$ $10.1 \mathrm{~Hz}), 128.8\left(\mathrm{~d}, J_{c-p}=12.8 \mathrm{~Hz}\right), 128.4,128.1,128.0,127.7\left(\mathrm{~d}, J_{c-p}=54.1 \mathrm{~Hz}\right), 127.3,127.3,126.9,126.9$, $126.2,124.8\left(\mathrm{~d}, J_{c-p}=67.8 \mathrm{~Hz}\right), 122.2,121.0,120.8,119.6\left(\mathrm{~d}, J_{c-p}=2.7 \mathrm{~Hz}\right), 118.3,55.5\left(\mathrm{~d}, J_{c-p}=4.6 \mathrm{~Hz}\right) .{ }^{31} \mathbf{p}$ NMR (CDCl, $200 \mathrm{MHz}) \delta=23.0,2.3$. HRMS (ESI): $\mathrm{m} / z$ calcd for $\mathrm{C}_{41} \mathrm{H}_{31} \mathrm{AuNO}_{4} \mathrm{P}_{2}{ }^{+} 860.1388\left[\mathrm{M}+\mathrm{MeCN}-\mathrm{NTf}_{2}\right]^{+}$, found, 860.1387. $[\alpha]_{D}=+28\left(c 1.83, \mathrm{CHCl}_{3}\right)$.

\section{Synthesis of enones 8 and indole 9}

\section{Synthesis of 2-iodocyclohex-2-en-1-one (SI-3) ${ }^{4}$}

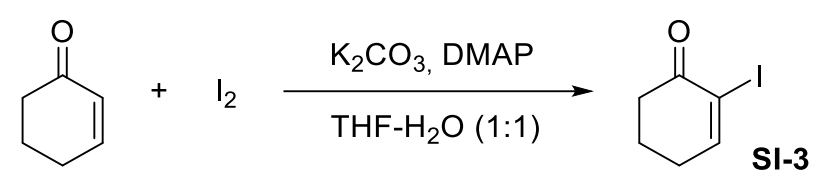

To a stirred solution of cyclohex-2-en-1-one ( $4.05 \mathrm{~mL}, 42 \mathrm{mmol}, 1.0$ equiv.) in a 1:1 mixture of THF- $\mathrm{H}_{2} \mathrm{O}$ (200 mL) was added $\mathrm{K}_{2} \mathrm{CO}_{3}\left(6.9 \mathrm{~g}, 50 \mathrm{mmol}, 1.2\right.$ equiv.), $\mathrm{I}_{2}(15.9 \mathrm{~g}, 63 \mathrm{mmol}, 1.5$ equiv.) and 4dimethylaminopyridine (DMAP) $(1.02 \mathrm{~g}, 8.3 \mathrm{mmol}, 0.2$ equiv.) successively. Then the reaction was stirred at room temperature for $2 \mathrm{~h}$. Upon completion, the reaction solution mixture was diluted with ethyl acetate and washed with saturated aqueous $\mathrm{Na}_{2} \mathrm{~S}_{2} \mathrm{O}_{3}$ and aqueous $0.1 \mathrm{M} \mathrm{HCl}$ successively. The mixture was subsequently extracted with ethyl acetate and dried over $\mathrm{MgSO}_{4}$. Crude product was purified by flash column chromatography (heptane to $20 \%$ EtOAc-Heptane), giving product SI-3 as a white solid $(7.17 \mathrm{~g}, 32$ $\mathrm{mmol}, 77 \%)$.

Synthesis of 2-iodocyclopent-2-en-1-one ${ }^{4,5}$ (SI-4)

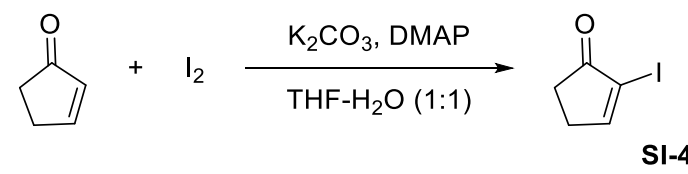

Compound SI-4 was prepared by following the general procedure for the synthesis of SI-3. To a stirred

\footnotetext{
${ }^{4}$ (a) T. Yao; Zhang, X.; Larock, R. C. J. Am. Chem. Soc. 2004, 126, 11164-11165; (b) M. Krafft; Cran, J. Synlett 2005, 1263-1266; (c) L.-E. Harim; Moises, R.-O.; F., O. H. Eur. J. Org. Chem. 2016, 51-54.

${ }^{5}$ H. Sundén; Schäfer, A.; Scheepstra, M.; Leysen, S.; Malo, M.; Ma, J.-N.; Burstein, E. S.; Ottmann, C.; Brunsveld, L.; Olsson, R. J. Med. Chem. 2016, 59, 1232-1238.
} 
solution of cyclopent-2-en-1-one $\left(0.42 \mathrm{~mL}, 5 \mathrm{mmol}, 1.0\right.$ equiv.) in a 1:1 mixture of THF- $\mathrm{H}_{2} \mathrm{O}(30 \mathrm{~mL})$ was added $\mathrm{K}_{2} \mathrm{CO}_{3}\left(0.83 \mathrm{~g}, 6 \mathrm{mmol}, 1.2\right.$ equiv.), $\mathrm{I}_{2}(1.9 \mathrm{~g}, 7.5 \mathrm{mmol}, 1.5$ equiv.) and 4-dimethylaminopyridine (DMAP) ( $0.13 \mathrm{~g}, 1 \mathrm{mmol}, 0.2$ equiv.) successively. Then the reaction was stirred at room temperature for 2 h. Upon completion, the reaction solution mixture was diluted with ethyl acetate and washed with saturated aqueous $\mathrm{Na}_{2} \mathrm{~S}_{2} \mathrm{O}_{3}$ and aqueous $0.1 \mathrm{M} \mathrm{HCl}$ successively. The mixture was subsequently extracted with ethyl acetate and dried over $\mathrm{MgSO}_{4}$. Crude product was purified by flash column chromatography (heptane to $20 \%$ EtOAc-Heptane), giving product SI-4 as a pale yellow solid (0.57 g, $2.7 \mathrm{mmol}, 55 \%$ ).

Synthesis of 2-iodocyclohept-2-en-1-one ${ }^{4,5}$ (SI-5)

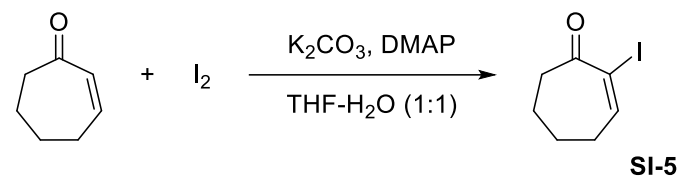

Compound SI-5 was prepared by following the general procedure for the synthesis of SI-3. To a stirred solution of cyclopent-2-en-1-one ( $0.34 \mathrm{~mL}, 3.0 \mathrm{mmol}, 1.0$ equiv.) in a 1:1 mixture of THF- $\mathrm{H}_{2} \mathrm{O}(14 \mathrm{~mL})$ was added $\mathrm{K}_{2} \mathrm{CO}_{3}\left(0.50 \mathrm{~g}, 3.6 \mathrm{mmol}, 1.2\right.$ equiv.), $\mathrm{I}_{2}(1.14 \mathrm{~g}, 4.5 \mathrm{mmol}, 1.5$ equiv.) and 4-dimethylaminopyridine (DMAP) ( $0.08 \mathrm{~g}, 0.6 \mathrm{mmol}, 0.2$ equiv.) successively. Then the reaction was stirred at room temperature for $2 \mathrm{~h}$. Upon completion, the reaction solution mixture was diluted with ethyl acetate and washed with saturated aqueous $\mathrm{Na}_{2} \mathrm{~S}_{2} \mathrm{O}_{3}$ and aqueous $0.1 \mathrm{M} \mathrm{HCl}$ successively. The mixture was subsequently extracted with ethyl acetate and dried over $\mathrm{MgSO}_{4}$. Crude product was purified by flash column chromatography (heptane to 20 \% EtOAc-Heptane), giving product SI-5 as a pale yellow oil (0.37 g, $1.6 \mathrm{mmol}, 52 \%$ ).

Synthesis of 2-(phenylethynyl)cyclohex-2-en-1-one ${ }^{4}(8 a)$

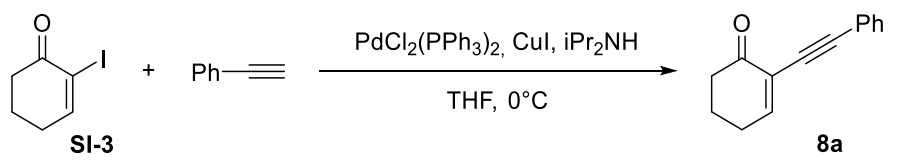

A solution of 2-iodo-cyclohexenone (430.0 mg, $1.94 \mathrm{mmol}, 1.0$ equiv.) in THF (5.1 mL) was treated with $\mathrm{PdCl}_{2}\left(\mathrm{PPh}_{3}\right)_{2}(69.0 \mathrm{mg}, 0.1 \mathrm{mmol}, 5 \mathrm{~mol} \%)$ and Cul $(37.0 \mathrm{mg}, 0.19 \mathrm{mmol}, 10 \mathrm{~mol} \%)$ and cooled down to 0 ${ }^{\circ} \mathrm{C}$ under an $\mathrm{Ar}$ atmosphere and in the dark. After $10 \mathrm{~min}$ of stirring, phenylacetylene $(0.44 \mathrm{~mL}, 409.2 \mathrm{mg}$, $4.01 \mathrm{mmol}$ ) and diisopropyl amine $(0.82 \mathrm{~mL}, 59.0 \mathrm{mg}, 5.85 \mathrm{mmol}$ ) were added, and the resulting yellow to dark brown solution was stirred at $0{ }^{\circ} \mathrm{C}$. The reaction mixture was partitioned between $\mathrm{CH}_{2} \mathrm{Cl}_{2}$ and $0.5 \mathrm{~N}$ aqueous $\mathrm{HCl}$ solution. The aqueous layer was extracted three times with $\mathrm{CH}_{2} \mathrm{Cl}_{2}$ and the combined organic phases were washed with brine, dried over $\mathrm{MgSO}_{4}$, filtered and concentrated under vacuum. The crude product was purified by flash column chromatography to yield alkyne $8 \mathrm{a}$ as a yellow solid (334 mg, 1.75 $\mathrm{mmol}, 88 \%)$. 


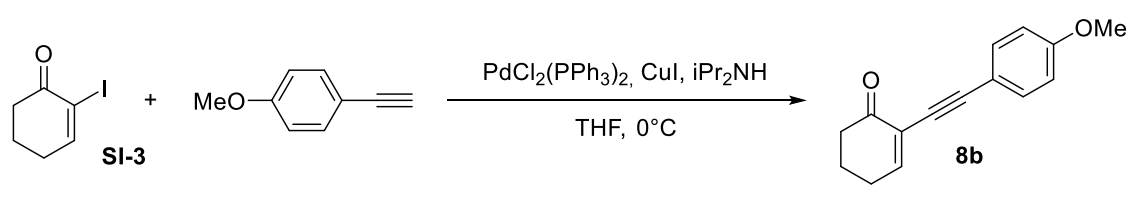

Compound $\mathbf{8} \mathbf{b}$ was prepared by following the general procedure for the synthesis of $\mathbf{8} \mathbf{a}$. A solution of 2-iodo-cyclohexenone (444.0 mg, $2 \mathrm{mmol}, 1.0$ equiv.) in THF (5 mL) was treated with $\mathrm{PdCl}_{2}\left(\mathrm{PPh}_{3}\right)_{2}(70.2 \mathrm{mg}$, $0.1 \mathrm{mmol}, 5 \mathrm{~mol} \%)$ and $\mathrm{Cul}(38.1 \mathrm{mg}, 0.2 \mathrm{mmol}, 10 \mathrm{~mol} \%)$ and cooled down to $0{ }^{\circ} \mathrm{C}$ under an Ar atmosphere. After $10 \mathrm{~min}$ of stirring, 1-ethynyl-4-methoxybenzene (528.6 mg, $4 \mathrm{mmol}, 2$ equiv.) and diisopropyl amine ( $0.87 \mathrm{~mL}, 627.4 \mathrm{mg}, 6.2 \mathrm{mmol}$ ) were added, and the resulting solution was stirred at $0{ }^{\circ} \mathrm{C}$. The reaction mixture was partitioned between $\mathrm{CH}_{2} \mathrm{Cl}_{2}$ and $0.5 \mathrm{~N}$ aqueous $\mathrm{HCl}$ solution. The aqueous layer was extracted three times with $\mathrm{CH}_{2} \mathrm{Cl}_{2}$ and the combined organic phases were washed with brine, dried over $\mathrm{MgSO}_{4}$, filtered and concentrated under vacuum. The crude product was purified by flash column chromatography to yield alkyne $8 \mathrm{~b}$ as a brown solid $(148.7 \mathrm{mg}, 0.66 \mathrm{mmol}, 33 \%)$.

Synthesis of 2-(phenylethynyl)cyclohex-2-en-1-one ${ }^{5}(8 c)$

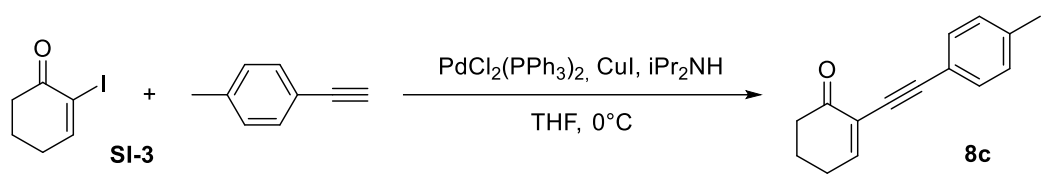

Compound $\mathbf{8 c}$ was prepared by following the general procedure for the synthesis of $\mathbf{8 a}$. A solution of 2-iodo-cyclohexenone (444.0 mg, 2 mmol, 1.0 equiv.) in THF (5 mL) was treated with $\mathrm{PdCl}_{2}\left(\mathrm{PPh}_{3}\right)_{2}(70.2 \mathrm{mg}$, $0.1 \mathrm{mmol}, 5 \mathrm{~mol} \%)$ and $\mathrm{Cul}(38.1 \mathrm{mg}, 0.2 \mathrm{mmol}, 10 \mathrm{~mol} \%)$ and cooled down to $0{ }^{\circ} \mathrm{C}$ under an Ar atmosphere. After 10 min of stirring, 1-ethynyl-4-methylbenzene ( $464.6 \mathrm{mg}, 4 \mathrm{mmol}, 2$ equiv.) and diisopropyl amine $(0.87 \mathrm{~mL}, 627.4 \mathrm{mg}, 6.2 \mathrm{mmol})$ were added, and the resulting solution was stirred at $0{ }^{\circ} \mathrm{C}$. The reaction mixture was partitioned between $\mathrm{CH}_{2} \mathrm{Cl}_{2}$ and $0.5 \mathrm{~N}$ aqueous $\mathrm{HCl}$ solution. The aqueous layer was extracted three times with $\mathrm{CH}_{2} \mathrm{Cl}_{2}$ and the combined organic phases were washed with brine, dried over $\mathrm{MgSO}_{4}$, filtered and concentrated under vacuum. The crude product was purified by flash column chromatography to yield alkyne $8 \mathrm{c}$ as a gray solid (313.9 $\mathrm{mg}, 1.49 \mathrm{mmol}, 75 \%$ ).

Synthesis of 2-(phenylethynyl)cyclohex-2-en-1-one (8d) ${ }^{7}$

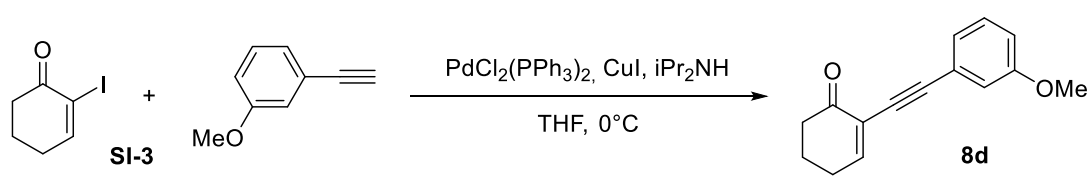

Compound $\mathbf{8} \mathbf{d}$ was prepared by following the general procedure for the synthesis of $\mathbf{8 a}$. A solution of 2-iodo-cyclohexenone (444.0 mg, $2 \mathrm{mmol}, 1.0$ equiv.) in THF (5 mL) was treated with $\mathrm{PdCl}_{2}\left(\mathrm{PPh}_{3}\right)_{2}(70.2 \mathrm{mg}$, $0.1 \mathrm{mmol}, 5 \mathrm{~mol} \%)$ and $\mathrm{Cul}\left(38.1 \mathrm{mg}, 0.2 \mathrm{mmol}, 10 \mathrm{~mol} \%\right.$ ) and cooled down to $0{ }^{\circ} \mathrm{C}$ under an Ar atmosphere.

\footnotetext{
${ }^{6}$ Rauniyar, V.; Wang, Z. J.; Burks, H. E.; Toste, F. D., J. Am. Chem. Soc. 2011, 133, 8486-8489.

7 Cho, C.-H.; Larock, R. C. ACS Comb. Sci. 2011, 13, 272-279.
} 
After $10 \mathrm{~min}$ of stirring, 1-ethynyl-4-methoxybenzene (528.6 mg, $4 \mathrm{mmol}, 2$ equiv.) and diisopropyl amine ( $0.87 \mathrm{~mL}, 627.4 \mathrm{mg}, 6.2 \mathrm{mmol}$ ) were added, and the resulting solution was stirred at $0{ }^{\circ} \mathrm{C}$. The reaction mixture was partitioned between $\mathrm{CH}_{2} \mathrm{Cl}_{2}$ and $0.5 \mathrm{~N}$ aqueous $\mathrm{HCl}$ solution. The aqueous layer was extracted three times with $\mathrm{CH}_{2} \mathrm{Cl}_{2}$ and the combined organic phases were washed with brine, dried over $\mathrm{MgSO}_{4}$, filtered and concentrated under vacuum. The crude product was purified by flash column chromatography to yield alkyne $8 \mathrm{~d}$ as a brown solid (366.6 $\mathrm{mg}, 1.62 \mathrm{mmol}, 53 \%$ ).

\section{Synthesis of 2-(Heptane-1-yn-1-yl)cyclohex-2-en-1-one (8e $)^{8}$}

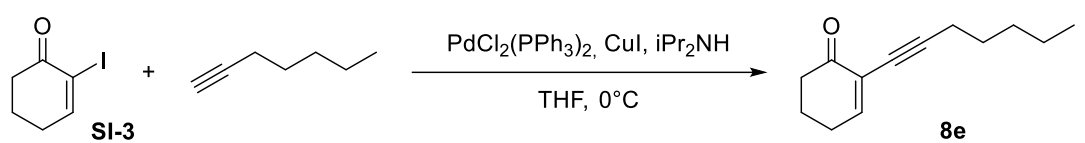

Compound $\mathbf{8} \mathbf{e}$ was prepared by following the general procedure for the synthesis of $\mathbf{8 a}$. A solution of 2-iodo-cyclohexenone (444 mg, $2 \mathrm{mmol}, 1.0$ equiv.) in THF (6 mL) was treated with $\mathrm{PdCl}_{2}\left(\mathrm{PPh}_{3}\right)_{2}(70.2 \mathrm{mg}$, $0.1 \mathrm{mmol}, 5 \mathrm{~mol} \%)$ and $\mathrm{Cul}(38.1 \mathrm{mg}, 0.2 \mathrm{mmol}, 10 \mathrm{~mol} \%)$ and cooled down to $0{ }^{\circ} \mathrm{C}$ under $\mathrm{Ar}$ atmosphere and in the dark. After $10 \mathrm{~min}$ of stirring, Hept-1-yne ( $0.53 \mathrm{~mL}, 4.0 \mathrm{mmol}, 2$ equiv.) and diisopropyl amine ( $0.83 \mathrm{~mL}, 6.3 \mathrm{mmol}, 3.1$ equiv.) were added, and the resulting yellow to dark brown solution was stirred from $0{ }^{\circ} \mathrm{C}$. The reaction mixture was partitioned between $\mathrm{CH}_{2} \mathrm{Cl}_{2}$ and $1.0 \mathrm{~N}$ aqueous $\mathrm{HCl}$ solution. The aqueous layer was extracted three times with $\mathrm{CH}_{2} \mathrm{Cl}_{2}$ and the combined organic phases were washed with brine, dried over $\mathrm{MgSO}_{4}$, filtered and concentrated under vacuum. The crude product was purified by flash column chromatography to yield product $8 \mathrm{e}$ as a white solid (231.9 mg, $1.2 \mathrm{mmol}, 59 \%$ ).

Synthesis of 2-(phenylethynyl)cyclopent-2-en-1-one ${ }^{9}$ (8f)

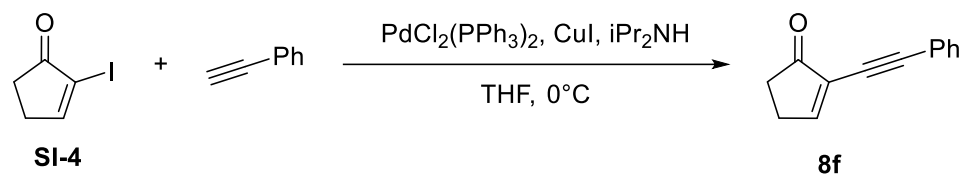

Compound $\mathbf{8 f}$ was prepared by following the general procedure for the synthesis of $\mathbf{8 a}$. A solution of 2iodocyclopent-2-en-1-one (186 mg, $0.89 \mathrm{mmol}, 1.0$ equiv.) in THF (3 mL) was treated with $\mathrm{PdCl}_{2}\left(\mathrm{PPh}_{3}\right)_{2}(31.4$ $\mathrm{mg}, 0.04 \mathrm{mmol}, 5 \mathrm{~mol} \%)$ and $\mathrm{Cul}(17.1 \mathrm{mg}, 0.09 \mathrm{mmol}, 10 \mathrm{~mol} \%)$ and cooled down to $0{ }^{\circ} \mathrm{C}$ under $\mathrm{Ar}$ atmosphere and in the dark. After $10 \mathrm{~min}$ of stirring, phenylacetylene $(0.2 \mathrm{~mL}, 1.79 \mathrm{mmol}, 2$ equiv.) and diisopropyl amine ( $0.39 \mathrm{~mL}, 2.77 \mathrm{mmol}, 3.1$ equiv.) were added, and the resulting yellow to dark brown solution was stirred from $0{ }^{\circ} \mathrm{C}$. The reaction mixture was partitioned between $\mathrm{CH}_{2} \mathrm{Cl}_{2}$ and $1.0 \mathrm{~N}$ aqueous $\mathrm{HCl}$ solution. The aqueous layer was extracted three times with $\mathrm{CH}_{2} \mathrm{Cl}_{2}$ and the combined organic phases were washed with brine, dried over $\mathrm{MgSO}_{4}$, filtered and concentrated under vacuum. The crude product was purified by flash column chromatography to yield product $8 \mathrm{f}$ as a pale yellow solid $(156.7 \mathrm{mg}, 0.86 \mathrm{mmol}$, $97 \%)$.

8 (a) Salacz, L.; Girard, N.; Suffert, J.; Blond, G. Molecules 2019, 24, 595. (b) Salacz, L.; Girard, N.; Blond, G.; Suffert, J. Org. Lett. 2018, 20, 3915-3918.

9 T. Yao; Zhang, X.; Larock, R. C. J. Org. Chem. 2005, 70, 7679-7685. 


\section{Synthesis of 2-(phenylethynyl)cyclohept-2-en-1-one ${ }^{9}(8 \mathrm{~g})$}

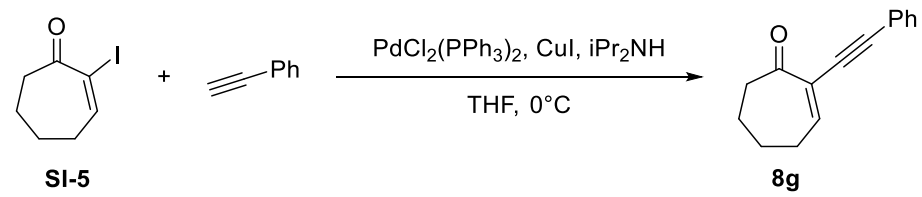

Compound $\mathbf{8 g}$ was prepared by following the general procedure for the synthesis of $\mathbf{8 a}$. A solution of 2-iodocyclopent-2-en-1-one ( $219.3 \mathrm{mg}, 0.93 \mathrm{mmol}, 1.0$ equiv.) in THF ( $3 \mathrm{~mL}$ ) was treated with $\mathrm{PdCl}_{2}\left(\mathrm{PPh}_{3}\right)_{2}$ (312.6 mg, $0.05 \mathrm{mmol}, 5 \mathrm{~mol} \%$ ) and $\mathrm{Cul}\left(17.7 \mathrm{mg}, 0.09 \mathrm{mmol}, 10 \mathrm{~mol} \%\right.$ ) and cooled down to $0{ }^{\circ} \mathrm{C}$ under $\mathrm{Ar}$ atmosphere and in the dark. After $10 \mathrm{~min}$ of stirring, phenylacetylene $(0.21 \mathrm{~mL}, 1.86 \mathrm{mmol}, 2$ equiv.) and diisopropyl amine ( $0.41 \mathrm{~mL}, 2.88 \mathrm{mmol}, 3.1$ equiv.) were added, and the resulting yellow to dark brown solution was stirred from $0{ }^{\circ} \mathrm{C}$. The reaction mixture was partitioned between $\mathrm{CH}_{2} \mathrm{Cl}_{2}$ and $1.0 \mathrm{~N}$ aqueous $\mathrm{HCl}$ solution. The aqueous layer was extracted three times with $\mathrm{CH}_{2} \mathrm{Cl}_{2}$ and the combined organic phases were washed with brine, dried over $\mathrm{MgSO}_{4}$, filtered and concentrated under vacuum. The crude product was purified by flash column chromatography to yield product $8 \mathrm{~g}$ as a pale yellow oil $(188.0 \mathrm{mg}, 0.89 \mathrm{mmol}, 96$ $\%)$.

Synthesis of 1-benzyl-1H-indole ${ }^{10}(9 \mathrm{~g})$

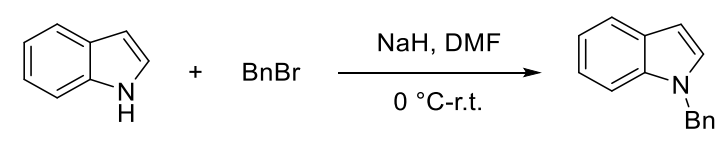

To a stirring solution of indole ( $352.5 \mathrm{mg}, 3 \mathrm{mmol}, 1$ equiv.) in dry DMF ( $3 \mathrm{~mL}$ ), NaH (144.0 mg, 3.6 mmol, 1.2 equiv.) was added portionwise under $\mathrm{N}_{2}$ at $0{ }^{\circ} \mathrm{C}$. The reaction mixture was then warmed to room temperature and stirred for $30 \mathrm{~min}$. After cooling to $0{ }^{\circ} \mathrm{C}, \mathrm{BnBr}(564.5 \mathrm{mg}, 3.3 \mathrm{mmol}, 1.1$ equiv.) (1.2 mL) was added dropewise to the reaction mixture. The resulting mixture was stirred at room temperature overnight. The reaction was quenched with water and extracted three times with ethyl acetate, the combined organic phases were dried over $\mathrm{MgSO}_{4}$, filtered and concentrated under vacuum. The crude product was purified by flash column chromatography to yield product $9 \mathrm{~g}$ as pale yellow oil $(553.4 \mathrm{mg}, 2.67$ mmol, $89 \%)$.

\section{Synthesis of 1,3-dimethyl-1H-indole ${ }^{11}$ (9i)}

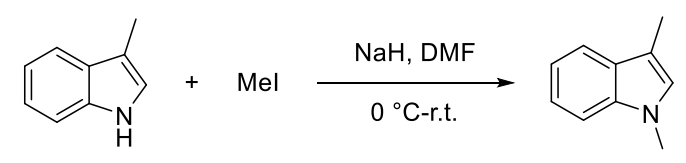

To a stirring solution of $60 \% \mathrm{NaH}(240 \mathrm{mg}, 6 \mathrm{mmol}, 2$ equiv.) in dry DMF (2.4 mL), 3-methylindole (393.5 mg, $3 \mathrm{mmol}, 1$ equiv.) in DMF (2.4) was added dropwise at $0{ }^{\circ} \mathrm{C}$. The mixture was allowed to warm to room temperature and stirred for $30 \mathrm{~min}$. After cooling to $0^{\circ} \mathrm{C}$, a solution of $\mathrm{Mel}(0.38 \mathrm{~mL}, 6 \mathrm{mmol}, 2$ equiv. $)$ in DMF (1.2 $\mathrm{mL})$ was added dropewise. The resulting mixture was stirred at room temperature overnight. The reaction was quenched with water and extracted three times with ethyl acetate, the combined organic

\footnotetext{
10 K. Nemoto; Tanaka, S.; Konno, M.; Onozawa, S.; Chiba, M.; Tanaka, Y.; Sasaki, Y.; Okubo, R.; Hattori, T. Tetrahedron 2016, 72, $734-745$.

11 C. Shao; Shi, G.; Zhang, Y.; Pan, S.; Guan, X. Org. Lett. 2015, 17, 2652-2655.
} 
phases were dried over $\mathrm{MgSO}_{4}$, filtered and concentrated under vacuum. The crude product was purified by flash column chromatography to yield product $9 \mathrm{i}$ as a clear oil (426.9 mg, $2.94 \mathrm{mmol}, 98 \%$ ).

\section{Synthesis of 3-isopropyl-1H-indole ${ }^{12}(9 k)$}

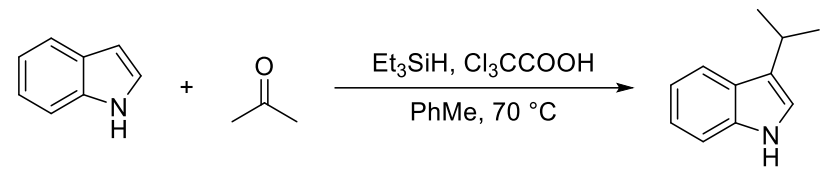

In a oven dried flask, triethylsilane $(5.78 \mathrm{~mL}, 36.0 \mathrm{mmol}, 2.4$ equiv.) and trichloroacetic acid ( $2.26 \mathrm{~g}$, $22.5 \mathrm{mmol}, 1.5$ equiv.) were dissolved in toluene $(7.50 \mathrm{~mL})$ and the resulting solution was heated to $70{ }^{\circ} \mathrm{C}$. A solution of indole ( $1.76 \mathrm{~g}, 15.0 \mathrm{mmol}, 1$ equiv.) and acetone $(1.23 \mathrm{~mL}, 16.5 \mathrm{mmol}, 1.1$ equiv.) in toluene $(7.50 \mathrm{~mL})$ were then added over $1 \mathrm{~h}$. After an additional $20 \mathrm{~min}$ at $70^{\circ} \mathrm{C}$ the solution was quenched with $10 \%$ aqueous solution of sodium carbonate, the organic layer was separated, dried over anhydrous sodium sulfate, concentrated under reduced pressure, and purified by column chromatography to afford indole $\mathbf{9 k}$ (1.61 $\mathrm{g}, 10.1 \mathrm{mmol}, 67 \%)$ as a colorless solid.

12 Y. Zhang; Stephens, D.; Hernandez, G.; Mendoza, R.; Larionov, O. V. Chem. Eur. J. 2012, 18, 16612-16615. 


\section{Influence of the protocol used for the activation of the catalysts}
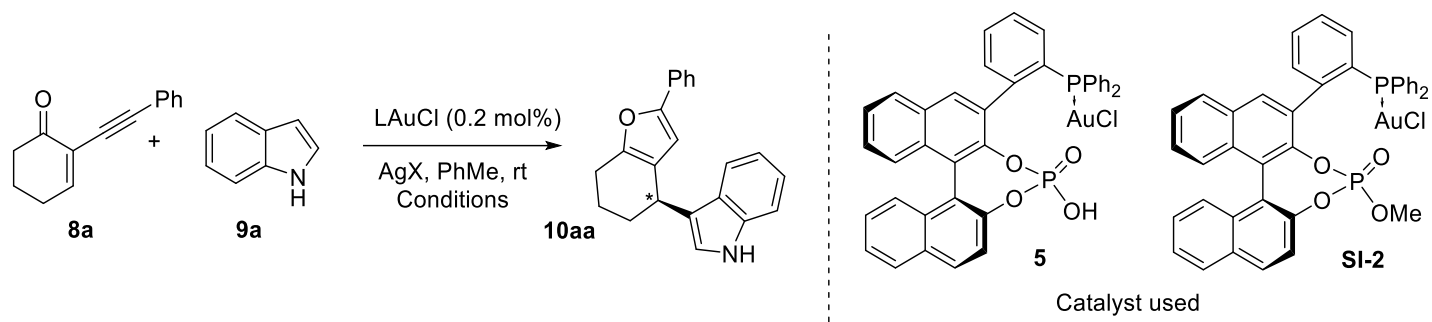

Protocol A: In situ activation of the active catalyst with the silver salt in the presence of all components of the reaction.

Protocol B: Activation of the active catalyst with the silver salt in $\mathrm{CDCl}_{3}$, filtration of the solution and use of the solution.

Protocol C: Formation of the active catalyst in $\mathrm{CDCl}_{3}$, filtration of the solution, evaporation and use of the resulting solid as the catalyst.

Experiments highlighted in grey are those reported within the manuscript.

Table S1: Influence of the protocol used for the activation of the precatalyst in the catalytic tests

\begin{tabular}{ccccccc} 
entry & $\mathrm{LAuCl}$ & $\mathrm{AgX}(\mathrm{mol} \%)$ & Actual catalyst & Yield (\%) & ee (\%) & protocol \\
\hline 1 & $(S)-5$ & $\mathrm{Ag}_{2} \mathrm{CO}_{3}(0.1)$ & $\mathbf{6}$ & 75 & 96 & $\mathrm{~A}$ \\
2 & $(S)-5$ & $\mathrm{Ag}_{2} \mathrm{CO}_{3}(0.1)$ & $\mathbf{6}$ & 61 & 79 & $\mathrm{~B}$ \\
3 & $(S)-5$ & $\mathrm{Ag}_{2} \mathrm{CO}_{3}(0.1)$ & $\mathbf{6}$ & 65 & 26 & $\mathrm{C}$ \\
4 & $(S)-5$ & $\mathrm{AgNTf}_{2}(0.2)$ & $\mathbf{7 a}$ & 71 & 7 & $\mathrm{~A}$ \\
5 & $(S)-5$ & $\mathrm{AgNTf}_{2}(0.2)$ & $\mathbf{7 a}$ & 85 & 29 & $\mathrm{~B}$ \\
6 & $(S)-5$ & $\mathrm{AgNTf}_{2}(0.2)$ & $\mathbf{7 a}$ & 80 & 60 & $\mathrm{C}$ \\
7 & $(S)-5$ & $\mathrm{AgSbF}_{6}(0.2)$ & $\mathbf{7 b}$ & 85 & 47 & $\mathrm{~A}$ \\
8 & $(S)-5$ & $\mathrm{AgSbF}_{6}(0.2)$ & $\mathbf{7 b}$ & 76 & 62 & $\mathrm{~B}$ \\
9 & $(S)-5$ & $\mathrm{AgSbF}_{6}(0.2)$ & $\mathbf{7 b}$ & 93 & 67 & $\mathrm{C}$ \\
10 & $\mathrm{SI}-\mathbf{2}$ & $\mathrm{AgNTf}_{2}(0.2)$ & $\mathbf{1 1 a}$ & 85 & 15 & $\mathrm{~B}$ \\
11 & $\mathrm{SI-2}$ & $\mathrm{AgSbF}_{6}(0.2)$ & $\mathbf{1 1 b}$ & 90 & 12 & $\mathrm{~B}$
\end{tabular}




\section{Scope of the reaction}

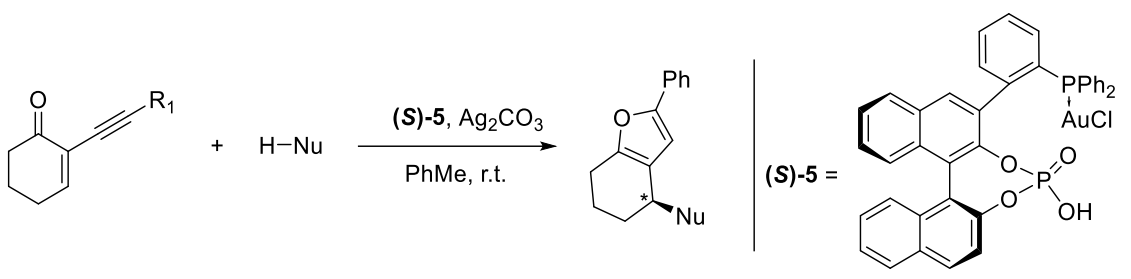

To a flask was added 2-(1-alkynyl)-2-alken-1-one ( $0.55 \mathrm{mmol}, 1.1$ equiv.), Nucleophile ( $\mathrm{NuH}, 0.5 \mathrm{mmol}$, 1.0 equiv.), (S)-5 (0.001 mmol, $0.2 \mathrm{~mol} \%), \mathrm{Ag}_{2} \mathrm{CO}_{3}(0.0005 \mathrm{mmol}, 0.1 \mathrm{~mol} \%)$ and toluene $(2.5 \mathrm{~mL})$. The resulting mixture was then stirred overnight at room temperature. The solvent was then removed under vacuum and the residue was purified by flash column chromatography.

\section{(R)-3-(2-phenyl-4,5,6,7-tetrahydrobenzofuran-4-yl)-1H-indole (10aa)}

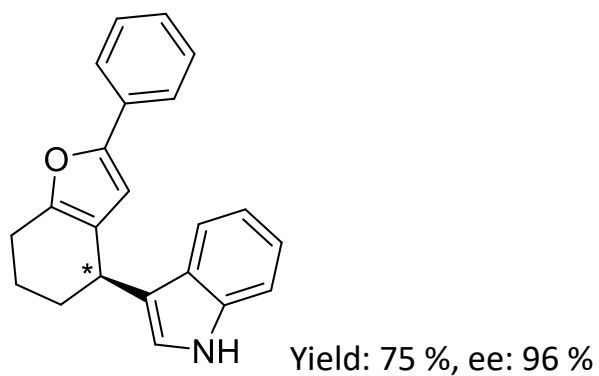

Compound 10aa was prepared according to the general procedure from $8 \mathrm{a}(107.9 \mathrm{mg}, 0.55 \mathrm{mmol}, 1.1$ equiv.), $9 a$ ( $58.8 \mathrm{mg}, 0.5 \mathrm{mmol}, 1.0$ equiv.), (S)-5 (0.8 mg, $0.001 \mathrm{mmol}, 0.2 \mathrm{~mol} \%$ ) and $\mathrm{Ag}_{2} \mathrm{CO}_{3}$ (0.1 mg, 0.0005 $\mathrm{mmol}, 0.1 \mathrm{~mol} \%)$ in toluene $(2.5 \mathrm{~mL})$, yielding product $10 \mathrm{aa}(116.5 \mathrm{mg}, 0.37 \mathrm{mmol}, 75 \%$ ) as a white solid for which the spectroscopical data correspond to those reported in the literature. ${ }^{13}[\alpha]_{D}=+38$ (c 0.89 , $\mathrm{CHCl}_{3}$ ). ee $=96 \%$, determined on a Chiralpak IA column (heptane:IPA = 95:5, $1 \mathrm{~mL} / \mathrm{min}$ ), retention times: $T_{\mathrm{R}}$ (minor): $19.7 \mathrm{~min}, \mathrm{~T}_{\mathrm{R}}$ (major): $25.6 \mathrm{~min}$.

10aa was alternatively obtained from the same protocol in the absence of $\mathrm{Ag}_{2} \mathrm{CO}_{3}$ in $80 \%$ yield and $92 \%$ ee.

m.p.: $158.8-160.3^{\circ} \mathrm{C} .{ }^{1}{ }^{H}$ NMR $\left(\mathrm{CDCl}_{3}, 500 \mathrm{MHz}\right) \delta=7.89(\mathrm{~s}, 1 \mathrm{H}), 7.62(\mathrm{~d}, J=7.6 \mathrm{~Hz}, 1 \mathrm{H}), 7.59(\mathrm{~d}, J=7.6$ $\mathrm{Hz}, 2 \mathrm{H}), 7.36(\mathrm{~d}, J=7.9 \mathrm{~Hz}, 1 \mathrm{H}), 7.33-7.30(\mathrm{~m}, 2 \mathrm{H}), 7.22-7.16(\mathrm{~m}, 2 \mathrm{H}), 7.11(\mathrm{t}, J=7.3 \mathrm{~Hz}, 1 \mathrm{H}), 6.86(\mathrm{~d}, J=1.8$ $\mathrm{Hz}, 1 \mathrm{H}), 6.44(\mathrm{~s}, 1 \mathrm{H}), 4.25(\mathrm{br}, 1 \mathrm{H}), 2.76-2.75(\mathrm{~m}, 2 \mathrm{H}), 2.18-2.13(\mathrm{~m}, 1 \mathrm{H}), 1.98-1.93(\mathrm{~m}, 2 \mathrm{H}), 1.88-1.84(\mathrm{~m}$, 1H). HRMS (ESI): $m / z$ : calcd for $\mathrm{C}_{22} \mathrm{H}_{20} \mathrm{NO}^{+} 314.1539[\mathrm{M}+\mathrm{H}]^{+}$, found, 314.1543 .

${ }^{13}$ (a) G. Force; Ki, Y. L. T.; Isaac, K.; Retailleau, P.; Marinetti, A.; Betzer, J.-F. Adv. Synth. Catal. 2018, 360, 3356-3366; (b) V. Rauniyar; Wang, Z. J.; Burks, H. E.; Toste, F. D. J. Am. Chem. Soc. 2011, 133, 8486-8489. 
<smiles>COc1ccc2[nH]cc([C@@H]3CCCc4oc(-c5ccccc5)cc43)c2c1</smiles>

Yield: $81 \%$, ee: $97 \%$.

Compound 10ab was prepared according to the general procedure from $8 \mathrm{a}(107.9 \mathrm{mg}, 0.55 \mathrm{mmol}, 1.1$ equiv.), 9c (73.6 mg, $0.5 \mathrm{mmol}, 1.0$ equiv.), (S)-5 (0.8 mg, $0.001 \mathrm{mmol}, 0.2 \mathrm{~mol} \%$ ) and $\mathrm{Ag}_{2} \mathrm{CO}_{3}$ (0.1 mg, 0.0005 $\mathrm{mmol}, 0.1 \mathrm{~mol} \%)$ in toluene $(2.5 \mathrm{~mL})$, yielding product $10 \mathrm{ab}(138.2 \mathrm{mg}, 0.40 \mathrm{mmol}, 81 \%)$ as a white solid for which the spectroscopical data correspond to those reported in the literature. ${ }^{13}[\alpha]_{D}=-7\left(c 0.98, \mathrm{CHCl}_{3}\right)$. ee $=97 \%$, determined on a Chiralpak IA column (heptane:IPA $=95: 5,1 \mathrm{~mL} / \mathrm{min}$ ), retention times: $T_{R}$ (minor): $29.2 \mathrm{~min}, \mathrm{~T}_{\mathrm{R}}$ (major): $41.4 \mathrm{~min}$.

10ab was alternatively obtained from the same protocol in the absence of $\mathrm{Ag}_{2} \mathrm{CO}_{3}$ in $73 \%$ yield and $94 \%$ ee.

${ }^{1}{ }^{H}$ NMR $\left(\mathrm{CDCl}_{3}, 500 \mathrm{MHz}\right) \delta=7.79(\mathrm{~s}, 1 \mathrm{H}), 7.58(\mathrm{~d}, J=7.6 \mathrm{~Hz}, 2 \mathrm{H}), 7.31(\mathrm{t}, J=7.6 \mathrm{~Hz}, 2 \mathrm{H}), 7.23(\mathrm{~d}, J=$ $8.2 \mathrm{~Hz}, 1 \mathrm{H}), 7.17(\mathrm{t}, J=7.3 \mathrm{~Hz}, 1 \mathrm{H}), 7.06(\mathrm{~s}, 1 \mathrm{H}), 6.86(\mathrm{~d}, J=8.5 \mathrm{~Hz}, 1 \mathrm{H}), 6.81(\mathrm{~s}, 1 \mathrm{H}), 6.44(\mathrm{~s}, 1 \mathrm{H}), 4.20(\mathrm{t}, J=$ $5.2 \mathrm{~Hz}, 1 \mathrm{H}), 3.84(\mathrm{~s}, 3 \mathrm{H}), 2.80-2.71(\mathrm{~m}, 2 \mathrm{H}), 2.16-2.11(\mathrm{~m}, 1 \mathrm{H}), 1.98-1.90(\mathrm{~m}, 2 \mathrm{H}), 1.87-1.82(\mathrm{~m}, 1 \mathrm{H})$. HRMS (ESI): $m / z$ : calculated for $\mathrm{C}_{23} \mathrm{H}_{22} \mathrm{NO}_{2}{ }^{+}: 344.1645[\mathrm{M}+\mathrm{H}]^{+}$, found, 344.1646 .

(R)-5-bromo-3-(2-phenyl-4,5,6,7-tetrahydrobenzofuran-4-yl)-1H-indole (10ac)

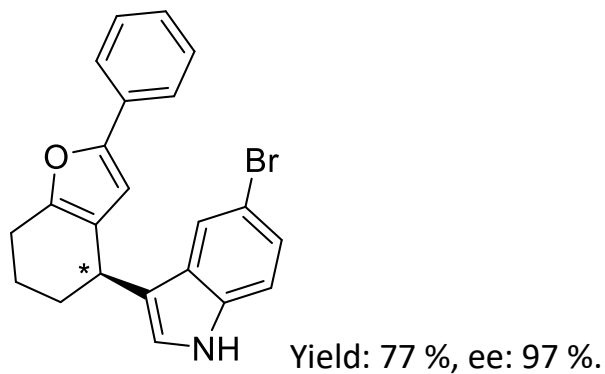

Compound 10ac was prepared according to the general procedure from $8 \mathrm{a}(107.9 \mathrm{mg}, 0.55 \mathrm{mmol}, 1.1$ equiv.), $9 \mathrm{~d}$ (98.0 mg, $0.5 \mathrm{mmol}, 1.0$ equiv.), $(S)-5(0.8 \mathrm{mg}, 0.001 \mathrm{mmol}, 0.2 \mathrm{~mol} \%)$ and $\mathrm{Ag}_{2} \mathrm{CO}_{3}(0.1 \mathrm{mg}$, $0.0005 \mathrm{mmol}, 0.1 \mathrm{~mol} \%)$ in toluene $(2.5 \mathrm{~mL})$, yielding product $10 \mathrm{ac}(150.9 \mathrm{mg}, 0.39 \mathrm{mmol}, 77 \%)$ as a white solid for which the spectroscopical data correspond to those reported in the literature. ${ }^{13}[\alpha]_{D}=-10$ (c 1.02, $\mathrm{CHCl}_{3}$ ). ee $=97 \%$, determined on a Chiralpak IA column (heptane:IPA $=95: 5,1 \mathrm{~mL} / \mathrm{min}$ ), retention times: $T_{\mathrm{R}}$ (minor): $19.4 \mathrm{~min}, \mathrm{~T}_{\mathrm{R}}$ (major): $26.7 \mathrm{~min}$.

10ac was alternatively obtained from the same protocol in the absence of $\mathrm{Ag}_{2} \mathrm{CO}_{3}$ in $30 \%$ yield and $75 \%$ ee.

${ }^{1}{ }^{H}$ NMR $\left(\mathrm{CDCl}_{3}, 300 \mathrm{MHz}\right) \delta=7.93(\mathrm{~s}, 1 \mathrm{H}), 7.74(\mathrm{~d}, J=1.3 \mathrm{~Hz}, 1 \mathrm{H}), 7.60-7.57(\mathrm{~m}, 2 \mathrm{H}), 7.34-7.16(\mathrm{~m}, 5 \mathrm{H})$, 
$6.83(\mathrm{~d}, J=2.3 \mathrm{~Hz}, 1 \mathrm{H}), 6.40(\mathrm{~s}, 1 \mathrm{H}), 4.17(\mathrm{t}, J=5.5 \mathrm{~Hz}, 1 \mathrm{H}), 2.76-2.73(\mathrm{~m}, 2 \mathrm{H}), 2.19-2.07(\mathrm{~m}, 1 \mathrm{H}), 1.96-1.79$ (m, 3H). HRMS (ESI): $m / z$ : calculated for $\mathrm{C}_{22} \mathrm{H}_{19} \mathrm{BrNO}^{+}: 392.0645[\mathrm{M}+\mathrm{H}]^{+}$, found, 392.0638 .

(R)-6-methyl-3-(2-phenyl-4,5,6,7-tetrahydrobenzofuran-4-yl)-1H-indole (10ad)<smiles>Cc1ccc2c(C3CCCc4oc(-c5ccccc5)cc43)c[nH]c2c1</smiles>

Yield: $44 \%$, ee: $96 \%$

Compound 10ad was prepared according to the general procedure from $8 \mathrm{a}(21.6 \mathrm{mg}, 0.11 \mathrm{mmol}, 1.1$ equiv.), $9 e$ (13.1 mg, $0.1 \mathrm{mmol}, 1.0$ equiv.), (S) -5 (0.2 mg, $0.0002 \mathrm{mmol}, 0.2 \mathrm{~mol} \%$ ) and $\mathrm{Ag}_{2} \mathrm{CO}_{3}(0.1 \mathrm{mg}$, $0.0001 \mathrm{mmol}, 0.1 \mathrm{~mol} \%)$ in toluene $(2.5 \mathrm{~mL})$, yielding product $10 \mathrm{ad}(14.4 \mathrm{mg}, 0.04 \mathrm{mmol}, 44 \%)$ as a white solid for which the spectroscopical data correspond to those reported in the literature. ${ }^{13}$

$[\alpha]_{\mathrm{D}}=+28\left(c\right.$ 1.21, $\left.\mathrm{CHCl}_{3}\right)$. ee $=96 \%$, determined on a Chiralpak IA column (heptane:IPA $=95: 5,1$ $\mathrm{mL} / \mathrm{min}$ ), retention times: $\mathrm{T}_{\mathrm{R}}$ (minor): $19.5 \mathrm{~min}, \mathrm{~T}_{\mathrm{R}}$ (major): $22.4 \mathrm{~min} .{ }^{1} \mathbf{H} \mathbf{N M R}\left(\mathrm{CDCl}_{3}, \mathbf{5 0 0} \mathbf{~ M H z}\right) \delta=7.71(\mathrm{~s}$, $1 \mathrm{H}), 7.57(\mathrm{~d}, J=7.9 \mathrm{~Hz}, 2 \mathrm{H}), 7.48(\mathrm{~d}, J=7.9 \mathrm{~Hz}, 1 \mathrm{H}), 7.30(\mathrm{t}, J=7.6 \mathrm{~Hz}, 2 \mathrm{H}), 7.18-7.12(\mathrm{~m}, 2 \mathrm{H}), 6.93(\mathrm{~d}, J=7.9$ $\mathrm{Hz}, 1 \mathrm{H}), 6.75(\mathrm{~s}, 1 \mathrm{H}), 6.42(\mathrm{~s}, 1 \mathrm{H}), 4.20(\mathrm{br}, 1 \mathrm{H}), 2.76-2.73(\mathrm{~m}, 2 \mathrm{H}), 2.45(\mathrm{~s}, 3 \mathrm{H}), 2.15-2.10(\mathrm{~m}, 1 \mathrm{H}), 1.98-1.90$ $(\mathrm{m}, 2 \mathrm{H}), 1.86-1.83(\mathrm{~m}, 1 \mathrm{H})$. HRMS (ESI): $\mathrm{m} / \mathrm{z}$ : calculated for $\mathrm{C}_{23} \mathrm{H}_{22} \mathrm{NO}^{+}: 328.1696[\mathrm{M}+\mathrm{H}]^{+}$, found, 328.1696 .

\section{(R)-2-methyl-3-(2-phenyl-4,5,6,7-tetrahydrobenzofuran-4-yl)-1H-indole (10ae)}

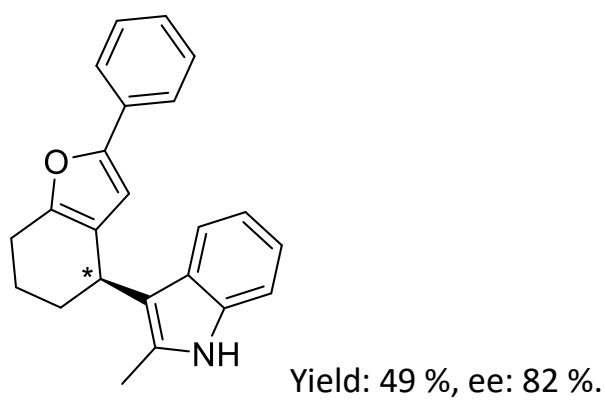

Compound 10ae was prepared according to the general procedure from $8 \mathrm{a}(107.9 \mathrm{mg}, 0.55 \mathrm{mmol}, 1.1$ equiv.), $9 f\left(65.6 \mathrm{mg}, 0.5 \mathrm{mmol}, 1.0\right.$ equiv.), ( $(S)-5$ ( $0.8 \mathrm{mg}, 0.001 \mathrm{mmol}, 0.2 \mathrm{~mol} \%$ ) and $\mathrm{Ag}_{2} \mathrm{CO}_{3}(0.1 \mathrm{mg}, 0.0005$ $\mathrm{mmol}, 0.1 \mathrm{~mol} \%)$ in toluene $(2.5 \mathrm{~mL})$, yielding product $10 \mathrm{ae}(80.0 \mathrm{mg}, 0.24 \mathrm{mmol}, 49 \%)$ as a white solid for which the spectroscopical data correspond to those reported in the literature..$^{13}$

$[\alpha]_{D}=+26\left(c 1.20, \mathrm{CHCl}_{3}\right)$. ee $=82 \%$, determined on a Chiralpak AD-H column (heptane:IPA = 90:10, 1

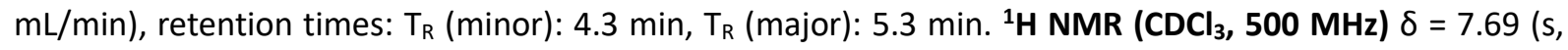
1H), $7.54(\mathrm{~d}, J=7.9 \mathrm{~Hz}, 2 \mathrm{H}), 7.30-7.24(\mathrm{~m}, 4 \mathrm{H}), 7.15(\mathrm{t}, J=7.3 \mathrm{~Hz}, 1 \mathrm{H}), 7.06(\mathrm{t}, J=7.3 \mathrm{~Hz}, 1 \mathrm{H}), 6.94(\mathrm{t}, J=7.6$ $\mathrm{Hz}, 1 \mathrm{H}), 6.28(\mathrm{~s}, 1 \mathrm{H}), 4.14(\mathrm{br}, 1 \mathrm{H}), 2.82(\mathrm{br}, 2 \mathrm{H}), 2.31(\mathrm{~s}, 3 \mathrm{H}), 2.11-2.04(\mathrm{~m}, 2 \mathrm{H}), 1.96-1.88(\mathrm{~m}, 2 \mathrm{H})$. HRMS (ESI): $\mathrm{m} / \mathrm{z}$ : calculated for $\mathrm{C}_{23} \mathrm{H}_{22} \mathrm{NO}^{+}: 328.1696[\mathrm{M}+\mathrm{H}]^{+}$, found, 328.1691 . 


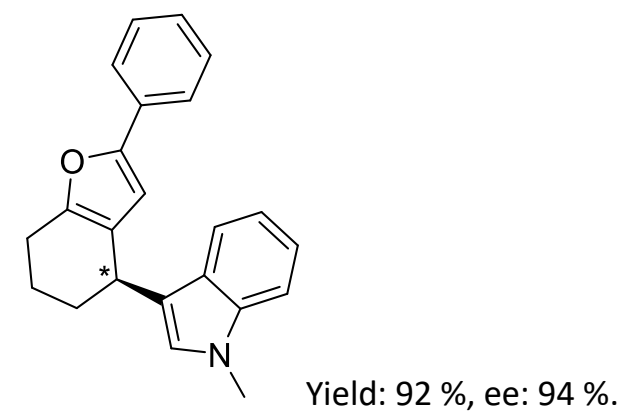

Compound 10af was prepared according to the general procedure from $8 \mathrm{a}(107.9 \mathrm{mg}, 0.55 \mathrm{mmol}, 1.1$ equiv.), 9b (65.6 mg, $0.5 \mathrm{mmol}, 1.0$ equiv.), (S)-5 (0.8 mg, $0.001 \mathrm{mmol}, 0.2 \mathrm{~mol} \%$ ) and $\mathrm{Ag}_{2} \mathrm{CO}_{3}(0.1 \mathrm{mg}$, $0.0005 \mathrm{mmol}, 0.1 \mathrm{~mol} \%)$ in toluene $(2.5 \mathrm{~mL})$, yielding product 10af (150.2 $\mathrm{mg}, 0.46 \mathrm{mmol}, 92 \%)$ as a white solid for which the spectroscopical data correspond to those reported in the literature. ${ }^{13,4 a}$

$[\alpha]_{D}=-5\left(c 0.78, \mathrm{CHCl}_{3}\right)$. ee: $94 \%$, determined on a Chiralpak IC column (heptane:IPA = 95:05, $\left.1 \mathrm{~mL} / \mathrm{min}\right)$, retention times: $\mathrm{T}_{\mathrm{R}}$ (minor): $4.7 \mathrm{~min}, \mathrm{~T}_{\mathrm{R}}$ (major): $5.1 \mathrm{~min} .{ }^{1} \mathbf{H} \mathbf{N M R}\left(\mathrm{CDCl}_{3}, 500 \mathrm{MHz}\right) \delta=7.60(\mathrm{t}, J=7.6 \mathrm{~Hz}$, $3 \mathrm{H}), 7.33-7.29(\mathrm{~m}, 3 \mathrm{H}), 7.24-7.21(\mathrm{~m}, 1 \mathrm{H}), 7.18(\mathrm{t}, J=7.3 \mathrm{~Hz}, 1 \mathrm{H}), 7.10(\mathrm{t}, J=7.3 \mathrm{~Hz}, 1 \mathrm{H}), 6.72(\mathrm{~s}, 1 \mathrm{H}), 6.44(\mathrm{~s}$, $1 \mathrm{H}), 4.24(\mathrm{t}, J=5.5 \mathrm{~Hz}, 1 \mathrm{H}), 3.71(\mathrm{~s}, 3 \mathrm{H}), 2.76-2.75(\mathrm{~m}, 2 \mathrm{H}), 2.16-2.12(\mathrm{~m}, 1 \mathrm{H}), 1.97-1.91(\mathrm{~m}, 2 \mathrm{H}), 1.87-1.84$ (m, 1H). HRMS (ESI): $m / z$ : calcd for $\mathrm{C}_{23} \mathrm{H}_{22} \mathrm{NO}^{+} 328.1696[\mathrm{M}+\mathrm{H}]^{+}$, found, 328.1696 .

(R)-1-benzyl-3-(2-phenyl-4,5,6,7-tetrahydrobenzofuran-4-yl)-1H-indole (10ag)

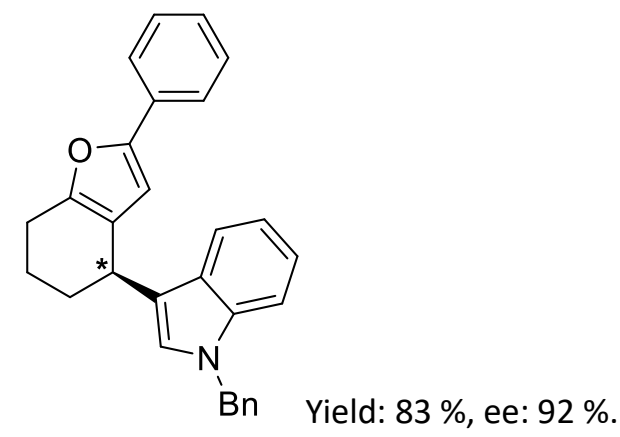

Compound 10ag was prepared according to the general procedure from $8 \mathrm{a}(21.6 \mathrm{mg}, 0.11 \mathrm{mmol}, 1.1$ equiv.), $9 \mathrm{~g}$ (20.7 mg, $0.1 \mathrm{mmol}, 1.0$ equiv.), (S)-5 (0.2 mg, $0.0002 \mathrm{mmol}, 0.2 \mathrm{~mol} \%$ ) and $\mathrm{Ag}_{2} \mathrm{CO}_{3}(0.1 \mathrm{mg}$, $0.0001 \mathrm{mmol}, 0.1 \mathrm{~mol} \%)$ in toluene $(0.5 \mathrm{~mL})$, yielding product $10 \mathrm{ag}(33.3 \mathrm{mg}, 0.08 \mathrm{mmol}, 83 \%)$ as a pale yellow solid

$[\alpha]_{D}=+34$ (c 1.00, $\mathrm{CHCl}_{3}$ ). ee: $92 \%$, determined on a Chiralpak IA column (heptane:IPA = 95:05, 1 $\mathrm{mL} / \mathrm{min}$ ), retention times: $\mathrm{T}_{\mathrm{R}}$ (major): $6.5 \mathrm{~min}, \mathrm{~T}_{\mathrm{R}}$ (minor): $11.3 \mathrm{~min} .{ }^{1} \mathrm{H} \mathbf{N M R}\left(\mathrm{CDCl}_{3}, 500 \mathrm{MHz}\right) \delta=7.54(\mathrm{~d}, J$ $=7.6 \mathrm{~Hz}, 1 \mathrm{H}), 7.49(\mathrm{~d}, J=7.9 \mathrm{~Hz}, 2 \mathrm{H}), 7.23-7.13(\mathrm{~m}, 6 \mathrm{H}), 7.08(\mathrm{t}, J=7.6 \mathrm{~Hz}, 2 \mathrm{H}), 7.02-7.97(\mathrm{~m}, 3 \mathrm{H}), 6.73(\mathrm{~s}$, $1 \mathrm{H}), 6.35(\mathrm{~s}, 1 \mathrm{H}), 5.14\left(\mathrm{dd}, J_{1}=21.3 \mathrm{~Hz}, J_{2}=16.2 \mathrm{~Hz}, 2 \mathrm{H}\right), 4.17(\mathrm{t}, J=6.1 \mathrm{~Hz}, 1 \mathrm{H}), 2.68-2.64(\mathrm{~m}, 2 \mathrm{H}), 2.10-2.05$ $(\mathrm{m}, 1 \mathrm{H}), 1.90-1.84(\mathrm{~m}, 2 \mathrm{H}), 1.80-1.73(\mathrm{~m}, 1 \mathrm{H}) .{ }^{13} \mathrm{C} \mathbf{N M R}\left(\mathrm{CDCl}_{3}, 125 \mathrm{MHz}\right) \delta=151.9,151.4,138.0,137.2$, $131.6,128.9,128.7,127.7,127.6,126.9,126.8,126.6,123.5,122.4,121.9,119.6,119.6,119.2,110.0,106.3$, 50.2, 31.6, 31.1, 23.6, 21.4. HRMS (ESI): $\mathrm{m} / \mathrm{z}$ : calcd for $\mathrm{C}_{29} \mathrm{H}_{24} \mathrm{NO}^{+} 402.1852\left[\mathrm{M}+\mathrm{H}-\mathrm{H}_{2}\right]^{+}$, found, 402.1858 . 


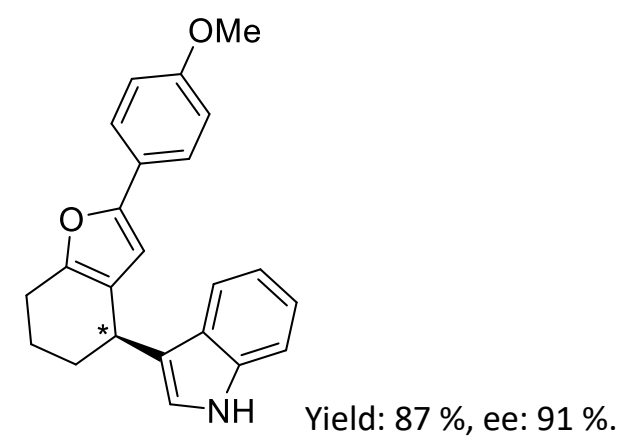

Compound 10ba was prepared according to the general procedure from $8 \mathbf{b}(24.9 \mathrm{mg}, 0.11 \mathrm{mmol}, 1.1$ equiv.), 9a (11.7 mg, $0.1 \mathrm{mmol}, 1.0$ equiv.), (S)-5 (0.2 mg, $0.0002 \mathrm{mmol}, 0.2 \mathrm{~mol} \%$ ) and $\mathrm{Ag}_{2} \mathrm{CO}_{3}(0.1 \mathrm{mg}$, $0.0001 \mathrm{mmol}, 0.1 \mathrm{~mol} \%)$ in toluene $(0.5 \mathrm{~mL})$, yielding product $10 \mathrm{ba}(29.8 \mathrm{mg}, 0.09 \mathrm{mmol}, 87 \%)$ as a pale yellow solid for which the spectroscopical data correspond to those reported in the literature. ${ }^{13}$

$[\alpha]_{\mathrm{D}}=+11\left(\mathrm{c} \mathrm{1.90}, \mathrm{CHCl}_{3}\right)$. ee $=91 \%$, determined on a Chiralpak IC column (heptane:IPA = 95:5, 1 $\mathrm{mL} / \mathrm{min}$ ), retention times: $\mathrm{T}_{\mathrm{R}}$ (minor): $9.5 \mathrm{~min}, \mathrm{~T}_{\mathrm{R}}$ (major): $10.5 \mathrm{~min} .{ }^{1} \mathbf{H} \mathbf{N M R}\left(\mathrm{CDCl}_{3}, \mathbf{5 0 0} \mathbf{M H z}\right) \delta=7.89(\mathrm{~s}$, $1 \mathrm{H}), 7.62(\mathrm{~d}, J=7.9 \mathrm{~Hz}, 1 \mathrm{H}), 7.51(\mathrm{~d}, J=8.5 \mathrm{~Hz}, 2 \mathrm{H}), 7.34(\mathrm{~d}, J=7.9 \mathrm{~Hz}, 1 \mathrm{H}), 7.19(\mathrm{t}, J=7.6 \mathrm{~Hz}, 1 \mathrm{H}), 7.10(\mathrm{t}, J$ $=7.6 \mathrm{~Hz}, 1 \mathrm{H}), 6.86-6.84(\mathrm{~m}, 3 \mathrm{H}), 6.29(\mathrm{~s}, 1 \mathrm{H}), 4.24-4.22(\mathrm{~m}, 1 \mathrm{H}), 3.79(\mathrm{~s}, 3 \mathrm{H}), 2.75-2.72(\mathrm{~m}, 2 \mathrm{H}), 2.61-2.11$ $(\mathrm{m}, 1 \mathrm{H}), 1.98-1.91(\mathrm{~m}, 2 \mathrm{H}), 1.86-1.81(\mathrm{~m}, 1 \mathrm{H})$. HRMS (ESI): $\mathrm{m} / \mathrm{z}$ : calculated for $\mathrm{C}_{23} \mathrm{H}_{22} \mathrm{NO}_{2}{ }^{+}: 344.1645[\mathrm{M}+\mathrm{H}]^{+}$, found, 344.1645 .

(R)-3-(2-(p-tolyl)-4,5,6,7-tetrahydrobenzofuran-4-yl)-1H-indole (10ca)

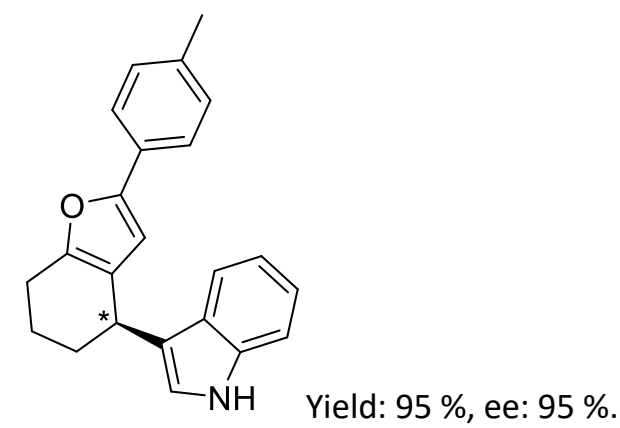

Compound 10ca was prepared according to the general procedure from $8 \mathrm{c}(23.1 \mathrm{mg}, 0.11 \mathrm{mmol}, 1.1$ equiv.), 9a (11.7 mg, $0.1 \mathrm{mmol}, 1.0$ equiv.), (S)-5 (0.2 mg, $0.0002 \mathrm{mmol}, 0.2 \mathrm{~mol} \%$ ) and $\mathrm{Ag}_{2} \mathrm{CO}_{3}(0.1 \mathrm{mg}$, $0.0001 \mathrm{mmol}, 0.1 \mathrm{~mol} \%)$ in toluene $(0.5 \mathrm{~mL})$, yielding product $10 \mathrm{ca}(31.0 \mathrm{mg}, 0.09 \mathrm{mmol}, 95 \%)$ as a pale yellow solid for which the spectroscopical data correspond to those reported in the literature. ${ }^{13}$

$[\alpha]_{D}=+68\left(c 1.01, \mathrm{CHCl}_{3}\right)$. ee $=95 \%$, determined on a Chiralpak IC column (heptane:IPA = 95:5, 1 $\mathrm{mL} / \mathrm{min}$ ), retention times: $\mathrm{T}_{\mathrm{R}}$ (minor): $6.3 \mathrm{~min}, \mathrm{~T}_{\mathrm{R}}$ (major): $7.2 \mathrm{~min} .{ }^{1} \mathrm{H} \mathrm{NMR}\left(\mathrm{CDCl}_{3}, \mathbf{5 0 0} \mathbf{M H z}\right) \delta=7.71(\mathrm{~s}$, $1 \mathrm{H}), 7.52(\mathrm{~d}, J=7.9 \mathrm{~Hz}, 1 \mathrm{H}), 7.38(\mathrm{~d}, J=7.9 \mathrm{~Hz}, 2 \mathrm{H}), 7.21(\mathrm{~d}, J=8.2 \mathrm{~Hz}, 1 \mathrm{H}), 7.09(\mathrm{t}, J=7.6 \mathrm{~Hz}, 1 \mathrm{H}), 7.03-7.00$ $(\mathrm{m}, 3 \mathrm{H}), 6.70-6.69(\mathrm{~m}, 1 \mathrm{H}), 6.27(\mathrm{~s}, 1 \mathrm{H}), 4.13(\mathrm{t}, J=5.5 \mathrm{~Hz}, 1 \mathrm{H}), 2.66-2.63(\mathrm{~m}, 2 \mathrm{H}), 2.22(\mathrm{~s}, 3 \mathrm{H}), 2.06-2.01(\mathrm{~m}$, 1H), 1.86-1.81 (m, 2H), 1.76-1.71 (m, 1H). HRMS (ESI): $\mathrm{m} / z$ : calculated for $\mathrm{C}_{23} \mathrm{H}_{20} \mathrm{NO}^{+}: 326.1539\left[\mathrm{M}+\mathrm{H}-\mathrm{H}_{2}\right]^{+}$, found, 326.1537 . 


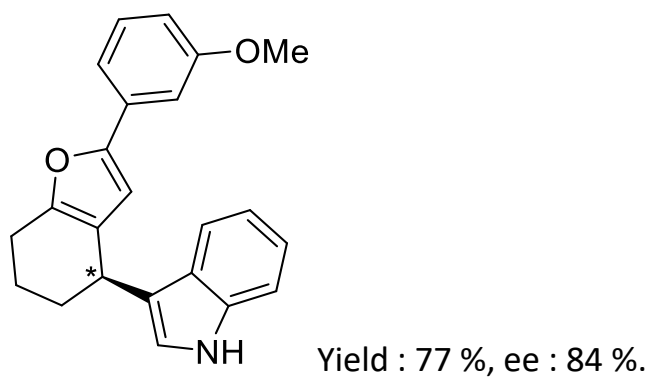

Compound 10da was prepared according to the general procedure from $8 \mathrm{~d}(24.9 \mathrm{mg}, 0.11 \mathrm{mmol}, 1.1$ equiv.), 9a (11.7 mg, $0.1 \mathrm{mmol}, 1.0$ equiv.), (S)-5 (0.2 mg, $0.0002 \mathrm{mmol}, 0.2 \mathrm{~mol} \%$ ) and $\mathrm{Ag}_{2} \mathrm{CO}_{3}(0.1 \mathrm{mg}$, $0.0001 \mathrm{mmol}, 0.1 \mathrm{~mol} \%)$ in toluene $(0.5 \mathrm{~mL})$, yielding product $10 \mathrm{da}(26.4 \mathrm{mg}, 0.08 \mathrm{mmol}, 77 \%$ ) as a pale yellow solid.

$[\alpha]_{\mathrm{D}}=+15\left(c\right.$ 1.29, $\left.\mathrm{CHCl}_{3}\right)$. ee $=84 \%$, determined on a Chiralpak IA column (heptane:IPA $=90: 10,1$ $\mathrm{mL} / \mathrm{min}$ ), retention times: $\mathrm{T}_{\mathrm{R}}$ (minor): $12.1 \mathrm{~min}, \mathrm{~T}_{\mathrm{R}}$ (major): $23.7 \mathrm{~min} .{ }^{1}{ }^{\mathrm{H} \mathrm{NMR}}\left(\mathrm{CDCl}_{3}, \mathbf{5 0 0} \mathbf{M H z}\right) \delta=7.92(\mathrm{~s}$, $1 \mathrm{H}), 7.61(\mathrm{~d}, J=7.9 \mathrm{~Hz}, 1 \mathrm{H}), 7.36(\mathrm{~d}, J=8.2 \mathrm{~Hz}, 1 \mathrm{H}), 7.24-7.09(\mathrm{~m}, 5 \mathrm{H}), 6.86(\mathrm{~d}, J=1.8 \mathrm{~Hz}, 1 \mathrm{H}), 6.74\left(\mathrm{dd}, J_{1}=\right.$ $\left.7.9 \mathrm{~Hz}, J_{2}=1.8 \mathrm{~Hz}, 1 \mathrm{H}\right), 6.43(\mathrm{~s}, 1 \mathrm{H}), 4.25(\mathrm{t}, J=5.5 \mathrm{~Hz}, 1 \mathrm{H}), 3.82(\mathrm{~s}, 3 \mathrm{H}), 2.78-2.74(\mathrm{~m}, 2 \mathrm{H}), 2.18-2.13(\mathrm{~m}, 1 \mathrm{H})$,

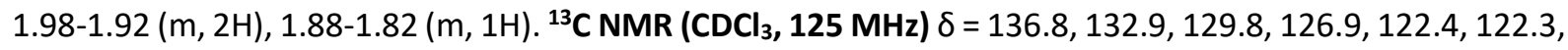
122.2, 120.5, 119.5, 119.4, 116.1, 112.9, 111.4, 108.6, 106.6, 160.1, 151.7, 151.5, 55.5, 31.4, 31.1, 23.6, 21.4. HRMS (ESI): $\mathrm{m} / \mathrm{z}$ : calculated for $\mathrm{C}_{23} \mathrm{H}_{22} \mathrm{NO}_{2}{ }^{+}: 344.1645[\mathrm{M}+\mathrm{H}]^{+}$, found, 344.1641 .

(R)-3-(2-pentyl-4,5,6,7-tetrahydrobenzofuran-4-yl)-1H-indole (10ea)

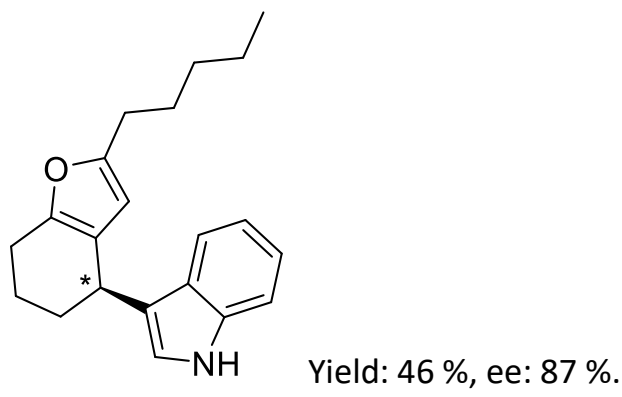

Compound 10ea was prepared according to the general procedure from $8 \mathrm{e}(20.9 \mathrm{mg}, 0.11 \mathrm{mmol}, 1.1$ equiv.), $9 a$ (11.7 mg, $0.1 \mathrm{mmol}, 1.0$ equiv.), ( $S$ )-5 (0.8 mg, $0.001 \mathrm{mmol}, 1 \mathrm{~mol} \%$ ) and $\mathrm{Ag}_{2} \mathrm{CO}_{3}$ (0.1 mg, 0.0005 $\mathrm{mmol}, 0.5 \mathrm{~mol} \%)$ in toluene $(0.5 \mathrm{~mL})$, yielding product $10 \mathrm{ea}(14 \mathrm{mg}, 0.05 \mathrm{mmol}, 46 \%)$ as a white solid.

$[\alpha]_{D}=+2\left(c \quad 0.14, \mathrm{CHCl}_{3}\right)$. ee $=87 \%$, determined on a Chiralpak IA column (heptane:IPA $=95: 5,1$

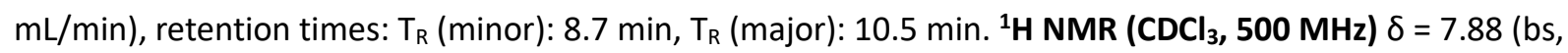
$1 \mathrm{H}), 7.59$ (d, $J=7.9 \mathrm{~Hz}, 1 \mathrm{H}), 7.34(\mathrm{~d}, J=7.9 \mathrm{~Hz}, 1 \mathrm{H}), 7.18(\mathrm{t}, J=7.6 \mathrm{~Hz}, 1 \mathrm{H}), 7.09(\mathrm{t}, J=7.6 \mathrm{~Hz}, 1 \mathrm{H}), 6.83(\mathrm{~s}$, $1 \mathrm{H}), 5.74(\mathrm{~s}, 1 \mathrm{H}), 4.17(\mathrm{t}, J=5.5 \mathrm{~Hz}, 1 \mathrm{H}), 2.67-2.60(\mathrm{~m}, 2 \mathrm{H}), 2.54(\mathrm{t}, J=7.6 \mathrm{~Hz}, 2 \mathrm{H}), 2.14-2.09(\mathrm{~m}, 1 \mathrm{H}), 1.94-$ $1.86(\mathrm{~m}, 2 \mathrm{H}), 1.82-1.76(\mathrm{~m}, 1 \mathrm{H}), 1.62-1.57(\mathrm{~m}, 2 \mathrm{H}), 1.32-1.28(\mathrm{~m}, 4 \mathrm{H}), 0.88(\mathrm{t}, J=6.7 \mathrm{~Hz}, 3 \mathrm{H}) .{ }^{13} \mathrm{C} \mathrm{NMR}\left(\mathrm{CDCl}_{3}\right.$, $125 \mathrm{MHz}) \delta=154.4,149.2,136.8,127.0,122.2,122.1,120.8,120.4,119.5,119.3,111.3,105.6,31.7,31.5$, 31.1, 28.4, 28.2, 23.5, 22.6, 21.5, 14.2. HRMS (ESI): $\mathrm{m} / \mathrm{z}$ : calculated for $\mathrm{C}_{21} \mathrm{H}_{24} \mathrm{NO}^{+}: 306.1852\left[\mathrm{M}+\mathrm{H}-\mathrm{H}_{2}\right]^{+}$, found, 306.1857. 


\section{(R)-3-(2-phenyl-5,6-dihydro-4H-cyclopenta[b]furan-4-yl)-1H-indole (10fa)}

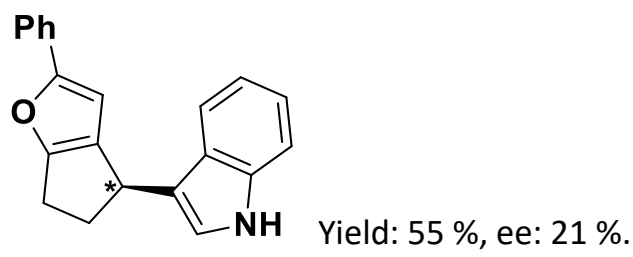

Compound 10fa was prepared according to the general procedure from $8 \mathrm{f}(20.1 \mathrm{mg}, 0.11 \mathrm{mmol}, 1.1$ equiv.), $9 a$ (11.7 mg, $0.1 \mathrm{mmol}, 1.0$ equiv.), (S)-5 (1.7 mg, $0.002 \mathrm{mmol}, 2 \mathrm{~mol} \%$ ) and $\mathrm{Ag}_{2} \mathrm{CO}_{3}$ (0.3 mg, 0.001 $\mathrm{mmol}, 1 \mathrm{~mol} \%)$ in toluene $(0.5 \mathrm{~mL})$, yielding product $10 \mathrm{fa}(16.4 \mathrm{mg}, 0.06 \mathrm{mmol}, 55 \%)$ as a white solid.

$[\alpha]_{\mathrm{D}}=-18\left(c \quad 0.75, \mathrm{CHCl}_{3}\right)$. ee $=21 \%$, determined on a Chiralpak IA column (heptane:IPA $=95: 05,1$

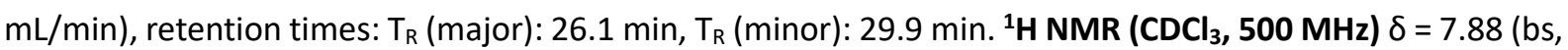
$1 \mathrm{H}), 7.61(\mathrm{~d}, J=7.3 \mathrm{~Hz}, 2 \mathrm{H}), 7.58(\mathrm{~d}, J=7.9 \mathrm{~Hz}, 1 \mathrm{H}), 7.36-7.32(\mathrm{~m}, 3 \mathrm{H}), 7.19(\mathrm{t}, J=7.6 \mathrm{~Hz}, 2 \mathrm{H}), 7.09(\mathrm{t}, J=7.6$ $\mathrm{Hz}, 1 \mathrm{H}), 6.93(\mathrm{~d}, J=1.9 \mathrm{~Hz}, 1 \mathrm{H}), 6.57(\mathrm{~s}, 1 \mathrm{H}), 4.47(\mathrm{t}, J=6.6 \mathrm{~Hz}, 1 \mathrm{H}), 3.07-3.00(\mathrm{~m}, 1 \mathrm{H}), 2.96-2.83(\mathrm{~m}, 2 \mathrm{H})$,

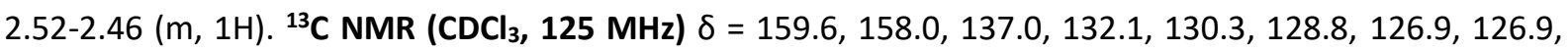
123.3, 122.3, 120.9, 120.7, 119.5 (2C), 111.4, 103.8, 37.7, 34.2, 25.0. HRMS (ESI): $\mathrm{m} / \mathrm{z}$ : calculated for $\mathrm{C}_{21} \mathrm{H}_{16} \mathrm{NO}^{+}: 298.1226\left[\mathrm{M}+\mathrm{H}-\mathrm{H}_{2}\right]^{+}$, found, 298.1227.

(R)-3-(2-phenyl-5,6,7,8-tetrahydro-4H-cyclohepta[b]furan-4-yl)-1H-indole (10ga)<smiles>CC(C)(C)OC(=O)c1ccccc1</smiles>

Compound 10ga was prepared according to the general procedure from $8 \mathrm{~g}(23.1 \mathrm{mg}, 0.11 \mathrm{mmol}, 1.1$ equiv.), $9 a$ (11.7 mg, $0.1 \mathrm{mmol}, 1.0$ equiv.), $(S)-5\left(0.17 \mathrm{mg}, 0.0002 \mathrm{mmol}, 0.2 \mathrm{~mol} \%\right.$ ) and $\mathrm{Ag}_{2} \mathrm{CO}_{3}(0.1 \mathrm{mg}$, $0.0001 \mathrm{mmol}, 0.1 \mathrm{~mol} \%)$ in toluene $(0.5 \mathrm{~mL})$, yielding product $10 \mathrm{ga}(13.5 \mathrm{mg}, 0.04 \mathrm{mmol}, 41 \%)$ as a white solid. [ $\alpha]_{D}=+107$ ( c 0.59, $\mathrm{CHCl}_{3}$ ). ee $=94 \%$, determined on a Chiralpak IA column (heptane:IPA = 95:05, 1 $\mathrm{mL} / \mathrm{min}$ ), retention times: $\mathrm{T}_{R}$ (minor): $14.8 \mathrm{~min}, \mathrm{~T}_{\mathrm{R}}$ (major): $16.8 \mathrm{~min}$.

10ga was alternatively obtained from the same protocol in the absence of $\mathrm{Ag}_{2} \mathrm{CO}_{3}$ in $55 \%$ yield and $95 \%$ ee.

${ }^{1}{ }^{H}$ NMR $\left(\mathrm{CDCl}_{3}, 500 \mathrm{MHz}\right) \delta=7.92(\mathrm{bs}, 1 \mathrm{H}), 7.61(\mathrm{~d}, J=7.9 \mathrm{~Hz}, 1 \mathrm{H}), 7.51(\mathrm{~d}, J=7.3 \mathrm{~Hz}, 2 \mathrm{H}), 7.36(\mathrm{~d}, J=$ $8.2 \mathrm{~Hz}, 1 \mathrm{H}), 7.28(\mathrm{t}, J=7.9 \mathrm{~Hz}, 2 \mathrm{H}), 7.19(\mathrm{t}, J=7.6 \mathrm{~Hz}, 1 \mathrm{H}), 7.14(\mathrm{t}, J=7.6 \mathrm{~Hz}, 1 \mathrm{H}), 7.10(\mathrm{t}, J=7.6 \mathrm{~Hz}, 1 \mathrm{H}), 6.81$ (d, J = $1.9 \mathrm{~Hz}, 1 \mathrm{H}), 6.25(\mathrm{~s}, 1 \mathrm{H}), 4.27\left(\mathrm{dd}, J_{1}=7.6 \mathrm{~Hz}, J_{2}=3.2 \mathrm{~Hz}, 1 \mathrm{H}\right), 2.95(\mathrm{t}, J=5.7 \mathrm{~Hz}, 2 \mathrm{H}), 2.33-2.28(\mathrm{~m}$,

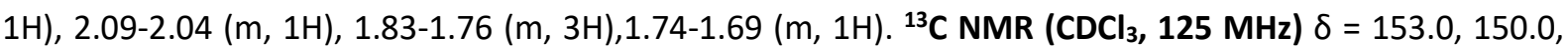
136.9, 131.4, 128.7, 126.8, 126.6, 126.2, 123.4, 122.6, 122.1, 120.0, 119.8, 119.4, 111.4, 109.1, 35.5, 34.6, 29.3, 27.6, 26.9. HRMS (ESI): $\mathrm{m} / \mathrm{z}$ : calculated for $\mathrm{C}_{23} \mathrm{H}_{22} \mathrm{NO}^{+}: 328.1696[\mathrm{M}+\mathrm{H}]^{+}$, found, 328.1696 . 


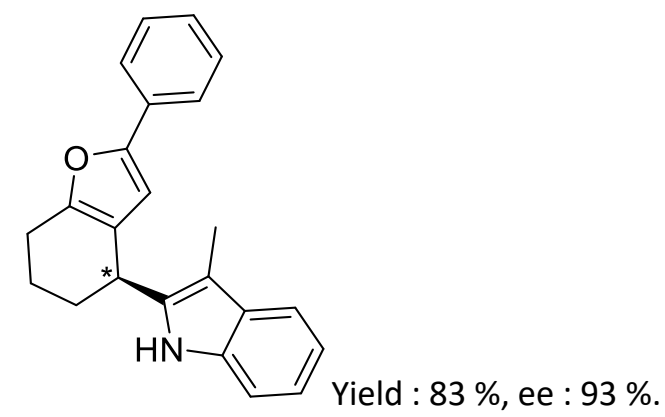

Compound 14ah was prepared according to the general procedure from $8 \mathrm{a}(21.6 \mathrm{mg}, 0.11 \mathrm{mmol}, 1.1$ equiv.), $9 \mathrm{~g}$ (13.1 mg, $0.1 \mathrm{mmol}, 1.0$ equiv.), (S)-5 (0.2 mg, $0.0002 \mathrm{mmol}, 0.2 \mathrm{~mol} \%)$ and $\mathrm{Ag}_{2} \mathrm{CO}_{3}(0.1 \mathrm{mg}$, $0.0001 \mathrm{mmol}, 0.1 \mathrm{~mol} \%)$ in toluene $(0.5 \mathrm{~mL})$, yielding product $14 \mathrm{ah}(27.1 \mathrm{mg}, 0.08 \mathrm{mmol}, 83 \%)$ as a pale yellow solid.

$[\alpha]_{\mathrm{D}}=+83\left(\right.$ c $\left.1.29, \mathrm{CHCl}_{3}\right)$. ee $=93 \%$, determined on a Chiralpak IA column (heptane:IPA $=95: 5,1$ $\mathrm{mL} / \mathrm{min})$, retention times: $\mathrm{T}_{\mathrm{R}}$ (minor): $7.1 \mathrm{~min}, \mathrm{~T}_{\mathrm{R}}$ (major): $10.3 \mathrm{~min} .{ }^{1} \mathrm{H} \mathbf{N M R}\left(\mathrm{CDCl}_{3}, \mathbf{5 0 0} \mathbf{M H z}\right) \delta=7.63(\mathrm{~s}$, $1 \mathrm{H}), 7.58(\mathrm{~d}, J=7.6 \mathrm{~Hz}, 2 \mathrm{H}), 7.54-7.52(\mathrm{~m}, 1 \mathrm{H}), 7.33(\mathrm{t}, J=7.6 \mathrm{~Hz}, 2 \mathrm{H}), 7.22-7.19(\mathrm{~m}, 2 \mathrm{H}), 7.12-7.07(\mathrm{~m}, 2 \mathrm{H})$, $6.35(\mathrm{~s}, 1 \mathrm{H}), 4.25\left(\mathrm{dd}, J_{1}=7.3 \mathrm{~Hz}, J_{2}=5.8 \mathrm{~Hz}, 1 \mathrm{H}\right), 2.78(\mathrm{t}, J=6.1 \mathrm{~Hz}, 2 \mathrm{H}), 2.33(\mathrm{~s}, 3 \mathrm{H}), 2.17-2.11(\mathrm{~m}, 1 \mathrm{H})$, 2.05-1.98 (m, 1H), 1.93-1.85 (m, 1H), 1.83-1.76 (m, 1H). $\left.{ }^{13} \mathrm{C} \mathrm{NMR}_{(\mathrm{CDCl}}, 125 \mathrm{MHz}\right) \delta=152.6,152.2,137.2$, 135.1, 131.2, 129.7, 129.0, 128.9, 128.7, 127.2, 123.5, 121.3, 120.1, 119.2, 118.3, 110.7, 107.0, 105.7, 31.8, 31.4, 23.4, 22.0, 8.7. HRMS (ESI): $\mathrm{m} / \mathrm{z}$ : calculated for $\mathrm{C}_{23} \mathrm{H}_{20} \mathrm{NO}^{+}: 326.1539\left[\mathrm{M}+\mathrm{H}-\mathrm{H}_{2}\right]^{+}$, found, 326.1541 .

\section{(S)-1,3-dimethyl-2-(2-phenyl-4,5,6,7-tetrahydrobenzofuran-4-yl)-1H-indole (14ai)}

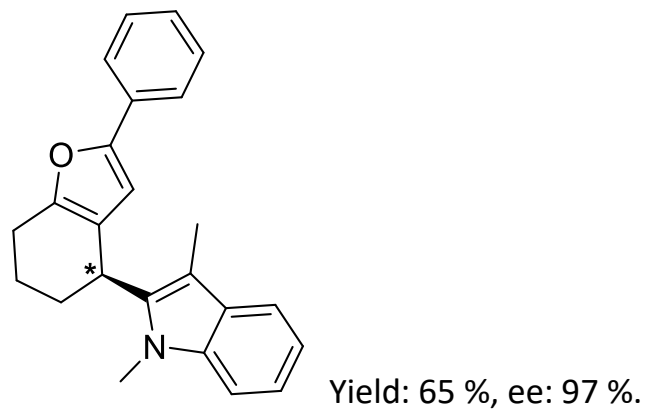

Compound 14ai was prepared according to the general procedure from $8 \mathrm{a}(21.6 \mathrm{mg}, 0.11 \mathrm{mmol}, 1.1$ equiv.), $9 \mathrm{i}$ (14.5 mg, $0.1 \mathrm{mmol}, 1.0$ equiv.), (S)-5 (0.2 mg, $0.0002 \mathrm{mmol}, 0.2 \mathrm{~mol} \%$ ) and $\mathrm{Ag}_{2} \mathrm{CO}_{3}(0.1 \mathrm{mg}$, $0.0001 \mathrm{mmol}, 0.1 \mathrm{~mol} \%)$ in toluene $(0.5 \mathrm{~mL})$, yielding product $14 a \mathrm{i}(20.5 \mathrm{mg}, 0.07 \mathrm{mmol}, 65 \%)$ as a white solid.

$[\alpha]_{\mathrm{D}}=+28\left(c\right.$ 0.94, $\left.\mathrm{CHCl}_{3}\right)$. ee $=97 \%$, determined on a Chiralpak IC column (heptane:IPA $=95: 5,1$ $\mathrm{mL} / \mathrm{min}$ ), retention times: $\mathrm{T}_{\mathrm{R}}$ (major): $4.4 \mathrm{~min}, \mathrm{~T}_{\mathrm{R}}$ (minor): $4.9 \mathrm{~min} .{ }^{1} \mathrm{H}$ NMR $\left(\mathrm{CDCl}_{3}, 500 \mathrm{MHz}\right) \delta=7.58(\mathrm{~d}, J$ $=7.6 \mathrm{~Hz}, 2 \mathrm{H}), 7.55(\mathrm{~d}, J=7.9 \mathrm{~Hz}, 1 \mathrm{H}), 7.33(\mathrm{t}, J=7.6 \mathrm{~Hz}, 2 \mathrm{H}), 7.26(\mathrm{~d}, J=8.2 \mathrm{~Hz}, 1 \mathrm{H}), 7.20\left(\mathrm{td}, J_{1}=7.3 \mathrm{~Hz}, J_{2}=\right.$ $3.1 \mathrm{~Hz}, 2 \mathrm{H}), 7.13(\mathrm{t}, J=7.3 \mathrm{~Hz}, 1 \mathrm{H}), 6.32(\mathrm{~s}, 1 \mathrm{H}), 4.37-4.35(\mathrm{~m}, 1 \mathrm{H}), 3.58(\mathrm{~s}, 3 \mathrm{H}), 2.84-2.81(\mathrm{~m}, 2 \mathrm{H}), 2.23(\mathrm{~s}$, $3 \mathrm{H}), 2.20-2.11(\mathrm{~m}, 2 \mathrm{H}), 2.00-1.87(\mathrm{~m}, 2 \mathrm{H}) .{ }^{13} \mathrm{C} \mathrm{NMR}\left(\mathrm{CDCl}_{3}, 125 \mathrm{MHz}\right) \delta=152.5,150.8,137.4,137.2,131.3$, $128.8,128.7,127.0,123.5,121.2,118.9,118.4,108.8,107.8,105.6,105.3,32.0,30.5,23.4,23.2,9.2$. HRMS (ESI): $\mathrm{m} / \mathrm{z}$ : calculated for $\mathrm{C}_{24} \mathrm{H}_{24} \mathrm{NO}^{+}: 342.1852[\mathrm{M}+\mathrm{H}]^{+}$, found, 342.1853 . 
methyl (S)-2-(2-(2-phenyl-4,5,6,7-tetrahydrobenzofuran-4-yl)-1H-indol-3-yl)acetate (14aj) and methyl (S)-2-(1-(2-phenyl-4,5,6,7-tetrahydrobenzofuran-4-yl)-1H-indol-3-yl)acetate (15aj)<smiles>CC(=O)Cc1c([C@@H]2CCCc3oc(-c4ccccc4)cc32)[nH]c2ccccc12</smiles>

14aj<smiles>CC(=O)Cc1cn([C@H]2CCCc3oc(-c4ccccc4)cc32)c2ccccc12</smiles>

15aj

Compound 14aj and 15aj were prepared according to the general procedure from $8 \mathrm{a}$ ( $23.1 \mathrm{mg}, 0.11$ mmol, 1.1 equiv.), 9j (18.9 mg, $0.1 \mathrm{mmol}, 1.0$ equiv.), (S)-5 (0.2 mg, $0.0002 \mathrm{mmol}, 0.2 \mathrm{~mol} \%$ ) and $\mathrm{Ag}_{2} \mathrm{CO}_{3}$ $(0.1 \mathrm{mg}, 0.0001 \mathrm{mmol}, 0.1 \mathrm{~mol} \%)$ in toluene $(0.5 \mathrm{~mL})$, yielding product 14 aj $(20 \mathrm{mg}, 0.05 \mathrm{mmol}, 52 \%$ ) as a white solid and 15ajk (16.7 mg, $0.05 \mathrm{mmol}, 46 \%$ ) as a white solid.

\section{Data for 14aj}

$[\alpha]_{\mathrm{D}}=+59$ (c 1.08, $\mathrm{CHCl}_{3}$ ). ee $=91 \%$, determined on a Chiralpak IC column (heptane:IPA $=98: 2,1$

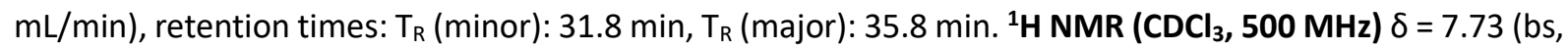
$1 \mathrm{H}), 7.54-7.50(\mathrm{~m}, 3 \mathrm{H}), 7.26(\mathrm{t}, J=7.9 \mathrm{~Hz}, 2 \mathrm{H}), 7.17-7.12(\mathrm{~m}, 2 \mathrm{H}), 7.07-7.03(\mathrm{~m}, 2 \mathrm{H}), 6.26(\mathrm{~s}, 1 \mathrm{H}), 4.22\left(\mathrm{dd}, J_{1}\right.$ $\left.=7.3 \mathrm{~Hz}, J_{2}=6.0 \mathrm{~Hz}, 1 \mathrm{H}\right), 3.73\left(\mathrm{dd}, J_{1}=22.8 \mathrm{~Hz}, J_{2}=15.4 \mathrm{~Hz}, 2 \mathrm{H}\right), 3.61(\mathrm{~s}, 3 \mathrm{H}), 2.72(\mathrm{t}, J=6.0 \mathrm{~Hz}, 2 \mathrm{H}), 2.16-$ $\left.2.11(\mathrm{~m}, 1 \mathrm{H}), 1.99-1.96(\mathrm{~m}, 1 \mathrm{H}), 1.88-1.80(\mathrm{~m}, 1 \mathrm{H}), 1.78-1.71(\mathrm{~m}, 1 \mathrm{H}) .{ }^{13} \mathrm{C} \mathrm{NMR}_{(\mathrm{CDCl}}, 125 \mathrm{MHz}\right) \delta=172.6$, 152.7, 152.3, 139.2, 135.1, 131.1, 128.9, 128.7, 128.7, 127.3, 123.5, 121.8, 119.9, 118.6, 110.9, 105.6, 104.5, 52.2, 31.9, 31.7, 30.4, 23.4, 22.1. HRMS (ESI): $\mathrm{m} / \mathrm{z}$ : calculated for $\mathrm{C}_{25} \mathrm{H}_{24} \mathrm{NO}_{3}{ }^{+}: 386.1751[\mathrm{M}+\mathrm{H}]^{+}$, found, 386.1751 .

\section{Data for 15aj}

$[\alpha]_{\mathrm{D}}=-4\left(\right.$ c $\left.0.94, \mathrm{CHCl}_{3}\right)$. ee $=95 \%$, determined on a Chiralpak IC column (heptane:IPA $=98: 2,1$ $\mathrm{mL} / \mathrm{min}$ ), retention times: $\mathrm{T}_{\mathrm{R}}$ (major): $23.1 \mathrm{~min}, \mathrm{~T}_{\mathrm{R}}$ (minor): $25.9 \mathrm{~min} .{ }^{1} \mathbf{H}$ NMR (CDCl, $\left.500 \mathrm{MHz}\right) \delta=7.63$ $(\mathrm{d}, J=7.9 \mathrm{~Hz}, 1 \mathrm{H}), 7.59(\mathrm{~d}, J=7.6 \mathrm{~Hz}, 2 \mathrm{H}), 7.39(\mathrm{~d}, J=8.5 \mathrm{~Hz}, 1 \mathrm{H}), 7.34(\mathrm{t}, J=7.9 \mathrm{~Hz}, 2 \mathrm{H}), 7.23-7.20(\mathrm{~m}, 2 \mathrm{H})$, $7.14(\mathrm{t}, J=7.6 \mathrm{~Hz}, 1 \mathrm{H}), 6.99(\mathrm{~s}, 1 \mathrm{H}), 6.40(\mathrm{~s}, 1 \mathrm{H}), 5.53(\mathrm{~d}, J=5.4 \mathrm{~Hz}, 1 \mathrm{H}), 3.72\left(\mathrm{dd}, J_{1}=18.6 \mathrm{~Hz}, J_{2}=16.1 \mathrm{~Hz}\right.$, $2 \mathrm{H}), 3.66(\mathrm{~s}, 3 \mathrm{H}), 2.85\left(\mathrm{dt}, J_{1}=16.7 \mathrm{~Hz}, J_{2}=6.3 \mathrm{~Hz}, 1 \mathrm{H}\right), 2.74\left(\mathrm{dt}, J_{1}=16.7 \mathrm{~Hz}, J_{2}=6.3 \mathrm{~Hz}, 1 \mathrm{H}\right), 2.19-2.13(\mathrm{~m}$,

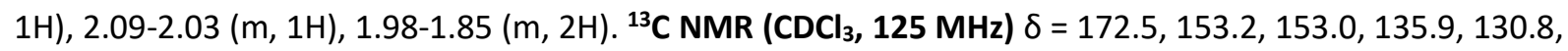
128.7, 128.4, 127.2, 125.4, 123.5, 121.6, 119.4, 119.3, 118.5, 109.7, 106.8, 104.8, 77.3, 77.0, 76.8, 51.9, 50.0, 31.3, 30.6, 23.1, 20.3. HRMS (ESI): $\mathrm{m} / \mathrm{z}$ : calculated for $\mathrm{C}_{25} \mathrm{H}_{24} \mathrm{NO}_{3}{ }^{+}: 386.1751[\mathrm{M}+\mathrm{H}]^{+}$, found, 386.1747. 
(S)-3-isopropyl-2-(2-phenyl-4,5,6,7-tetrahydrobenzofuran-4-yl)-1H-indole (14ak) and (S)-3-isopropyl-1(2-phenyl-4,5,6,7-tetrahydrobenzofuran-4-yl)-1H-indole (15ak)
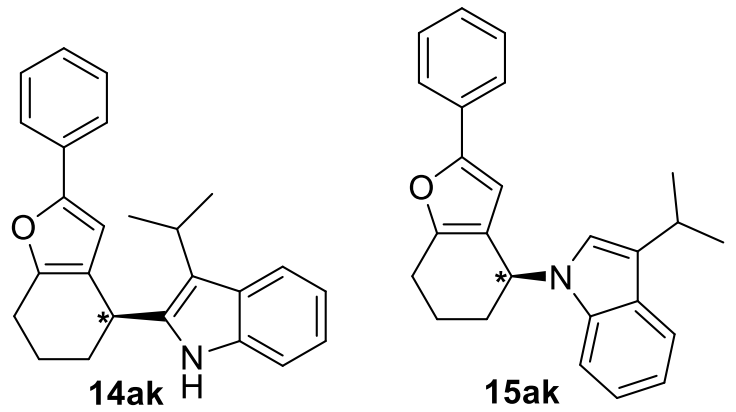

Compound 14ak and 15ak were prepared according to the general procedure from $8 \mathrm{a}(23.1 \mathrm{mg}, 0.11$ mmol, 1.1 equiv.), 9k (15.9 mg, 0.1 mmol, 1.0 equiv.), (S)-5 (0.2 mg, 0.0002 mmol, $0.2 \mathrm{~mol} \%$ ) and $\mathrm{Ag}_{2} \mathrm{CO}_{3}$ $(0.1 \mathrm{mg}, 0.0001 \mathrm{mmol}, 0.1 \mathrm{~mol} \%)$ in toluene $(0.5 \mathrm{~mL})$, yielding product $14 \mathrm{ak}(7.4 \mathrm{mg}, 0.06 \mathrm{mmol}, 21 \%)$ as a pale yellow solid and 15 ak $(20.7 \mathrm{mg}, 0.02 \mathrm{mmol}, 59 \%)$ as a pale yellow solid.

\section{Data for 14ak}

$[\alpha]_{\mathrm{D}}=\mathbf{- 1 4}\left(\right.$ c 1.00, $\left.\mathrm{CHCl}_{3}\right)$. ee $=49 \%$, determined on a Chiralpak IA column (heptane:IPA = 95:5, 1 $\mathrm{mL} / \mathrm{min}$ ), retention times: $\mathrm{T}_{\mathrm{R}}$ (minor): $5.2 \mathrm{~min}, \mathrm{~T}_{\mathrm{R}}$ (major): $6.9 \mathrm{~min} .{ }^{1} \mathrm{H}_{\mathrm{NMR}}\left(\mathrm{CDCl}_{3}, \mathbf{5 0 0} \mathbf{~ M H z} \delta=7.66(\mathrm{~d}, J\right.$ $=7.0 \mathrm{~Hz}, 1 \mathrm{H}), 7.51(\mathrm{~d}, J=7.2 \mathrm{~Hz}, 2 \mathrm{H}), 7.26(\mathrm{t}, J=7.7 \mathrm{~Hz}, 2 \mathrm{H}), 7.18-7.11(\mathrm{~m}, 2 \mathrm{H}), 7.05-7.6 .96(\mathrm{~m}, 2 \mathrm{H}), 6.27(\mathrm{~s}$, $1 \mathrm{H}), 4.22\left(\mathrm{dd}, J_{1}=7.5 \mathrm{~Hz}, J_{2}=5.6 \mathrm{~Hz}, 1 \mathrm{H}\right), 3.22(\mathrm{~m}, J=7.1 \mathrm{~Hz}, 1 \mathrm{H}), 2.73-2.70(\mathrm{~m}, 2 \mathrm{H}), 2.12-2.02(\mathrm{~m}, 1 \mathrm{H}), 2.02-$ $1.92(\mathrm{~m}, 1 \mathrm{H}), 1.89-1.79(\mathrm{~m}, 1 \mathrm{H}), 1.76-1.65(\mathrm{~m}, 1 \mathrm{H}), 1.43(\mathrm{t}, J=7.3 \mathrm{~Hz}, 6 \mathrm{H}) .{ }^{13} \mathrm{C} \mathrm{NMR}\left(\mathrm{CDCl}_{3}, 125 \mathrm{MHz}\right) \delta=$ 152.6, 152.3, 135.9, 135.6, 131.1, 128.9, 127.4, 127.2, 123.5, 121.0, 120.3, 120.2, 118.9, 118.1, 111.0, 105.7, 32.3, 31.9, 26.1, 23.7, 23.6, 23.5, 22.1. HRMS (ESI): $\mathrm{m} / \mathrm{z}$ : calculated for $\mathrm{C}_{25} \mathrm{H}_{26} \mathrm{NO}^{+}: 356.2009$ [M+H] ${ }^{+}$, found, 356.2009 .

\section{Data for 15ak}

$[\alpha]_{D}=+16\left(c\right.$ 0.50, $\left.\mathrm{CHCl}_{3}\right)$. ee $=92 \%$, determined on a Chiralpak IA column (heptane:IPA = 95:5, 1 $\mathrm{mL} / \mathrm{min}$ ), retention times: $\mathrm{T}_{\mathrm{R}}$ (minor): $4.2 \mathrm{~min}, \mathrm{~T}_{\mathrm{R}}$ (major): $4.6 \mathrm{~min} .{ }^{1} \mathrm{H} \mathbf{N M R}\left(\mathrm{CDCl}_{3}, \mathbf{5 0 0} \mathbf{M H z}\right) \delta=7.71(\mathrm{~d}, J$ $=7.9 \mathrm{~Hz}, 1 \mathrm{H}), 7.65(\mathrm{~d}, J=7.9 \mathrm{~Hz}, 2 \mathrm{H}), 7.42(\mathrm{~d}, J=8.2 \mathrm{~Hz}, 1 \mathrm{H}), 7.39(\mathrm{t}, J=7.6 \mathrm{~Hz}, 2 \mathrm{H}), 7.28-7.22(\mathrm{~m}, 2 \mathrm{H}), 7.15$ $(\mathrm{d}, J=7.6 \mathrm{~Hz}, 1 \mathrm{H}), 6.81(\mathrm{~s}, 1 \mathrm{H}), 6.45(\mathrm{~s}, 1 \mathrm{H}), 5.56(\mathrm{t}, J=5.5,1 \mathrm{H}), 3.21(\mathrm{~m}, J=6.7 \mathrm{~Hz}, 1 \mathrm{H}), 2.93-2.88(\mathrm{~m}, 1 \mathrm{H})$, 2.82-2.77 (m, $1 \mathrm{H}), 2.23-2.17(\mathrm{~m}, 1 \mathrm{H}), 2.12-2.06(\mathrm{~m}, 1 \mathrm{H}), 2.03-1.92(\mathrm{~m}, 2 \mathrm{H}), 1.35\left(\mathrm{dd}, J_{1}=6.7 \mathrm{~Hz}, J_{2}=4.6 \mathrm{~Hz}\right.$, 6H). ${ }^{13} \mathrm{C}$ NMR $\left(\mathrm{CDCl}_{3}, 125 \mathrm{MHz}\right) \delta=153.2,153.0,136.5,131.1,128.9,128.0,127.3,123.7,122.6,121.7$, 121.4, 119.9, 119.2, 118.8, 109.8, 105.1, 50.1, 30.9, 25.8, 23.7, 23.6, 23.3, 20.7. HRMS (ESI): $\mathrm{m} / \mathrm{z}$ : calculated for $\mathrm{C}_{25} \mathrm{H}_{26} \mathrm{NO}^{+}: 356.2009[\mathrm{M}+\mathrm{H}]^{+}$, found, 356.2010. 
<smiles>O=C(NC1CCCc2oc(-c3ccccc3)cc21)OCc1ccccc1</smiles>

Compound 16a was prepared according to a modified general procedure from $8 \mathrm{a}(107.9 \mathrm{mg}, 0.55 \mathrm{mmol}$, 1.1 equiv.), benzyl carbamate ( $75.6 \mathrm{mg}, 0.5 \mathrm{mmol}, 1.0$ equiv.), (S)-5 (8.4 mg, $0.01 \mathrm{mmol}, 2 \mathrm{~mol} \%$ ) and $\mathrm{Ag}_{2} \mathrm{CO}_{3}$ $(1.4 \mathrm{mg}, 0.005 \mathrm{mmol}, 1 \mathrm{~mol} \%)$ in toluene $(2.5 \mathrm{~mL})$, yielding product $16 \mathrm{a}(151.7 \mathrm{mg}, 0.44 \mathrm{mmol}, 88 \%)$ as a white solid for which the spectroscopical data correspond to those reported in the literature. ${ }^{13}$

$[\alpha]_{D}=-3\left(c 1.15, \mathrm{CHCl}_{3}\right)$. ee $=94 \%$, determined on a Chiralpak IB column (heptane:IPA $=97: 3,1 \mathrm{~mL} / \mathrm{min}$ ), retention times: $\mathrm{T}_{\mathrm{R}}$ (minor): $17.7 \mathrm{~min}, \mathrm{~T}_{\mathrm{R}}$ (major): $22.5 \mathrm{~min} .{ }^{1} \mathrm{H} \mathbf{N M R}\left(\mathrm{CDCl}_{3}, 500 \mathrm{MHz}\right) \delta=7.56(\mathrm{~d}, J=7.6 \mathrm{~Hz}$, 2H), 7.33-7.29 (m, 7H), $7.18(\mathrm{t}, J=7.3 \mathrm{~Hz}, 1 \mathrm{H}), 6.53(\mathrm{~s}, 1 \mathrm{H}), 5.11(\mathrm{~s}, 2 \mathrm{H}), 5.03(\mathrm{~d}, J=7.9 \mathrm{~Hz}, 1 \mathrm{H}), 4.75-4.74$ (m, $1 \mathrm{H}), 2.63-2.54(\mathrm{~m}, 2 \mathrm{H}), 2.00-1.96(\mathrm{~m}, 1 \mathrm{H}), 1.88-1.80(\mathrm{~m}, 2 \mathrm{H}), 1.69-1.66(\mathrm{~m}, 1 \mathrm{H})$. HRMS (ESI): $\mathrm{m} / \mathrm{z}$ : calculated for $\mathrm{C}_{22} \mathrm{H}_{22} \mathrm{NO}_{3}{ }^{+}: 348.1594[\mathrm{M}+\mathrm{H}]^{+}$, found, 348.1597.

(S)-4-methyl-N-(2-phenyl-4,5,6,7-tetrahydrobenzofuran-4-yl)benzenesulfonamide (16b)<smiles>FNC1CCCc2oc(-c3ccccc3)cc21</smiles>

Yield: $80 \%$, ee: $88 \%$.

Compound 16b was prepared according to the general procedure from $8 \mathrm{a}$ (107.9 $\mathrm{mg}, 0.55 \mathrm{mmol}, 1.1$ equiv.), tosylamide ( $85.6 \mathrm{mg}, 0.5 \mathrm{mmol}, 1.0$ equiv.), $(\mathrm{S})-5$ (0.8 mg, $0.001 \mathrm{mmol}, 0.2 \mathrm{~mol} \%$ ) and $\mathrm{Ag}_{2} \mathrm{CO}_{3}(0.1$ $\mathrm{mg}, 0.0005 \mathrm{mmol}, 0.1 \mathrm{~mol} \%)$ in toluene $(2.5 \mathrm{~mL})$, yielding product $16 \mathrm{~b}(146.4 \mathrm{mg}, 0.40 \mathrm{mmol}, 80 \%)$ as a white solid for which the spectroscopical data correspond to those reported in the literature. ${ }^{13}$

When the reaction was performed with 0.2 mol\% of catalyst $(S)-5$, the obtained ee was $94 \%$ but with a decrease of the yield to $17 \%$.

16b was alternatively obtained from the same protocol in the absence of $\mathrm{Ag}_{2} \mathrm{CO}_{3}$ in $41 \%$ yield and $88 \%$ ee.

$[\alpha]_{\mathrm{D}}=-99\left(\right.$ c 1.14, $\left.\mathrm{CHCl}_{3}\right)$. ee $=87 \%$, determined on a Chiralpak IC column (heptane:IPA $=90: 10,1$

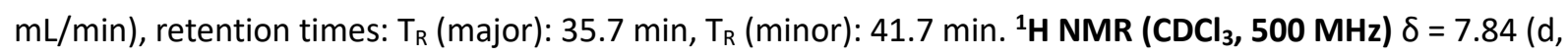
$J=8.2 \mathrm{~Hz}, 2 \mathrm{H}), 7.46(\mathrm{~d}, J=7.3 \mathrm{~Hz}, 2 \mathrm{H}), 7.37(\mathrm{~d}, J=8.2 \mathrm{~Hz}, 2 \mathrm{H}), 7.33(\mathrm{t}, J=7.6 \mathrm{~Hz}, 2 \mathrm{H}), 7.21(\mathrm{t}, J=7.3 \mathrm{~Hz}, 1 \mathrm{H})$, 5.86-5.85 (m, $1 \mathrm{H}), 4.59(\mathrm{~d}, J=8.2 \mathrm{~Hz}, 1 \mathrm{H}), 4.38-4.36(\mathrm{~m}, 1 \mathrm{H}), 2.66-2.61(\mathrm{~m}, 1 \mathrm{H}), 2.58-2.52(1 \mathrm{H}), 2.48(\mathrm{~s}, 3 \mathrm{H})$, 
1.90-1.73 (m, 4H). HRMS (ESI): $\mathrm{m} / \mathrm{z}$ : calculated for $\mathrm{C}_{21} \mathrm{H}_{20} \mathrm{NO}_{3} \mathrm{~S}^{-}: 366.1169$ [M-H]', found, 366.1172.

(S)-N,2-diphenyl-4,5,6,7-tetrahydrobenzofuran-4-amine (16c)

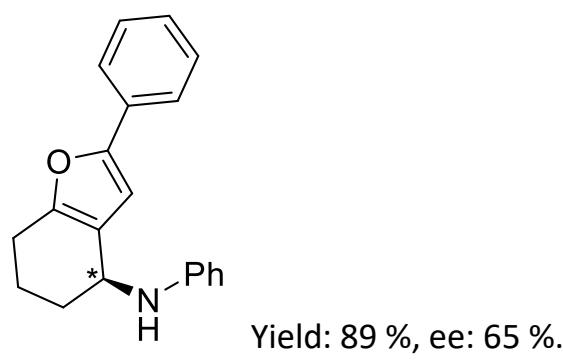

Compound 16c was prepared according to the general procedure from $8 \mathrm{a}(21.6 \mathrm{mg}, 0.11 \mathrm{mmol}, 1.1$ equiv.), aniline (0.009 mL, $0.1 \mathrm{mmol}, 1.0$ equiv.), (S)-5 (8.4 mg, $0.01 \mathrm{mmol}, 2 \mathrm{~mol} \%$ ) and $\mathrm{Ag}_{2} \mathrm{CO}_{3}(1.4 \mathrm{mg}$, $0.005 \mathrm{mmol}, 1 \mathrm{~mol} \%)$ in toluene $(1 \mathrm{~mL})$, yielding product $16 \mathrm{c}(25.6 \mathrm{mg}, 0.09 \mathrm{mmol}, 89 \%)$ as a white solid for which the spectroscopical data correspond to those reported in the literature. ${ }^{13}$

$[\alpha]_{\mathrm{D}}=+4\left(c\right.$ 0.95, $\left.\mathrm{CHCl}_{3}\right)$. ee $=65 \%$, determined on a Chiralpak IB column (heptane:IPA $=98: 02,1$ $\mathrm{mL} / \mathrm{min}$ ), retention times: $\mathrm{T}_{\mathrm{R}}$ (major): $10.5 \mathrm{~min}, \mathrm{~T}_{\mathrm{R}}$ (minor): $14.0 \mathrm{~min} .{ }^{1}{ }^{\mathrm{H} \mathrm{NMR}}$ (CDCl, $\left.500 \mathrm{MHz}\right) \delta=7.59$ (t, $J=7.3 \mathrm{~Hz}, 2 \mathrm{H}), 7.33(\mathrm{t}, J=7.6 \mathrm{~Hz}, 2 \mathrm{H}), 7.24-7.19(\mathrm{~m}, 3 \mathrm{H}), 6.74-6.68(\mathrm{~m}, 3 \mathrm{H}), 6.58(\mathrm{~s}, 1 \mathrm{H}), 4.56(\mathrm{br}, 1 \mathrm{H}), 3.79$ (bs, $1 \mathrm{H}), 2.75-2.62(\mathrm{~m}, 2 \mathrm{H}), 2.02-1.95(\mathrm{~m}, 2 \mathrm{H}), 1.88-1.80(\mathrm{~m}, 2 \mathrm{H})$. HRMS (ESI): $\mathrm{m} / \mathrm{z}$ : calculated for $\mathrm{C}_{20} \mathrm{H}_{18} \mathrm{NO}^{+}$: $288.1383\left[\mathrm{M}-\mathrm{H}_{2}+\mathrm{H}\right]^{+}$, found, 288.1393.

\section{(S)-4-phenoxy-2-phenyl-4,5,6,7-tetrahydrobenzofuran (16d)}

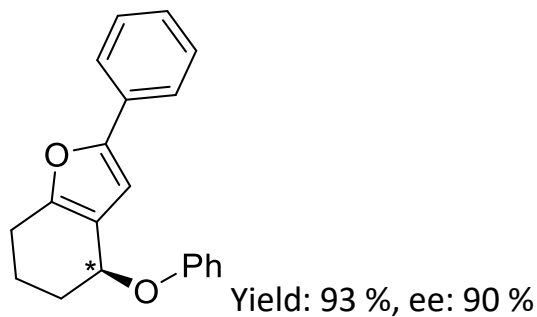

Compound 16d was prepared according to the general procedure from $8 \mathrm{a}(107.9 \mathrm{mg}, 0.55 \mathrm{mmol}, 1.1$ equiv.), phenol (47.1 mg, $0.5 \mathrm{mmol}, 1.0$ equiv.), (S)-5 (0.8 mg, $0.001 \mathrm{mmol}, 0.2 \mathrm{~mol} \%$ ) and $\mathrm{Ag}_{2} \mathrm{CO}_{3}(0.1 \mathrm{mg}$, $0.0005 \mathrm{mmol}, 0.1 \mathrm{~mol} \%)$ in toluene $(2.5 \mathrm{~mL})$, yielding product $16 \mathrm{~d}(133.9 \mathrm{mg}, 0.46 \mathrm{mmol}, 93 \%)$ as a white solid for which the spectroscopical data correspond to those reported in the literature. ${ }^{13}$

$[\alpha]_{\mathrm{D}}=-21\left(c\right.$ 0.99, $\left.\mathrm{CHCl}_{3}\right)$. ee $=90 \%$, determined on a Chiralpak IB column (heptane:IPA $=98: 02,1$ $\mathrm{mL} / \mathrm{min}$ ), retention times: $\mathrm{T}_{\mathrm{R}}$ (major): $6.1 \mathrm{~min}, \mathrm{~T}_{\mathrm{R}}$ (minor): $\left.9.2 \mathrm{~min} .{ }^{1} \mathrm{H} \mathrm{NMR} \mathrm{CDCl}_{3}, \mathbf{5 0 0} \mathbf{M H z}\right) \delta=7.60(\mathrm{~d}, J$ $=7.3 \mathrm{~Hz}, 2 \mathrm{H}), 7.35-7.30(\mathrm{~m}, 4 \mathrm{H}), 7.21(\mathrm{t}, J=7.3 \mathrm{~Hz}, 1 \mathrm{H}), 7.03(\mathrm{~d}, J=7.9 \mathrm{~Hz}, 2 \mathrm{H}), 6.98(\mathrm{t}, J=7.3 \mathrm{~Hz}, 1 \mathrm{H}), 6.58$ $(\mathrm{s}, 1 \mathrm{H}), 5.36(\mathrm{t}, J=4.3 \mathrm{~Hz}, 1 \mathrm{H}), 2.80\left(\mathrm{dt}, J_{1}=16.8, J_{2}=5.2 \mathrm{~Hz}, 1 \mathrm{H}\right), 2.69-2.63(\mathrm{~m}, 1 \mathrm{H}), 2.16-2.08(\mathrm{~m}, 2 \mathrm{H}), 2.00-$ $1.93(\mathrm{~m}, 1 \mathrm{H}), 1.92-1.85(\mathrm{~m}, 1 \mathrm{H})$. HRMS (ESI): $\mathrm{m} / \mathrm{z}$ : calculated for $\mathrm{C}_{20} \mathrm{H}_{17} \mathrm{O}_{2}{ }^{+}: 289.1223\left[\mathrm{M}-\mathrm{H}_{2}+\mathrm{H}\right]^{+}$, found, 289.1224. 
(S)-2-phenyl-4,5,6,7-tetrahydrobenzofuran-4-ol (16e), (4S,4'S)-4,4'-oxybis(2-phenyl-4,5,6,7tetrahydrobenzofuran) (16eb) and (S)-2-phenyl-4-(((R)-2-phenyl-4,5,6,7-tetrahydrobenzofuran-4yl)oxy)-4,5,6,7-tetrahydrobenzofuran (16ec)<smiles>O[C@H]1CCCc2oc(-c3ccccc3)cc21</smiles>

16ea

Yield: $32 \%$ ee: $79 \%$

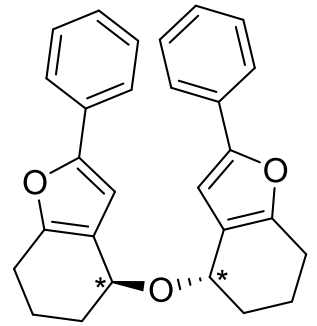

$16 \mathrm{eb}$

Yield: $50 \%$

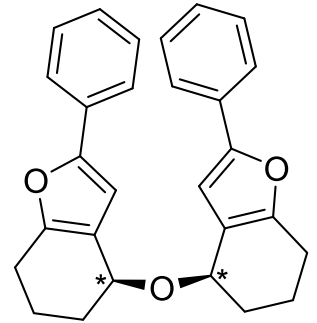

$16 \mathrm{ec}$

Yield: $18 \%$

Compound 16e was prepared according to the general procedure from $8 \mathrm{a}(98.1 \mathrm{mg}, 0.5 \mathrm{mmol}, 1.0$ equiv.), water ( $0.03 \mathrm{~mL}, 1.5 \mathrm{mmol}, 3.0$ equiv.), (S)-5 (0.8 mg, $0.001 \mathrm{mmol}, 0.2 \mathrm{~mol} \%$ ) and $\mathrm{Ag}_{2} \mathrm{CO}_{3}(0.1 \mathrm{mg}$, $0.0005 \mathrm{mmol}, 0.1 \mathrm{~mol} \%$ ) in toluene ( $2.5 \mathrm{~mL}$ ), yielding products $16 \mathrm{ea}$ ( $34.3 \mathrm{mg}, 0.16 \mathrm{mmol}, 32 \%), 16 \mathrm{eb}$ (50.9 $\mathrm{mg}, 0.25 \mathrm{mmol}, 50 \%)$ and $16 \mathrm{ec}(18.1 \mathrm{mg}, 0.09 \mathrm{mmol}, 18 \%)$ as white solids.

\section{Data for 16ea: ${ }^{13}$}

$[\alpha]_{\mathrm{D}}=+12\left(c\right.$ 1.44, $\left.\mathrm{CHCl}_{3}\right)$. ee $=97 \%$, determined on a Chiralpak IB column (heptane:IPA $=98: 2,1$

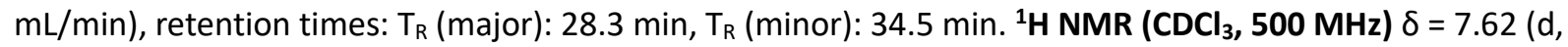
$J=7.3 \mathrm{~Hz}, 2 \mathrm{H}), 7.35(\mathrm{t}, J=7.6 \mathrm{~Hz}, 2 \mathrm{H}), 7.22(\mathrm{t}, J=7.6 \mathrm{~Hz}, 1 \mathrm{H}), 6.66(\mathrm{~s}, 1 \mathrm{H}), 4.77-4.75(\mathrm{~m}, 1 \mathrm{H}), 2.75-2.69(\mathrm{~m}$, $1 \mathrm{H}), 2.64-2.58(\mathrm{~m}, 1 \mathrm{H}), 2.08-2.00(\mathrm{~m}, 1 \mathrm{H}), 1.98-1.92(\mathrm{~m}, 1 \mathrm{H}), 1.89-1.78(\mathrm{~m}, 2 \mathrm{H}), 1.62(\mathrm{~s}, 1 \mathrm{H})$. HRMS (ESI): $\mathrm{m} / \mathrm{z}$ : calculated for $\mathrm{C}_{14} \mathrm{H}_{13} \mathrm{O}_{2}{ }^{+}: 213.0910\left[\mathrm{M}-\mathrm{H}_{2}+\mathrm{H}\right]^{+}$, found, 213.0912 .

\section{Data for 16eb:}

$[\alpha]_{\mathrm{D}}=+75\left(\mathrm{c} 0.95, \mathrm{CHCl}_{3}\right) .{ }^{1} \mathrm{H} \mathrm{NMR}\left(\mathrm{CDCl}_{3}, 500 \mathrm{MHz}\right) \delta=7.61(\mathrm{~d}, J=7.9 \mathrm{~Hz}, 4 \mathrm{H}), 7.34(\mathrm{t}, J=7.9 \mathrm{~Hz}, 4 \mathrm{H})$, $7.20(\mathrm{t}, J=7.3 \mathrm{~Hz}, 2 \mathrm{H}), 6.61(\mathrm{~s}, 2 \mathrm{H}), 4.62(\mathrm{t}, J=3.8 \mathrm{~Hz}, 2 \mathrm{H}), 2.74\left(\mathrm{dt}, J_{1}=16.4 \mathrm{~Hz}, J_{2}=5.0 \mathrm{~Hz}, 2 \mathrm{H}\right), 2.64-2.58$ $\left.(\mathrm{m}, 2 \mathrm{H}), 2.16-2.11(\mathrm{~m}, 2 \mathrm{H}), 2.03-1.99(\mathrm{~m}, 2 \mathrm{H}), 1.92-1.87(\mathrm{~m}, 4 \mathrm{H}) .{ }^{13} \mathrm{C} \mathrm{NMR}_{(\mathrm{CDCl}}, 125 \mathrm{MHz}\right) \delta=153.0,131.4$, 128.9, 128.8, 127.1, 123.7, 120.9, 105.3, 69.2, 29.8, 23.5, 19.2. HRMS (ESI): $m / z$ : calculated for $\mathrm{C}_{28} \mathrm{H}_{27} \mathrm{O}_{3}{ }^{+}$: $411.1955[\mathrm{M}+\mathrm{H}]^{+}$, found, 411.1956 .

\section{Data for 16ec:}

${ }^{1} \mathrm{H} \mathrm{NMR}\left(\mathrm{CDCl}_{3}, 500 \mathrm{MHz}\right) \delta=7.62(\mathrm{~d}, J=7.9 \mathrm{~Hz}, 4 \mathrm{H}), 7.34(\mathrm{t}, J=7.6 \mathrm{~Hz}, 4 \mathrm{H}), 7.20(\mathrm{t}, J=7.3 \mathrm{~Hz}, 2 \mathrm{H})$, $6.61(\mathrm{~s}, 2 \mathrm{H}), 4.70(\mathrm{t}, J=4.1 \mathrm{~Hz}, 2 \mathrm{H}), 2.75\left(\mathrm{dt}, J_{1}=16.4 \mathrm{~Hz}, J_{2}=5.3 \mathrm{~Hz}, 2 \mathrm{H}\right), 2.66-2.60(\mathrm{~m}, 2 \mathrm{H}), 2.16-2.10(\mathrm{~m}$, $2 \mathrm{H}), 1.96-1.93(\mathrm{~m}, 4 \mathrm{H}), 1.88-1.84(\mathrm{~m}, 2 \mathrm{H}) .{ }^{13} \mathrm{C} \mathrm{NMR}_{\left(\mathrm{CDCl}_{3}, 125 \mathrm{MHz}\right)} \delta=152.9,131.4,128.8,127.1,123.8$, 123.7, 121.1, 105.3, 69.3, 30.2, 23.5, 19.5. HRMS (ESI): $\mathrm{m} / \mathrm{z}$ : calculated for $\mathrm{C}_{28} \mathrm{H}_{27} \mathrm{O}_{3}{ }^{+}: 411.1955[\mathrm{M}+\mathrm{H}]^{+}$, found, 411.1953 . 
(S)-3-((2-phenyl-4,5,6,7-tetrahydrobenzofuran-4-yl)oxy)cyclohex-2-en-1-one (16f)<smiles>O=C1C=C(OC2CCCC(OC3CCCCC3)c3cc(-c4ccccc4)oc32)CCC1</smiles>

$\mathrm{Na}$

Yield: $77 \%$, ee: $89 \%$.

Compound $16 \mathrm{f}$ was prepared according to the general procedure from $8 \mathrm{a}(21.6 \mathrm{mg}, 0.11 \mathrm{mmol}, 1.1$ equiv.), $9 b$ (11.2 mg, $0.1 \mathrm{mmol}, 1.0$ equiv.), (S)-5 (0.2 mg, $0.0002 \mathrm{mmol}, 0.2 \mathrm{~mol} \%$ ) and $\mathrm{Ag}_{2} \mathrm{CO}_{3}(0.1 \mathrm{mg}$, $0.0001 \mathrm{mmol}, 0.1 \mathrm{~mol} \%)$ in toluene $(0.5 \mathrm{~mL})$, yielding product $16 \mathrm{f}(23.6 \mathrm{mg}, 0.77 \mathrm{mmol}, 77 \%)$ as a white solid for which the spectroscopical data correspond to those reported in the literature. ${ }^{13}$

$[\alpha]_{\mathrm{D}}=-150\left(c\right.$ 0.92, $\left.\mathrm{CHCl}_{3}\right)$. ee: $89 \%$, determined on a Chiralpak IA column (heptane:IPA $=98: 02,1$ $\mathrm{mL} / \mathrm{min}$ ), retention times: $\mathrm{T}_{\mathrm{R}}$ (major): $22.8 \mathrm{~min}, \mathrm{~T}_{\mathrm{R}}$ (minor): $27.1 \mathrm{~min} .{ }^{1} \mathbf{H} \mathbf{N M R}\left(\mathrm{CDCl}_{3}, \mathbf{3 0 0} \mathbf{M H z}\right) \delta=7.54(\mathrm{~d}$, $J=7.3 \mathrm{~Hz}, 2 \mathrm{H}), 7.28(\mathrm{t}, J=7.5 \mathrm{~Hz}, 2 \mathrm{H}), 7.16(\mathrm{t}, J=7.7 \mathrm{~Hz}, 1 \mathrm{H}), 6.53(\mathrm{~s}, 1 \mathrm{H}), 5.54(\mathrm{~s}, 1 \mathrm{H}), 5.19(\mathrm{t}, J=3.6 \mathrm{~Hz}$, 1H), $2.72\left(\mathrm{dt}, J_{1}=16.8 \mathrm{~Hz}, J_{2}=4.7 \mathrm{~Hz}, 1 \mathrm{H}\right), 2.63-2.53(\mathrm{~m}, 1 \mathrm{H}), 2.37-2.29(\mathrm{~m}, 4 \mathrm{H}), 2.05-1.79(\mathrm{~m}, 6 \mathrm{H}) . \mathbf{H R M S}$ (ESI): $\mathrm{m} / z$ : calcd for $\mathrm{C}_{40} \mathrm{H}_{40} \mathrm{NaO}_{6}{ }^{+} 639.2723[2 \mathrm{M}+\mathrm{Na}]^{+}$, found, 639.2717 . 


\section{X-ray Crystallography}

X-ray diffraction data were measured for 10 aa and (S)-5 using a RIGAKU diffractometer constituted by a MM007 HF rotating-anode generator, delivering copper radiation through Osmic CMF confocal optics, and a Rapid II curved Image Plate detector. Fs_process ${ }^{14}$ software under the CrystalClear $2.0^{15}$ suite was used to integrate and scale the data, applying multi-scan $R E Q A B^{6}$ for the absorption correction. Regarding SI-1, the data collection was carried out with a RIGAKU XtaLabPro diffractometer equipped with a Mo microfocus sealed tube generator coupled to a double-bounce confocal Max-Flux ${ }^{\circledast}$ multilayer optic and a HPAD PILATUS3R 200K detector. CrysAlisPro 1.171.39.46 16 was employed for the data processing, with SCALE3 ABSPACK scaling algorithm implemented for the empirical absorption correction using spherical harmonics. The structures were solved either by dual-space direct methods (SHELXD program), ${ }_{1}^{17}$ (regarding the two first ones) or by intrinsic phasing methods (SHELXT program), ${ }^{18}$ and all were refined by full-matrix leastsquares methods on $F^{2}$ using SHELX-L. ${ }^{19}$ All non-hydrogen atoms of the molecules of interest improved by anisotropic refinement. Most of the $\mathrm{H}$ atoms were identified in difference maps. Methyl $\mathrm{H}$ atoms of the solvent isopropanol molecule present in the $(\boldsymbol{S})-5$ structure were idealized and included as rigid groups allowed to rotate but not tip, and refined with $U_{\text {iso }}$ set to $1.5 U_{\text {eq }}(C)$ of the parent carbon atom. All other $\mathrm{H}$ atoms bound to carbon atoms were positioned geometrically and refined with $U_{\text {iso }}$ set to $1.2 U_{\mathrm{eq}}(\mathrm{C})$ of the parent carbon atom. The position of the hydrogen atom in the $\mathrm{NH}$ group in 10 aa was refined freely with $U_{\text {iso }}$ set to $1.2 U_{\text {eq }}(\mathrm{N})$. The $\mathrm{NH}$ group is pointing perpendicularly toward the six-membered moiety of the indole at $x+0.5,0.5-y, 1-z$ at a $2.60 \AA$ distance. The two hydroxy $H$ atoms in $(S)-5$ were allowed to change the torsion angle (instruction AFIX 147) and their displacement parameters were related with $U_{\text {iso }}$ set to $1.5 U_{\text {eq }}(O)$. A BUMP instruction was nevertheless applied to prevent steric clashes between them, the hydroxyl group of the solvent molecule bridging two phosphate moieties at $x, y, z$ and $x-0.5,1.5-y, 1-z$ respectively, via O-H...O $\mathrm{H}$-bonds generating the chain motifs $\mathrm{C}^{2}{ }_{2}(6) .{ }^{20}$ The anisotropic displacement parameters of atoms in a phenyl group were restrained using the RIGU ${ }^{21}$ instruction. Crystal data, data collection and structure refinement details are summarized in Table $\mathbf{S 2}$. The three structures have in common to belong to the same space group P $2_{1} 2_{1} 2_{1}$ and only the third one has more than one molecule in the asymmetric unit (ie 2). The absolute structures for (S)-5 and SI-1 were straightforwardly confirmed by dispersion effects, mainly provided by the Au $M$ (at the Cu source excitation) and $L$ (with the Mo source) far-absorption edges (> 5e-): both compounds synthesized from pure aS binol conserved that axial chirality. Moreover the tetracoordinate phosphorus stereogenic centre in SI-1 is $\left(R_{P}\right)$. Absence of strong anomalous scatterers in 10aa could have hampered its absolute structure determination on $\mathrm{C} 1(R)$ but highly redundant data quality coupled with intense microfocused copper radiation led to significant Flack ${ }^{22}$ parameter estimate in favour of that assignment. This was corroborated later on by Bayesian-type analysis ${ }^{23}$ upon $100 \%$ Bijvoet pairs of reflections, as implemented within the PLATON program ${ }^{24}$ (see Table S2).

\footnotetext{
14 Rigaku - Higashi T. FS Process, Rigaku Corporation, 1998, Tokyo, Japan.

15 Rigaku. CrystalClear-SM Expert 2.0 r4 Rigaku Corporation, 2009, Tokyo, Japan.

16 Rigaku OD CrysAlis PRO. Rigaku Oxford Diffraction, Yarnton, Oxfordshire, 2015, England.

17 Sheldrick, G. M. Acta Crystallogr., 2008, A64, 112-122

18 Sheldrick, G. M. Acta Crystallogr., 2015, C71, 3-8.

19 Sheldrick, G. M. Acta Crystallogr., 2015, A71, 3-8.

20 Bernstein, J., Davis, R. E., Shimoni, L. \& Chang, N. L. Angew. Chem. Int. Ed. Engl. 1995, 34, 1555-1573.

21 Thorn, A., Dittrich, B. \& Sheldrick, G. M. Acta Crystallogr., 2012, A68, 448-451.

22 Flack, H.D. Acta Crystallogr., 1983, A39, 876-881.

23 Hooft, R.W.W., Straver, L.H., Spek, A.L. J. Appl. Cryst., 2008, 41, 96-103.

24 Spek, A. L. Acta Crystallogr., 2009, D65, 148-155.
} 


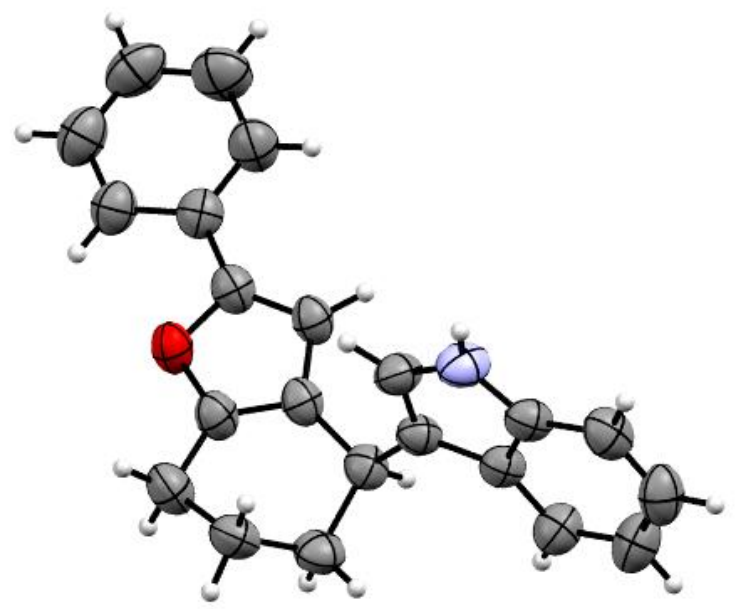

Figure S1. X-ray Molecular Structure of 10aa

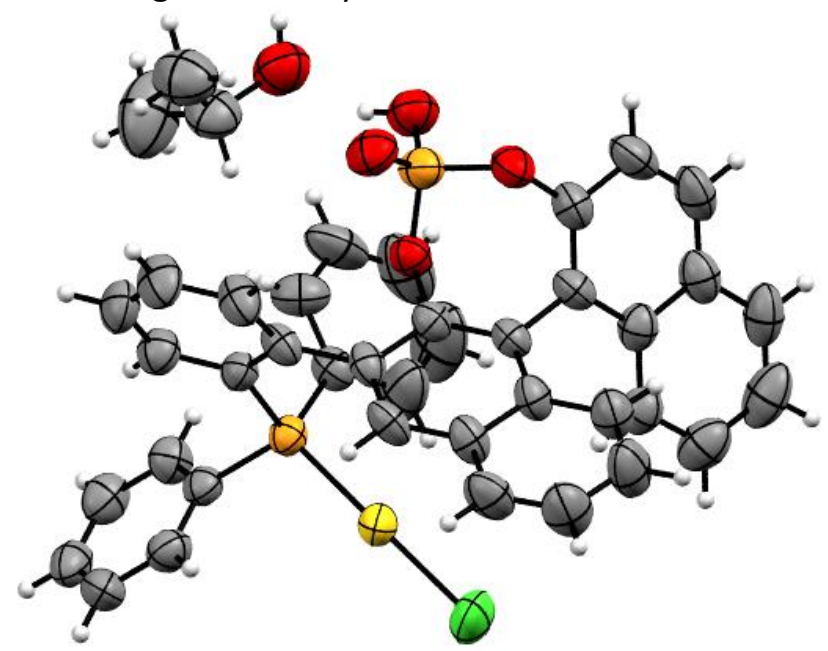

Figure S2. X-ray Molecular Structure of (S)-5

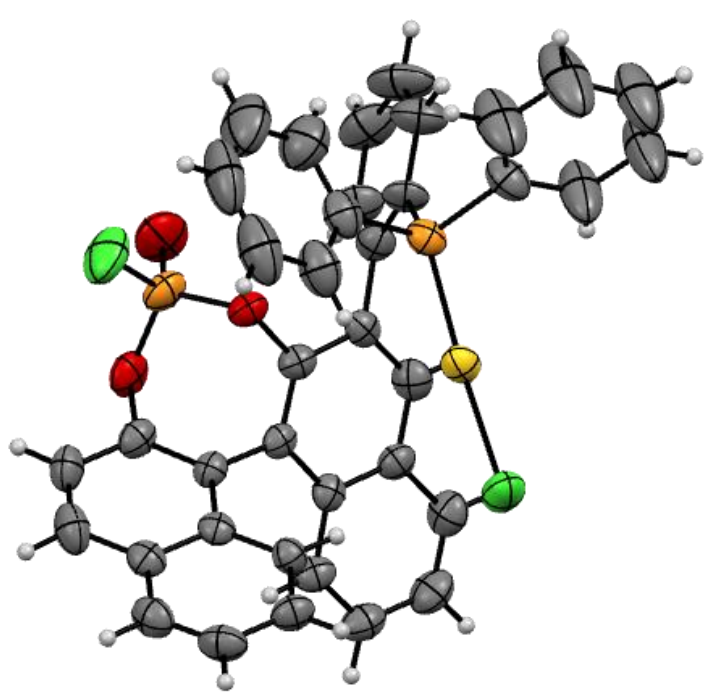

Figure S3. X-ray Molecular Structure of SI-1 
Table S2: Crystal data, data collection and structure refinement details for the 3 compounds.

\begin{tabular}{|c|c|c|c|c|}
\hline Compound & & 10aa & $(S)-5$ & SI-1 \\
\hline Empirical formula & & $\mathrm{C}_{22} \mathrm{H}_{19} \mathrm{NO}$ & $\begin{array}{c}\mathrm{C}_{38} \mathrm{H}_{26} \mathrm{AuClO}_{4} \mathrm{P}_{2} \\
\mathrm{C}_{3} \mathrm{H}_{8} \mathrm{O}\end{array}$ & $\mathrm{C}_{38} \mathrm{H}_{25} \mathrm{AuCl}_{2} \mathrm{O}_{3} \mathrm{P}_{2}$ \\
\hline Formula weight & & 313.38 & 901.04 & 859.39 \\
\hline Temperature (K) & & $293(2)$ & $293(2)$ & $293(2)$ \\
\hline Wavelength (Å) & & 1.54187 & 1.54187 & 0.71073 \\
\hline $\begin{array}{l}\text { Crystal system, } \\
\text { space group }\end{array}$ & & $\begin{array}{l}\text { Orthorhombic, } \\
\quad P 2_{1} 2_{1} 2_{1}\end{array}$ & $\begin{array}{l}\text { Orthorhombic, } \\
\quad P 2_{1} 2_{1} 2_{1}\end{array}$ & $\begin{array}{l}\text { Orthorhombic, } \\
\quad P 2_{1} 2_{1} 2_{1}\end{array}$ \\
\hline \multirow{6}{*}{ Unit cell dimensions } & a $(\AA ̊)$ & $6.6896(3)$ & $10.8015(2)$ & $11.26267(18)$ \\
\hline & $b(\AA ̊)$ & $8.6995(3)$ & $17.6790(3)$ & $19.0781(4)$ \\
\hline & $c(\AA)$ & $28.863(2)$ & 19.6851(14) & $31.7278(6)$ \\
\hline & $\alpha\left(\left(^{\circ}\right)\right.$ & 90 & 90 & 90 \\
\hline & $\beta\left(\left(^{\circ}\right)\right.$ & 90 & 90 & 90 \\
\hline & $\gamma\left(^{\circ}\right)$ & 90 & 90 & 90 \\
\hline Volume $\left(\AA^{3}\right)$ & & $1679.72(15)$ & $3759.1(3)$ & $6817.4(2)$ \\
\hline $\mathrm{Z}$ & & 4 & 4 & 8 \\
\hline Calculated density $\left(\mathrm{mg} / \mathrm{m}^{3}\right)$ & & 1.239 & 1.592 & 1.675 \\
\hline Absorption coefficient $\left(\mathrm{mm}^{-1}\right)$ & & 0.588 & 9.165 & 4.603 \\
\hline$F(000)$ & & 664 & 1784 & 3360 \\
\hline Crystal size $(\mathrm{mm})$ & & $0.42 \times 0.34 \times 0.29$ & $0.35 \times 0.26 \times 0.22$ & $0.30 \times 0.18 \times 0.10$ \\
\hline$\theta$ range for data collection $\left({ }^{\circ}\right)$ & & 3.062 to 68.230 & 4.492 to 68.235 & 3.618 to 29.083 \\
\hline Limiting indices & & $\begin{array}{l}-7 \leq h \leq 7 \\
-10 \leq k \leq 7 \\
-34 \leq 1 \leq 34\end{array}$ & $\begin{array}{c}-12 \leq h \leq 12 \\
-21 \leq k \leq 18 \\
-20 \leq 1 \leq 23\end{array}$ & $\begin{array}{c}-11 \leq h \leq 15 \\
-21 \leq k \leq 25 \\
-35 \leq 1 \leq 42\end{array}$ \\
\hline $\begin{array}{l}\text { Reflections collected / unique } \\
{[R \text { (int) }}\end{array}$ & & $\begin{array}{c}21364 / 3065 \\
0.0303\end{array}$ & 32647 / 68350.0462 & $\begin{array}{c}78149 / 15931 \\
0.0614\end{array}$ \\
\hline Completeness to $\theta$ full (\%) & & 99.7 & 99.0 & 99.5 \\
\hline Absorption correction & & \multicolumn{3}{|c|}{ Semi-empirical from equivalents } \\
\hline Max. and Min. transmission & & 1.000 and 0.815 & 1.000 and 0.605 & 1.000 and 0.264 \\
\hline Refinement method & & \multicolumn{3}{|c|}{ Full-matrix least-squares on $F^{2}$} \\
\hline Data / restraints / parameters & & $3063 / 0 / 221$ & 6802 / 39 / 456 & 15910 / 0 / 829 \\
\hline Goodness-of-fit on $F^{2}$ & & 1.074 & 1.079 & 1.045 \\
\hline Final $R$ indices & $\mathrm{R} 1$ & 0.0297 & 0.0361 & 0.0437 \\
\hline$[1>2 \sigma(I)]$ & wR2 & 0.0746 & 0.0837 & 0.0874 \\
\hline $\mathrm{R}$ indices & $\mathrm{R} 1$ & 0.0326 & 0.0386 & 0.0661 \\
\hline (all data) & wR2 & 0.0788 & 0.0867 & 0.0927 \\
\hline Absolute structure parameters ${ }^{16}$ & & & & \\
\hline Flack $\mathrm{x}^{14}$ & & $0.03(6)$ & $-0.023(3)$ & $-0.005(3)$ \\
\hline Parsons z 25 & & $0.00(7)$ & $-0.023(4)$ & $-0.004(3)$ \\
\hline Hooft y ${ }^{15}$ & & $0.02(5)$ & $-0.027(4)$ & $-0.011(4)$ \\
\hline P2 (true) & & $100 \%$ & $100 \%$ & $100 \%$ \\
\hline P3 (true) & & $100 \%$ & $100 \%$ & $100 \%$ \\
\hline Extinction coefficient & & $0.0017(6)$ & - & - \\
\hline Largest diff. peak and hole $\left(e . \AA^{-3}\right)$ & & 0.104 and -0.123 & 1.607 and -0.460 & 1.700 and -1.761 \\
\hline
\end{tabular}

\footnotetext{
25 Parsons, Flack and Wagner, Acta Crystallogr., 2013, B69 249-259.
} 
CCDC 1884441-1884443 (compounds 10aa, (S)-5, and SI-1 respectively) contain the supplementary crystallographic data for this paper. These data can be obtained free of charge from The Cambridge Crystallographic Data Centre via www.ccdc.cam.ac.uk/data request/cif 


\section{Computational experiments}

Calculations were carried out with the Gaussian09 package ${ }^{26}$ and all structures were fully optimized without any symmetry constraints at the DFT level by means of the M06 functional. ${ }^{27}$ The def2-SVP basis set $^{28}$ was applied for all atoms and solvent effects are accounted for by continuum solvation method (integral equation formalism version of the polarizable continuum model (IEFPCM) for toluene). Each stationary point has been characterized with frequency analysis and shows the correct number of negative eigenvalues (zero for a local minimum and one for a transition state). All transition states were verified by stepping along the reaction coordinate (intrinsic reaction coordinate calculations) and confirming that they transformed into the corresponding reactants/products. Final energy calculations at the M06 level associated with the def2-TZVPP basis set, including solvation effect, have been achieved on the IEFPCM(toluene)-M06/def2-SVP geometries. To get accurate geometries and energies, the SCF convergence criterion was systematically tightened to $10^{-8} \mathrm{au}$, and the force minimizations were carried out until the rms force became smaller that (at least) $1 \times 10^{-5}$ au ("tight" optimization keyword in Gaussian 09). The "UltraFine" grid (99 radial shells and 590 angular points per shell) was used throughout the calculations, as recommended when using Gaussian 09. The Gibbs free energies presented in this article are IEFPCM(toluene)-M06/def2-TZVPP//IEFPCM(toluene)-M06/def2-SVP electronic energies (which include solvation-energy corrections from the IEFPCM method) modified with thermal and entropy corrections from IEFPCM(toluene)-M06/def2-SVP calculations. Due to the well-known errors associated with entropy calculations, we apply a scaling factor of 0.5 to the entropic contributions as recommended in the literature. ${ }^{29}$ Therefore, the calculated $\Delta G$ values reported in this study include the ZPE, enthalpic temperature correction, solvation energy, and half the entropy. Note that application of this scheme only slightly modifies the activation barriers and the general profile, but changes the interaction between 2 molecules or the formation of two separate molecules from a single one.

${ }^{26}$ Frisch, M. J.; Trucks, G. W.; Schlegel, H. B.; Scuseria, G. E.; Robb, M. A.; Cheeseman, J. R.; Scalmani, G.; Barone, V.; Mennucci, B.; Petersson, G. A.; Nakatsuji, H.; Caricato, M.; Li, X.; Hratchian, H. P.; Izmaylov, A. F.; Bloino, J.; Zheng, G.; Sonnenberg, J. L.; Hada, M.; Ehara, M.; Toyota, K.; Fukuda, R.; Hasegawa, J.; Ishida, M.; Nakajima, T.; Honda, Y.; Kitao, O.; Nakai, H.; Vreven, T.; Montgomery, J. A. Jr.; Peralta, J. E.; Ogliaro, F.; Bearpark, M.; Heyd, J. J.; Brothers, E.; Kudin, K. N.; Staroverov, V. N.; Kobayashi, R.; Normand, J.; Raghavachari, K.; Rendell, A.; Burant, J. C.; Iyengar, S. S.; Tomasi, J.; Cossi, M.; Rega, N.; Millam, J. M.; Klene, M.; Knox, J. E.; Cross, J. B.; Bakken, V.; Adamo, C.; Jaramillo, J.; Gomperts, R.; Stratmann, R. E.; Yazyev, O.; Austin, A. J.; Cammi, R.; Pomelli, C.; Ochterski, J. W.; Martin, R. L.; Morokuma, K.; Zakrzewski, V. G.; Voth, G. A.; Salvador, P.; Dannenberg, J. J.; Dapprich, S.; Daniels, A. D.; Farkas, Ö.; Foresman, J. B.; Ortiz, J. V.; Cioslowski, J.; Fox, D. J. Gaussian, Inc., Wallingford CT, Gaussian 09, Revision D.01, 2013.

27 Zhao, Y.; Truhlar, D. G. Theor. Chem. Acc. 2008, 120, 215-241.

28 Weigend, F.; Ahlrichs, R. Phys. Chem. Chem. Phys. 2005, 7, 3297-3305.

${ }_{29}$ (a) Cooper, J.; Ziegler, T. A Inorg. Chem. 2002, 41, 6614-6622. (b) Lau, J. K. C.; Deudel, D. V. J. Chem. Theory Comput. 2006, 2, $103-106$. (c) Li, H.; Lu, G.; Jiang, J.; Huang, F.; Wang, Z. X. Organometallics 2011, 30, 2349-2363. (d) Hua, J.; Krizner, H. E.; De Haan, D. O. J. Phys. Chem. A 2011, 115, 1667-1675. 
Table S3. Absolute electronic energies and thermal corrections for all molecules.

\begin{tabular}{|c|c|c|c|c|}
\hline & $\begin{array}{c}\text { E } \\
\text { (IEFPCM(toluene)- } \\
\text { M06/def2-SVP) }\end{array}$ & $\begin{array}{l}\text { Thermal } \\
\text { correction to } \\
\text { Enthalpy }\end{array}$ & $\begin{array}{l}\text { Thermal } \\
\text { correction to } \\
\text { Gibbs Free } \\
\text { Energy }\end{array}$ & $\begin{array}{c}\text { E } \\
\text { (IEFPCM(toluene)- } \\
\text { M06/def2-TZVPP) }^{a}\end{array}$ \\
\hline $6 a$ & -2579.873720 & 0.563652 & 0.457837 & -2582.064036 \\
\hline $6 b$ & -2579.858139 & 0.563051 & 0.458189 & -2582.048093 \\
\hline $8 a$ & -614.973838 & 0.231001 & 0.175881 & -615.647943 \\
\hline la & -3194.881553 & 0.796223 & 0.661093 & -3197.736882 \\
\hline TS- & & & & \\
\hline I-Ila & -3194.866164 & 0.794745 & 0.659083 & -3197.721182 \\
\hline Ila & -3194.889867 & 0.797009 & 0.663051 & -3197.742211 \\
\hline Ib & -3194.876723 & 0.795906 & 0.658906 & -3197.733102 \\
\hline $\begin{array}{l}\text { TS- } \\
\text { I-IIb }\end{array}$ & -3194.859562 & 0.794646 & 0.658623 & -3197.716113 \\
\hline IIb & -3194.875312 & 0.796585 & 0.660517 & -3197.730015 \\
\hline II'a & -3558.190359 & 0.935523 & 0.783008 & -3561.433449 \\
\hline $\begin{array}{l}\text { TS- } \\
\text { II'-IIla }\end{array}$ & -3558.181394 & 0.935577 & 0.785308 & -3561.424148 \\
\hline IIIa & -3558.206139 & 0.937570 & 0.788999 & -3561.445807 \\
\hline $9 a$ & -363.281615 & 0.136580 & 0.099133 & -363.675534 \\
\hline $10 a$ & -978.341694 & 0.372484 & 0.305026 & -979.395817 \\
\hline 14 & -2581.082261 & 0.585818 & 0.476289 & \\
\hline
\end{tabular}

a Energies computed at the IEFPCM(toluene)-M06/def2-SVP geometries.

Comment: The main steps of the postulated reaction mechanism have been modelled in order to confirm the relevance of the mechanistic hypothesis and to estimate the influence of the tethered phosphate group (Figure S4). Coordination of the alkyne 8a to the catalyst 6a led to several conformers of intermediate I. Two of them, la and Ib, are represented in Figure S1. Both display in-plane coordination of the alkyne unit. In la the enone moiety is close to the phosphate group, while in Ib, the two groups are remote. la is favored by $6.7 \mathrm{~kJ}_{\mathrm{mol}}{ }^{-1}$ with respect to $\mathbf{l b}$. Starting from la, the exoergonic cycloisomerization step leads to the carbocationic intermediate lla which features an electrostatic pairing between the carbocation and the phosphate group. The absence of this electrostatic interaction, as modeled in path $b$, leads to a higher energy barrier (42.6 vs $36.6 \mathrm{~kJ}_{\mathrm{mol}}{ }^{-1}$ ) and ends up to an endergonic process. The interaction

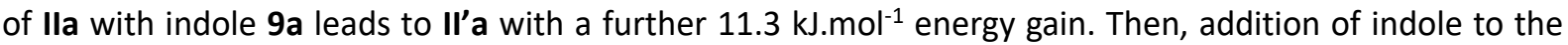
carbocation though its C3-carbon goes across a $27.5 \mathrm{~kJ} \mathrm{~mol}^{-1}$ activation barrier. It results in IIla that lies at $71.0 \mathrm{~kJ} . \mathrm{mol}^{-1}$ on the free energy surface. 


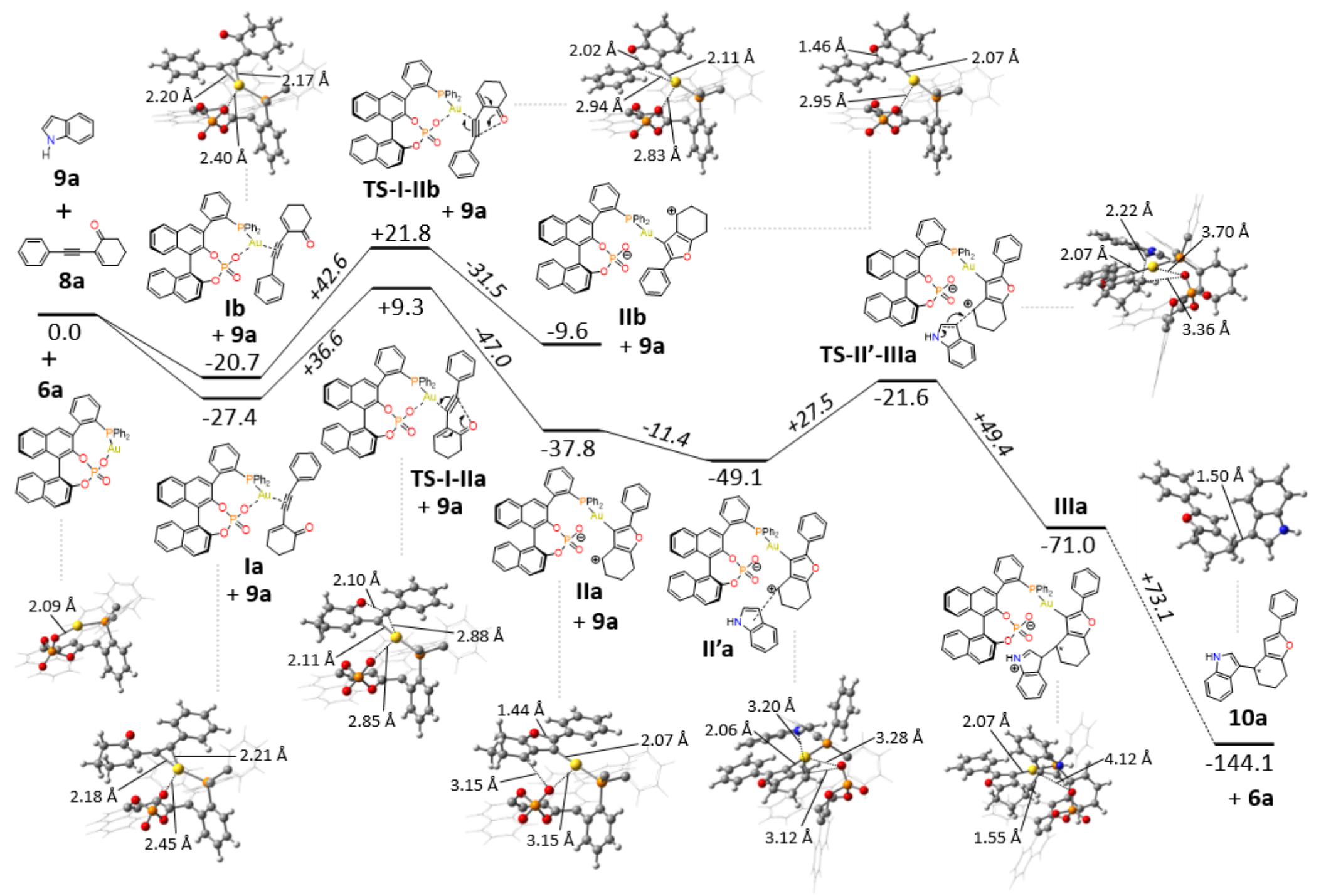

Figure S4. Computed mechanistic patterns 


\section{Cartesian coordinates of the optimized geometries}

Structure 6a

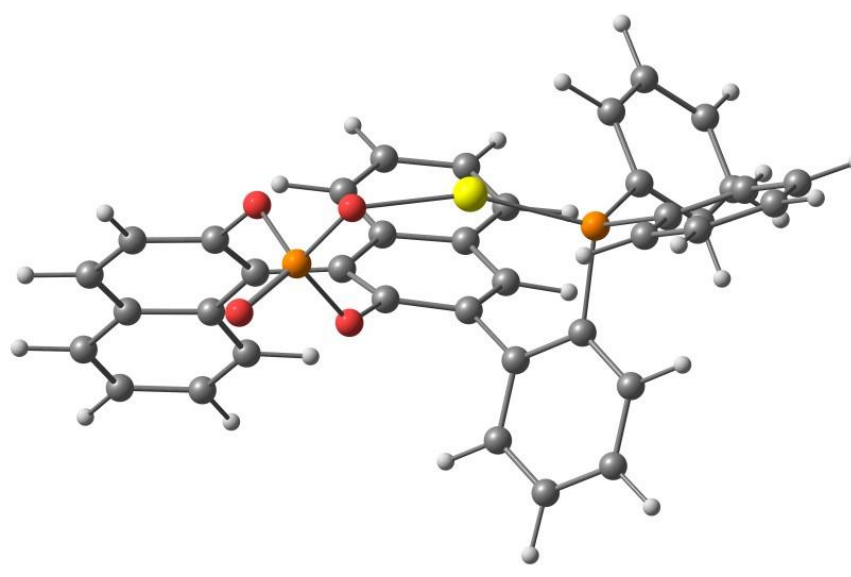

\begin{tabular}{lrrr} 
C & 3.739782000 & -0.304895000 & -0.214439000 \\
C & 4.992686000 & 0.075989000 & 0.371057000 \\
C & 3.707050000 & -1.429720000 & -1.015394000 \\
C & 5.118028000 & 1.141855000 & 1.301108000 \\
C & 6.169235000 & -0.666571000 & 0.040517000 \\
C & 4.859754000 & -2.179777000 & -1.326645000 \\
C & 6.337884000 & 1.477597000 & 1.840648000 \\
H & 4.224863000 & 1.697131000 & 1.600024000 \\
C & 7.413733000 & -0.287503000 & 0.605303000 \\
C & 6.071683000 & -1.788614000 & -0.822775000 \\
C & 7.503644000 & 0.765826000 & 1.482660000 \\
H & 6.403528000 & 2.300010000 & 2.558916000 \\
H & 8.303713000 & -0.864263000 & 0.332292000 \\
H & 6.980552000 & -2.351047000 & -1.058976000 \\
H & 8.468991000 & 1.045075000 & 1.914064000 \\
C & 2.482710000 & 0.434603000 & 0.055963000 \\
C & 2.373840000 & 1.831141000 & -0.247548000 \\
C & 1.390890000 & -0.224011000 & 0.615623000 \\
C & 3.354486000 & 2.531089000 & -0.998944000 \\
C & 1.221792000 & 2.551189000 & 0.181201000 \\
C & 0.265789000 & 0.506871000 & 1.121176000 \\
C & 3.205598000 & 3.867824000 & -1.287392000 \\
H & 4.230172000 & 1.989896000 & -1.367164000 \\
C & 1.101843000 & 3.932917000 & -0.114541000 \\
C & 0.223569000 & 1.869031000 & 0.911315000 \\
C & 2.073941000 & 4.583140000 & -0.835230000 \\
H & 3.969348000 & 4.380702000 & -1.878980000 \\
H & 0.207736000 & 4.463093000 & 0.233102000 \\
H & -0.602825000 & 2.451919000 & 1.330730000 \\
& & & \\
\hline
\end{tabular}




\begin{tabular}{|c|c|c|c|}
\hline $\mathrm{H}$ & 1.972338000 & 5.646994000 & -1.067317000 \\
\hline 0 & 2.505240000 & -1.830892000 & -1.529374000 \\
\hline 0 & 1.391614000 & -1.579998000 & 0.749928000 \\
\hline$P$ & 1.507304000 & -2.664033000 & -0.523167000 \\
\hline 0 & 2.092352000 & -3.914050000 & -0.006556000 \\
\hline 0 & 0.188867000 & -2.676537000 & -1.308334000 \\
\hline C & -0.761719000 & -0.078900000 & 2.029575000 \\
\hline C & -2.151203000 & -0.050853000 & 1.753504000 \\
\hline C & -0.341726000 & -0.547578000 & 3.277716000 \\
\hline C & -3.063996000 & -0.419453000 & 2.748075000 \\
\hline C & -1.257474000 & -0.934789000 & 4.252230000 \\
\hline C & -2.622265000 & -0.846452000 & 3.997775000 \\
\hline $\mathrm{H}$ & -4.138259000 & -0.398342000 & 2.537505000 \\
\hline $\mathrm{H}$ & -3.349035000 & -1.130108000 & 4.763635000 \\
\hline $\mathrm{H}$ & -0.899133000 & -1.296176000 & 5.219945000 \\
\hline $\mathrm{H}$ & 0.731916000 & -0.588003000 & 3.486197000 \\
\hline $\mathrm{H}$ & 4.744998000 & -3.060058000 & -1.963784000 \\
\hline$P$ & -2.728567000 & 0.190209000 & 0.020308000 \\
\hline $\mathrm{Au}$ & -1.290479000 & -1.227729000 & -1.036011000 \\
\hline C & -2.724695000 & 1.965735000 & -0.368406000 \\
\hline C & -3.187533000 & 2.897296000 & 0.570045000 \\
\hline C & -2.243018000 & 2.400411000 & -1.605399000 \\
\hline C & -3.170979000 & 4.255024000 & 0.265287000 \\
\hline $\mathrm{H}$ & -3.549440000 & 2.558984000 & 1.548566000 \\
\hline C & -2.228444000 & 3.761289000 & -1.906081000 \\
\hline H & -1.861388000 & 1.671105000 & -2.328662000 \\
\hline C & -2.692230000 & 4.686041000 & -0.973254000 \\
\hline $\mathrm{H}$ & -3.530361000 & 4.982109000 & 0.998682000 \\
\hline $\mathrm{H}$ & -1.844249000 & 4.099663000 & -2.871994000 \\
\hline $\mathrm{H}$ & -2.676685000 & 5.753853000 & -1.209132000 \\
\hline C & -4.474937000 & -0.339287000 & 0.000114000 \\
\hline C & -5.536042000 & 0.561647000 & -0.127593000 \\
\hline C & -4.741803000 & -1.712712000 & 0.096529000 \\
\hline C & -6.849748000 & 0.093645000 & -0.143622000 \\
\hline $\mathrm{H}$ & -5.344827000 & 1.634603000 & -0.222959000 \\
\hline C & -6.053378000 & -2.172870000 & 0.089258000 \\
\hline $\mathrm{H}$ & -3.913627000 & -2.426652000 & 0.181141000 \\
\hline C & -7.109751000 & -1.269034000 & -0.031170000 \\
\hline $\mathrm{H}$ & -7.674686000 & 0.803777000 & -0.247537000 \\
\hline $\mathrm{H}$ & -6.252337000 & -3.244886000 & 0.169245000 \\
\hline H & -8.141037000 & -1.632454000 & -0.044555000 \\
\hline
\end{tabular}

Structure $\mathbf{6 b}$ 


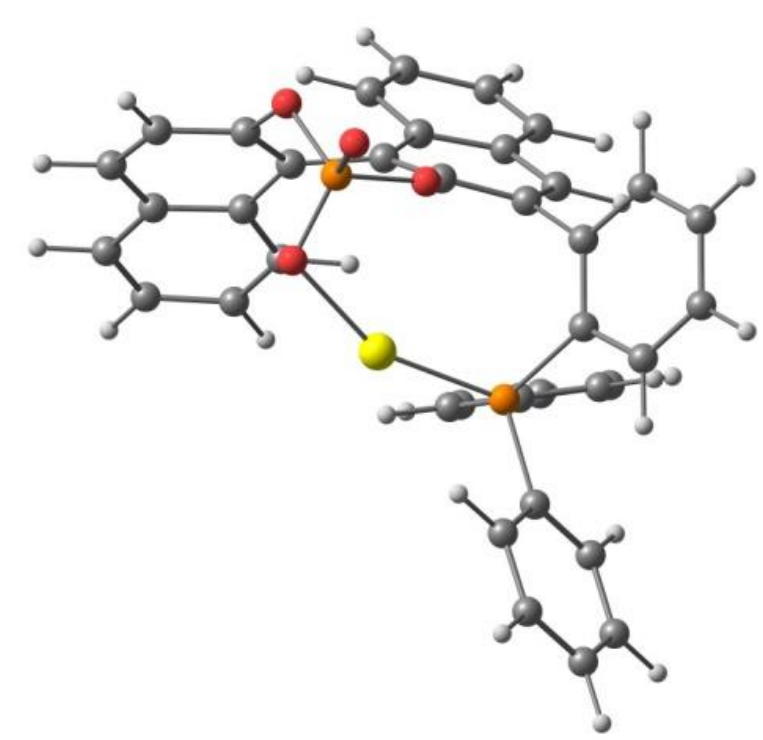

\begin{tabular}{|c|c|c|c|}
\hline C & -2.946902000 & -0.642379000 & 0.232663000 \\
\hline C & -3.411901000 & -0.131271000 & -1.036362000 \\
\hline C & -3.273003000 & -1.954100000 & 0.531280000 \\
\hline C & -2.951742000 & 1.091034000 & -1.599225000 \\
\hline C & -4.337300000 & -0.897230000 & -1.813440000 \\
\hline C & -4.189139000 & -2.710050000 & -0.228477000 \\
\hline C & -3.407944000 & 1.542334000 & -2.816336000 \\
\hline $\mathrm{H}$ & -2.209114000 & 1.684939000 & -1.060813000 \\
\hline C & -4.811623000 & -0.391371000 & -3.050774000 \\
\hline C & -4.743038000 & -2.177192000 & -1.359208000 \\
\hline C & -4.364616000 & 0.807763000 & -3.548017000 \\
\hline $\mathrm{H}$ & -3.023816000 & 2.484824000 & -3.219524000 \\
\hline H & -5.533313000 & -0.994550000 & -3.611608000 \\
\hline $\mathrm{H}$ & -5.462905000 & -2.748125000 & -1.953812000 \\
\hline $\mathrm{H}$ & -4.731241000 & 1.183452000 & -4.507249000 \\
\hline C & -2.095436000 & 0.210545000 & 1.110002000 \\
\hline C & -2.523701000 & 1.566798000 & 1.357315000 \\
\hline C & -0.867583000 & -0.169388000 & 1.663361000 \\
\hline C & -3.867113000 & 1.984438000 & 1.155605000 \\
\hline C & -1.605123000 & 2.537505000 & 1.852418000 \\
\hline C & 0.092199000 & 0.816113000 & 2.05644200 \\
\hline C & -4.254880000 & 3.284554000 & 1.381274000 \\
\hline $\mathrm{H}$ & -4.610882000 & 1.251014000 & 0.833988000 \\
\hline C & -2.021354000 & 3.879525000 & 2.043057000 \\
\hline C & -0.280826000 & 2.134415000 & 2.136398000 \\
\hline C & -3.321066000 & 4.254562000 & 1.806730000 \\
\hline $\mathrm{H}$ & -5.301183000 & 3.567124000 & 1.233556000 \\
\hline H & -1.281664000 & 4.605956000 & 2.397167000 \\
\hline $\mathrm{H}$ & 0.453810000 & 2.887524000 & 2.44340500 \\
\hline $\mathrm{H}$ & -3.638277000 & 5.289074000 & 1.964669 \\
\hline
\end{tabular}




\begin{tabular}{|c|c|c|c|}
\hline O & -2.658527000 & -2.602957000 & 1.567218000 \\
\hline 0 & -0.430001000 & -1.434014000 & 1.864874000 \\
\hline$P$ & -1.052799000 & -2.899567000 & 1.385494000 \\
\hline 0 & -0.758539000 & -2.965469000 & -0.126886000 \\
\hline 0 & -0.608296000 & -3.939839000 & 2.325686000 \\
\hline C & 1.499578000 & 0.435175000 & 2.377019000 \\
\hline C & 2.504572000 & 0.252200000 & 1.394512000 \\
\hline C & 1.856721000 & 0.329099000 & 3.723258000 \\
\hline C & 3.816320000 & -0.027151000 & 1.797458000 \\
\hline C & 3.162681000 & 0.042684000 & 4.112293000 \\
\hline C & 4.146308000 & -0.131594000 & 3.146448000 \\
\hline $\mathrm{H}$ & 4.601239000 & -0.168351000 & 1.048306000 \\
\hline $\mathrm{H}$ & 5.177077000 & -0.352232000 & 3.436207000 \\
\hline $\mathrm{H}$ & 3.408328000 & -0.043152000 & 5.174220000 \\
\hline $\mathrm{H}$ & 1.076779000 & 0.471299000 & 4.477845000 \\
\hline $\mathrm{H}$ & -4.418082000 & -3.722061000 & 0.114462000 \\
\hline$P$ & 2.118201000 & 0.273424000 & -0.410739000 \\
\hline $\mathrm{Au}$ & 0.665274000 & -1.467698000 & -0.728372000 \\
\hline C & 3.723723000 & 0.119808000 & -1.261619000 \\
\hline C & 4.499398000 & 1.235592000 & -1.594254000 \\
\hline C & 4.199237000 & -1.165472000 & -1.549255000 \\
\hline C & 5.741682000 & 1.062982000 & -2.200444000 \\
\hline $\mathrm{H}$ & 4.134115000 & 2.246381000 & -1.387132000 \\
\hline C & 5.444928000 & -1.332880000 & -2.146871000 \\
\hline $\mathrm{H}$ & 3.589384000 & -2.041750000 & -1.299390000 \\
\hline C & 6.216383000 & -0.218497000 & -2.473077000 \\
\hline $\mathrm{H}$ & 6.342066000 & 1.938317000 & -2.462909000 \\
\hline $\mathrm{H}$ & 5.811249000 & -2.339464000 & -2.365410000 \\
\hline $\mathrm{H}$ & 7.192038000 & -0.349780000 & -2.949196000 \\
\hline C & 1.488293000 & 1.942045000 & -0.773540000 \\
\hline C & 1.977731000 & 3.055584000 & -0.078738000 \\
\hline C & 0.505599000 & 2.111371000 & -1.752760000 \\
\hline C & 1.480780000 & 4.324771000 & -0.358295000 \\
\hline $\mathrm{H}$ & 2.738158000 & 2.925871000 & 0.700538000 \\
\hline C & 0.008530000 & 3.384527000 & -2.026279000 \\
\hline $\mathrm{H}$ & 0.112258000 & 1.242268000 & -2.293287000 \\
\hline C & 0.491583000 & 4.488605000 & -1.328121000 \\
\hline $\mathrm{H}$ & 1.861625000 & 5.191073000 & 0.189928000 \\
\hline $\mathrm{H}$ & -0.767074000 & 3.511322000 & -2.787162000 \\
\hline $\mathrm{H}$ & 0.092877000 & 5.484819000 & -1.538511000 \\
\hline
\end{tabular}

Structure 8a 


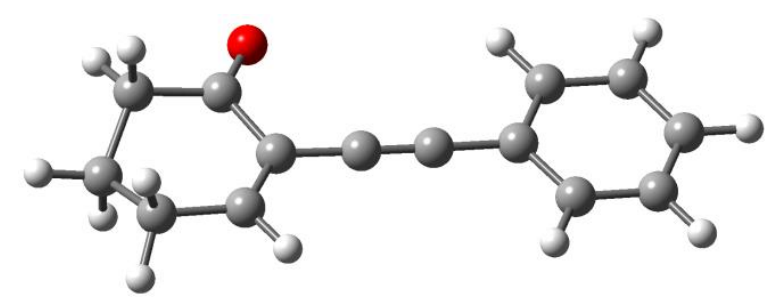

\begin{tabular}{|c|c|c|c|}
\hline C & -0.893928000 & -0.197365000 & -0.020421000 \\
\hline C & 0.322760000 & -0.239620000 & -0.035905000 \\
\hline C & -2.317944000 & -0.125893000 & -0.001625000 \\
\hline C & -3.099295000 & -1.294413000 & -0.003084000 \\
\hline C & -2.960351000 & 1.124674000 & 0.018396000 \\
\hline C & -4.486811000 & -1.210583000 & 0.014779000 \\
\hline $\mathrm{H}$ & -2.600516000 & -2.267789000 & -0.018381000 \\
\hline C & -4.348053000 & 1.199562000 & 0.036418000 \\
\hline $\mathrm{H}$ & -2.350266000 & 2.032353000 & 0.019734000 \\
\hline C & -5.115063000 & 0.034441000 & 0.034551000 \\
\hline $\mathrm{H}$ & -5.085219000 & -2.126136000 & 0.013423000 \\
\hline $\mathrm{H}$ & -4.837631000 & 2.177475000 & 0.052047000 \\
\hline $\mathrm{H}$ & -6.206898000 & 0.096964000 & 0.048640000 \\
\hline C & 1.746568000 & -0.268619000 & -0.054874000 \\
\hline C & 2.446188000 & -1.429780000 & -0.098975000 \\
\hline $\mathrm{H}$ & 1.883502000 & -2.369565000 & -0.151528000 \\
\hline C & 2.457463000 & 1.048831000 & -0.058512000 \\
\hline $\mathrm{O}$ & 1.856251000 & 2.097826000 & 0.01172900 \\
\hline C & 3.956715000 & 0.984937000 & -0.215341000 \\
\hline $\mathrm{H}$ & 4.375457000 & 1.934689000 & 0.15116600 \\
\hline $\mathrm{H}$ & 4.155414000 & 0.953531000 & -1.305765000 \\
\hline C & 3.932036000 & -1.505896000 & -0.087390000 \\
\hline $\mathrm{H}$ & 4.280334000 & -1.699361000 & -1.123908000 \\
\hline $\mathrm{H}$ & 4.252945000 & -2.392340000 & 0.486129000 \\
\hline C & 4.565312000 & -0.235118000 & 0.452169000 \\
\hline $\mathrm{H}$ & 5.657560000 & -0.252905000 & 0.310777000 \\
\hline $\mathrm{H}$ & 4.391727000 & -0.180456000 & 1.542707000 \\
\hline
\end{tabular}

Structure la 


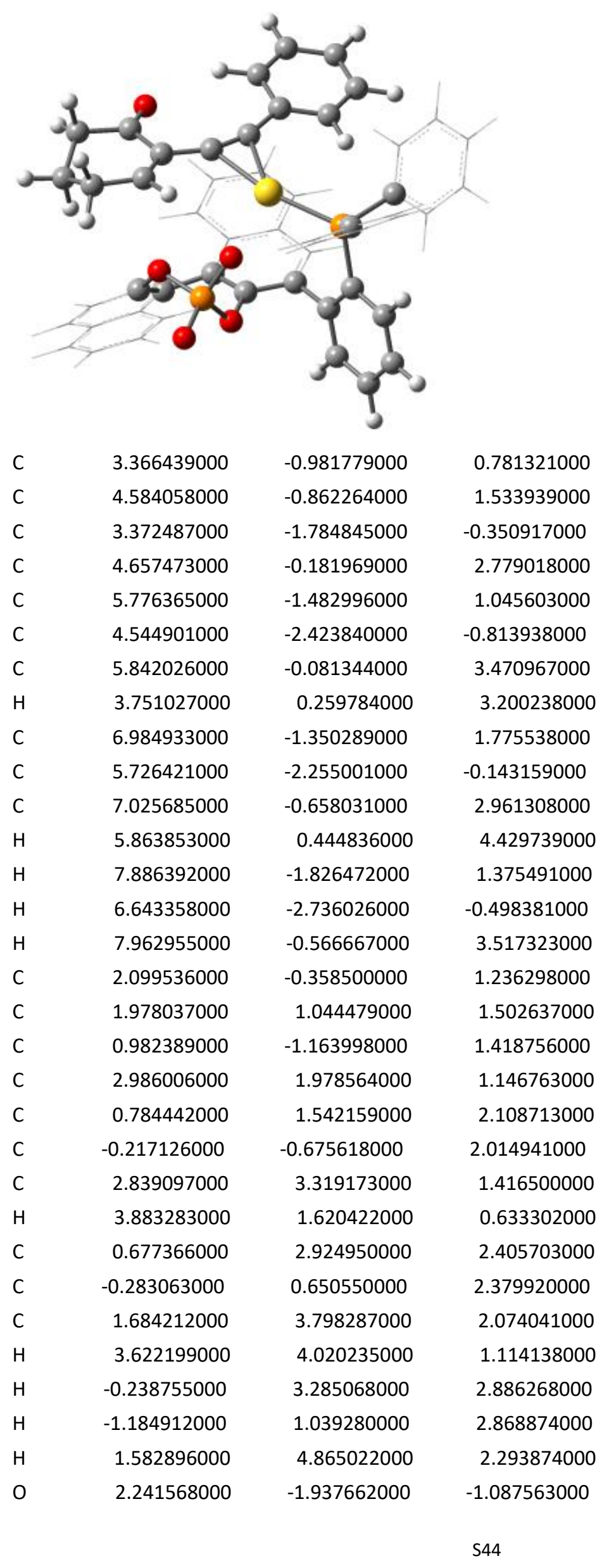




\begin{tabular}{|c|c|c|c|}
\hline 0 & 1.013113000 & -2.471687000 & 1.050663000 \\
\hline$P$ & 0.952655000 & -2.861811000 & -0.570526000 \\
\hline 0 & 1.220459000 & -4.308130000 & -0.700462000 \\
\hline 0 & -0.240338000 & -2.172431000 & -1.207957000 \\
\hline C & -1.304159000 & -1.640197000 & 2.338411000 \\
\hline C & -2.516565000 & -1.741891000 & 1.630975000 \\
\hline C & -1.094805000 & -2.493366000 & 3.430746000 \\
\hline C & -3.486843000 & -2.666456000 & 2.045409000 \\
\hline C & -2.061269000 & -3.405390000 & 3.834272000 \\
\hline C & -3.268287000 & -3.488711000 & 3.142901000 \\
\hline $\mathrm{H}$ & -4.428196000 & -2.749173000 & 1.492056000 \\
\hline $\mathrm{H}$ & -4.035999000 & -4.203855000 & 3.450126000 \\
\hline $\mathrm{H}$ & -1.869569000 & -4.057893000 & 4.690504000 \\
\hline $\mathrm{H}$ & -0.141898000 & -2.429546000 & 3.965499000 \\
\hline $\mathrm{H}$ & 4.466385000 & -3.045456000 & -1.710378000 \\
\hline$P$ & -2.875772000 & -0.712234000 & 0.158855000 \\
\hline $\mathrm{Au}$ & -0.918827000 & 0.170563000 & -0.965565000 \\
\hline C & -3.984064000 & 0.614040000 & 0.758350000 \\
\hline C & -4.221557000 & 0.832705000 & 2.118298000 \\
\hline C & -4.536439000 & 1.491452000 & -0.186311000 \\
\hline C & -4.997625000 & 1.918388000 & 2.527266000 \\
\hline $\mathrm{H}$ & -3.800458000 & 0.153369000 & 2.867309000 \\
\hline C & -5.325171000 & 2.560203000 & 0.223694000 \\
\hline $\mathrm{H}$ & -4.335285000 & 1.341296000 & -1.254224000 \\
\hline C & -5.552573000 & 2.778650000 & 1.583854000 \\
\hline $\mathrm{H}$ & -5.174429000 & 2.084880000 & 3.593556000 \\
\hline $\mathrm{H}$ & -5.754980000 & 3.235335000 & -0.521451000 \\
\hline $\mathrm{H}$ & -6.165680000 & 3.624646000 & 1.907226000 \\
\hline C & -3.933091000 & -1.768095000 & -0.897813000 \\
\hline C & -5.330738000 & -1.686399000 & -0.881242000 \\
\hline C & -3.297553000 & -2.710724000 & -1.717921000 \\
\hline C & -6.087261000 & -2.538029000 & -1.683317000 \\
\hline $\mathrm{H}$ & -5.836046000 & -0.958059000 & -0.238262000 \\
\hline C & -4.063924000 & -3.560903000 & -2.511640000 \\
\hline $\mathrm{H}$ & -2.201690000 & -2.777315000 & -1.721076000 \\
\hline C & -5.455082000 & -3.474880000 & -2.498586000 \\
\hline $\mathrm{H}$ & -7.178741000 & -2.469330000 & -1.667877000 \\
\hline $\mathrm{H}$ & -3.565520000 & -4.297632000 & -3.147825000 \\
\hline H & -6.051232000 & -4.142429000 & -3.127456000 \\
\hline C & -0.087477000 & 2.198431000 & -1.276545000 \\
\hline C & 0.623679000 & 1.386428000 & -1.918897000 \\
\hline C & -0.569460000 & 3.433753000 & -0.725430000 \\
\hline C & 0.161717000 & 4.596651000 & -1.030867000 \\
\hline C & -1.693714000 & 3.522018000 & 0.105744000 \\
\hline
\end{tabular}




$\begin{array}{lrrr}\text { C } & -0.241688000 & 5.821787000 & -0.514415000 \\ \text { H } & 1.052303000 & 4.503652000 & -1.659733000 \\ \text { C } & -2.091906000 & 4.754703000 & 0.612152000 \\ \text { H } & -2.249623000 & 2.615434000 & 0.361208000 \\ \text { C } & -1.368592000 & 5.906391000 & 0.304995000 \\ \text { H } & 0.330631000 & 6.722652000 & -0.753732000 \\ \text { H } & -2.975334000 & 4.810977000 & 1.255630000 \\ \text { H } & -1.683254000 & 6.874295000 & 0.705778000 \\ \text { C } & 1.726178000 & 0.920127000 & -2.705760000 \\ \text { C } & 1.786798000 & -0.327493000 & -3.235419000 \\ \text { C } & 2.812937000 & 1.913154000 & -2.941025000 \\ \text { O } & 2.762383000 & 3.043751000 & -2.501013000 \\ \text { C } & 3.948723000 & 1.445269000 & -3.816541000 \\ \text { H } & 4.827414000 & 2.067398000 & -3.586696000 \\ \text { H } & 3.656948000 & 1.690220000 & -4.857960000 \\ \text { C } & 2.958586000 & -0.839169000 & -3.987873000 \\ \text { H } & 2.721118000 & -0.807103000 & -5.071872000 \\ \text { H } & 3.087385000 & -1.909543000 & -3.752886000 \\ \text { C } & 4.217616000 & -0.042993000 & -3.690249000 \\ \text { H } & 5.040153000 & -0.348161000 & -4.356244000 \\ \text { H } & 4.547775000 & -0.271489000 & -2.658726000 \\ \text { H } & 0.933639000 & -1.000030000 & -3.084989000\end{array}$

Structure TS-I-Ila

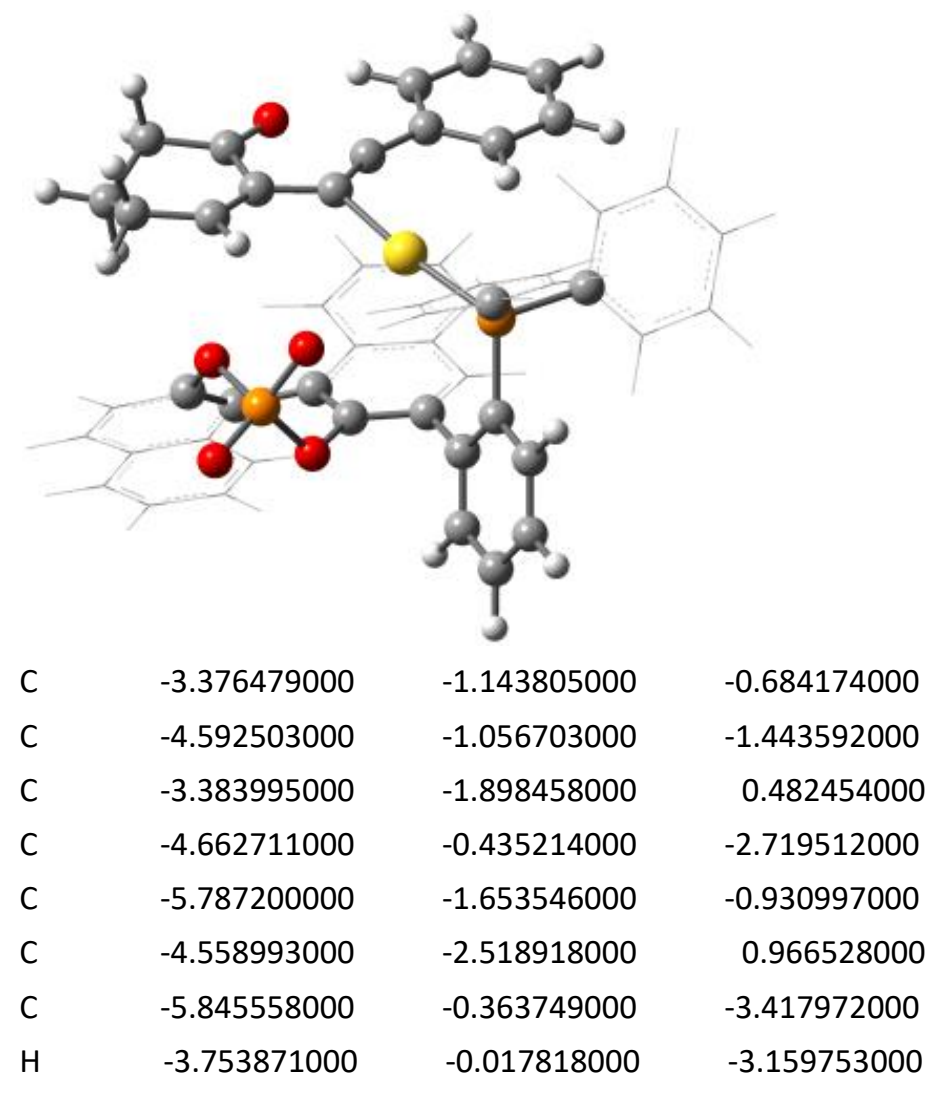




\begin{tabular}{|c|c|c|c|}
\hline c & -6.994204000 & -1.551135000 & -1.668542000 \\
\hline C & -5.739772000 & -2.376290000 & 0.288460000 \\
\hline C & -7.031833000 & -0.913114000 & -2.884516000 \\
\hline $\mathrm{H}$ & -5.863788000 & 0.116281000 & -4.400812000 \\
\hline $\mathrm{H}$ & -7.897054000 & -2.008331000 & -1.249621000 \\
\hline $\mathrm{H}$ & -6.657277000 & -2.843910000 & 0.660119000 \\
\hline $\mathrm{H}$ & -7.967876000 & -0.845949000 & -3.446127000 \\
\hline C & -2.104522000 & -0.548643000 & -1.163048000 \\
\hline C & -1.975440000 & 0.836591000 & -1.504872000 \\
\hline C & -0.993050000 & -1.372725000 & -1.302920000 \\
\hline C & -2.985652000 & 1.792545000 & -1.218191000 \\
\hline C & -0.776498000 & 1.296393000 & -2.131048000 \\
\hline C & 0.213345000 & -0.917879000 & -1.912608000 \\
\hline C & -2.841914000 & 3.111330000 & -1.582359000 \\
\hline $\mathrm{H}$ & -3.887430000 & 1.466736000 & -0.690584000 \\
\hline C & -0.668529000 & 2.657078000 & -2.516807000 \\
\hline C & 0.291536000 & 0.388921000 & -2.340331000 \\
\hline C & -1.681902000 & 3.547970000 & -2.260065000 \\
\hline $\mathrm{H}$ & -3.631078000 & 3.829548000 & -1.340128000 \\
\hline $\mathrm{H}$ & 0.252981000 & 2.986683000 & -3.009406000 \\
\hline $\mathrm{H}$ & 1.201095000 & 0.746540000 & -2.839270000 \\
\hline $\mathrm{H}$ & -1.581765000 & 4.597196000 & -2.553253000 \\
\hline $\mathrm{O}$ & -2.256570000 & -2.020744000 & 1.222502000 \\
\hline $\mathrm{O}$ & -1.043770000 & -2.661791000 & -0.893200000 \\
\hline$P$ & -0.982840000 & -3.014181000 & 0.746696000 \\
\hline 0 & -1.351979000 & -4.439070000 & 0.894454000 \\
\hline $\mathrm{O}$ & 0.221785000 & -2.354986000 & 1.362214000 \\
\hline C & 1.303905000 & -1.896473000 & -2.164178000 \\
\hline C & 2.547021000 & -1.879421000 & -1.505133000 \\
\hline C & 1.072713000 & -2.889528000 & -3.126312000 \\
\hline C & 3.524243000 & -2.830049000 & -1.837287000 \\
\hline C & 2.047047000 & -3.823299000 & -3.452549000 \\
\hline C & 3.283933000 & -3.790736000 & -2.810571000 \\
\hline $\mathrm{H}$ & 4.487765000 & -2.821396000 & -1.316185000 \\
\hline $\mathrm{H}$ & 4.056941000 & -4.523569000 & -3.056907000 \\
\hline $\mathrm{H}$ & 1.838103000 & -4.585446000 & -4.208267000 \\
\hline $\mathrm{H}$ & 0.096626000 & -2.918876000 & -3.620085000 \\
\hline $\mathrm{H}$ & -4.480410000 & -3.110070000 & 1.883444000 \\
\hline$P$ & 2.929415000 & -0.677796000 & -0.179117000 \\
\hline $\mathrm{Au}$ & 1.087140000 & 0.320550000 & 0.907336000 \\
\hline C & 3.981281000 & 0.603618000 & -0.956199000 \\
\hline C & 4.410595000 & 0.531444000 & -2.284570000 \\
\hline C & 4.342045000 & 1.711616000 & -0.175922000 \\
\hline C & 5.190340000 & 1.555266000 & -2.824093000 \\
\hline
\end{tabular}




\begin{tabular}{|c|c|c|c|}
\hline $\mathrm{H}$ & 4.133380000 & -0.325847000 & -2.906899000 \\
\hline C & 5.129360000 & 2.724401000 & -0.713524000 \\
\hline $\mathrm{H}$ & 4.001117000 & 1.777887000 & 0.865042000 \\
\hline C & 5.551711000 & 2.648651000 & -2.041947000 \\
\hline $\mathrm{H}$ & 5.517868000 & 1.493432000 & -3.865668000 \\
\hline $\mathrm{H}$ & 5.410554000 & 3.581015000 & -0.094198000 \\
\hline $\mathrm{H}$ & 6.164548000 & 3.448087000 & -2.467912000 \\
\hline C & 4.028789000 & -1.592359000 & 0.956356000 \\
\hline C & 5.413736000 & -1.403743000 & 0.992837000 \\
\hline C & 3.419876000 & -2.528571000 & 1.804604000 \\
\hline C & 6.190790000 & -2.150574000 & 1.877396000 \\
\hline $\mathrm{H}$ & 5.892207000 & -0.674724000 & 0.329779000 \\
\hline C & 4.207573000 & -3.273695000 & 2.677833000 \\
\hline $\mathrm{H}$ & 2.329409000 & -2.669208000 & 1.770258000 \\
\hline C & 5.589218000 & -3.084900000 & 2.717562000 \\
\hline $\mathrm{H}$ & 7.273978000 & -2.001311000 & 1.906725000 \\
\hline $\mathrm{H}$ & 3.735109000 & -4.007670000 & 3.336682000 \\
\hline $\mathrm{H}$ & 6.201601000 & -3.670017000 & 3.409857000 \\
\hline C & -0.505015000 & 2.673702000 & 1.384817000 \\
\hline C & -0.474746000 & 1.444978000 & 1.768641000 \\
\hline C & -0.077447000 & 3.884110000 & 0.759436000 \\
\hline C & -0.829521000 & 5.068727000 & 0.813670000 \\
\hline C & 1.144028000 & 3.879503000 & 0.055330000 \\
\hline C & -0.367958000 & 6.215194000 & 0.176660000 \\
\hline $\mathrm{H}$ & -1.775474000 & 5.071583000 & 1.356529000 \\
\hline C & 1.591456000 & 5.032290000 & -0.575160000 \\
\hline $\mathrm{H}$ & 1.727827000 & 2.954549000 & -0.003346000 \\
\hline C & 0.839163000 & 6.205512000 & -0.519937000 \\
\hline $\mathrm{H}$ & -0.963414000 & 7.131152000 & 0.227430000 \\
\hline $\mathrm{H}$ & 2.540630000 & 5.008026000 & -1.119049000 \\
\hline $\mathrm{H}$ & 1.194892000 & 7.111752000 & -1.017835000 \\
\hline C & -1.551931000 & 0.925825000 & 2.583473000 \\
\hline C & -1.674053000 & -0.301075000 & 3.148709000 \\
\hline C & -2.553174000 & 1.974430000 & 2.795657000 \\
\hline 0 & -2.336772000 & 3.087152000 & 2.316863000 \\
\hline C & -3.748670000 & 1.662446000 & 3.636533000 \\
\hline $\mathrm{H}$ & -4.585678000 & 2.307047000 & 3.327069000 \\
\hline $\mathrm{H}$ & -3.485976000 & 1.973367000 & 4.667949000 \\
\hline C & -2.873314000 & -0.677171000 & 3.939369000 \\
\hline $\mathrm{H}$ & -2.617571000 & -0.586074000 & 5.016100000 \\
\hline $\mathrm{H}$ & -3.081335000 & -1.748137000 & 3.781417000 \\
\hline C & -4.084138000 & 0.179517000 & 3.597708000 \\
\hline $\mathrm{H}$ & -4.920178000 & -0.039921000 & 4.279586000 \\
\hline $\mathrm{H}$ & -4.429453000 & -0.088083000 & 2.581035000 \\
\hline
\end{tabular}


Structure Ila

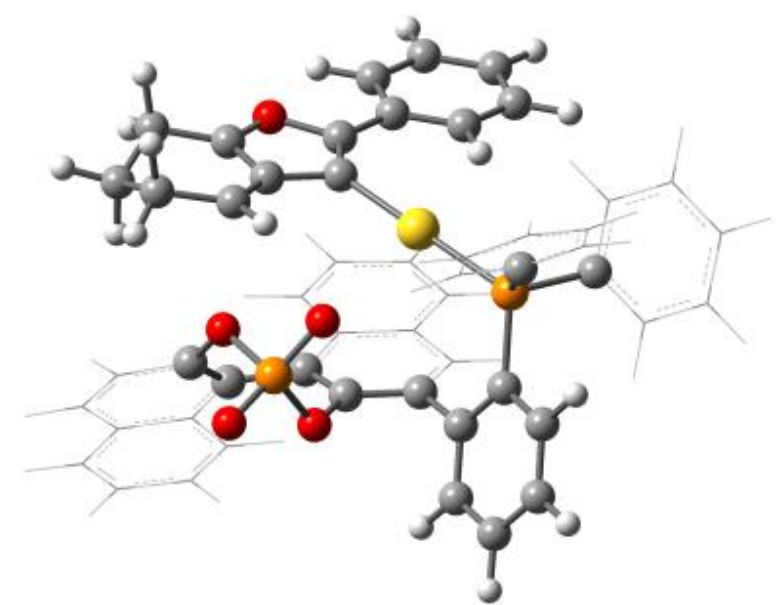

\begin{tabular}{|c|c|c|c|}
\hline C & 3.366841000 & -1.167193000 & 0.676343000 \\
\hline C & 4.577367000 & -1.109087000 & 1.445833000 \\
\hline C & 3.390153000 & -1.852091000 & -0.531214000 \\
\hline C & 4.632762000 & -0.552980000 & 2.752004000 \\
\hline C & 5.779511000 & -1.669688000 & 0.910210000 \\
\hline C & 4.570659000 & -2.440903000 & -1.040018000 \\
\hline C & 5.809852000 & -0.510286000 & 3.462180000 \\
\hline $\mathrm{H}$ & 3.717201000 & -0.163475000 & 3.203856000 \\
\hline C & 6.980224000 & -1.597837000 & 1.661517000 \\
\hline C & 5.745553000 & -2.328428000 & -0.345746000 \\
\hline C & 7.003350000 & -1.024261000 & 2.909603000 \\
\hline $\mathrm{H}$ & 5.818339000 & -0.081323000 & 4.468408000 \\
\hline $\mathrm{H}$ & 7.889708000 & -2.026265000 & 1.226937000 \\
\hline $\mathrm{H}$ & 6.667929000 & -2.773442000 & -0.732642000 \\
\hline $\mathrm{H}$ & 7.934590000 & -0.980783000 & 3.481411000 \\
\hline C & 2.082305000 & -0.621697000 & 1.178369000 \\
\hline C & 1.923682000 & 0.745756000 & 1.571658000 \\
\hline C & 0.986813000 & -1.473635000 & 1.267782000 \\
\hline C & 2.918640000 & 1.730988000 & 1.334926000 \\
\hline C & 0.703500000 & 1.159833000 & 2.186765000 \\
\hline C & -0.237198000 & -1.066418000 & 1.874708000 \\
\hline C & 2.733420000 & 3.037196000 & 1.725479000 \\
\hline $\mathrm{H}$ & 3.841117000 & 1.440295000 & 0.82271900 \\
\hline C & 0.551526000 & 2.508408000 & 2.598321000 \\
\hline C & -0.346705000 & 0.222581000 & 2.346672000 \\
\hline C & 1.546210000 & 3.430557000 & 2.381500000 \\
\hline $\mathrm{H}$ & 3.510018000 & 3.780706000 & 1.52093500 \\
\hline $\mathrm{H}$ & -0.390933000 & 2.804763000 & 3.072239000 \\
\hline $\mathrm{H}$ & -1.273149000 & 0.545540000 & 2.837808000 \\
\hline $\mathrm{H}$ & 1.411006000 & 4.471611000 & 2.6890080 \\
\hline
\end{tabular}




\begin{tabular}{|c|c|c|c|}
\hline 0 & 2.259018000 & -1.950485000 & -1.274569000 \\
\hline $\mathrm{O}$ & 1.074335000 & -2.744486000 & 0.806008000 \\
\hline$P$ & 1.041555000 & -3.055276000 & -0.839618000 \\
\hline 0 & 1.536152000 & -4.437477000 & -1.025109000 \\
\hline 0 & -0.188669000 & -2.463150000 & -1.460664000 \\
\hline C & -1.329585000 & -2.057155000 & 2.046296000 \\
\hline C & -2.582893000 & -1.954059000 & 1.413841000 \\
\hline C & -1.097931000 & -3.145440000 & 2.899411000 \\
\hline C & -3.569732000 & -2.918822000 & 1.669598000 \\
\hline C & -2.082813000 & -4.090817000 & 3.150820000 \\
\hline C & -3.330221000 & -3.974352000 & 2.539334000 \\
\hline $\mathrm{H}$ & -4.540733000 & -2.845104000 & 1.167562000 \\
\hline $\mathrm{H}$ & -4.112006000 & -4.715280000 & 2.727172000 \\
\hline $\mathrm{H}$ & -1.874553000 & -4.927169000 & 3.823792000 \\
\hline $\mathrm{H}$ & -0.115007000 & -3.238748000 & 3.370797000 \\
\hline $\mathrm{H}$ & 4.500961000 & -2.987530000 & -1.985126000 \\
\hline$P$ & -2.956871000 & -0.633301000 & 0.198213000 \\
\hline $\mathrm{Au}$ & -1.131244000 & 0.480011000 & -0.825949000 \\
\hline C & -4.033689000 & 0.561064000 & 1.075388000 \\
\hline C & -4.534639000 & 0.341377000 & 2.361882000 \\
\hline C & -4.345924000 & 1.756418000 & 0.411925000 \\
\hline C & -5.338197000 & 1.303819000 & 2.973969000 \\
\hline $\mathrm{H}$ & -4.293861000 & -0.584063000 & 2.895447000 \\
\hline C & -5.157887000 & 2.708262000 & 1.020032000 \\
\hline $\mathrm{H}$ & -3.948269000 & 1.938323000 & -0.594545000 \\
\hline C & -5.652539000 & 2.484050000 & 2.305371000 \\
\hline $\mathrm{H}$ & -5.721046000 & 1.126116000 & 3.982935000 \\
\hline $\mathrm{H}$ & -5.400441000 & 3.634113000 & 0.490540000 \\
\hline $\mathrm{H}$ & -6.283553000 & 3.235695000 & 2.788098000 \\
\hline C & -4.045727000 & -1.464804000 & -1.012912000 \\
\hline C & -5.423130000 & -1.240554000 & -1.088769000 \\
\hline C & -3.427443000 & -2.365646000 & -1.892685000 \\
\hline C & -6.183911000 & -1.915642000 & -2.043148000 \\
\hline $\mathrm{H}$ & -5.909138000 & -0.539072000 & -0.401829000 \\
\hline C & -4.198525000 & -3.039932000 & -2.835262000 \\
\hline $\mathrm{H}$ & -2.342715000 & -2.538645000 & -1.828285000 \\
\hline C & -5.573381000 & -2.814801000 & -2.914162000 \\
\hline $\mathrm{H}$ & -7.261646000 & -1.737888000 & -2.102425000 \\
\hline $\mathrm{H}$ & -3.718742000 & -3.747126000 & -3.517882000 \\
\hline $\mathrm{H}$ & -6.172676000 & -3.344010000 & -3.660865000 \\
\hline C & 0.911032000 & 2.780003000 & -1.506152000 \\
\hline C & 0.462848000 & 1.504112000 & -1.650784000 \\
\hline C & 0.430304000 & 3.988181000 & -0.851403000 \\
\hline C & 1.205859000 & 5.157825000 & -0.824582000 \\
\hline
\end{tabular}




$\begin{array}{lrrr}\text { C } & -0.826283000 & 4.004055000 & -0.224697000 \\ \text { C } & 0.740283000 & 6.302910000 & -0.184784000 \\ \text { H } & 2.187251000 & 5.166029000 & -1.305270000 \\ \text { C } & -1.287020000 & 5.149443000 & 0.411366000 \\ \text { H } & -1.437146000 & 3.094546000 & -0.231362000 \\ \text { C } & -0.506746000 & 6.306246000 & 0.436617000 \\ \text { H } & 1.360639000 & 7.203993000 & -0.173858000 \\ \text { H } & -2.268250000 & 5.138192000 & 0.895543000 \\ \text { H } & -0.871604000 & 7.207757000 & 0.937111000 \\ \text { C } & 1.490753000 & 0.859520000 & -2.449293000 \\ \text { C } & 1.572792000 & -0.382653000 & -3.041733000 \\ \text { C } & 2.493439000 & 1.816133000 & -2.706870000 \\ \text { O } & 2.172516000 & 2.950920000 & -2.172499000 \\ \text { C } & 3.682510000 & 1.608814000 & -3.548833000 \\ \text { H } & 4.538996000 & 2.186805000 & -3.165859000 \\ \text { H } & 3.459388000 & 2.028329000 & -4.551709000 \\ \text { C } & 2.719999000 & -0.713257000 & -3.917454000 \\ \text { H } & 2.364196000 & -0.551850000 & -4.958938000 \\ \text { H } & 2.926281000 & -1.793736000 & -3.856392000 \\ \text { C } & 3.970554000 & 0.112207000 & -3.630591000 \\ \text { H } & 4.739037000 & -0.085757000 & -4.392296000 \\ \text { H } & 4.390765000 & -0.222408000 & -2.664607000 \\ \text { H } & 0.725452000 & -1.077829000 & -2.944472000\end{array}$

Structure Ib

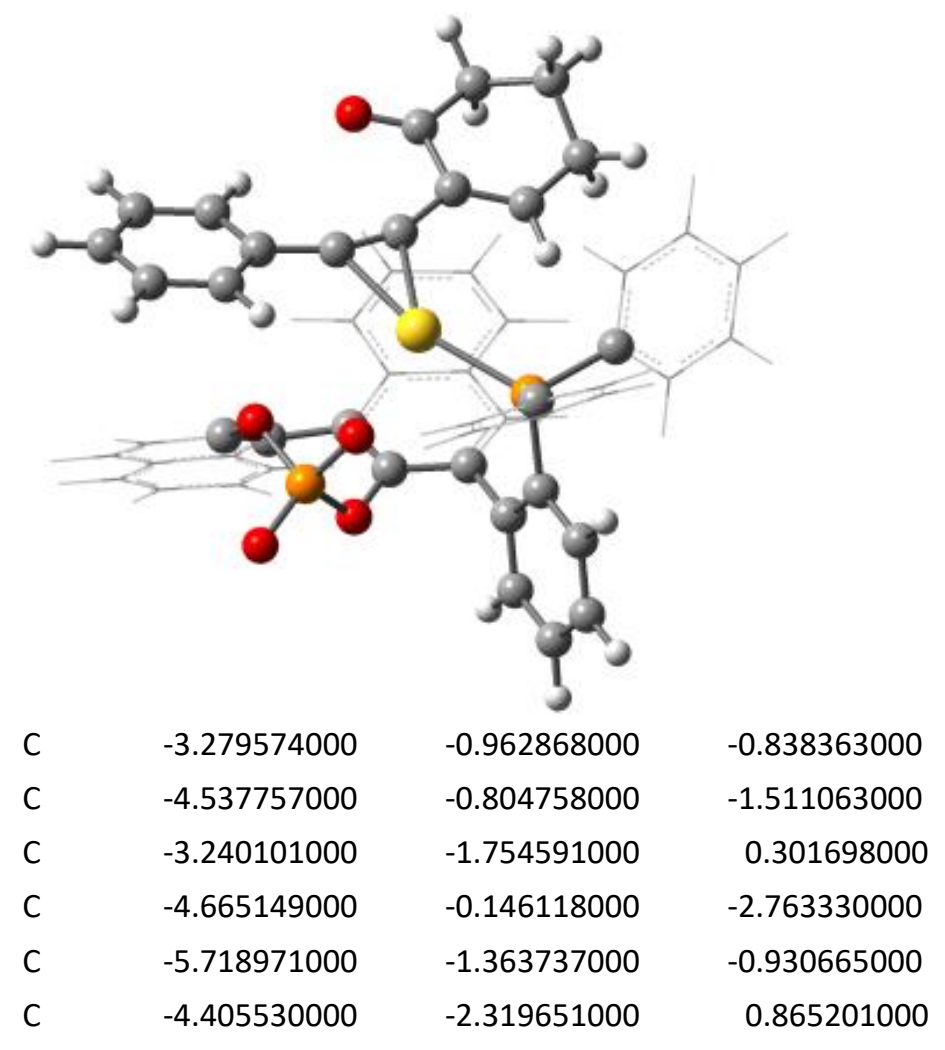




\begin{tabular}{|c|c|c|c|}
\hline C & -5.887591000 & -0.008632000 & -3.378775000 \\
\hline $\mathrm{H}$ & -3.769910000 & 0.246151000 & -3.252790000 \\
\hline C & -6.966570000 & -1.193546000 & -1.583158000 \\
\hline C & -5.621459000 & -2.107635000 & 0.273103000 \\
\hline C & -7.057441000 & -0.523976000 & -2.779241000 \\
\hline $\mathrm{H}$ & -5.951443000 & 0.497844000 & -4.346326000 \\
\hline $\mathrm{H}$ & -7.858669000 & -1.621734000 & -1.113769000 \\
\hline $\mathrm{H}$ & -6.533501000 & -2.531188000 & 0.705787000 \\
\hline $\mathrm{H}$ & -8.025186000 & -0.403819000 & -3.274305000 \\
\hline C & -2.026130000 & -0.362908000 & -1.355221000 \\
\hline C & -1.900492000 & 1.041438000 & -1.617457000 \\
\hline C & -0.922057000 & -1.178234000 & -1.577377000 \\
\hline C & -2.893874000 & 1.983500000 & -1.239196000 \\
\hline C & -0.720379000 & 1.534086000 & -2.252620000 \\
\hline C & 0.277055000 & -0.683516000 & -2.169348000 \\
\hline C & -2.758370000 & 3.320192000 & -1.536329000 \\
\hline H & -3.776110000 & 1.633717000 & -0.695819000 \\
\hline C & -0.621463000 & 2.912015000 & -2.573046000 \\
\hline C & 0.3433880000 & 0.642300000 & -2.531346000 \\
\hline C & -1.623801000 & 3.789623000 & -2.235988000 \\
\hline $\mathrm{H}$ & -3.536566000 & 4.024407000 & -1.227408000 \\
\hline $\mathrm{H}$ & 0.280517000 & 3.260113000 & -3.090480000 \\
\hline $\mathrm{H}$ & 1.242643000 & 1.029413000 & -3.025629000 \\
\hline $\mathrm{H}$ & -1.540199000 & 4.850212000 & -2.495055000 \\
\hline $\mathrm{O}$ & -2.064535000 & -1.963340000 & 0.944670000 \\
\hline $\mathrm{O}$ & -0.963490000 & -2.495097000 & -1.254383000 \\
\hline$P$ & -0.880187000 & -2.961438000 & 0.349429000 \\
\hline $\mathrm{O}$ & -1.239497000 & -4.391152000 & 0.422980000 \\
\hline $\mathrm{O}$ & 0.385089000 & -2.384956000 & 0.963649000 \\
\hline C & 1.390502000 & -1.624209000 & -2.463180000 \\
\hline C & 2.583733000 & -1.667873000 & -1.717703000 \\
\hline C & 1.245054000 & -2.493630000 & -3.551040000 \\
\hline C & 3.604882000 & -2.551083000 & -2.096169000 \\
\hline C & 2.261174000 & -3.367982000 & -3.916756000 \\
\hline C & 3.451175000 & -3.390745000 & -3.192502000 \\
\hline $\mathrm{H}$ & 4.532006000 & -2.591107000 & -1.515087000 \\
\hline $\mathrm{H}$ & 4.257268000 & -4.074368000 & -3.472016000 \\
\hline $\mathrm{H}$ & 2.121913000 & -4.037599000 & -4.769880000 \\
\hline $\mathrm{H}$ & 0.305632000 & -2.474250000 & -4.112327000 \\
\hline $\mathrm{H}$ & -4.292460000 & -2.916210000 & 1.774172000 \\
\hline$P$ & 2.820993000 & -0.635954000 & -0.220717000 \\
\hline $\mathrm{Au}$ & 0.787607000 & -0.018422000 & 0.981066000 \\
\hline C & 3.784642000 & 0.821326000 & -0.763885000 \\
\hline C & 4.047560000 & 1.092062000 & -2.109325000 \\
\hline
\end{tabular}




\begin{tabular}{|c|c|c|c|}
\hline C & 4.198556000 & 1.737014000 & 0.215143000 \\
\hline C & 4.705973000 & 2.268760000 & -2.471557000 \\
\hline $\mathrm{H}$ & 3.739245000 & 0.380567000 & -2.883313000 \\
\hline C & 4.877495000 & 2.895161000 & -0.146903000 \\
\hline $\mathrm{H}$ & 3.979228000 & 1.540771000 & 1.272284000 \\
\hline C & 5.124915000 & 3.167253000 & -1.494247000 \\
\hline $\mathrm{H}$ & 4.902060000 & 2.475349000 & -3.527460000 \\
\hline $\mathrm{H}$ & 5.207285000 & 3.596052000 & 0.625549000 \\
\hline $\mathrm{H}$ & 5.649437000 & 4.083215000 & -1.780862000 \\
\hline C & 3.983242000 & -1.601780000 & 0.812161000 \\
\hline C & 5.359789000 & -1.348704000 & 0.836245000 \\
\hline C & 3.453030000 & -2.656402000 & 1.568817000 \\
\hline C & 6.199499000 & -2.140663000 & 1.617018000 \\
\hline $\mathrm{H}$ & 5.784579000 & -0.533618000 & 0.241007000 \\
\hline C & 4.302151000 & -3.445118000 & 2.341118000 \\
\hline $\mathrm{H}$ & 2.374560000 & -2.858528000 & 1.536783000 \\
\hline C & 5.672011000 & -3.188046000 & 2.369526000 \\
\hline $\mathrm{H}$ & 7.274245000 & -1.938139000 & 1.633331000 \\
\hline $\mathrm{H}$ & 3.885985000 & -4.268872000 & 2.927751000 \\
\hline $\mathrm{H}$ & 6.333411000 & -3.808310000 & 2.981322000 \\
\hline C & -0.813619000 & 1.022491000 & 2.082259000 \\
\hline C & -0.162111000 & 1.880719000 & 1.432681000 \\
\hline C & -1.814475000 & 0.427106000 & 2.921893000 \\
\hline C & -3.005342000 & 1.141275000 & 3.143999000 \\
\hline C & -1.629976000 & -0.825794000 & 3.524556000 \\
\hline C & -3.994691000 & 0.597452000 & 3.954909000 \\
\hline $\mathrm{H}$ & -3.121244000 & 2.125646000 & 2.682164000 \\
\hline C & -2.621832000 & -1.355449000 & 4.340001000 \\
\hline $\mathrm{H}$ & -0.713771000 & -1.388737000 & 3.321914000 \\
\hline C & -3.804884000 & -0.647896000 & 4.554380000 \\
\hline $\mathrm{H}$ & -4.921436000 & 1.152807000 & 4.124436000 \\
\hline $\mathrm{H}$ & -2.474549000 & -2.333883000 & 4.805331000 \\
\hline $\mathrm{H}$ & -4.585747000 & -1.071166000 & 5.193025000 \\
\hline C & 0.184685000 & 3.192013000 & 0.966606000 \\
\hline C & 1.198998000 & 3.463975000 & 0.110929000 \\
\hline C & -0.731390000 & 4.282447000 & 1.429671000 \\
\hline 0 & -1.680869000 & 4.061210000 & 2.149681000 \\
\hline C & -0.446498000 & 5.658144000 & 0.881482000 \\
\hline $\mathrm{H}$ & -0.893601000 & 6.392783000 & 1.568553000 \\
\hline $\mathrm{H}$ & -1.016717000 & 5.727592000 & -0.067453000 \\
\hline C & 1.576545000 & 4.835955000 & -0.316275000 \\
\hline $\mathrm{H}$ & 1.196145000 & 4.992103000 & -1.347978000 \\
\hline $\mathrm{H}$ & 2.675493000 & 4.896760000 & -0.408682000 \\
\hline C & 1.027753000 & 5.900767000 & 0.617077000 \\
\hline
\end{tabular}




$\begin{array}{rrrr}H & 1.188000000 & 6.907112000 & 0.199188000 \\ H & 1.582780000 & 5.866589000 & 1.572582000 \\ H & 1.778374000 & 2.626018000 & -0.296667000\end{array}$

Structure TS-I-IIb

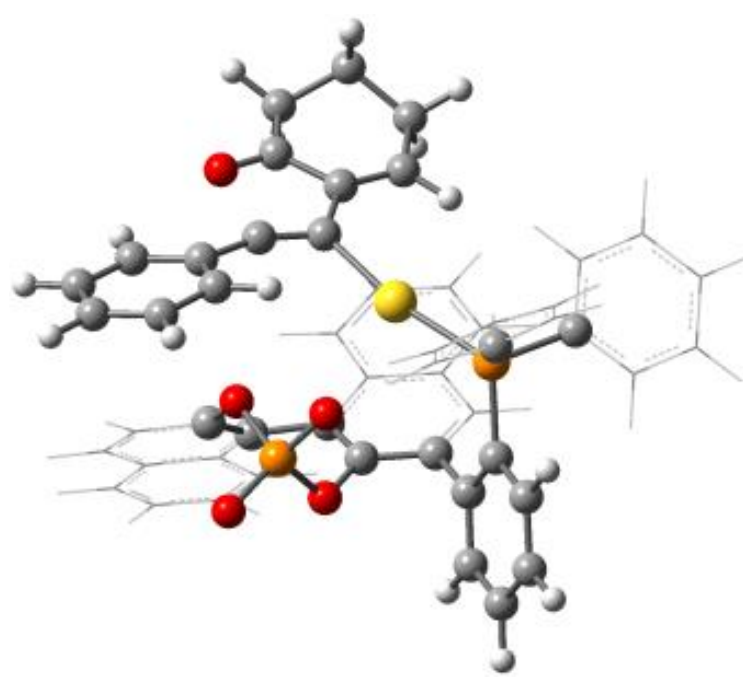

\begin{tabular}{lrrr} 
C & -3.038215000 & -0.765903000 & -1.301903000 \\
C & -4.271049000 & -0.583324000 & -2.010570000 \\
C & -3.006049000 & -1.676802000 & -0.252781000 \\
C & -4.377173000 & 0.220493000 & -3.177423000 \\
C & -5.445345000 & -1.266913000 & -1.563765000 \\
C & -4.162570000 & -2.371661000 & 0.168606000 \\
C & -5.576172000 & 0.375155000 & -3.833773000 \\
H & -3.481888000 & 0.713504000 & -3.566073000 \\
C & -6.669070000 & -1.076774000 & -2.254621000 \\
C & -5.359590000 & -2.153513000 & -0.459176000 \\
C & -6.741966000 & -0.268305000 & -3.363372000 \\
H & -5.623950000 & 0.995219000 & -4.733899000 \\
H & -7.557041000 & -1.603963000 & -1.889305000 \\
H & -6.263600000 & -2.680385000 & -0.136484000 \\
H & -7.691178000 & -0.135621000 & -3.890347000 \\
C & -1.779170000 & -0.087564000 & -1.690728000 \\
C & -1.652526000 & 1.337469000 & -1.754659000 \\
C & -0.656047000 & -0.869170000 & -1.943384000 \\
C & -2.693661000 & 2.217184000 & -1.352497000 \\
C & -0.420710000 & 1.916198000 & -2.190148000 \\
C & 0.578700000 & -0.294333000 & -2.364852000 \\
C & -2.544040000 & 3.582915000 & -1.430826000 \\
H & -3.627751000 & 1.790968000 & -0.975003000 \\
C & -0.295210000 & 3.327411000 & -2.261028000 \\
C & 0.669221000 & 1.070648000 & -2.511453000 \\
\hline & -1.337765000 & 4.149167000 & -1.902605000
\end{tabular}




\begin{tabular}{|c|c|c|c|}
\hline $\mathrm{H}$ & -3.367967000 & 4.236937000 & -1.125884000 \\
\hline $\mathrm{H}$ & 0.656269000 & 3.7466600000 & -2.607477000 \\
\hline $\mathrm{H}$ & 1.608891000 & 1.523292000 & -2.850528000 \\
\hline $\mathrm{H}$ & -1.233858000 & 5.236486000 & -1.974814000 \\
\hline 0 & -1.852447000 & -1.897942000 & 0.416611000 \\
\hline 0 & -0.710335000 & -2.210429000 & -1.794864000 \\
\hline$P$ & -0.654542000 & -2.869570000 & -0.239384000 \\
\hline 0 & -1.117305000 & -4.271554000 & -0.349476000 \\
\hline 0 & 0.608152000 & -2.414362000 & 0.435271000 \\
\hline C & 1.731584000 & -1.190475000 & -2.626314000 \\
\hline C & 2.859091000 & -1.224435000 & -1.785907000 \\
\hline C & 1.689762000 & -2.044667000 & -3.733651000 \\
\hline C & 3.920639000 & -2.089825000 & -2.082918000 \\
\hline C & 2.749363000 & -2.894708000 & -4.026109000 \\
\hline C & 3.872492000 & -2.913863000 & -3.201926000 \\
\hline $\mathrm{H}$ & 4.794753000 & -2.126010000 & -1.423948000 \\
\hline $\mathrm{H}$ & 4.708709000 & -3.583480000 & -3.420805000 \\
\hline $\mathrm{H}$ & 2.694834000 & -3.552484000 & -4.897901000 \\
\hline $\mathrm{H}$ & 0.796954000 & -2.036931000 & -4.366259000 \\
\hline $\mathrm{H}$ & -4.048870000 & -3.075099000 & 0.998390000 \\
\hline$P$ & 2.947563000 & -0.171248000 & -0.289260000 \\
\hline $\mathrm{Au}$ & 0.950246000 & 0.360680000 & 0.874970000 \\
\hline C & 3.830663000 & 1.342489000 & -0.825994000 \\
\hline C & 4.611387000 & 1.382527000 & -1.986265000 \\
\hline C & 3.715958000 & 2.493800000 & -0.036861000 \\
\hline C & 5.263911000 & 2.559236000 & -2.351115000 \\
\hline $\mathrm{H}$ & 4.705812000 & 0.491903000 & -2.616621000 \\
\hline C & 4.376008000 & 3.665097000 & -0.398714000 \\
\hline $\mathrm{H}$ & 3.101221000 & 2.465447000 & 0.871676000 \\
\hline C & 5.147672000 & 3.700039000 & -1.559717000 \\
\hline $\mathrm{H}$ & 5.867074000 & 2.583319000 & -3.263065000 \\
\hline $\mathrm{H}$ & 4.283715000 & 4.557744000 & 0.226529000 \\
\hline $\mathrm{H}$ & 5.659361000 & 4.622094000 & -1.849906000 \\
\hline C & 4.114334000 & -1.046417000 & 0.811523000 \\
\hline C & 5.409915000 & -0.587560000 & 1.064964000 \\
\hline C & 3.646858000 & -2.218171000 & 1.426332000 \\
\hline C & 6.239901000 & -1.299299000 & 1.931196000 \\
\hline $\mathrm{H}$ & 5.778026000 & 0.327200000 & 0.587992000 \\
\hline C & 4.487264000 & -2.923869000 & 2.282117000 \\
\hline $\mathrm{H}$ & 2.625642000 & -2.567987000 & 1.216556000 \\
\hline C & 5.780650000 & -2.465777000 & 2.537578000 \\
\hline $\mathrm{H}$ & 7.253105000 & -0.938238000 & 2.130156000 \\
\hline $\mathrm{H}$ & 4.126921000 & -3.840796000 & 2.757186000 \\
\hline $\mathrm{H}$ & 6.434336000 & -3.022506000 & 3.215389000 \\
\hline
\end{tabular}




$\begin{array}{llll}\text { C } & -1.431938000 & 0.357243000 & 2.596806000 \\ \text { C } & -0.668628000 & 1.176970000 & 1.948248000 \\ \text { C } & -1.838642000 & -0.894843000 & 3.139839000 \\ \text { C } & -3.176366000 & -1.176759000 & 3.458015000 \\ \text { C } & -0.862436000 & -1.900904000 & 3.267889000 \\ \text { C } & -3.529201000 & -2.449332000 & 3.888583000 \\ \text { H } & -3.927462000 & -0.390702000 & 3.360405000 \\ \text { C } & -1.235873000 & -3.175739000 & 3.667477000 \\ \text { H } & 0.173447000 & -1.688684000 & 2.987072000 \\ \text { C } & -2.566098000 & -3.453772000 & 3.981267000 \\ \text { H } & -4.571758000 & -2.664164000 & 4.139818000 \\ \text { H } & -0.479713000 & -3.962798000 & 3.727386000 \\ \text { H } & -2.853192000 & -4.459693000 & 4.299856000 \\ \text { C } & -1.035299000 & 2.580952000 & 1.983158000 \\ \text { C } & -0.429835000 & 3.655814000 & 1.425860000 \\ \text { C } & -2.296978000 & 2.733119000 & 2.723590000 \\ \text { O } & -2.793547000 & 1.715320000 & 3.203654000 \\ \text { C } & -2.936576000 & 4.077516000 & 2.798140000 \\ \text { H } & -3.605225000 & 4.120736000 & 3.670834000 \\ \text { H } & -3.585238000 & 4.148887000 & 1.900934000 \\ \text { C } & -0.952668000 & 5.038636000 & 1.589923000 \\ \text { H } & -1.474837000 & 5.307670000 & 0.647294000 \\ \text { H } & -0.112218000 & 5.749652000 & 1.657362000 \\ \text { C } & -1.890519000 & 5.181869000 & 2.779637000 \\ \text { H } & -2.379172000 & 6.168019000 & 2.769090000 \\ \text { H } & -1.300323000 & 5.135511000 & 3.712613000 \\ \text { H } & 0.467390000 & 3.494965000 & 0.815088000 \\ & & & \\ & & & \end{array}$

Structure Ilb

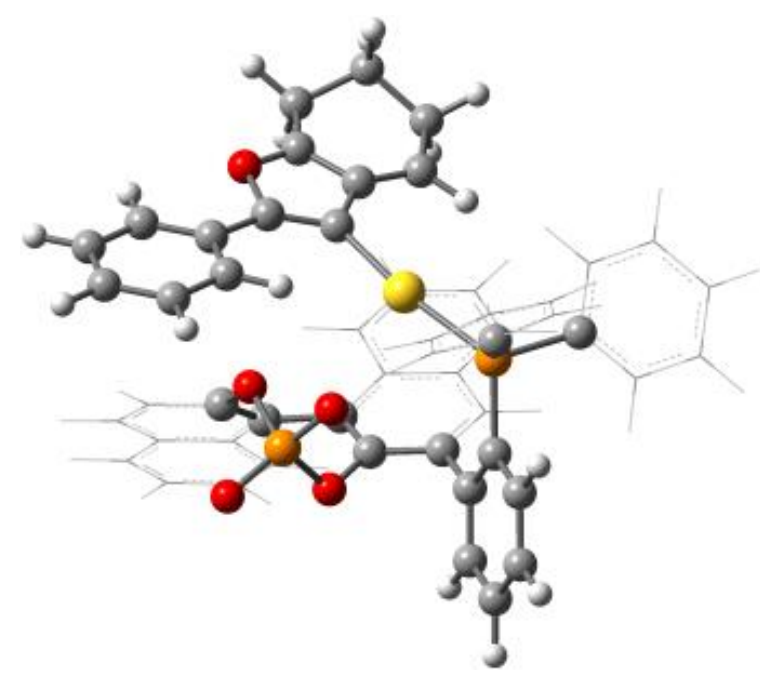
C $\quad 2.954516000$
$-0.671926000$
1.446294000
C $\quad 4.204934000$
$-0.450153000$
2.109701000 


\begin{tabular}{|c|c|c|c|}
\hline c & 2.891546000 & -1.650930000 & 0.460603000 \\
\hline C & 4.340038000 & 0.419555000 & 3.225169000 \\
\hline C & 5.367701000 & -1.158230000 & 1.670901000 \\
\hline c & 4.040024000 & -2.359374000 & 0.038164000 \\
\hline C & 5.555341000 & 0.610926000 & 3.840499000 \\
\hline $\mathrm{H}$ & 3.453093000 & 0.932481000 & 3.607851000 \\
\hline C & 6.608635000 & -0.929099000 & 2.318004000 \\
\hline C & 5.254195000 & -2.101424000 & 0.616492000 \\
\hline C & 6.709172000 & -0.058943000 & 3.376964000 \\
\hline $\mathrm{H}$ & 5.625944000 & 1.280358000 & 4.703009000 \\
\hline $\mathrm{H}$ & 7.487686000 & -1.476387000 & 1.960583000 \\
\hline $\mathrm{H}$ & 6.151795000 & -2.638789000 & 0.293074000 \\
\hline $\mathrm{H}$ & 7.671505000 & 0.102482000 & 3.871174000 \\
\hline C & 1.710118000 & 0.054689000 & 1.792343000 \\
\hline C & 1.606929000 & 1.481817000 & 1.730424000 \\
\hline C & 0.571439000 & -0.684086000 & 2.099426000 \\
\hline C & 2.673364000 & 2.310879000 & 1.287609000 \\
\hline C & 0.373975000 & 2.114522000 & 2.080304000 \\
\hline C & -0.666643000 & -0.055974000 & 2.4238890000 \\
\hline C & 2.547665000 & 3.681187000 & 1.253875000 \\
\hline $\mathrm{H}$ & 3.611359000 & 1.843467000 & 0.974605000 \\
\hline C & 0.270811000 & 3.528418000 & 2.029752000 \\
\hline C & -0.740073000 & 1.317156000 & 2.438803000 \\
\hline C & 1.337800000 & 4.303474000 & 1.639266000 \\
\hline $\mathrm{H}$ & 3.395813000 & 4.294557000 & 0.930136000 \\
\hline $\mathrm{H}$ & -0.683082000 & 3.988417000 & 2.311989000 \\
\hline $\mathrm{H}$ & -1.682556000 & 1.812256000 & 2.701759000 \\
\hline $\mathrm{H}$ & 1.251329000 & 5.394614000 & 1.620302000 \\
\hline $\mathrm{O}$ & 1.717888000 & -1.921114000 & -0.148187000 \\
\hline $\mathrm{O}$ & 0.613746000 & -2.032637000 & 2.093895000 \\
\hline$P$ & 0.550508000 & -2.853488000 & 0.616160000 \\
\hline $\mathrm{O}$ & 1.057908000 & -4.222303000 & 0.864717000 \\
\hline $\mathrm{O}$ & -0.731694000 & -2.504357000 & -0.079314000 \\
\hline C & -1.843019000 & -0.909462000 & 2.720811000 \\
\hline C & -2.928662000 & -1.014140000 & 1.832895000 \\
\hline C & -1.864031000 & -1.650459000 & 3.907012000 \\
\hline C & -4.012215000 & -1.839270000 & 2.160923000 \\
\hline C & -2.945885000 & -2.461502000 & 4.228325000 \\
\hline C & -4.026773000 & -2.553137000 & 3.354593000 \\
\hline $\mathrm{H}$ & -4.855275000 & -1.932063000 & 1.468094000 \\
\hline $\mathrm{H}$ & -4.880065000 & -3.193383000 & 3.594819000 \\
\hline $\mathrm{H}$ & -2.941219000 & -3.031857000 & 5.161295000 \\
\hline $\mathrm{H}$ & -1.002414000 & -1.586918000 & 4.578701000 \\
\hline $\mathrm{H}$ & 3.908456000 & -3.102932000 & -0.754052000 \\
\hline
\end{tabular}




\begin{tabular}{|c|c|c|c|}
\hline$P$ & -2.933991000 & -0.100404000 & 0.241458000 \\
\hline $\mathrm{Au}$ & -0.914271000 & 0.309076000 & -0.961653000 \\
\hline C & -3.804428000 & 1.470413000 & 0.626069000 \\
\hline C & -4.615602000 & 1.624763000 & 1.755633000 \\
\hline C & -3.646020000 & 2.549077000 & -0.253331000 \\
\hline C & -5.253496000 & 2.839716000 & 2.001745000 \\
\hline $\mathrm{H}$ & -4.746276000 & 0.792610000 & 2.455509000 \\
\hline C & -4.291879000 & 3.758787000 & -0.010889000 \\
\hline $\mathrm{H}$ & -3.007828000 & 2.432067000 & -1.138391000 \\
\hline C & -5.093362000 & 3.907025000 & 1.120585000 \\
\hline $\mathrm{H}$ & -5.880745000 & 2.951613000 & 2.890704000 \\
\hline $\mathrm{H}$ & -4.166116000 & 4.592944000 & -0.707284000 \\
\hline $\mathrm{H}$ & -5.594561000 & 4.859257000 & 1.316578000 \\
\hline C & -4.109206000 & -1.037941000 & -0.801551000 \\
\hline C & -5.385477000 & -0.566925000 & -1.122409000 \\
\hline C & -3.667656000 & -2.272513000 & -1.301943000 \\
\hline C & -6.221079000 & -1.327280000 & -1.940538000 \\
\hline $\mathrm{H}$ & -5.735070000 & 0.396066000 & -0.734944000 \\
\hline C & -4.513326000 & -3.026167000 & -2.110609000 \\
\hline $\mathrm{H}$ & -2.663920000 & -2.633250000 & -1.035254000 \\
\hline C & -5.787110000 & -2.555377000 & -2.432986000 \\
\hline $\mathrm{H}$ & -7.218985000 & -0.955716000 & -2.191269000 \\
\hline $\mathrm{H}$ & -4.172482000 & -3.991632000 & -2.495500000 \\
\hline $\mathrm{H}$ & -6.444922000 & -3.150646000 & -3.073132000 \\
\hline C & 1.703549000 & 0.228211000 & -2.719212000 \\
\hline C & 0.743779000 & 0.914181000 & -2.048139000 \\
\hline C & 1.957977000 & -1.151795000 & -3.091073000 \\
\hline C & 3.194357000 & -1.549284000 & -3.623890000 \\
\hline C & 0.964898000 & -2.121633000 & -2.883709000 \\
\hline C & 3.432695000 & -2.886606000 & -3.927453000 \\
\hline $\mathrm{H}$ & 3.979832000 & -0.807798000 & -3.789260000 \\
\hline C & 1.213322000 & -3.454979000 & -3.174900000 \\
\hline $\mathrm{H}$ & 0.006755000 & -1.832598000 & -2.441140000 \\
\hline C & 2.447092000 & -3.844618000 & -3.698202000 \\
\hline $\mathrm{H}$ & 4.402238000 & -3.182890000 & -4.338397000 \\
\hline H & 0.438604000 & -4.199867000 & -2.972306000 \\
\hline $\mathrm{H}$ & 2.640342000 & -4.897198000 & -3.924060000 \\
\hline C & 1.165526000 & 2.303798000 & -2.141577000 \\
\hline C & 0.673050000 & 3.482804000 & -1.658033000 \\
\hline C & 2.399887000 & 2.329313000 & -2.857685000 \\
\hline O & 2.739700000 & 1.143473000 & -3.196973000 \\
\hline C & 3.210369000 & 3.531432000 & -3.096756000 \\
\hline $\mathrm{H}$ & 3.828293000 & 3.419308000 & -4.000859000 \\
\hline H & 3.919099000 & 3.613307000 & -2.245110000 \\
\hline
\end{tabular}




$\begin{array}{lrrr}\mathrm{C} & 1.330266000 & 4.779017000 & -1.950889000 \\ \mathrm{H} & 1.867827000 & 5.067062000 & -1.021331000 \\ \mathrm{H} & 0.564973000 & 5.563888000 & -2.073898000 \\ \mathrm{C} & 2.281635000 & 4.741153000 & -3.141863000 \\ \mathrm{H} & 2.870041000 & 5.669023000 & -3.188092000 \\ \mathrm{H} & 1.687897000 & 4.698155000 & -4.072234000 \\ \mathrm{H} & -0.212100000 & 3.462402000 & -1.007100000\end{array}$

Structure II'a

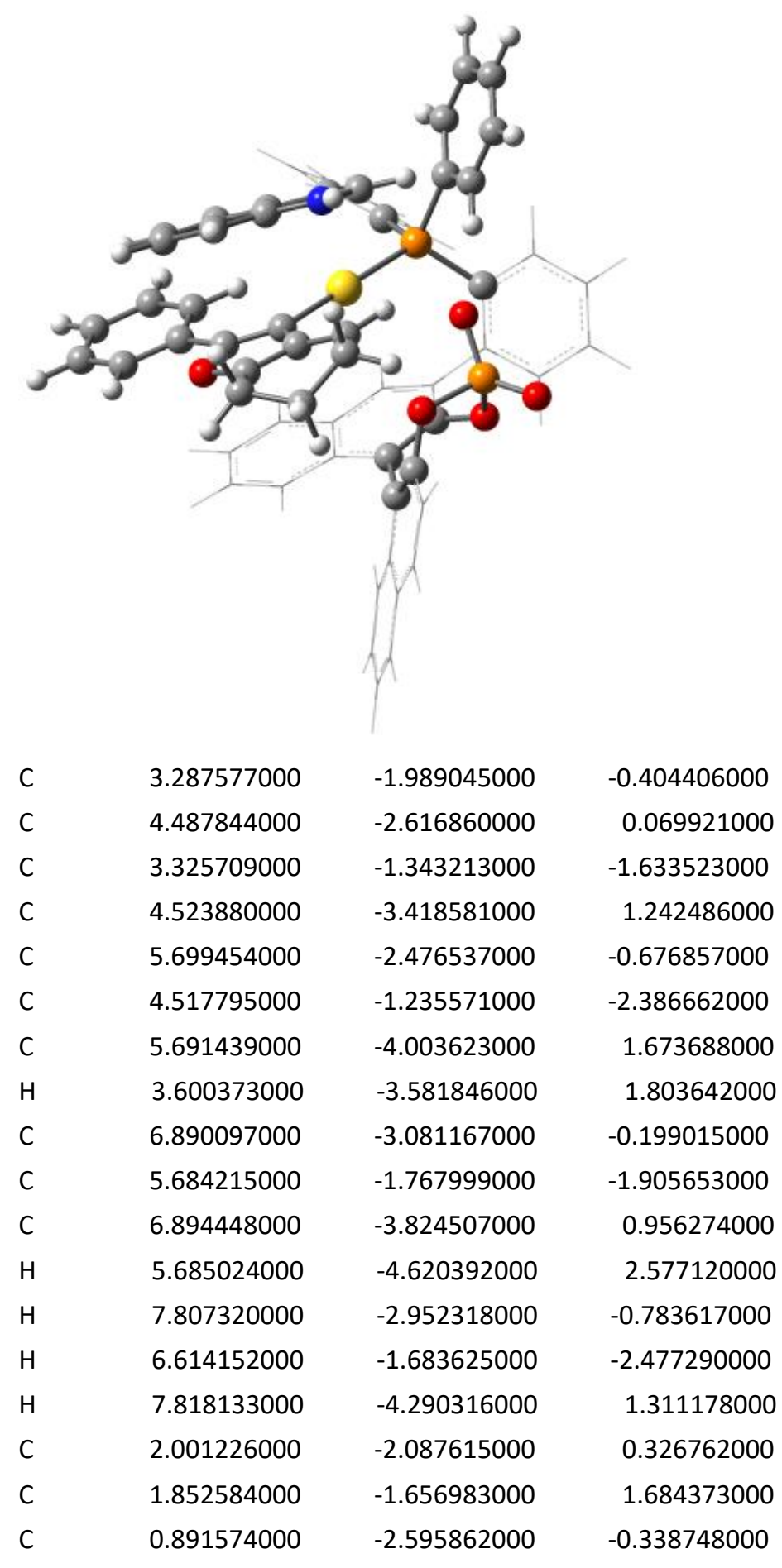




\begin{tabular}{|c|c|c|c|}
\hline c & 2.868910000 & -0.949039000 & 2.378651000 \\
\hline C & 0.618604000 & -1.894387000 & 2.362278000 \\
\hline C & -0.350340000 & -2.819100000 & 0.325004000 \\
\hline C & 2.688239000 & -0.538220000 & 3.679070000 \\
\hline $\mathrm{H}$ & 3.803508000 & -0.717928000 & 1.858663000 \\
\hline C & 0.469905000 & -1.477244000 & 3.709573000 \\
\hline C & -0.453277000 & -2.500171000 & 1.660993000 \\
\hline C & 1.483150000 & -0.816911000 & 4.361068000 \\
\hline $\mathrm{H}$ & 3.480970000 & 0.018711000 & 4.187938000 \\
\hline $\mathrm{H}$ & -0.485634000 & -1.668520000 & 4.210978000 \\
\hline $\mathrm{H}$ & -1.393450000 & -2.688087000 & 2.194578000 \\
\hline $\mathrm{H}$ & 1.349306000 & -0.479983000 & 5.393320000 \\
\hline $\mathrm{O}$ & 2.205045000 & -0.766884000 & -2.134279000 \\
\hline $\mathrm{O}$ & 0.979289000 & -2.918665000 & -1.652313000 \\
\hline $\mathrm{P}$ & 0.973182000 & -1.714263000 & -2.815447000 \\
\hline 0 & 1.453627000 & -2.326838000 & -4.074296000 \\
\hline $\mathrm{O}$ & -0.246650000 & -0.853558000 & -2.662992000 \\
\hline C & -1.480855000 & -3.420770000 & -0.424576000 \\
\hline C & -2.703599000 & -2.757842000 & -0.646708000 \\
\hline C & -1.325105000 & -4.720694000 & -0.926705000 \\
\hline C & -3.733170000 & -3.414484000 & -1.338168000 \\
\hline C & -2.352698000 & -5.364131000 & -1.602706000 \\
\hline C & -3.568121000 & -4.711776000 & -1.804670000 \\
\hline $\mathrm{H}$ & -4.678736000 & -2.892519000 & -1.522685000 \\
\hline $\mathrm{H}$ & -4.382374000 & -5.207648000 & -2.340000000 \\
\hline $\mathrm{H}$ & -2.202773000 & -6.379549000 & -1.979824000 \\
\hline $\mathrm{H}$ & -0.367116000 & -5.226685000 & -0.775630000 \\
\hline $\mathrm{H}$ & 4.464198000 & -0.730267000 & -3.355409000 \\
\hline$P$ & -2.987206000 & -1.020471000 & -0.133471000 \\
\hline $\mathrm{Au}$ & -1.092922000 & 0.344951000 & 0.269608000 \\
\hline C & -4.081670000 & -1.106408000 & 1.332746000 \\
\hline C & -4.655987000 & -2.296238000 & 1.790331000 \\
\hline C & -4.330507000 & 0.086752000 & 2.026234000 \\
\hline C & -5.471526000 & -2.290377000 & 2.922139000 \\
\hline $\mathrm{H}$ & -4.463659000 & -3.237576000 & 1.265316000 \\
\hline C & -5.154998000 & 0.091136000 & 3.146821000 \\
\hline $\mathrm{H}$ & -3.874027000 & 1.022079000 & 1.678637000 \\
\hline C & -5.724655000 & -1.099915000 & 3.598277000 \\
\hline $\mathrm{H}$ & -5.911996000 & -3.226876000 & 3.275828000 \\
\hline $\mathrm{H}$ & -5.348304000 & 1.028654000 & 3.675863000 \\
\hline $\mathrm{H}$ & -6.365507000 & -1.098846000 & 4.484563000 \\
\hline C & -4.026593000 & -0.335464000 & -1.474878000 \\
\hline C & -5.380683000 & -0.027998000 & -1.318695000 \\
\hline C & -3.387050000 & -0.102915000 & -2.702484000 \\
\hline
\end{tabular}




\begin{tabular}{|c|c|c|c|}
\hline C & -6.096558000 & 0.515318000 & -2.385753000 \\
\hline $\mathrm{H}$ & -5.884141000 & -0.211258000 & -0.363209000 \\
\hline C & -4.114115000 & 0.432649000 & -3.761868000 \\
\hline $\mathrm{H}$ & -2.321912000 & -0.352926000 & -2.823890000 \\
\hline C & -5.465150000 & 0.745572000 & -3.605439000 \\
\hline $\mathrm{H}$ & -7.156043000 & 0.756988000 & -2.260574000 \\
\hline $\mathrm{H}$ & -3.619330000 & 0.605793000 & -4.722076000 \\
\hline $\mathrm{H}$ & -6.029314000 & 1.170727000 & -4.440736000 \\
\hline C & 1.082999000 & 2.157423000 & 1.680956000 \\
\hline C & 0.564346000 & 1.530341000 & 0.591958000 \\
\hline C & 0.665579000 & 2.349659000 & 3.060814000 \\
\hline C & 1.482740000 & 3.023861000 & 3.982218000 \\
\hline c & -0.576432000 & 1.861952000 & 3.499911000 \\
\hline C & 1.073909000 & 3.191032000 & 5.302558000 \\
\hline $\mathrm{H}$ & 2.451589000 & 3.412744000 & 3.658848000 \\
\hline C & -0.980938000 & 2.034438000 & 4.817226000 \\
\hline$H$ & -1.220815000 & 1.329341000 & 2.791489000 \\
\hline C & -0.157584000 & 2.697648000 & 5.728426000 \\
\hline $\mathrm{H}$ & 1.726802000 & 3.715780000 & 6.006372000 \\
\hline $\mathrm{H}$ & -1.951472000 & 1.643709000 & 5.138075000 \\
\hline $\mathrm{H}$ & -0.477341000 & 2.832263000 & 6.765721000 \\
\hline C & 1.504527000 & 1.835623000 & -0.469126000 \\
\hline C & 1.475551000 & 1.611172000 & -1.829813000 \\
\hline C & 2.524151000 & 2.631481000 & 0.083475000 \\
\hline $\mathrm{O}$ & 2.305286000 & 2.823085000 & $1.34856700 \mathrm{C}$ \\
\hline C & 3.621998000 & 3.255269000 & -0.670366000 \\
\hline $\mathrm{H}$ & 4.533554000 & 3.334224000 & -0.056722000 \\
\hline $\mathrm{H}$ & 3.303117000 & 4.298095000 & -0.892022000 \\
\hline C & 2.532089000 & 2.181292000 & -2.693887000 \\
\hline $\mathrm{H}$ & 2.096867000 & 3.117767000 & -3.112802000 \\
\hline $\mathrm{H}$ & 2.6888490000 & 1.525867000 & -3.565741000 \\
\hline C & 3.839048000 & 2.467451000 & -1.960273000 \\
\hline $\mathrm{H}$ & 4.535939000 & 3.004958000 & -2.620760000 \\
\hline $\mathrm{H}$ & 4.316733000 & 1.500517000 & -1.714915000 \\
\hline $\mathrm{H}$ & 0.623783000 & 1.076209000 & -2.274340000 \\
\hline c & -1.135356000 & 3.412831000 & -2.249448000 \\
\hline C & -0.784021000 & 3.614538000 & -3.559559000 \\
\hline C & -0.396677000 & 4.363626000 & -1.470692000 \\
\hline $\mathrm{H}$ & -1.836071000 & 2.662107000 & -1.880399000 \\
\hline $\mathrm{N}$ & 0.140004000 & 4.631703000 & -3.642602000 \\
\hline C & 0.400284000 & 5.110646000 & -2.377318000 \\
\hline $\mathrm{H}$ & -1.123781000 & 3.099257000 & -4.457429000 \\
\hline C & -0.305517000 & 4.639328000 & -0.096410000 \\
\hline C & 0.574916000 & 5.620743000 & 0.335403000 \\
\hline
\end{tabular}




$\begin{array}{lrrr}\text { H } & -0.912609000 & 4.076215000 & 0.620161000 \\ \text { H } & 0.663590000 & 5.830868000 & 1.406087000 \\ \text { C } & 1.281134000 & 6.108395000 & -1.947039000 \\ \text { C } & 1.358916000 & 6.349630000 & -0.580841000 \\ \text { H } & 1.884467000 & 6.678458000 & -2.660085000 \\ \text { H } & 2.037087000 & 7.124326000 & -0.210489000 \\ \text { H } & 0.525480000 & 5.003390000 & -4.502406000\end{array}$

Structure TS-II'-IIIa

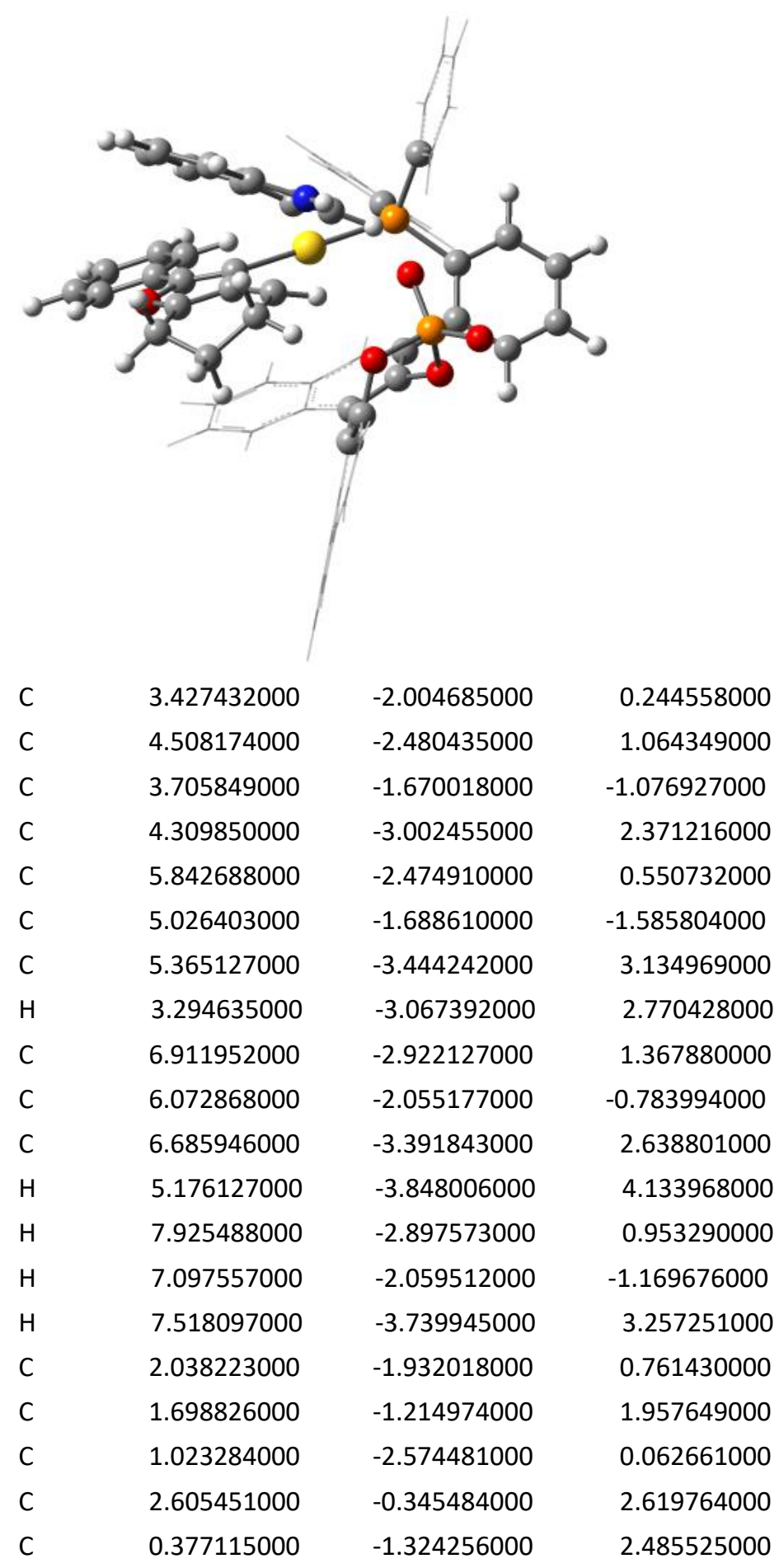




\begin{tabular}{|c|c|c|c|}
\hline C & -0.306084000 & -2.670701000 & 0.570551000 \\
\hline C & 2.228284000 & 0.358868000 & 3.741007000 \\
\hline $\mathrm{H}$ & 3.615713000 & -0.225308000 & 2.216816000 \\
\hline C & 0.028842000 & -0.616966000 & 3.663696000 \\
\hline C & -0.589966000 & -2.086986000 & 1.784726000 \\
\hline C & 0.930511000 & 0.215822000 & 4.279973000 \\
\hline $\mathrm{H}$ & 2.940035000 & 1.040344000 & 4.217314000 \\
\hline $\mathrm{H}$ & -0.992893000 & -0.716061000 & 4.047765000 \\
\hline $\mathrm{H}$ & -1.595452000 & -2.181879000 & 2.214264000 \\
\hline $\mathrm{H}$ & 0.638938000 & 0.783469000 & 5.169266000 \\
\hline $\mathrm{O}$ & 2.734070000 & -1.233655000 & -1.911277000 \\
\hline $\mathrm{O}$ & 1.292862000 & -3.154354000 & -1.133968000 \\
\hline$P$ & 1.460652000 & -2.179232000 & -2.481923000 \\
\hline 0 & 1.917752000 & -3.038549000 & -3.596587000 \\
\hline $\mathrm{O}$ & 0.319873000 & -1.200213000 & -2.550489000 \\
\hline C & -1.336518000 & -3.406648000 & -0.198362000 \\
\hline C & -2.550768000 & -2.827803000 & -0.617652000 \\
\hline C & -1.096174000 & -4.753189000 & -0.514183000 \\
\hline C & -3.484401000 & -3.608289000 & -1.319077000 \\
\hline C & -2.031235000 & -5.517087000 & -1.196960000 \\
\hline C & -3.237379000 & -4.944710000 & -1.599380000 \\
\hline $\mathrm{H}$ & -4.421195000 & -3.153238000 & -1.659762000 \\
\hline $\mathrm{H}$ & -3.978926000 & -5.535196000 & -2.144202000 \\
\hline $\mathrm{H}$ & -1.815639000 & -6.565061000 & -1.422703000 \\
\hline $\mathrm{H}$ & -0.146171000 & -5.199440000 & -0.207995000 \\
\hline $\mathrm{H}$ & 5.167782000 & -1.401822000 & -2.631490000 \\
\hline$P$ & -2.974488000 & -1.058951000 & -0.371741000 \\
\hline $\mathrm{Au}$ & -1.329788000 & 0.540810000 & 0.261909000 \\
\hline C & -4.328645000 & -1.041025000 & 0.862833000 \\
\hline C & -4.860403000 & -2.199811000 & 1.436561000 \\
\hline C & -4.805601000 & 0.210844000 & 1.280431000 \\
\hline C & -5.859226000 & -2.107176000 & 2.406287000 \\
\hline $\mathrm{H}$ & -4.491501000 & -3.184333000 & 1.131682000 \\
\hline C & -5.811415000 & 0.298934000 & 2.237110000 \\
\hline $\mathrm{H}$ & -4.382478000 & 1.126095000 & 0.847348000 \\
\hline C & -6.338256000 & -0.862386000 & 2.804202000 \\
\hline $\mathrm{H}$ & -6.264390000 & -3.019620000 & 2.852767000 \\
\hline $\mathrm{H}$ & -6.181439000 & 1.280259000 & 2.547608000 \\
\hline $\mathrm{H}$ & -7.122577000 & -0.794021000 & 3.563329000 \\
\hline C & -3.740358000 & -0.597203000 & -1.968129000 \\
\hline C & -5.093938000 & -0.288743000 & -2.121375000 \\
\hline C & -2.873917000 & -0.538335000 & -3.070012000 \\
\hline C & -5.582213000 & 0.0844490000 & -3.373957000 \\
\hline $\mathrm{H}$ & -5.774798000 & -0.339792000 & -1.264902000 \\
\hline
\end{tabular}




\begin{tabular}{|c|c|c|c|}
\hline C & -3.373251000 & -0.170138000 & -4.315612000 \\
\hline $\mathrm{H}$ & -1.810386000 & -0.796502000 & -2.950239000 \\
\hline C & -4.724213000 & 0.145429000 & -4.468981000 \\
\hline $\mathrm{H}$ & -6.642460000 & 0.326292000 & -3.492174000 \\
\hline $\mathrm{H}$ & -2.698787000 & -0.129295000 & -5.176004000 \\
\hline $\mathrm{H}$ & -5.110268000 & 0.438597000 & -5.449650000 \\
\hline C & 0.247340000 & 2.785335000 & 1.857341000 \\
\hline C & 0.091025000 & 1.943774000 & 0.790477000 \\
\hline C & -0.530578000 & 3.075828000 & 3.052982000 \\
\hline C & -0.028615000 & 3.925333000 & 4.051983000 \\
\hline C & -1.786665000 & 2.480037000 & 3.255815000 \\
\hline C & -0.753415000 & 4.158613000 & 5.217265000 \\
\hline $\mathrm{H}$ & 0.946972000 & 4.397931000 & 3.91336900 \\
\hline C & -2.505951000 & 2.714274000 & 4.420686000 \\
\hline $\mathrm{H}$ & -2.189801000 & 1.810384000 & 2.487886000 \\
\hline C & -1.993394000 & 3.553405000 & 5.411715000 \\
\hline $\mathrm{H}$ & -0.341319000 & 4.821038000 & 5.984494000 \\
\hline $\mathrm{H}$ & -3.479306000 & 2.232815000 & 4.557010000 \\
\hline $\mathrm{H}$ & -2.559592000 & 3.735099000 & 6.329724000 \\
\hline C & 1.308990000 & 2.115307000 & 0.033602000 \\
\hline C & 1.790725000 & 1.459960000 & -1.123539000 \\
\hline C & 2.094802000 & 3.043841000 & 0.696671000 \\
\hline $\mathrm{O}$ & 1.483549000 & 3.455972000 & 1.78988800 \\
\hline C & 3.477119000 & 3.423567000 & $0.33810100 c$ \\
\hline $\mathrm{H}$ & 4.058614000 & 3.665597000 & 1.24261800 \\
\hline $\mathrm{H}$ & 3.465169000 & 4.341368000 & -0.285474000 \\
\hline C & 3.164628000 & 1.828530000 & -1.596858000 \\
\hline $\mathrm{H}$ & 3.094800000 & 2.681385000 & -2.302659000 \\
\hline $\mathrm{H}$ & 3.594620000 & 0.986551000 & -2.161111000 \\
\hline C & 4.084088000 & 2.264202000 & -0.453196000 \\
\hline $\mathrm{H}$ & 5.067127000 & 2.548282000 & -0.860637000 \\
\hline $\mathrm{H}$ & 4.256494000 & 1.407318000 & 0.22477000 \\
\hline $\mathrm{H}$ & 1.390483000 & 0.468282000 & -1.365133000 \\
\hline C & 0.337629000 & 2.197108000 & -2.625339000 \\
\hline C & 0.934628000 & 1.578694000 & -3.728351000 \\
\hline C & 0.659939000 & 3.598285000 & -2.721826000 \\
\hline $\mathrm{H}$ & -0.465395000 & 1.742059000 & -2.039535000 \\
\hline $\mathrm{N}$ & 1.644521000 & 2.486955000 & -4.425546000 \\
\hline C & 1.513079000 & 3.744391000 & -3.838099000 \\
\hline $\mathrm{H}$ & 0.898918000 & 0.513962000 & -3.975103000 \\
\hline C & 0.393858000 & 4.706830000 & -1.909259000 \\
\hline C & 0.972436000 & 5.926371000 & -2.243086000 \\
\hline $\mathrm{H}$ & -0.258742000 & 4.608731000 & -1.035518000 \\
\hline $\mathrm{H}$ & 0.772316000 & 6.806735000 & -1.625933000 \\
\hline
\end{tabular}




$\begin{array}{llll}\text { C } & 2.096061000 & 4.962452000 & -4.180523000 \\ \text { C } & 1.810248000 & 6.052041000 & -3.364316000 \\ \text { H } & 2.755744000 & 5.057129000 & -5.047302000 \\ \text { H } & 2.247423000 & 7.026206000 & -3.600604000 \\ \text { H } & 2.219749000 & 2.276891000 & -5.235239000\end{array}$

Structure IIla

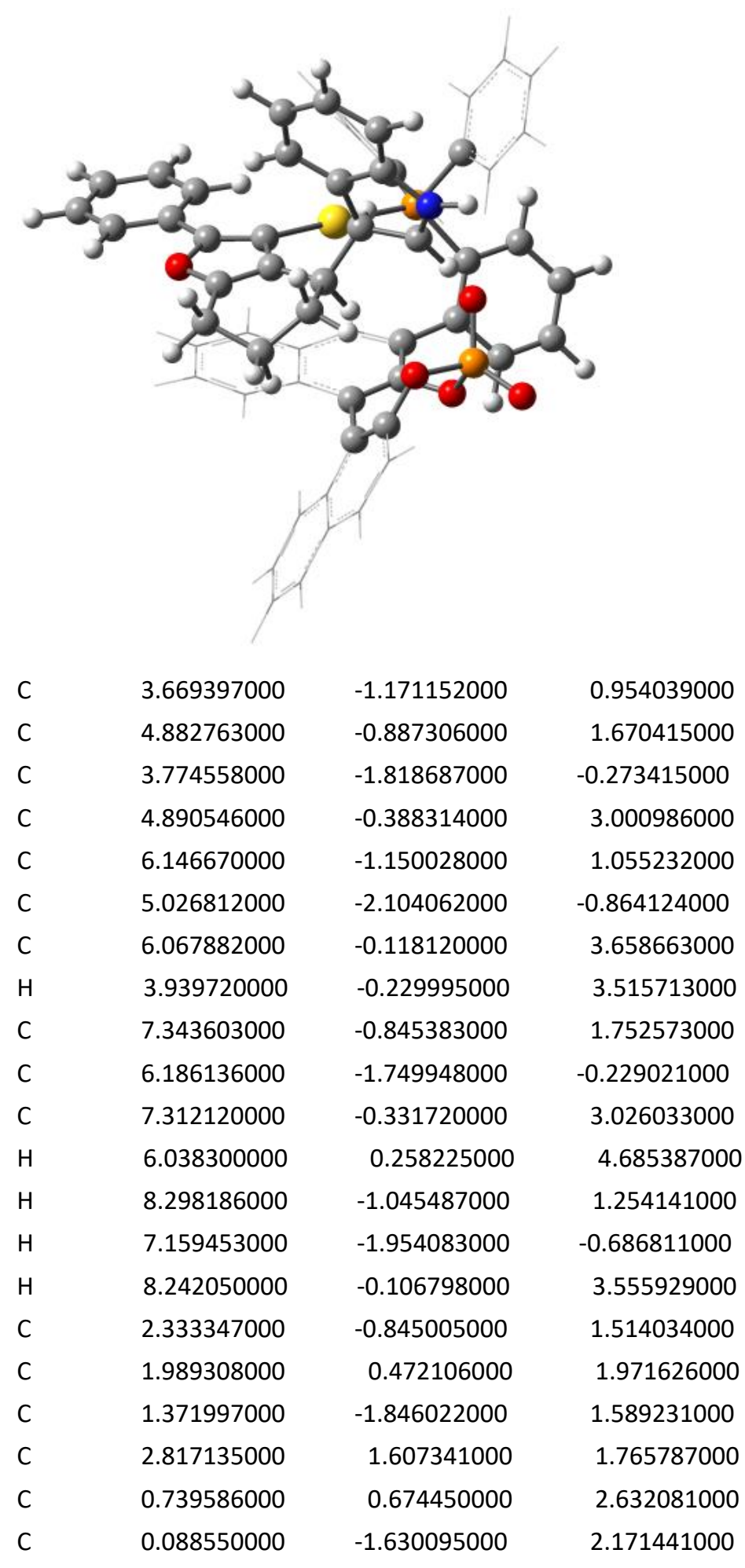




\begin{tabular}{|c|c|c|c|}
\hline C & 2.447492000 & 2.851522000 & 2.223551000 \\
\hline $\mathrm{H}$ & 3.758441000 & 1.488674000 & 1.220513000 \\
\hline C & 0.403217000 & 1.959458000 & 3.126923000 \\
\hline C & -0.181028000 & -0.397657000 & 2.722898000 \\
\hline C & 1.237959000 & 3.031186000 & 2.930088000 \\
\hline $\mathrm{H}$ & 3.092660000 & 3.713792000 & 2.027372000 \\
\hline $\mathrm{H}$ & -0.561059000 & 2.087882000 & 3.631807000 \\
\hline $\mathrm{H}$ & -1.140213000 & -0.222759000 & 3.226894000 \\
\hline $\mathrm{H}$ & 0.952210000 & 4.025451000 & 3.287584000 \\
\hline $\mathrm{O}$ & 2.666548000 & -2.150617000 & -0.983974000 \\
\hline $\mathrm{O}$ & 1.661119000 & -3.089927000 & 1.127386000 \\
\hline$P$ & 1.643301000 & -3.398422000 & -0.506316000 \\
\hline $\mathrm{O}$ & 2.285258000 & -4.714271000 & -0.720976000 \\
\hline 0 & 0.318498000 & -2.976894000 & -1.088546000 \\
\hline C & -0.915789000 & -2.717758000 & 2.183842000 \\
\hline C & -2.203668000 & -2.577026000 & 1.628488000 \\
\hline C & -0.578611000 & -3.941262000 & 2.784146000 \\
\hline C & -3.110597000 & -3.647359000 & 1.704294000 \\
\hline C & -1.486982000 & -4.986708000 & 2.861565000 \\
\hline C & -2.764857000 & -4.840151000 & 2.323041000 \\
\hline $\mathrm{H}$ & -4.107061000 & -3.544057000 & 1.260194000 \\
\hline $\mathrm{H}$ & -3.487297000 & -5.659291000 & 2.373428000 \\
\hline $\mathrm{H}$ & -1.195048000 & -5.924869000 & 3.341513000 \\
\hline $\mathrm{H}$ & 0.426015000 & -4.059232000 & 3.198193000 \\
\hline $\mathrm{H}$ & 5.024186000 & -2.607966000 & -1.834385000 \\
\hline$P$ & -2.778840000 & -1.084424000 & 0.724425000 \\
\hline $\mathrm{Au}$ & -1.321564000 & 0.654481000 & -0.035053000 \\
\hline C & -4.061620000 & -0.326437000 & 1.793203000 \\
\hline C & -4.445926000 & -0.859671000 & 3.027466000 \\
\hline C & -4.632111000 & 0.881486000 & 1.362941000 \\
\hline C & -5.393251000 & -0.201153000 & 3.811695000 \\
\hline $\mathrm{H}$ & -4.000747000 & -1.792750000 & 3.386785000 \\
\hline C & -5.587176000 & 1.527779000 & 2.140857000 \\
\hline $\mathrm{H}$ & -4.322108000 & 1.319269000 & 0.405589000 \\
\hline C & -5.967649000 & 0.987177000 & 3.369665000 \\
\hline $\mathrm{H}$ & -5.682033000 & -0.624235000 & 4.777945000 \\
\hline $\mathrm{H}$ & -6.030058000 & 2.464276000 & 1.790011000 \\
\hline $\mathrm{H}$ & -6.710770000 & 1.499770000 & 3.986963000 \\
\hline C & -3.670397000 & -1.809336000 & -0.703691000 \\
\hline C & -5.033157000 & -1.624247000 & -0.944426000 \\
\hline C & -2.891456000 & -2.548433000 & -1.607548000 \\
\hline C & -5.617983000 & -2.169277000 & -2.088456000 \\
\hline $\mathrm{H}$ & -5.648722000 & -1.056636000 & -0.238677000 \\
\hline C & -3.486614000 & -3.093222000 & -2.741319000 \\
\hline
\end{tabular}




\begin{tabular}{|c|c|c|c|}
\hline H & -1.821779000 & -2.716514000 & -1.407233000 \\
\hline C & -4.847414000 & -2.901246000 & -2.987273000 \\
\hline $\mathrm{H}$ & -6.686237000 & -2.022621000 & -2.272358000 \\
\hline $\mathrm{H}$ & -2.881993000 & -3.682159000 & -3.437981000 \\
\hline $\mathrm{H}$ & -5.309546000 & -3.330319000 & -3.881141000 \\
\hline C & 0.195472000 & 3.435253000 & -0.301177000 \\
\hline C & -0.020960000 & 2.128082000 & -0.680385000 \\
\hline C & -0.542174000 & 4.379782000 & 0.524415000 \\
\hline C & -0.031378000 & 5.662687000 & 0.785106000 \\
\hline C & -1.766105000 & 4.024429000 & 1.116573000 \\
\hline C & -0.710431000 & 6.545113000 & 1.620380000 \\
\hline $\mathrm{H}$ & 0.918830000 & 5.958677000 & 0.332706000 \\
\hline C & -2.438643000 & 4.905533000 & 1.953777000 \\
\hline $\mathrm{H}$ & -2.179698000 & 3.027546000 & 0.926497000 \\
\hline C & -1.915020000 & 6.172909000 & 2.215381000 \\
\hline $\mathrm{H}$ & -0.289748000 & 7.537268000 & 1.811690000 \\
\hline $\mathrm{H}$ & -3.384629000 & 4.597119000 & 2.410302000 \\
\hline $\mathrm{H}$ & -2.444210000 & 6.865755000 & 2.876049000 \\
\hline C & 1.140402000 & 1.794488000 & -1.453634000 \\
\hline C & 1.502670000 & 0.501975000 & -2.101016000 \\
\hline C & 1.968189000 & 2.875703000 & -1.460126000 \\
\hline $\mathrm{O}$ & 1.405493000 & 3.893678000 & -0.782724000 \\
\hline C & 3.352702000 & 2.931515000 & -1.986558000 \\
\hline $\mathrm{H}$ & 4.001959000 & 3.489537000 & -1.288017000 \\
\hline $\mathrm{H}$ & 3.401792000 & 3.475548000 & -2.951118000 \\
\hline C & 2.810088000 & 0.630187000 & -2.888014000 \\
\hline H & 2.606110000 & 1.087278000 & -3.877528000 \\
\hline $\mathrm{H}$ & 3.221517000 & -0.378005000 & -3.079229000 \\
\hline C & 3.839606000 & 1.490605000 & -2.161180000 \\
\hline $\mathrm{H}$ & 4.798495000 & 1.471971000 & -2.704498000 \\
\hline $\mathrm{H}$ & 4.036076000 & 1.046603000 & -1.165034000 \\
\hline $\mathrm{H}$ & 1.623359000 & -0.272992000 & -1.320515000 \\
\hline C & 0.331967000 & -0.000017000 & -2.985451000 \\
\hline C & 0.467081000 & -1.382356000 & -3.459584000 \\
\hline C & 0.009834000 & 0.752466000 & -4.236322000 \\
\hline $\mathrm{H}$ & -0.552808000 & -0.026221000 & -2.291456000 \\
\hline N & 0.264502000 & -1.441839000 & -4.746565000 \\
\hline C & -0.016600000 & -0.163080000 & -5.293388000 \\
\hline $\mathrm{H}$ & 0.623519000 & -2.251683000 & -2.789181000 \\
\hline C & -0.238377000 & 2.095871000 & -4.495202000 \\
\hline C & -0.504684000 & 2.473965000 & -5.812709000 \\
\hline $\mathrm{H}$ & -0.227036000 & 2.829177000 & -3.683397000 \\
\hline $\mathrm{H}$ & -0.703125000 & 3.525052000 & -6.038520000 \\
\hline C & -0.282813000 & 0.186498000 & -6.608513000 \\
\hline & & & S67 \\
\hline
\end{tabular}




$\begin{array}{lrrr}\mathrm{C} & -0.527232000 & 1.537450000 & -6.851814000 \\ \mathrm{H} & -0.300410000 & -0.554165000 & -7.411686000 \\ \mathrm{H} & -0.741317000 & 1.868041000 & -7.871438000 \\ \mathrm{H} & 0.276253000 & -2.306919000 & -5.287373000\end{array}$

Structure 9a

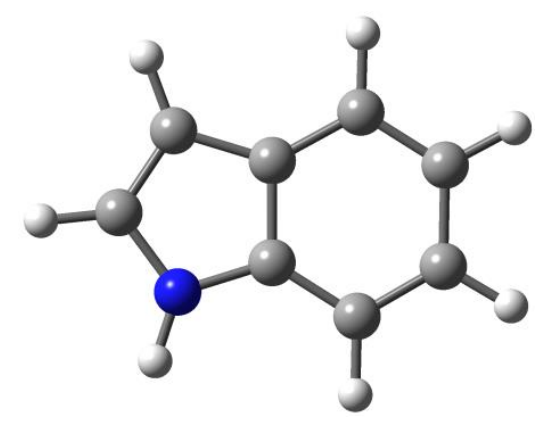

$\begin{array}{lrrr}\mathrm{C} & -0.249254000 & 0.751594000 & 0.000010000 \\ \mathrm{C} & -0.251769000 & -0.668662000 & -0.000070000 \\ \mathrm{C} & 0.930144000 & -1.415997000 & 0.000020000 \\ \mathrm{C} & 2.130231000 & -0.720334000 & 0.000197000 \\ \mathrm{C} & 2.156697000 & 0.688081000 & 0.000280000 \\ \mathrm{C} & 0.983272000 & 1.426374000 & 0.000187000 \\ \mathrm{C} & -1.621843000 & 1.167653000 & -0.000114000 \\ \mathrm{C} & -2.382256000 & 0.028092000 & -0.000258000 \\ \mathrm{H} & 0.907265000 & -2.509926000 & -0.000046000 \\ \mathrm{H} & 3.073187000 & -1.275142000 & 0.000271000 \\ \mathrm{H} & 3.121437000 & 1.204124000 & 0.000420000 \\ \mathrm{H} & 1.013488000 & 2.520417000 & 0.000254000 \\ \mathrm{H} & -1.998365000 & 2.189523000 & -0.000098000 \\ \mathrm{H} & -3.465562000 & -0.087725000 & -0.000376000 \\ \mathrm{H} & -1.877250000 & -2.039846000 & -0.000269000 \\ \mathrm{~N} & -1.563647000 & -1.077463000 & -0.000239000\end{array}$

Structure 10a

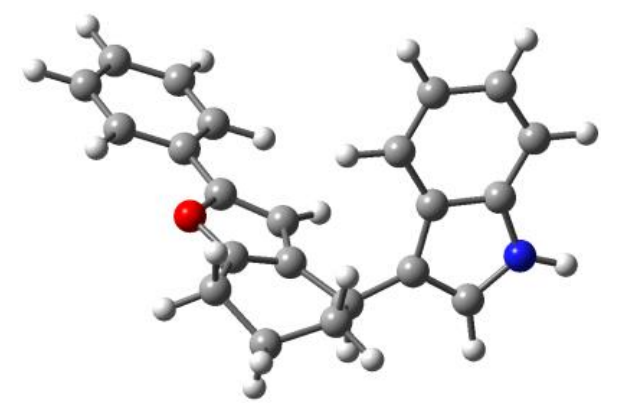

$\begin{array}{lrrr}\text { C } & 1.957706000 & 0.391158000 & -0.238417000 \\ \text { C } & 0.758385000 & 0.183862000 & -0.870382000 \\ C & 3.207197000 & -0.350712000 & -0.214666000 \\ \text { C } & 4.309333000 & 0.105558000 & 0.524547000\end{array}$




\begin{tabular}{|c|c|c|c|}
\hline C & 3.331945000 & -1.548365000 & -0.937176000 \\
\hline C & 5.498776000 & -0.616532000 & 0.537545000 \\
\hline $\mathrm{H}$ & 4.223160000 & 1.036255000 & 1.091321000 \\
\hline C & 4.521769000 & -2.265431000 & -0.920786000 \\
\hline $\mathrm{H}$ & 2.481771000 & -1.920535000 & -1.516932000 \\
\hline C & 5.612822000 & -1.803764000 & -0.183384000 \\
\hline $\mathrm{H}$ & 6.348081000 & -0.246173000 & 1.119434000 \\
\hline $\mathrm{H}$ & 4.599158000 & -3.196708000 & -1.489554000 \\
\hline $\mathrm{H}$ & 6.548854000 & -2.369289000 & -0.171028000 \\
\hline C & -0.090387000 & 1.268588000 & -0.496132000 \\
\hline C & -1.517604000 & 1.580624000 & -0.845052000 \\
\hline C & 0.655332000 & 2.056595000 & 0.332225000 \\
\hline 0 & 1.892736000 & 1.538644000 & 0.496407000 \\
\hline C & 0.235209000 & 3.304267000 & 1.008634000 \\
\hline $\mathrm{H}$ & 1.027560000 & 4.070686000 & 0.947192000 \\
\hline $\mathrm{H}$ & 0.073886000 & 3.120568000 & 2.089699000 \\
\hline C & -2.044165000 & 2.638734000 & 0.139829000 \\
\hline $\mathrm{H}$ & -2.251266000 & 2.147871000 & 1.110745000 \\
\hline $\mathrm{H}$ & -3.013926000 & 3.018041000 & -0.224206000 \\
\hline C & -1.059052000 & 3.782884000 & 0.350592000 \\
\hline $\mathrm{H}$ & -1.519010000 & 4.579674000 & 0.957057000 \\
\hline $\mathrm{H}$ & -0.819351000 & 4.235627000 & -0.630293000 \\
\hline $\mathrm{H}$ & -1.539824000 & 2.027689000 & -1.861261000 \\
\hline C & -2.387249000 & 0.361098000 & -0.882579000 \\
\hline C & -3.264247000 & 0.016360000 & -1.879557000 \\
\hline C & -2.514851000 & -0.653235000 & 0.133138000 \\
\hline $\mathrm{H}$ & 0.499229000 & -0.655051000 & -1.516597000 \\
\hline$N$ & -3.931063000 & -1.141663000 & -1.549363000 \\
\hline C & -3.492107000 & -1.576657000 & -0.322928000 \\
\hline $\mathrm{H}$ & -3.464458000 & 0.526643000 & -2.822526000 \\
\hline C & -1.893150000 & -0.881082000 & 1.373412000 \\
\hline C & -2.258604000 & -1.993277000 & 2.116651000 \\
\hline $\mathrm{H}$ & -1.124097000 & -0.193390000 & 1.740851000 \\
\hline $\mathrm{H}$ & -1.779385000 & -2.180186000 & 3.082142000 \\
\hline C & -3.861417000 & -2.699300000 & 0.424390000 \\
\hline C & -3.234672000 & -2.893118000 & 1.646935000 \\
\hline $\mathrm{H}$ & -4.616693000 & -3.399839000 & 0.055498000 \\
\hline $\mathrm{H}$ & -3.501339000 & -3.762529000 & 2.255257000 \\
\hline $\mathrm{H}$ & -4.622621000 & -1.606407000 & -2.123560000 \\
\hline
\end{tabular}

Structure 14 


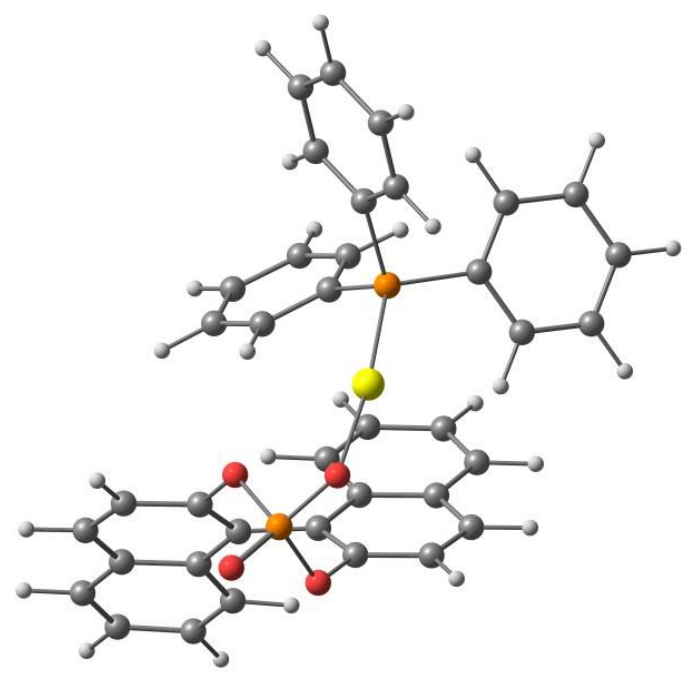

$\begin{array}{lccc}\text { C } & 3.168245000 & -0.245108000 & -0.108811000 \\ \text { C } & 4.368111000 & 0.400213000 & -0.558828000 \\ \text { C } & 2.801297000 & -1.436833000 & -0.720528000 \\ \text { C } & 4.920342000 & 1.532050000 & 0.098188000 \\ \text { C } & 5.072184000 & -0.127502000 & -1.685506000 \\ \text { C } & 3.508124000 & -1.970768000 & -1.819563000 \\ \text { C } & 6.070610000 & 2.131824000 & -0.358322000 \\ \text { H } & 4.425780000 & 1.921253000 & 0.992081000 \\ \text { C } & 6.247585000 & 0.522660000 & -2.141428000 \\ \text { C } & 4.606327000 & -1.313676000 & -2.308009000 \\ \text { C } & 6.737622000 & 1.633805000 & -1.499483000 \\ \text { H } & 6.476973000 & 2.997012000 & 0.173426000 \\ \text { H } & 6.765589000 & 0.106641000 & -3.011987000 \\ \text { H } & 5.158117000 & -1.711375000 & -3.165698000 \\ \text { H } & 7.648777000 & 2.121903000 & -1.856599000 \\ \text { C } & 2.343952000 & 0.284385000 & 1.004682000 \\ \text { C } & 1.787625000 & 1.608226000 & 1.000896000 \\ \text { C } & 2.040033000 & -0.547015000 & 2.074159000 \\ \text { C } & 1.916295000 & 2.491152000 & -0.104037000 \\ \text { H } & 1.031204000 & 2.058228000 & 2.127833000 \\ \text { C } & 1.292588000 & -0.103571000 & 3.187560000 \\ \text { C } & 1.352135000 & 3.745023000 & -0.087232000 \\ \text { H } & 2.462368000 & 2.158276000 & -0.990382000 \\ \text { C } & 0.188319000 & 5.195887000 & 1.044082000 \\ \text { C } & 1.691615000 & -2.115369000 & -0.301589000\end{array}$




\begin{tabular}{|c|c|c|c|}
\hline 0 & 2.474545000 & -1.841551000 & 2.079451000 \\
\hline$P$ & 1.691957000 & -2.948666000 & 1.139446000 \\
\hline 0 & 2.470478000 & -4.197772000 & 1.103074000 \\
\hline $\mathrm{O}$ & 0.206333000 & -2.939412000 & 1.529899000 \\
\hline C & -2.653800000 & 1.668016000 & 1.885341000 \\
\hline C & -3.210691000 & 1.292421000 & 0.658432000 \\
\hline C & -3.293628000 & 2.615533000 & 2.679813000 \\
\hline C & -4.417164000 & 1.864420000 & 0.237026000 \\
\hline C & -4.492399000 & 3.186587000 & 2.256310000 \\
\hline C & -5.053463000 & 2.810514000 & 1.036744000 \\
\hline $\mathrm{H}$ & -4.865111000 & 1.566804000 & -0.717594000 \\
\hline $\mathrm{H}$ & -5.996977000 & 3.253691000 & 0.706549000 \\
\hline $\mathrm{H}$ & -4.997838000 & 3.925216000 & 2.884741000 \\
\hline $\mathrm{H}$ & -2.856736000 & 2.901929000 & 3.641103000 \\
\hline $\mathrm{H}$ & 3.148373000 & -2.908474000 & -2.250402000 \\
\hline$P$ & -2.315858000 & 0.088261000 & -0.374681000 \\
\hline $\mathrm{Au}$ & -1.080432000 & -1.478677000 & 0.727647000 \\
\hline C & -1.237823000 & 1.048136000 & -1.490704000 \\
\hline C & -1.541715000 & 2.364174000 & -1.855029000 \\
\hline C & -0.123320000 & 0.410157000 & -2.050498000 \\
\hline C & -0.750074000 & 3.024317000 & -2.792596000 \\
\hline $\mathrm{H}$ & -2.400956000 & 2.876966000 & -1.409050000 \\
\hline C & 0.664726000 & 1.076199000 & -2.985422000 \\
\hline $\mathrm{H}$ & 0.136424000 & -0.612832000 & -1.749316000 \\
\hline C & 0.346759000 & 2.379985000 & -3.362528000 \\
\hline $\mathrm{H}$ & -0.992193000 & 4.052037000 & -3.078117000 \\
\hline $\mathrm{H}$ & 1.537878000 & 0.572497000 & -3.412142000 \\
\hline $\mathrm{H}$ & 0.965644000 & 2.902645000 & -4.097575000 \\
\hline C & -3.592469000 & -0.650244000 & -1.450789000 \\
\hline C & -3.699635000 & -0.342351000 & -2.809858000 \\
\hline C & -4.493613000 & -1.550922000 & -0.867967000 \\
\hline C & -4.707104000 & -0.928004000 & -3.576410000 \\
\hline $\mathrm{H}$ & -2.994483000 & 0.352604000 & -3.277581000 \\
\hline C & -5.500042000 & -2.126152000 & -1.635617000 \\
\hline $\mathrm{H}$ & -4.403847000 & -1.803863000 & 0.195226000 \\
\hline C & -5.606665000 & -1.815158000 & -2.991742000 \\
\hline $\mathrm{H}$ & -4.785467000 & -0.687887000 & -4.640319000 \\
\hline $\mathrm{H}$ & -6.201014000 & -2.827888000 & -1.175634000 \\
\hline $\mathrm{H}$ & -6.393804000 & -2.273494000 & -3.596885000 \\
\hline $\mathrm{H}$ & 1.115961000 & -0.815007000 & 3.998479000 \\
\hline $\mathrm{H}$ & -1.715101000 & 1.210354000 & 2.220059000 \\
\hline
\end{tabular}




\section{HPLC traces}<smiles>c1ccc(-c2cc3c(o2)CCCC3c2c[nH]c3ccccc23)cc1</smiles>

HPLC Analysis for 10aa: HPLC Chiralpak IA column [heptane:IPA $=95: 05,1 \mathrm{~mL} / \mathrm{min}, \lambda=277 \mathrm{~nm}$, retention times: $T_{R}$ (minor): $19.7 \mathrm{~min}, T_{R}$ (major): $25.6 \mathrm{~min}$ ].

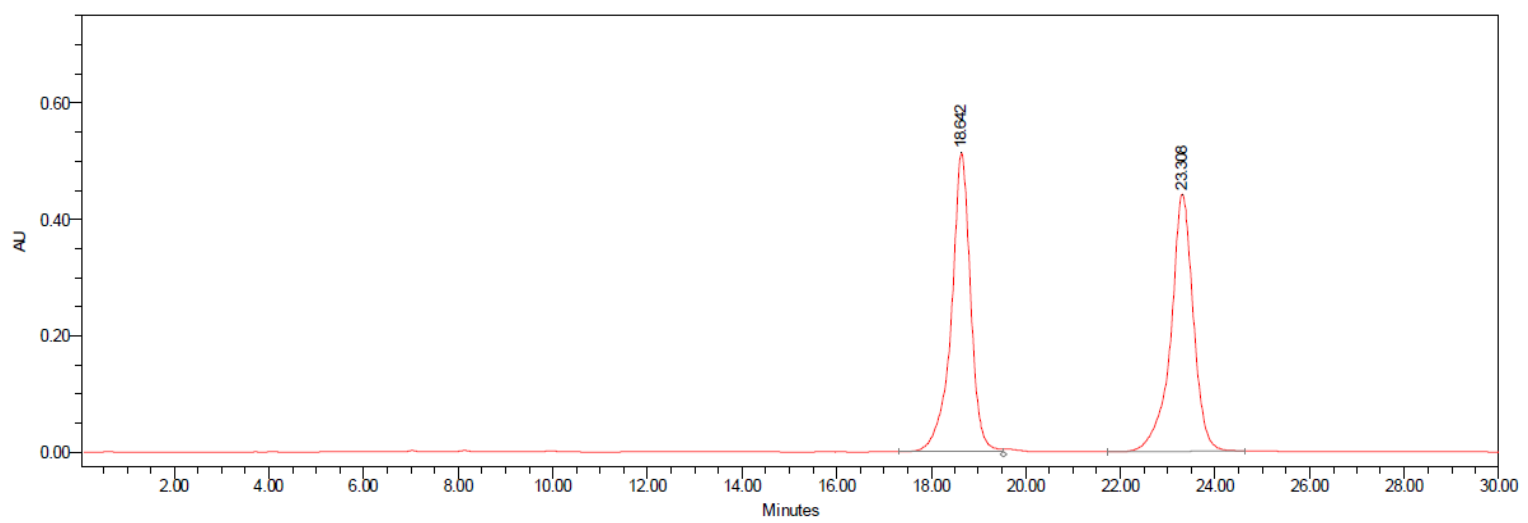

\begin{tabular}{|c|c|c|c|r|}
\hline & Channel Description & RT & Area & $\%$ Area \\
\hline 1 & PDA 200.0 to $400.0 \mathrm{~nm}$ at $2.4 \mathrm{~nm}$ & 18.642 & 14502314 & 49.78 \\
\hline 2 & PDA 200.0 to $400.0 \mathrm{~nm}$ at $2.4 \mathrm{~nm}$ & 23.308 & 14628255 & 50.22 \\
\hline
\end{tabular}

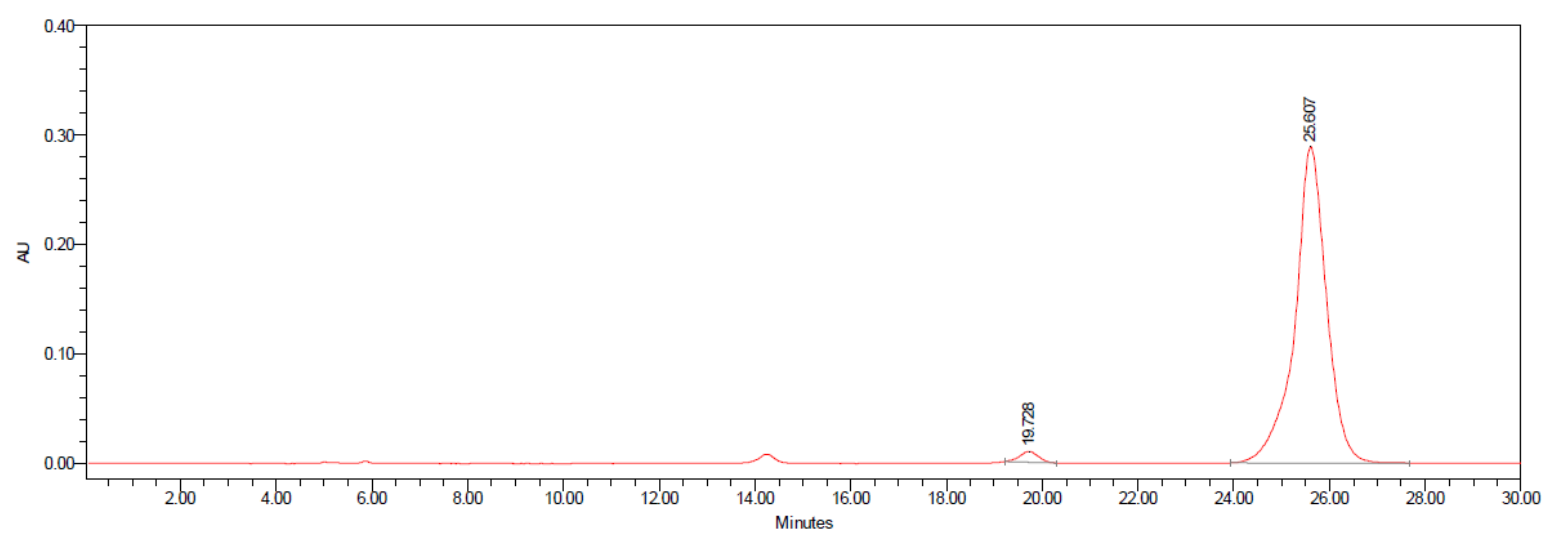

\begin{tabular}{|l|c|c|r|r|}
\hline & Channel Des cription & RT & Area & \% Area \\
\hline 1 & PDA 200.0 to $400.0 \mathrm{~nm}$ at $2.4 \mathrm{~nm}$ & 19.728 & 261081 & 1.93 \\
\hline 2 & PDA 200.0 to $400.0 \mathrm{~nm}$ at $2.4 \mathrm{~nm}$ & 25.607 & 13248494 & 98.07 \\
\hline
\end{tabular}


<smiles>COc1ccc2[nH]cc(C3CCCc4oc(-c5ccccc5)cc43)c2c1</smiles>

HPLC Analysis for 10ab: HPLC Chiralpak IA column [heptane:IPA $=95: 05,1 \mathrm{~mL} / \mathrm{min}, \lambda=277 \mathrm{~nm}$, retention times: $T_{R}$ (minor): $29.2 \mathrm{~min}, \mathrm{~T}_{\mathrm{R}}$ (major): $41.4 \mathrm{~min}$ ].

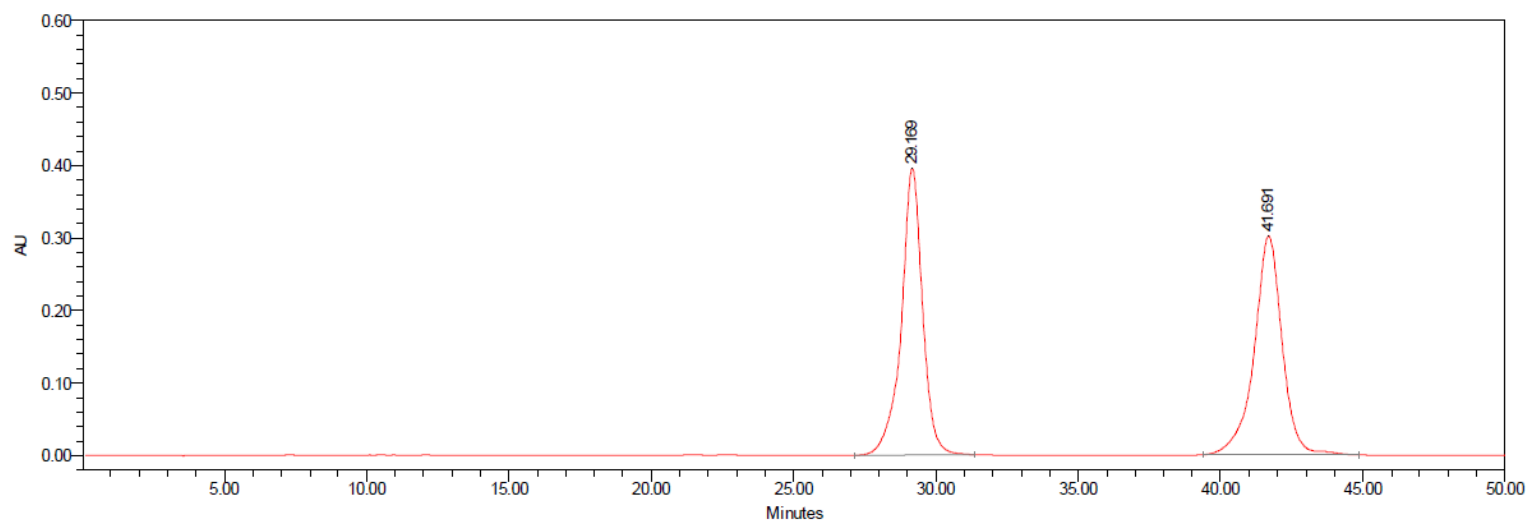

\begin{tabular}{|c|c|c|c|r|}
\hline & Channel Des cription & RT & Area & $\%$ Area \\
\hline 1 & PDA 200.0 to $400.0 \mathrm{~nm}$ at $2.4 \mathrm{~nm}$ & 29.169 & 20533331 & 49.83 \\
\hline 2 & PDA 200.0 to $400.0 \mathrm{~nm}$ at $2.4 \mathrm{~nm}$ & 41.691 & 20670930 & 50.17 \\
\hline
\end{tabular}

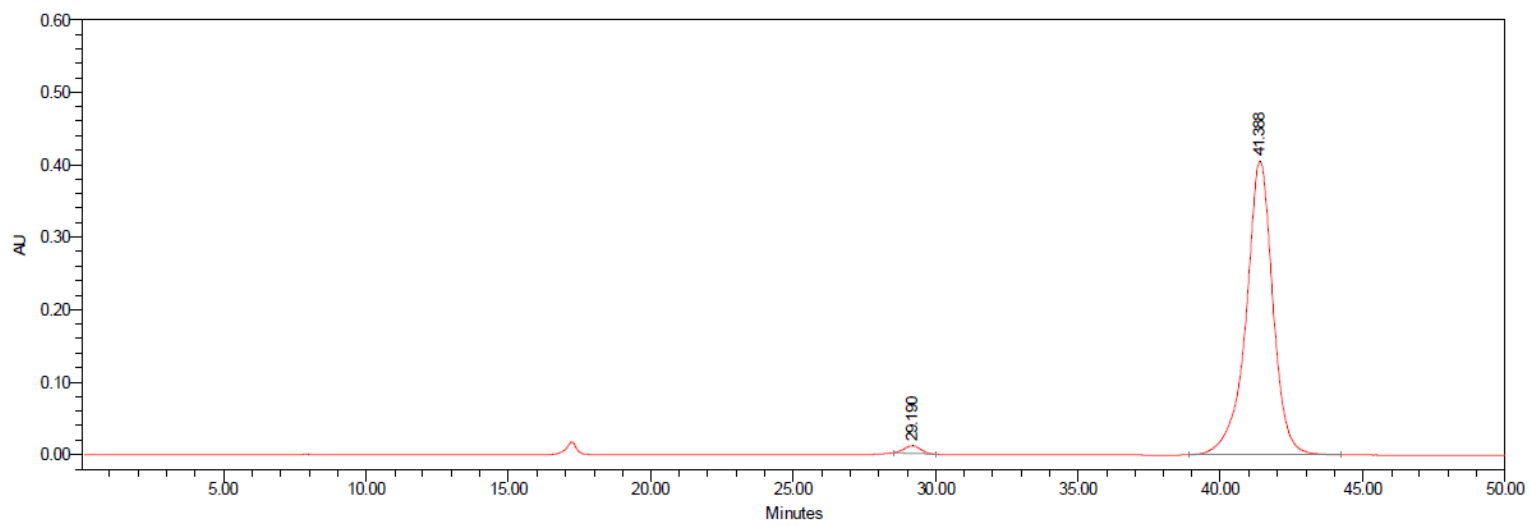

\begin{tabular}{|l|c|c|r|r|}
\hline & Channel Description & RT & Area & $\%$ Area \\
\hline 1 & PDA 200.0 to $400.0 \mathrm{~nm}$ at $2.4 \mathrm{~nm}$ & 29.190 & 407299 & 1.51 \\
\hline 2 & PDA 200.0 to $400.0 \mathrm{~nm}$ at $2.4 \mathrm{~nm}$ & 41.388 & 26563035 & 98.49 \\
\hline
\end{tabular}


<smiles></smiles>

HPLC Analysis for 10ac: HPLC Chiralpak IA column [heptane:IPA $=95: 05,1 \mathrm{~mL} / \mathrm{min}, \lambda=277 \mathrm{~nm}$, retention times: $T_{R}$ (minor): $19.4 \mathrm{~min}, \mathrm{~T}_{\mathrm{R}}$ (major): $26.7 \mathrm{~min}$ ].

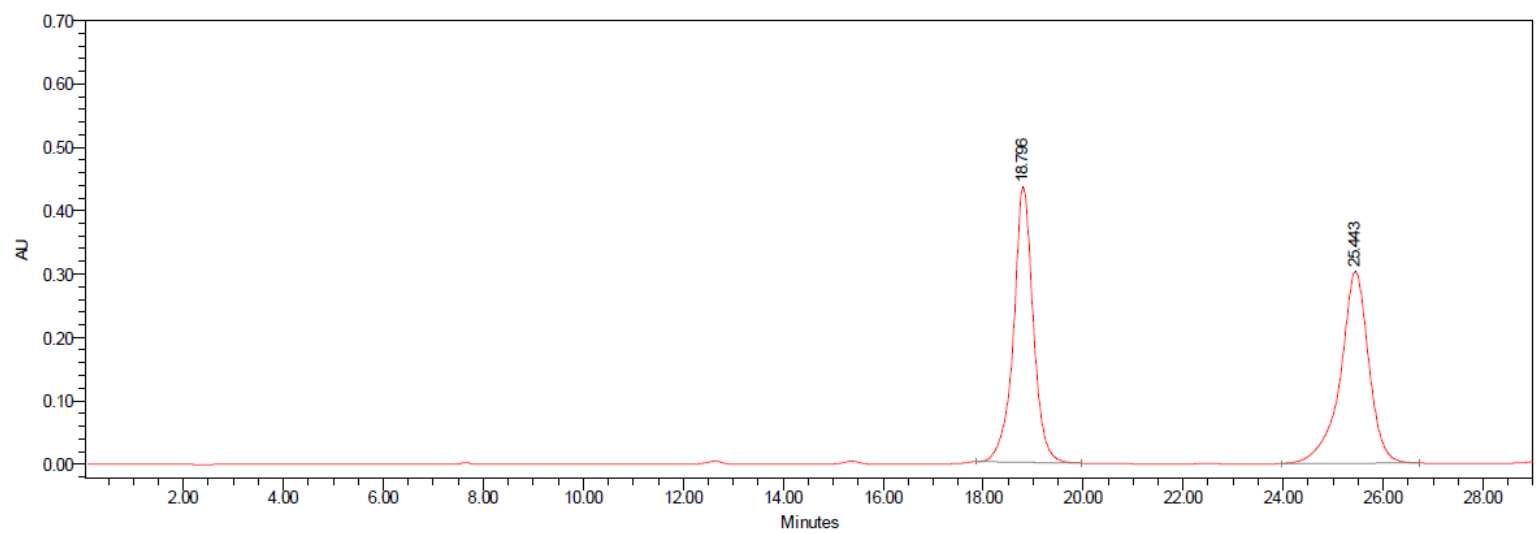

\begin{tabular}{|c|c|c|c|r|}
\hline & Channel Des cription & RT & Area & $\%$ Area \\
\hline 1 & PDA 200.0 to $400.0 \mathrm{~nm}$ at $2.4 \mathrm{~nm}$ & 18.796 & 11907602 & 49.92 \\
\hline 2 & PDA 200.0 to $400.0 \mathrm{~nm}$ at $2.4 \mathrm{~nm}$ & 25.443 & 11944609 & 50.08 \\
\hline
\end{tabular}

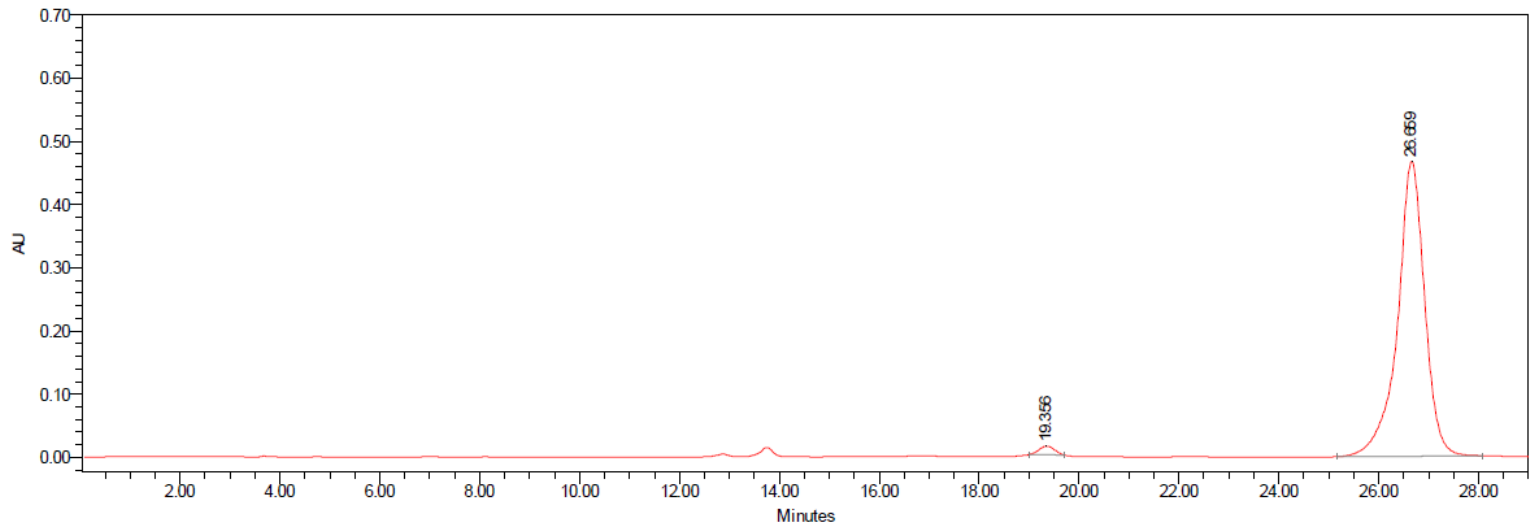

\begin{tabular}{|l|c|c|c|r|}
\hline & Channel Des cription & RT & \multicolumn{1}{c|}{ Area } & $\%$ Area \\
\hline 1 & PDA 200.0 to $400.0 \mathrm{~nm}$ at $2.4 \mathrm{~nm}$ & 19.356 & 280233 & 1.56 \\
\hline 2 & PDA 200.0 to $400.0 \mathrm{~nm}$ at $2.4 \mathrm{~nm}$ & 26.659 & 17640550 & 98.44 \\
\hline
\end{tabular}




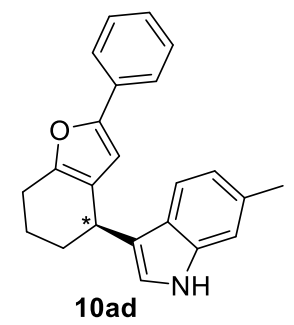

HPLC Analysis for 10ad: HPLC Chiralpak IA column [heptane:IPA = 95:05, $1 \mathrm{~mL} / \mathrm{min}, \lambda=277 \mathrm{~nm}$, retention times: $\mathrm{T}_{\mathrm{R}}$ (minor): $19.5 \mathrm{~min}, \mathrm{~T}_{\mathrm{R}}$ (major): $22.4 \mathrm{~min}$ ].

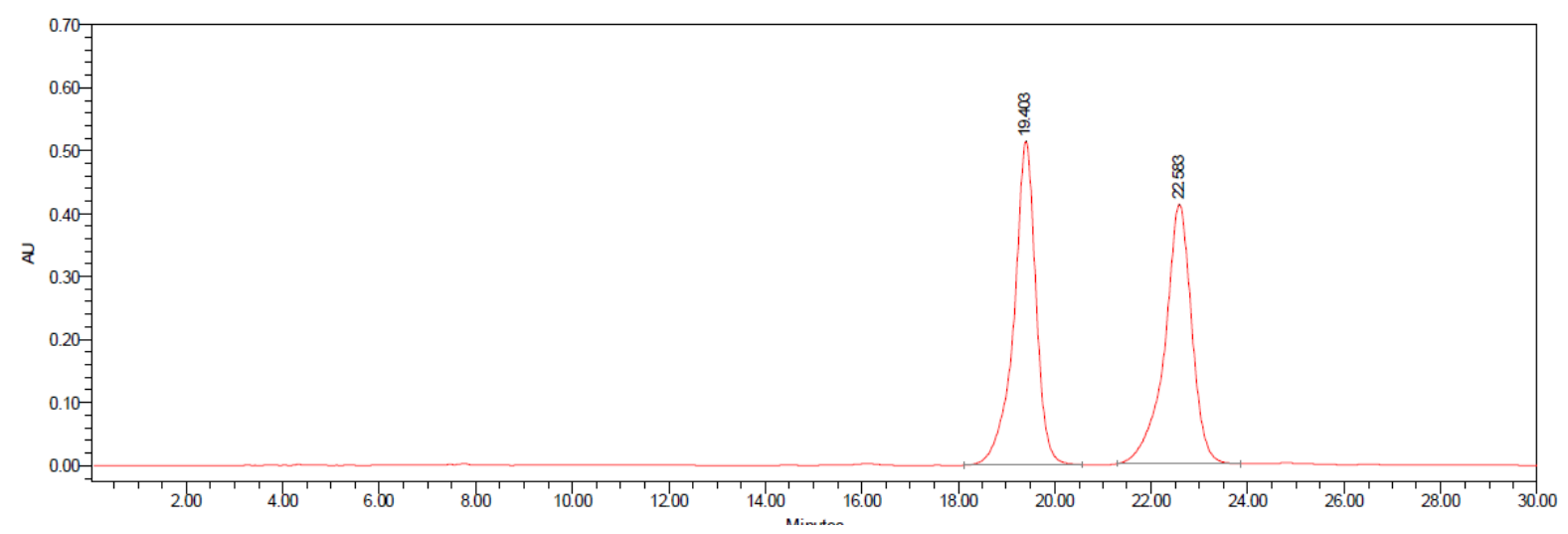

\begin{tabular}{|l|c|c|c|r|}
\hline & Channel Des cription & RT & Area & $\%$ Area \\
\hline 1 & PDA 200.0 to $400.0 \mathrm{~nm}$ at $2.4 \mathrm{~nm}$ & 19.403 & 16316844 & 50.42 \\
\hline 2 & PDA 200.0 to $400.0 \mathrm{~nm}$ at $2.4 \mathrm{~nm}$ & 22.583 & 16047118 & 49.58 \\
\hline
\end{tabular}

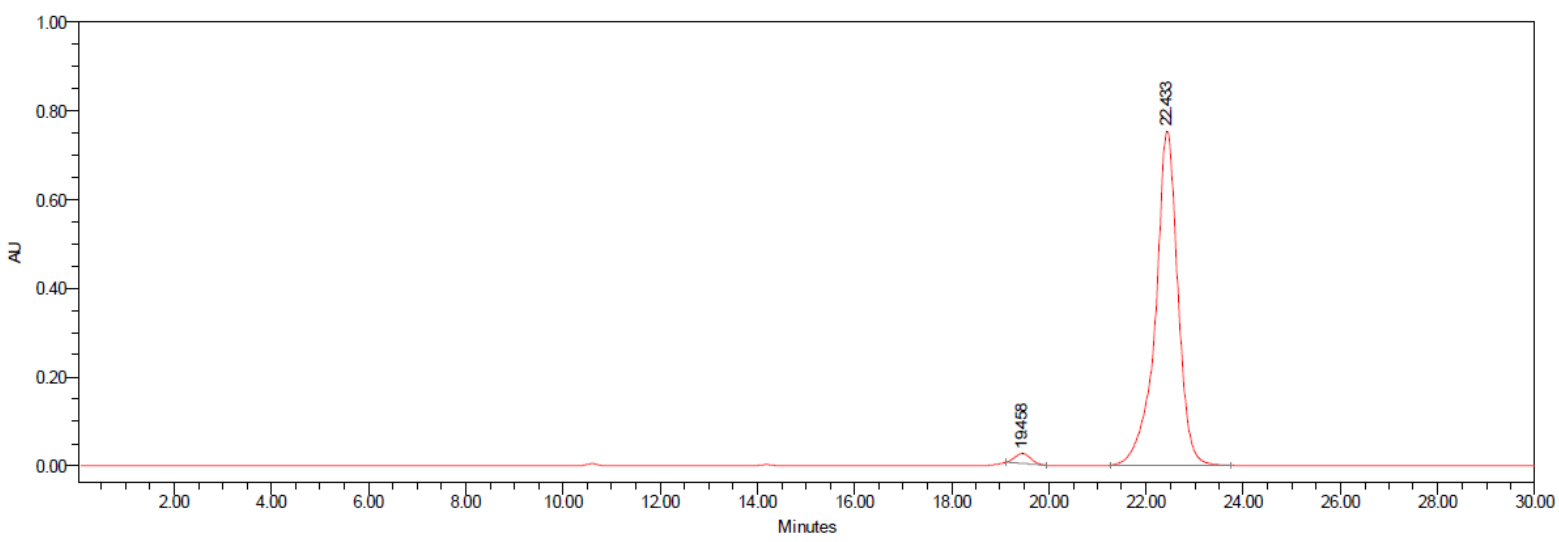

\begin{tabular}{|l|c|c|r|r|}
\hline & Channel Des cription & RT & Area & $\%$ Area \\
\hline 1 & PDA 200.0 to $400.0 \mathrm{~nm}$ at $2.4 \mathrm{~nm}$ & 19.458 & 497201 & 2.02 \\
\hline 2 & PDA 200.0 to $400.0 \mathrm{~nm}$ at $2.4 \mathrm{~nm}$ & 22.433 & 24129004 & 97.98 \\
\hline
\end{tabular}




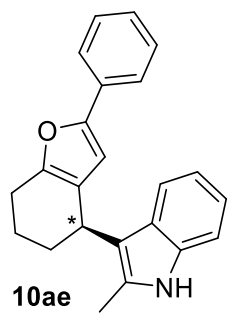

HPLC Analysis for 10ae: HPLC Chiralpak AD-H column [heptane:IPA = 90:10, $1 \mathrm{~mL} / \mathrm{min}, \lambda=277 \mathrm{~nm}$, retention times: $T_{R}$ (minor): $4.3 \mathrm{~min}, T_{R}$ (major): $5.3 \mathrm{~min}$ ].

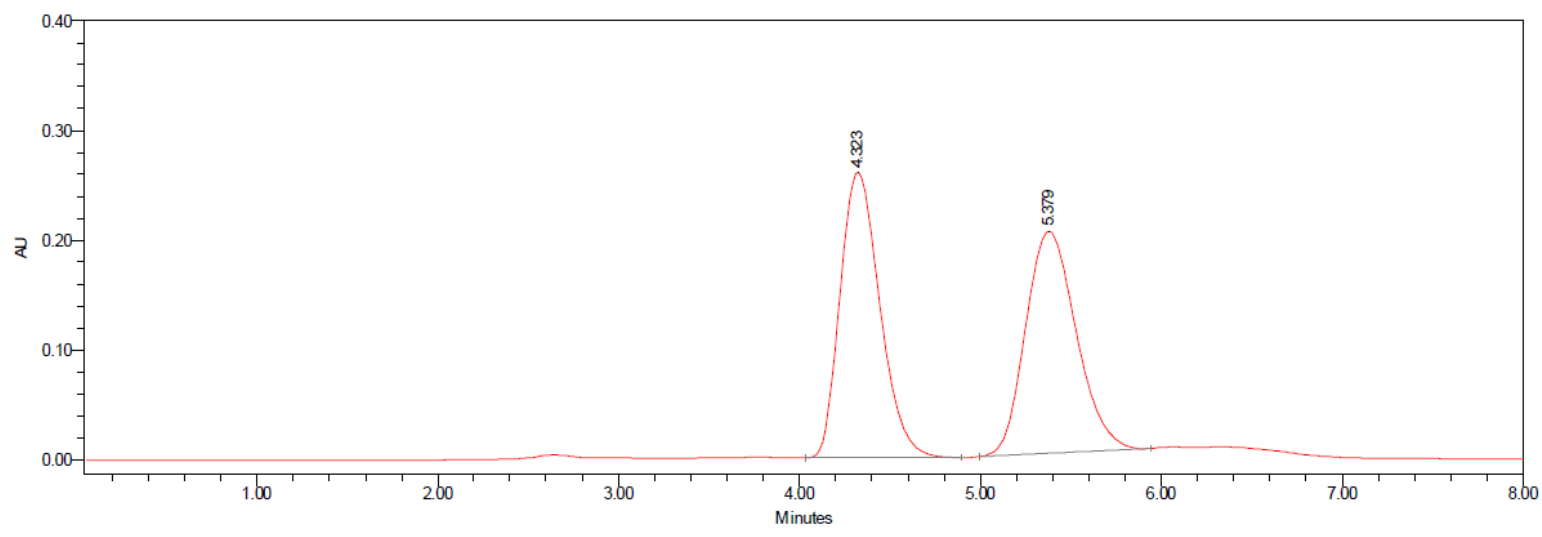

\begin{tabular}{|c|c|c|c|r|}
\hline & Channel Des cription & RT & Area & $\%$ Area \\
\hline 1 & PDA 200.0 to $400.0 \mathrm{~nm}$ at $2.4 \mathrm{~nm}$ & 4.323 & 3967340 & 50.30 \\
\hline 2 & PDA 200.0 to $400.0 \mathrm{~nm}$ at $2.4 \mathrm{~nm}$ & 5.379 & 3920104 & 49.70 \\
\hline
\end{tabular}

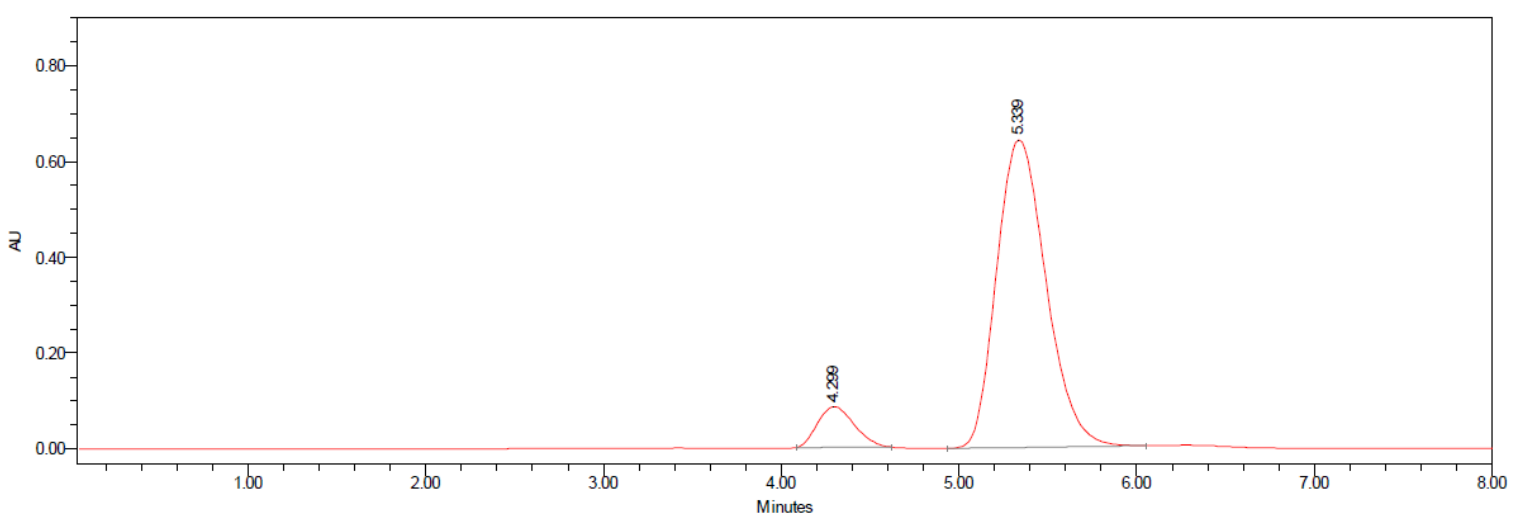

\begin{tabular}{|l|c|c|c|r|}
\hline & Channel Des cription & RT & Area & \% Area \\
\hline 1 & PDA 200.0 to $400.0 \mathrm{~nm}$ at $2.4 \mathrm{~nm}$ & 4.299 & 1217284 & 8.92 \\
\hline 2 & PDA 200.0 to $400.0 \mathrm{~nm}$ at $2.4 \mathrm{~nm}$ & 5.339 & 12435400 & 91.08 \\
\hline
\end{tabular}


<smiles>Cn1cc(C2CCCc3oc(-c4ccccc4)cc32)c2ccccc21</smiles>

HPLC Analysis for 10af: HPLC Chiralpak IC column [heptane:IPA $=95: 05,1 \mathrm{~mL} / \mathrm{min}, \lambda=277 \mathrm{~nm}$, retention times: $T_{R}$ (minor): $4.7 \mathrm{~min}, \mathrm{~T}_{R}$ (major): $5.1 \mathrm{~min}$ ].

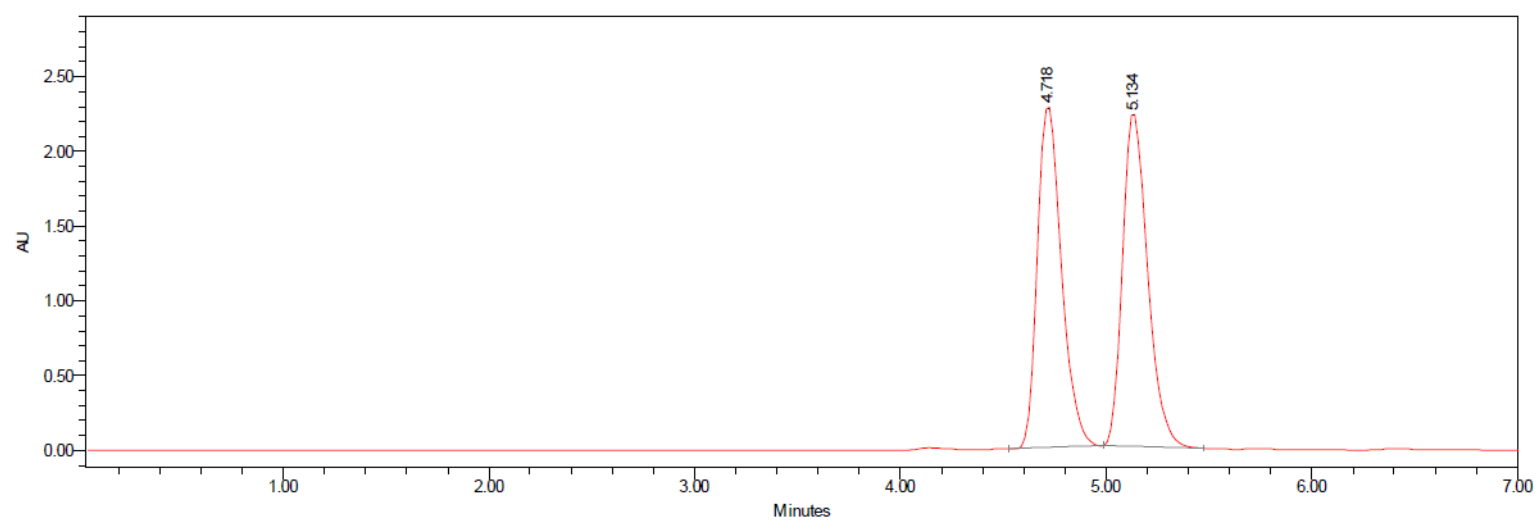

\begin{tabular}{|c|c|c|c|r|}
\hline & Channel Des cription & RT & Area & \% Area \\
\hline 1 & PDA 200.0 to $400.0 \mathrm{~nm}$ at $2.4 \mathrm{~nm}$ & 4.718 & 18557092 & 49.53 \\
\hline 2 & PDA 200.0 to $400.0 \mathrm{~nm}$ at $2.4 \mathrm{~nm}$ & 5.134 & 18910286 & 50.47 \\
\hline
\end{tabular}

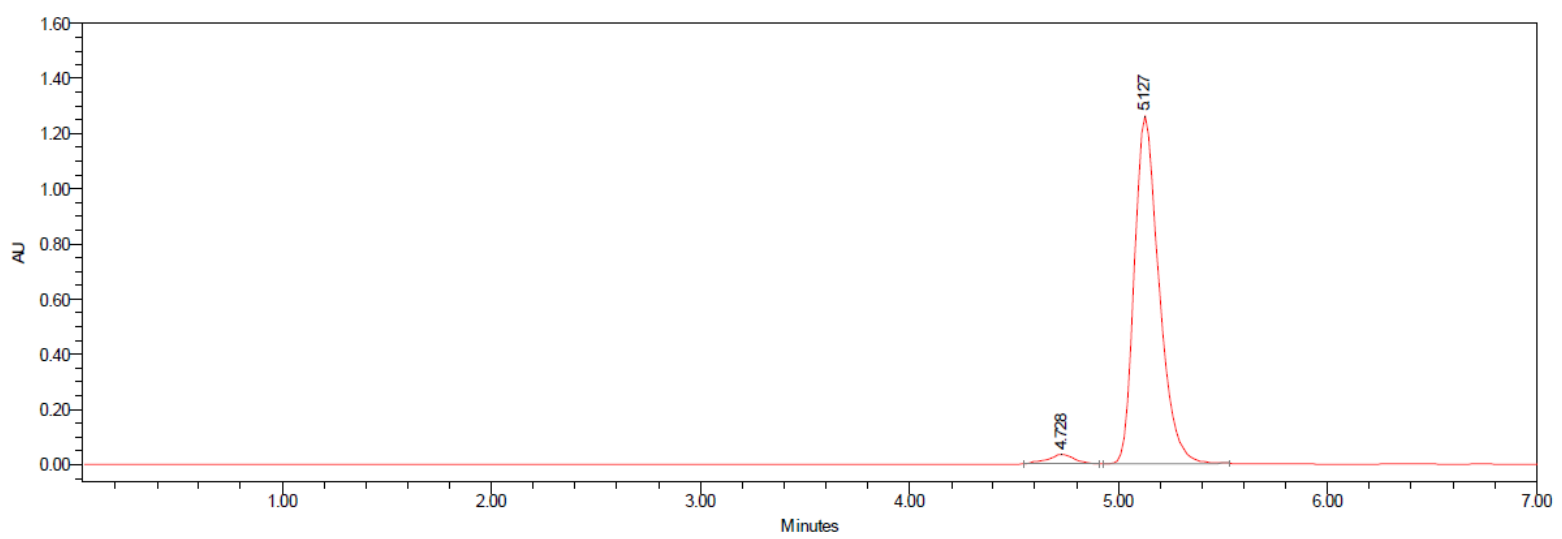

\begin{tabular}{|l|c|c|c|r|}
\hline & Channel Des cription & RT & Area & \% Area \\
\hline 1 & PDA 200.0 to $400.0 \mathrm{~nm}$ at $2.4 \mathrm{~nm}$ & 4.728 & 305767 & 2.89 \\
\hline 2 & PDA 200.0 to $400.0 \mathrm{~nm}$ at $2.4 \mathrm{~nm}$ & 5.127 & 10285879 & 97.11 \\
\hline
\end{tabular}


<smiles>Brn1cc(C2CCCc3oc(-c4ccccc4)cc32)c2ccccc21</smiles>

HPLC Analysis for 10ag: HPLC Chiralpak IA column [heptane:IPA $=95: 05,1 \mathrm{~mL} / \mathrm{min}, \lambda=277 \mathrm{~nm}$, retention times: $T_{R}$ (minor): $6.5 \mathrm{~min}, \mathrm{~T}_{R}$ (major): $11.3 \mathrm{~min}$ ].

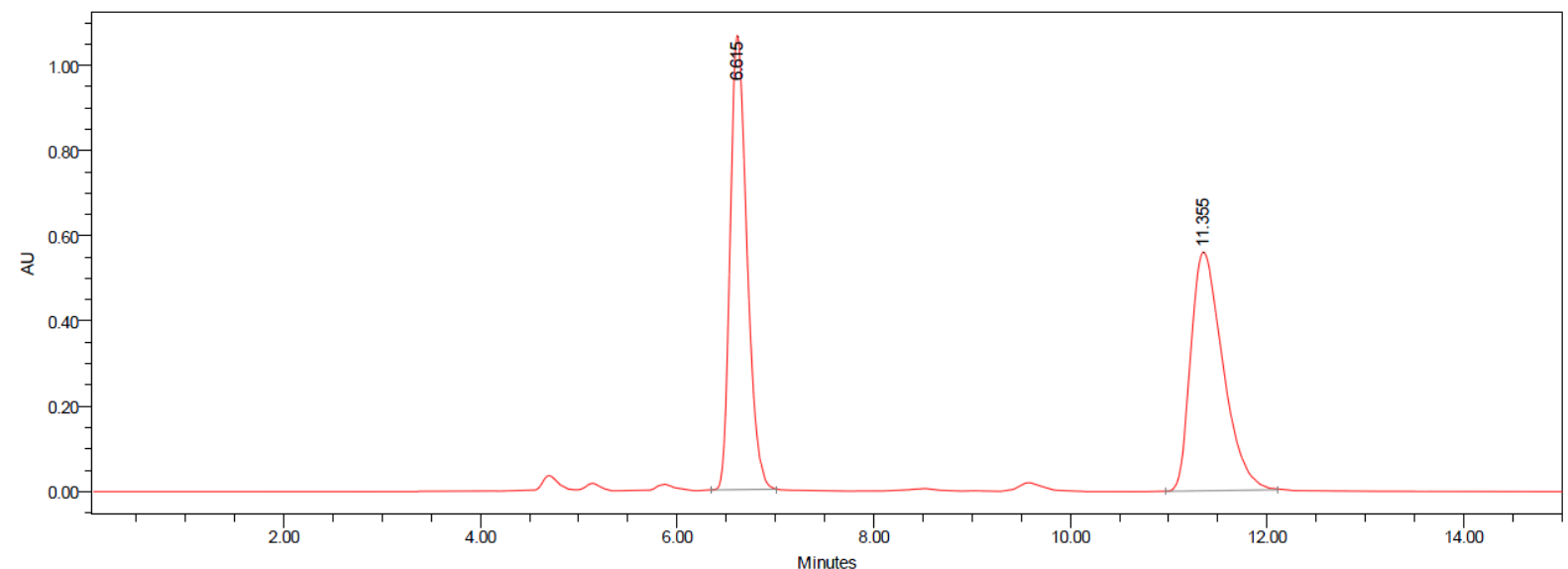

\begin{tabular}{|c|c|c|c|r|}
\hline & Channel Des cription & RT & Area & $\%$ Area \\
\hline 1 & PDA 200.0 to $400.0 \mathrm{~nm}$ at $2.4 \mathrm{~nm}$ & 6.615 & 12628104 & 50.00 \\
\hline 2 & PDA 200.0 to $400.0 \mathrm{~nm}$ at $2.4 \mathrm{~nm}$ & 11.355 & 12630238 & 50.00 \\
\hline
\end{tabular}

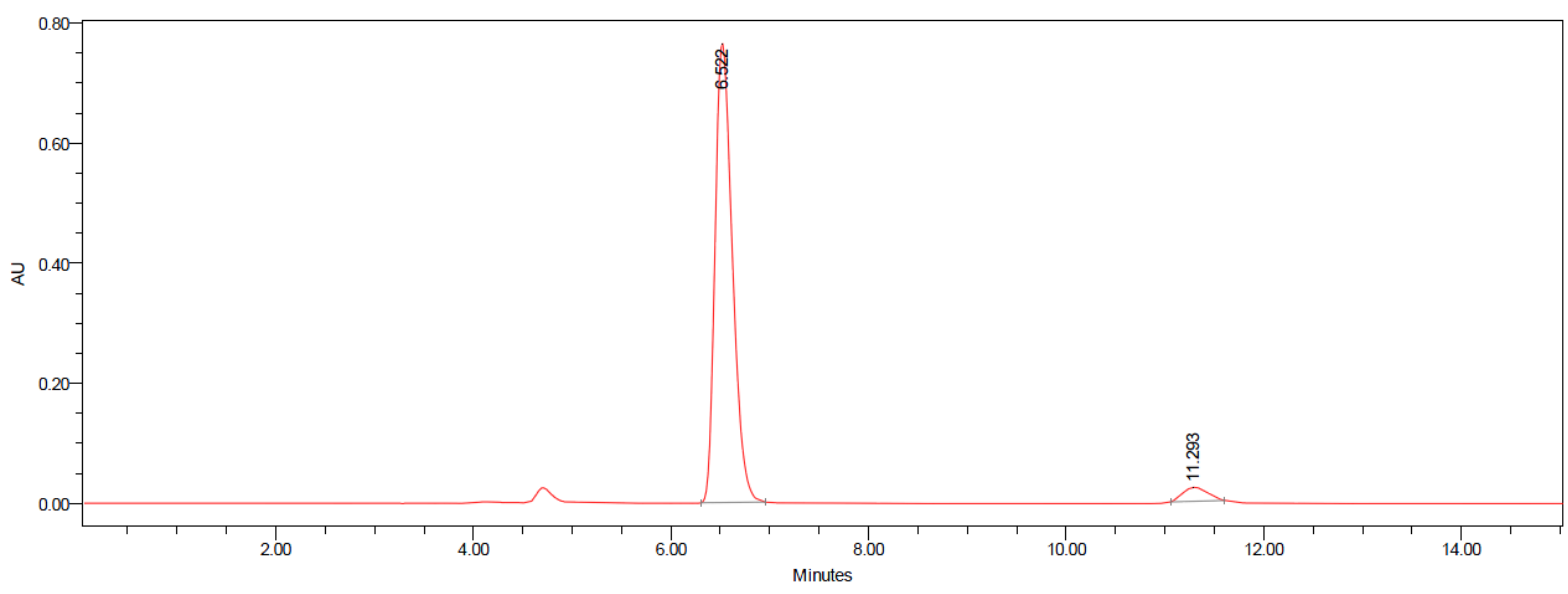

\begin{tabular}{|c|c|c|c|r|}
\hline & Channel Des cription & RT & Area & $\%$ Area \\
\hline 1 & PDA 200.0 to $400.0 \mathrm{~nm}$ at $2.4 \mathrm{~nm}$ & 6.522 & 9323364 & 95.85 \\
\hline 2 & PDA 200.0 to $400.0 \mathrm{~nm}$ at $2.4 \mathrm{~nm}$ & 11.293 & 403790 & 4.15 \\
\hline
\end{tabular}




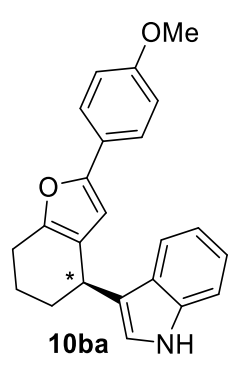

HPLC Analysis for 10ba: HPLC Chiralpak IC column [heptane:IPA $=95: 05,1 \mathrm{~mL} / \mathrm{min}, \lambda=277 \mathrm{~nm}$, retention times: $T_{R}$ (minor): $9.5 \mathrm{~min}, T_{R}$ (major): $10.5 \mathrm{~min}$ ].

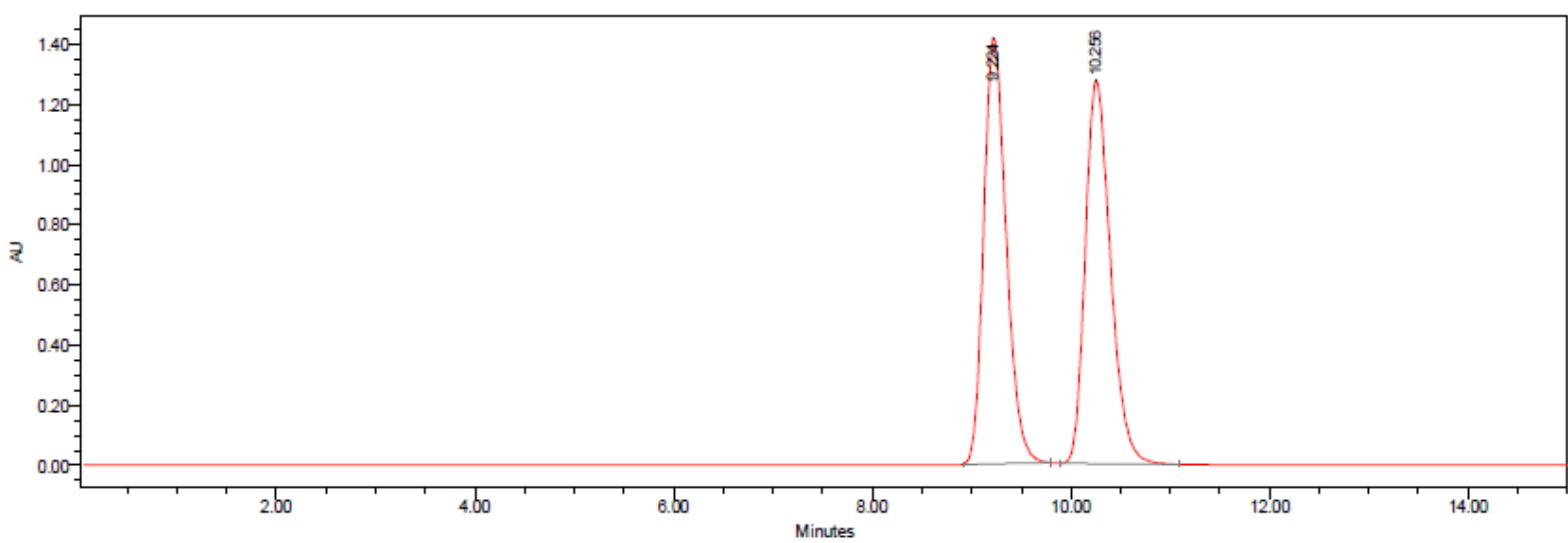

\begin{tabular}{|c|c|c|c|r|}
\hline & Channel Des cription & \multicolumn{1}{c|}{ RT } & Area & $\%$ Area \\
\hline 1 & PDA 200.0 to $400.0 \mathrm{~nm}$ at $2.4 \mathrm{~nm}$ & 9.224 & 22196993 & 49.76 \\
\hline 2 & PDA 200.0 to $400.0 \mathrm{~nm}$ at $2.4 \mathrm{~nm}$ & 10.256 & 22410909 & 50.24 \\
\hline
\end{tabular}

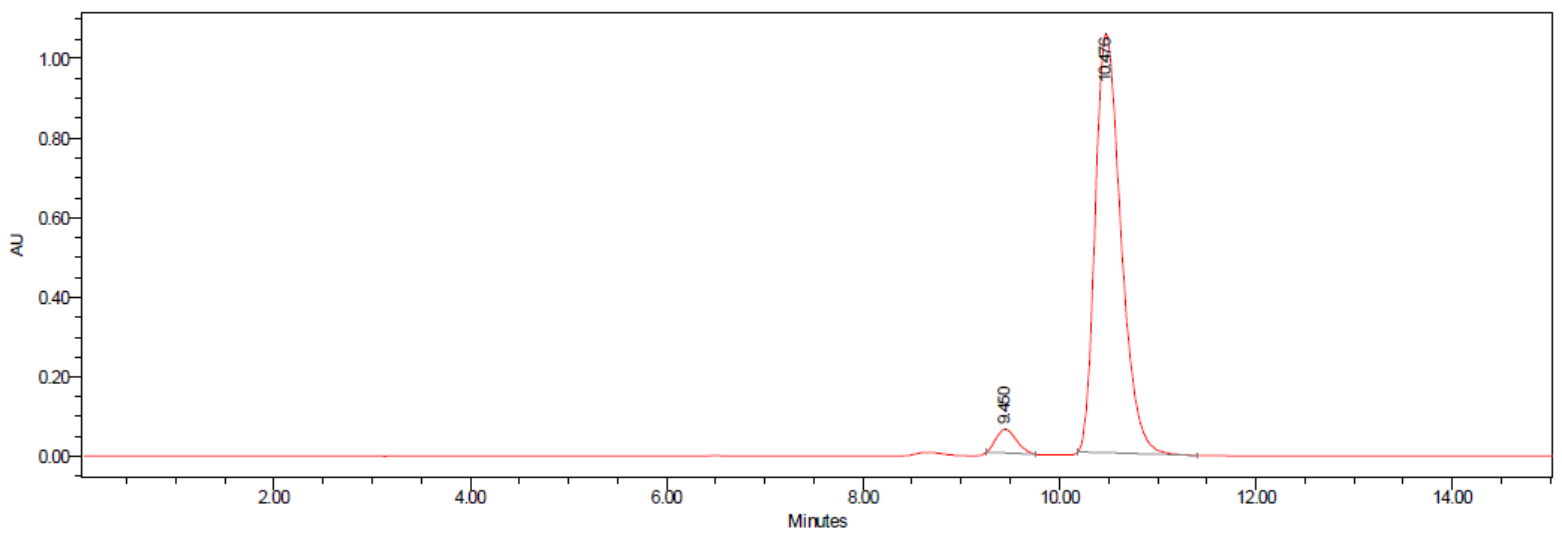

\begin{tabular}{|c|c|r|r|r|}
\hline & Channel Des cription & \multicolumn{1}{c|}{ RT } & \multicolumn{1}{c|}{ Area } & \% Area \\
\hline 1 & PDA 200.0 to $400.0 \mathrm{~nm}$ at $2.4 \mathrm{~nm}$ & 9.450 & 859446 & 4.31 \\
\hline 2 & PDA 200.0 to $400.0 \mathrm{~nm}$ at $2.4 \mathrm{~nm}$ & 10.476 & 19073662 & 95.69 \\
\hline
\end{tabular}




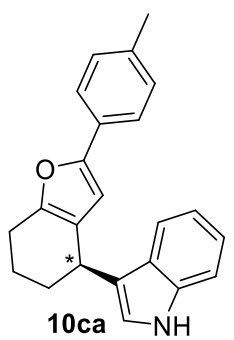

HPLC Analysis for 10ca: HPLC Chiralpak IC column [heptane:IPA $=95: 05,1 \mathrm{~mL} / \mathrm{min}, \lambda=277 \mathrm{~nm}$, retention times: $T_{R}$ (minor): $6.3 \mathrm{~min}, T_{R}$ (major): $7.2 \mathrm{~min}$ ].

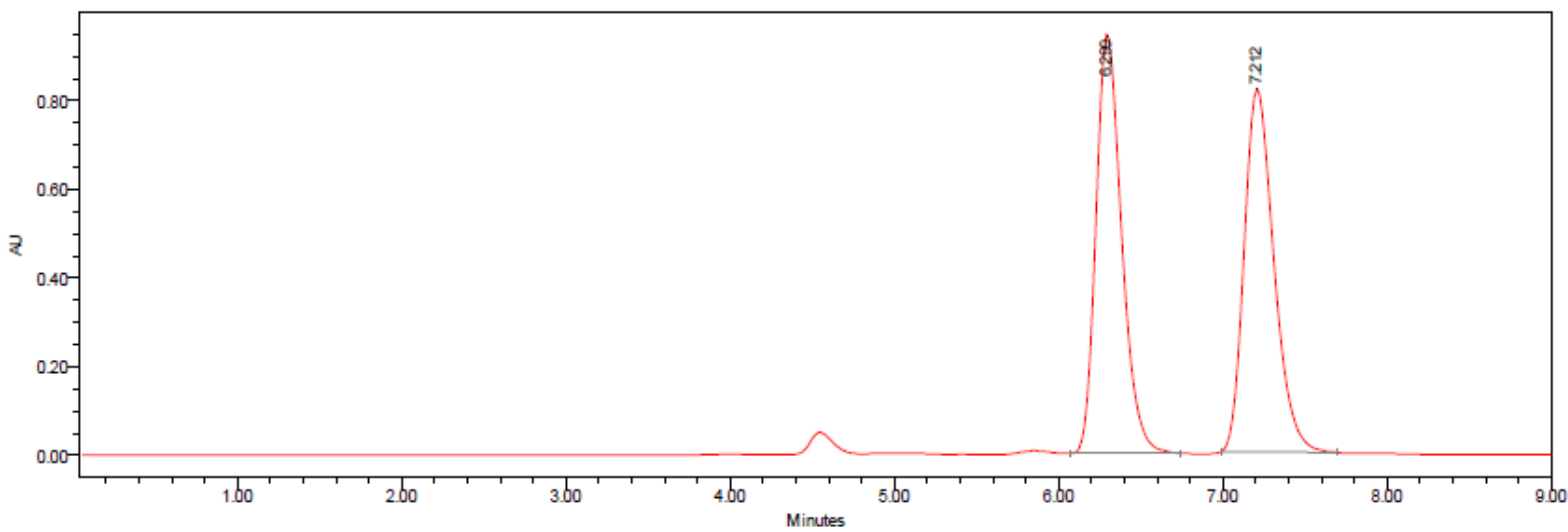

\begin{tabular}{|c|c|c|c|r|}
\hline & Channel Description & RT & Area & \% Area \\
\hline 1 & PDA 200.0 to $400.0 \mathrm{~nm}$ at $2.4 \mathrm{~nm}$ & 6.299 & 10263174 & 49.94 \\
\hline 2 & PDA 200.0 to $400.0 \mathrm{~nm}$ at $2.4 \mathrm{~nm}$ & 7.212 & 10289115 & 50.06 \\
\hline
\end{tabular}

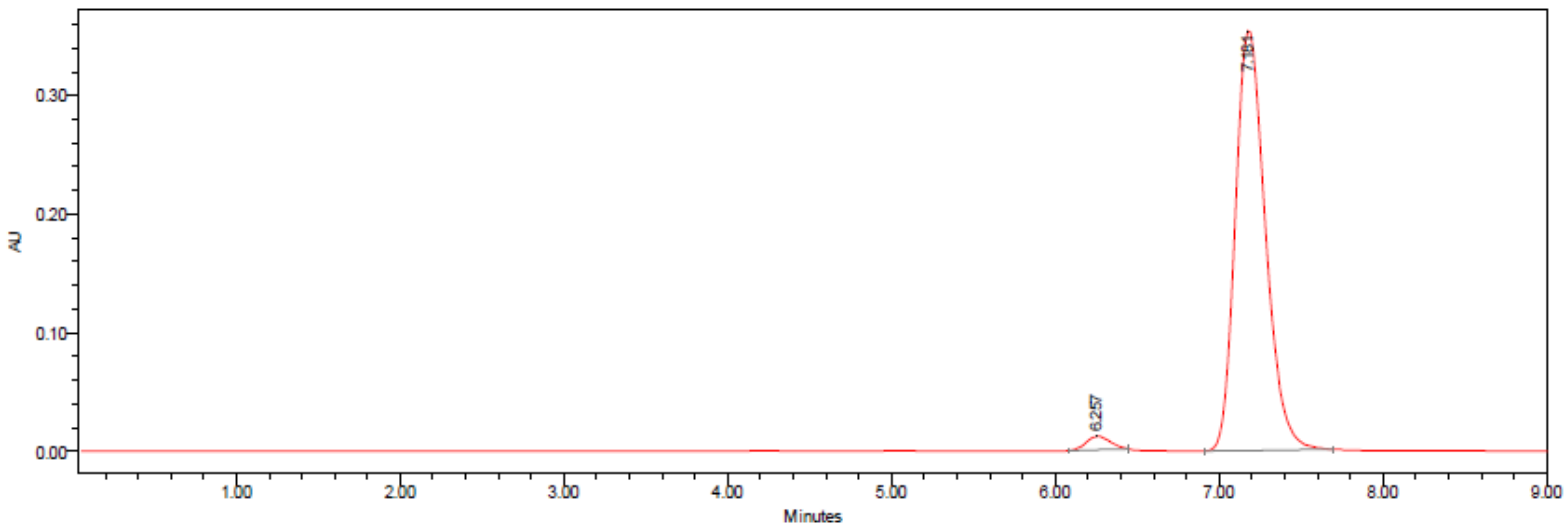

\begin{tabular}{|c|c|c|r|r|}
\hline & Channel Des cription & RT & Area & \% Area \\
\hline 1 & PDA 200.0 to $400.0 \mathrm{~nm}$ at $2.4 \mathrm{~nm}$ & 6.257 & 115884 & 2.54 \\
\hline 2 & PDA 200.0 to $400.0 \mathrm{~nm}$ at $2.4 \mathrm{~nm}$ & 7.181 & 4446473 & 97.46 \\
\hline
\end{tabular}




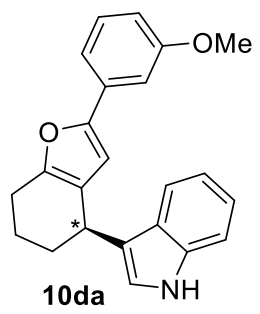

HPLC Analysis for 10da: HPLC Chiralpak IA column [heptane:IPA = 90:10, $1 \mathrm{~mL} / \mathrm{min}, \lambda=277 \mathrm{~nm}$, retention times: $\mathrm{T}_{\mathrm{R}}$ (minor): $12.1 \mathrm{~min}, \mathrm{~T}_{\mathrm{R}}$ (major): $23.7 \mathrm{~min}$ ].

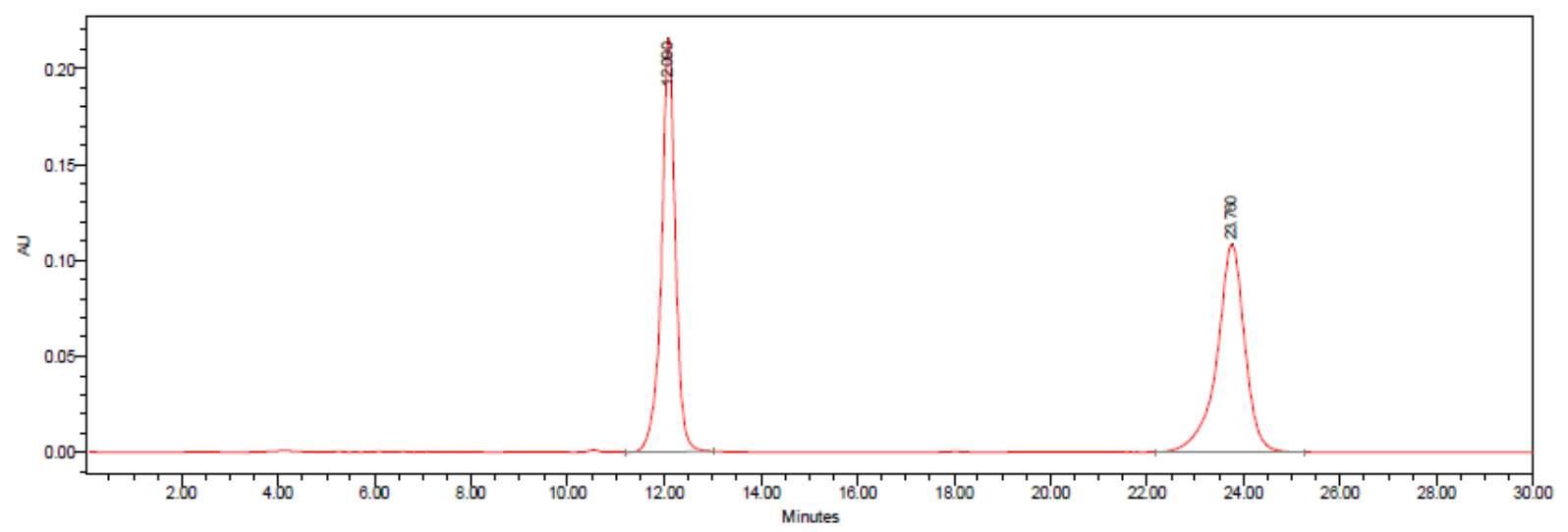

\begin{tabular}{|c|c|c|c|r|}
\hline & Channel Des cription & RT & Area & $\%$ Area \\
\hline 1 & PDA 200.0 to $400.0 \mathrm{~nm}$ at $2.4 \mathrm{~nm}$ & 12.090 & 4312360 & 49.84 \\
\hline 2 & PDA 200.0 to $400.0 \mathrm{~nm}$ at $2.4 \mathrm{~nm}$ & 23.760 & 4339656 & 50.16 \\
\hline
\end{tabular}

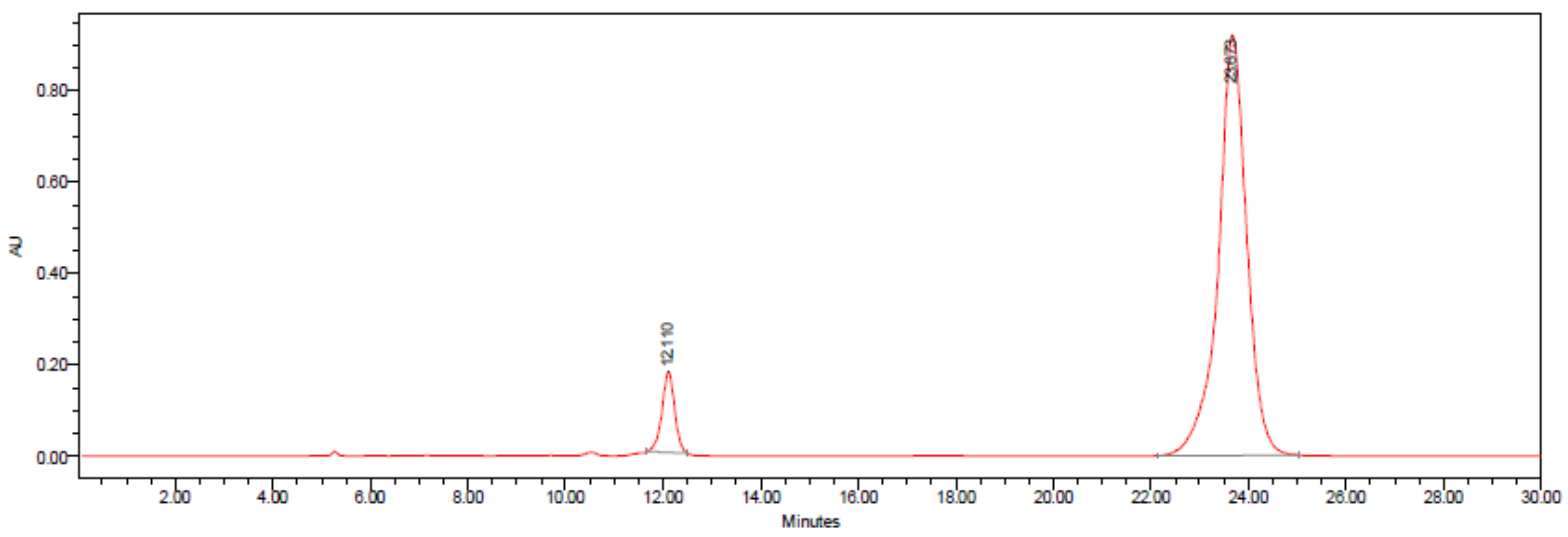

\begin{tabular}{|c|c|c|c|r|}
\hline & Channel Des cription & RT & Area & \% Area \\
\hline 1 & PDA 200.0 to $400.0 \mathrm{~nm}$ at $2.4 \mathrm{~nm}$ & 12.110 & 3174489 & 7.83 \\
\hline 2 & PDA 200.0 to $400.0 \mathrm{~nm}$ at $2.4 \mathrm{~nm}$ & 23.673 & 37371650 & 92.17 \\
\hline
\end{tabular}




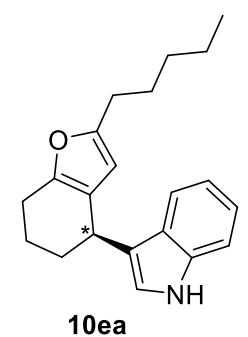

HPLC Analysis for 10ea: HPLC Chiralpak IA column [heptane:IPA $=95: 05,1 \mathrm{~mL} / \mathrm{min}, \lambda=277 \mathrm{~nm}$, retention times: $T_{R}$ (minor): $8.7 \mathrm{~min}, T_{R}$ (major): $10.5 \mathrm{~min}$ ].

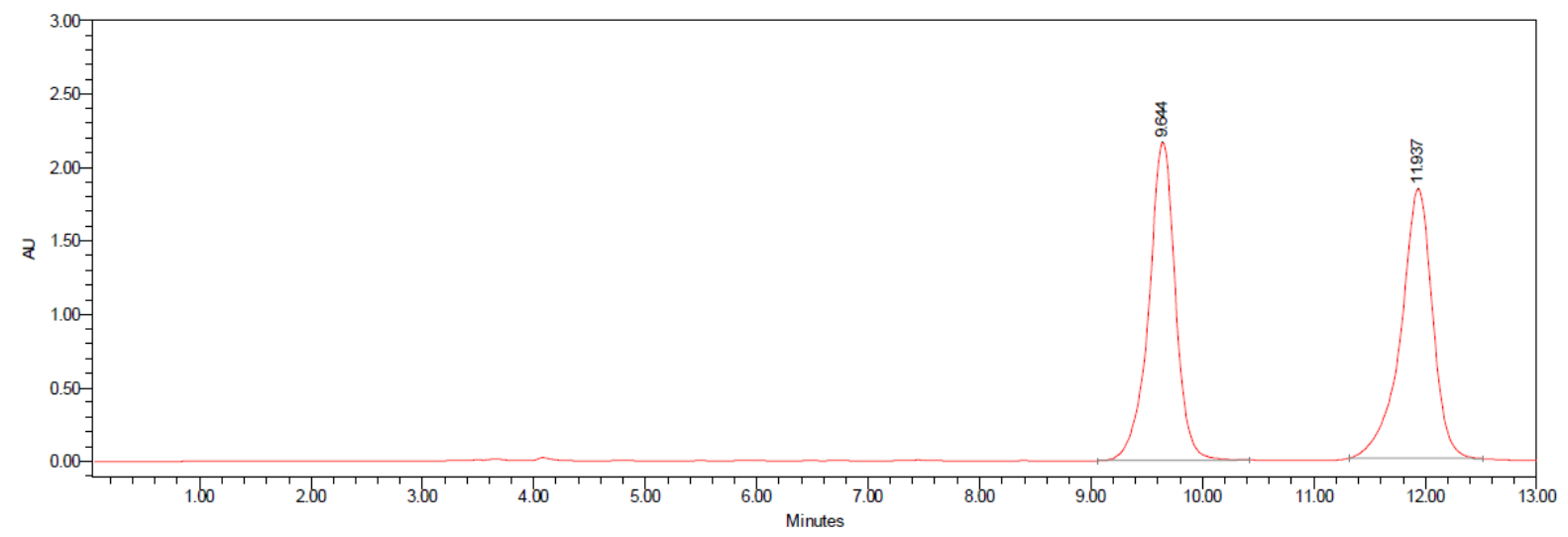

\begin{tabular}{|c|c|c|c|r|}
\hline & Channel Des cription & RT & Area & $\%$ Area \\
\hline 1 & PDA 200.0 to $400.0 \mathrm{~nm}$ at $2.4 \mathrm{~nm}$ & 9.644 & 35915017 & 49.72 \\
\hline 2 & PDA 200.0 to $400.0 \mathrm{~nm}$ at $2.4 \mathrm{~nm}$ & 11.937 & 36319260 & 50.28 \\
\hline
\end{tabular}

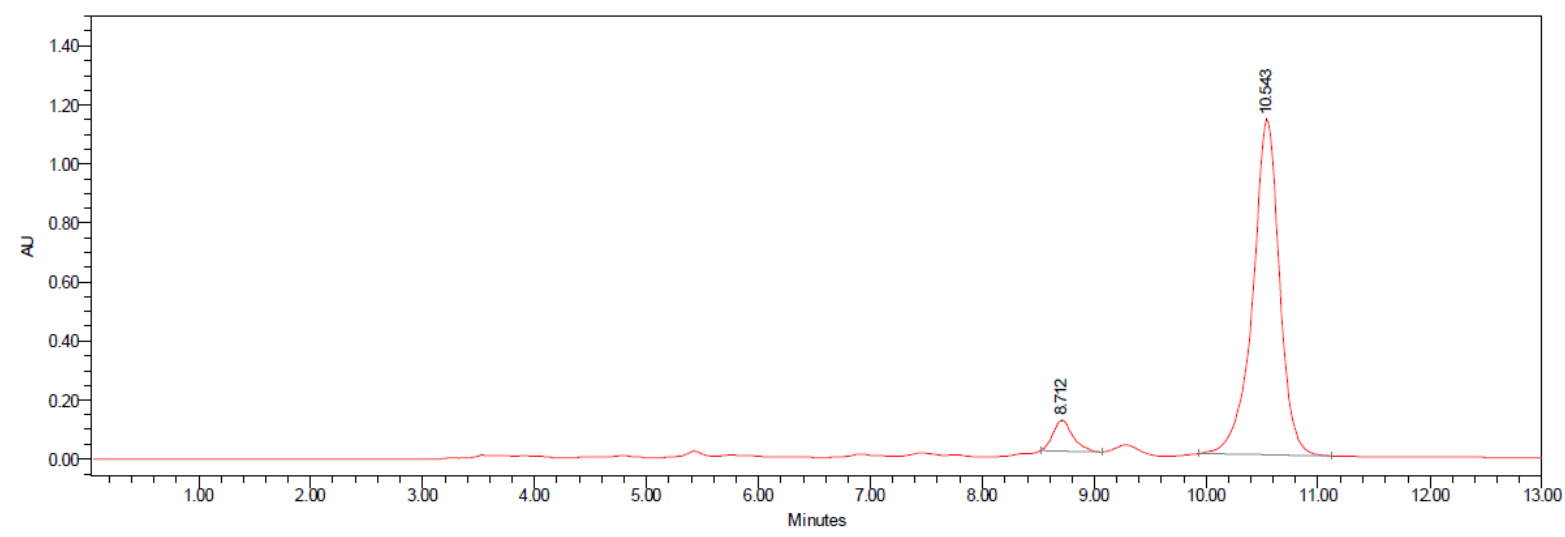

\begin{tabular}{|l|c|c|r|r|}
\hline & Channel Des cription & RT & Area & $\%$ Area \\
\hline 1 & PDA 200.0 to $400.0 \mathrm{~nm}$ at $2.4 \mathrm{~nm}$ & 8.712 & 1275487 & 6.34 \\
\hline 2 & PDA 200.0 to $400.0 \mathrm{~nm}$ at $2.4 \mathrm{~nm}$ & 10.543 & 18829441 & 93.66 \\
\hline
\end{tabular}




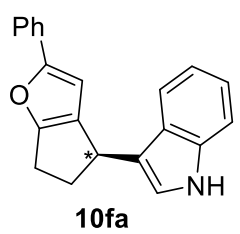

HPLC Analysis for 10fa: HPLC Chiralpak IA column [heptane:IPA = 95:05, $1 \mathrm{~mL} / \mathrm{min}, \lambda=277 \mathrm{~nm}$, retention times: $T_{R}$ (major): $26.1 \mathrm{~min}, \mathrm{~T}_{\mathrm{R}}$ (minor): $29.9 \mathrm{~min}$ ].

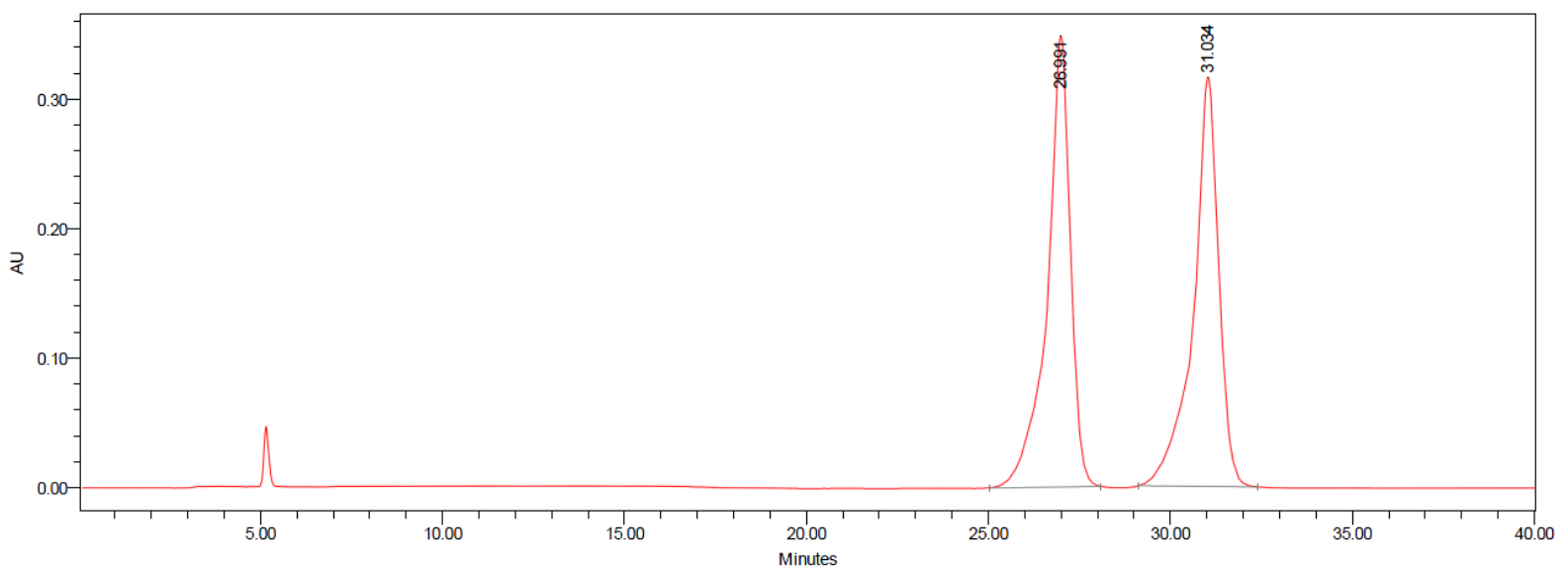

\begin{tabular}{|c|c|c|c|r|}
\hline & Channel Description & RT & Area & $\%$ Area \\
\hline 1 & PDA 200.0 to $400.0 \mathrm{~nm}$ at $2.4 \mathrm{~nm}$ & 26.991 & 15348618 & 50.11 \\
\hline 2 & PDA 200.0 to $400.0 \mathrm{~nm}$ at $2.4 \mathrm{~nm}$ & 31.034 & 15283917 & 49.89 \\
\hline
\end{tabular}

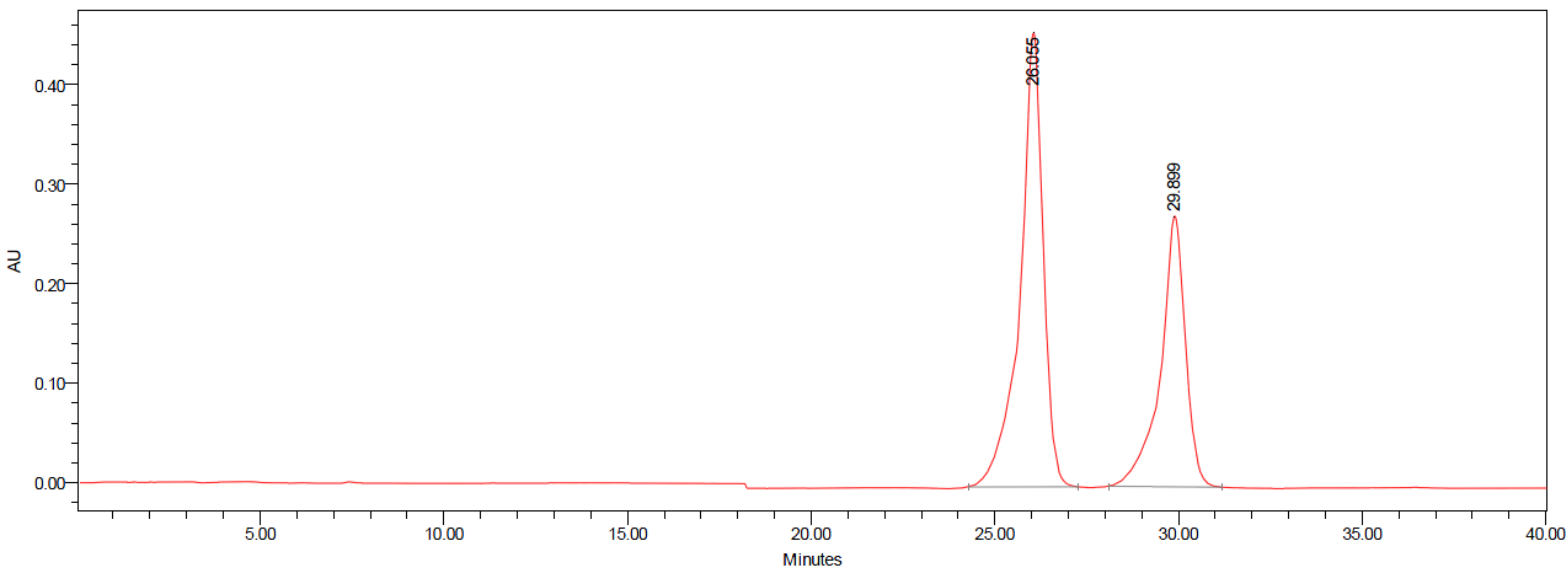

\begin{tabular}{|c|c|c|c|r|}
\hline & Channel Description & RT & Area & $\%$ Area \\
\hline 1 & PDA 200.0 to $400.0 \mathrm{~nm}$ at $2.4 \mathrm{~nm}$ & 26.055 & 19740973 & 60.60 \\
\hline 2 & PDA 200.0 to $400.0 \mathrm{~nm}$ at $2.4 \mathrm{~nm}$ & 29.899 & 12836253 & 39.40 \\
\hline
\end{tabular}




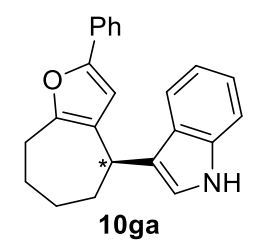

HPLC Analysis for 10ga: HPLC Chiralpak IA column [heptane:IPA $=95: 05,1 \mathrm{~mL} / \mathrm{min}, \lambda=277 \mathrm{~nm}$, retention times: $\mathrm{T}_{\mathrm{R}}$ (minor): $14.8 \mathrm{~min}, \mathrm{~T}_{\mathrm{R}}$ (major): $16.8 \mathrm{~min}$ ].

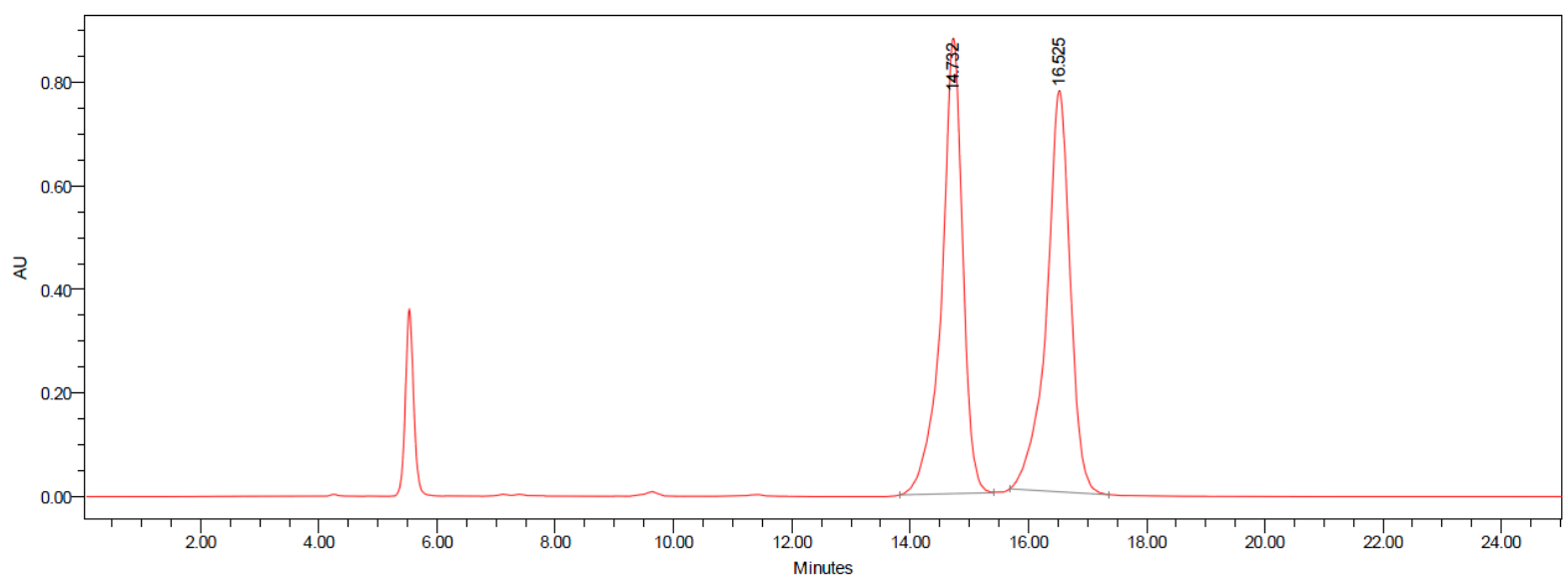

\begin{tabular}{|c|c|c|c|r|}
\hline & Channel Des cription & RT & Area & $\%$ Area \\
\hline 1 & PDA 200.0 to $400.0 \mathrm{~nm}$ at $2.4 \mathrm{~nm}$ & 14.732 & 21581390 & 50.25 \\
\hline 2 & PDA 200.0 to $400.0 \mathrm{~nm}$ at $2.4 \mathrm{~nm}$ & 16.525 & 21364017 & 49.75 \\
\hline
\end{tabular}

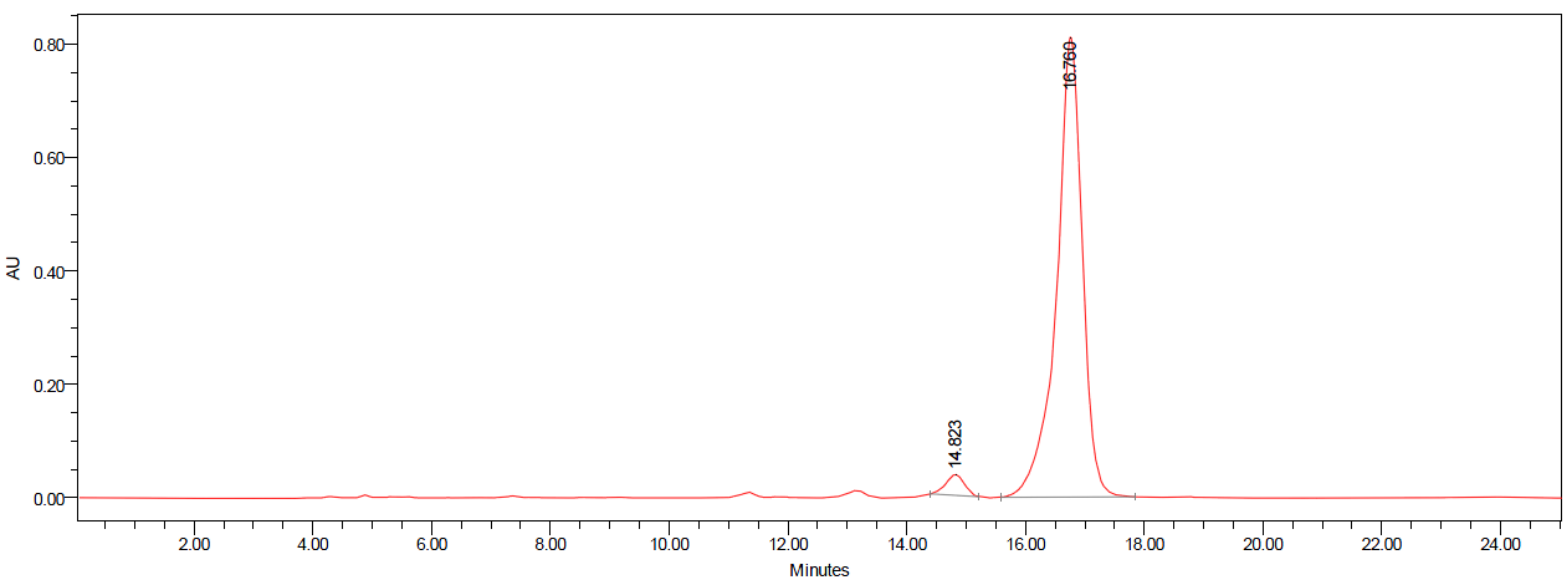

\begin{tabular}{|c|c|c|c|r|}
\hline & Channel Des cription & RT & Area & $\%$ Area \\
\hline 1 & PDA 200.0 to $400.0 \mathrm{~nm}$ at $2.4 \mathrm{~nm}$ & 14.823 & 794521 & 3.18 \\
\hline 2 & PDA 200.0 to $400.0 \mathrm{~nm}$ at $2.4 \mathrm{~nm}$ & 16.760 & 24213709 & 96.82 \\
\hline
\end{tabular}




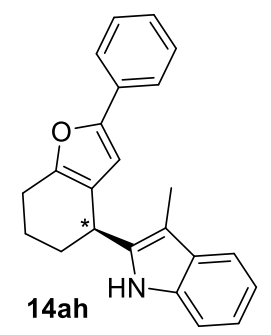

HPLC Analysis for 14ah: HPLC Chiralpak IA column [heptane:IPA = 95:05, $1 \mathrm{~mL} / \mathrm{min}, \lambda=277 \mathrm{~nm}$, retention times: $\mathrm{T}_{\mathrm{R}}$ (minor): $7.1 \mathrm{~min}, \mathrm{~T}_{\mathrm{R}}$ (major): $10.3 \mathrm{~min}$ ].

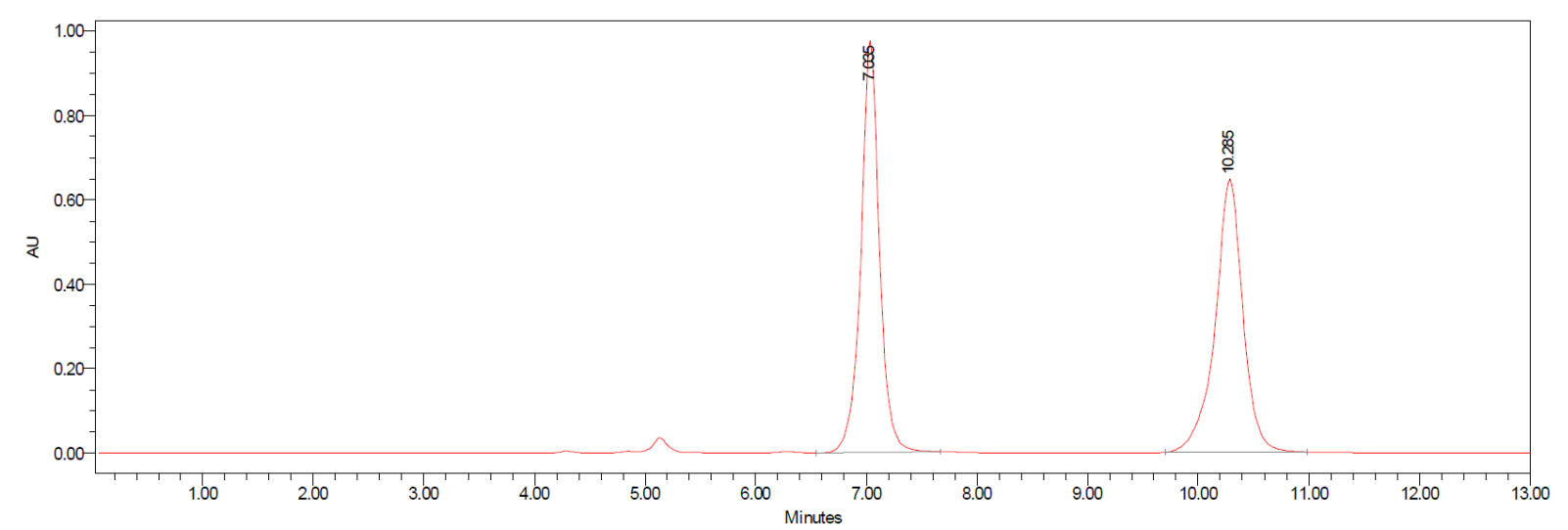

\begin{tabular}{|c|c|c|c|r|}
\hline & Channel Des cription & RT & Area & $\%$ Area \\
\hline 1 & PDA 200.0 to $400.0 \mathrm{~nm}$ at $2.4 \mathrm{~nm}$ & 7.035 & 11343652 & 50.11 \\
\hline 2 & PDA 200.0 to $400.0 \mathrm{~nm}$ at $2.4 \mathrm{~nm}$ & 10.285 & 11294023 & 49.89 \\
\hline
\end{tabular}

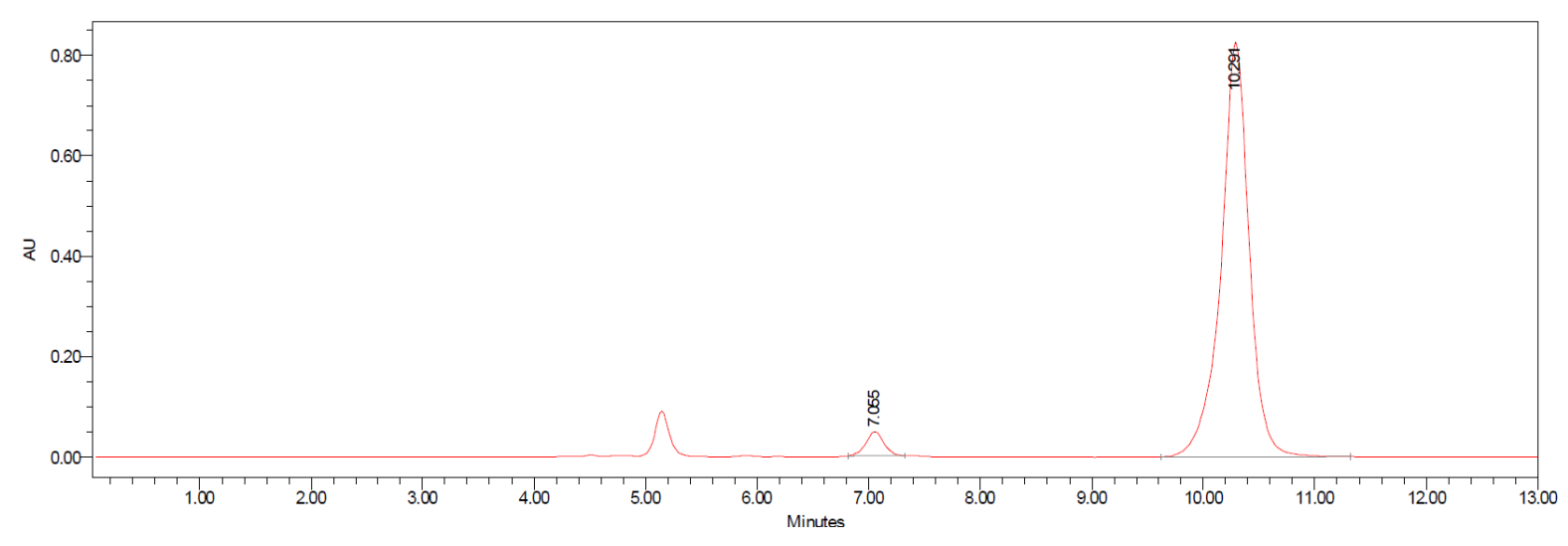

\begin{tabular}{|l|c|r|r|r|}
\hline & Channel Des cription & \multicolumn{1}{c|}{ RT } & Area & $\%$ Area \\
\hline 1 & PDA 200.0 to $400.0 \mathrm{~nm}$ at $2.4 \mathrm{~nm}$ & 7.055 & 535381 & 3.56 \\
\hline 2 & PDA 200.0 to $400.0 \mathrm{~nm}$ at $2.4 \mathrm{~nm}$ & 10.291 & 14502158 & 96.44 \\
\hline
\end{tabular}




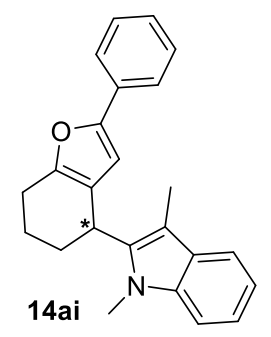

HPLC Analysis for 14ai: HPLC Chiralpak IC column [heptane:IPA = 95:05, $1 \mathrm{~mL} / \mathrm{min}, \lambda=277 \mathrm{~nm}$, retention times: $T_{R}$ (major): $4.4 \mathrm{~min}, \mathrm{~T}_{\mathrm{R}}$ (minor): $4.9 \mathrm{~min}$ ].

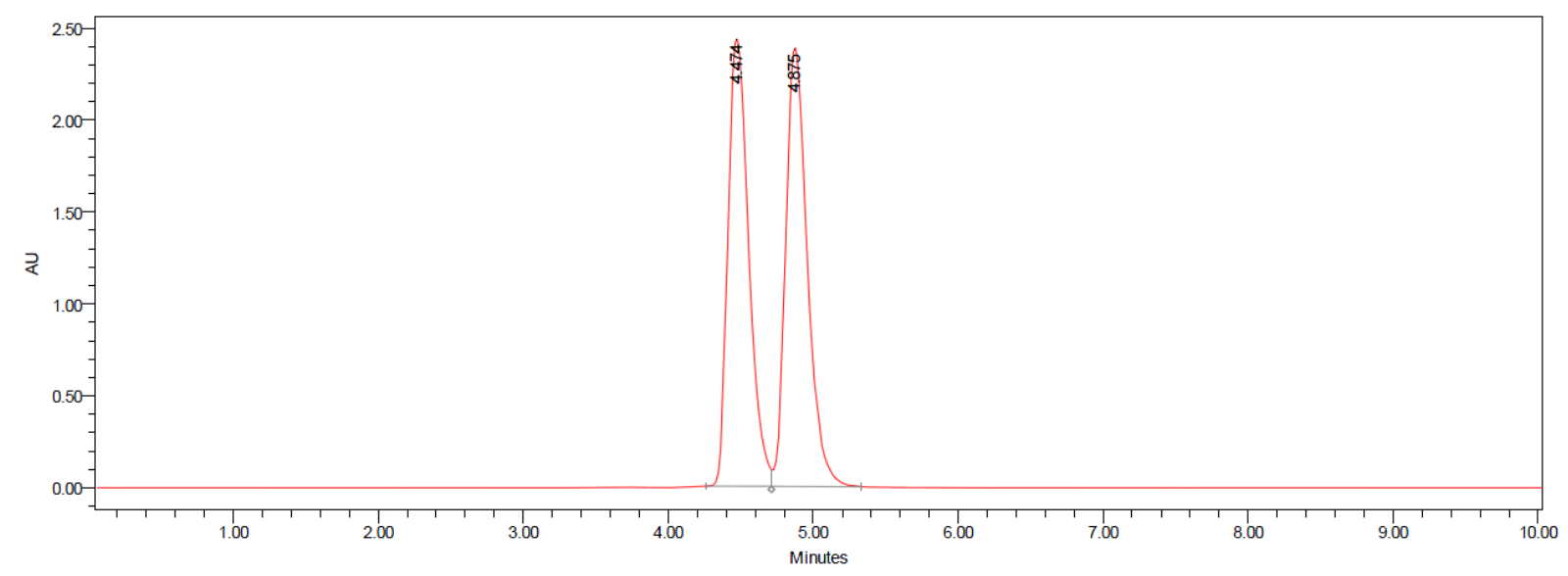

\begin{tabular}{|c|c|c|c|r|}
\hline & Channel Description & RT & Area & $\%$ Area \\
\hline 1 & PDA 200.0 to $400.0 \mathrm{~nm}$ at $2.4 \mathrm{~nm}$ & 4.474 & 24143180 & 49.41 \\
\hline 2 & PDA 200.0 to $400.0 \mathrm{~nm}$ at $2.4 \mathrm{~nm}$ & 4.875 & 24717306 & 50.59 \\
\hline
\end{tabular}

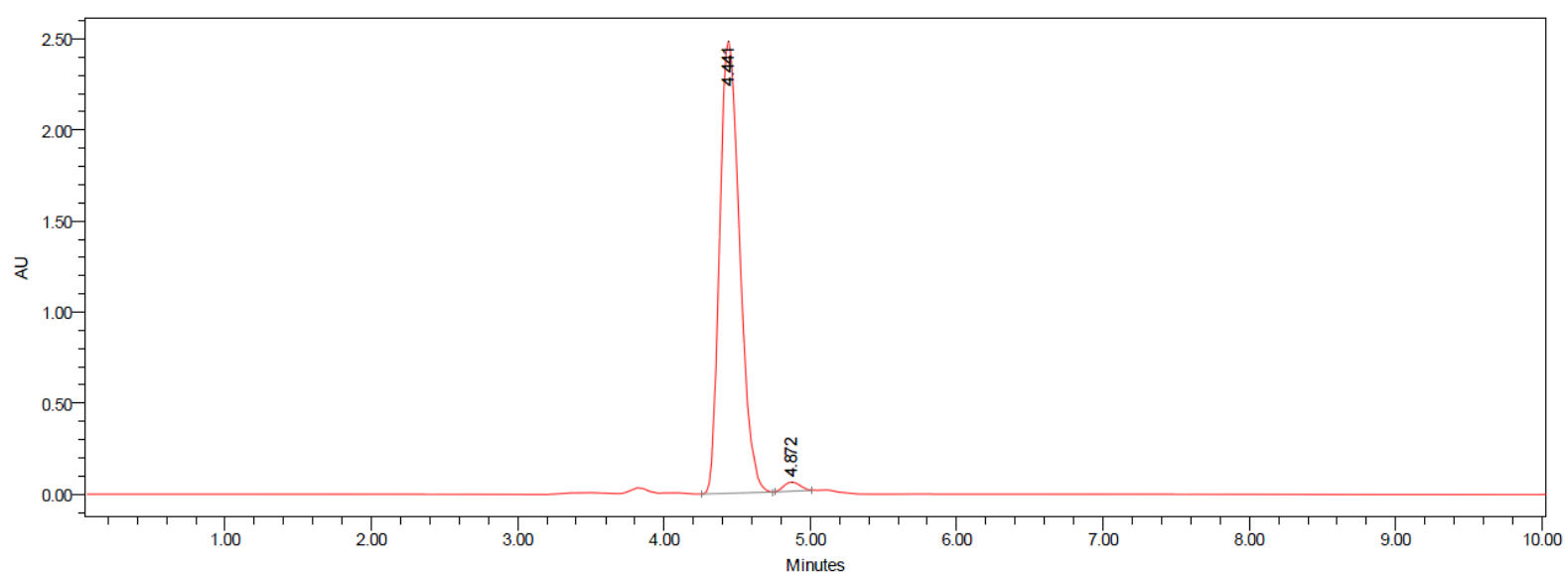

\begin{tabular}{|c|c|c|r|r|}
\hline & Channel Des cription & RT & Area & $\%$ Area \\
\hline 1 & PDA 200.0 to $400.0 \mathrm{~nm}$ at $2.4 \mathrm{~nm}$ & 4.441 & 23702749 & 98.39 \\
\hline 2 & PDA 200.0 to $400.0 \mathrm{~nm}$ at $2.4 \mathrm{~nm}$ & 4.872 & 387660 & 1.61 \\
\hline
\end{tabular}




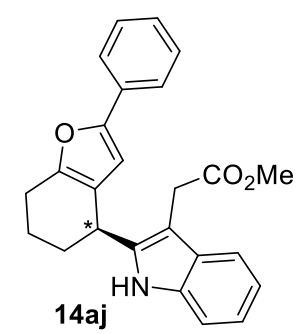

HPLC Analysis for 14aj: HPLC Chiralpak IC column [heptane:IPA $=98: 02,1 \mathrm{~mL} / \mathrm{min}, \lambda=277 \mathrm{~nm}$, retention times: $T_{R}$ (minor): $31.8 \mathrm{~min}, \mathrm{~T}_{\mathrm{R}}$ (major): $35.8 \mathrm{~min}$ ].

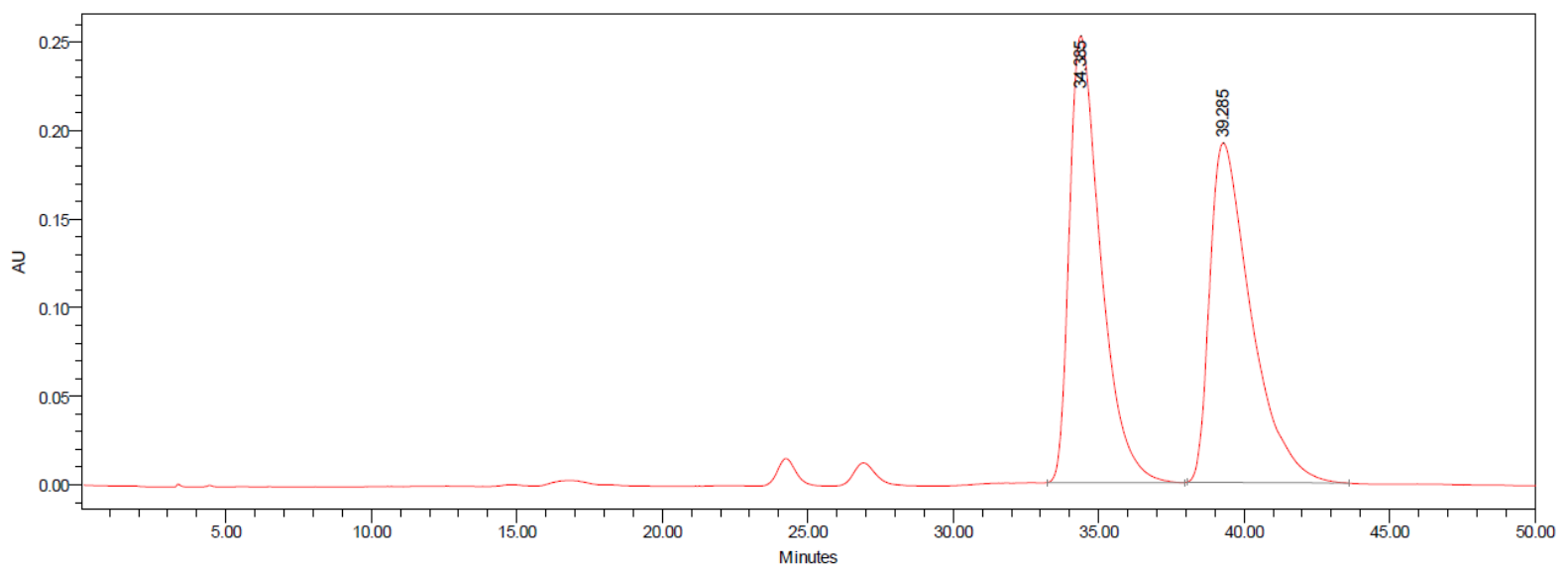

\begin{tabular}{|c|c|c|c|r|}
\hline & Channel Des cription & RT & Area & $\%$ Area \\
\hline 1 & PDA 200.0 to $400.0 \mathrm{~nm}$ at $2.4 \mathrm{~nm}$ & 34.385 & 18922963 & 50.07 \\
\hline 2 & PDA 200.0 to $400.0 \mathrm{~nm}$ at $2.4 \mathrm{~nm}$ & 39.285 & 18871860 & 49.93 \\
\hline
\end{tabular}

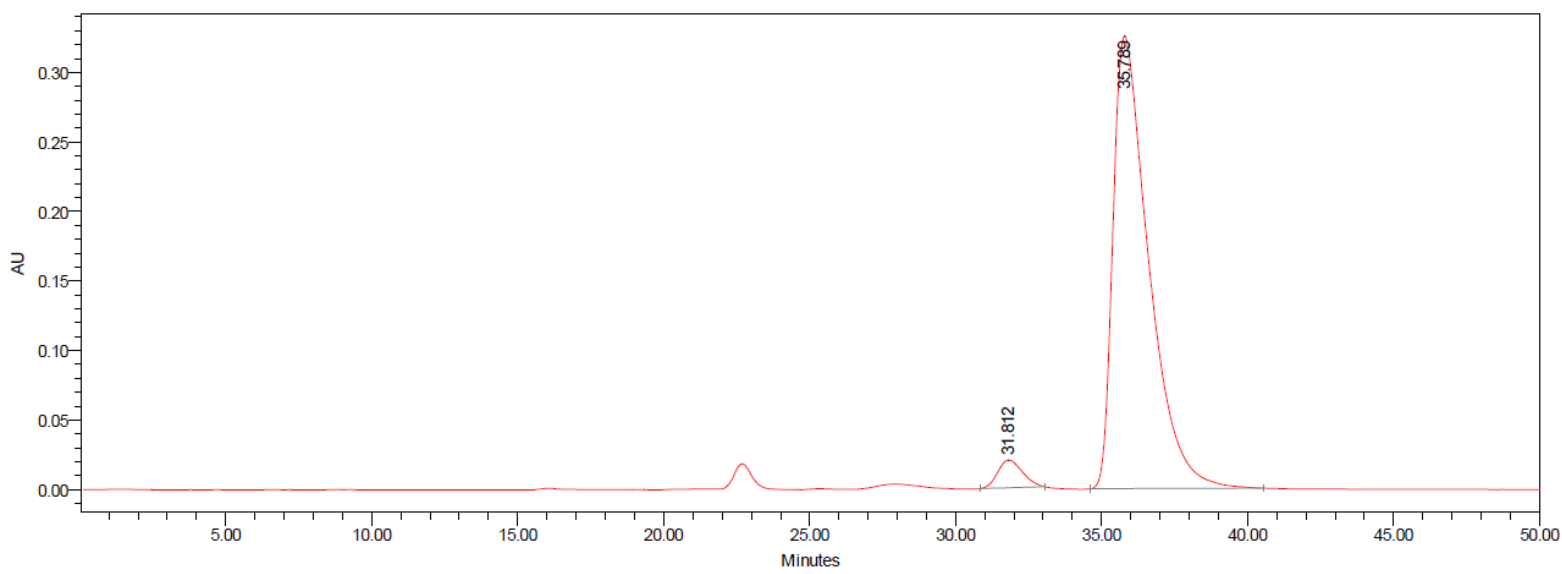

\begin{tabular}{|c|c|c|c|r|}
\hline & Channel Description & RT & Area & \% Area \\
\hline 1 & PDA 200.0 to $400.0 \mathrm{~nm}$ at $2.4 \mathrm{~nm}$ & 31.812 & 1166654 & 4.05 \\
\hline 2 & PDA 200.0 to $400.0 \mathrm{~nm}$ at $2.4 \mathrm{~nm}$ & 35.789 & 27660033 & 95.95 \\
\hline
\end{tabular}


<smiles>CC(=O)Cc1cn([C@@H]2CCCc3oc(-c4ccccc4)cc32)c2ccccc12</smiles>

HPLC Analysis for 15aj: HPLC Chiralpak IC column [heptane:IPA $=98: 02,1 \mathrm{~mL} / \mathrm{min}, \lambda=277 \mathrm{~nm}$, retention times: $T_{R}$ (major): $23.1 \mathrm{~min}, \mathrm{~T}_{R}$ (minor): $25.9 \mathrm{~min}$ ].

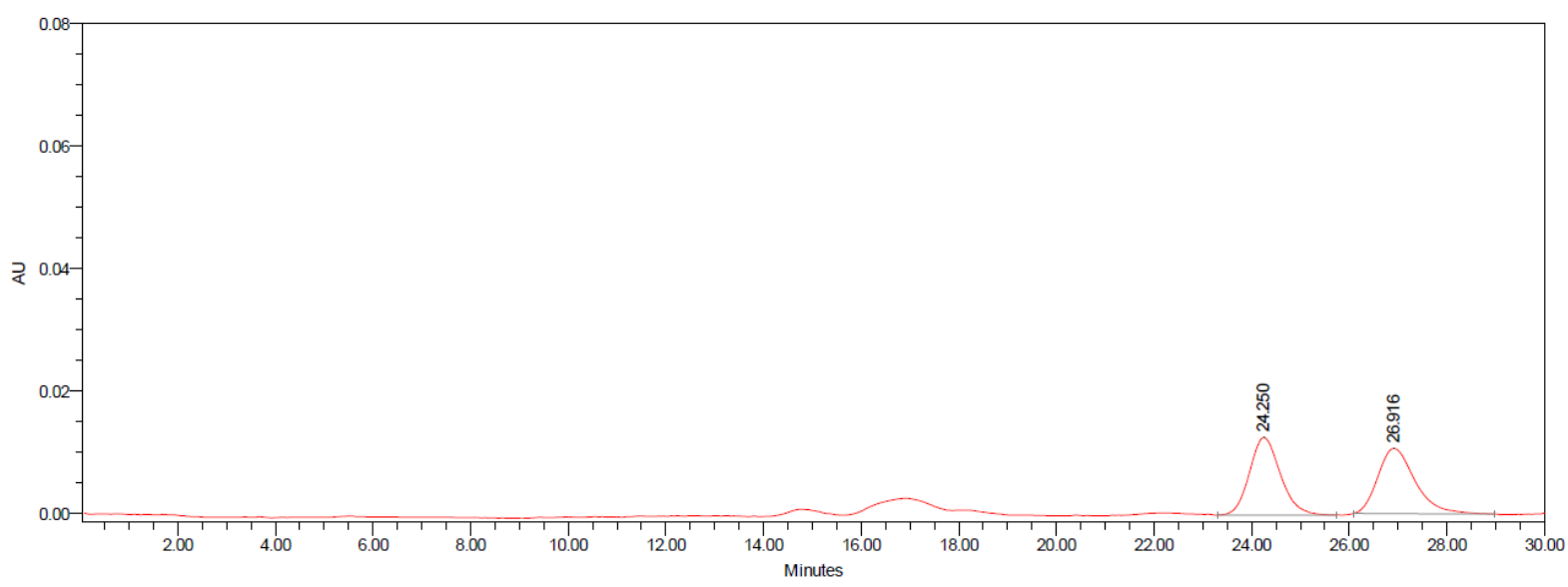

\begin{tabular}{|c|c|c|c|r|}
\hline & Channel Des cription & RT & Area & \% Area \\
\hline 1 & PDA 200.0 to $400.0 \mathrm{~nm}$ at $2.4 \mathrm{~nm}$ & 24.250 & 556623 & 49.69 \\
\hline 2 & PDA 200.0 to $400.0 \mathrm{~nm}$ at $2.4 \mathrm{~nm}$ & 26.916 & 563529 & 50.31 \\
\hline
\end{tabular}

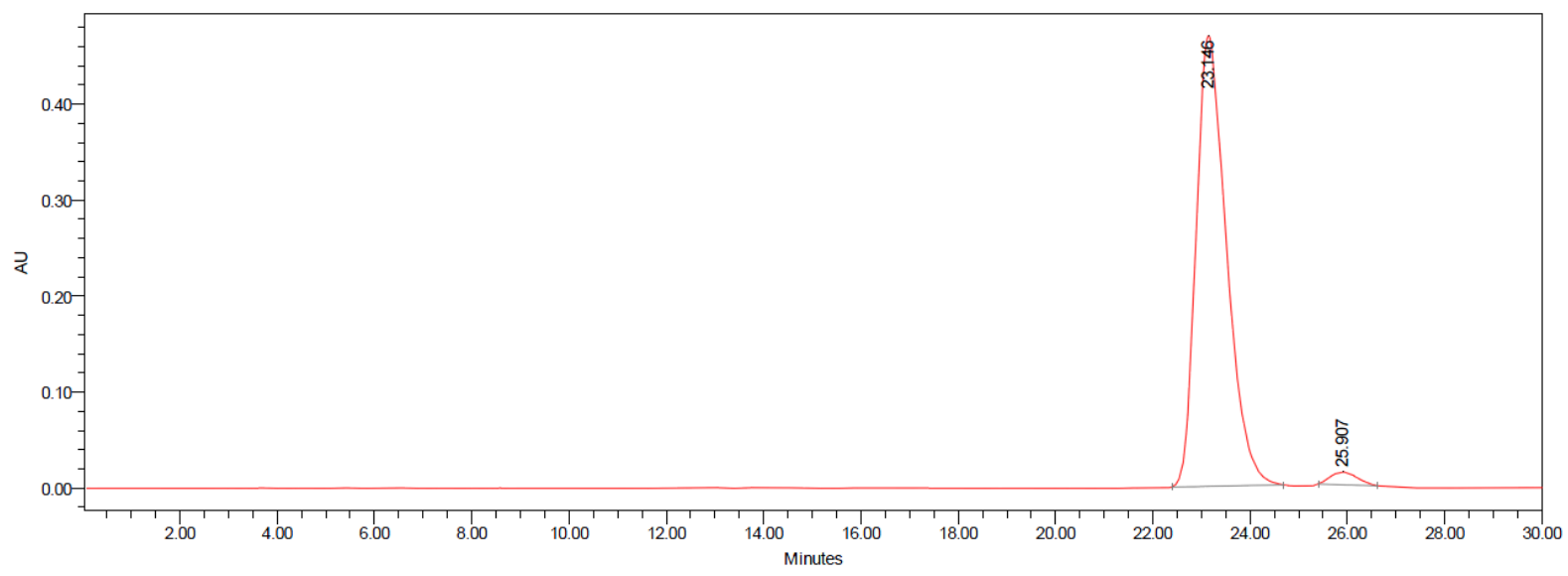

\begin{tabular}{|l|c|c|r|r|}
\hline & Channel Description & RT & Area & $\%$ Area \\
\hline 1 & PDA 200.0 to $400.0 \mathrm{~nm}$ at $2.4 \mathrm{~nm}$ & 23.146 & 20303913 & 97.56 \\
\hline 2 & PDA 200.0 to $400.0 \mathrm{~nm}$ at $2.4 \mathrm{~nm}$ & 25.907 & 508204 & 2.44 \\
\hline
\end{tabular}




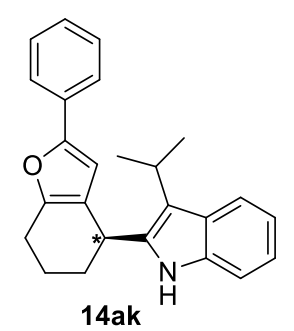

HPLC Analysis for 14ak: HPLC Chiralpak IA column [heptane:IPA $=95: 05,1 \mathrm{~mL} / \mathrm{min}, \lambda=277 \mathrm{~nm}$, retention times: $T_{R}$ (minor): $5.2 \mathrm{~min}, \mathrm{~T}_{R}$ (major): $6.9 \mathrm{~min}$ ].

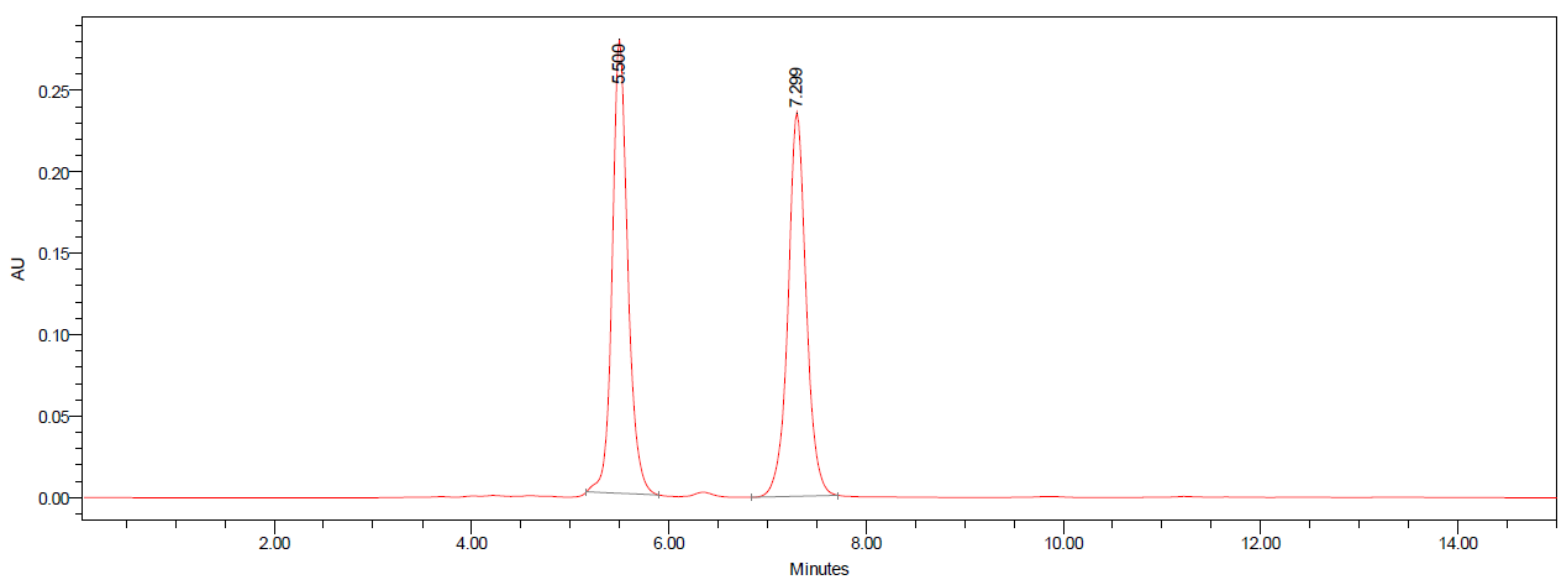

\begin{tabular}{|c|c|c|c|r|}
\hline & Channel Des cription & RT & Area & $\%$ Area \\
\hline 1 & PDA 200.0 to $400.0 \mathrm{~nm}$ at $2.4 \mathrm{~nm}$ & 5.500 & 3042828 & 50.23 \\
\hline 2 & PDA 200.0 to $400.0 \mathrm{~nm}$ at $2.4 \mathrm{~nm}$ & 7.299 & 3015266 & 49.77 \\
\hline
\end{tabular}

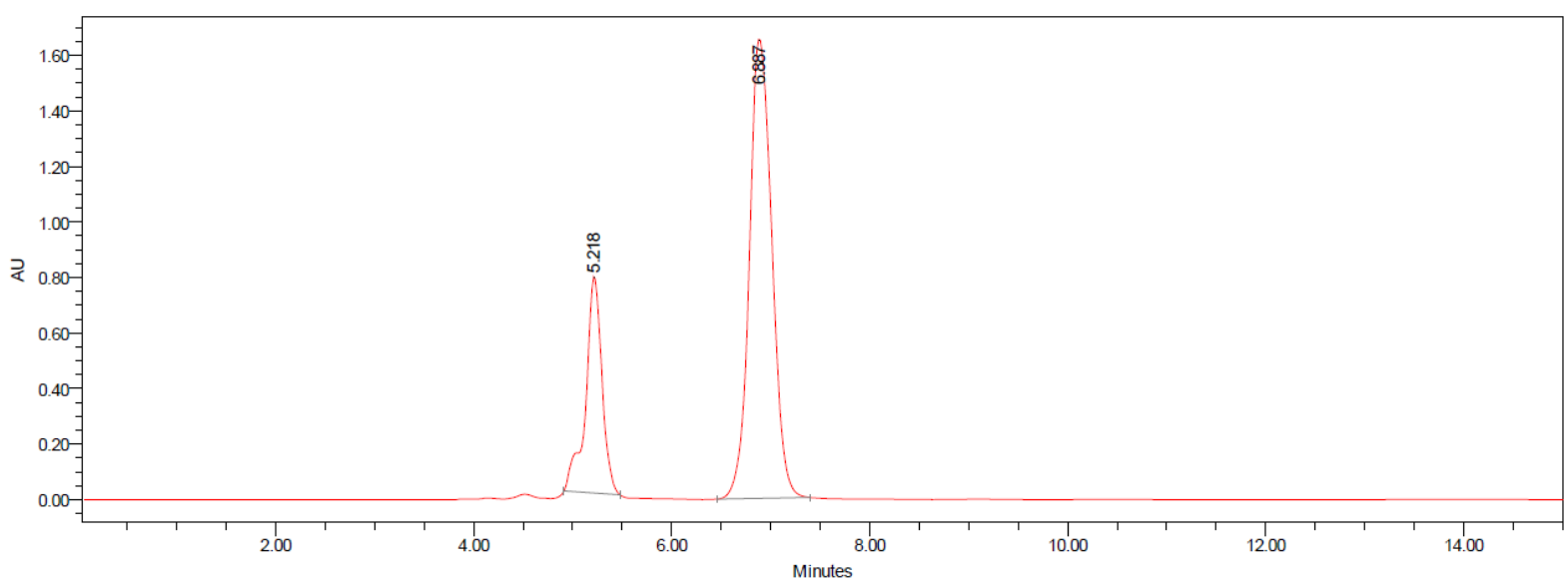

\begin{tabular}{|c|c|c|c|r|}
\hline & Channel Description & RT & Area & $\%$ Area \\
\hline 1 & PDA 200.0 to $400.0 \mathrm{~nm}$ at $2.4 \mathrm{~nm}$ & 5.218 & 8917970 & 25.43 \\
\hline 2 & PDA 200.0 to $400.0 \mathrm{~nm}$ at $2.4 \mathrm{~nm}$ & 6.887 & 26156996 & 74.57 \\
\hline
\end{tabular}




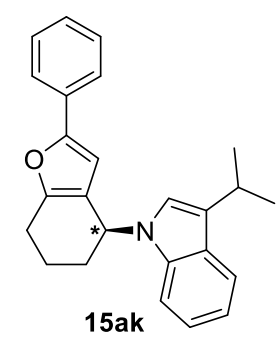

HPLC Analysis for 15ak: HPLC Chiralpak IA column [heptane:IPA = 95:05, $1 \mathrm{~mL} / \mathrm{min}, \lambda=277 \mathrm{~nm}$, retention times: $\mathrm{T}_{\mathrm{R}}$ (minor): $4.2 \mathrm{~min}, \mathrm{~T}_{\mathrm{R}}$ (major): $4.6 \mathrm{~min}$ ].

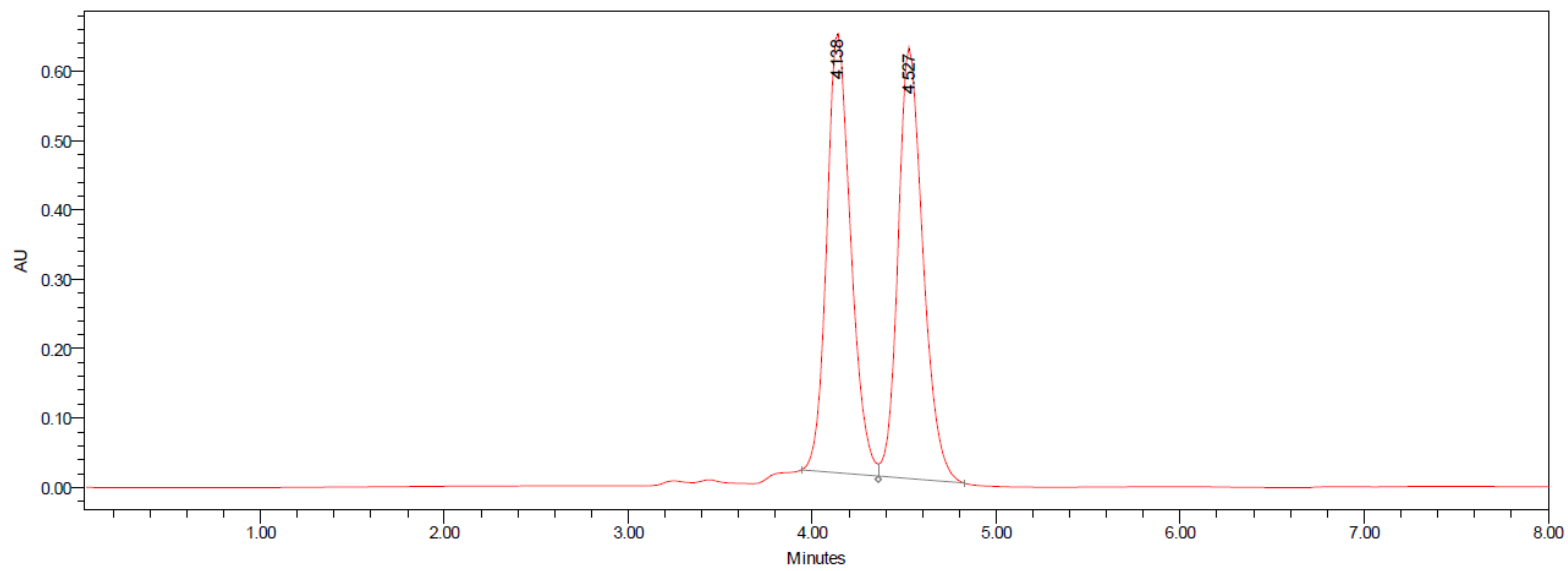

\begin{tabular}{|c|c|c|c|r|}
\hline & Channel Des cription & RT & Area & $\%$ Area \\
\hline 1 & PDA 200.0 to $400.0 \mathrm{~nm}$ at $2.4 \mathrm{~nm}$ & 4.138 & 5764973 & 49.61 \\
\hline 2 & PDA 200.0 to $400.0 \mathrm{~nm}$ at $2.4 \mathrm{~nm}$ & 4.527 & 5856614 & 50.39 \\
\hline
\end{tabular}

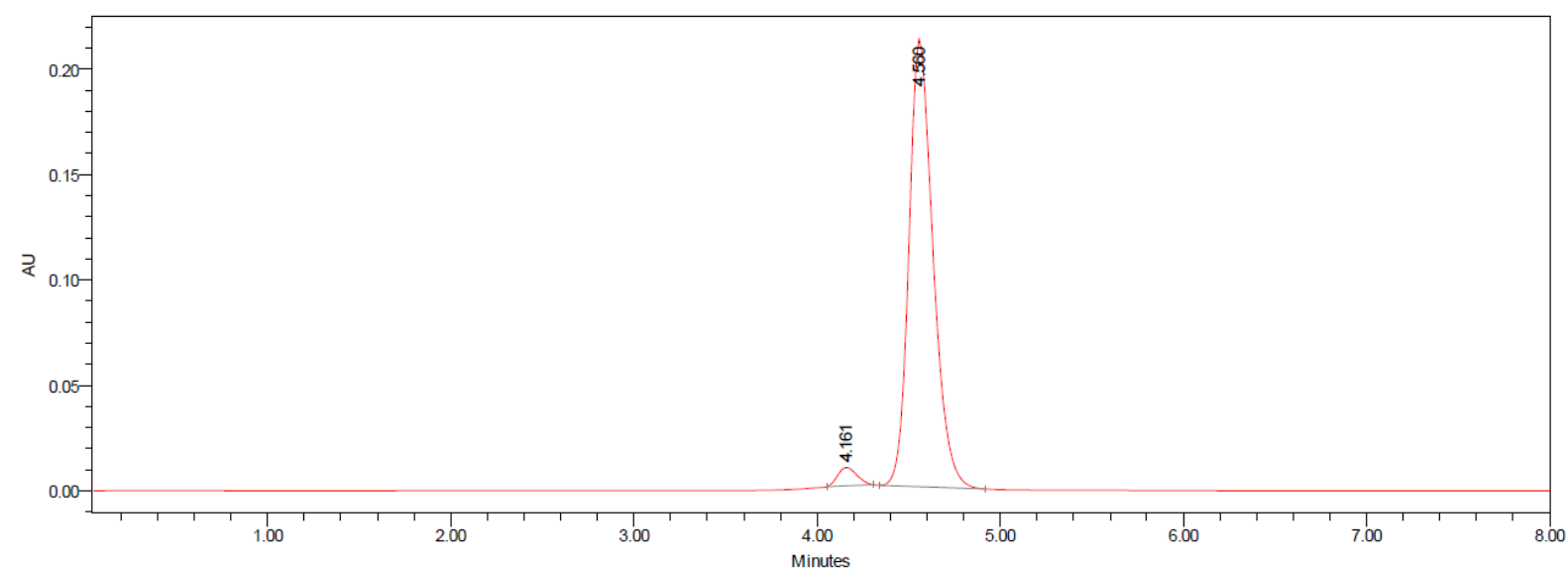

\begin{tabular}{|c|c|c|c|r|}
\hline & Channel Des cription & RT & Area & $\%$ Area \\
\hline 1 & PDA 200.0 to $400.0 \mathrm{~nm}$ at $2.4 \mathrm{~nm}$ & 4.161 & 62349 & 3.10 \\
\hline 2 & PDA 200.0 to $400.0 \mathrm{~nm}$ at $2.4 \mathrm{~nm}$ & 4.560 & 1946577 & 96.90 \\
\hline
\end{tabular}




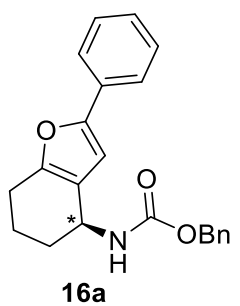

$16 a$

HPLC Analysis for 16a: HPLC Chiralpak IB column [heptane:IPA = 97:03, $1 \mathrm{~mL} / \mathrm{min}, \lambda=277 \mathrm{~nm}$, retention times: $T_{R}$ (minor): $17.7 \mathrm{~min}, \mathrm{~T}_{\mathrm{R}}$ (major): $22.5 \mathrm{~min}$ ].

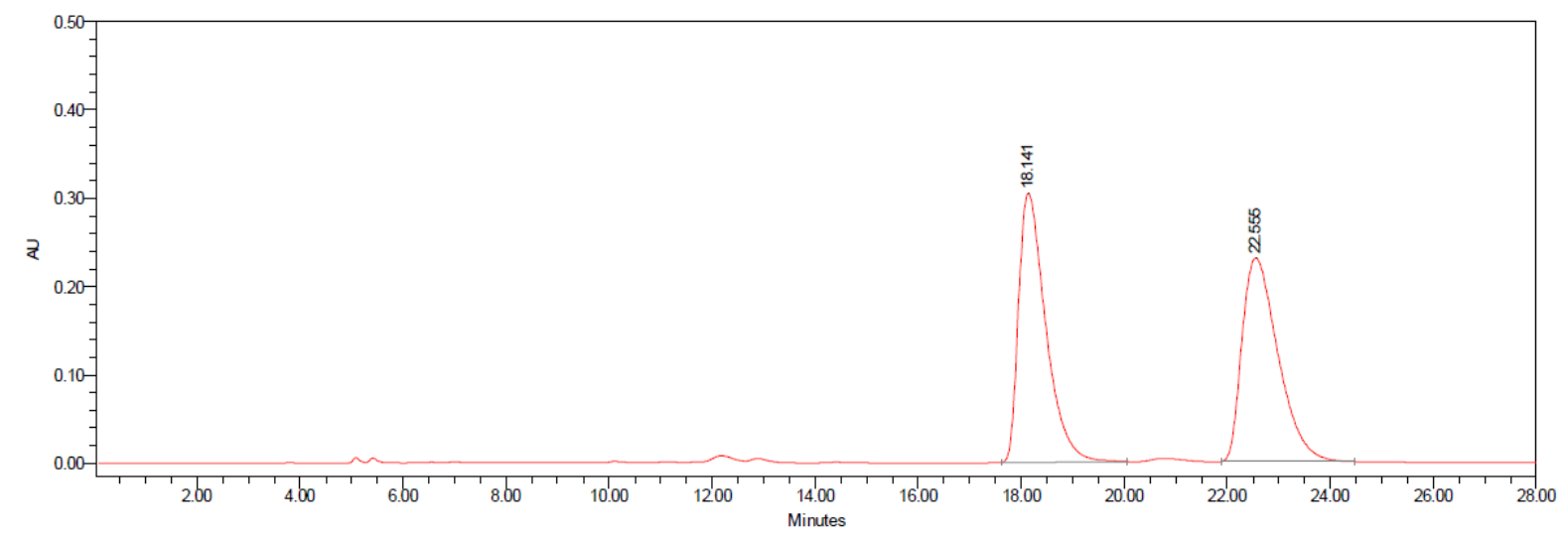

\begin{tabular}{|c|c|c|c|r|}
\hline & Channel Des cription & RT & Area & $\%$ Area \\
\hline 1 & PDA 200.0 to $400.0 \mathrm{~nm}$ at $2.4 \mathrm{~nm}$ & 18.141 & 11199041 & 49.99 \\
\hline 2 & PDA 200.0 to $400.0 \mathrm{~nm}$ at $2.4 \mathrm{~nm}$ & 22.555 & 11201882 & 50.01 \\
\hline
\end{tabular}

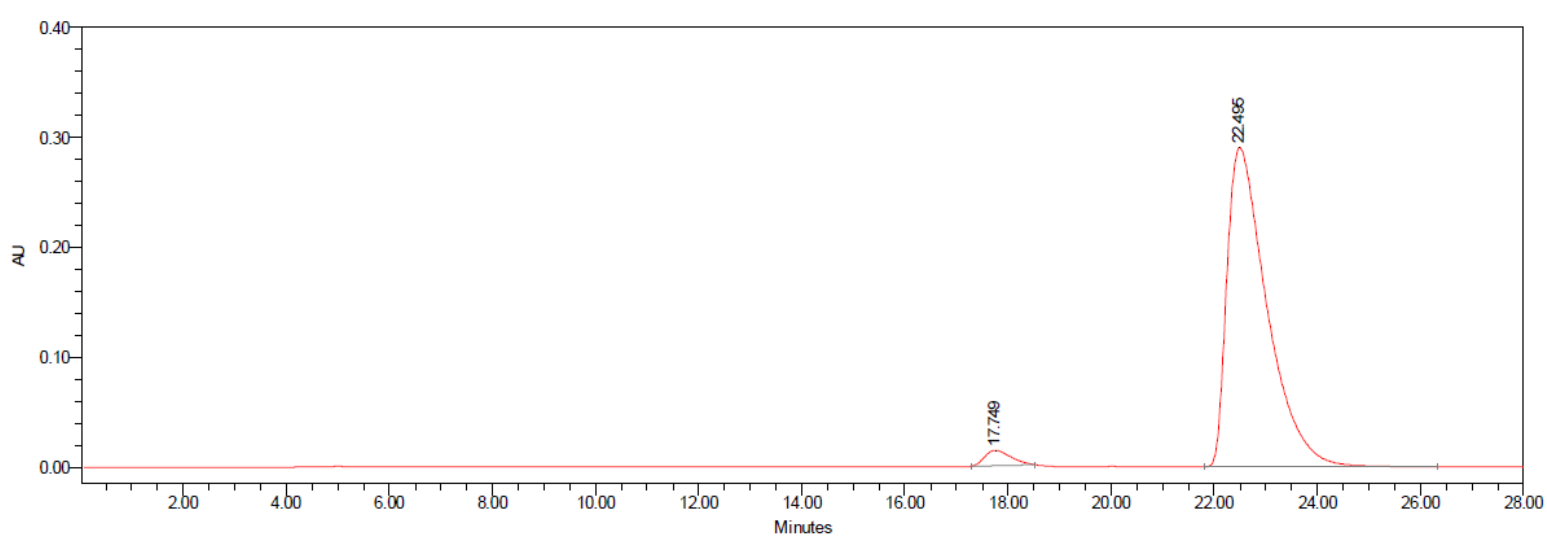

\begin{tabular}{|l|c|c|r|r|}
\hline & Channel Des cription & RT & Area & $\%$ Area \\
\hline 1 & PDA 200.0 to $400.0 \mathrm{~nm}$ at $2.4 \mathrm{~nm}$ & 17.749 & 486446 & 3.02 \\
\hline 2 & PDA 200.0 to $400.0 \mathrm{~nm}$ at $2.4 \mathrm{~nm}$ & 22.495 & 15613303 & 96.98 \\
\hline
\end{tabular}




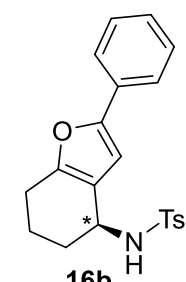

HPLC Analysis for 16b: HPLC Chiralpak IC column [heptane:IPA = 90:10, $1 \mathrm{~mL} / \mathrm{min}, \lambda=277 \mathrm{~nm}$, retention times: $\mathrm{T}_{\mathrm{R}}$ (major): $35.7 \mathrm{~min}, \mathrm{~T}_{\mathrm{R}}$ (minor): $41.7 \mathrm{~min}$ ].

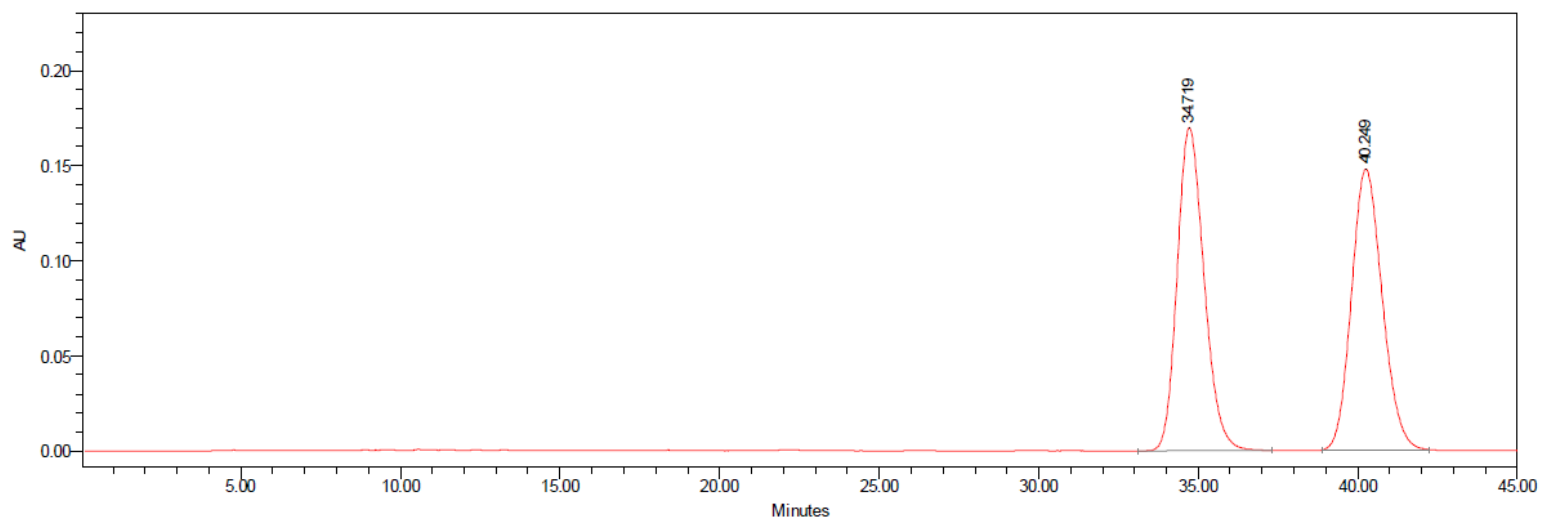

\begin{tabular}{|c|c|c|c|r|}
\hline & Channel Des cription & RT & Area & $\%$ Area \\
\hline 1 & PDA 200.0 to $400.0 \mathrm{~nm}$ at $2.4 \mathrm{~nm}$ & 34.719 & 9948385 & 50.17 \\
\hline 2 & PDA 200.0 to $400.0 \mathrm{~nm}$ at $2.4 \mathrm{~nm}$ & 40.249 & 9882336 & 49.83 \\
\hline
\end{tabular}

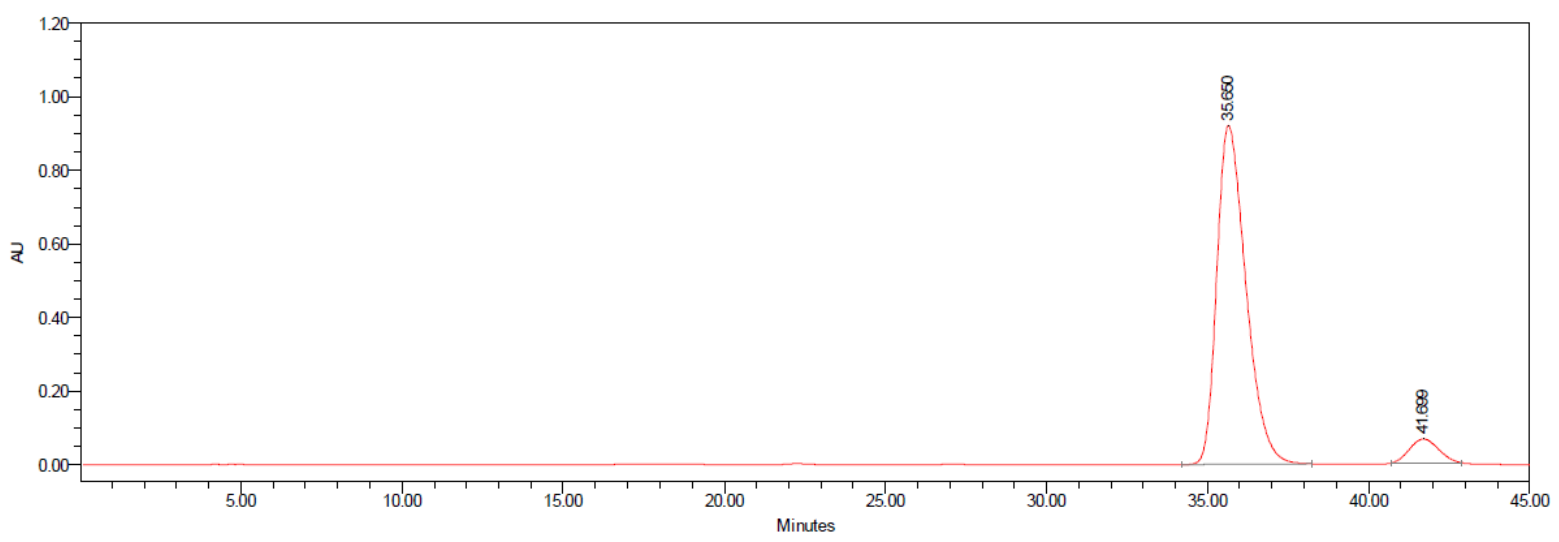

\begin{tabular}{|c|c|c|c|r|}
\hline & Channel Des cription & RT & Area & \% Area \\
\hline 1 & PDA 200.0 to $400.0 \mathrm{~nm}$ at $2.4 \mathrm{~nm}$ & 35.650 & 57606806 & 93.50 \\
\hline 2 & PDA 200.0 to $400.0 \mathrm{~nm}$ at $2.4 \mathrm{~nm}$ & 41.699 & 4006807 & 6.50 \\
\hline
\end{tabular}




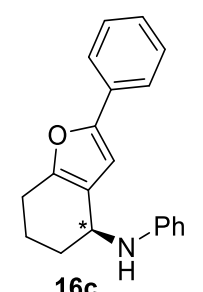

HPLC Analysis for 16c: HPLC Chiralpak IB column [heptane:IPA = 98:02, $1 \mathrm{~mL} / \mathrm{min}, \lambda=277 \mathrm{~nm}$, retention times: $\mathrm{T}_{\mathrm{R}}$ (major): $10.5 \mathrm{~min}, \mathrm{~T}_{\mathrm{R}}$ (minor): $14.0 \mathrm{~min}$ ].

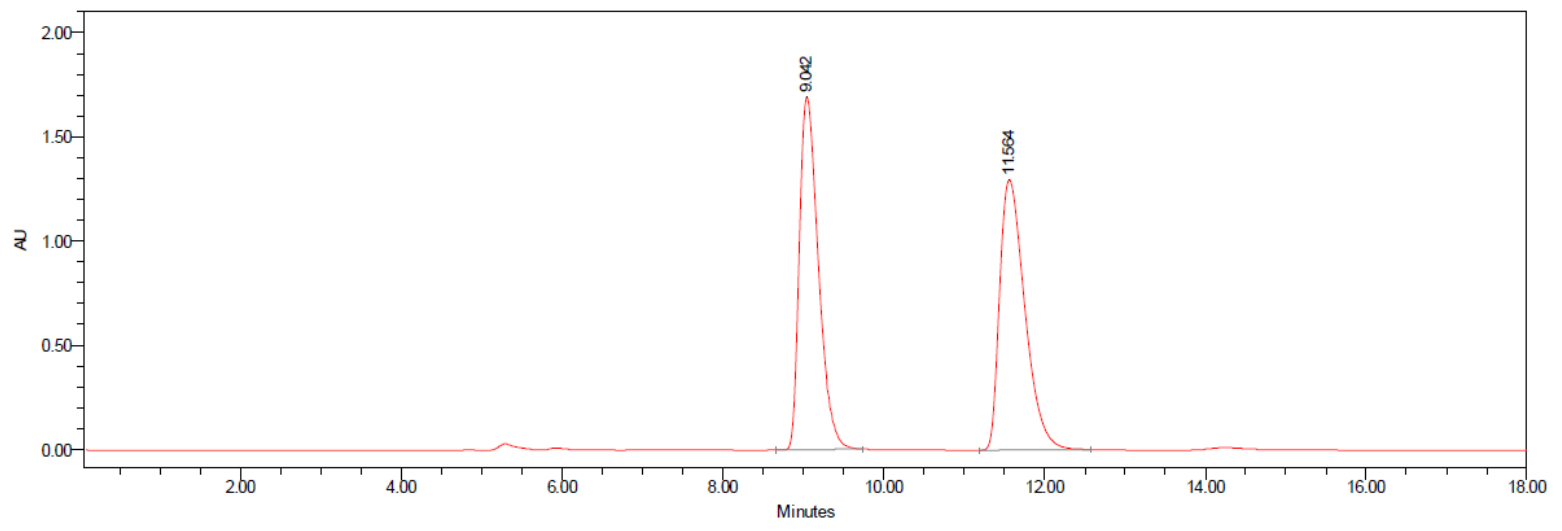

\begin{tabular}{|c|c|c|c|r|}
\hline & Channel Des cription & RT & Area & $\%$ Area \\
\hline 1 & PDA 200.0 to $400.0 \mathrm{~nm}$ at $2.4 \mathrm{~nm}$ & 9.042 & 27706662 & 49.68 \\
\hline 2 & PDA 200.0 to $400.0 \mathrm{~nm}$ at $2.4 \mathrm{~nm}$ & 11.564 & 28064942 & 50.32 \\
\hline
\end{tabular}

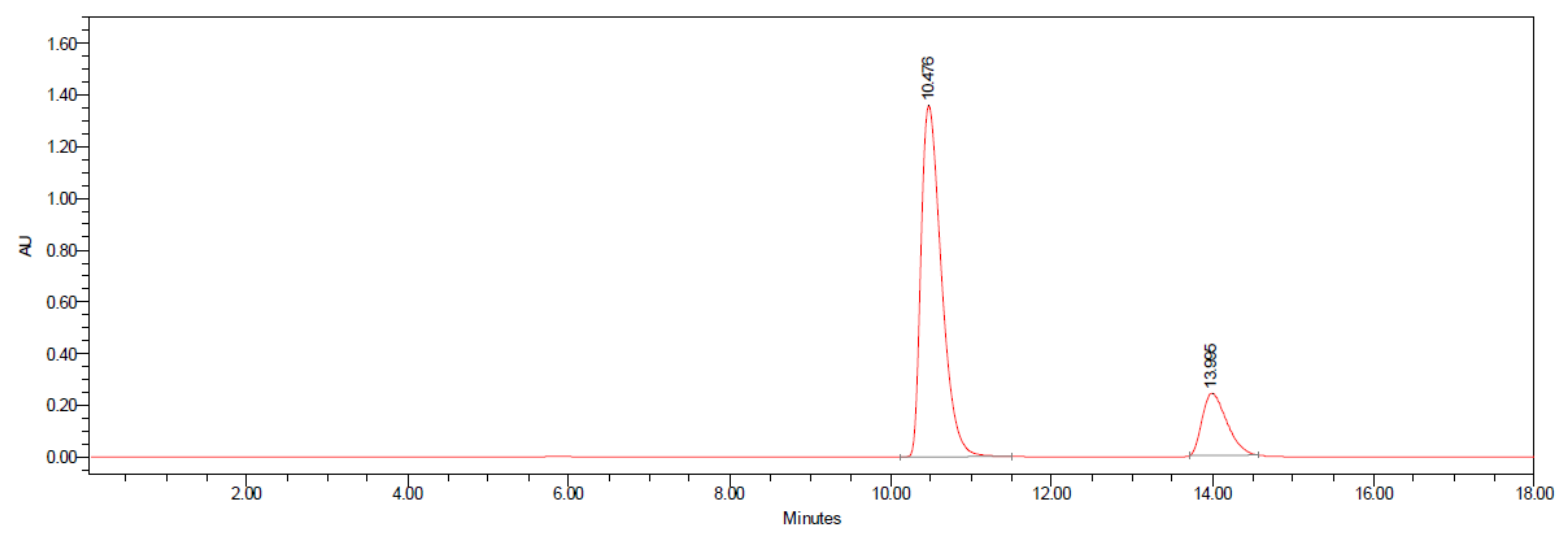

\begin{tabular}{|c|c|c|c|r|}
\hline & Channel Des cription & RT & Area & \% Area \\
\hline 1 & PDA 200.0 to $400.0 \mathrm{~nm}$ at $2.4 \mathrm{~nm}$ & 10.476 & 23784879 & 82.29 \\
\hline 2 & PDA 200.0 to $400.0 \mathrm{~nm}$ at $2.4 \mathrm{~nm}$ & 13.995 & 5120127 & 17.71 \\
\hline
\end{tabular}




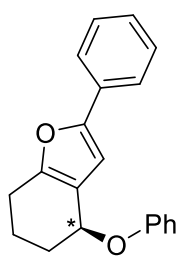

$16 d$

HPLC Analysis for 16d: HPLC Chiralpak IB column [heptane:IPA = 98:02, $1 \mathrm{~mL} / \mathrm{min}, \lambda=277 \mathrm{~nm}$, retention times: $T_{R}$ (major): $6.1 \mathrm{~min}, \mathrm{~T}_{\mathrm{R}}$ (minor): $9.2 \mathrm{~min}$ ].

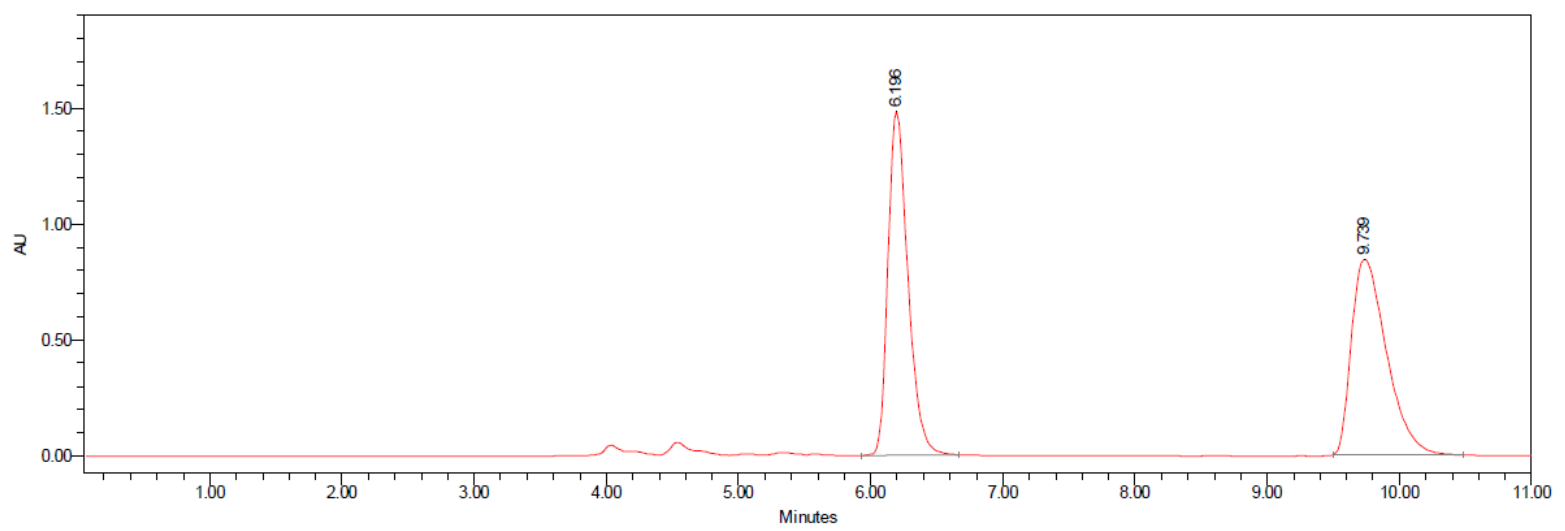

\begin{tabular}{|c|c|c|c|r|}
\hline & Channel Des cription & RT & Area & $\%$ Area \\
\hline 1 & PDA 200.0 to $400.0 \mathrm{~nm}$ at $2.4 \mathrm{~nm}$ & 6.196 & 15525085 & 50.00 \\
\hline 2 & PDA 200.0 to $400.0 \mathrm{~nm}$ at $2.4 \mathrm{~nm}$ & 9.739 & 15527509 & 50.00 \\
\hline
\end{tabular}

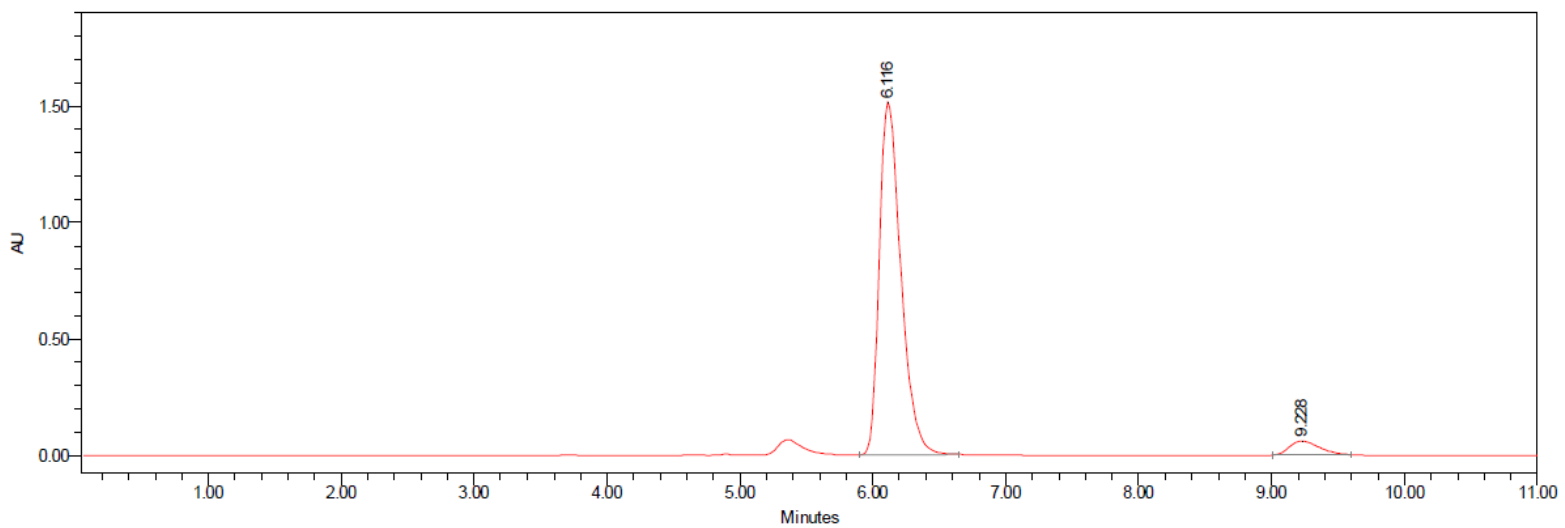

\begin{tabular}{|c|c|c|r|r|}
\hline & Channel Des cription & RT & Area & \% Area \\
\hline 1 & PDA 210.0 to $400.0 \mathrm{~nm}$ at $1.2 \mathrm{~nm}$ & 6.116 & 17023277 & 94.90 \\
\hline 2 & PDA 210.0 to $400.0 \mathrm{~nm}$ at $1.2 \mathrm{~nm}$ & 9.228 & 914783 & 5.10 \\
\hline
\end{tabular}




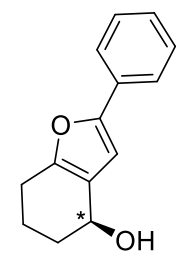

$16 e$

HPLC Analysis for 16e: HPLC Chiralpak IB column [heptane:IPA = 98:02, $1 \mathrm{~mL} / \mathrm{min}, \lambda=277 \mathrm{~nm}$, retention times: $\mathrm{T}_{\mathrm{R}}$ (major): $28.3 \mathrm{~min}, \mathrm{~T}_{\mathrm{R}}$ (minor): $34.5 \mathrm{~min}$ ].

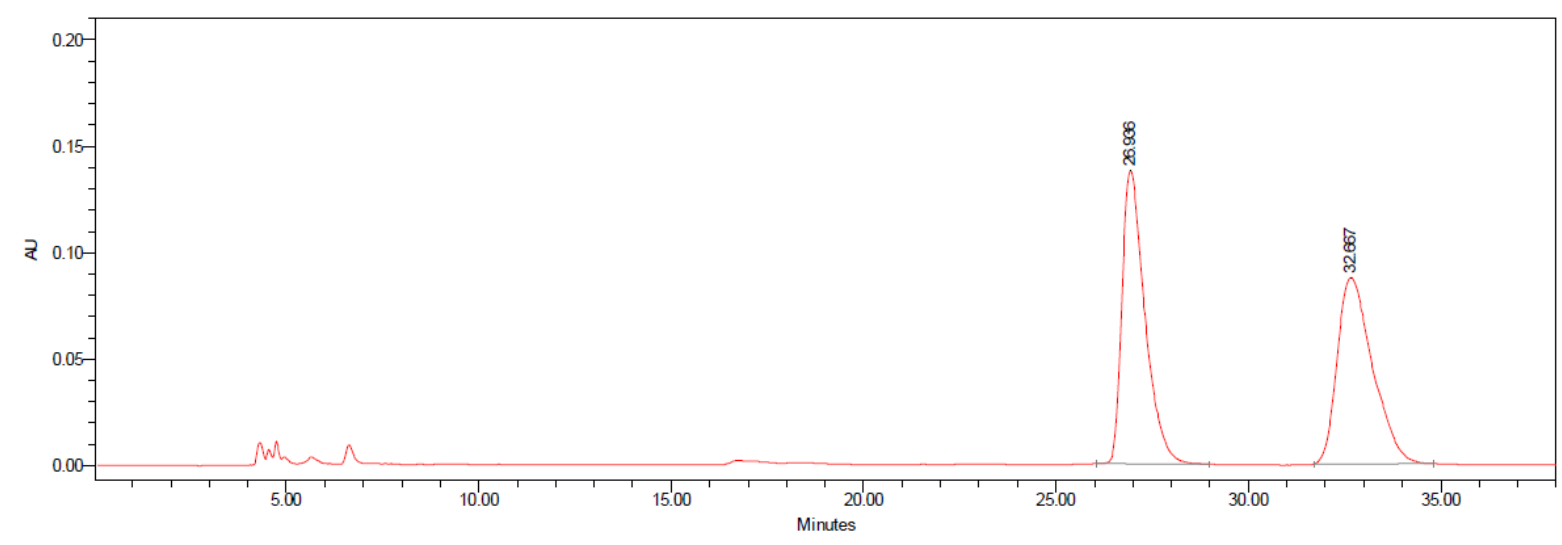

\begin{tabular}{|c|c|c|c|r|}
\hline & Channel Des cription & RT & Area & $\%$ Area \\
\hline 1 & PDA 200.0 to $400.0 \mathrm{~nm}$ at $2.4 \mathrm{~nm}$ & 26.936 & 5708912 & 50.56 \\
\hline 2 & PDA 200.0 to $400.0 \mathrm{~nm}$ at $2.4 \mathrm{~nm}$ & 32.667 & 5583065 & 49.44 \\
\hline
\end{tabular}

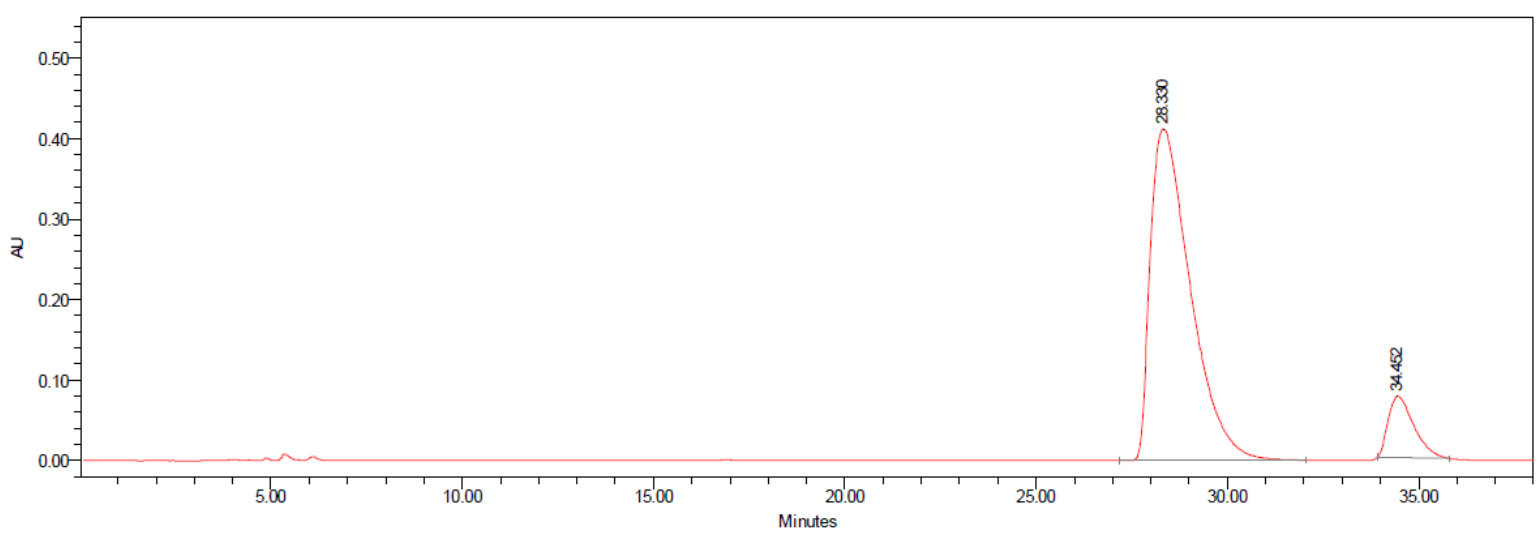

\begin{tabular}{|c|c|c|r|r|}
\hline & Channel Des cription & RT & Area & $\%$ Area \\
\hline 1 & PDA 200.0 to $400.0 \mathrm{~nm}$ at $2.4 \mathrm{~nm}$ & 28.330 & 29948802 & 89.41 \\
\hline 2 & PDA 200.0 to $400.0 \mathrm{~nm}$ at $2.4 \mathrm{~nm}$ & 34.452 & 3547065 & 10.59 \\
\hline
\end{tabular}


<smiles>O=C1C=C(OC2CCCc3cc(-c4ccccc4)oc32)CCC1</smiles>

HPLC Analysis for 16f: HPLC Chiralpak IA column [heptane:IPA $=95: 05,1 \mathrm{~mL} / \mathrm{min}, \lambda=277 \mathrm{~nm}$, retention times: $T_{R}$ (minor): $22.8 \mathrm{~min}, \mathrm{~T}_{\mathrm{R}}$ (major): $27.1 \mathrm{~min}$ ].

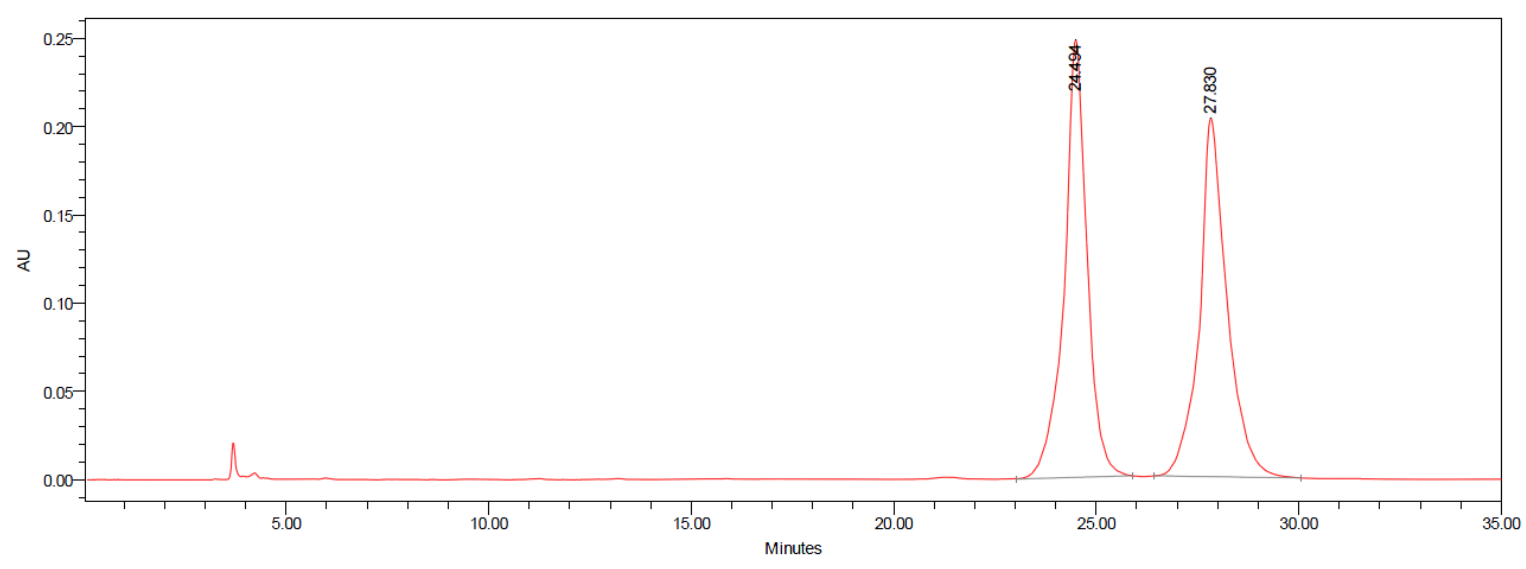

\begin{tabular}{|c|c|c|c|r|}
\hline & Channel Des cription & RT & Area & \% Area \\
\hline 1 & PDA 200.0 to $400.0 \mathrm{~nm}$ at $2.4 \mathrm{~nm}$ & 24.494 & 9643182 & 50.20 \\
\hline 2 & PDA 200.0 to $400.0 \mathrm{~nm}$ at $2.4 \mathrm{~nm}$ & 27.830 & 9565098 & 49.80 \\
\hline
\end{tabular}

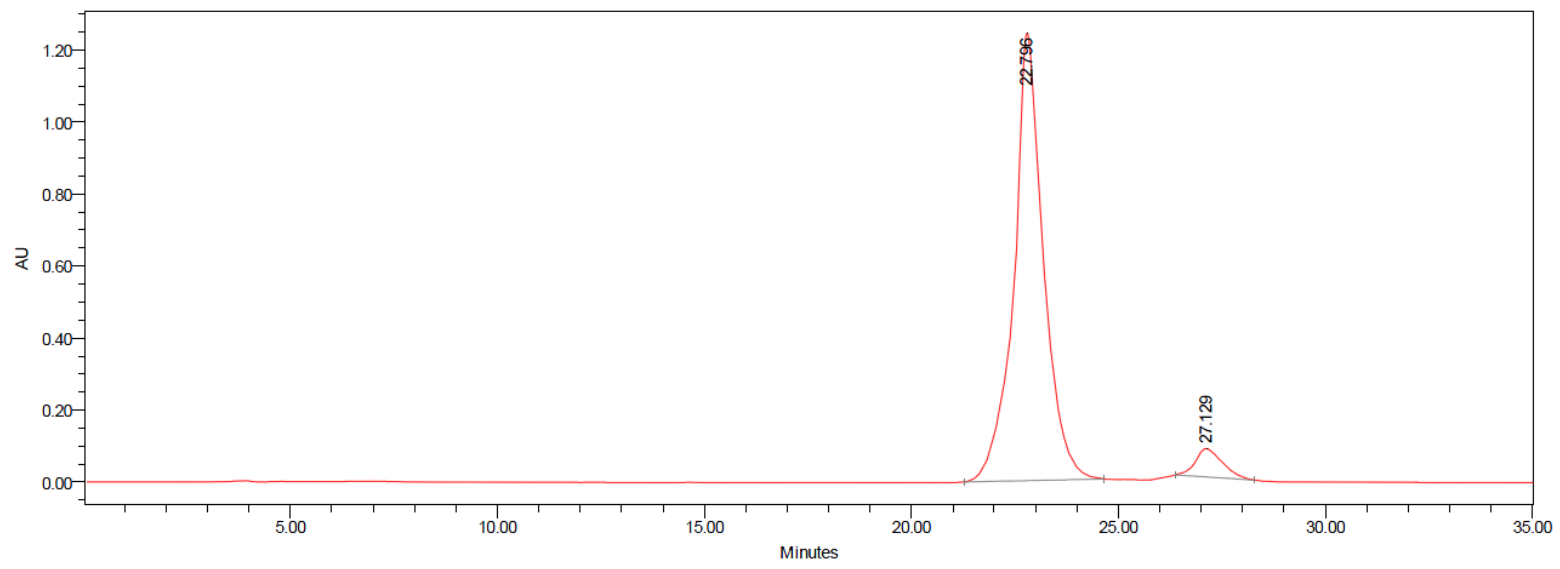

\begin{tabular}{|c|c|c|c|r|}
\hline & Channel Des cription & RT & Area & $\%$ Area \\
\hline 1 & PDA 200.0 to $400.0 \mathrm{~nm}$ at $2.4 \mathrm{~nm}$ & 22.796 & 61326122 & 94.50 \\
\hline 2 & PDA 200.0 to $400.0 \mathrm{~nm}$ at $2.4 \mathrm{~nm}$ & 27.129 & 3572509 & 5.50 \\
\hline
\end{tabular}




\section{Copies of NMR Spectra}

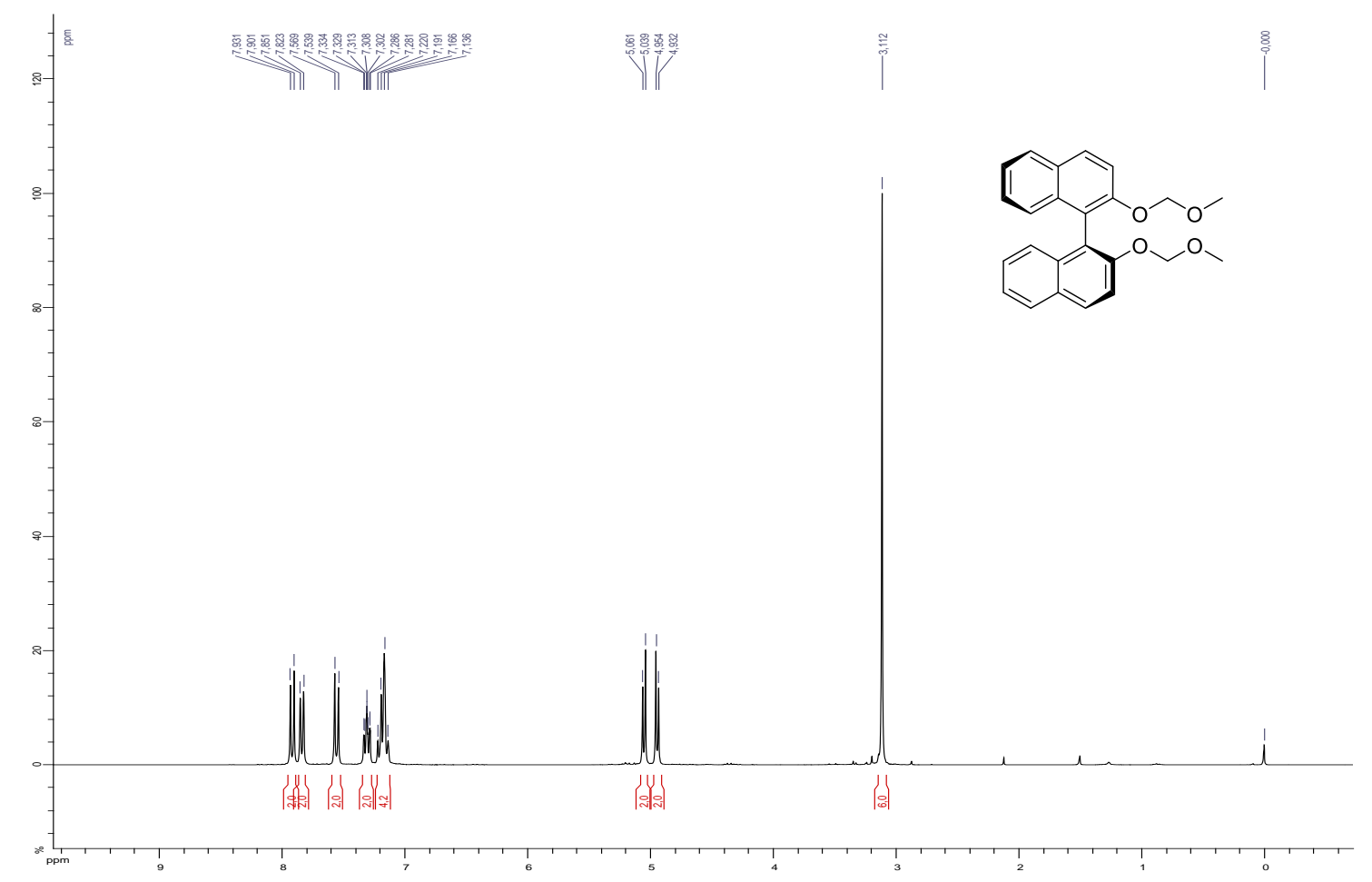

${ }^{1} \mathbf{H}$ NMR ( $\mathrm{CDCl}_{3}, 300 \mathrm{MHz}$ ) spectrum of (S)-2,2'-bis(methoxymethoxy)-1,1'-binaphthalene

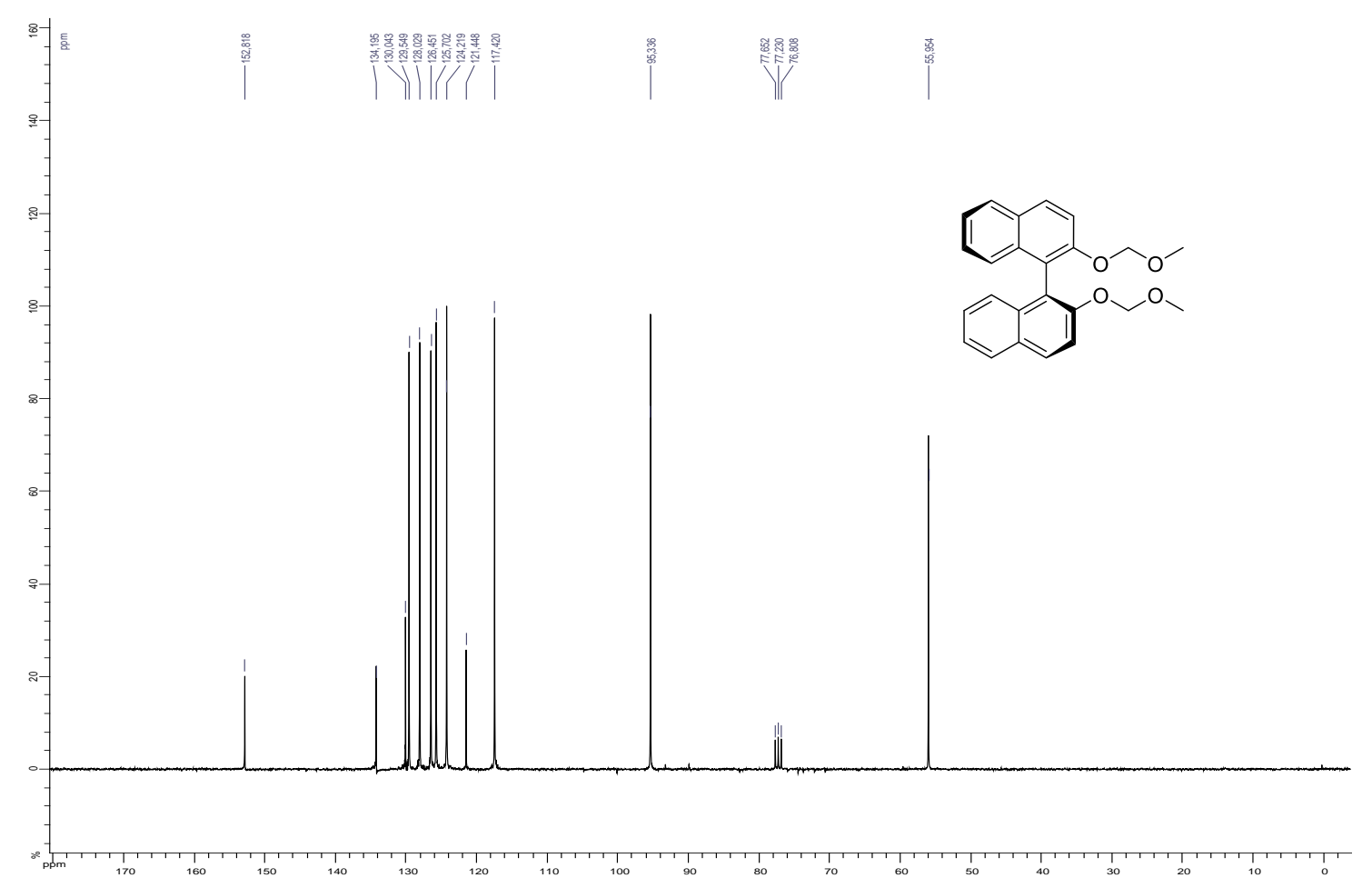

${ }^{13} \mathrm{C} \mathrm{NMR}\left(\mathrm{CDCl}_{3}, 75 \mathrm{MHz}\right.$ ) spectrum of (S)-2,2'-bis(methoxymethoxy)-1,1'-binaphthalene 


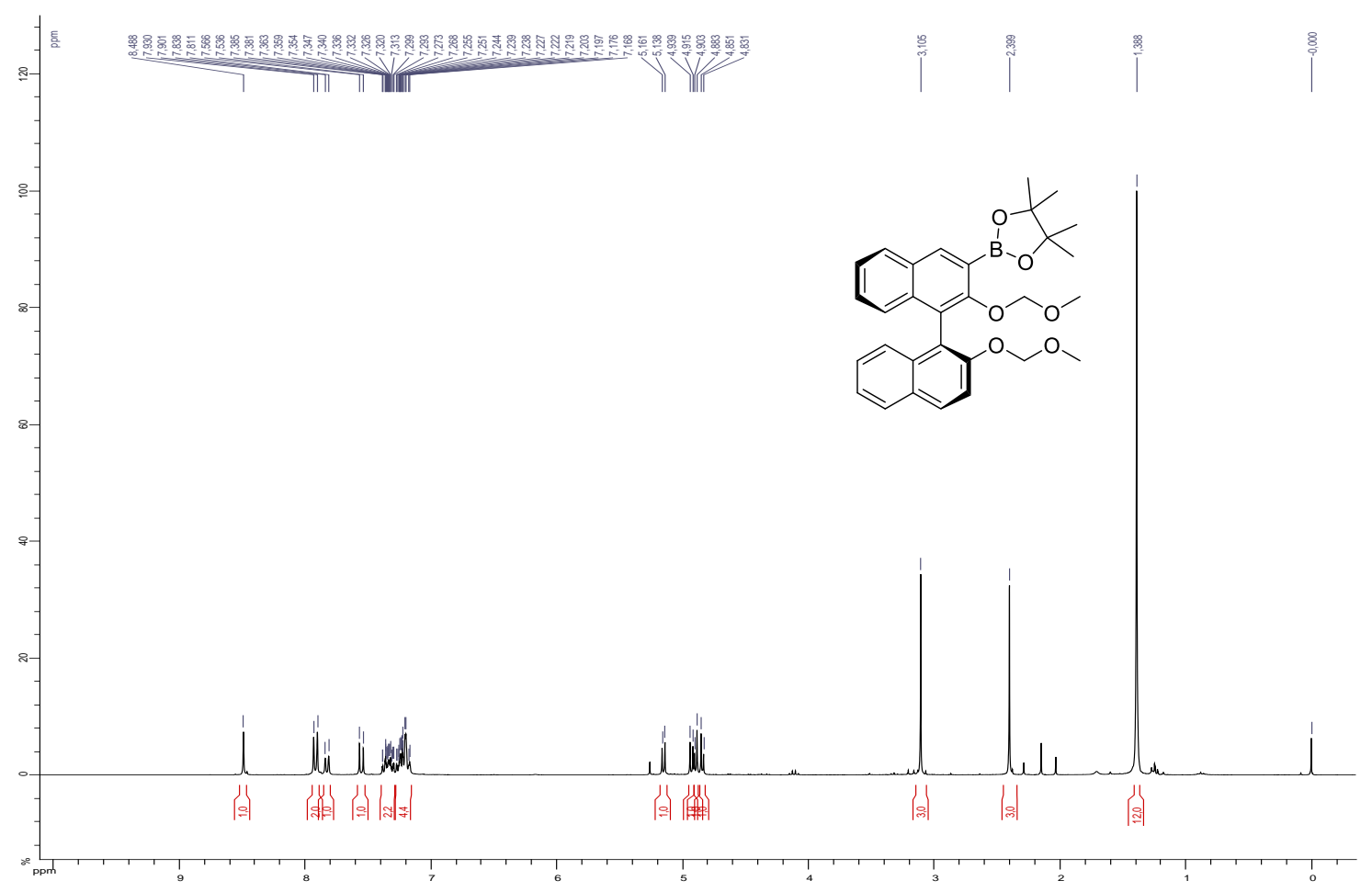

${ }^{1} \mathrm{H}$ NMR (CDCl $3,300 \mathrm{MHz}$ ) spectrum of (S)-2- (2,2'-bis(methoxymethoxy)- [1,1'-binaphthalen]-3-yl)- 4,4,5,5-tetramethyl1,3,2-dioxaborolane $[(\boldsymbol{S})-1]$

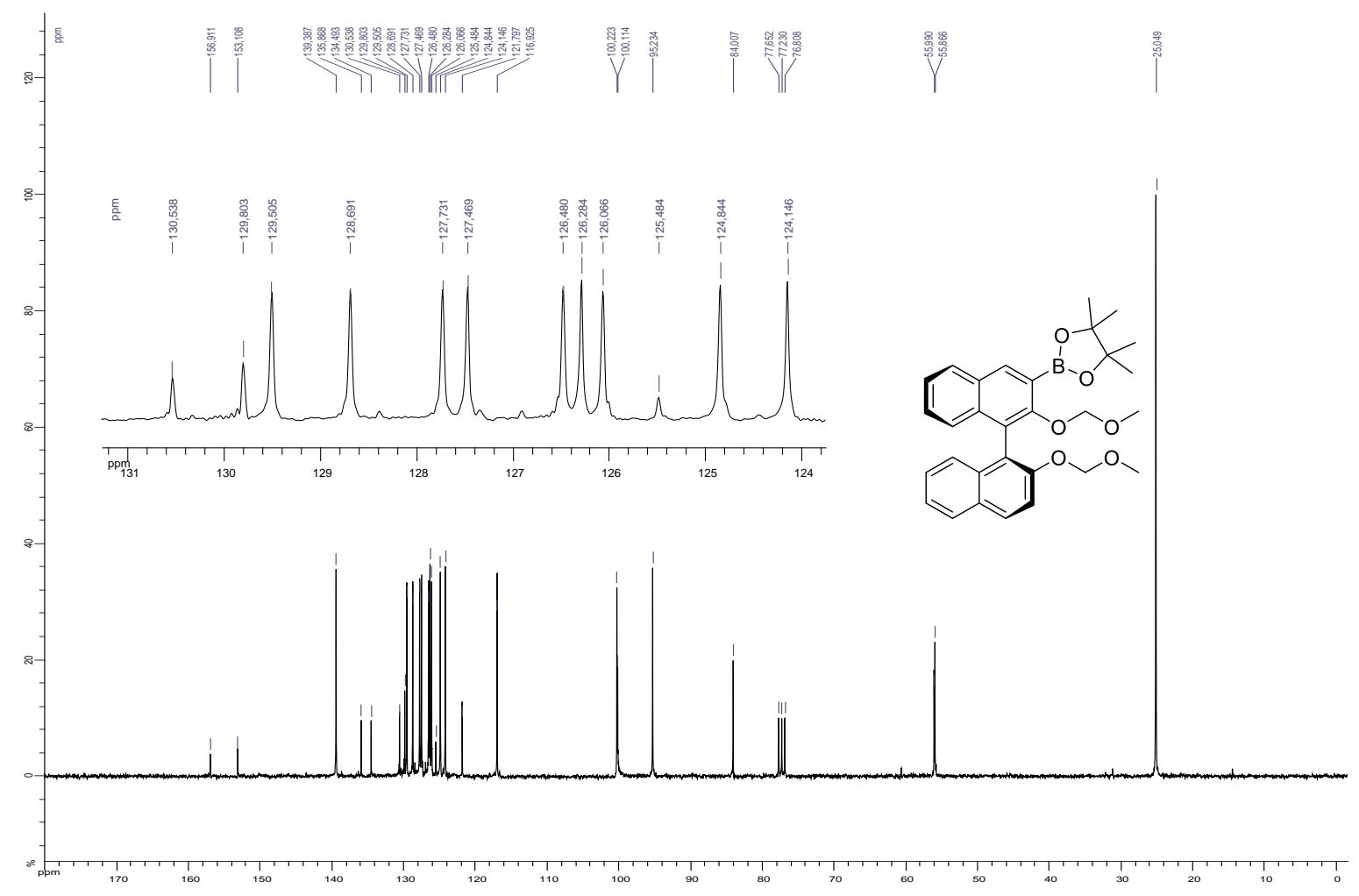

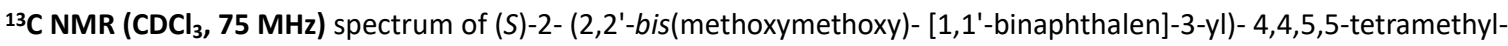
1,3,2-dioxaborolane $[(\boldsymbol{S})-1]$ 


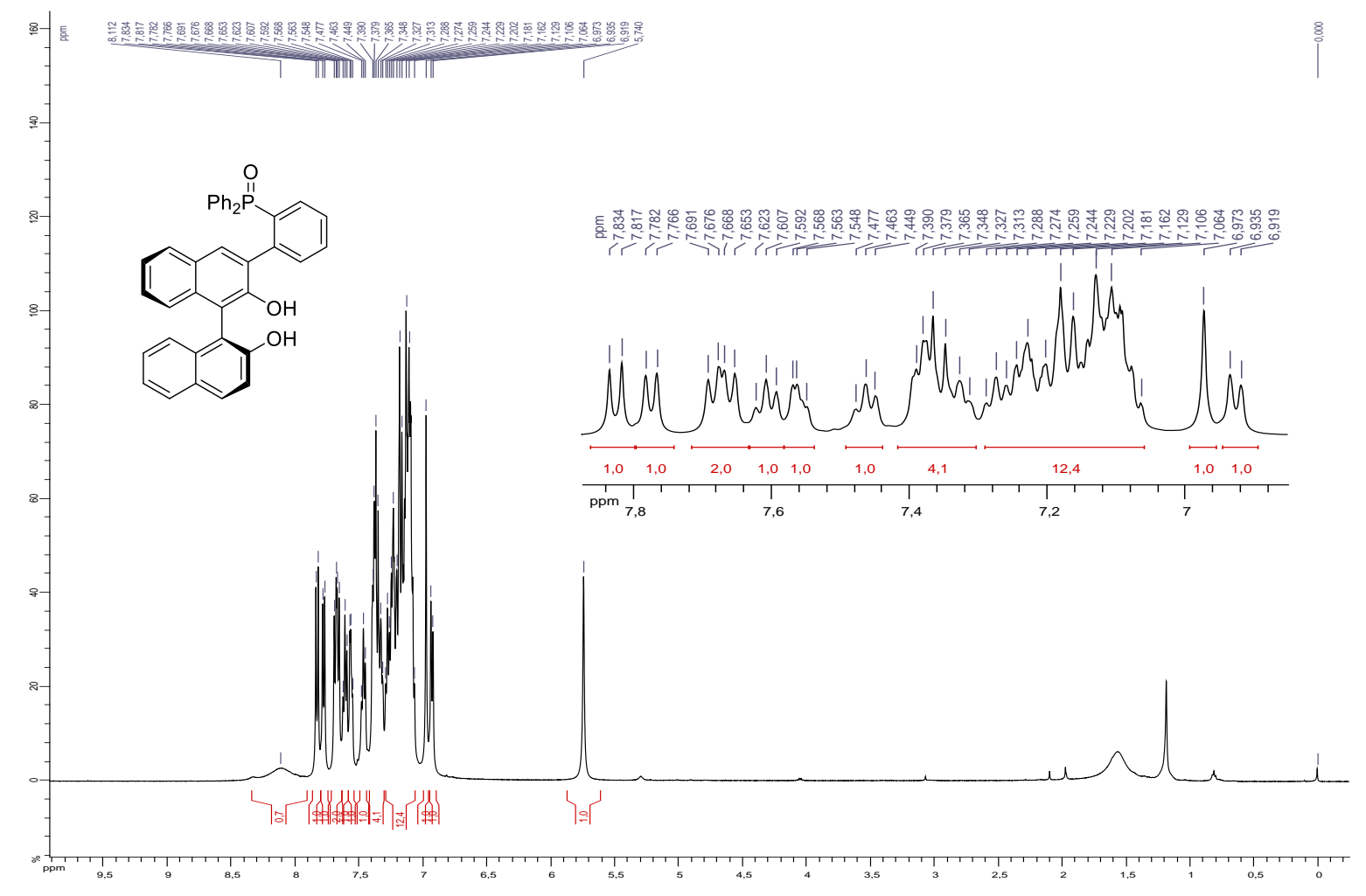

${ }^{1} \mathrm{H}$ NMR $\left(\mathrm{CDCl}_{3}, 500 \mathrm{MHz}\right)$ spectrum of (S)-(2-(2,2'-dihydroxy-[1,1'-binaphthalen]-3-yl)phenyl)diphenylphosphine oxide [(S)2]

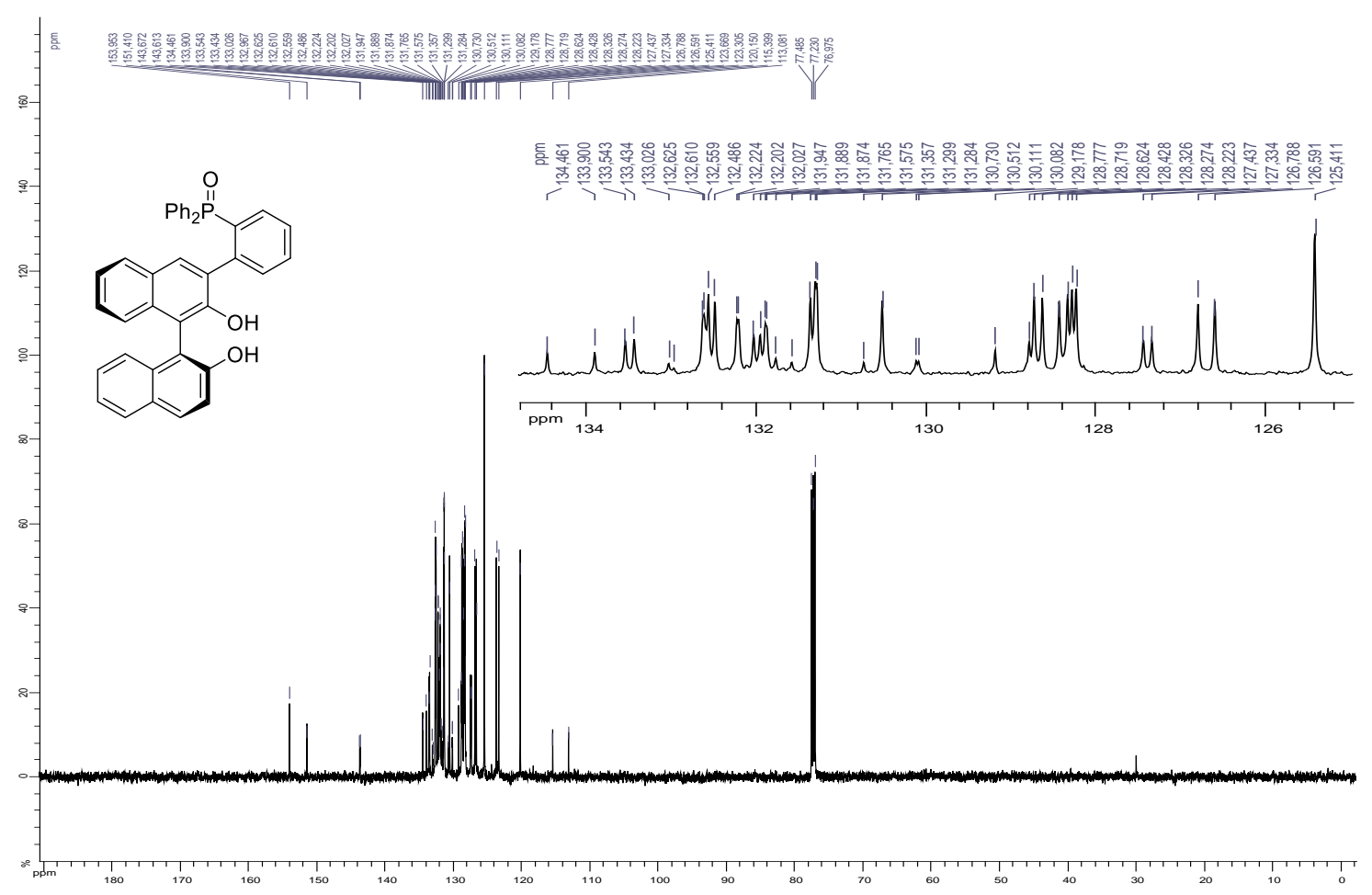

${ }^{13} \mathrm{C} \mathrm{NMR}\left(\mathrm{CDCl}_{3}, 125 \mathrm{MHz}\right)$ spectrum of (S)-(2-(2,2'-dihydroxy-[1,1'-binaphthalen]-3-yl)phenyl)diphenylphosphine oxide [(S)- 


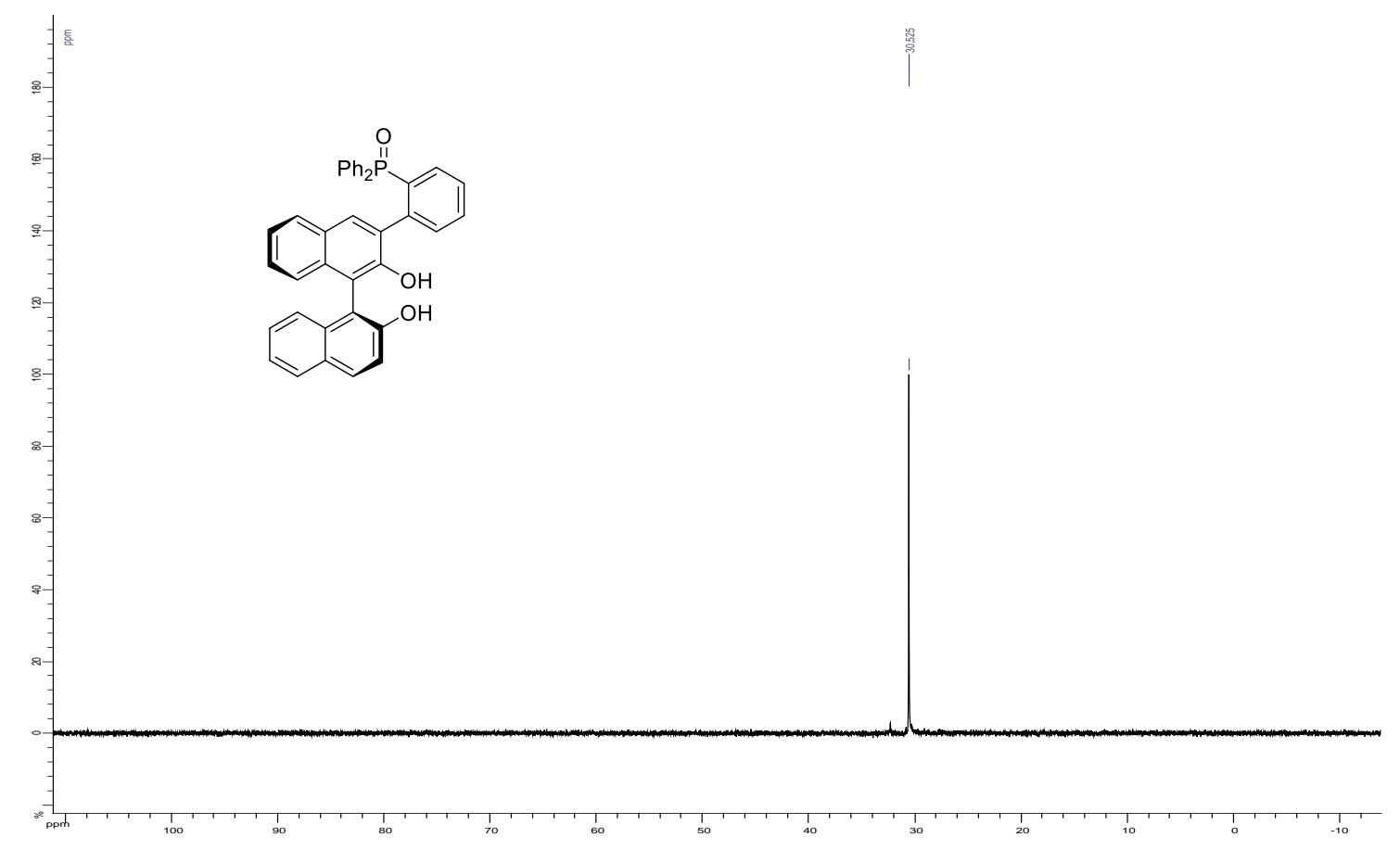

31P NMR ( $\left.\mathrm{CDCl}_{3}, 200 \mathrm{MHz}\right)$ spectrum of (S)-(2-(2,2'-dihydroxy-[1,1'-binaphthalen]-3-yl)phenyl)diphenylphosphine oxide [(S)2]

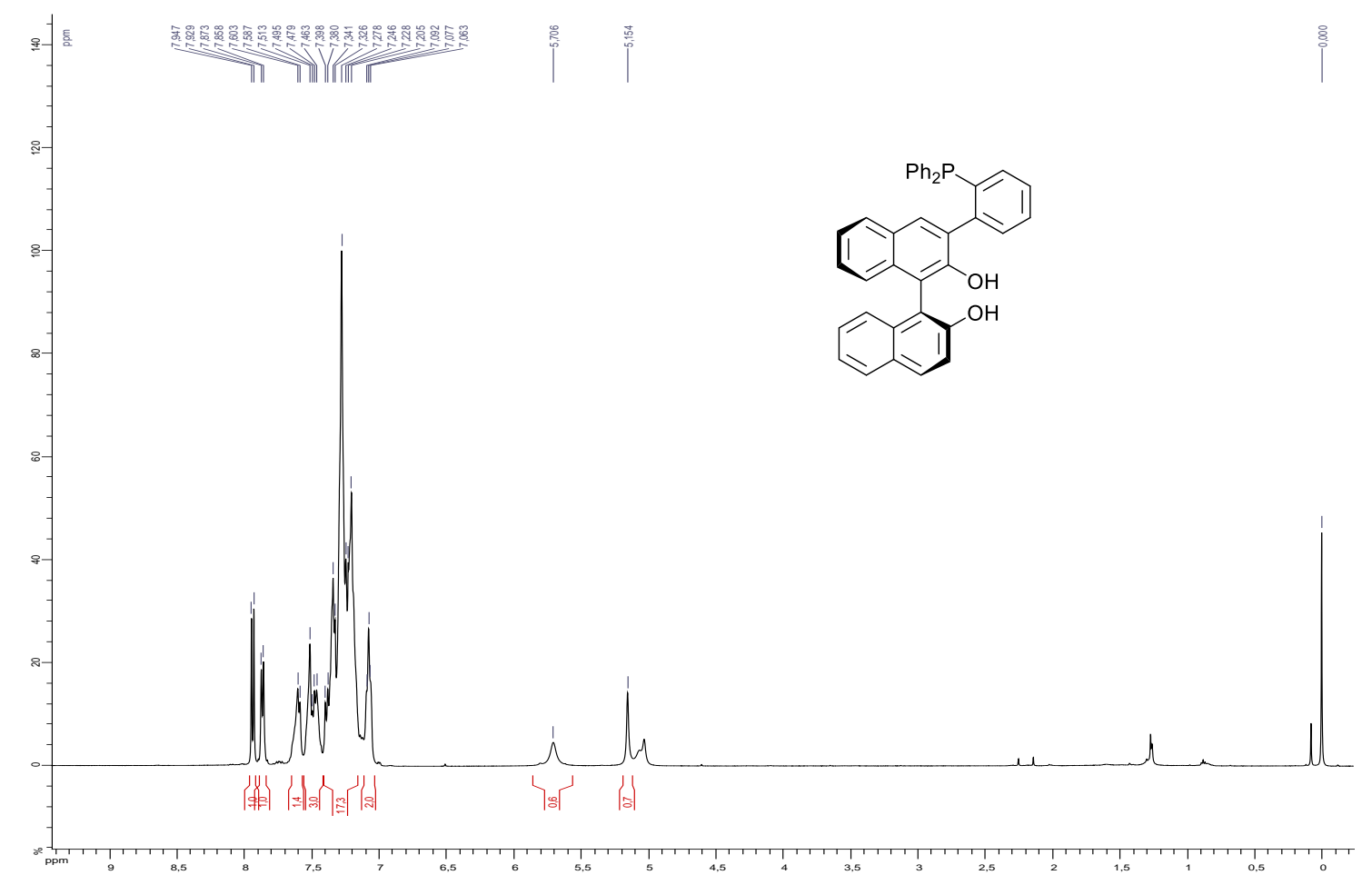

${ }^{1} \mathrm{H}$ NMR $\left(\mathrm{CDCl}_{3}, 500 \mathrm{MHz}\right.$ ) spectrum of (S)-3-(2-(diphenylphosphaneyl)phenyl)-[1,1'-binaphthalene]-2,2'-diol [(S)-3] 


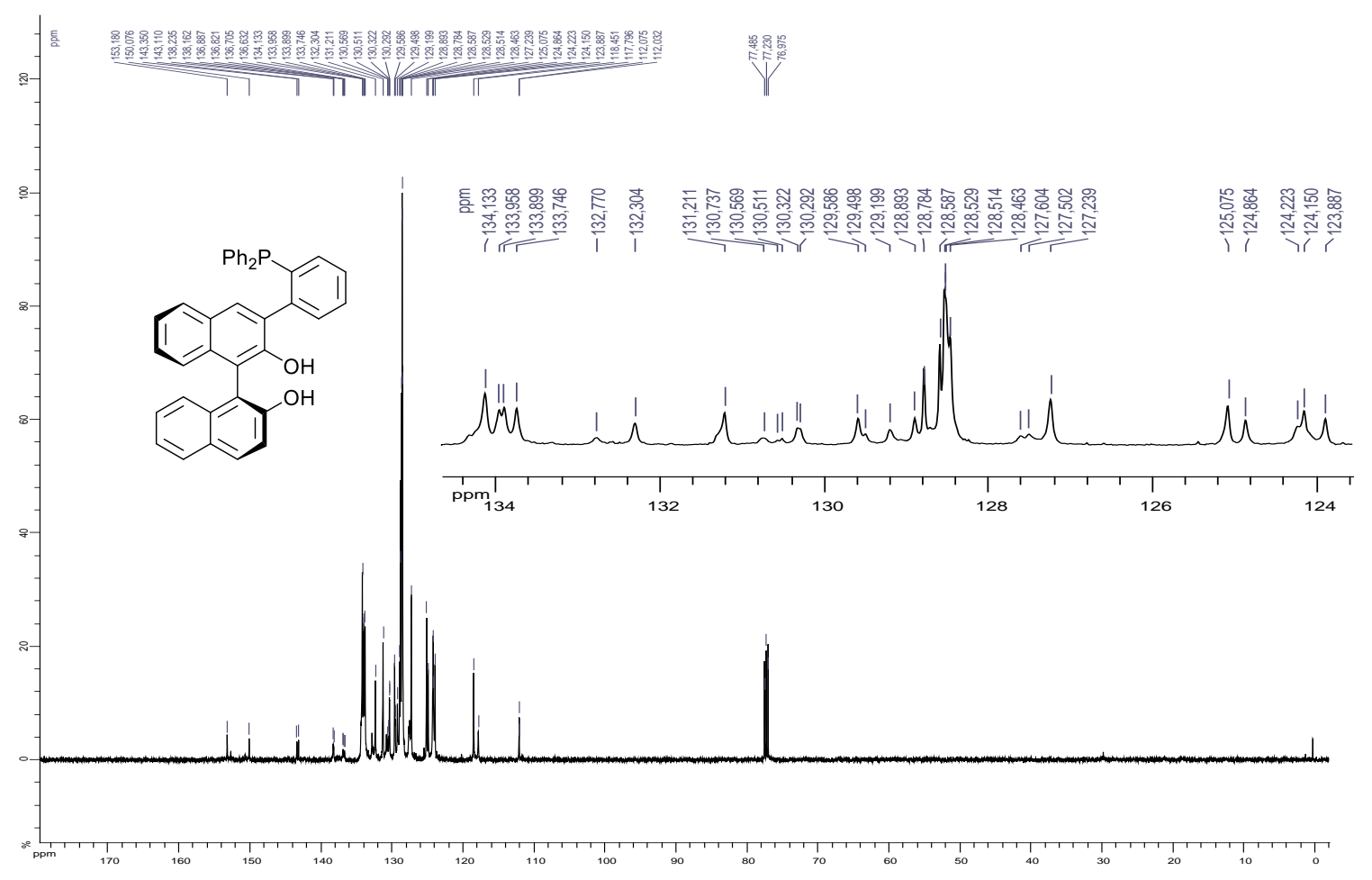

${ }^{13} \mathrm{C} \mathrm{NMR}\left(\mathrm{CDCl}_{3}, 125 \mathrm{MHz}\right.$ ) spectrum of (S)-3-(2-(diphenylphosphaneyl)phenyl)-[1,1'-binaphthalene]-2,2'-diol [(S)-3]

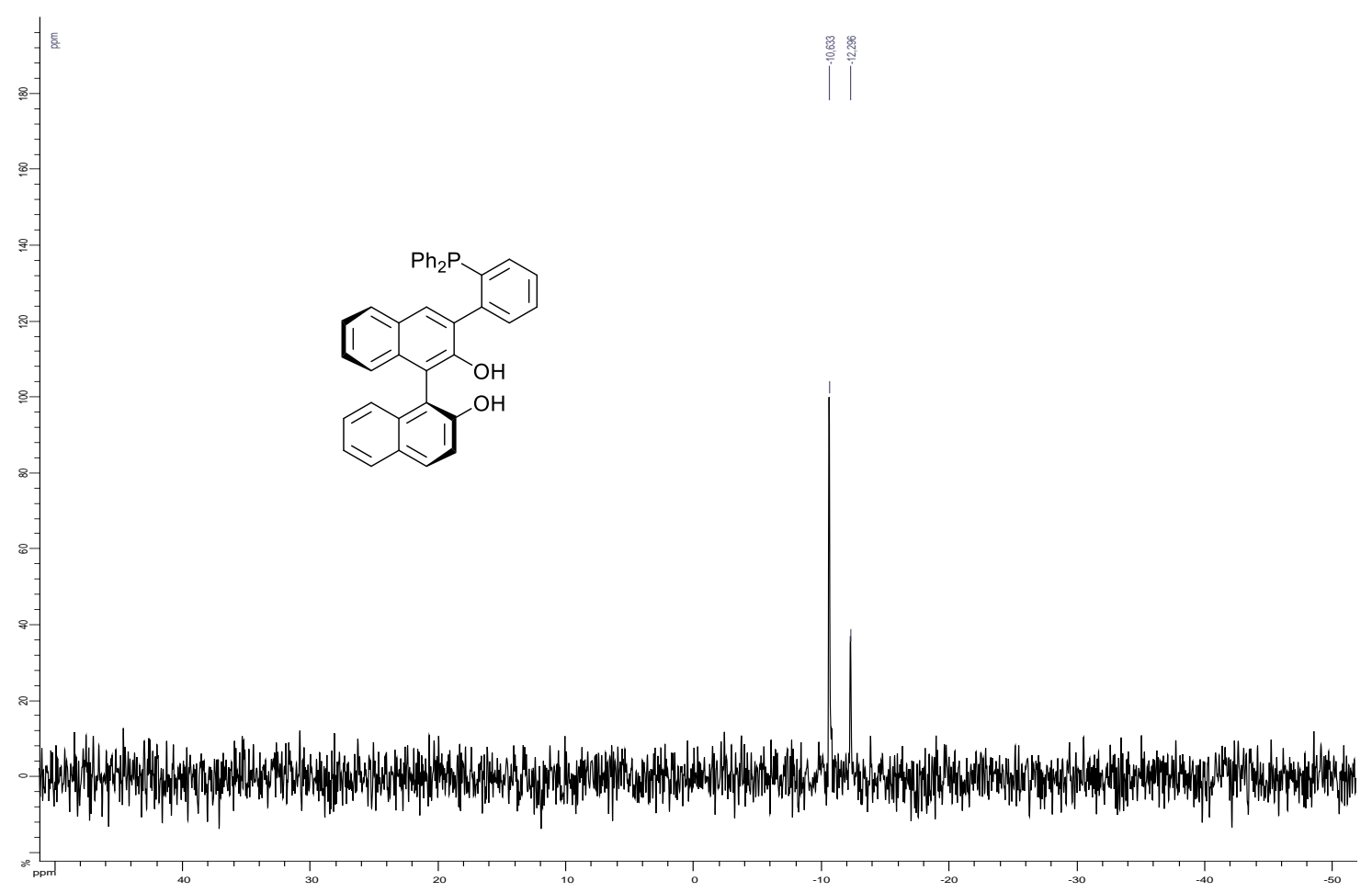

31P NMR ( $\left.\mathrm{CDCl}_{3}, 120 \mathrm{MHz}\right)$ spectrum of (S)-3-(2-(diphenylphosphaneyl)phenyl)-[1,1'-binaphthalene]-2,2'-diol [(S)-3] 


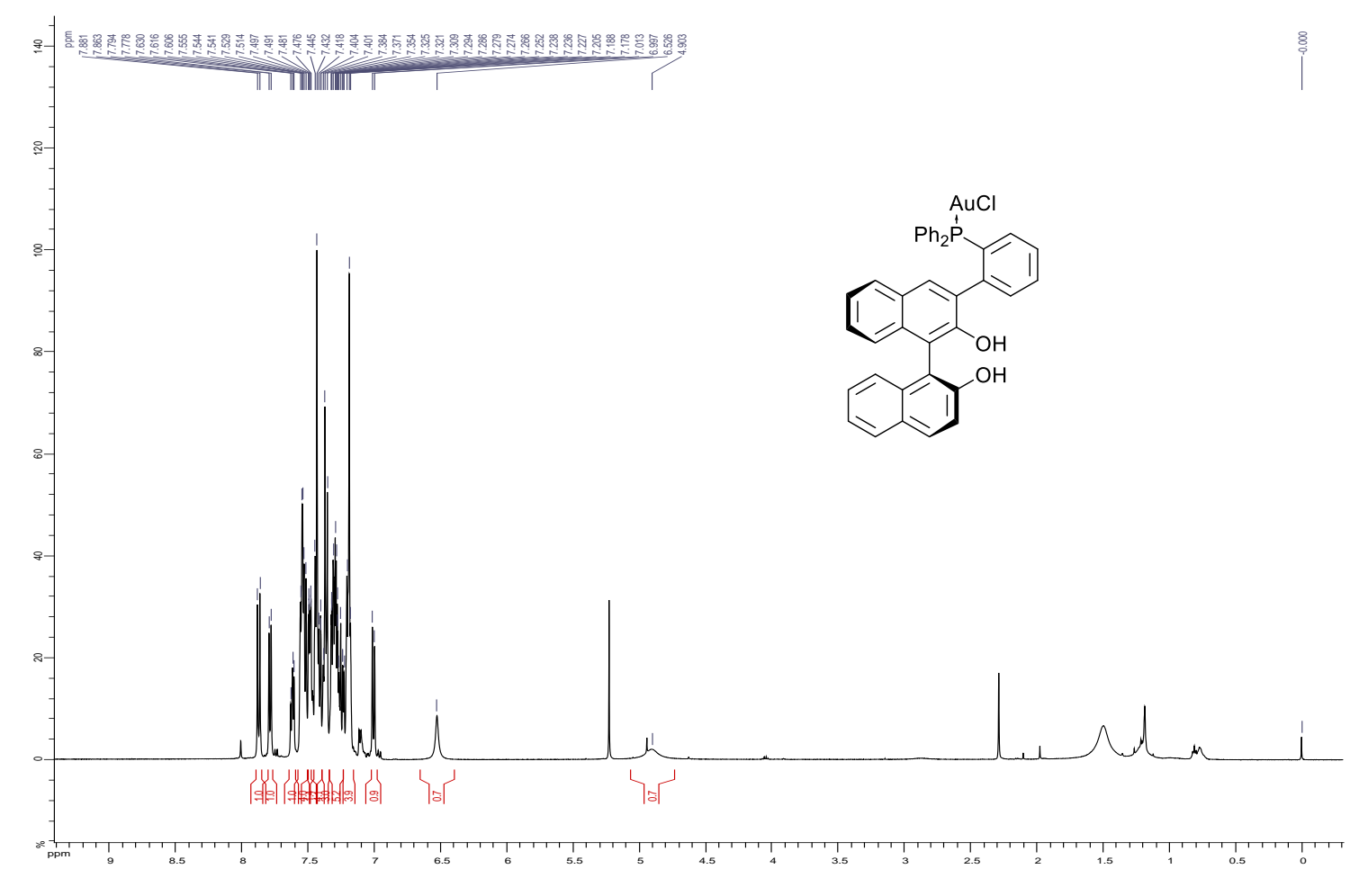

${ }^{1} \mathrm{H}$ NMR $\left(\mathrm{CDCl}_{3}, 500 \mathrm{MHz}\right)$ spectrum of (S)-(2-(2,2'-dihydroxy-[1,1'-binaphthalen]-3-yl)phenyl)diphenylphosphine gold(I) chloride $[(S)-4]$

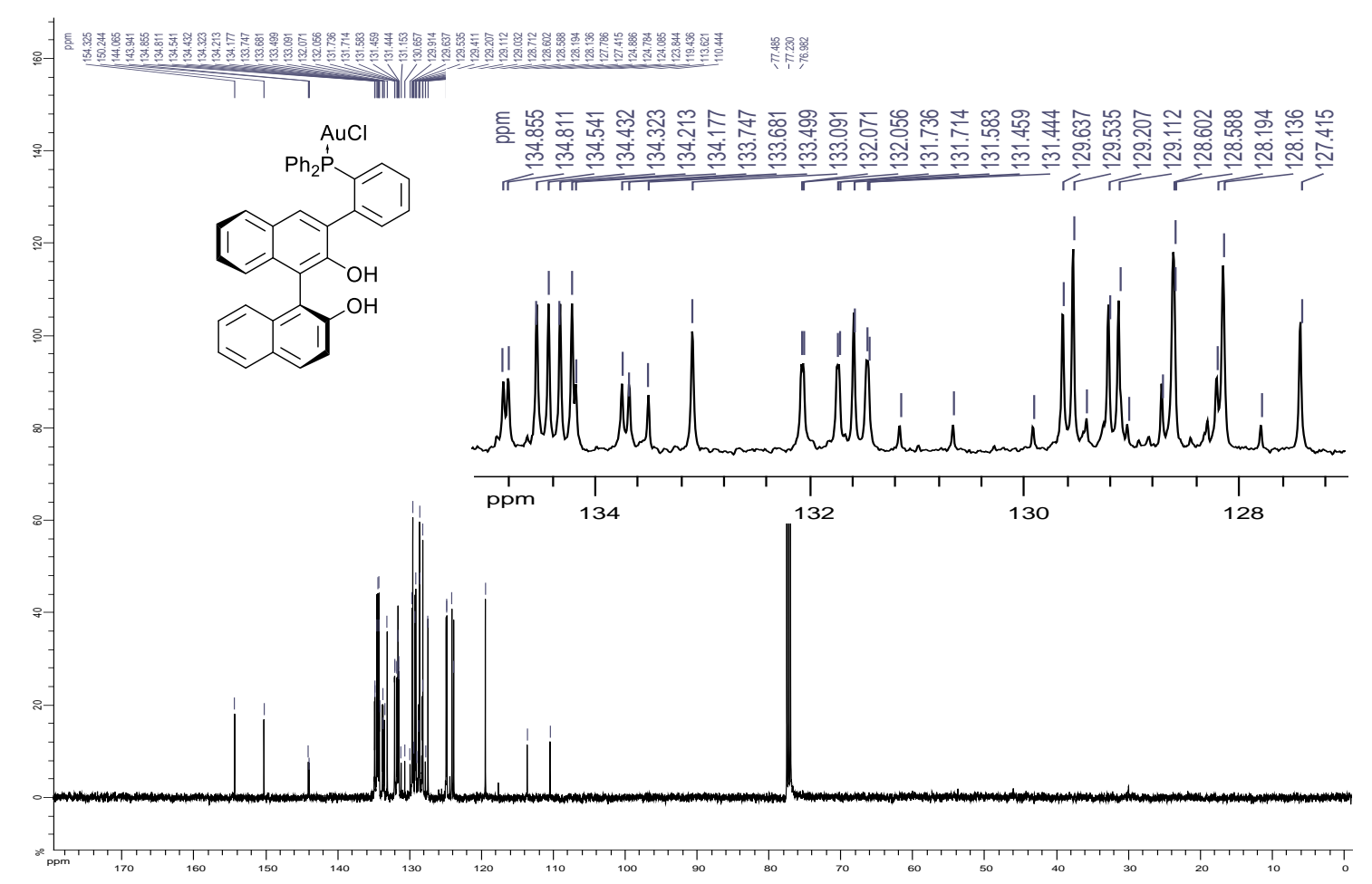

${ }^{13} \mathrm{C} \mathrm{NMR}\left(\mathrm{CDCl}_{3}, 125 \mathrm{MHz}\right)$ spectrum of (S)-(2-(2,2'-dihydroxy-[1,1'-binaphthalen]-3-yl)phenyl)diphenylphosphine gold(I) chloride $[(S)-4]$ 


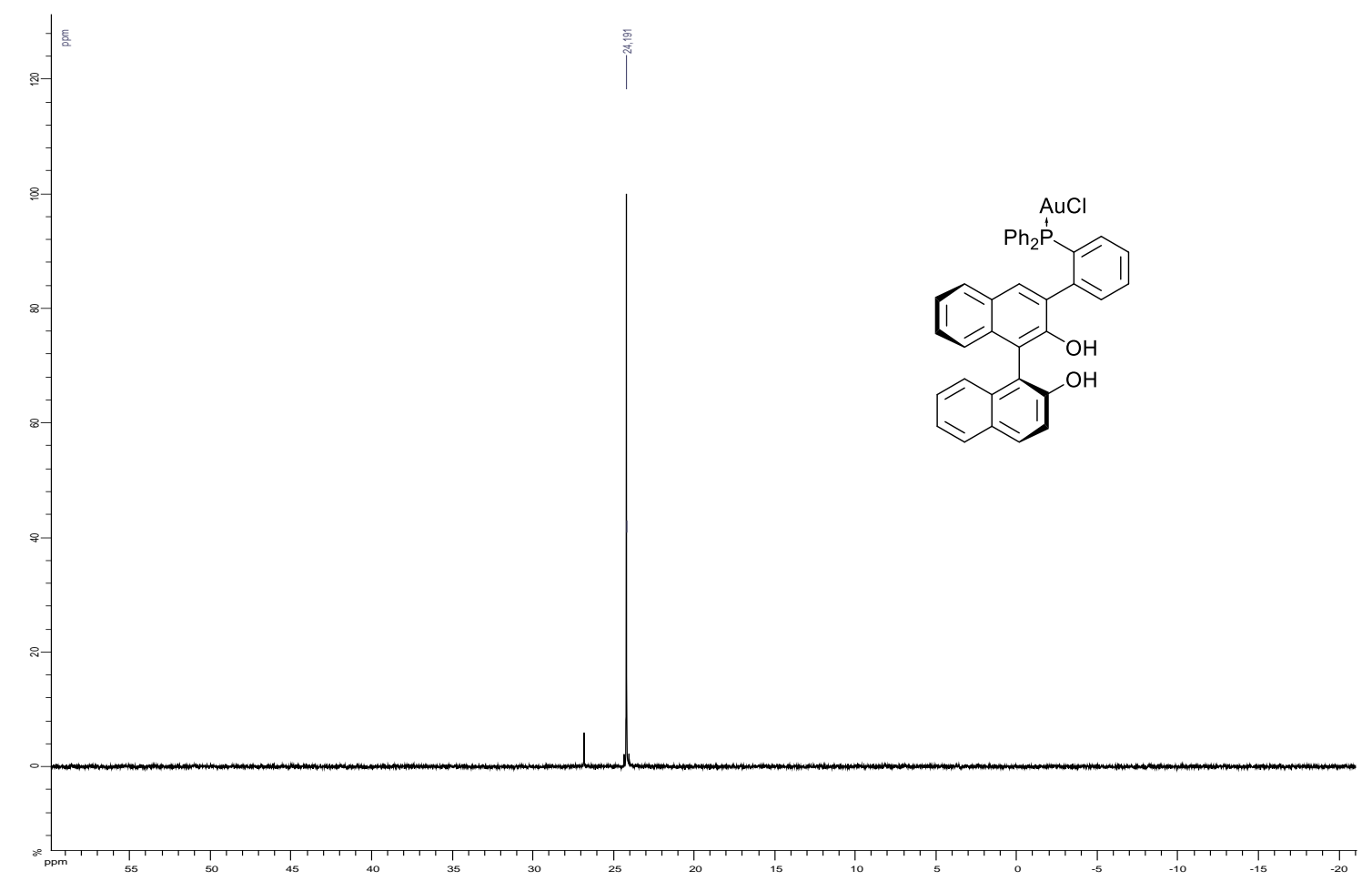

31P NMR ( $\left.\mathrm{CDCl}_{3}, 200 \mathrm{MHz}\right)$ spectrum of (S)-(2-(2,2'-dihydroxy-[1,1'-binaphthalen]-3-yl)phenyl)diphenylphosphine gold(I) chloride $[(S)-4]$ 


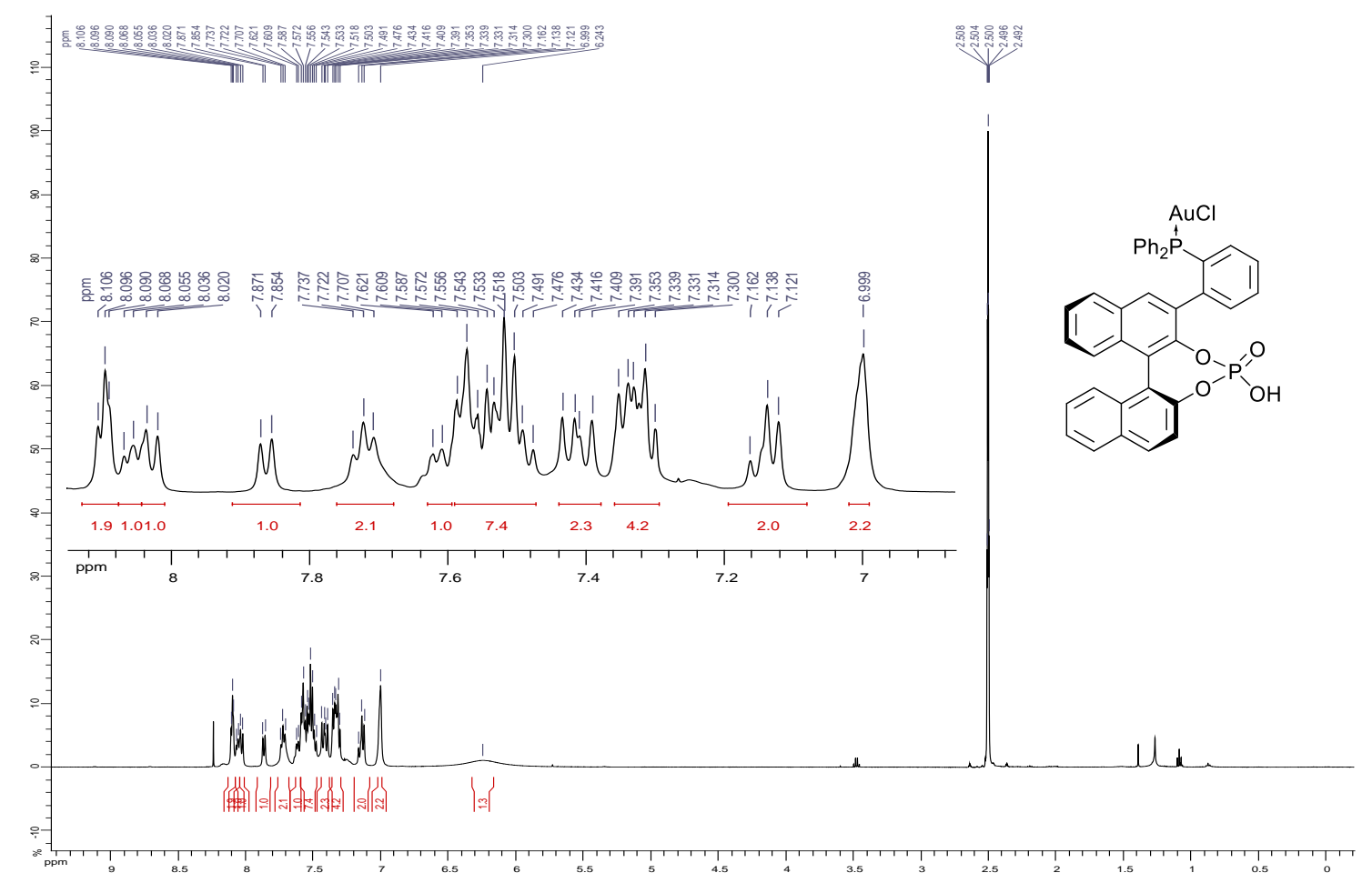

${ }^{1} \mathrm{H}$ NMR (DMSO, 343K, $500 \mathrm{MHz}$ ) spectrum of (S)-(2-(2,2'-diyl hydrogen phosphate-[1,1'-binaphthalen]-3-yl)phenyl) diphenylphosphine gold(I) chloride [(S)-5]

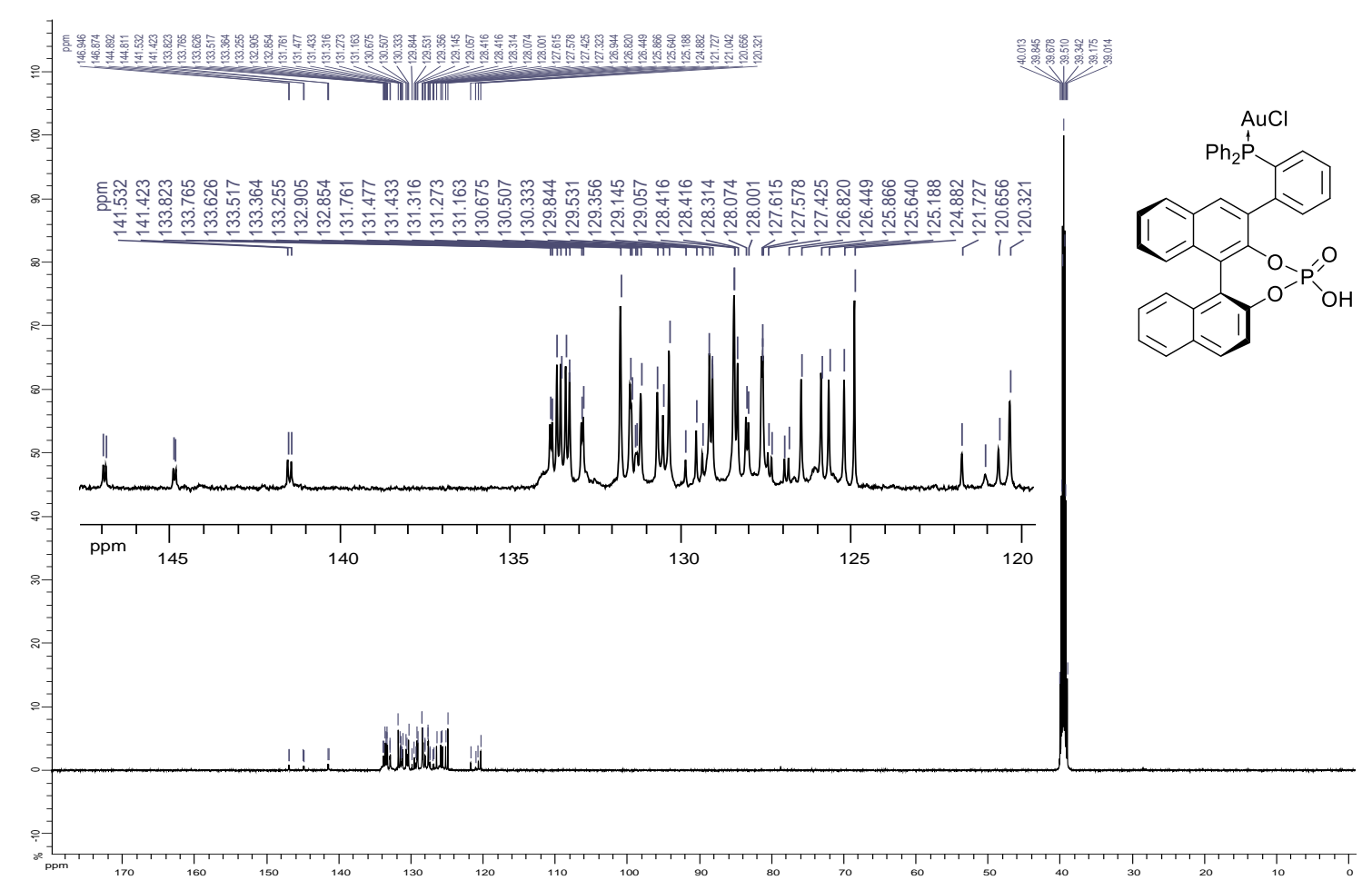

${ }^{13} \mathrm{C}$ NMR (DMSO, 343K, $125 \mathrm{MHz}$ ) spectrum of (S)-(2-(2,2'-diyl hydrogen phosphate-[1,1'-binaphthalen]-3-yl)phenyl) diphenylphosphine gold(I) chloride [(S)-5] 


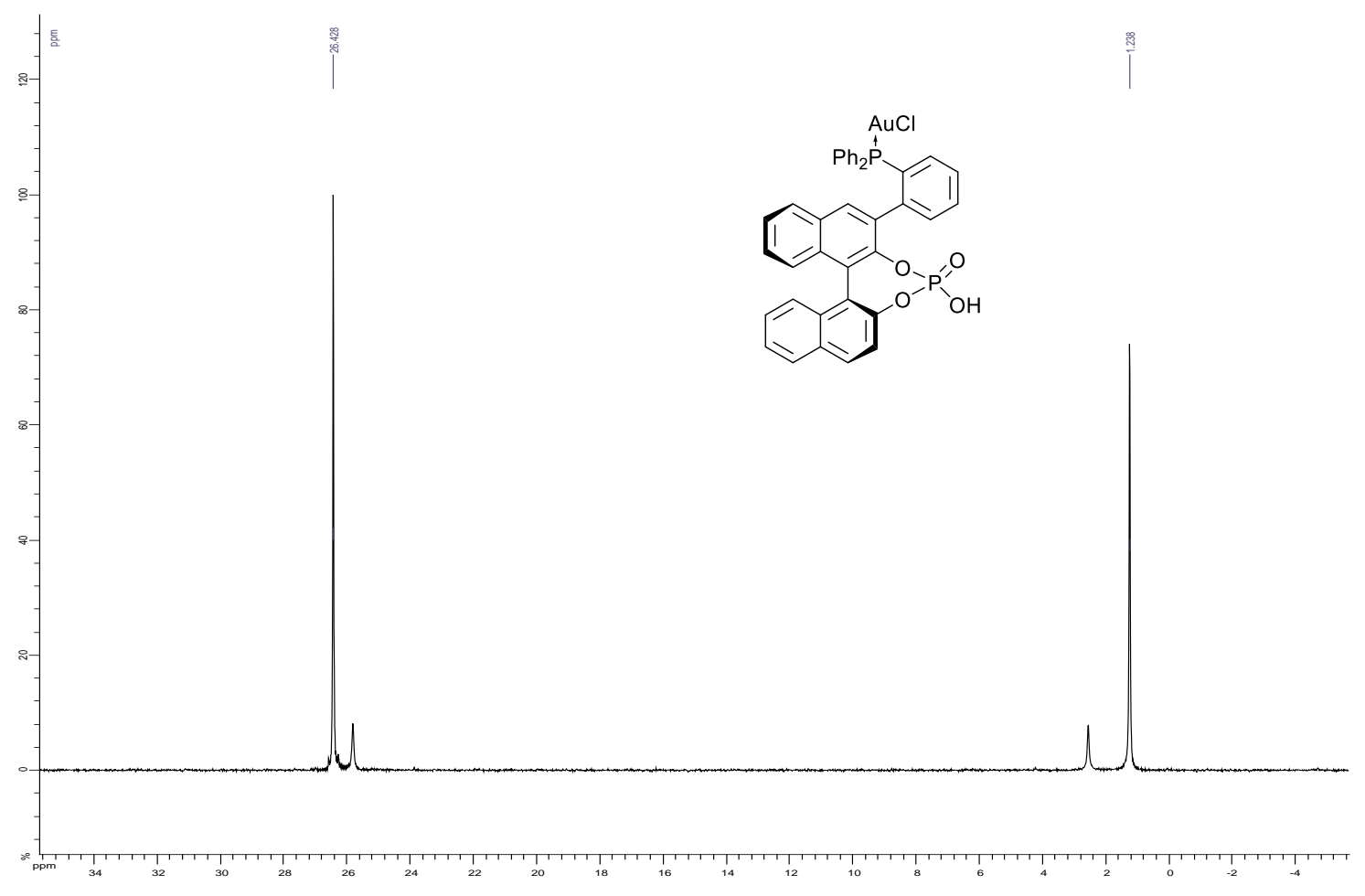

31P NMR (DMSO, 343K, 200 MHz) spectrum of (S)-(2-(2,2'-diyl hydrogen phosphate-[1,1'-binaphthalen]-3-yl)phenyl) diphenylphosphine gold(I) chloride [(S)-5]

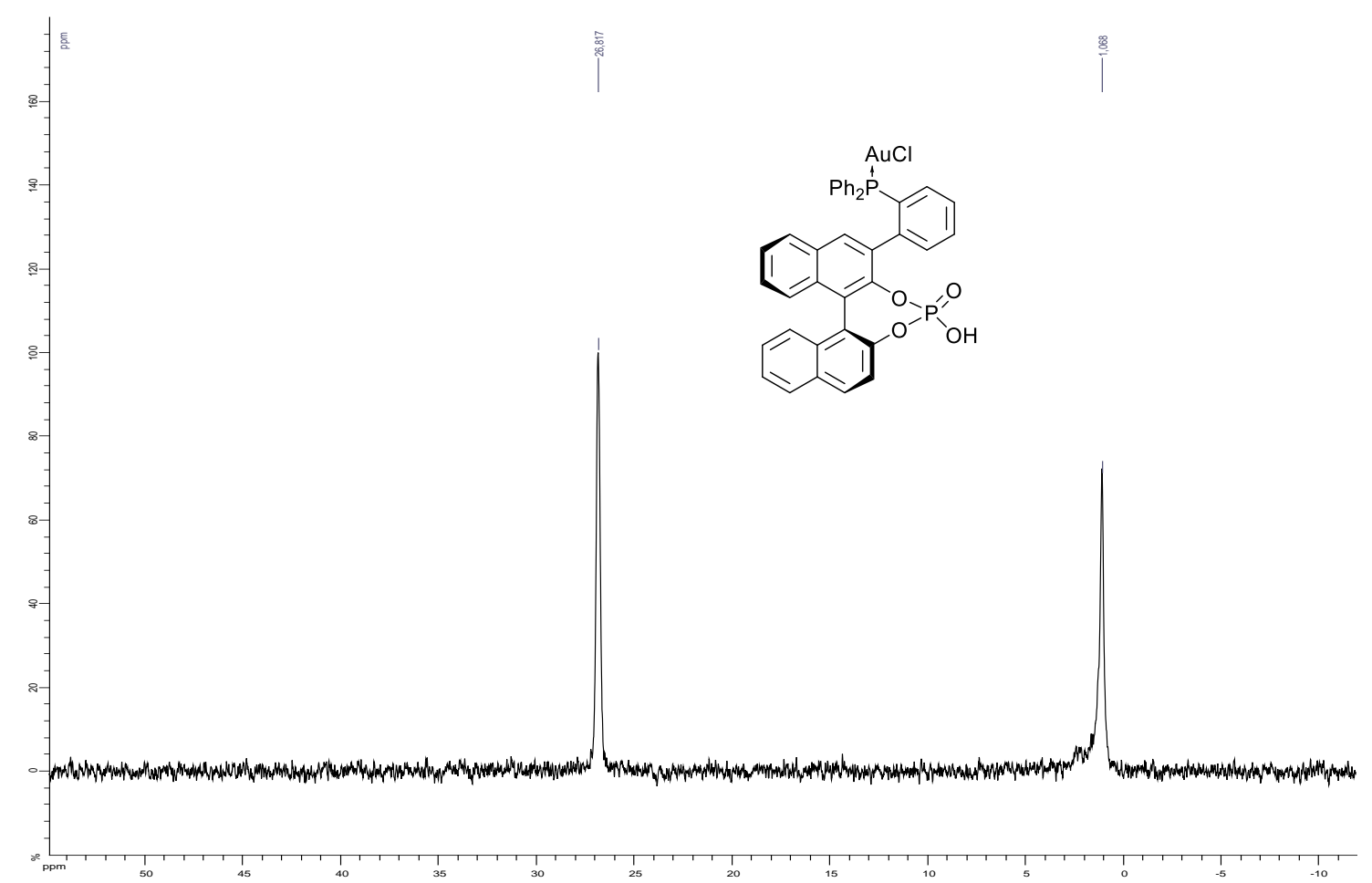

31P NMR (DMSO, 383K, $200 \mathrm{MHz}$ ) spectrum of (S)-(2-(2,2'-diyl hydrogen phosphate-[1,1'-binaphthalen]-3-yl)phenyl) diphenylphosphine gold $(I)$ chloride $[(S)-5]$ 


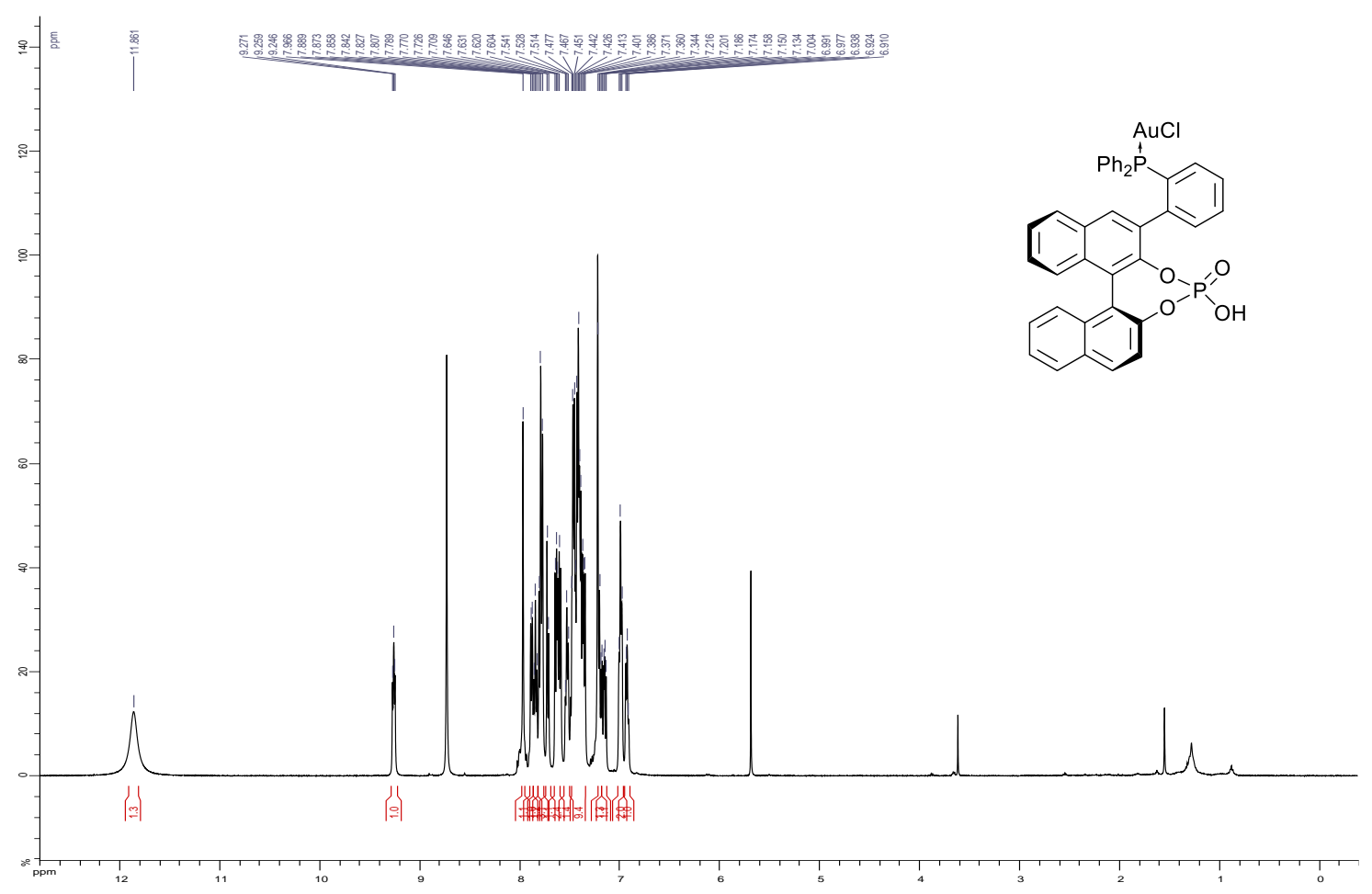

${ }^{1} \mathrm{H}$ NMR (Pyridine, $500 \mathrm{MHz}$ ) spectrum of $(S)$-(2-(2,2'-diyl hydrogen phosphate-[1,1'-binaphthalen]-3-yl)phenyl) diphenylphosphine gold(I) chloride [(S)-5]

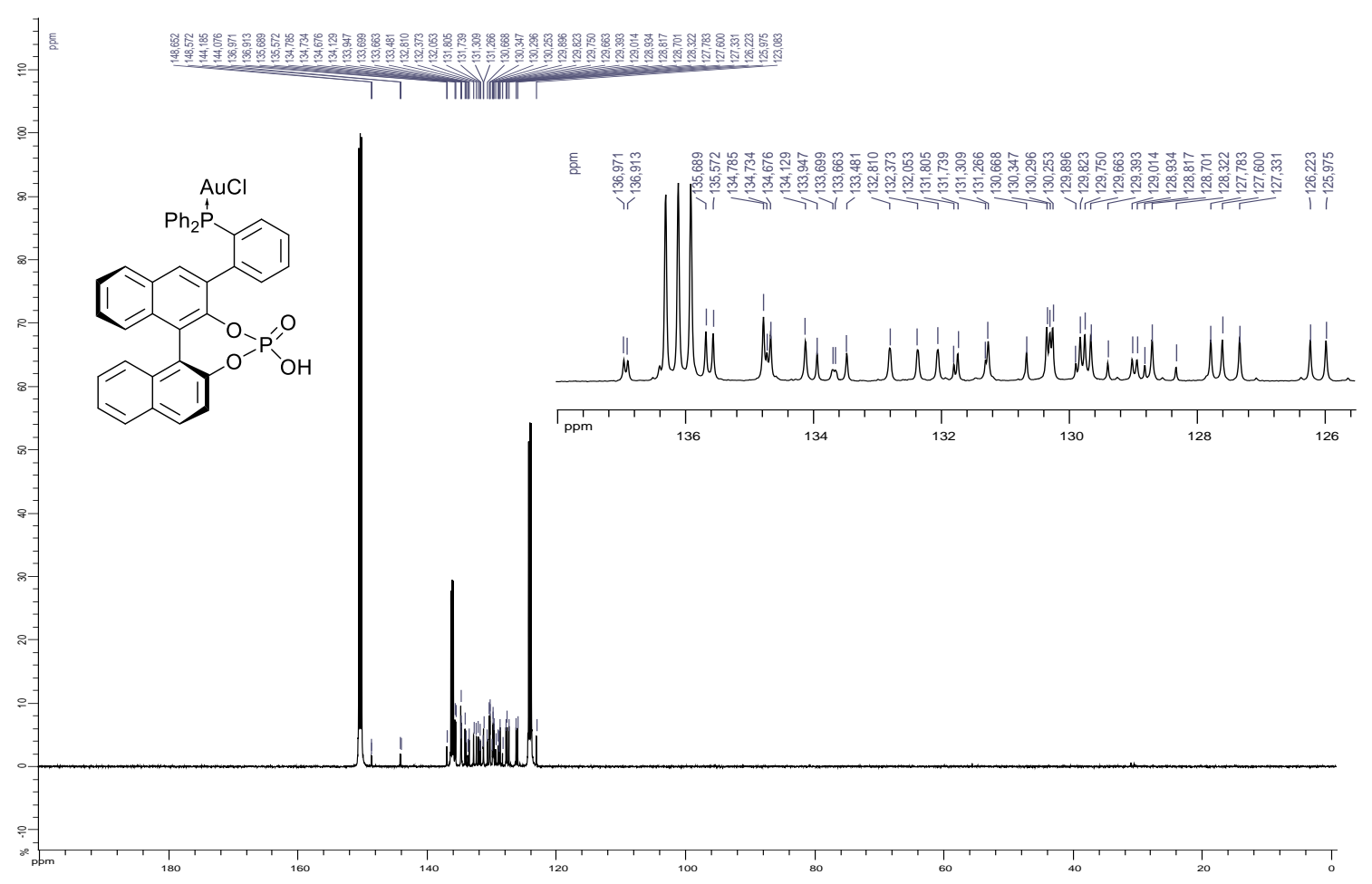

${ }^{13} \mathrm{C}$ NMR (Pyridine, $125 \mathrm{MHz}$ ) spectrum of (S)-(2-(2,2'-diyl hydrogen phosphate-[1,1'-binaphthalen]-3-yl)phenyl) diphenylphosphine gold $(\mathrm{I})$ chloride [(S)-5] 


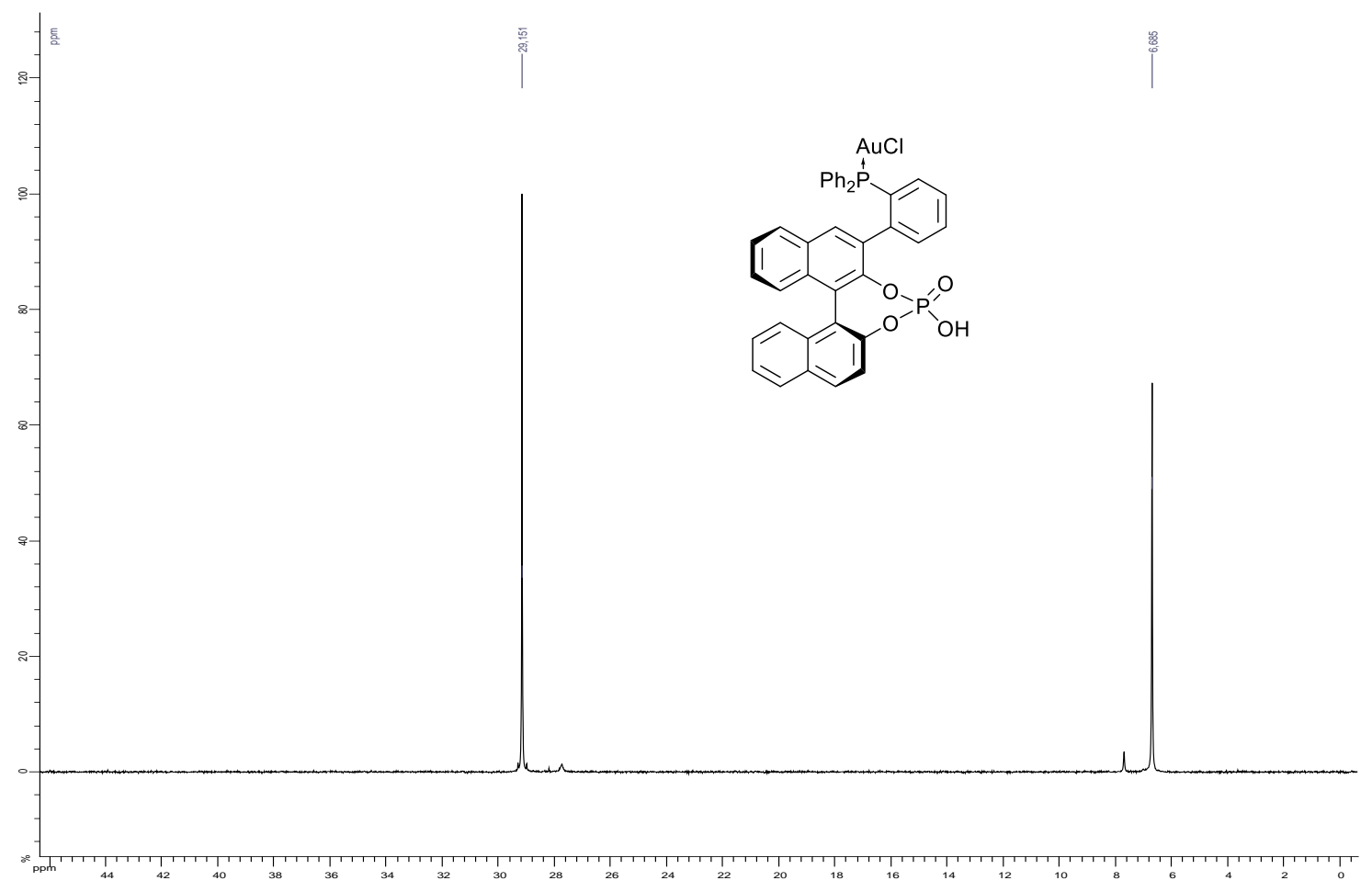

31P NMR (Pyridine, 200 MHz) spectrum of (S)-(2-(2,2'-diyl hydrogen phosphate-[1,1'-binaphthalen]-3-yl)phenyl) diphenylphosphine gold(I) chloride [(S)-5] 


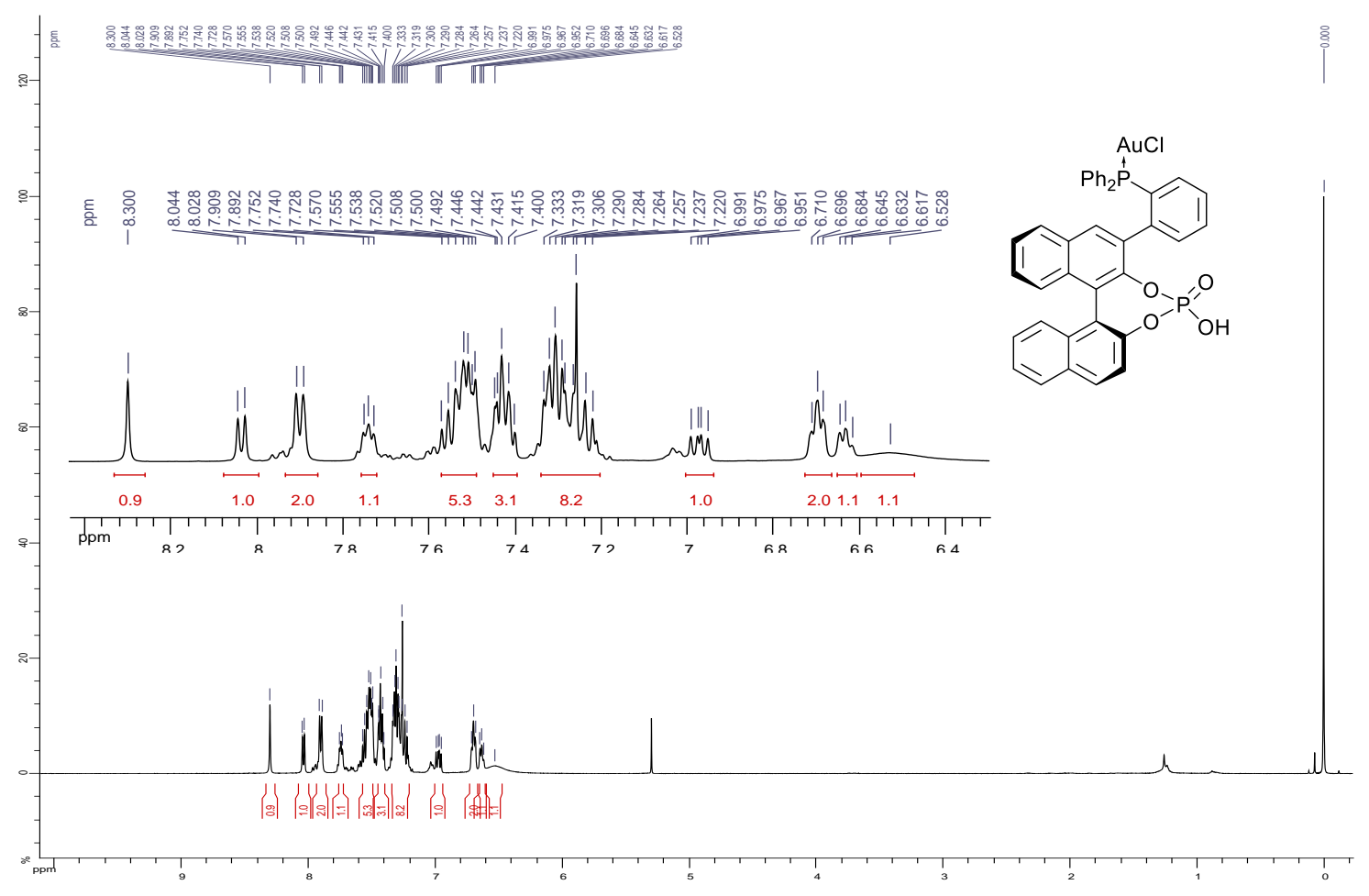

${ }^{1} \mathrm{H}$ NMR ( $\left.\mathrm{CDCl}_{3}, 500 \mathrm{MHz}\right)$ spectrum of (S)-(2-(2,2'-diyl hydrogen phosphate-[1,1'-binaphthalen]-3-yl)phenyl) diphenylphosphine gold(I) chloride [(S)-5]

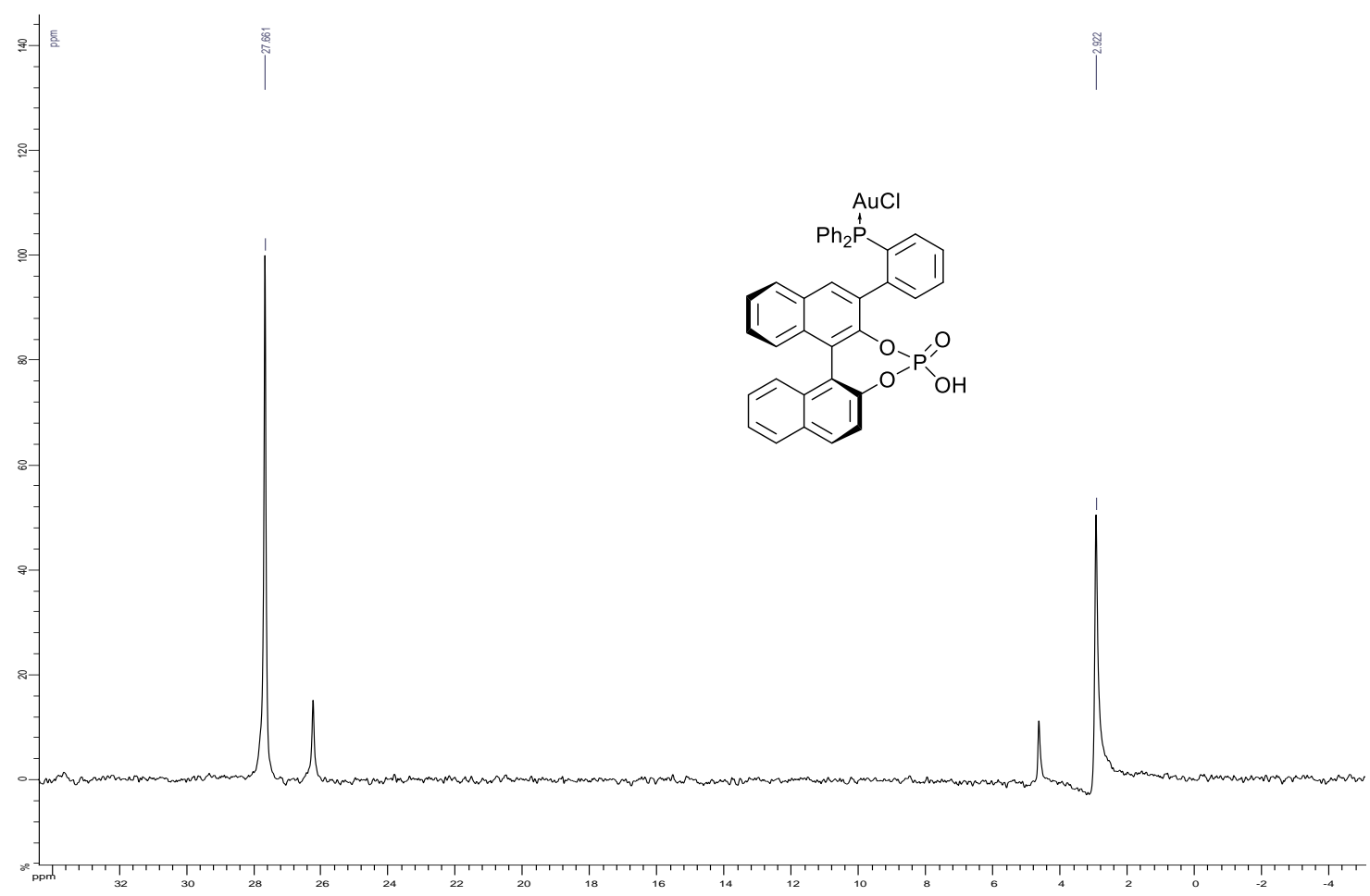

31P NMR ( $\left.\mathrm{CDCl}_{3}, 200 \mathrm{MHz}\right)$ spectrum of (S)-(2-(2,2'-diyl hydrogen phosphate-[1,1'-binaphthalen]-3-yl)phenyl) diphenylphosphine gold(I) chloride [(S)-5] 


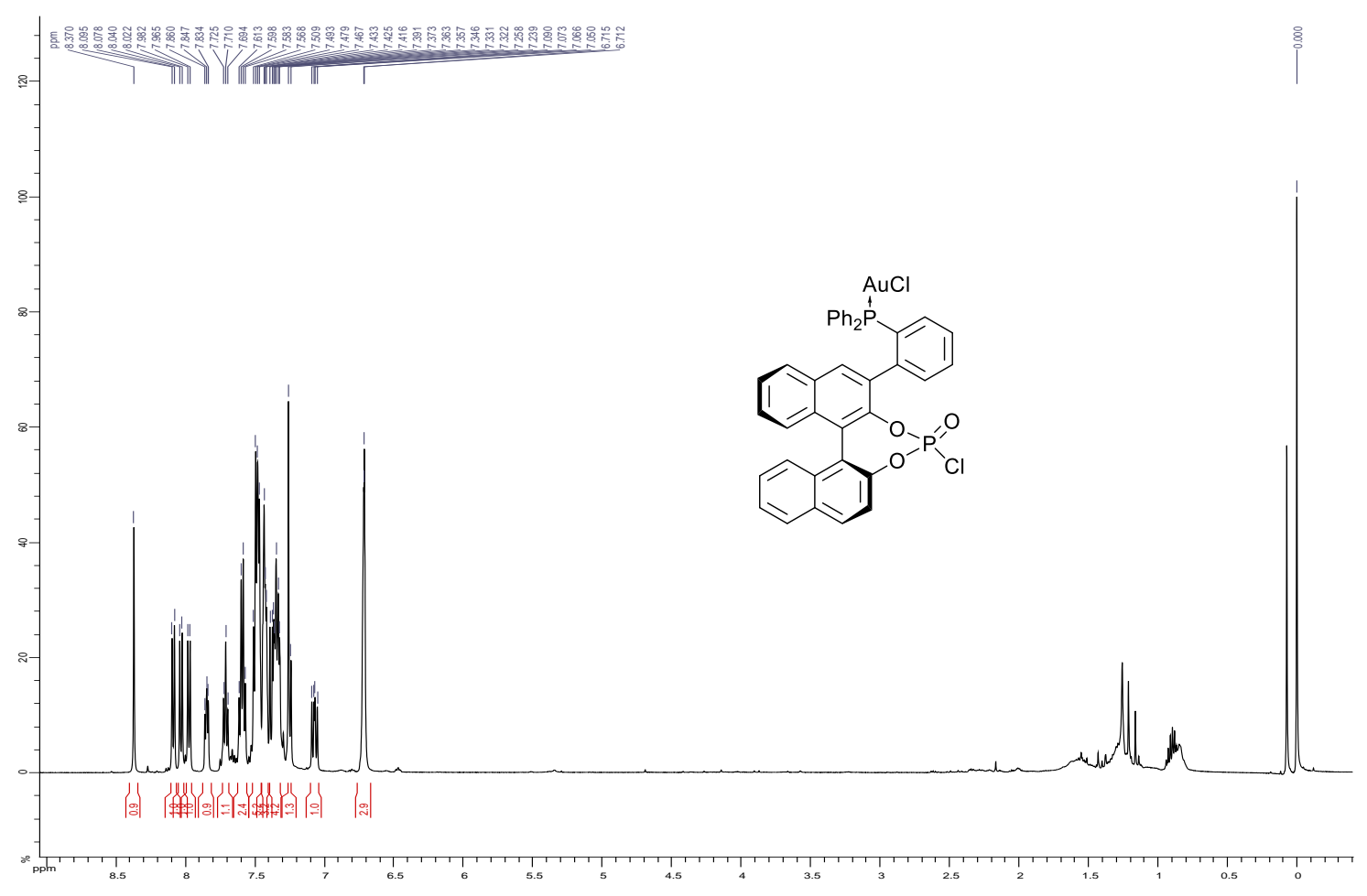

${ }^{1} \mathrm{H}$ NMR $\left(\mathrm{CDCl}_{3}, 500 \mathrm{MHz}\right)$ spectrum of $(S)$-(2-(2,2'-diylchlorogenphosphate-[1,1'-binaphthalen]-3-yl)phenyl) diphenylphosphine gold(I) chloride [SI-1]

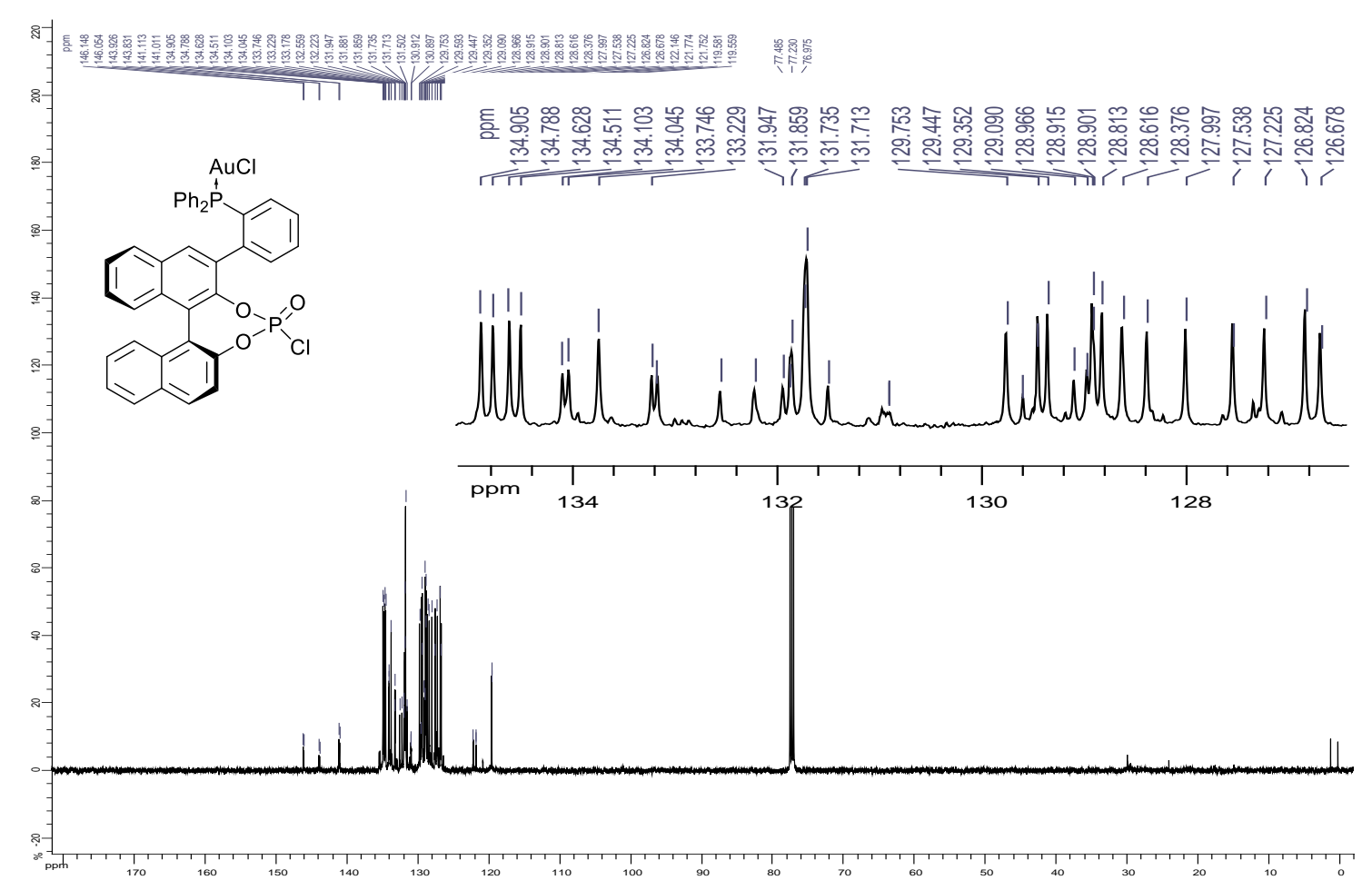

${ }^{13} \mathrm{C} \mathrm{NMR}\left(\mathrm{CDCl}_{3}, 500 \mathrm{MHz}\right)$ spectrum of (S)-(2-(2,2'-diylchlorogenphosphate-[1,1'-binaphthalen]-3-yl)phenyl) diphenylphosphine gold(I) chloride [SI-1] 


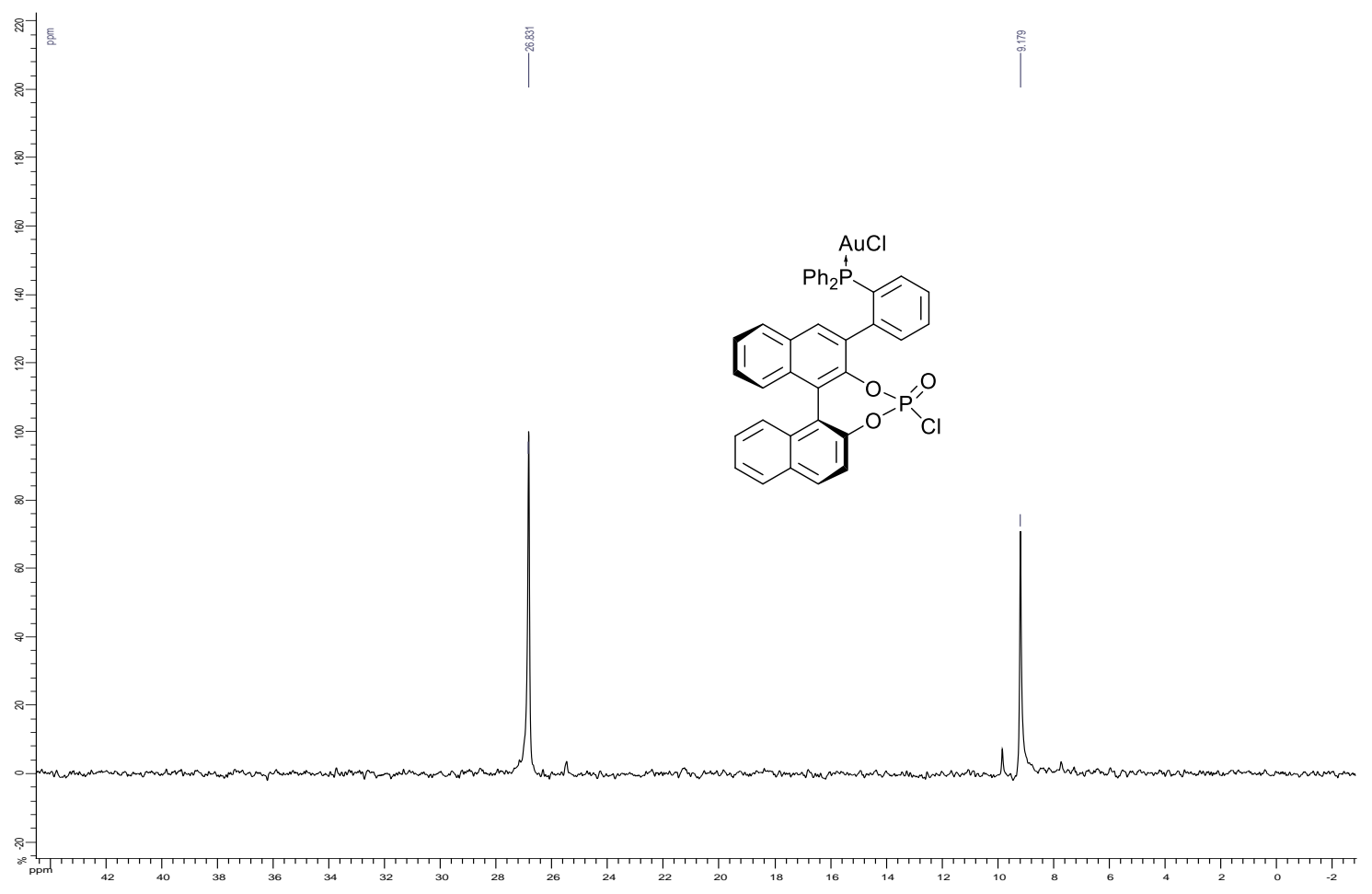

31P NMR ( $\left.\mathrm{CDCl}_{3}, 200 \mathrm{MHz}\right)$ spectrum of (S)-(2-(2,2'-diylchlorogenphosphate-[1,1'-binaphthalen]-3-yl)phenyl) diphenylphosphine gold(I) chloride $[(\boldsymbol{S})-5 \mathbf{b}]$ 


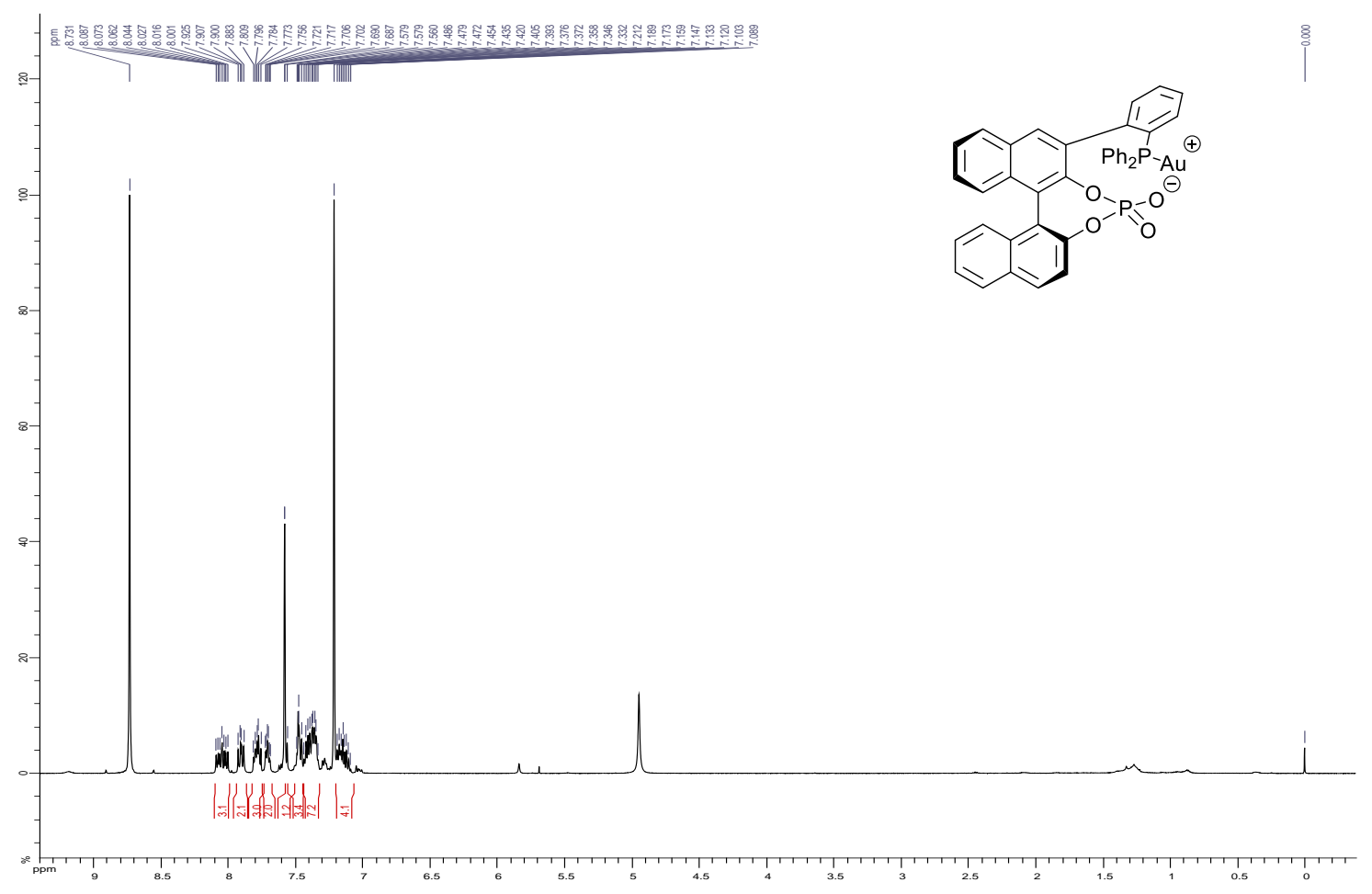

${ }^{1} \mathrm{H}$ NMR (Pyridine, $500 \mathrm{MHz}$ ) spectrum of $(S)-6$

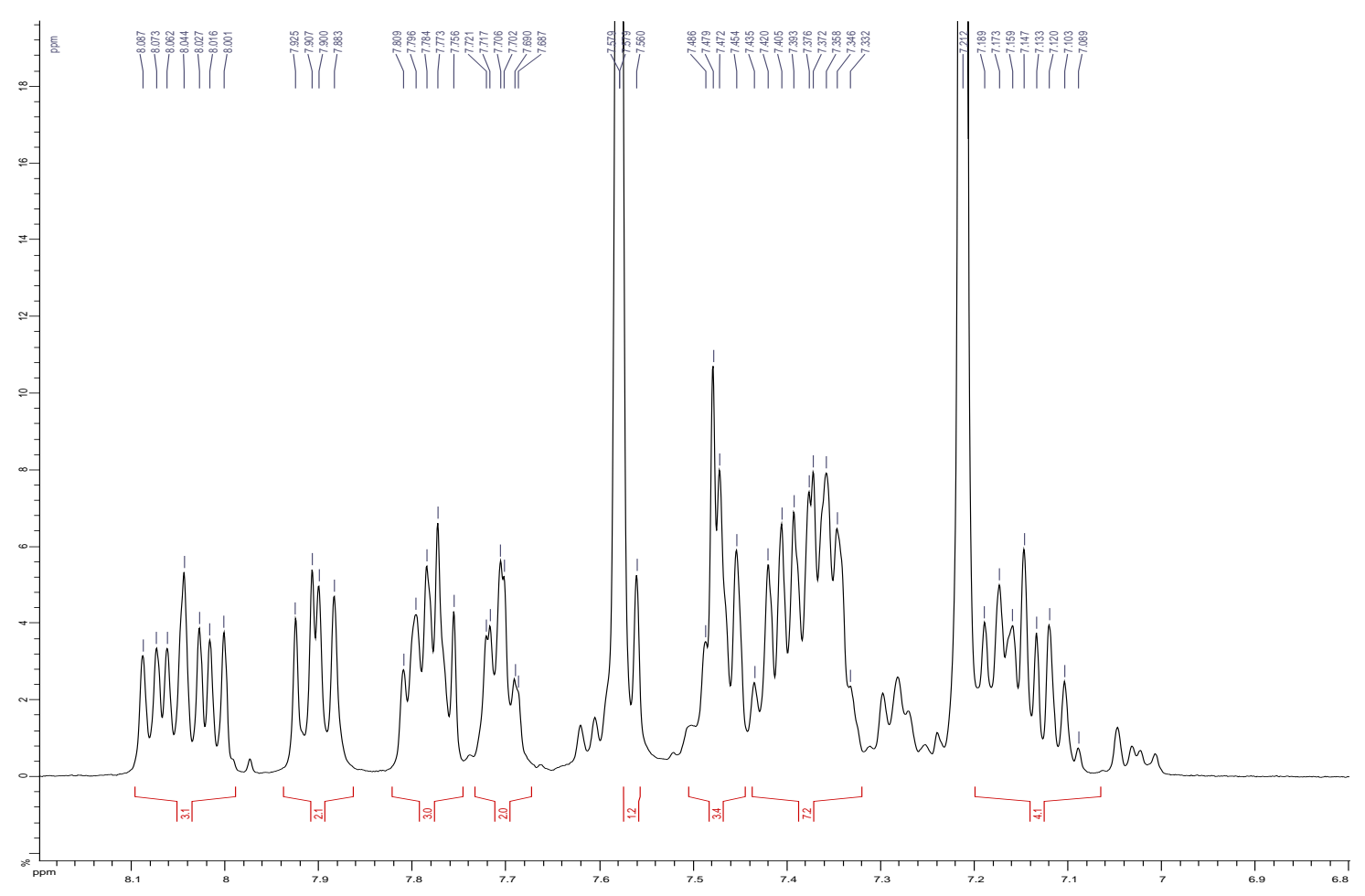

${ }^{1} \mathrm{H}$ NMR (Pyridine, $500 \mathrm{MHz}$ ) spectrum of (S)-6 (zoom 6.8-8.2 ppm) 


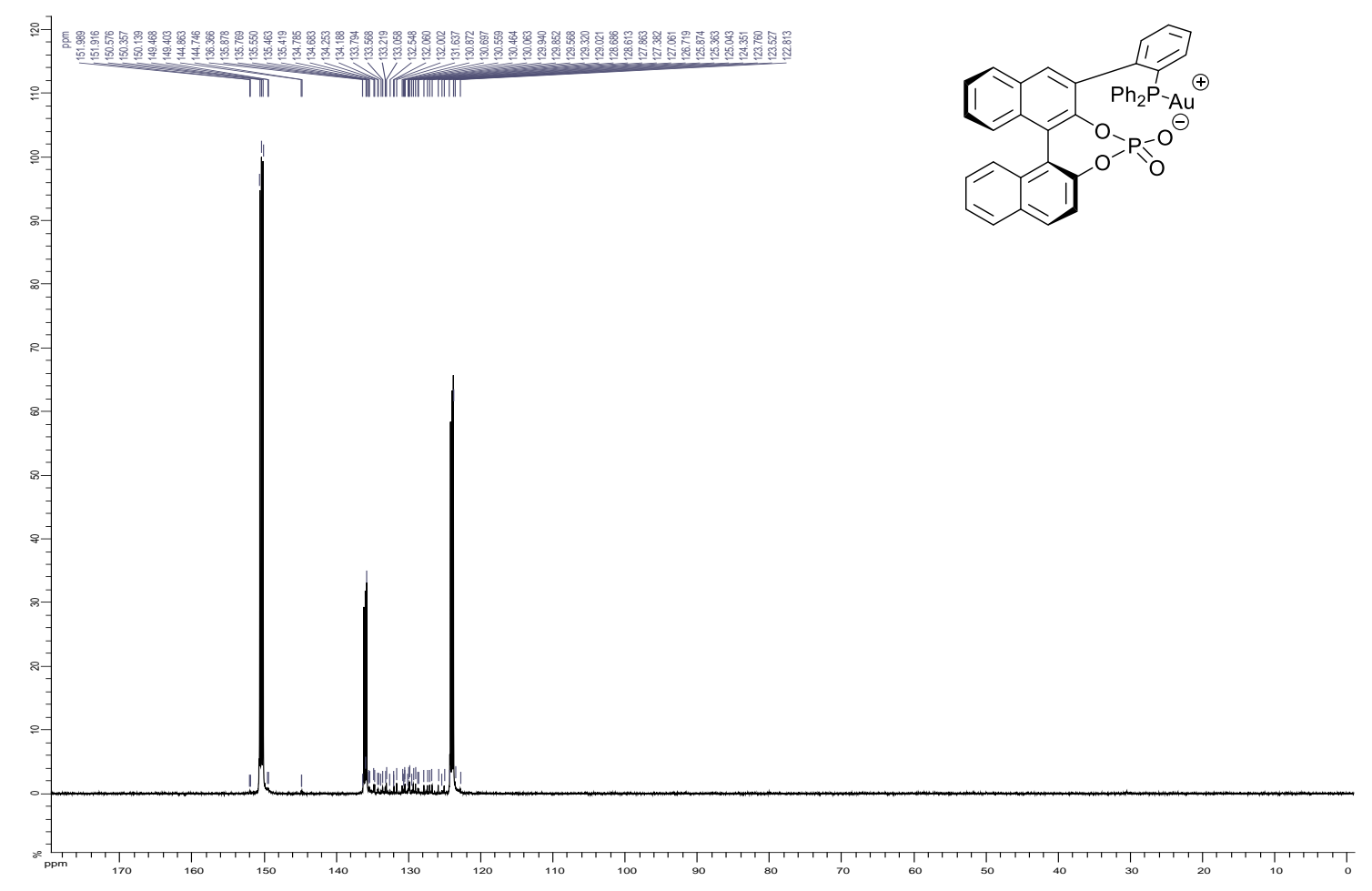

${ }^{13} \mathrm{C}$ NMR (Pyridine, $125 \mathrm{MHz}$ ) spectrum of $(S)-6$

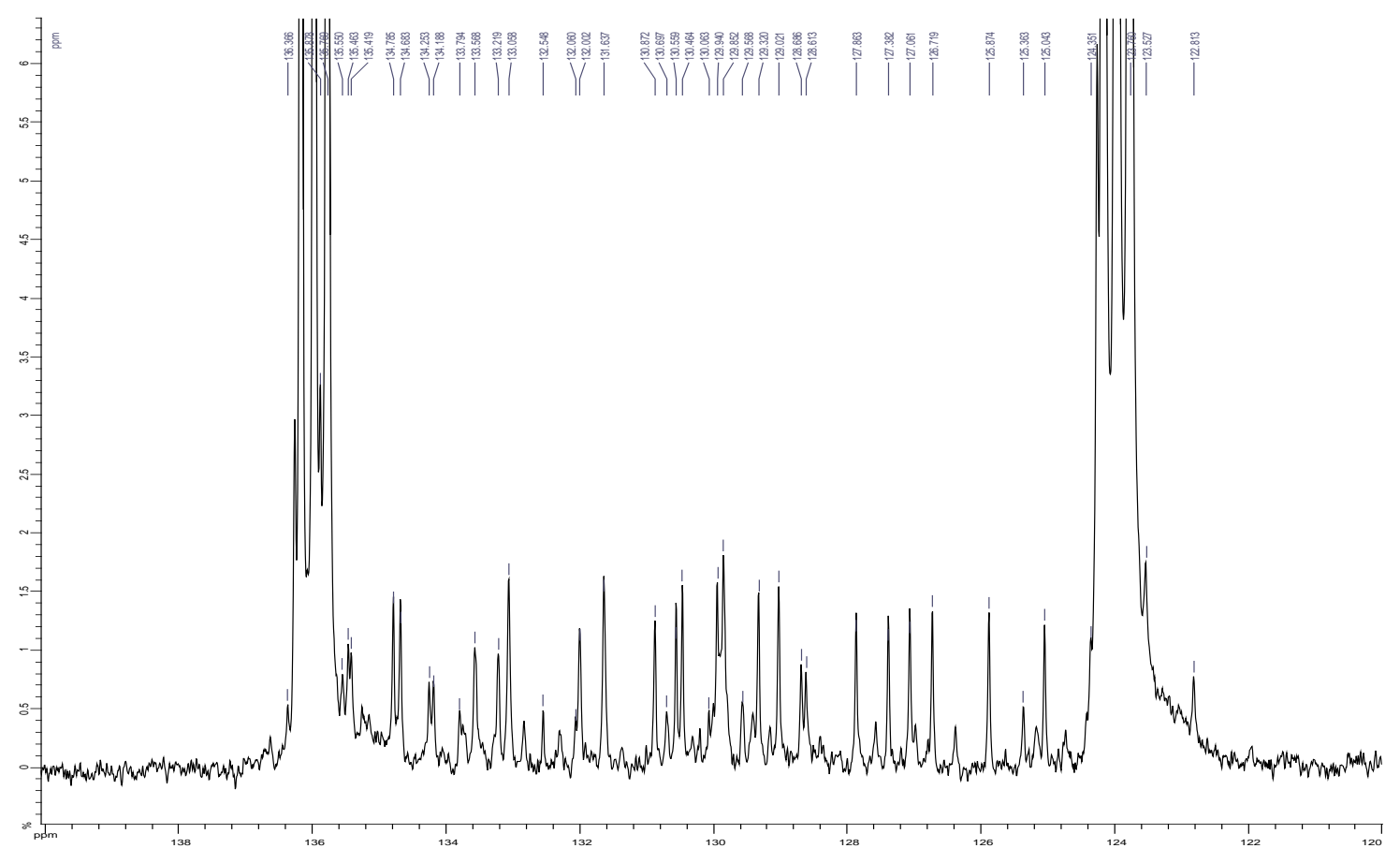

${ }^{13}$ C NMR (Pyridine, 125 MHz) spectrum of (S)-6 (zoom 120-140 ppm) 


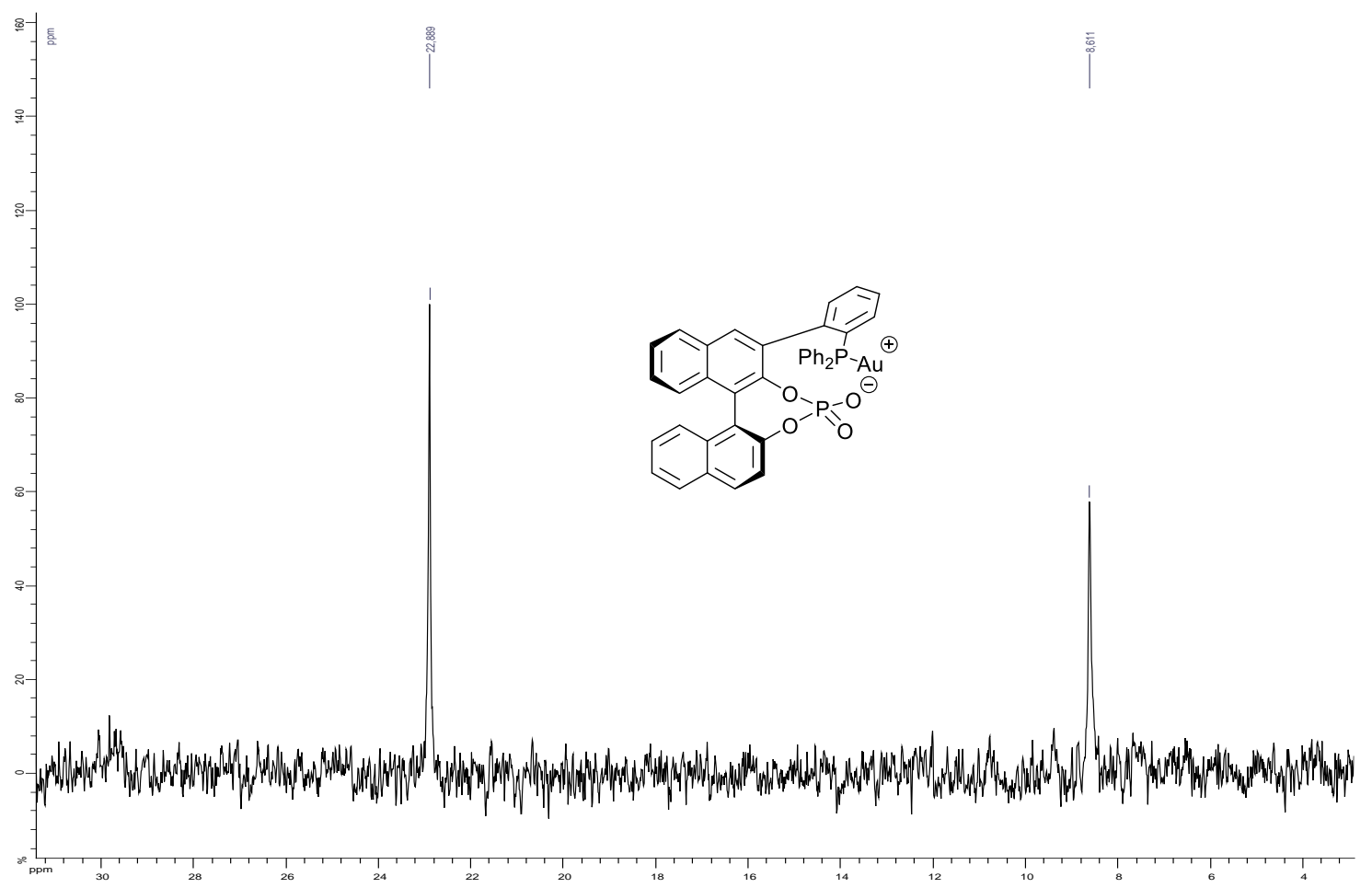

31P NMR (Pyridine, $200 \mathrm{MHz}$ ) spectrum of (S)-6 


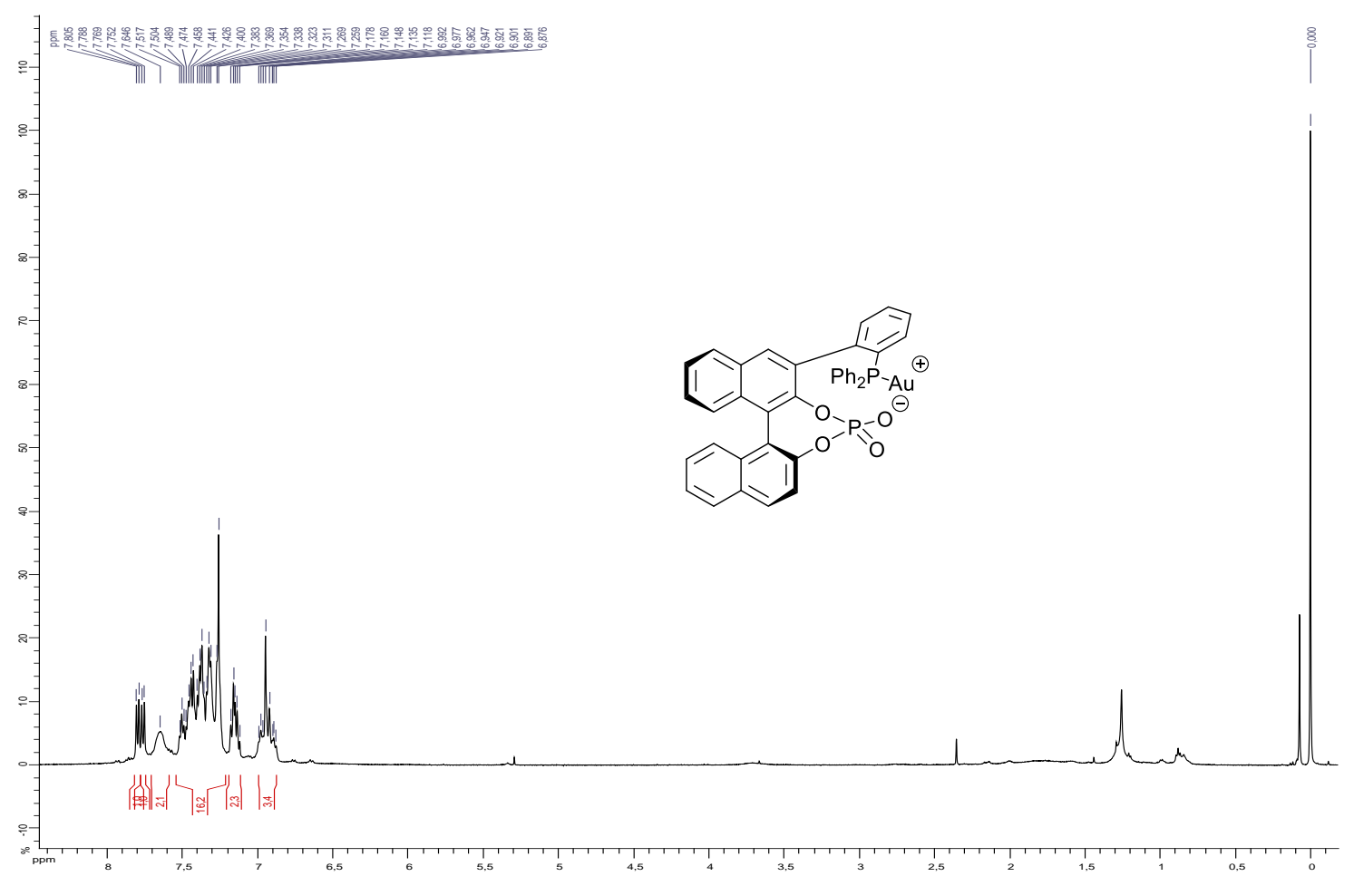

${ }^{1} \mathrm{H}$ NMR $\left(\mathrm{CDCl}_{3}, 500 \mathrm{MHz}\right)$ spectrum of (S)-6

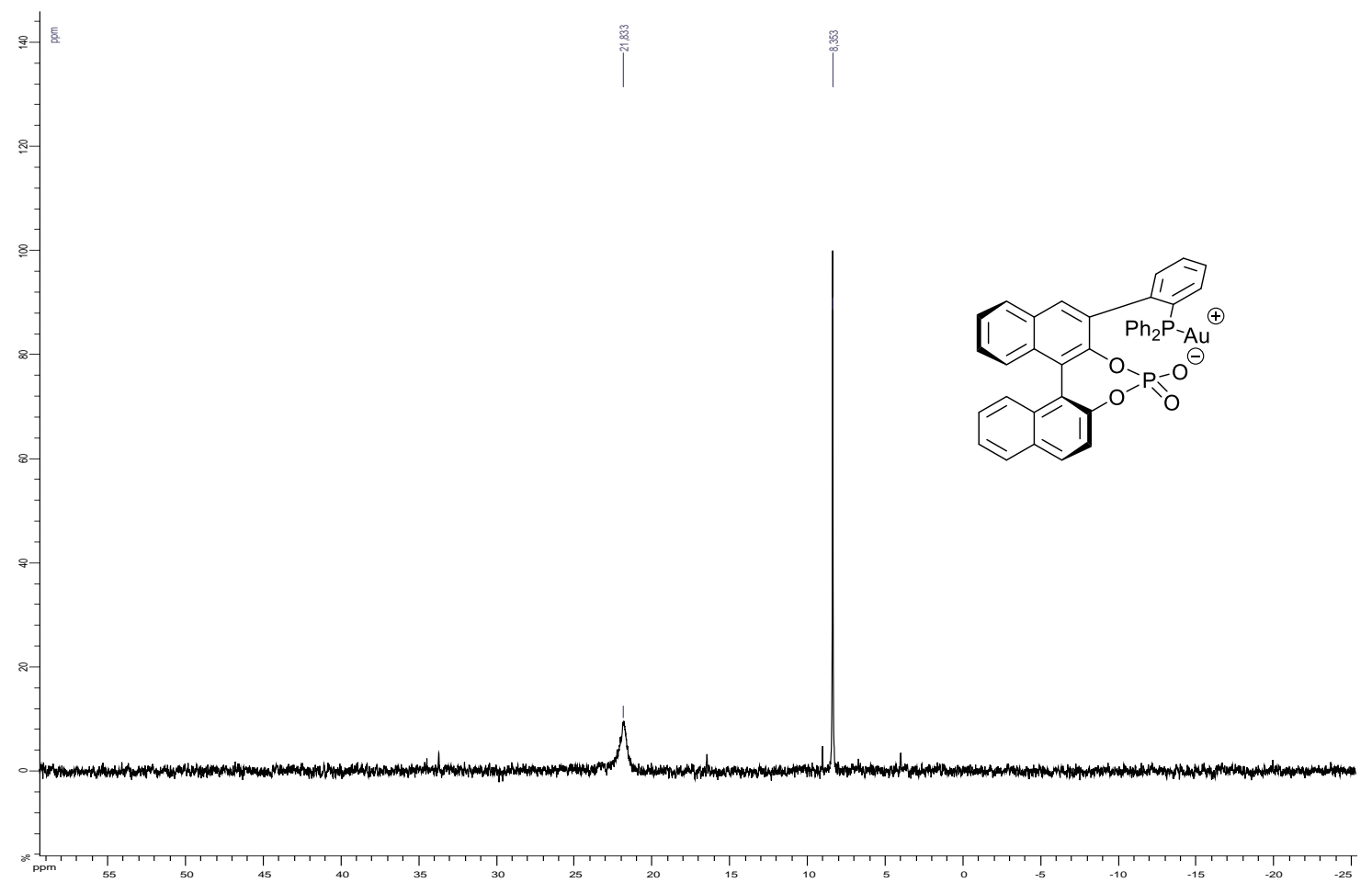

31P NMR ( $\left.\mathrm{CDCl}_{3}, 200 \mathrm{MHz}\right)$ spectrum of $(S)-6$ 


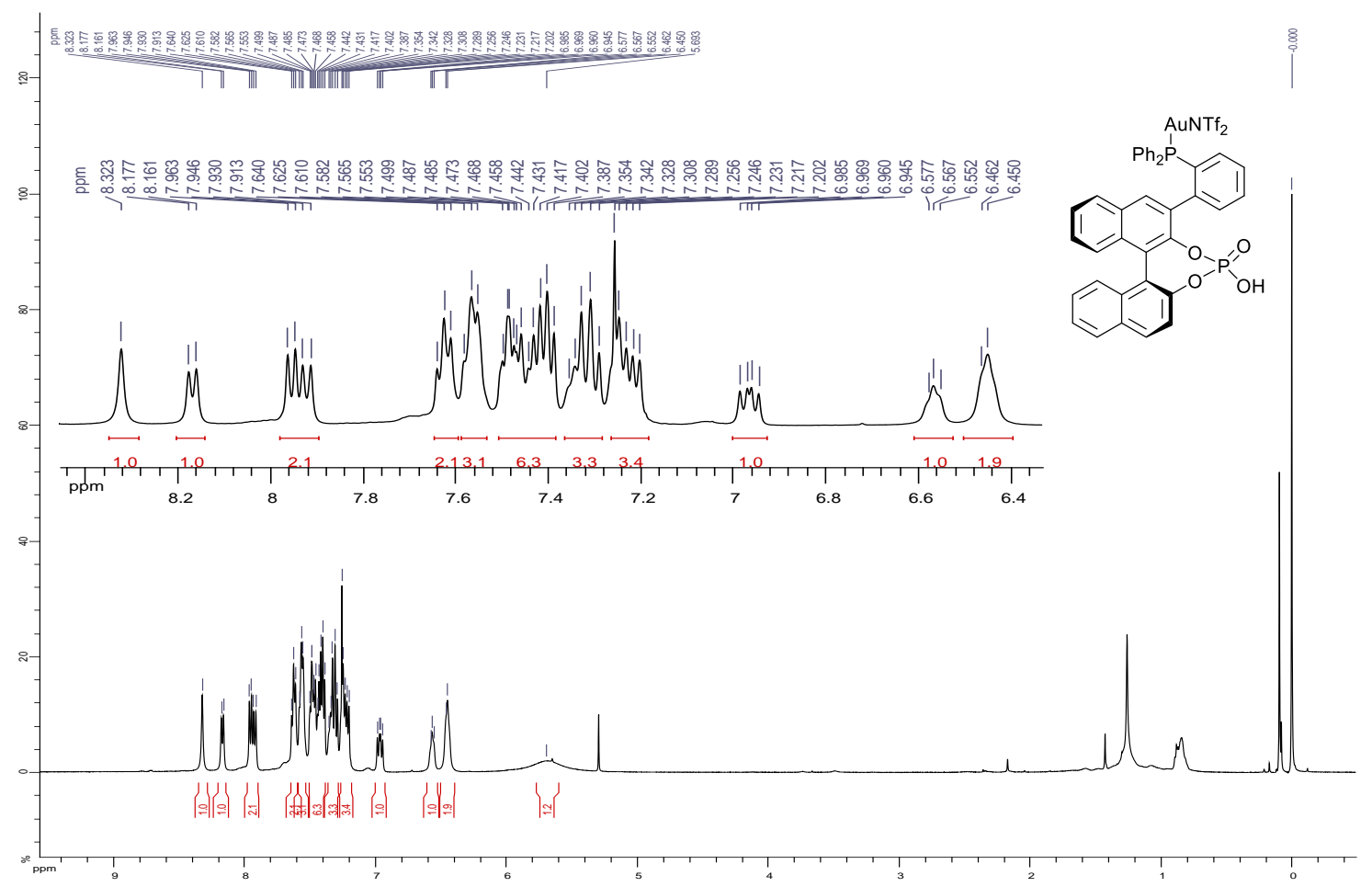

${ }^{1} \mathrm{H}$ NMR $\left(\mathrm{CDCl}_{3}, 500 \mathrm{MHz}\right)$ spectrum of (S)-(2-(2,2'-diyl hydrogen phosphate-[1,1'-binaphthalen]-3-yl)phenyl) diphenylphosphine gold(I) bis(trifluoromethanesulfonyl)imide [(S)-7a]

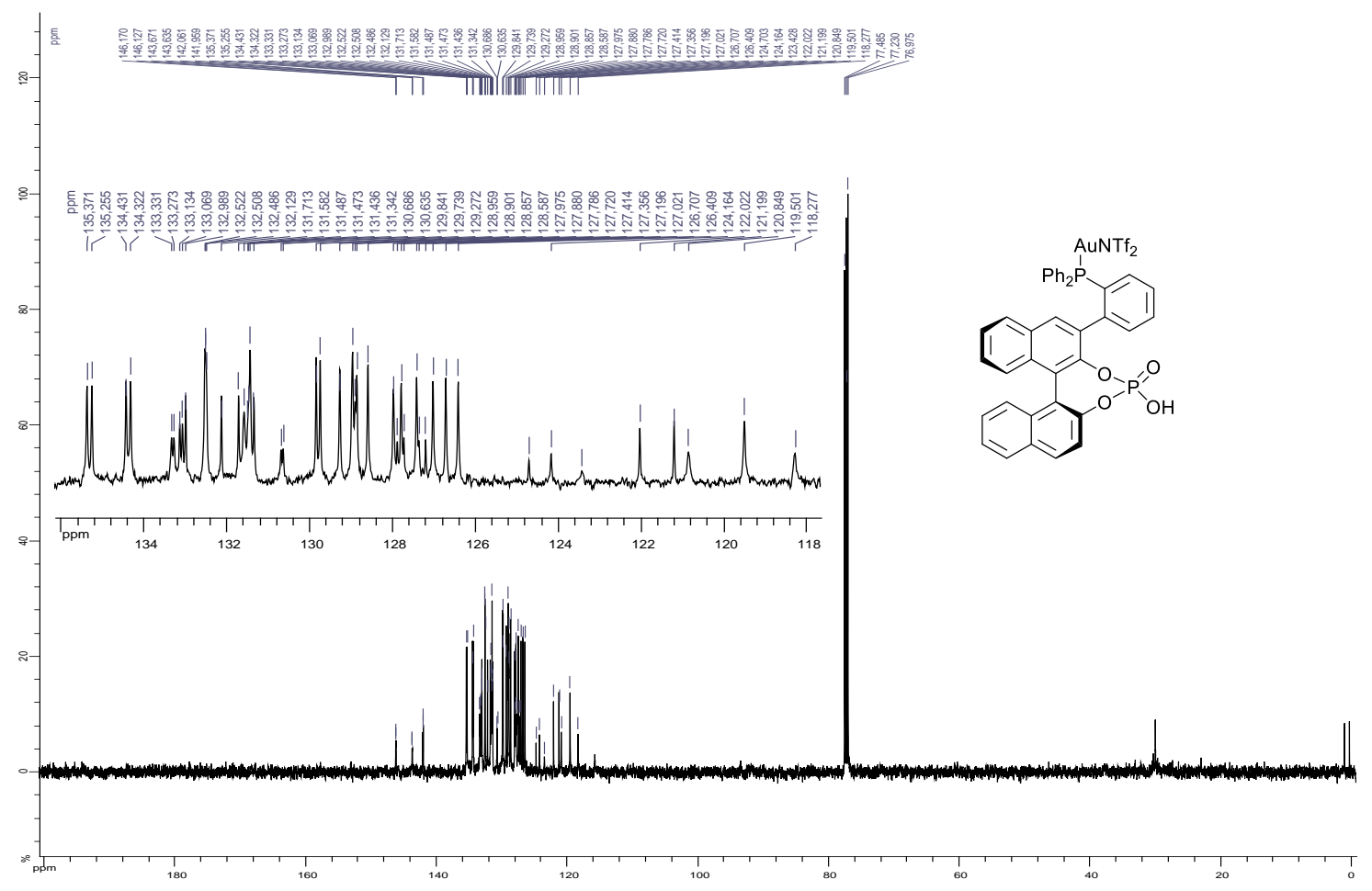

$\left.{ }^{13} \mathrm{C} \mathrm{NMR} \mathrm{(} \mathrm{CDCl}_{3}, 125 \mathrm{MHz}\right)$ spectrum of (S)-(2-(2,2'-diyl hydrogen phosphate-[1,1'-binaphthalen]-3-yl)phenyl) diphenylphosphine gold(I) bis(trifluoromethanesulfonyl)imide [(S)-7a] 


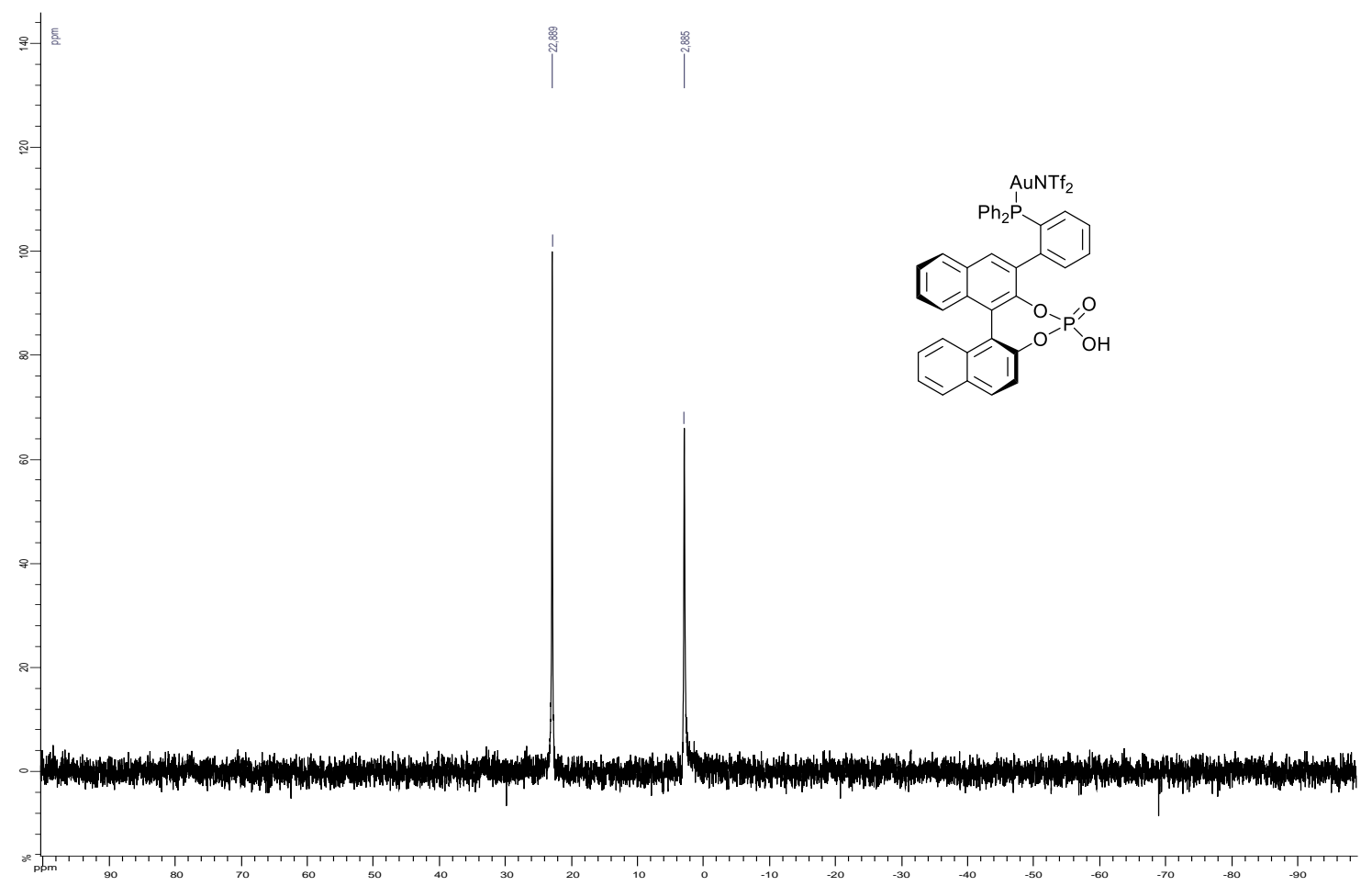

31P NMR ( $\mathrm{CDCl}_{3}, 200 \mathrm{MHz}$ ) spectrum of (S)-(2-(2,2'-diyl hydrogen phosphate-[1,1'-binaphthalen]-3-yl)phenyl) diphenylphosphine gold(I) bis(trifluoromethanesulfonyl)imide [(S)-7a] 


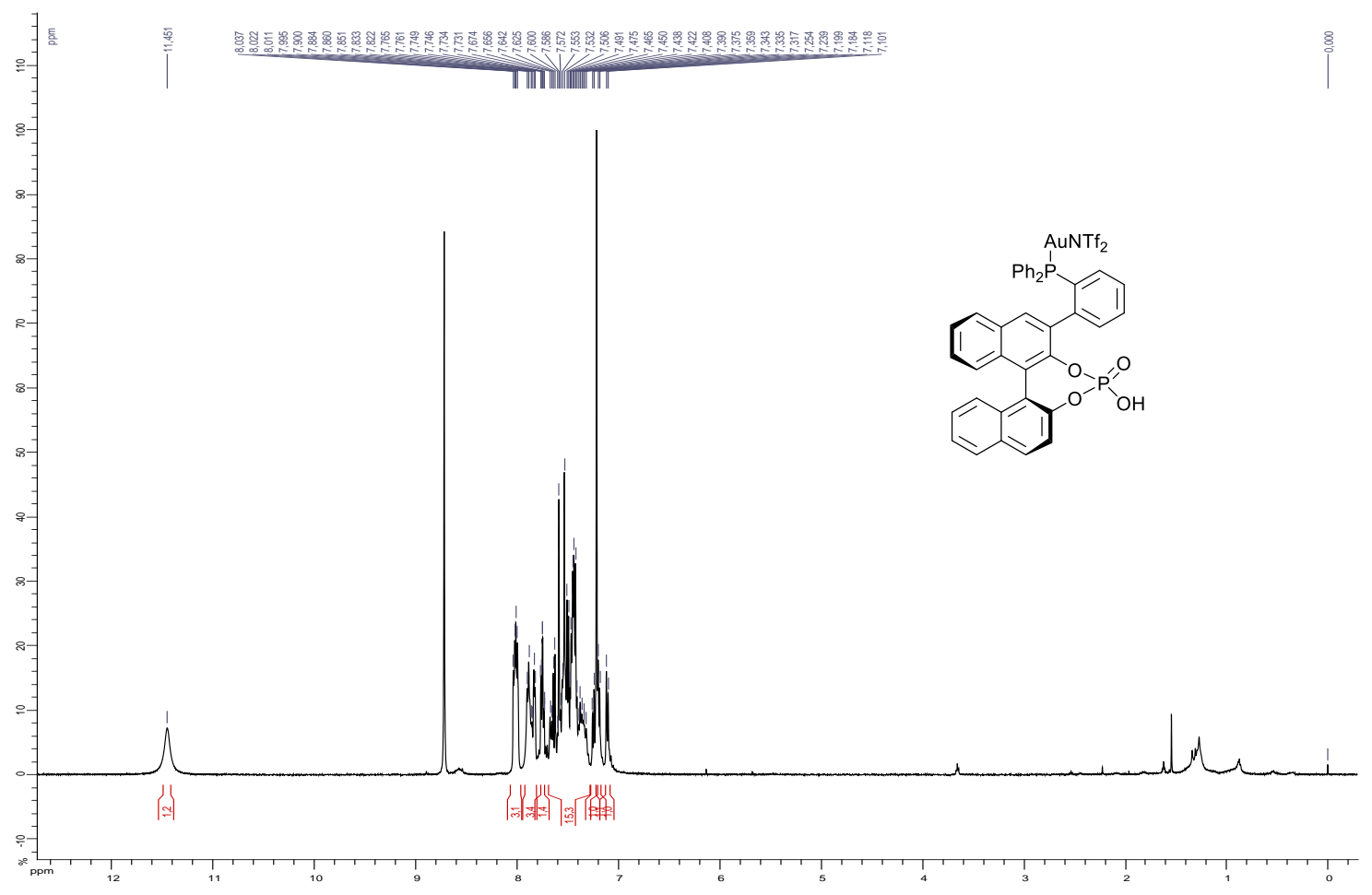

${ }^{1} \mathrm{H}$ NMR (Pyridine, $500 \mathrm{MHz}$ ) spectrum of (S)-(2-(2,2'-diyl hydrogen phosphate-[1,1'-binaphthalen]-3-yl)phenyl) diphenylphosphine gold(I) bis(trifluoromethanesulfonyl)imide [(S)-7a]

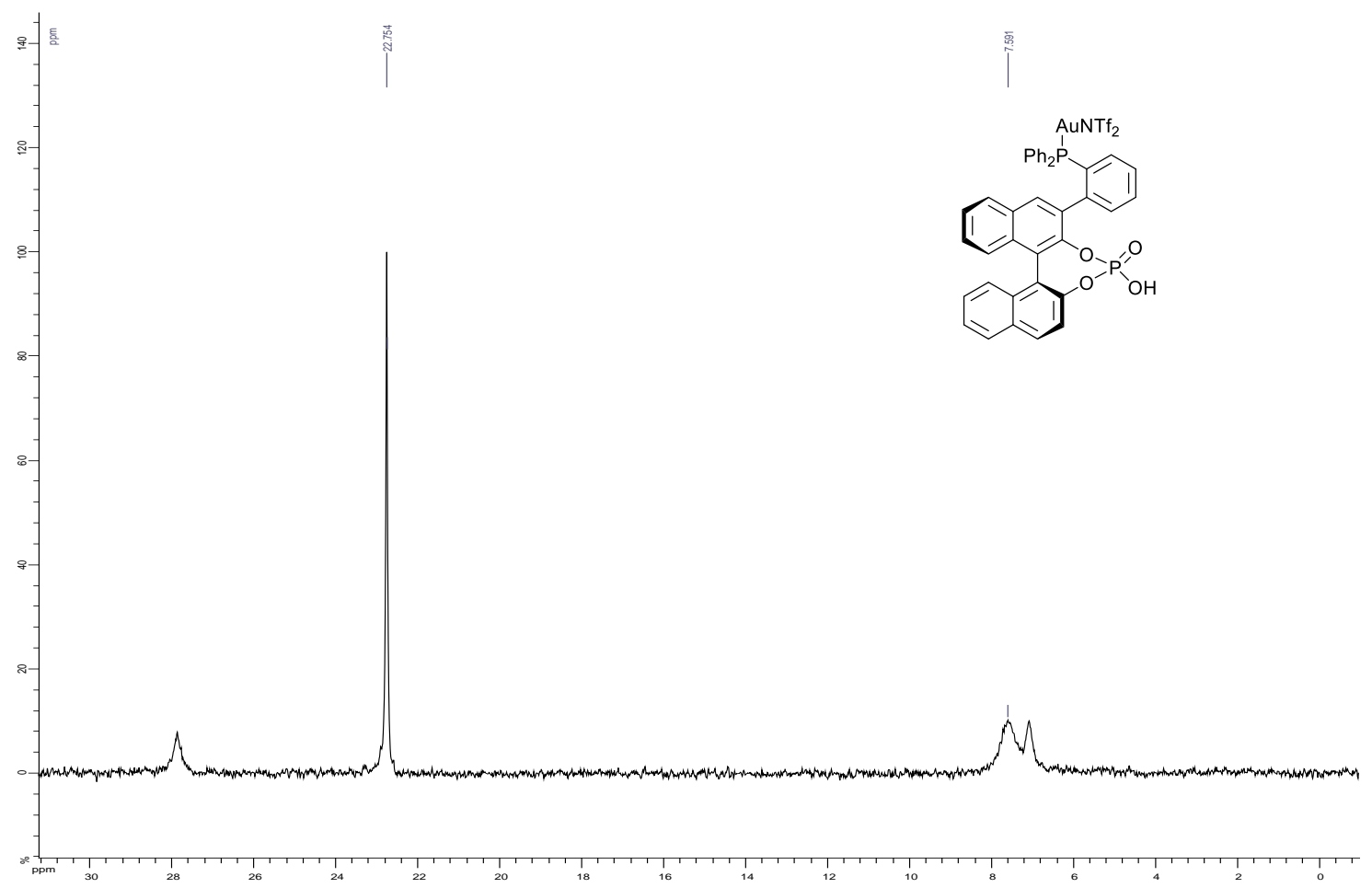

31P NMR (Pyridine, 200 MHz) spectrum of (S)-(2-(2,2'-diyl hydrogen phosphate-[1,1'-binaphthalen]-3-yl)phenyl) diphenylphosphine gold(I) bis(trifluoromethanesulfonyl)imide [(S)-7a] 


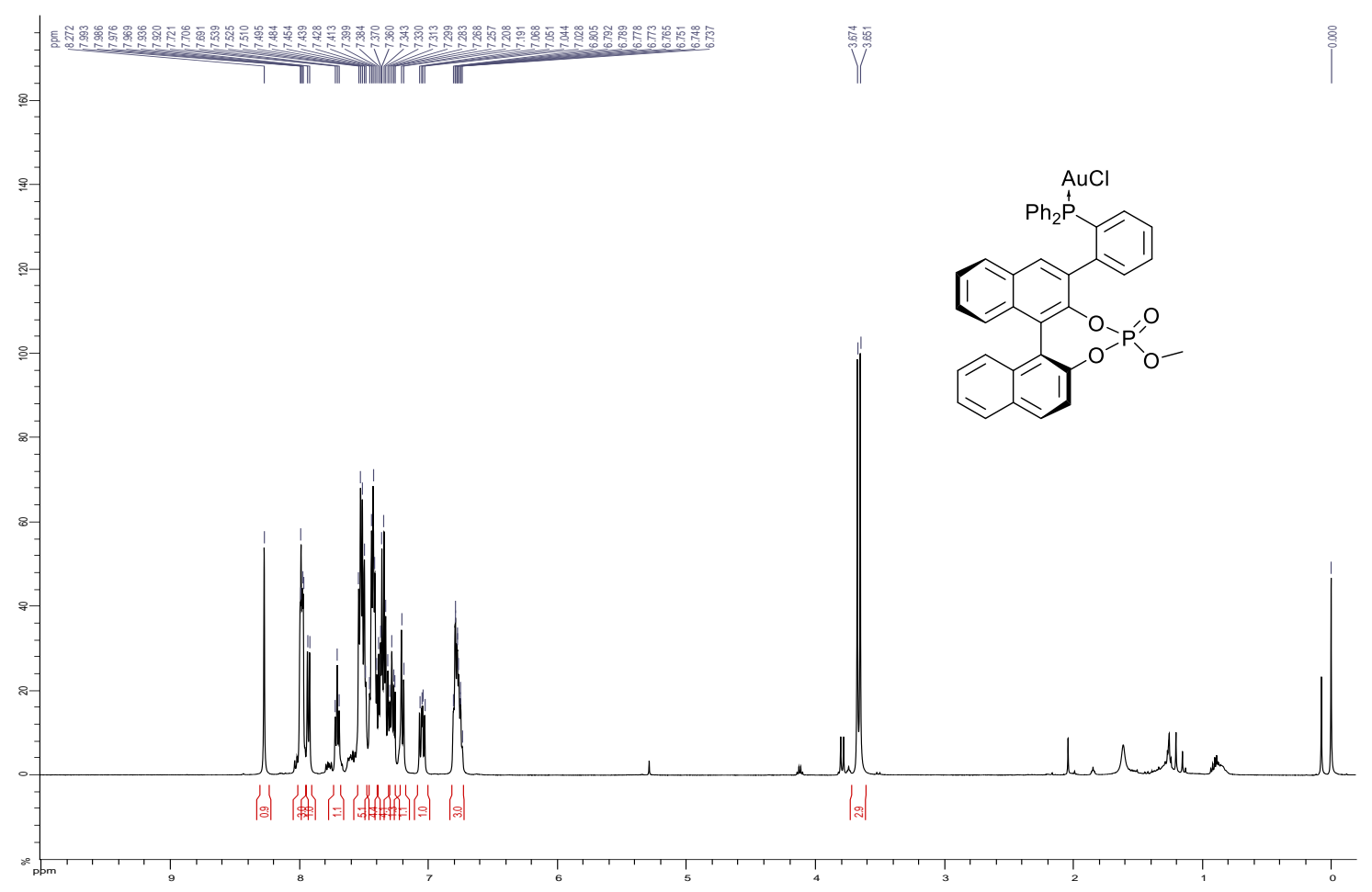

${ }^{1} \mathrm{H} \mathrm{NMR}\left(\mathrm{CDCl}_{3}, 500 \mathrm{MHz}\right)$ spectrum of $\mathrm{SI}-2$

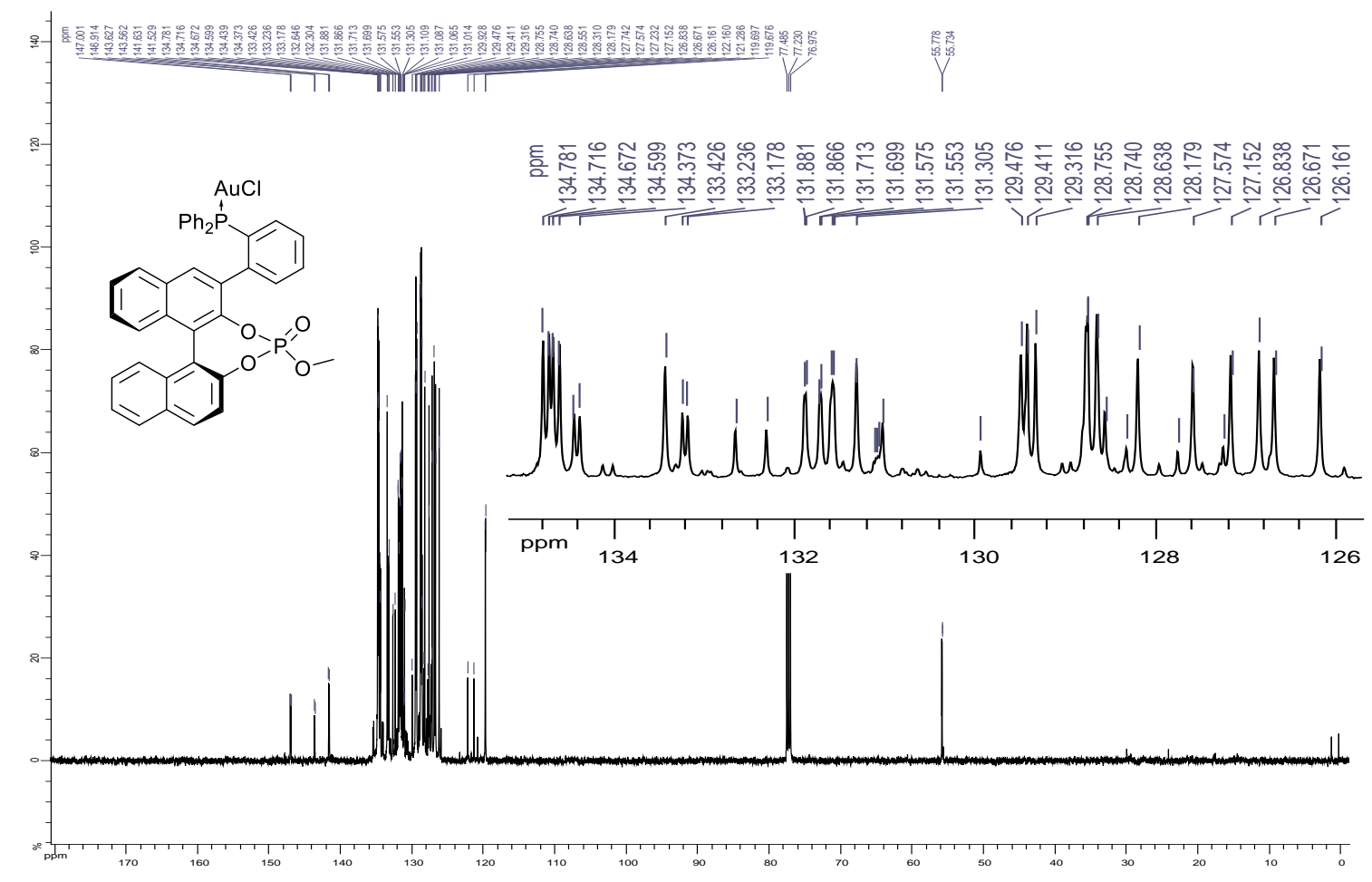

${ }^{13} \mathrm{C} \mathrm{NMR}\left(\mathrm{CDCl}_{3}, 125 \mathrm{MHz}\right)$ spectrum of SI-2 


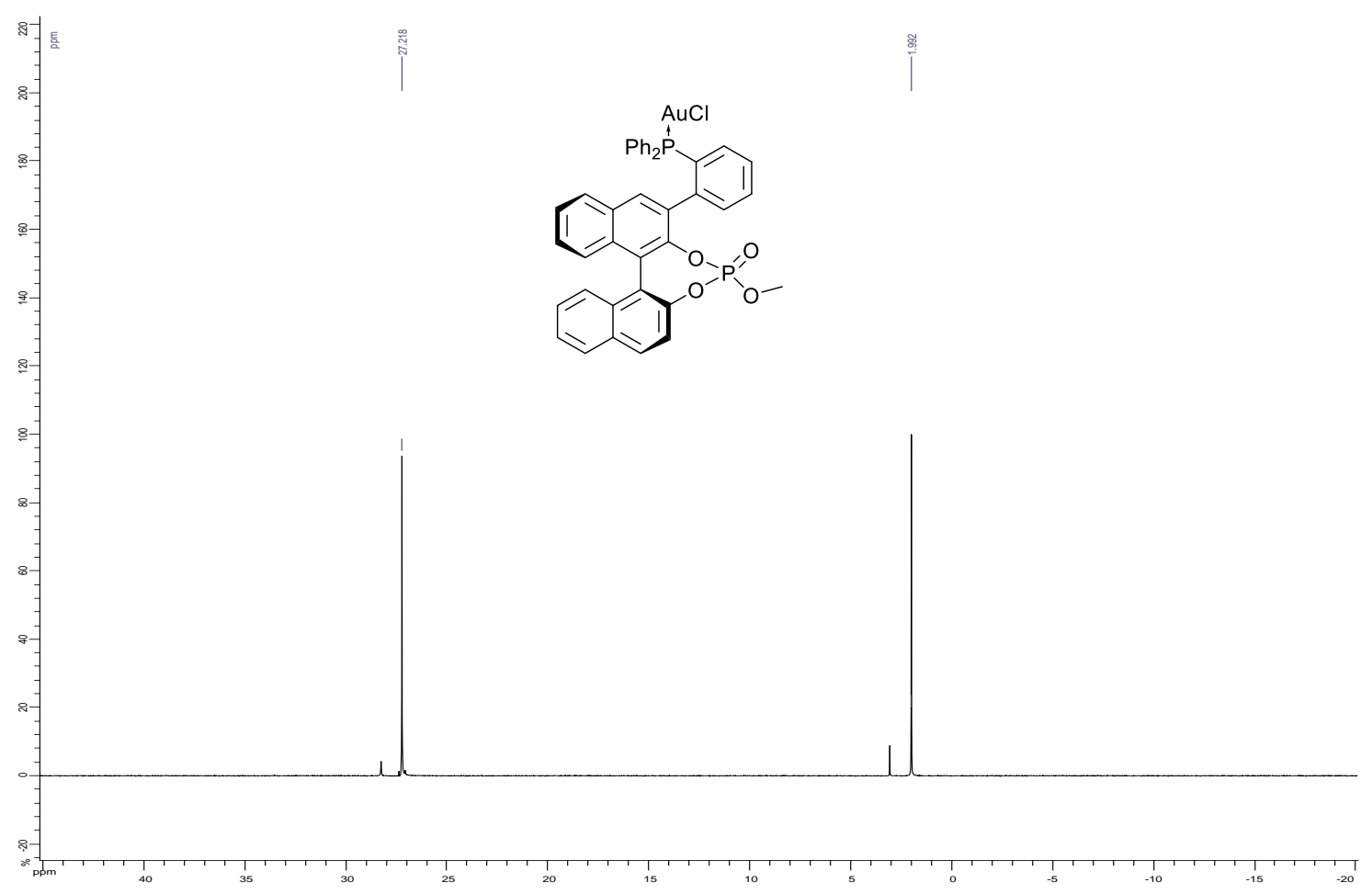

31P NMR ( $\left.\mathrm{CDCl}_{3}, 200 \mathrm{MHz}\right)$ spectrum of SI-2 


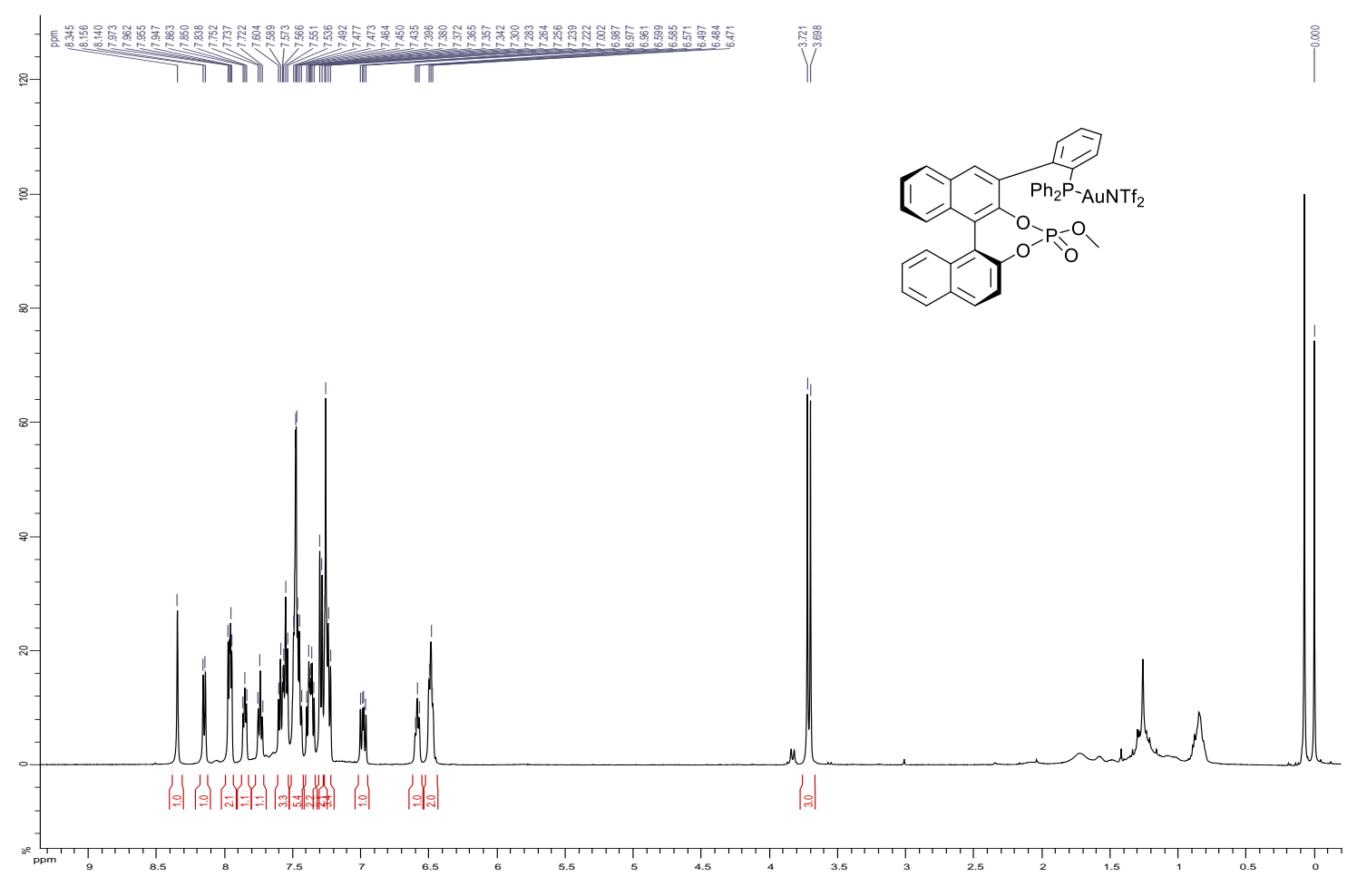

${ }^{1} \mathrm{H}$ NMR $\left(\mathrm{CDCl}_{3}, 500 \mathrm{MHz}\right)$ spectrum of $(S)$-11a

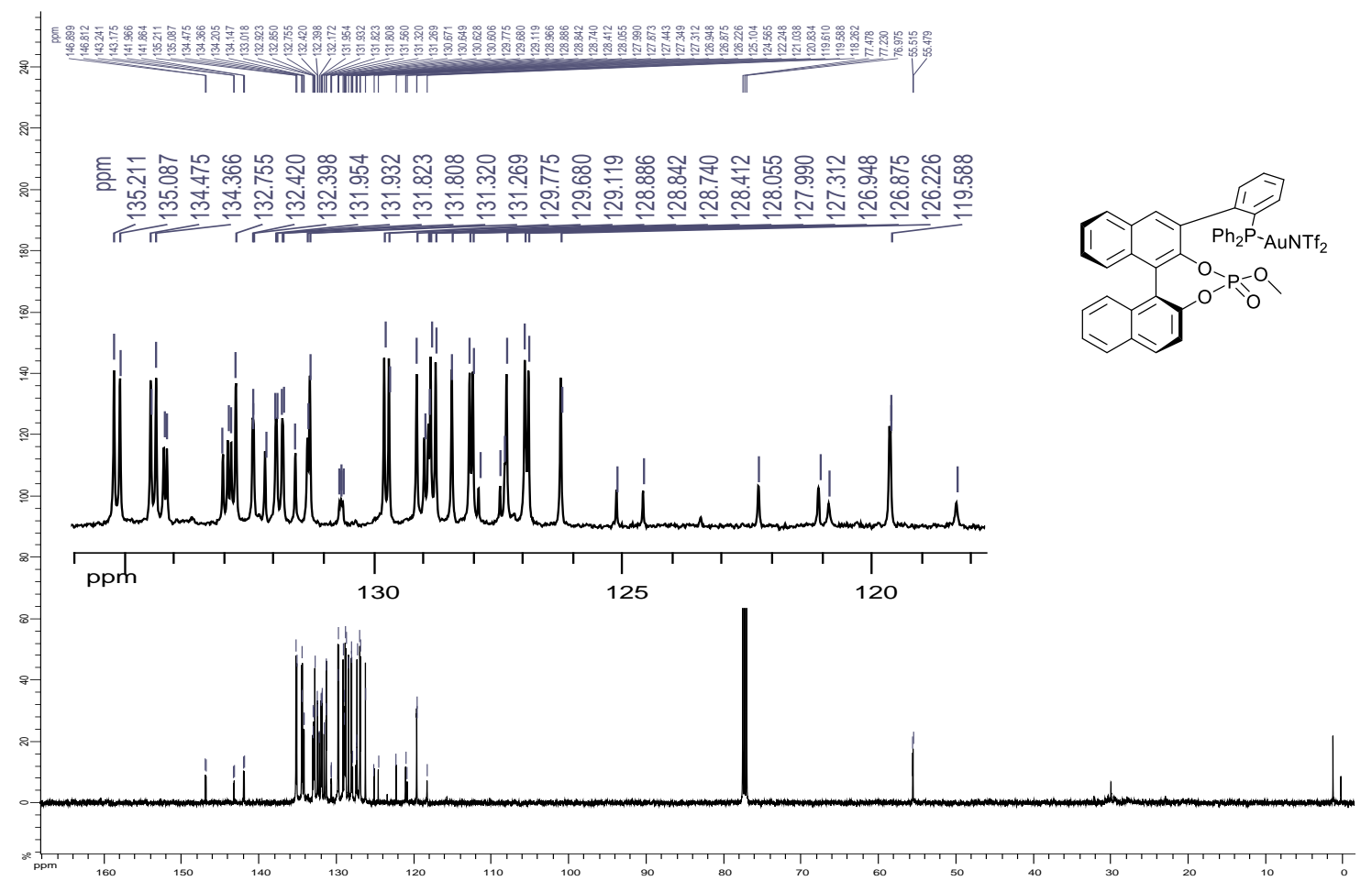

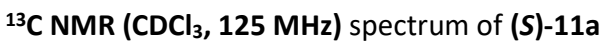




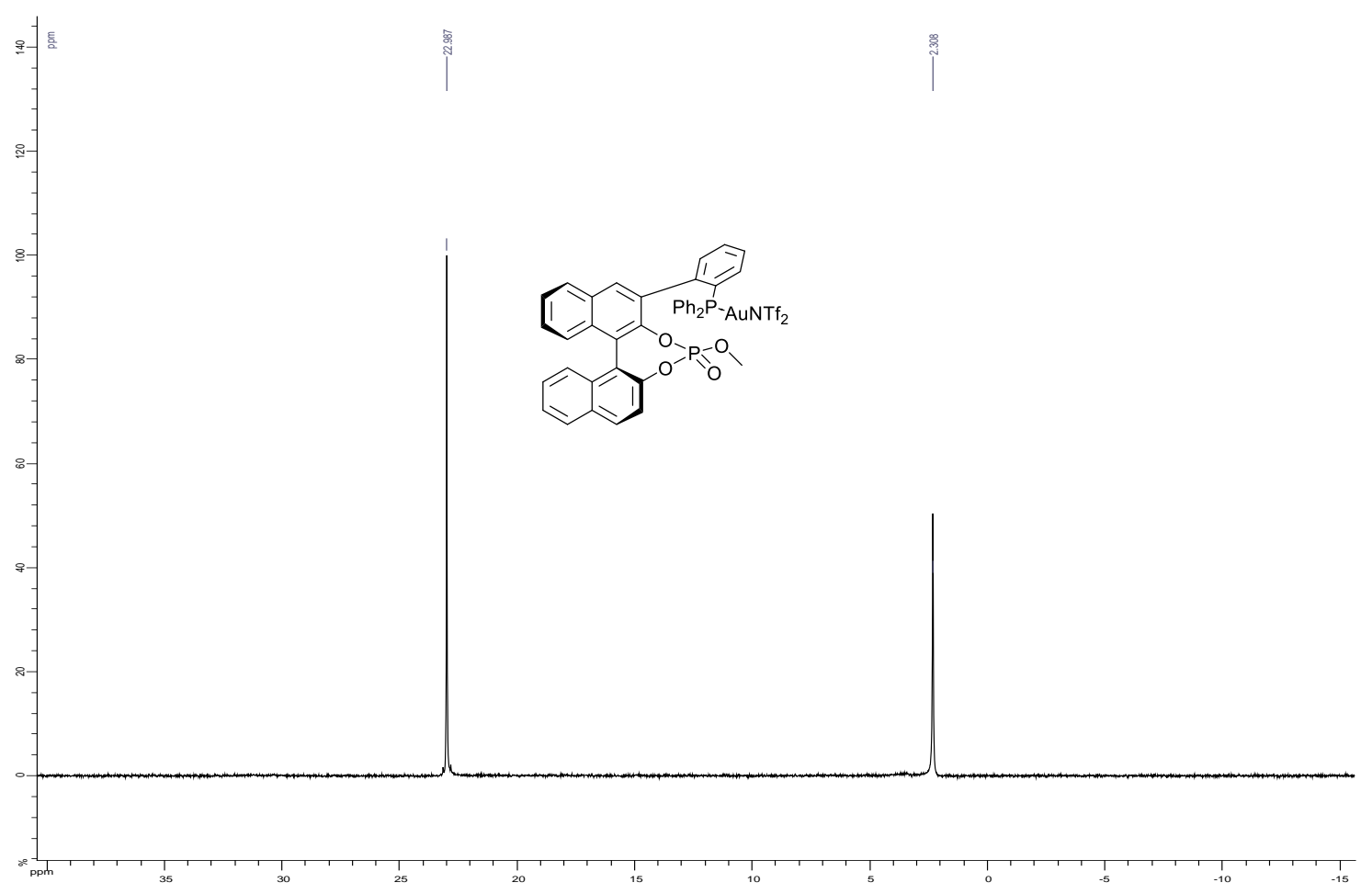

31P NMR ( $\left.\mathrm{CDCl}_{3}, 200 \mathrm{MHz}\right)$ spectrum of $(S)-11 \mathrm{a}$ 


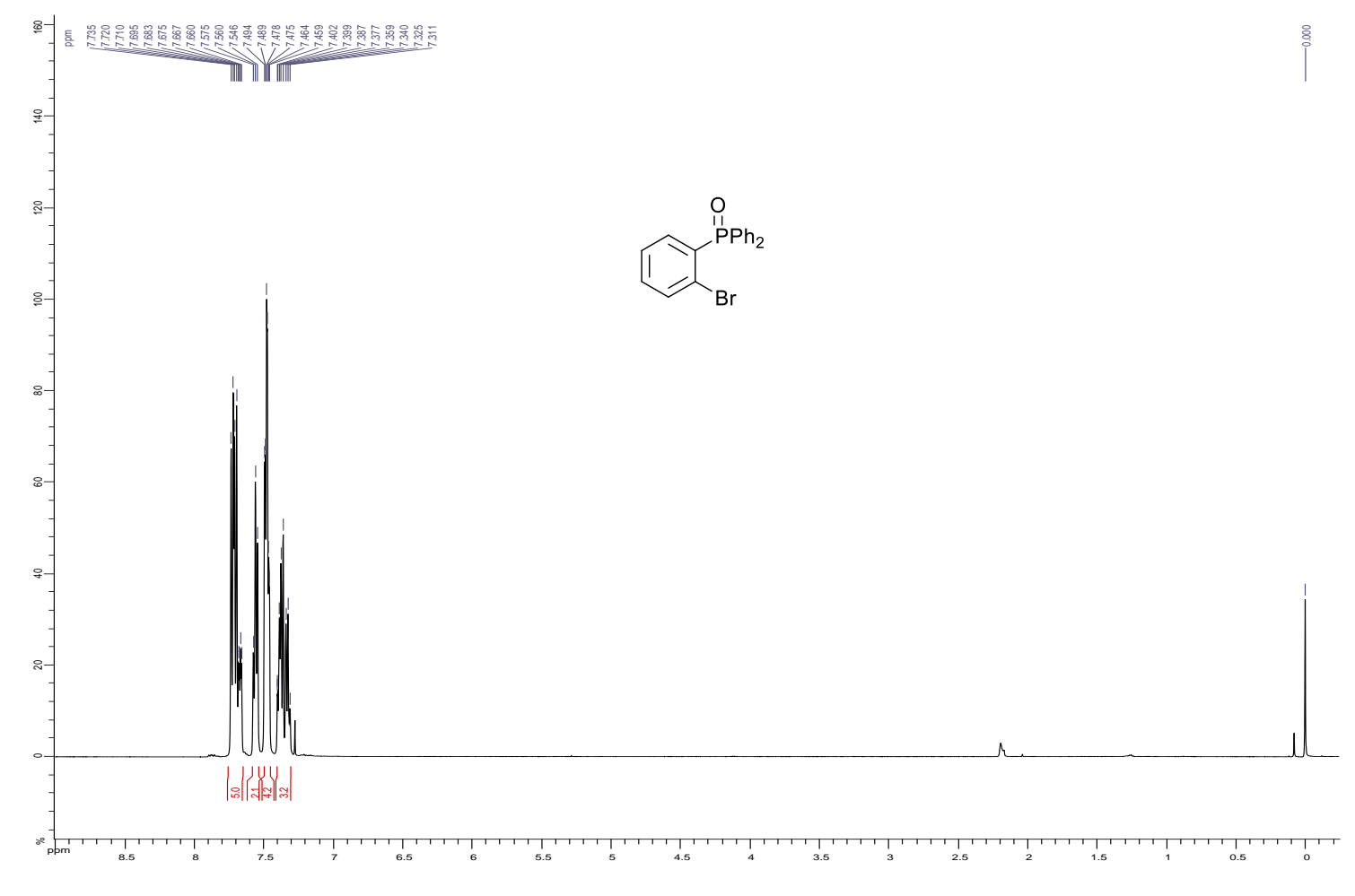

${ }^{1} \mathrm{H} \mathrm{NMR}\left(\mathrm{CDCl}_{3}, 500 \mathrm{MHz}\right)$ spectrum of (2-bromophenyl)diphenylphosphine oxide

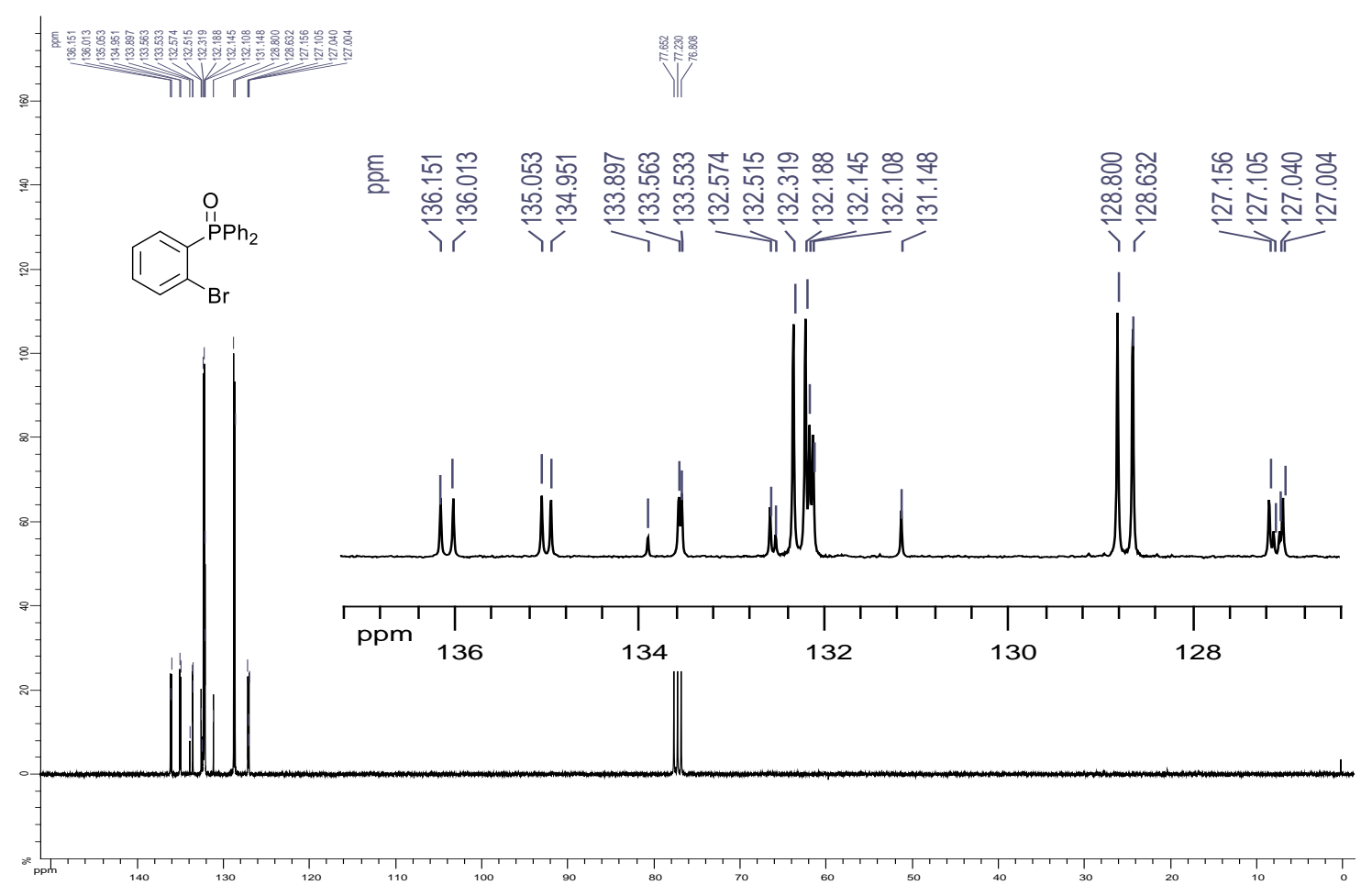

$\left.{ }^{13} \mathrm{C} \mathrm{NMR} \mathrm{(} \mathrm{CDCl}_{3}, 75 \mathrm{MHz}\right)$ spectrum of (2-bromophenyl)diphenylphosphine oxide 


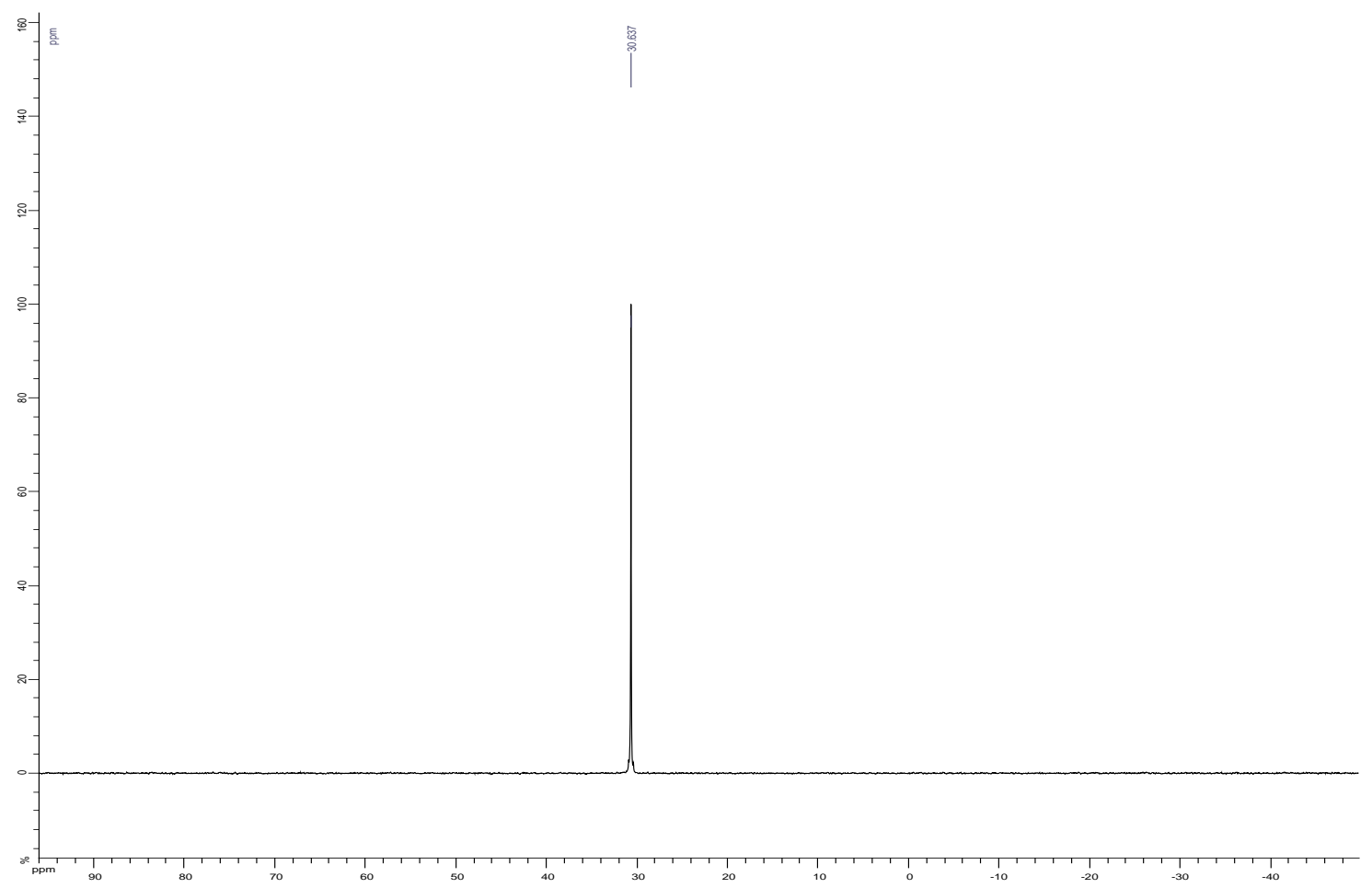

31P NMR ( $\left.\mathrm{CDCl}_{3}, 200 \mathrm{MHz}\right)$ spectrum of (2-bromophenyl)diphenylphosphine oxide 


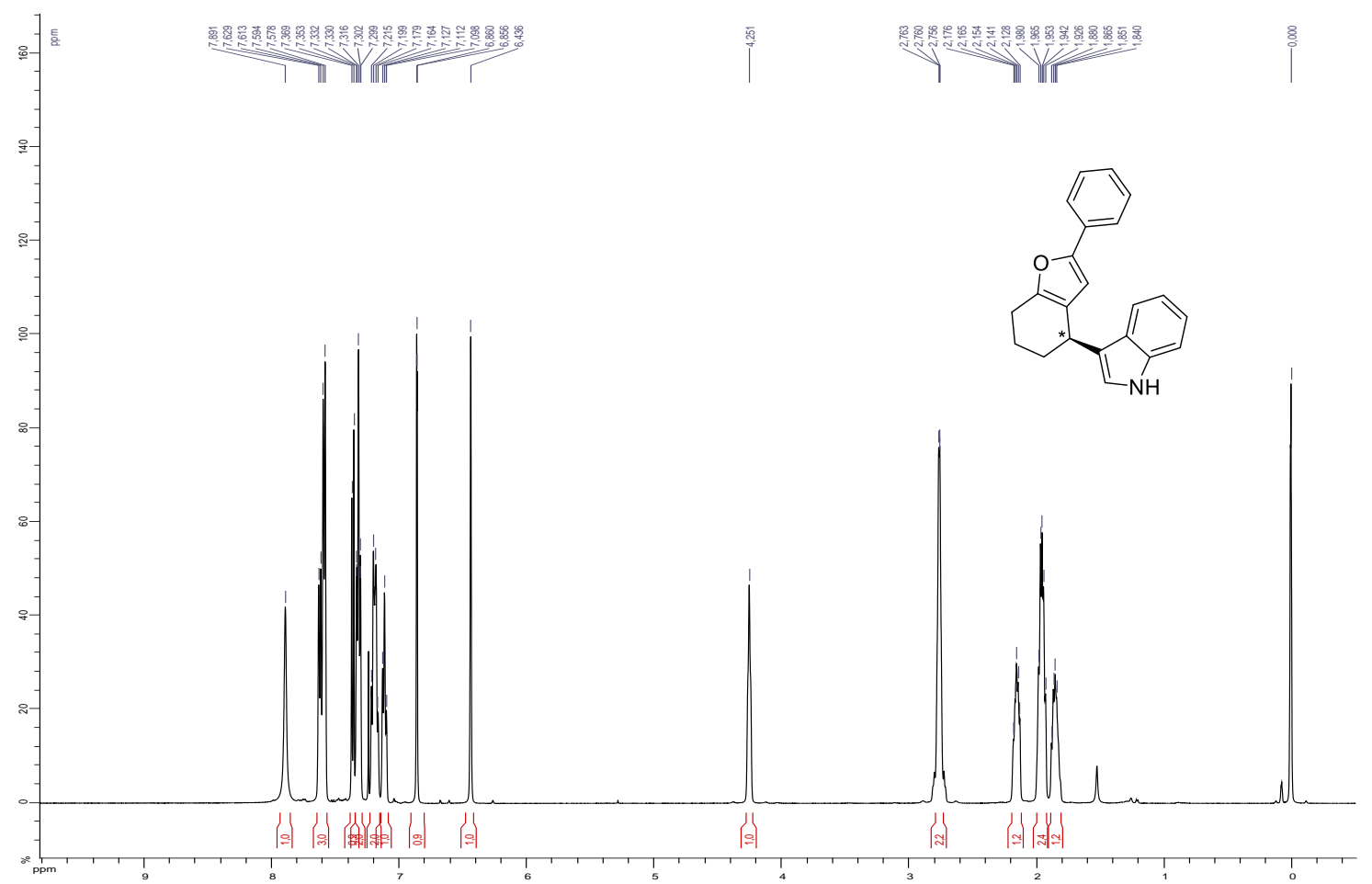

${ }^{1} \mathrm{H}$ NMR $\left(\mathrm{CDCl}_{3}, 500 \mathrm{MHz}\right)$ spectrum of $(R)$-3-(2-phenyl-4,5,6,7-tetrahydrobenzofuran-4-yl)-1H-indole (10aa)

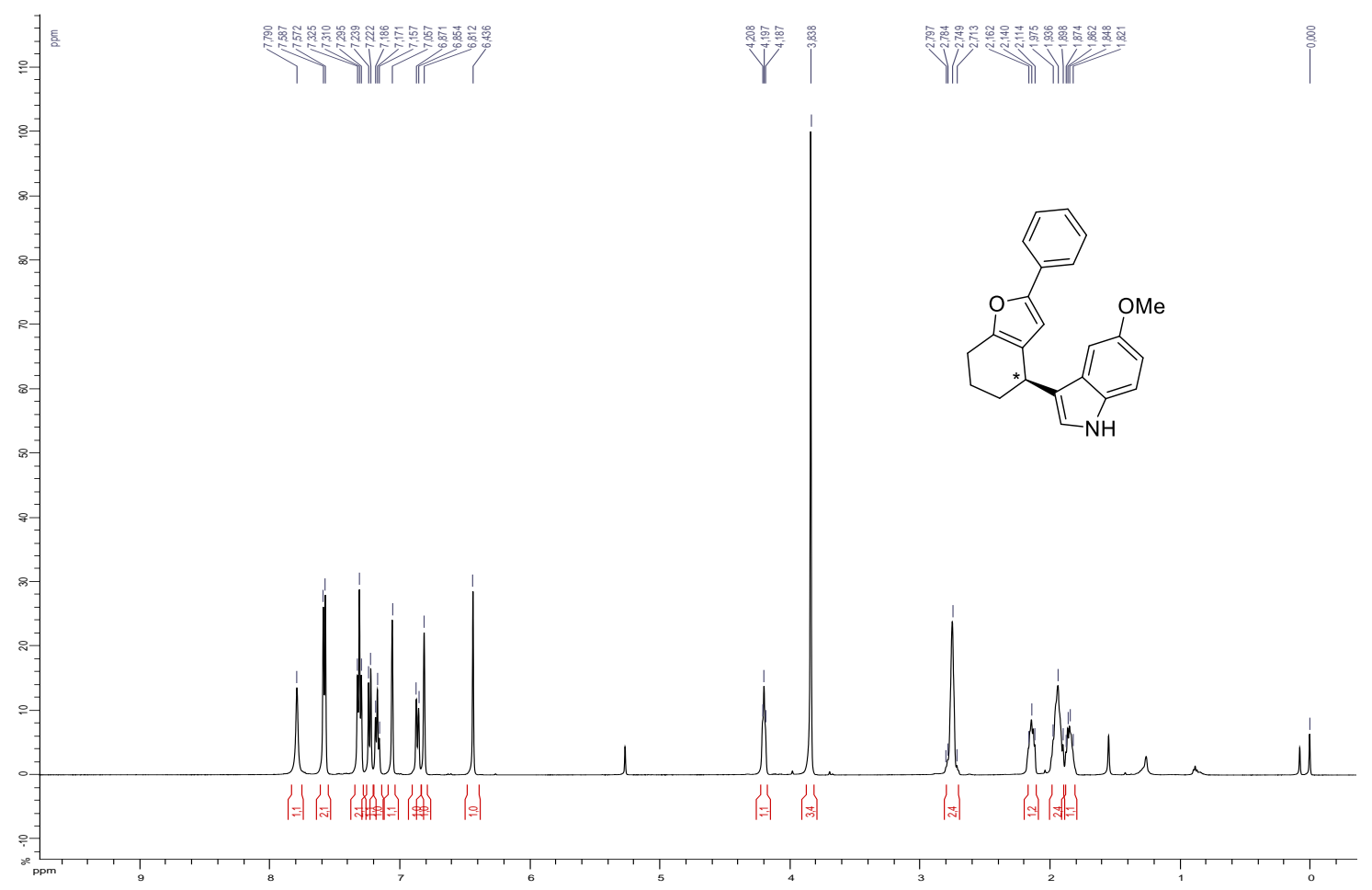

${ }^{1} \mathrm{H}$ NMR $\left(\mathrm{CDCl}_{3}, 500 \mathrm{MHz}\right)$ spectrum of $(R)$-5-methoxy-3-(2-phenyl-4,5,6,7-tetrahydrobenzofuran-4-yl)-1H-indole (10ab) 


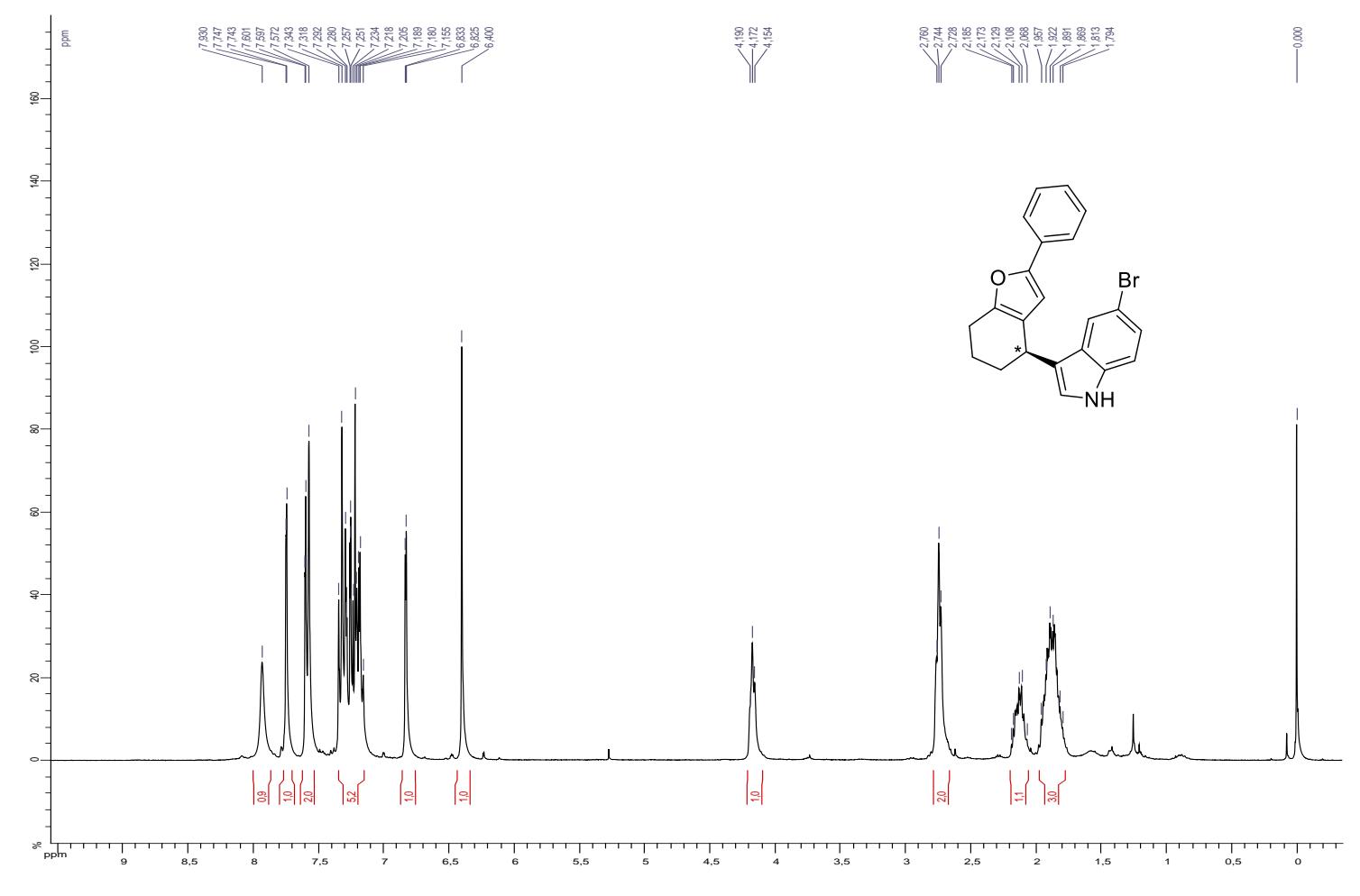

${ }^{1} \mathrm{H}$ NMR $\left(\mathrm{CDCl}_{3}, 300 \mathrm{MHz}\right)$ spectrum of $(R)$-5-bromo-3-(2-phenyl-4,5,6,7-tetrahydrobenzofuran-4-yl)-1H-indole (10ac)

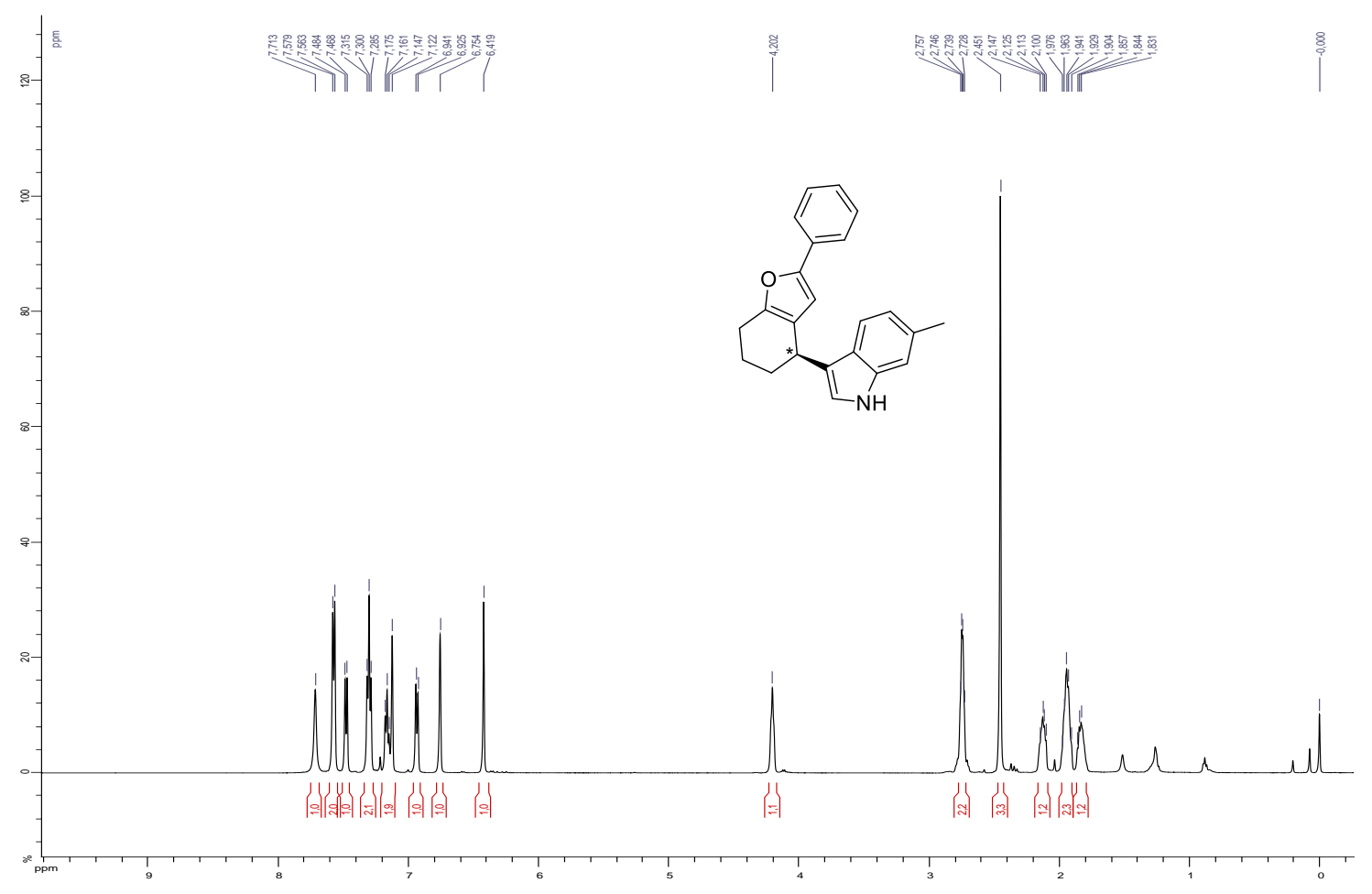

${ }^{1} \mathrm{H}$ NMR ( $\left.\mathrm{CDCl}_{\mathbf{3}}, 500 \mathrm{MHz}\right)$ spectrum of $(R)$-6-methyl-3-(2-phenyl-4,5,6,7-tetrahydrobenzofuran-4-yl)-1H-indole (10ad) 


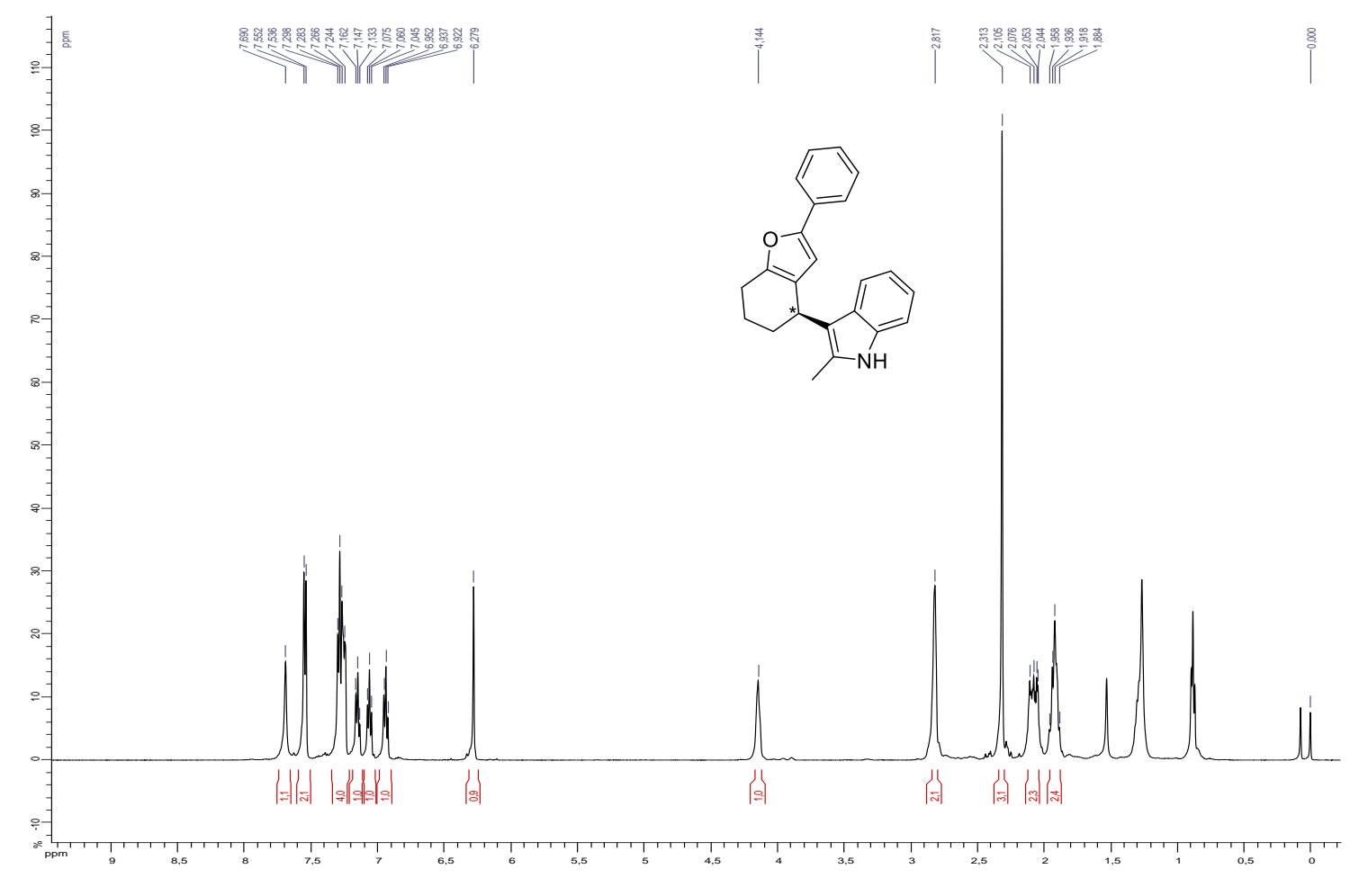

${ }^{1} \mathrm{H}$ NMR ( $\mathrm{CDCl}_{3}, 500 \mathrm{MHz}$ ) spectrum of (R)-2-methyl-3-(2-phenyl-4,5,6,7-tetrahydrobenzofuran-4-yl)-1H-indole (10ae)

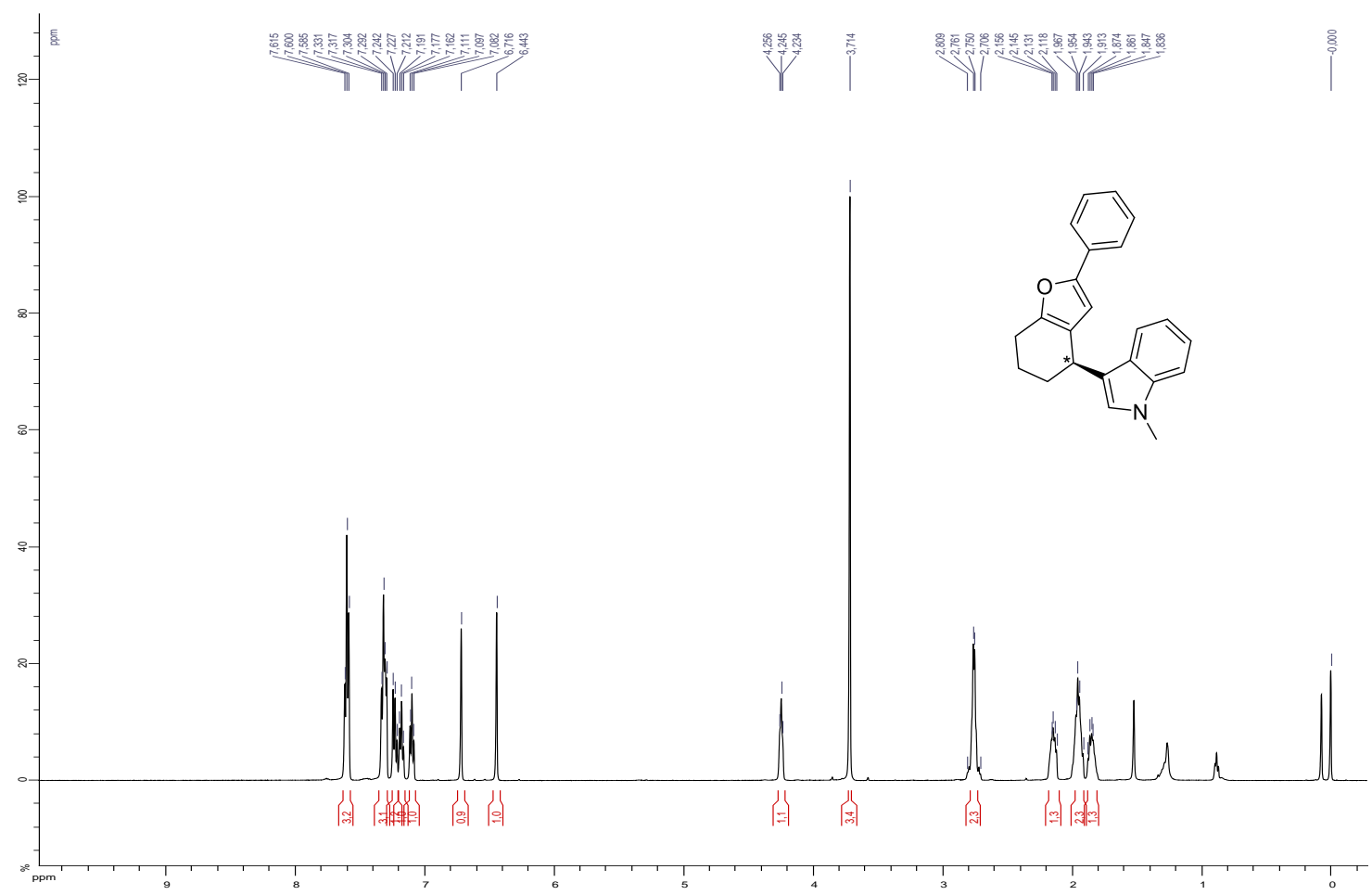

${ }^{1} \mathrm{H}$ NMR ( $\mathrm{CDCl}_{3}, 500 \mathrm{MHz}$ ) spectrum of $(R)$-1-methyl-3-(2-phenyl-4,5,6,7-tetrahydrobenzofuran-4-yl)-indole (10af) 


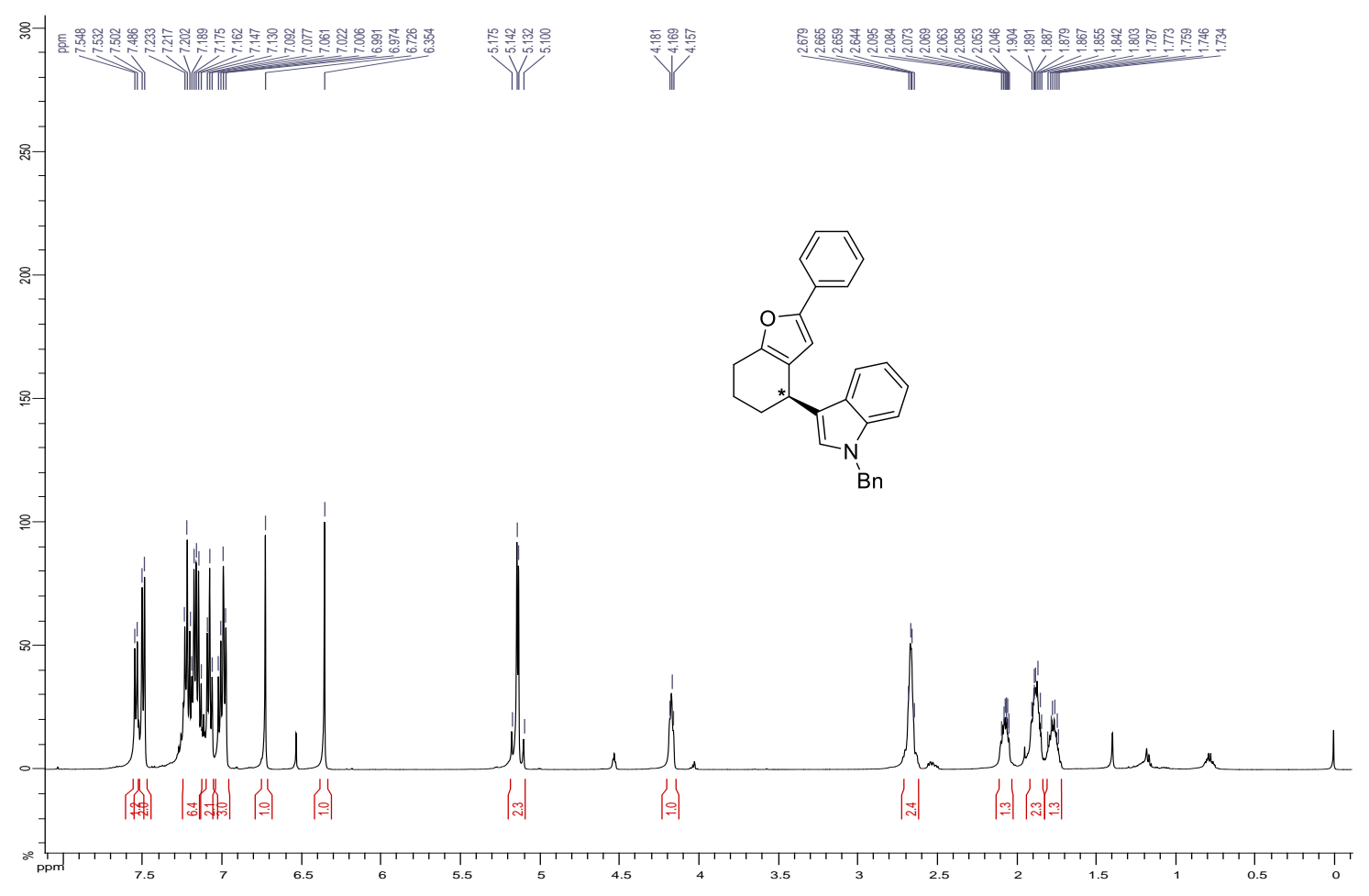

${ }^{1} \mathrm{H}$ NMR $\left(\mathrm{CDCl}_{3}, 500 \mathrm{MHz}\right.$ ) spectrum of (R)-1-benzyl-3-(2-phenyl-4,5,6,7-tetrahydrobenzofuran-4-yl)-indole (10ag)

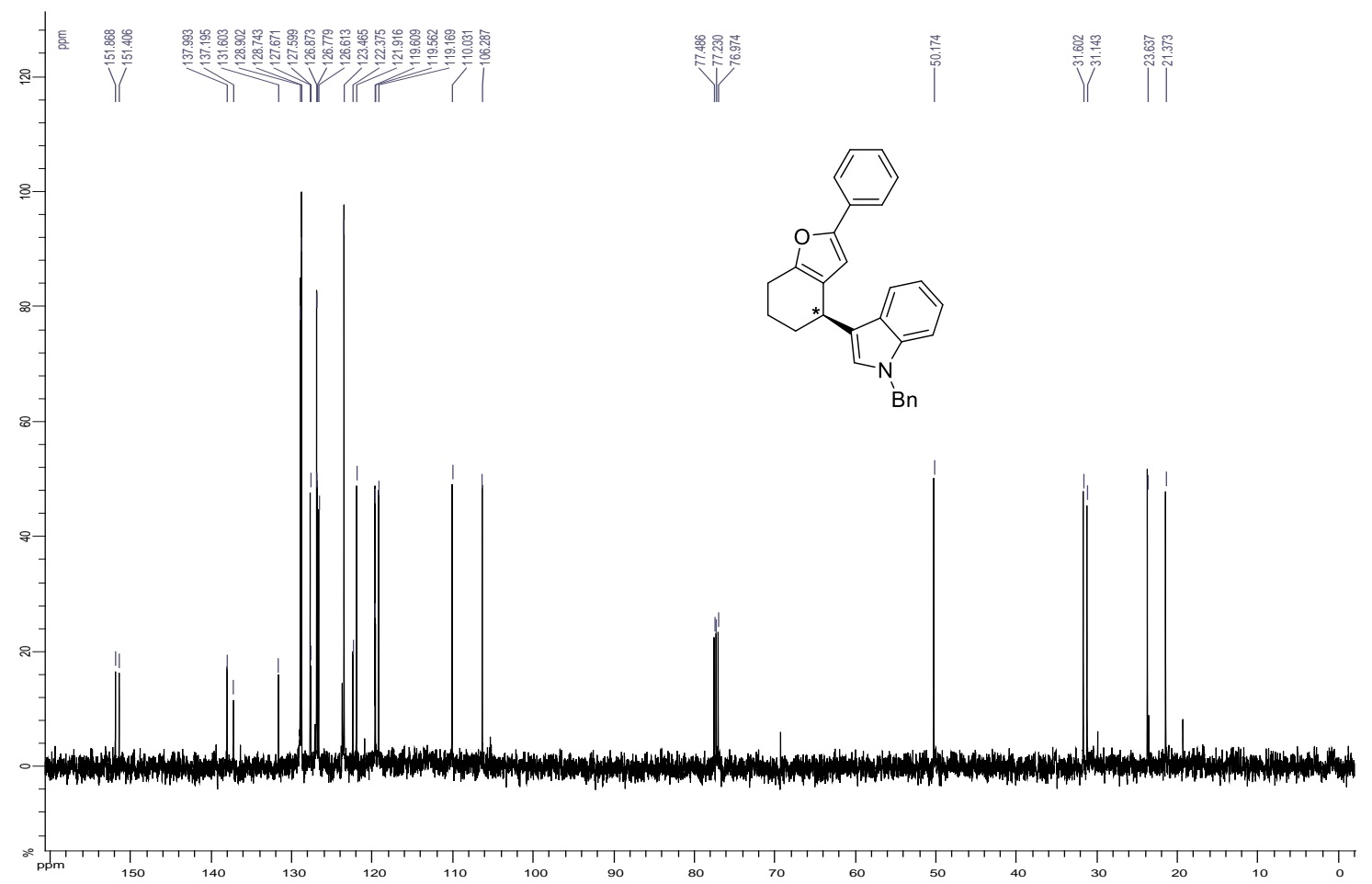

${ }^{13} \mathrm{C} \mathrm{NMR}\left(\mathrm{CDCl}_{3}, 125 \mathrm{MHz}\right)$ spectrum of (R)-1-benzyl-3-(2-phenyl-4,5,6,7-tetrahydrobenzofuran-4-yl)-1H-indole (10ag) 


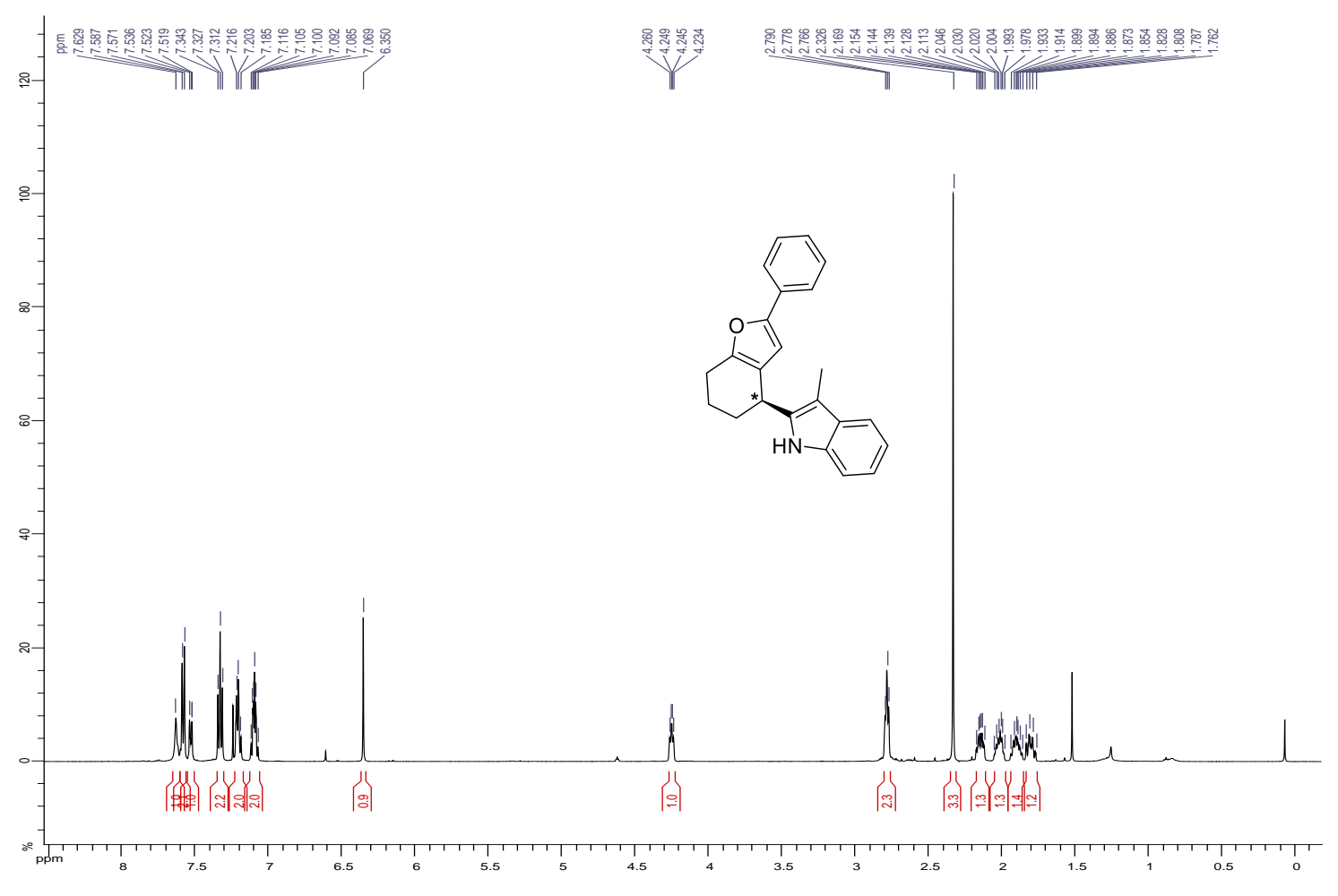

${ }^{1} \mathrm{H} \mathrm{NMR}\left(\mathrm{CDCl}_{3}, 500 \mathrm{MHz}\right.$ ) spectrum of (S)-3-methyl-2-(2-phenyl-4,5,6,7-tetrahydrobenzofuran-4-yl)-indole (14ah)

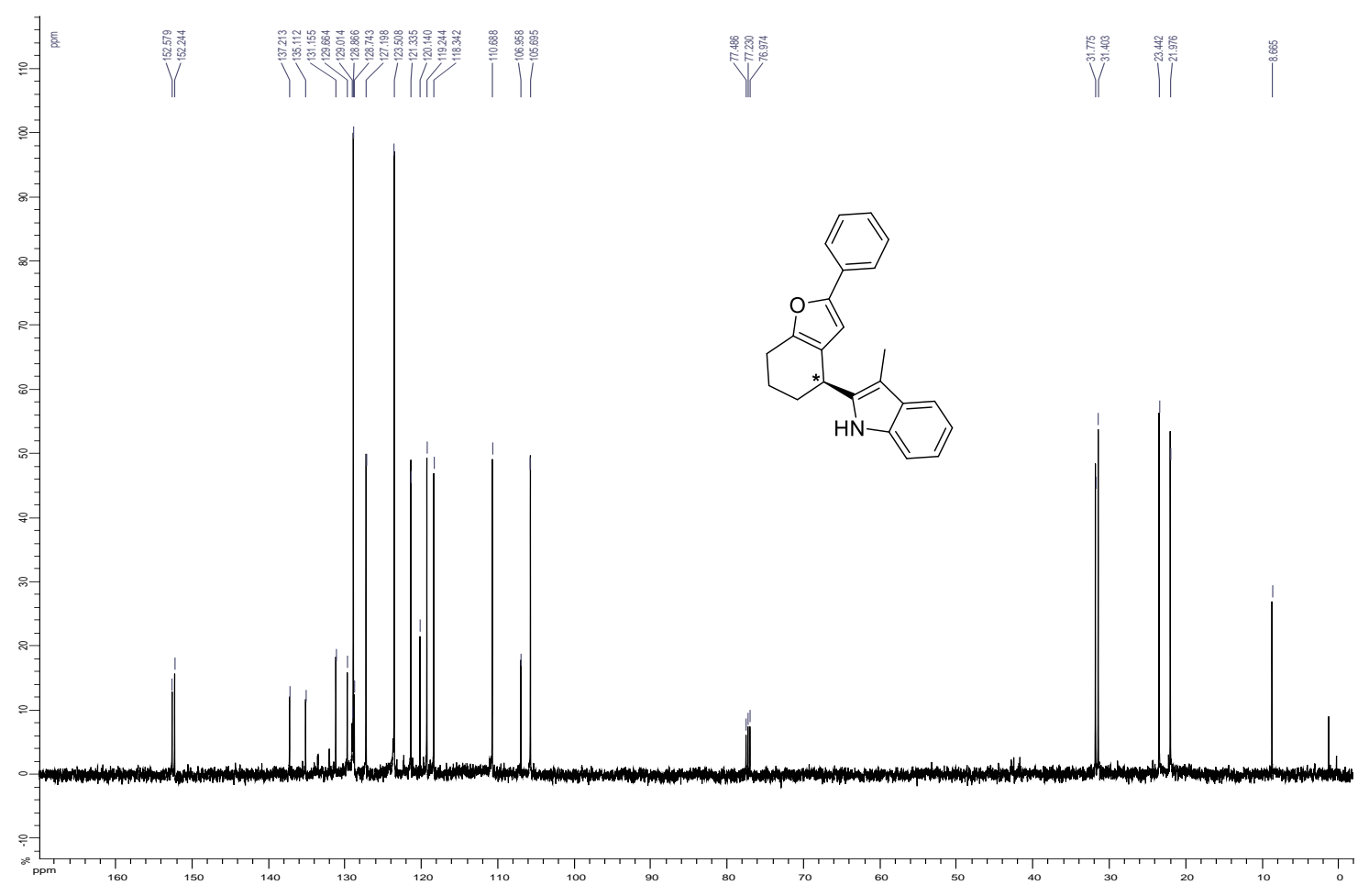

${ }^{13} \mathrm{C} \mathrm{NMR}\left(\mathrm{CDCl}_{3}, 125 \mathrm{MHz}\right.$ ) spectrum of (S)-3-methyl-2-(2-phenyl-4,5,6,7-tetrahydrobenzofuran-4-yl)-1H-indole (14ah) 


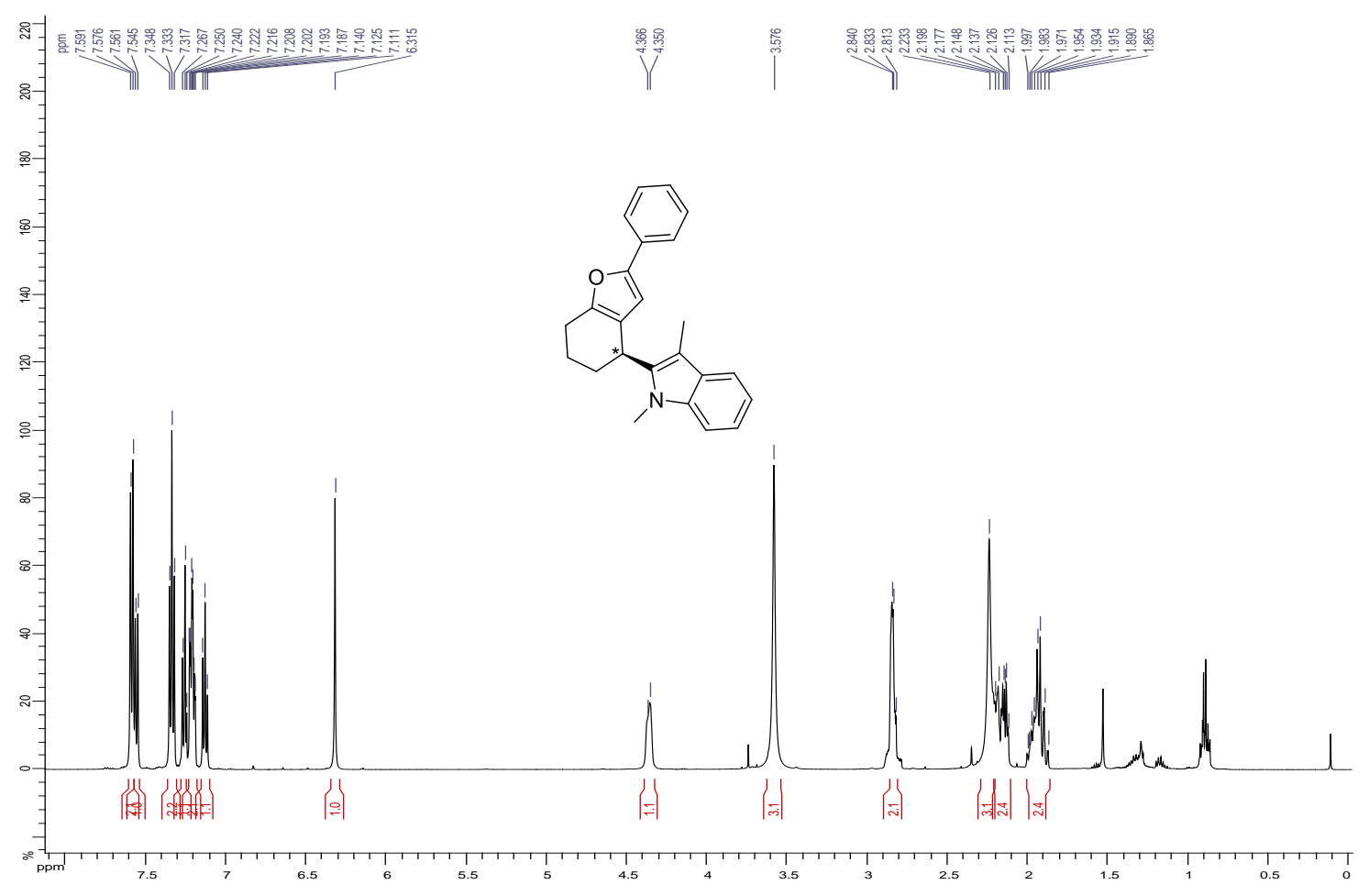

${ }^{1} \mathrm{H}$ NMR (CDCl, 500 MHz) spectrum of (S)-1,3-dimethyl-2-(2-phenyl-4,5,6,7-tetrahydrobenzofuran-4-yl)-indole (14ai)

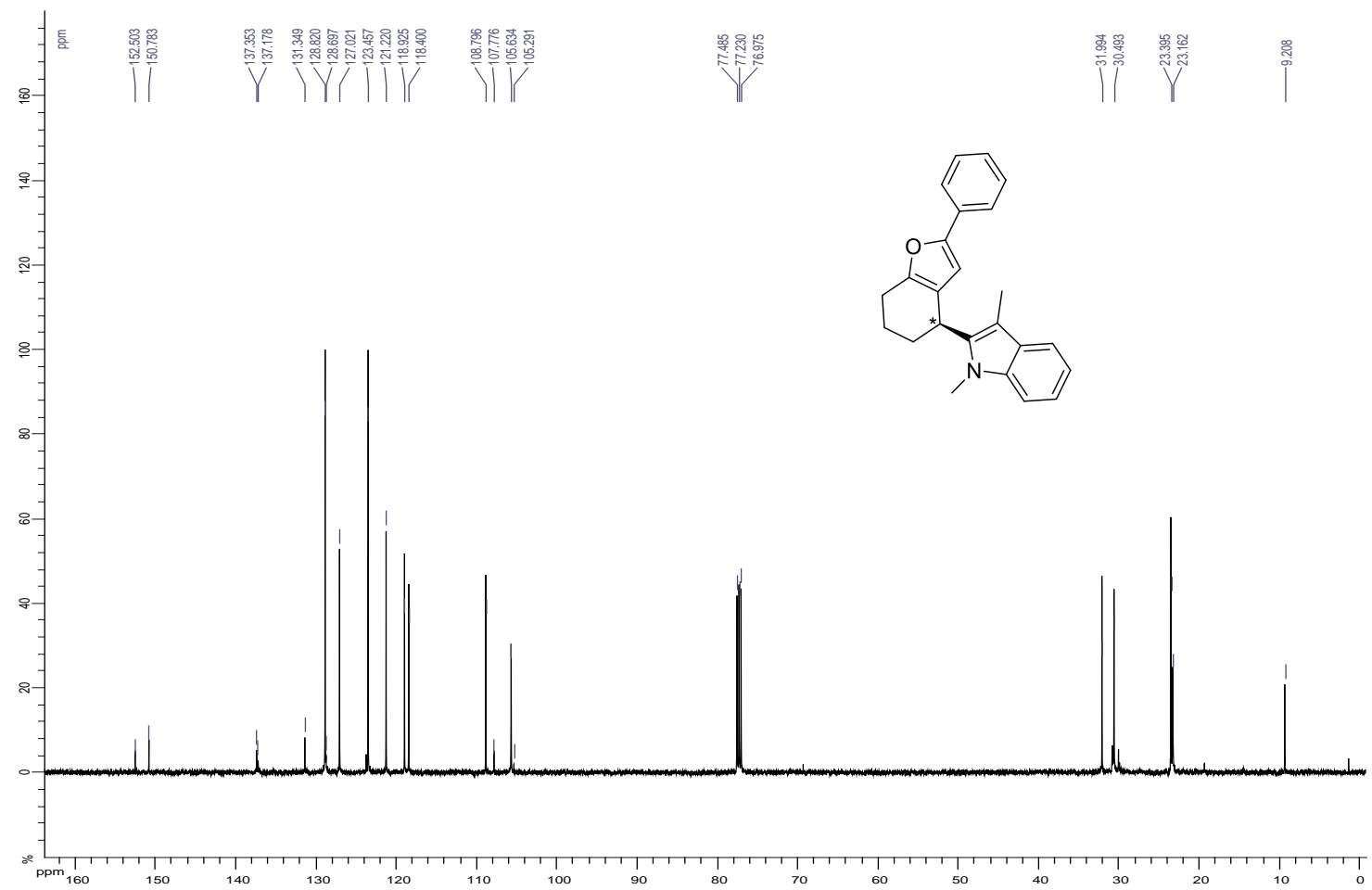

${ }^{13} \mathrm{C} \mathrm{NMR}\left(\mathrm{CDCl}_{3}, 125 \mathrm{MHz}\right)$ spectrum of (S)-1,3-dimethyl-2-(2-phenyl-4,5,6,7-tetrahydrobenzofuran-4-yl)-indole (14ai) 


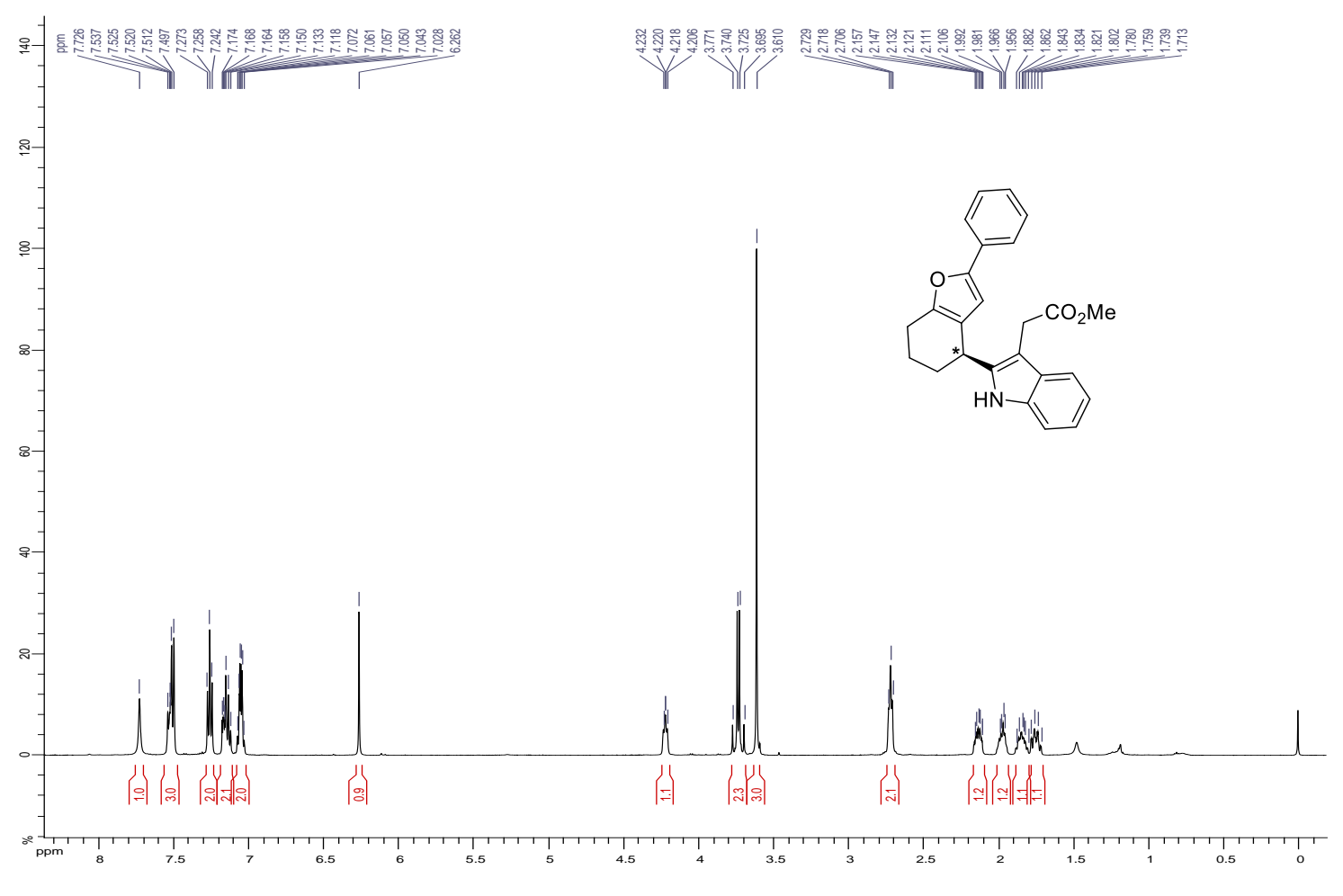

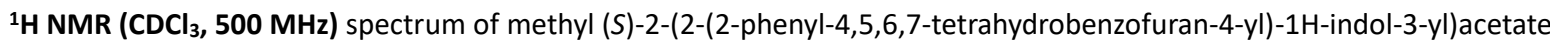
(14aj)

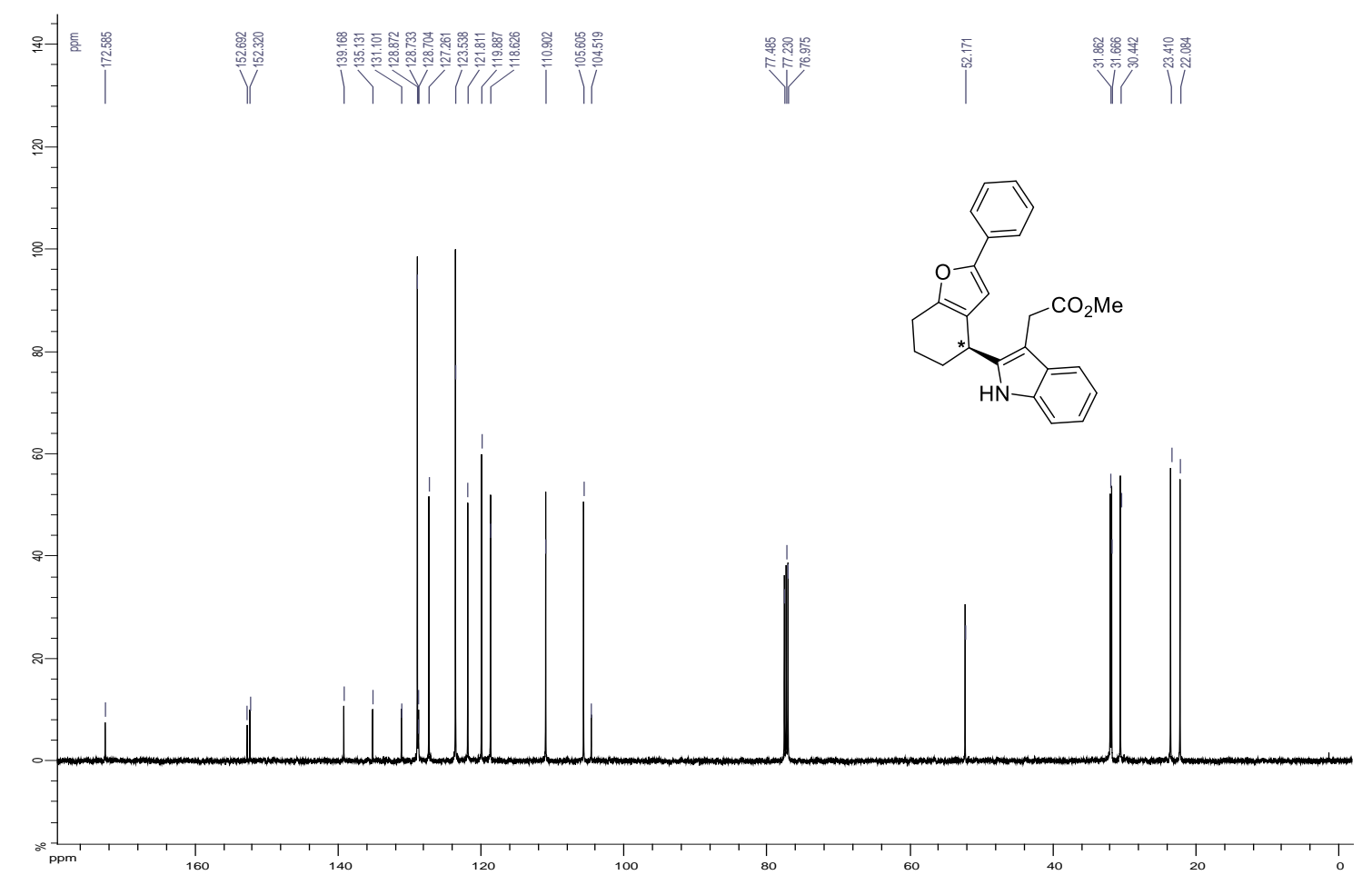

${ }^{13} \mathrm{C} \mathrm{NMR}\left(\mathrm{CDCl}_{3}, 125 \mathrm{MHz}\right.$ ) spectrum of methyl (S)-2-(2-(2-phenyl-4,5,6,7-tetrahydrobenzofuran-4-yl)-1H-indol-3-yl)acetate (14aj) 


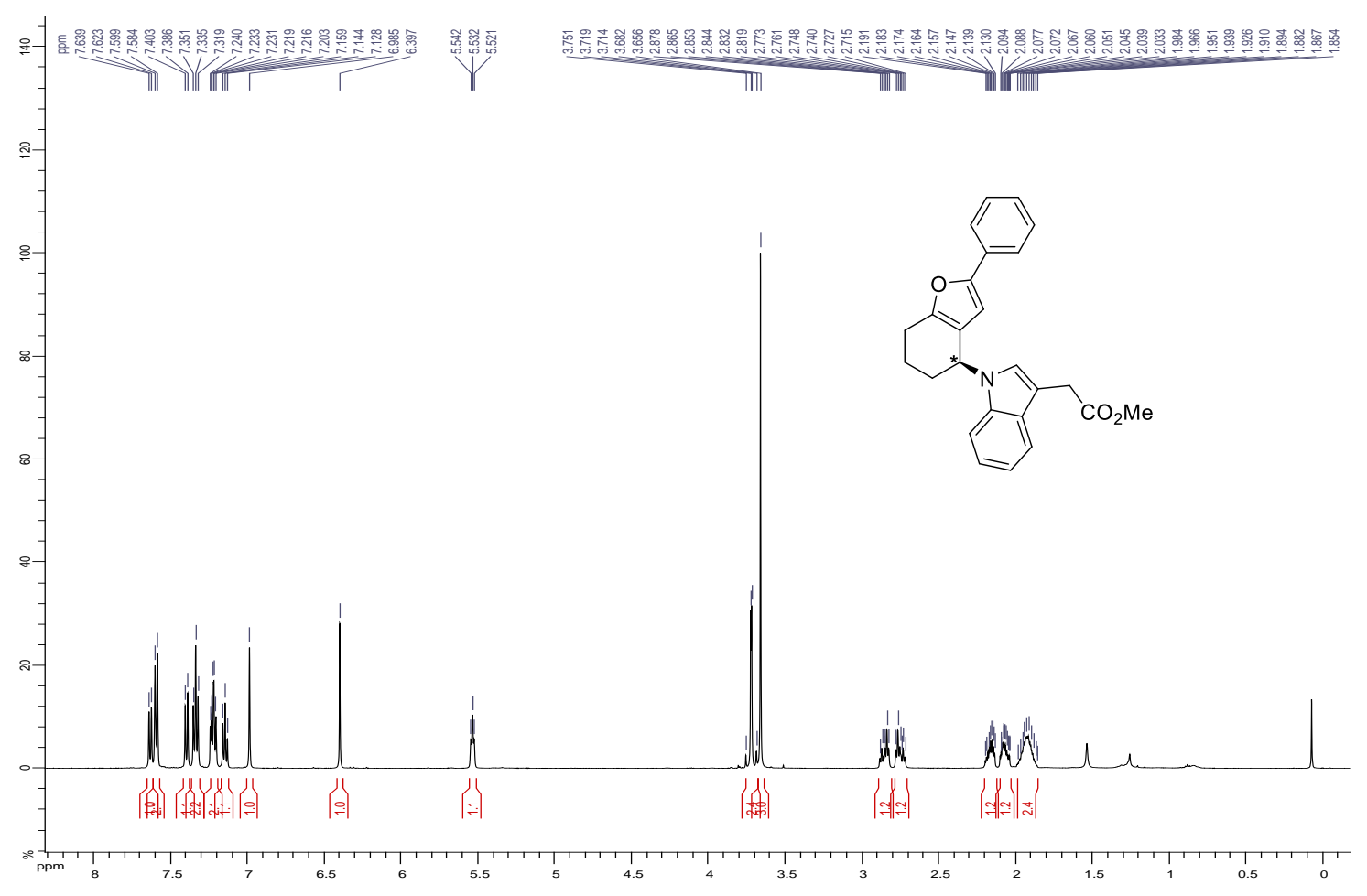

${ }^{1} \mathrm{H} \mathrm{NMR}\left(\mathrm{CDCl}_{3}, 500 \mathrm{MHz}\right.$ ) spectrum of methyl (S)-2-(1-(2-phenyl-4,5,6,7-tetrahydrobenzofuran-4-yl)-indol-3-yl)acetate (15aj)

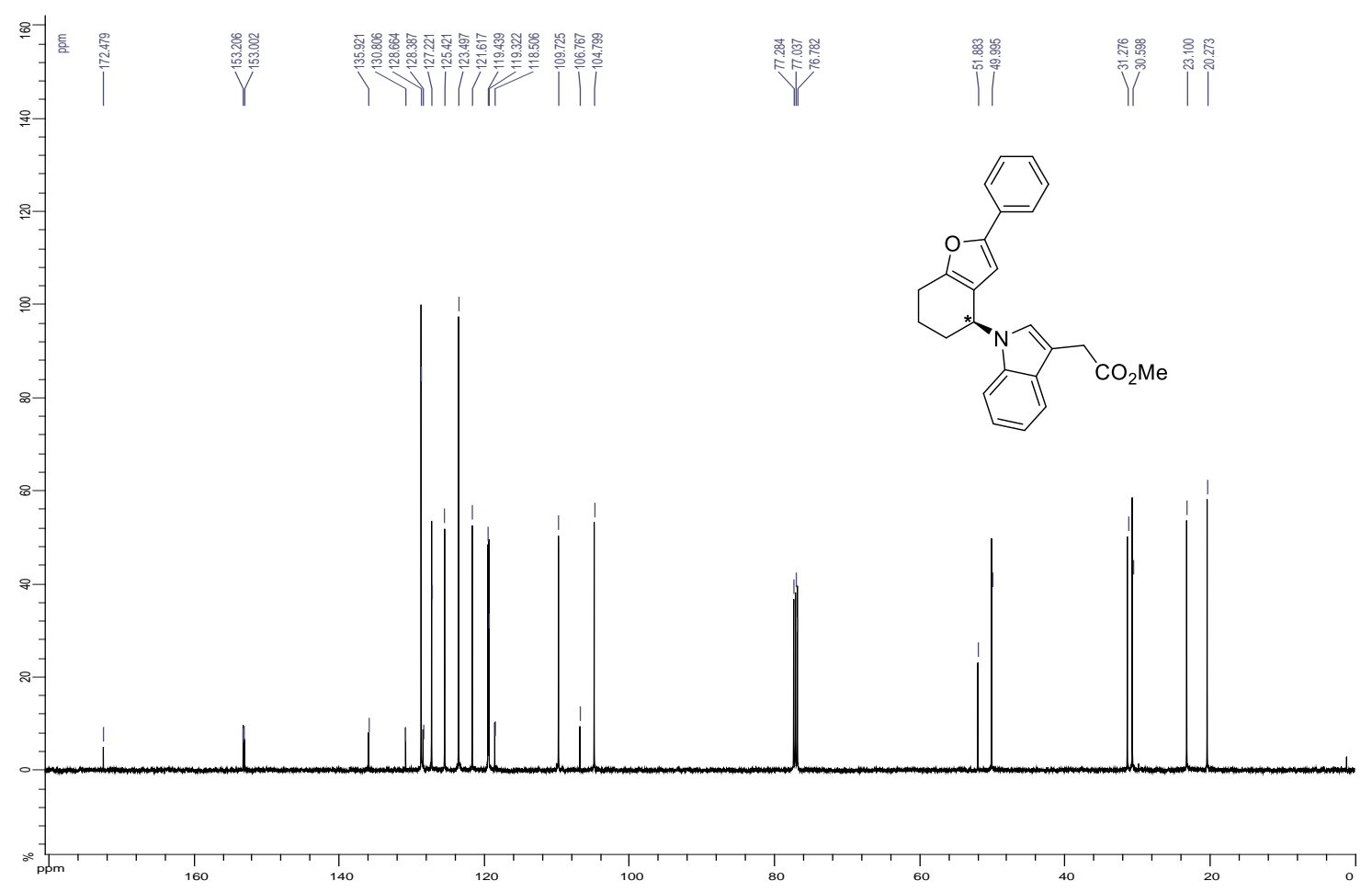

${ }^{13} \mathrm{C} \mathrm{NMR}\left(\mathrm{CDCl}_{3}, 125 \mathrm{MHz}\right.$ ) spectrum of methyl (S)-2-(1-(2-phenyl-4,5,6,7-tetrahydrobenzofuran-4-yl)-indol-3-yl)acetate (15aj) 


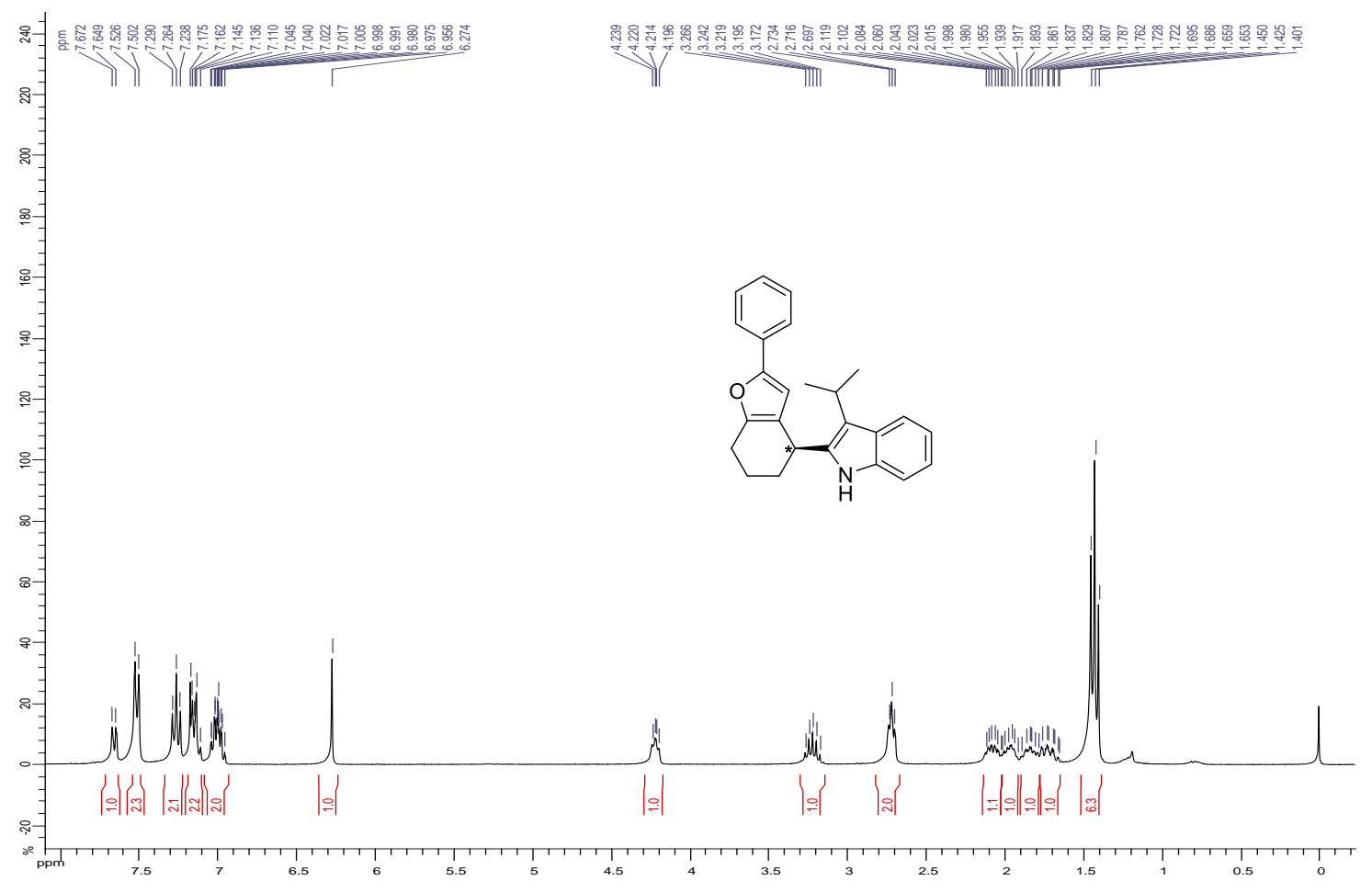

${ }^{1} \mathrm{H} \mathrm{NMR}\left(\mathrm{CDCl}_{3}, 500 \mathrm{MHz}\right.$ ) spectrum of (S)-3-isopropyl-2-(2-phenyl-4,5,6,7-tetrahydrobenzofuran-4-yl)-1H-indole (14ak)

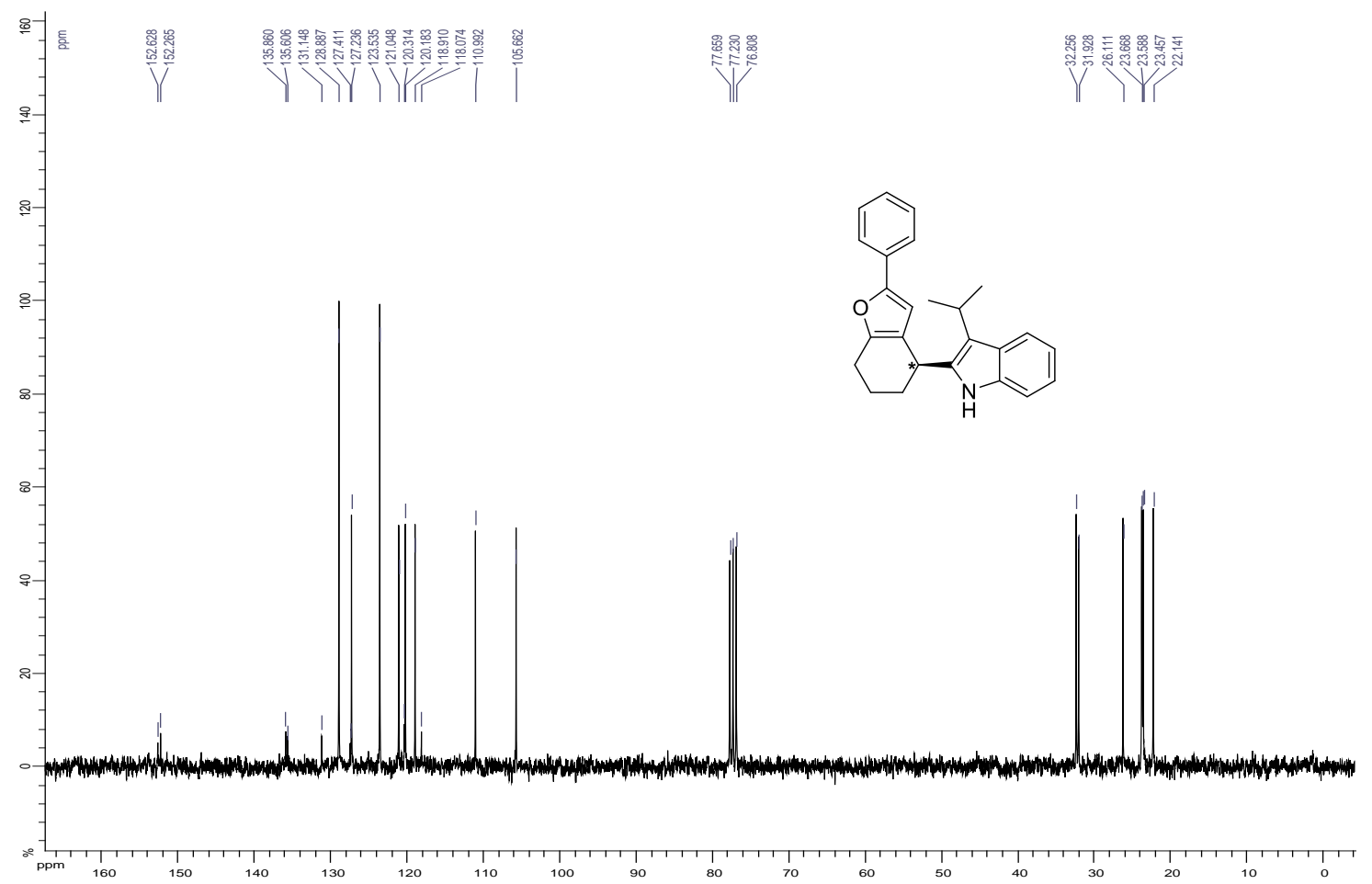

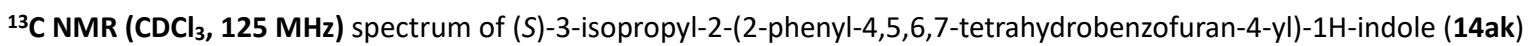




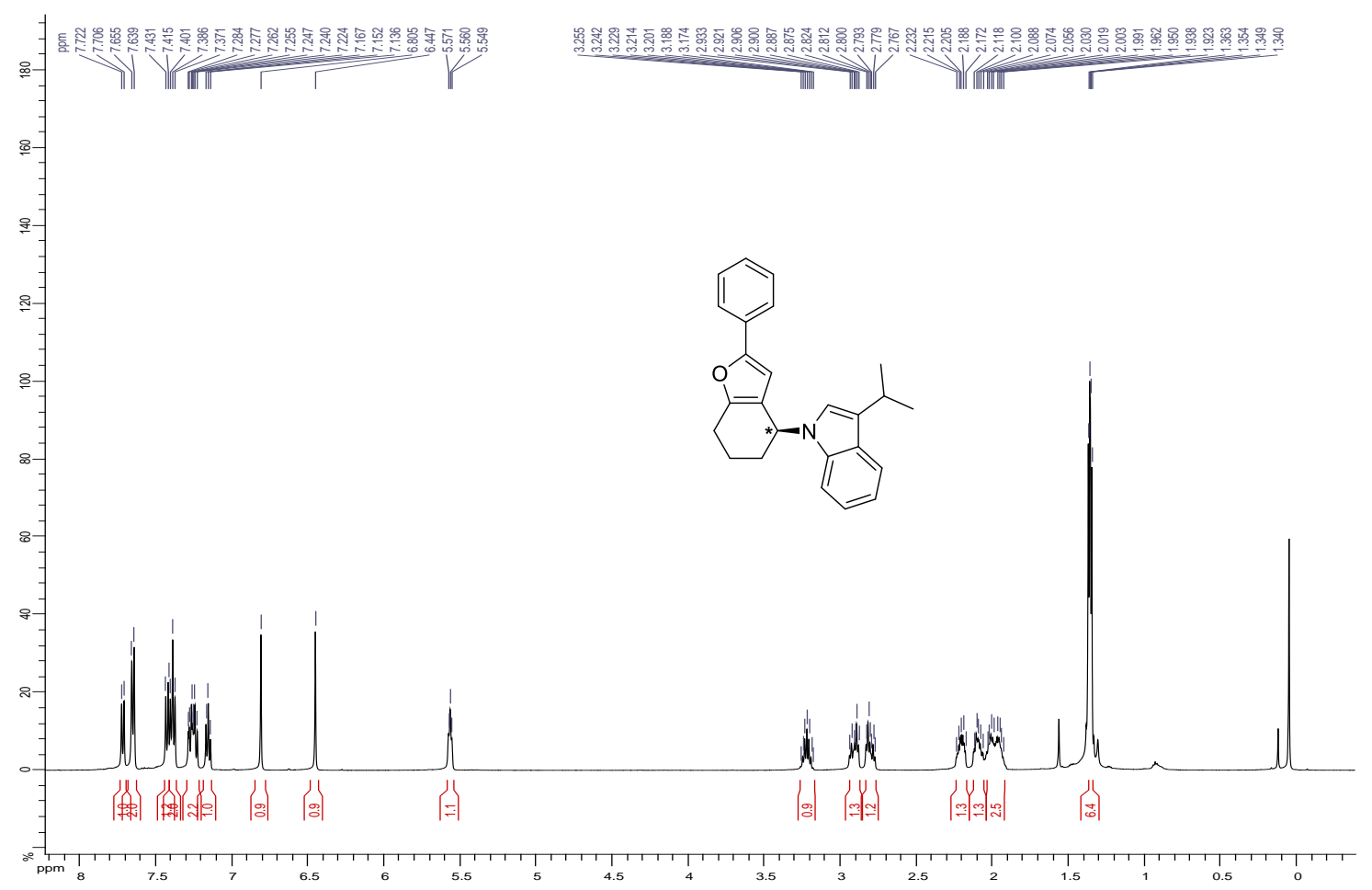

${ }^{1} \mathrm{H} \mathrm{NMR} \mathrm{(C}{ }_{3}, 500 \mathrm{MHz}$ ) spectrum of (S)-3-isopropyl-1-(2-phenyl-4,5,6,7-tetrahydrobenzofuran-4-yl)-indole (15ak)

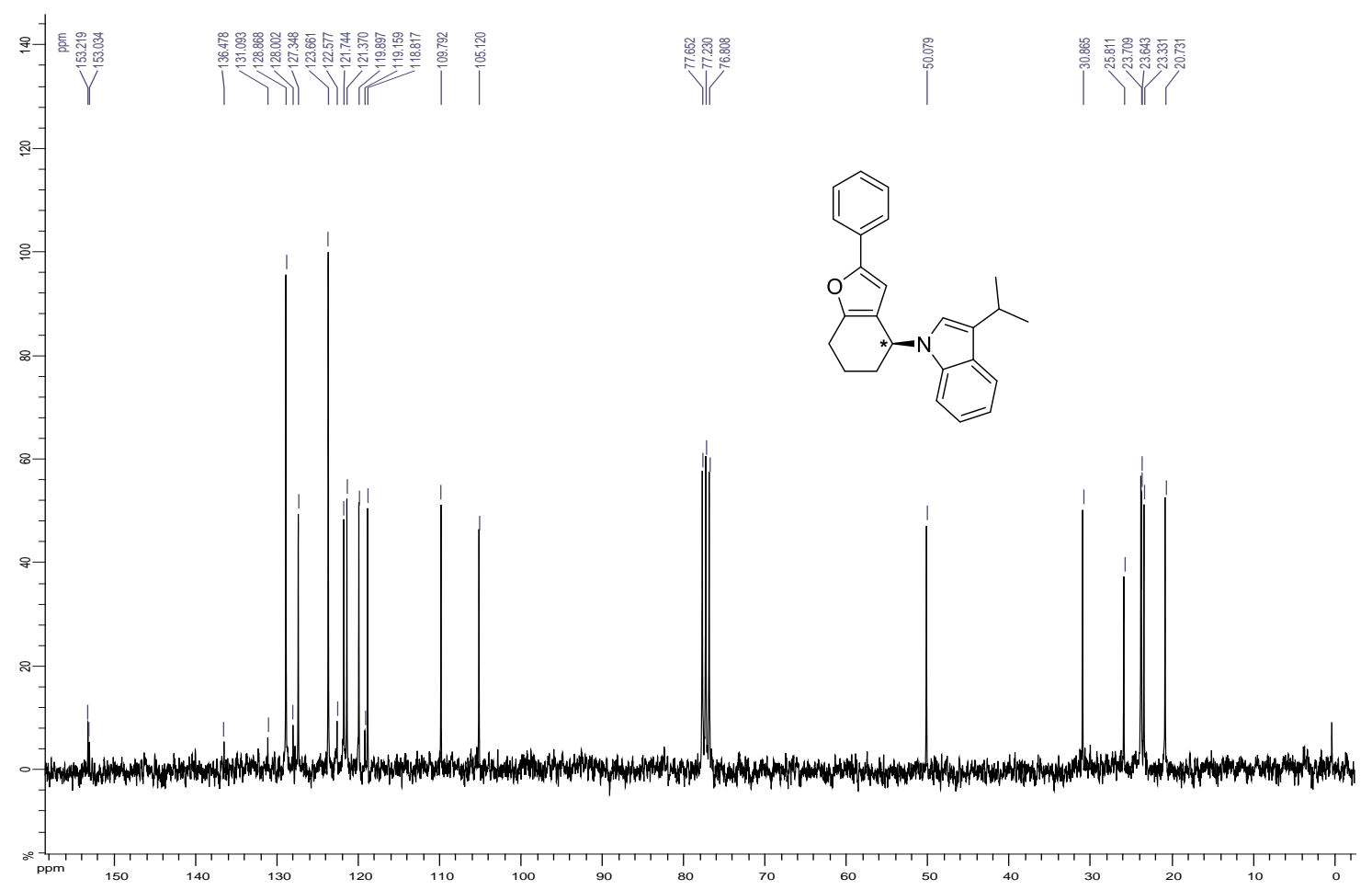

${ }^{13} \mathrm{C} \mathrm{NMR}\left(\mathrm{CDCl}_{3}, 125 \mathrm{MHz}\right.$ ) spectrum of (S)-3-isopropyl-1-(2-phenyl-4,5,6,7-tetrahydrobenzofuran-4-yl)-indole (15ak) 


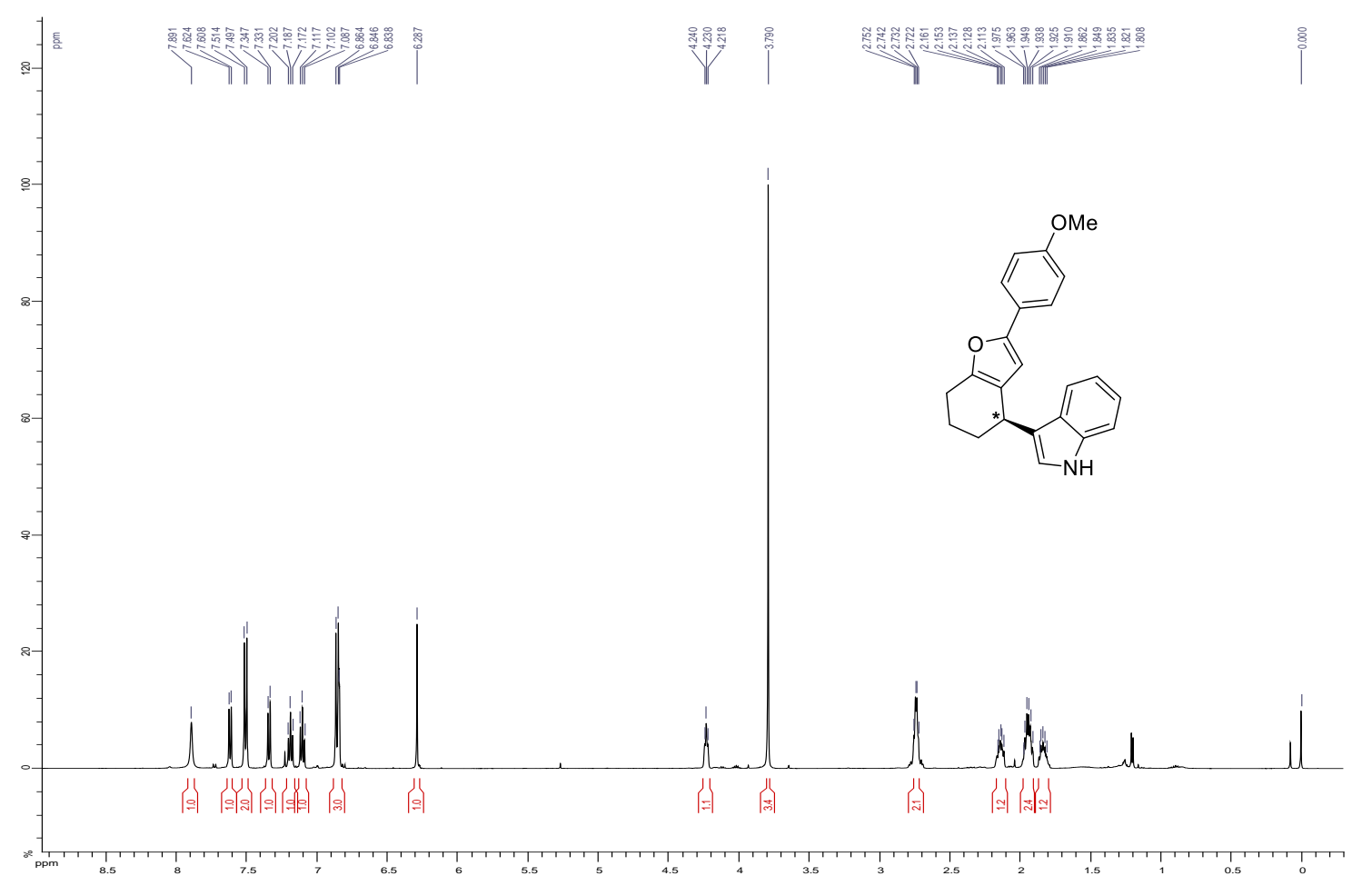

${ }^{1} \mathrm{H} \mathrm{NMR}\left(\mathrm{CDCl}_{3}, 500 \mathrm{MHz}\right.$ ) spectrum of (R)-3-(2-(4-methoxyphenyl)-4,5,6,7-tetrahydrobenzofuran-4-yl)-1H-indole (10ba)

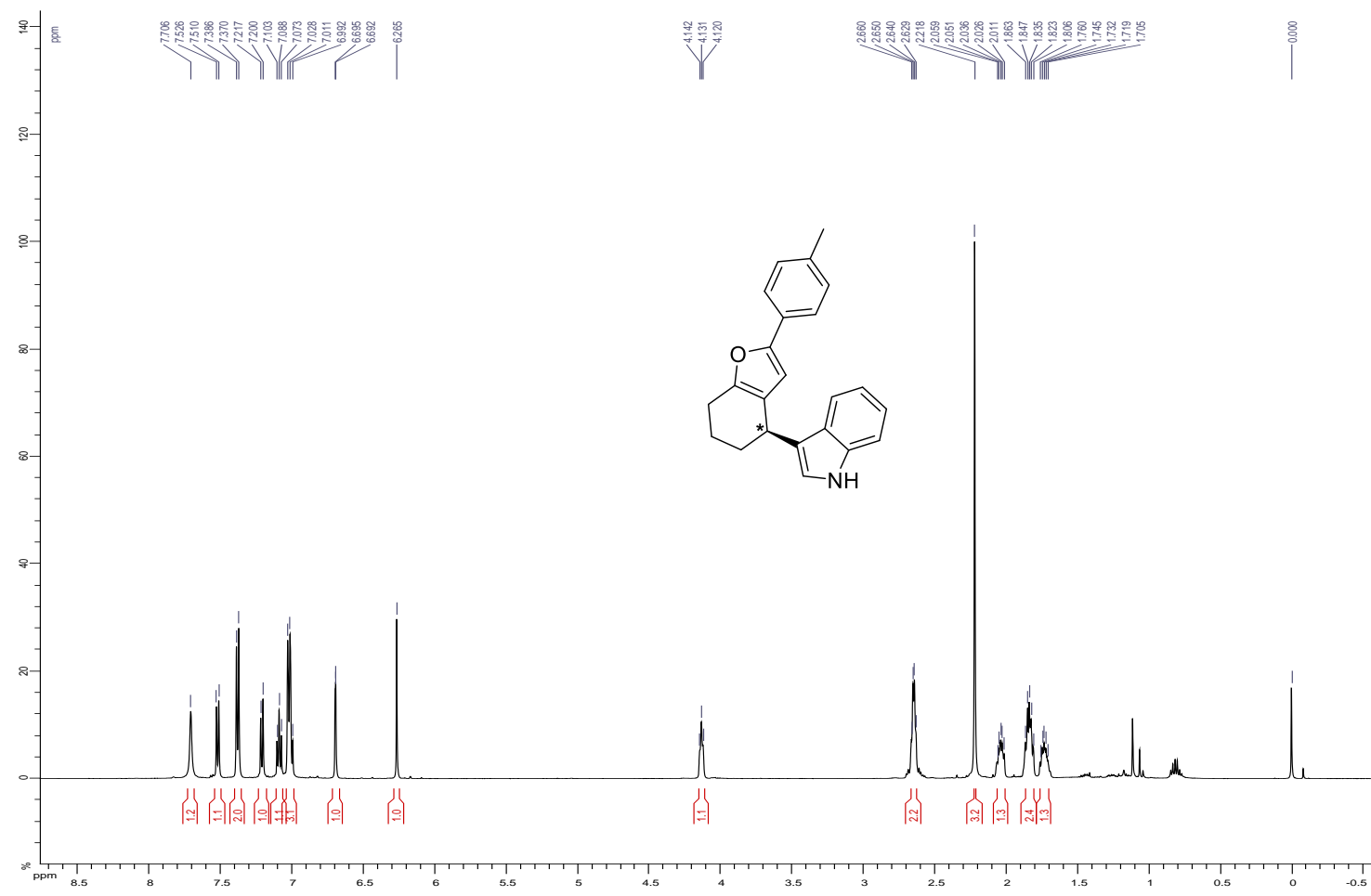

${ }^{1} \mathrm{H}$ NMR $\left(\mathrm{CDCl}_{3}, 500 \mathrm{MHz}\right)$ spectrum of $(R)$-3-(2-(p-tolyl)-4,5,6,7-tetrahydrobenzofuran-4-yl)-1H-indole (10ca) 


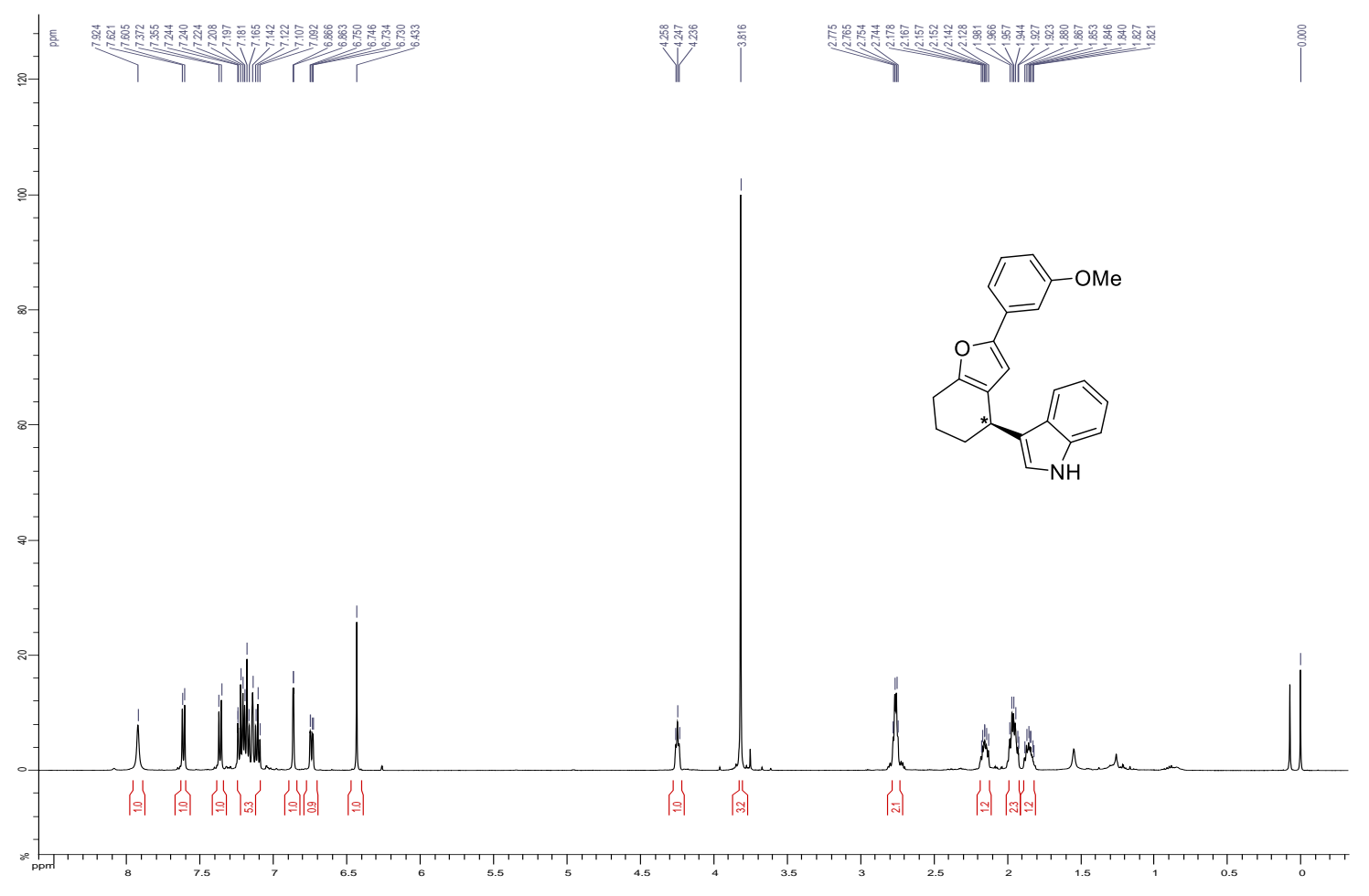

${ }^{1} \mathrm{H} \mathrm{NMR}\left(\mathrm{CDCl}_{3}, 500 \mathrm{MHz}\right.$ ) spectrum of (R)-3-(2-(3-methoxyphenyl)-4,5,6,7-tetrahydrobenzofuran-4-yl)-1H-indole (10da)

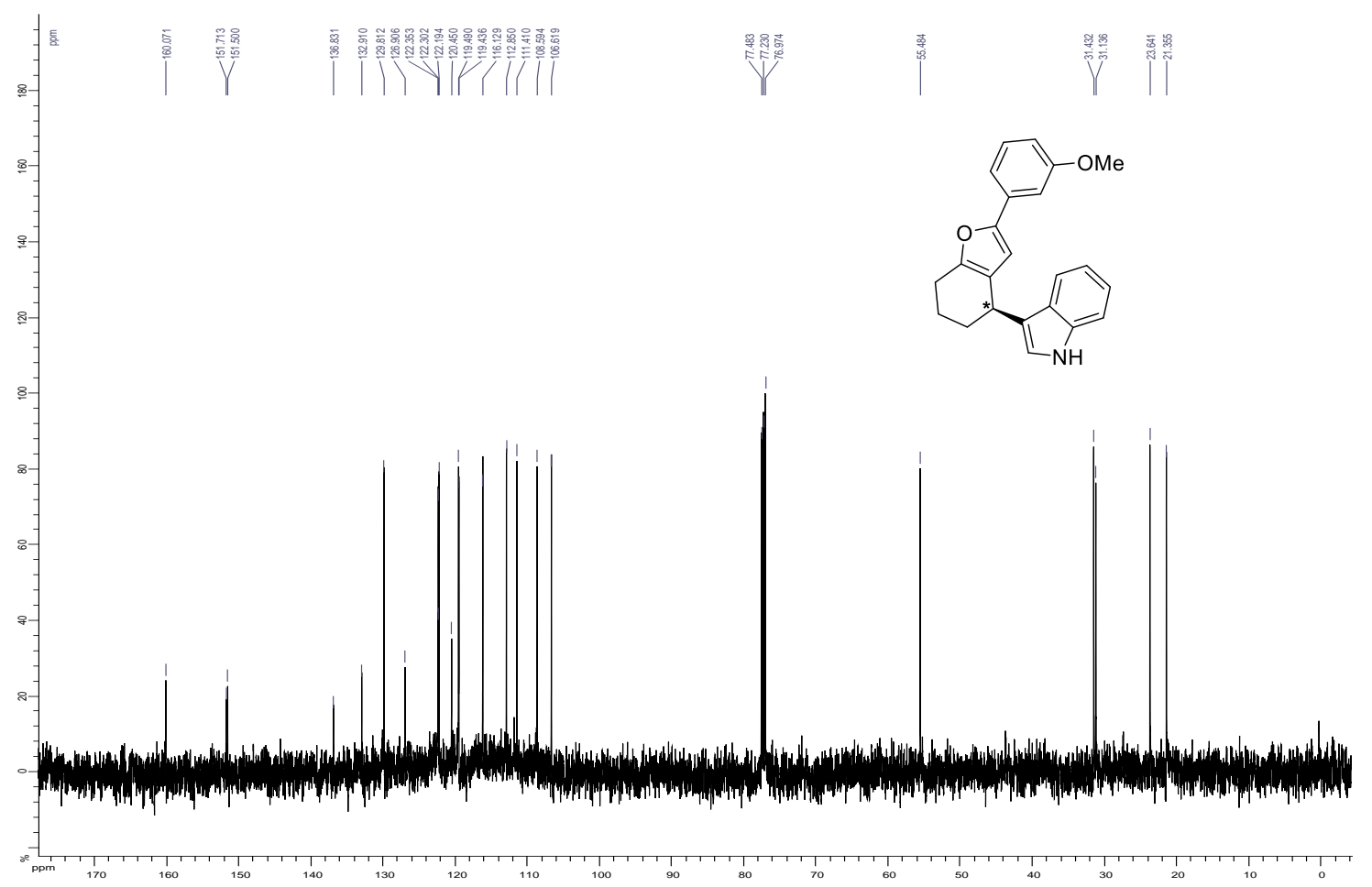

${ }^{13} \mathrm{C} \mathrm{NMR}\left(\mathrm{CDCl}_{3}, 125 \mathrm{MHz}\right.$ ) spectrum of (R)-3-(2-(3-methoxyphenyl)-4,5,6,7-tetrahydrobenzofuran-4-yl)-1H-indole (10da) 


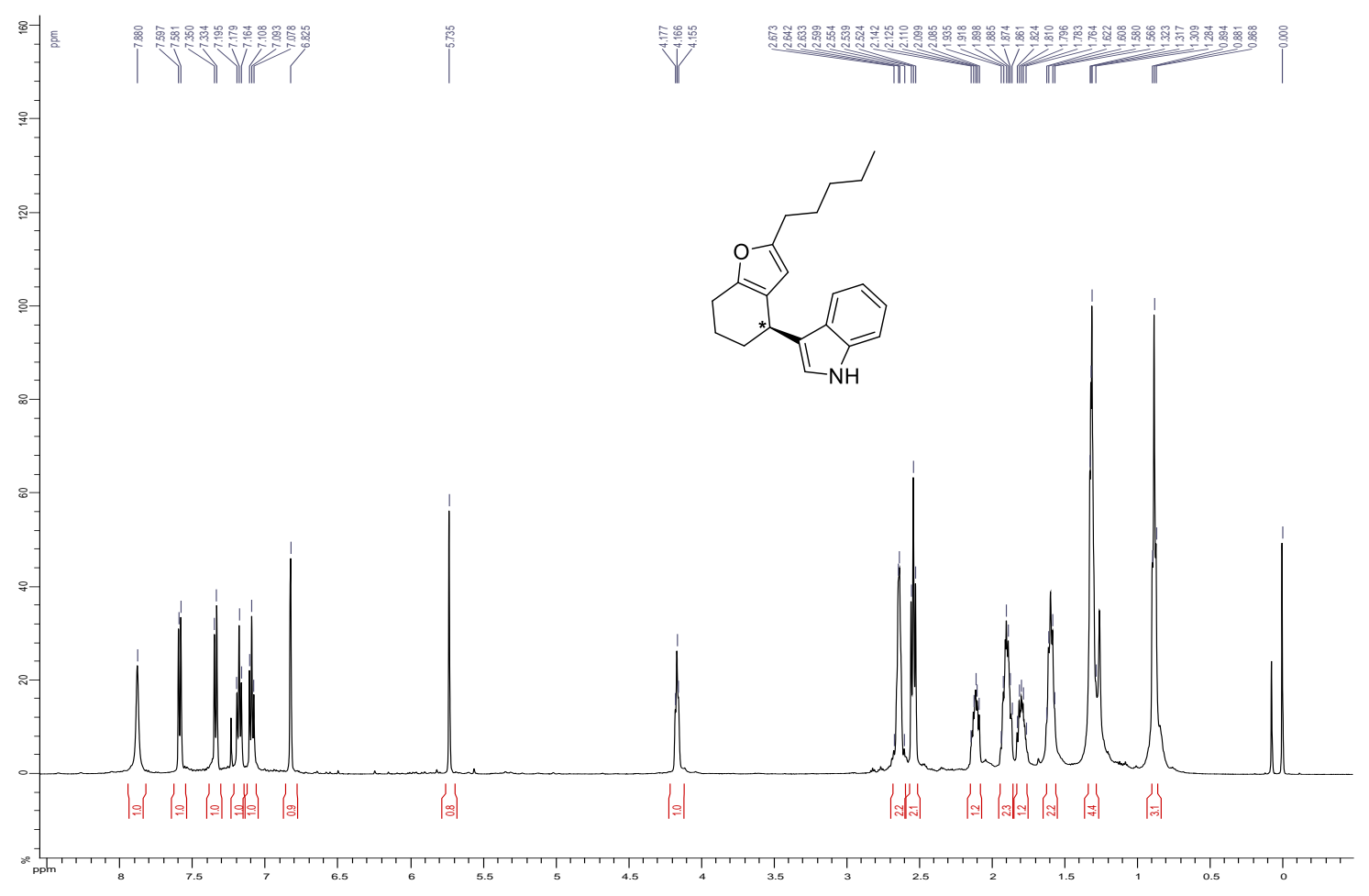

${ }^{1} \mathrm{H} \mathrm{NMR}\left(\mathrm{CDCl}_{3}, 500 \mathrm{MHz}\right.$ ) spectrum of (R)-3-(2-pentyl-4,5,6,7-tetrahydrobenzofuran-4-yl)-1H-indole (10ea)

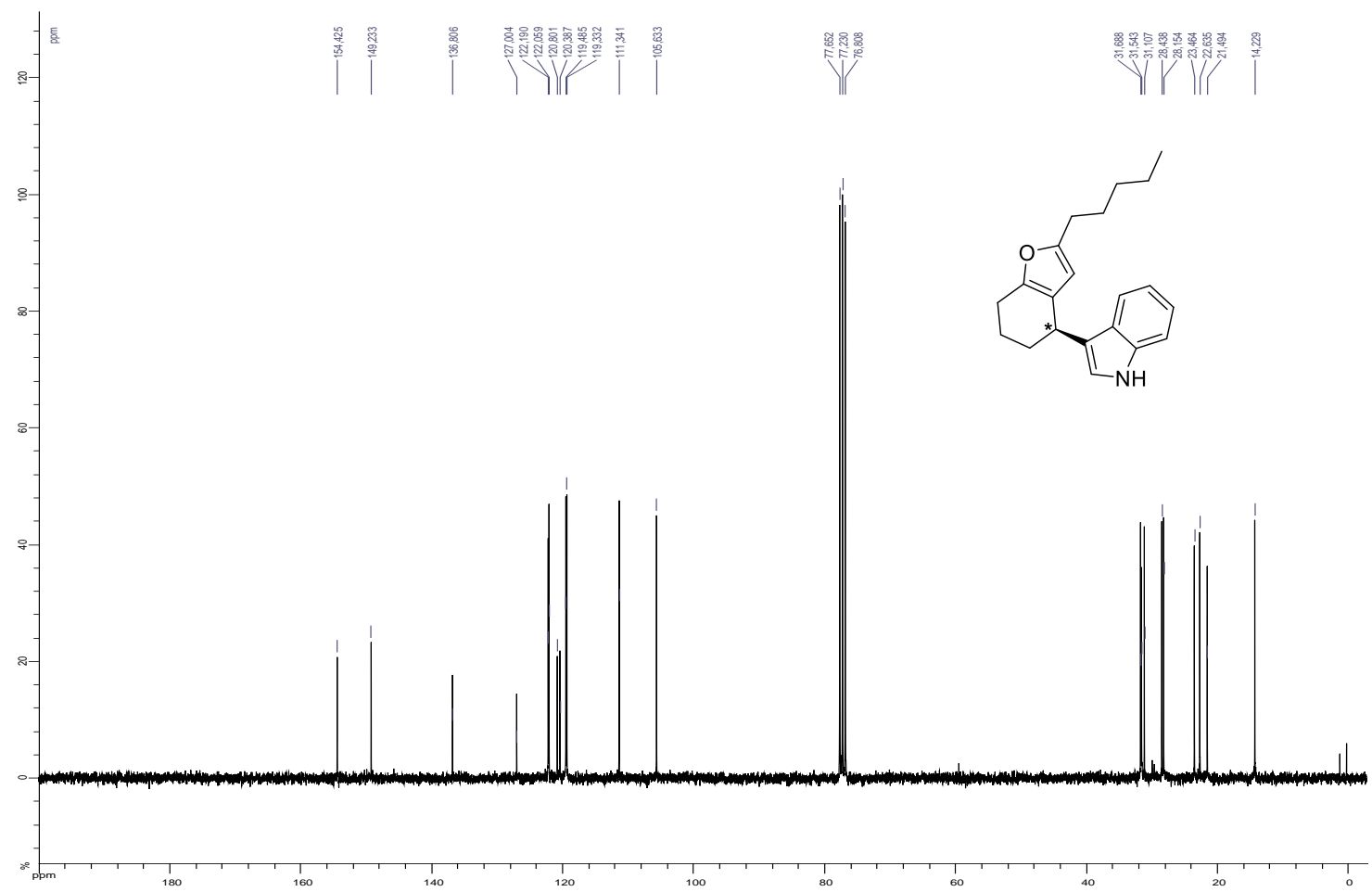

${ }^{13} \mathrm{C} \mathrm{NMR}\left(\mathrm{CDCl}_{3}, 75 \mathrm{MHz}\right.$ ) spectrum of (R)-3-(2-pentyl-4,5,6,7-tetrahydrobenzofuran-4-yl)-1H-indole (10ea) 


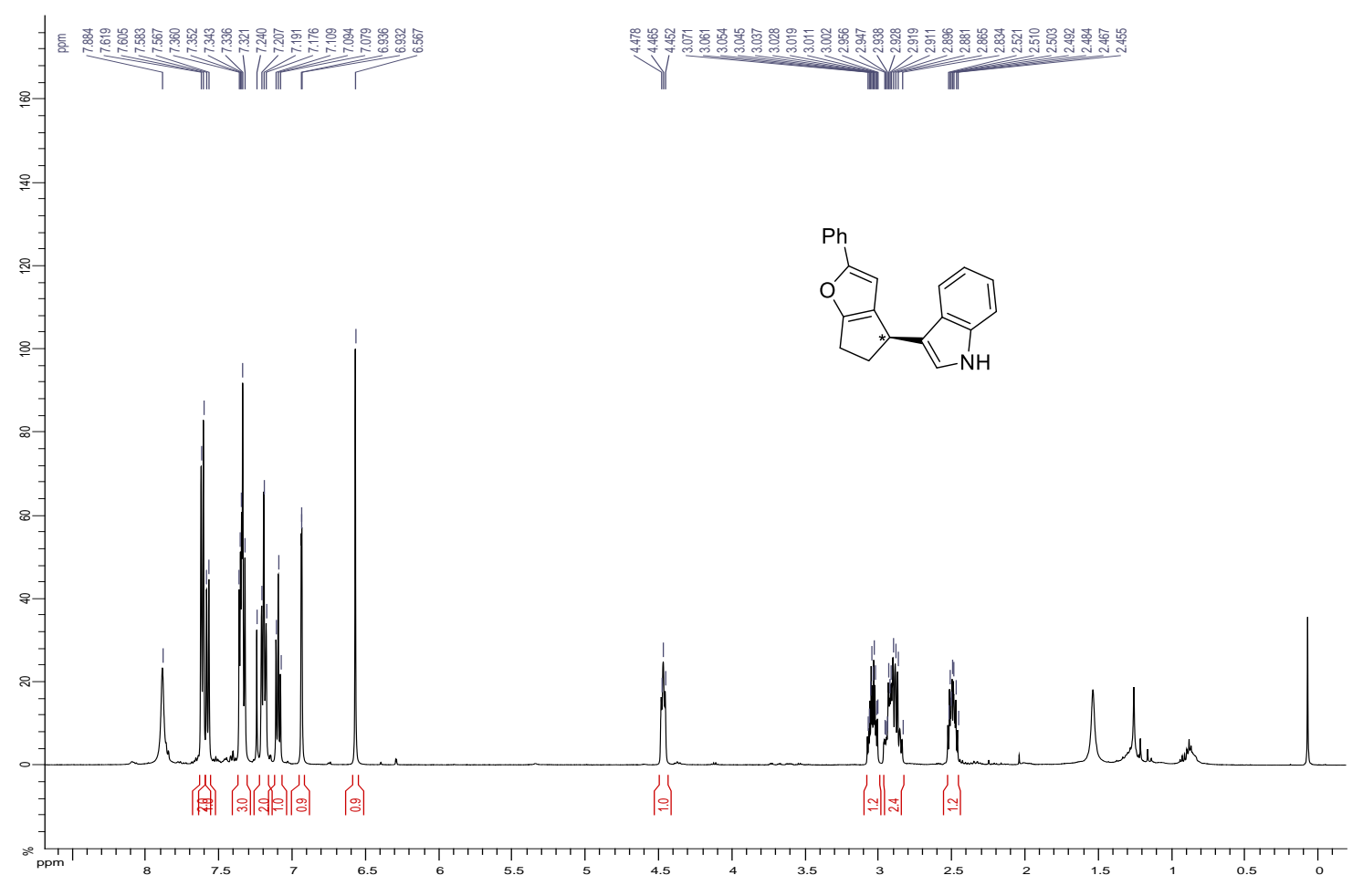

${ }^{1} \mathrm{H}$ NMR ( $\mathrm{CDCl}_{3}, 500 \mathrm{MHz}$ ) spectrum of (R)-3-(2-phenyl-5,6-dihydro-4H-cyclopenta[b]furan-4-yl)-1H-indole (10fa)

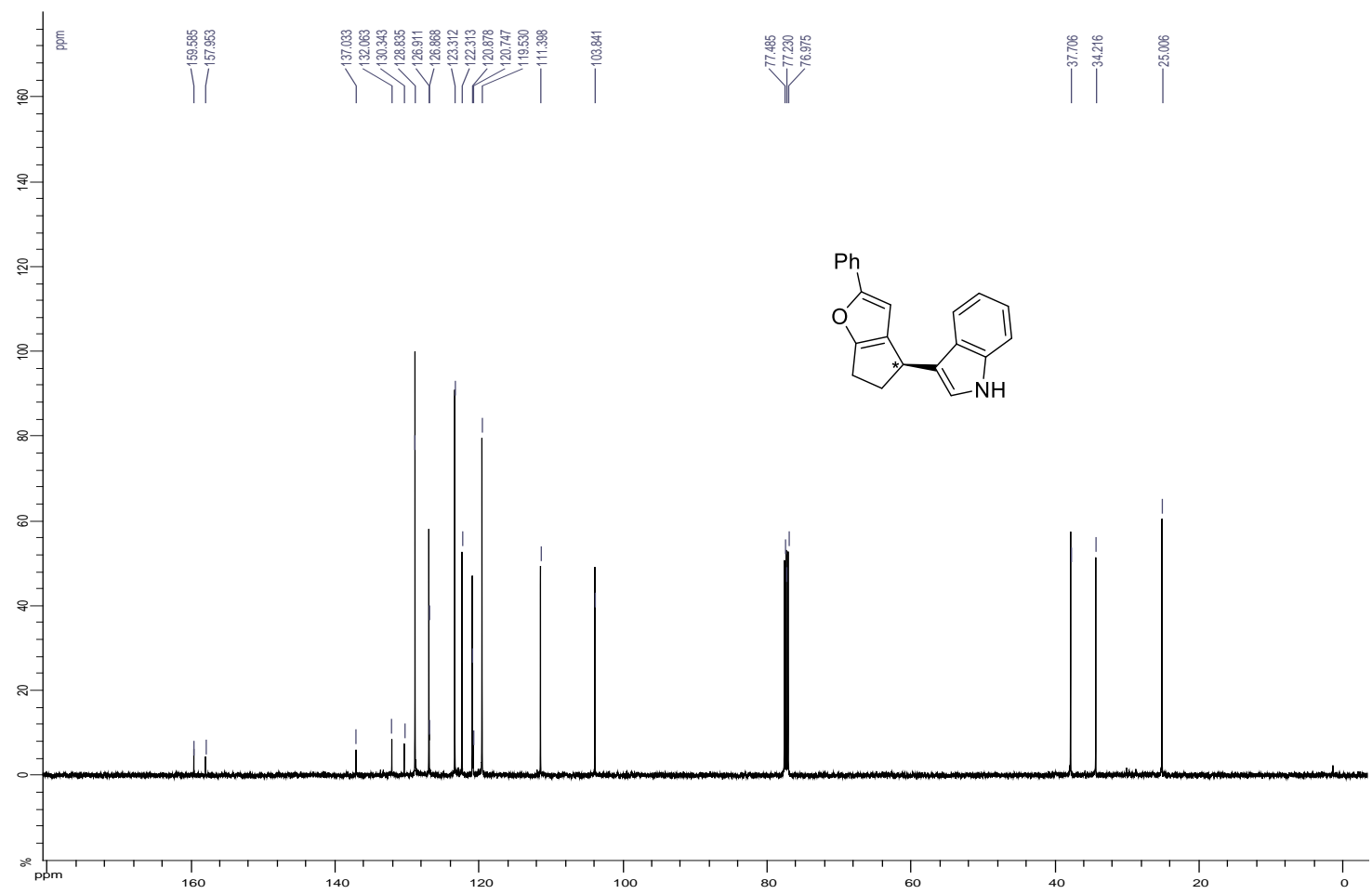

${ }^{13} \mathrm{C} \mathrm{NMR}\left(\mathrm{CDCl}_{3}, 125 \mathrm{MHz}\right.$ ) spectrum of (R)-3-(2-phenyl-5,6-dihydro-4H-cyclopenta[b]furan-4-yl)-1H-indole (10fa) 


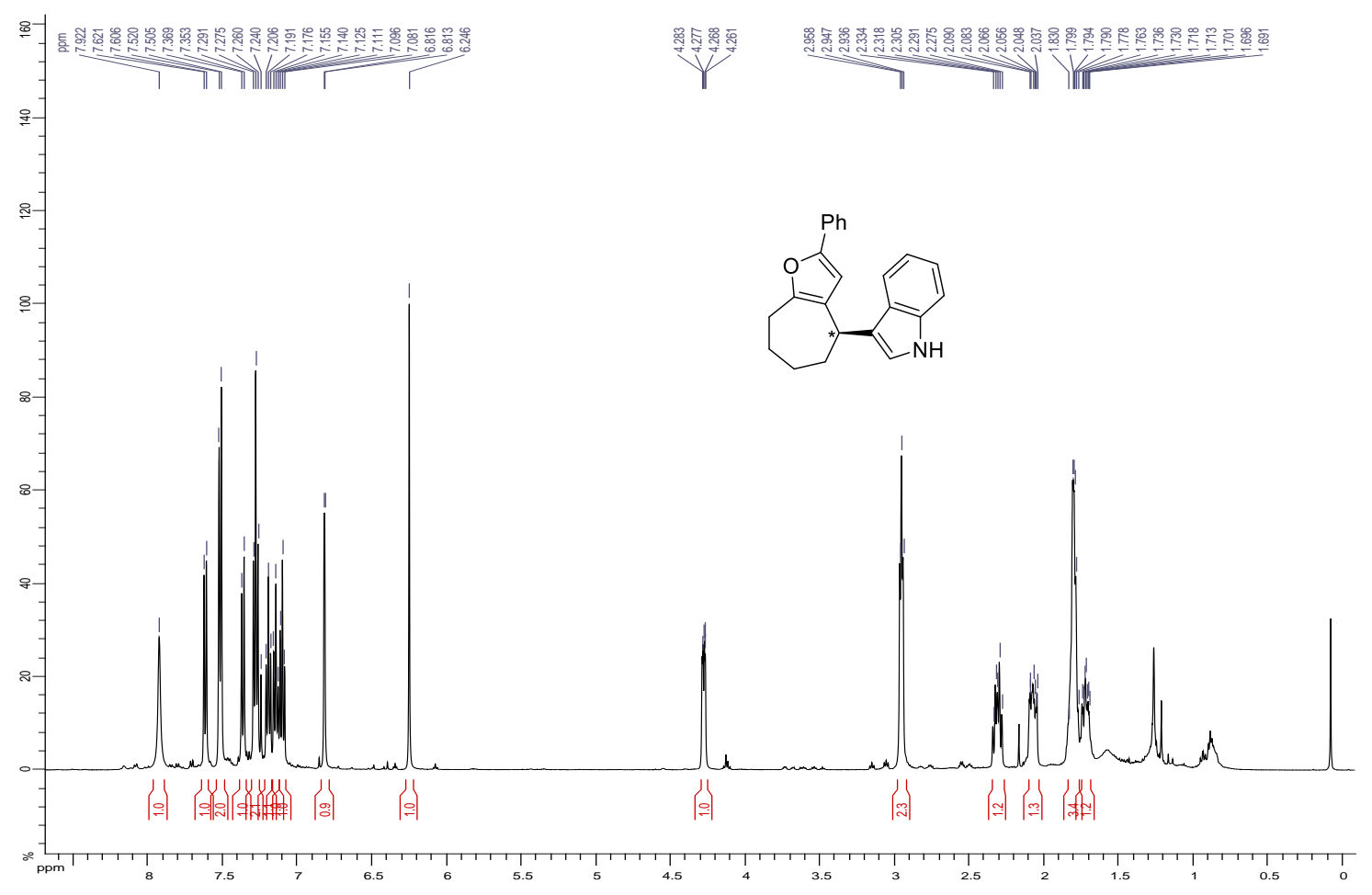

${ }^{1} \mathrm{H}$ NMR $\left(\mathrm{CDCl}_{3}, 500 \mathrm{MHz}\right.$ ) spectrum of (R)-3-(2-phenyl-5,6,7,8-tetrahydro-4H-cyclohepta[b]furan-4-yl)-1H-indole (10ga)

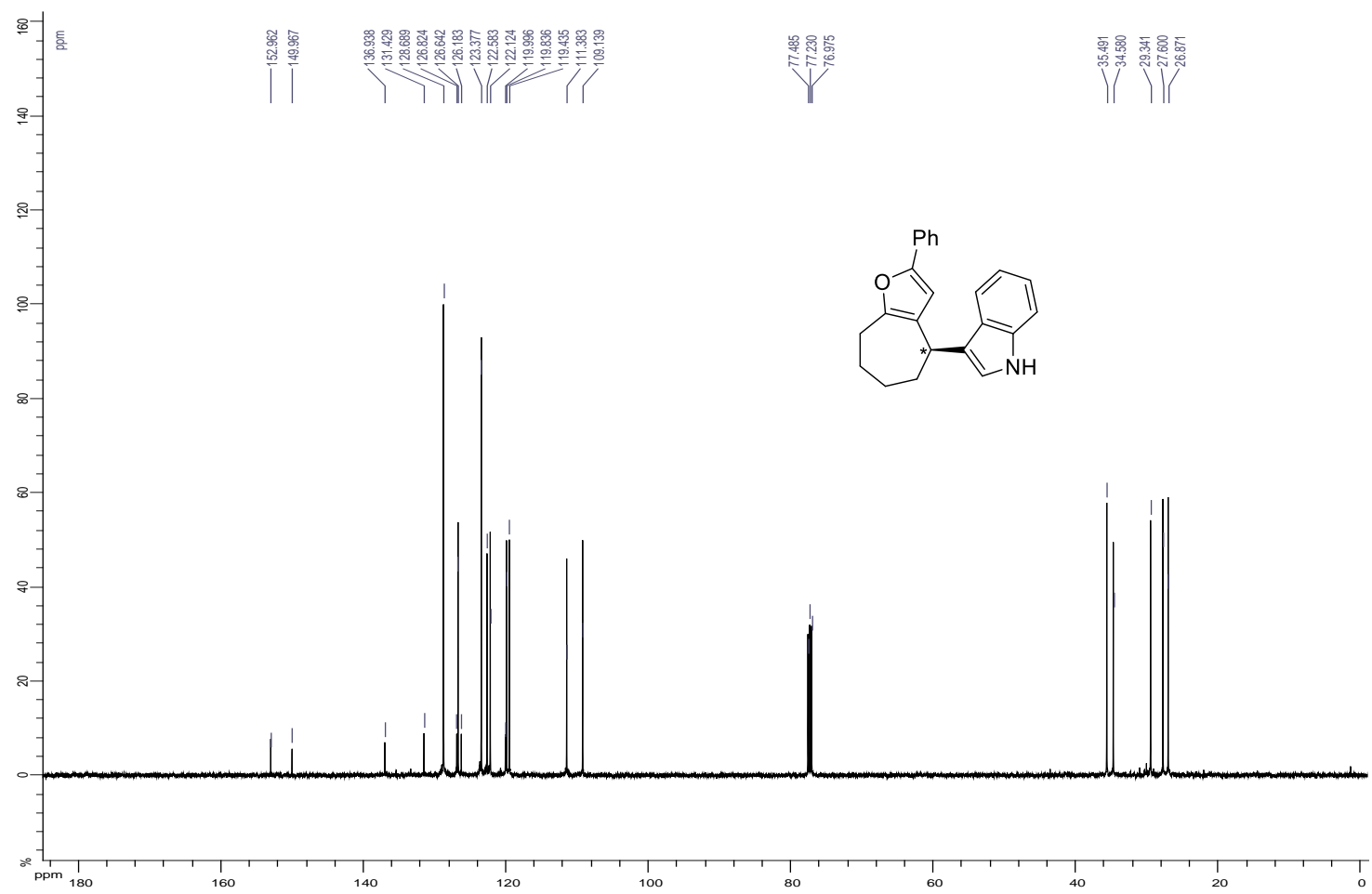

${ }^{13} \mathrm{C} \mathrm{NMR}\left(\mathrm{CDCl}_{3}, 125 \mathrm{MHz}\right.$ ) spectrum of (R)-3-(2-phenyl-5,6,7,8-tetrahydro-4H-cyclohepta[b]furan-4-yl)-1H-indole (10ga) 


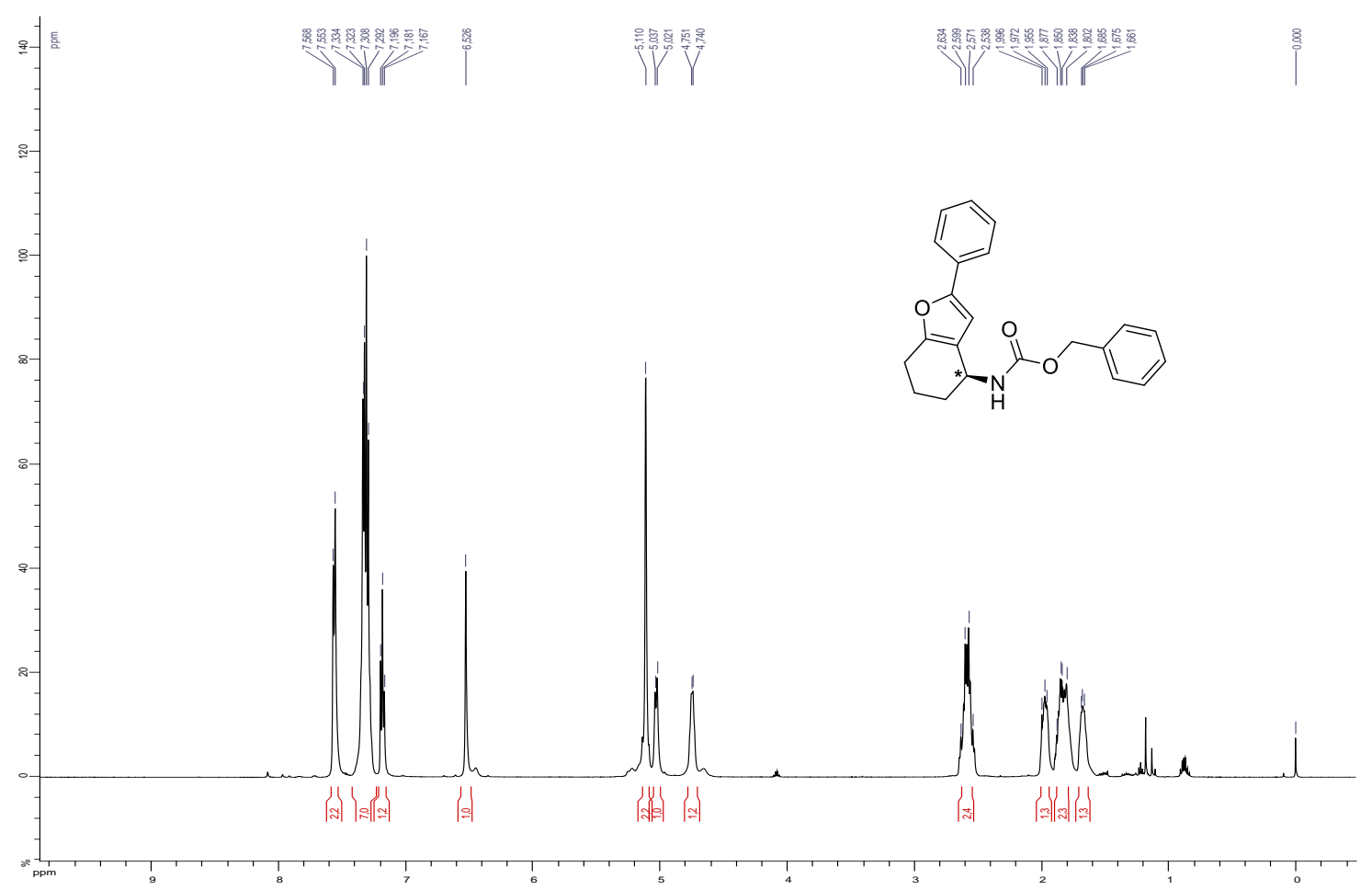

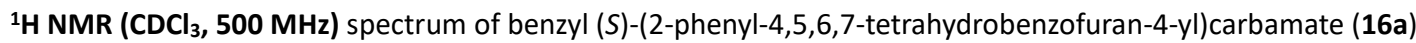

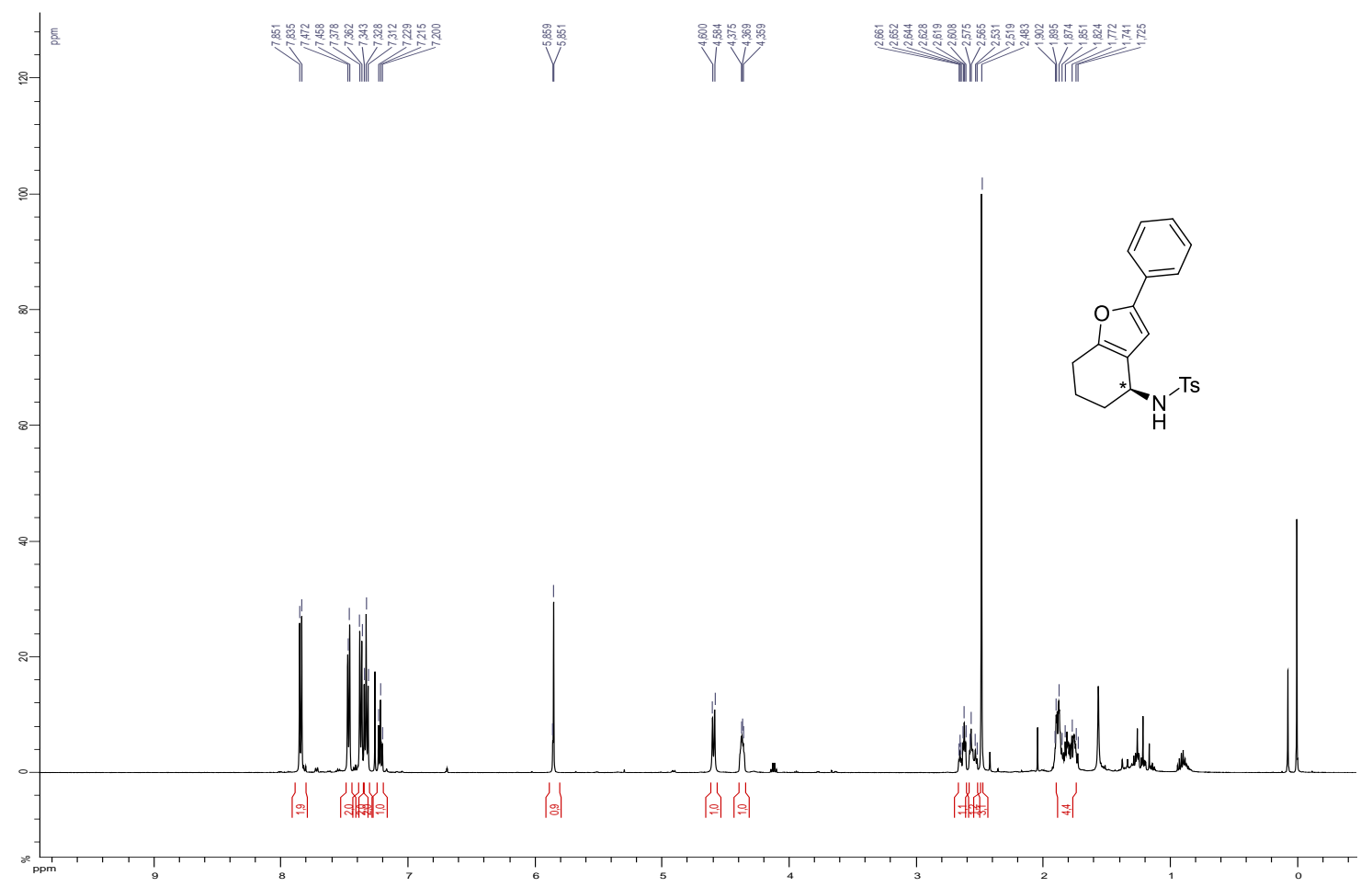

${ }^{1}{ }^{H}$ NMR $\left(\mathrm{CDCl}_{3}, 500 \mathrm{MHz}\right.$ ) spectrum of (S)-4-methyl-N-(2-phenyl-4,5,6,7-tetrahydrobenzofuran-4-yl)benzenesulfonamide (16b) 


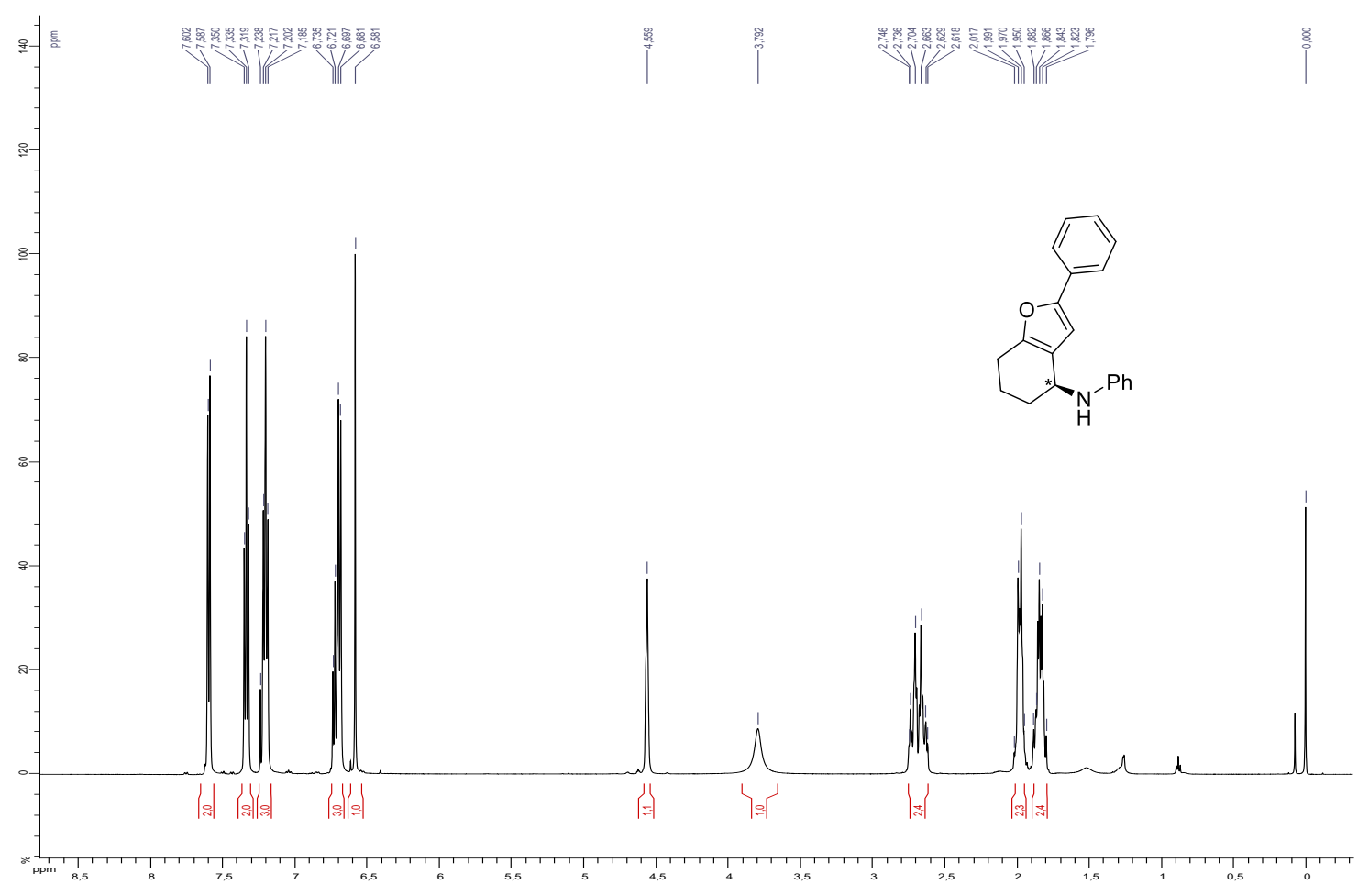

${ }^{1} \mathrm{H}$ NMR $\left(\mathrm{CDCl}_{3}, 500 \mathrm{MHz}\right.$ ) spectrum of (S)-N,2-diphenyl-4,5,6,7-tetrahydrobenzofuran-4-amine (16c)

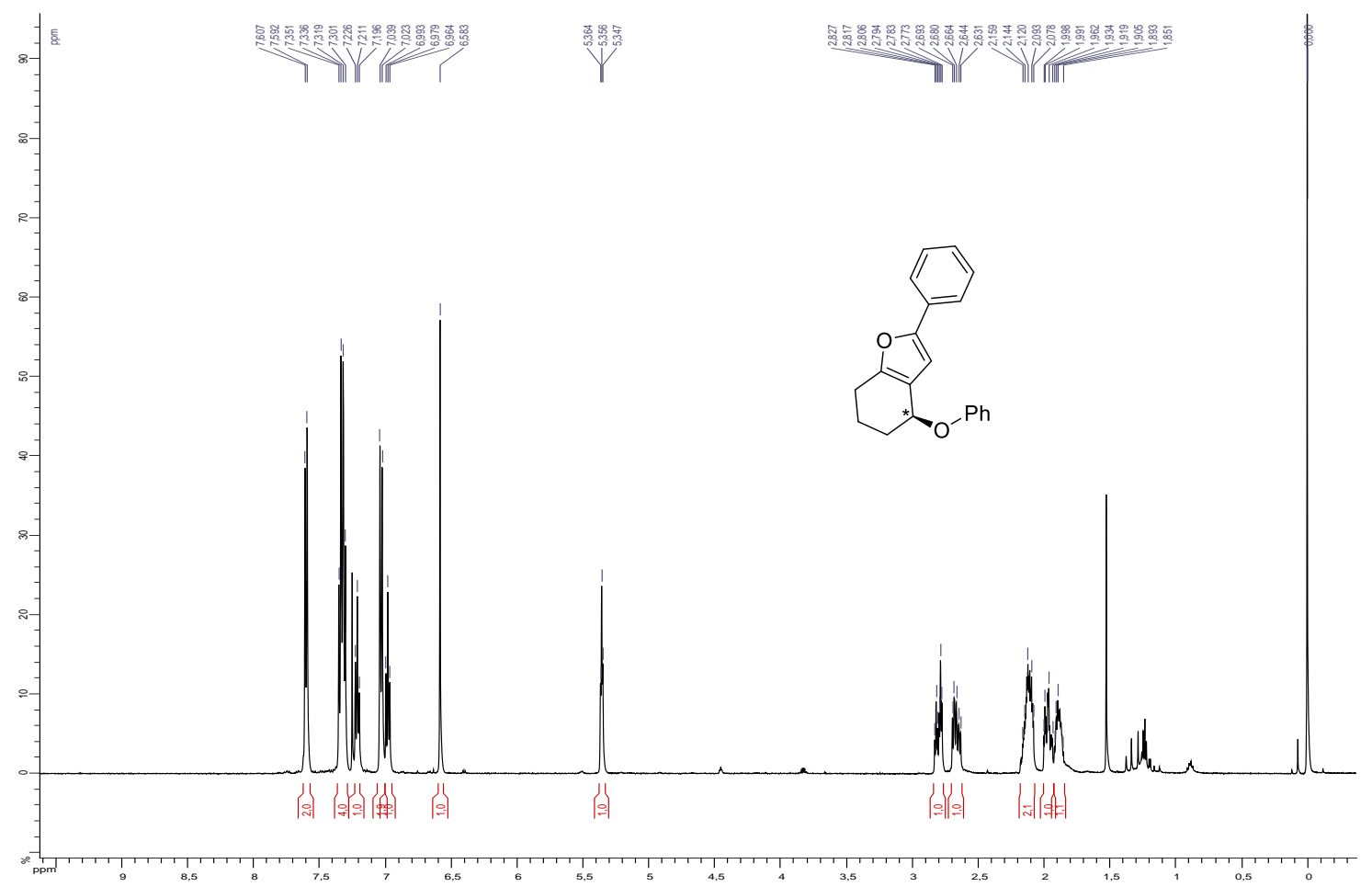

${ }^{1} \mathrm{H}$ NMR $\left(\mathrm{CDCl}_{3}, \mathbf{5 0 0} \mathrm{MHz}\right)$ spectrum of (S)-4-phenoxy-2-phenyl-4,5,6,7-tetrahydrobenzofuran (16d) 


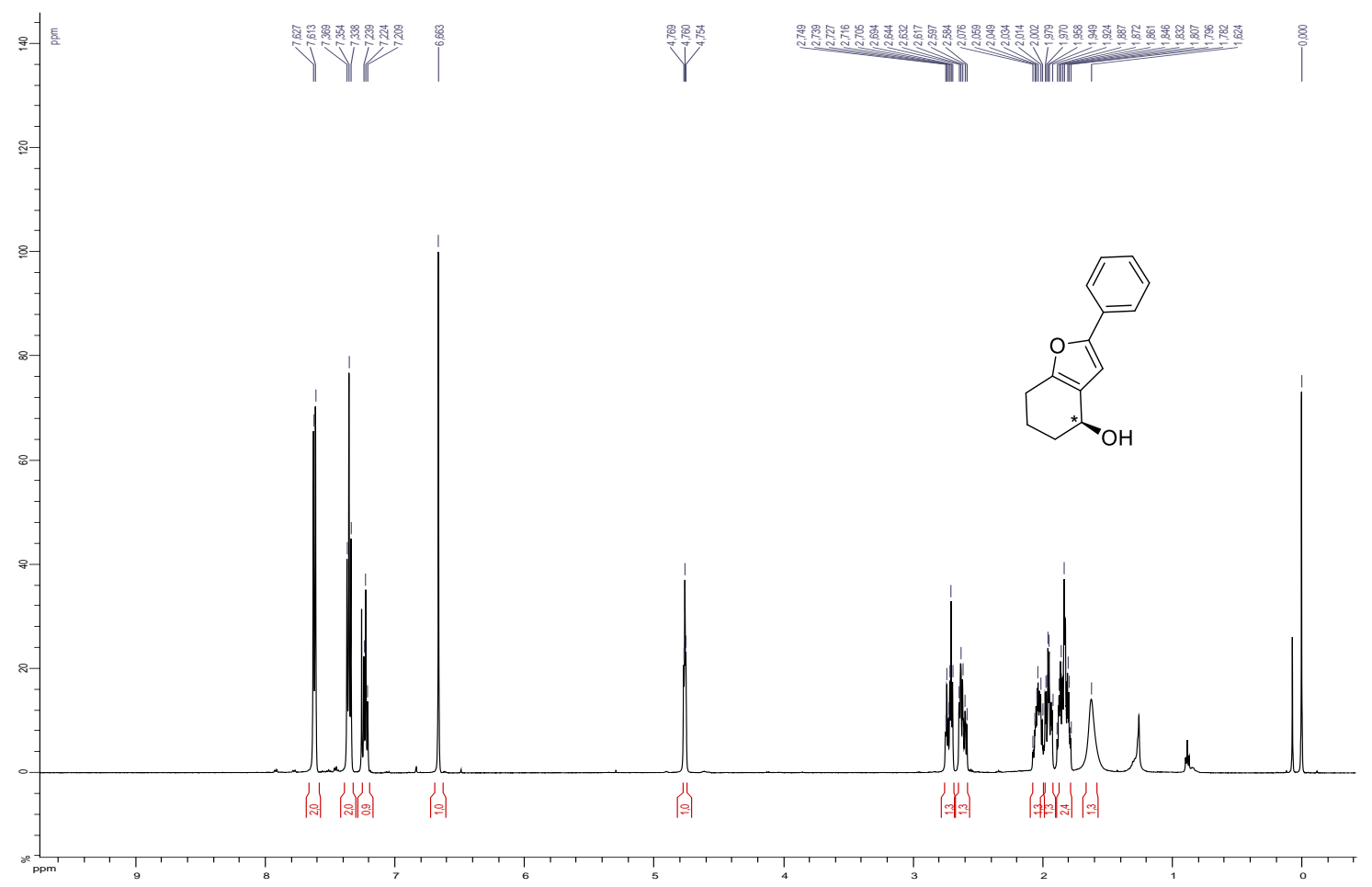

${ }^{1} \mathrm{H}$ NMR $\left(\mathrm{CDCl}_{3}, 500 \mathrm{MHz}\right.$ ) spectrum of (S)-2-phenyl-4,5,6,7-tetrahydrobenzofuran-4-ol (16e) 


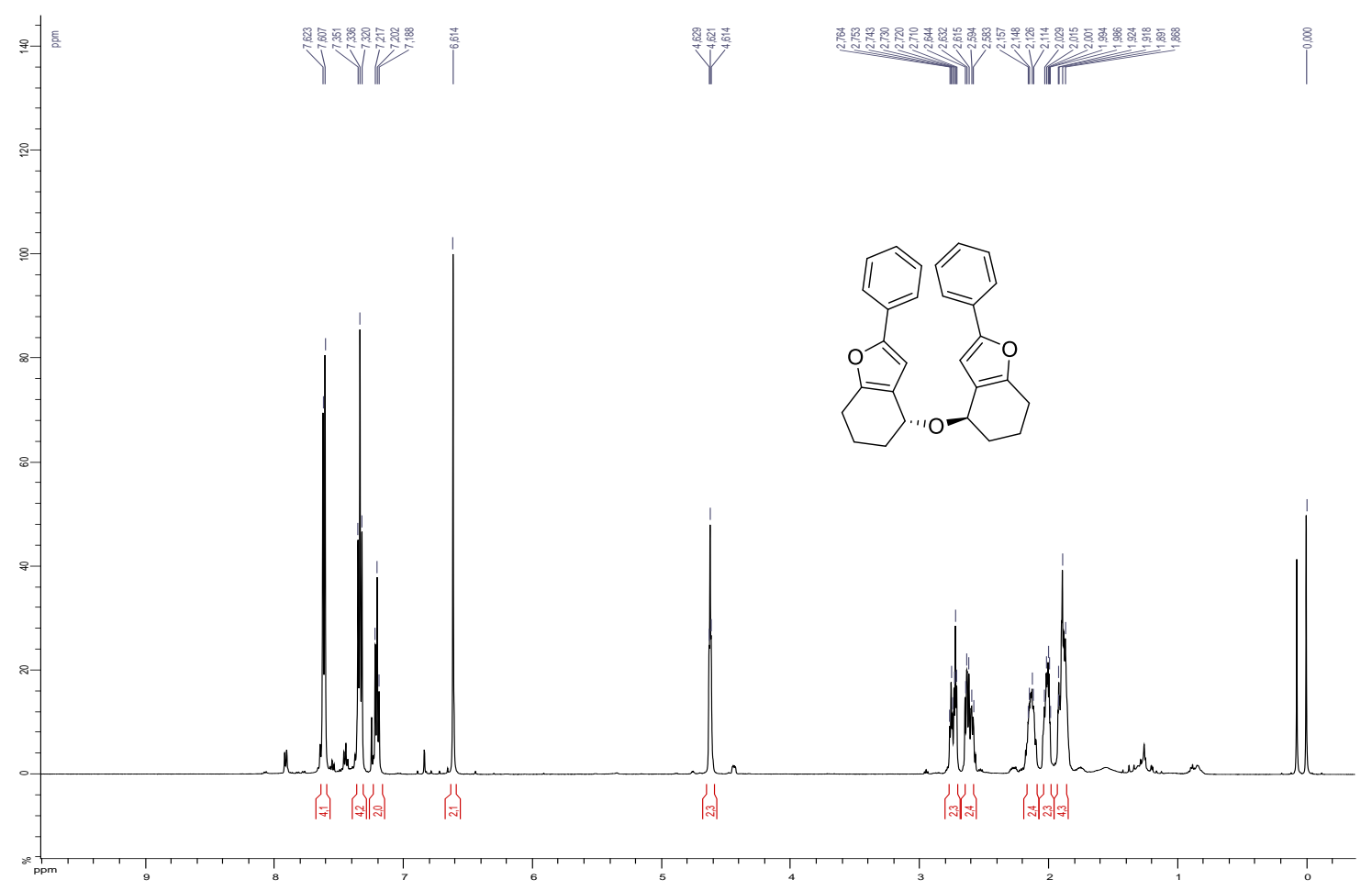

${ }^{1} \mathrm{H} \mathrm{NMR}\left(\mathrm{CDCl}_{3}, 500 \mathrm{MHz}\right)$ spectrum of (S)-2-phenyl-4-(((R)-2-phenyl-4,5,6,7-tetrahydrobenzofuran-4-yl)oxy)-4,5,6,7tetrahydrobenzofuran (16eb)

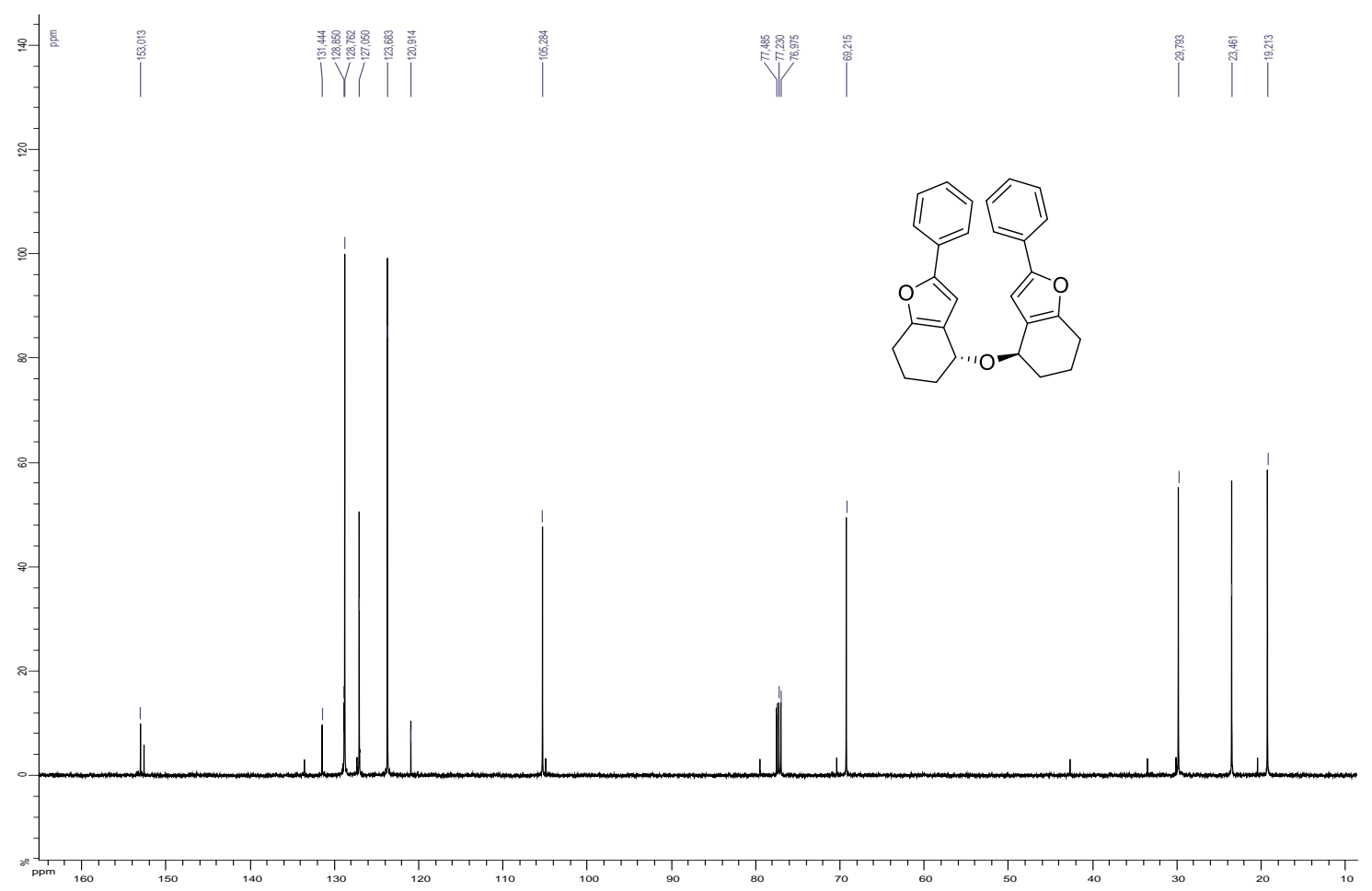

${ }^{13} \mathrm{C} \mathrm{NMR}\left(\mathrm{CDCl}_{3}, 125 \mathrm{MHz}\right)$ spectrum of (S)-2-phenyl-4-(((R)-2-phenyl-4,5,6,7-tetrahydrobenzofuran-4-yl)oxy)-4,5,6,7tetrahydrobenzofuran (16eb) 


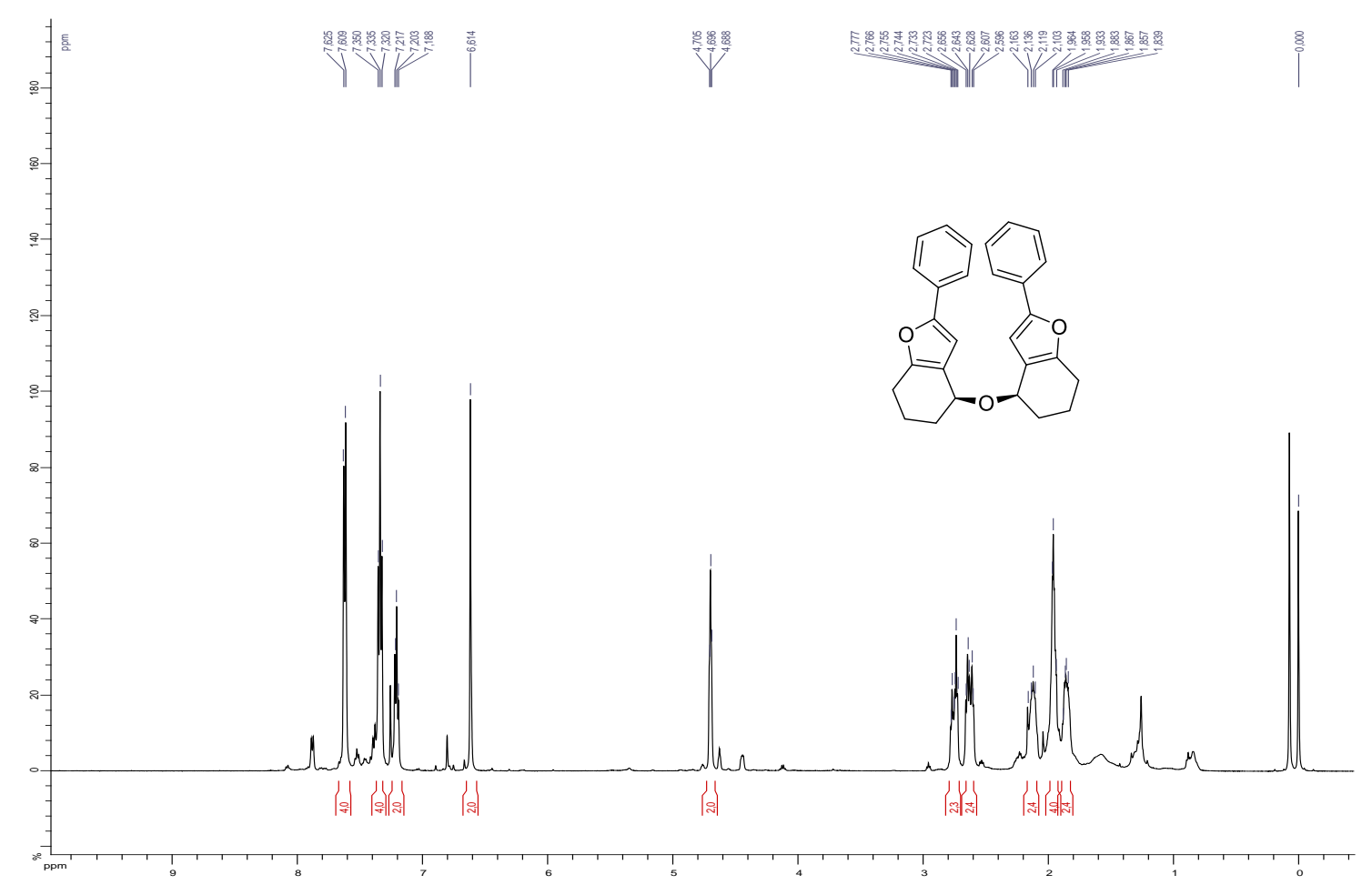

${ }^{1} \mathrm{H}$ NMR $\left(\mathrm{CDCl}_{3}, 500 \mathrm{MHz}\right)$ spectrum of $\left(4 R, 4^{\prime} R\right)-4,4^{\prime}$-oxybis(2-phenyl-4,5,6,7-tetrahydrobenzofuran) (16ec)

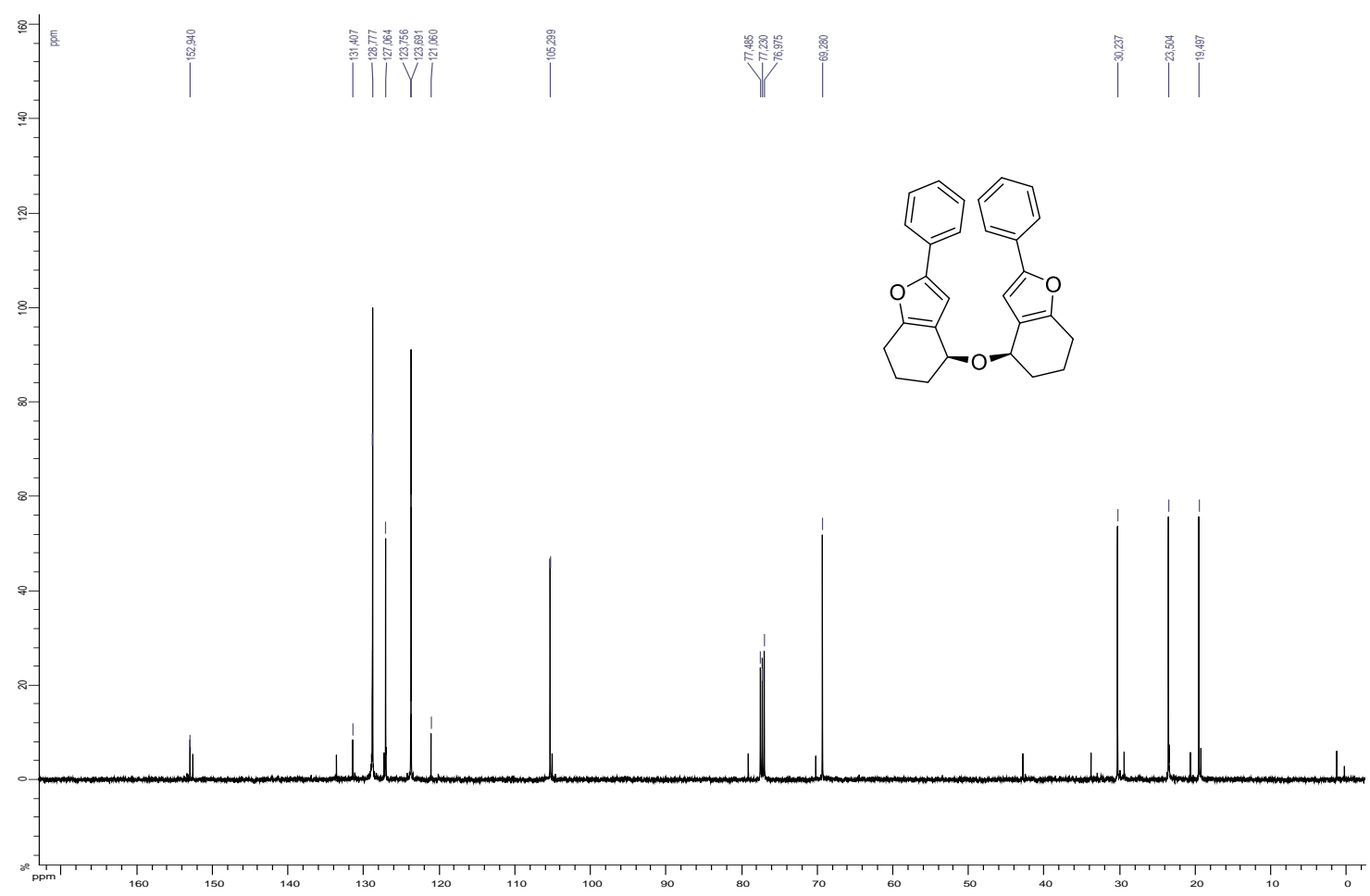

${ }^{13} \mathrm{C} \mathrm{NMR}\left(\mathrm{CDCl}_{3}, 125 \mathrm{MHz}\right)$ spectrum of $\left(4 R, 4^{\prime} R\right)-4,4^{\prime}$-oxybis(2-phenyl-4,5,6,7-tetrahydrobenzofuran) (16ec) 


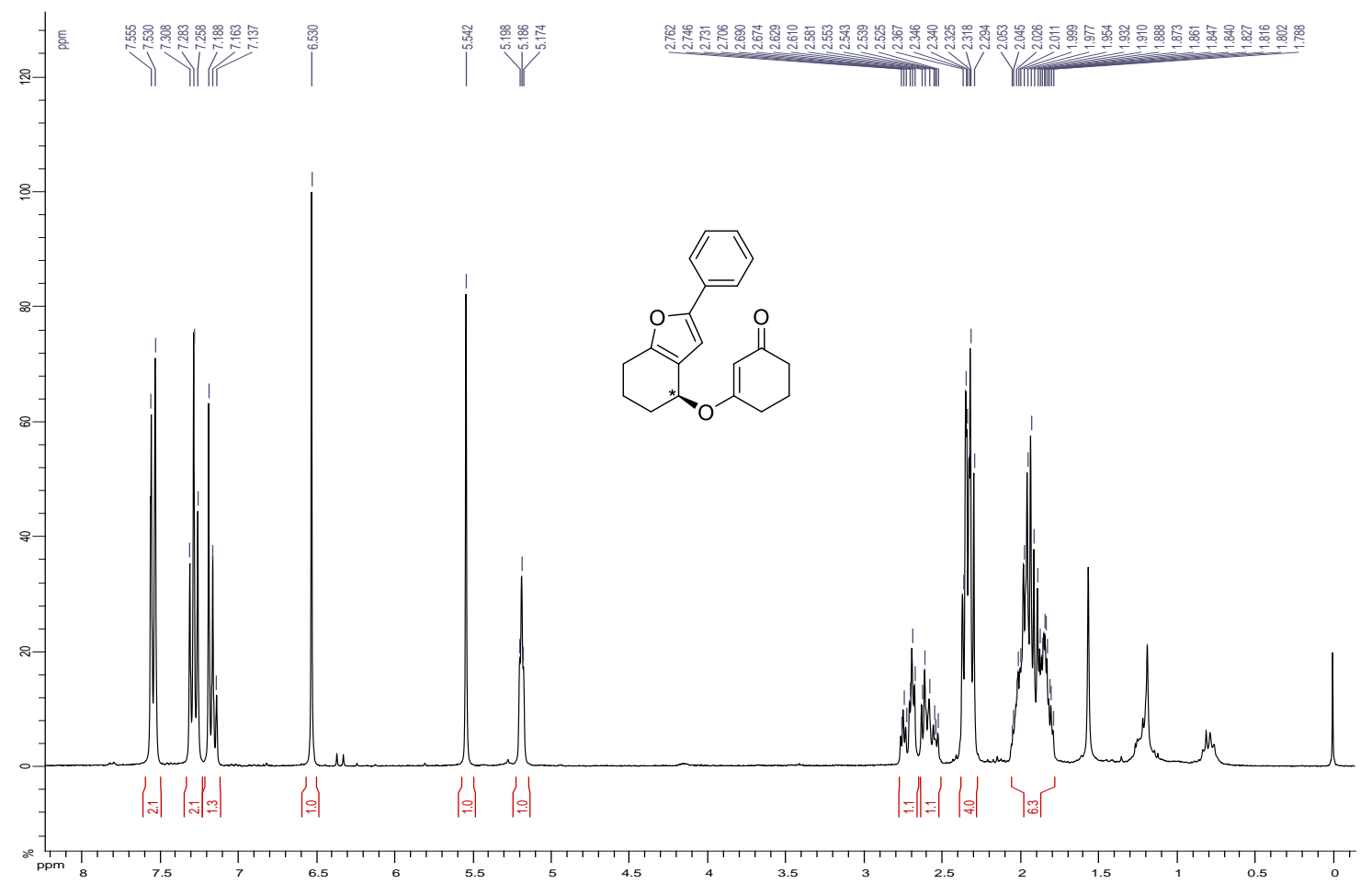

${ }^{1} \mathrm{H}$ NMR ( $\mathrm{CDCl}_{3}, 300 \mathrm{MHz}$ ) spectrum of (R)-2-(2-phenyl-4,5,6,7-tetrahydrobenzofuran-4-yl)cyclohexane-1,3-dione (16f) 